2. To: (Receiving Organization)

Distribution

5. Proj./Prog./Dept./Div.: $D 289$,

W-320 TWRS/TCPN \# GZNAH

8. Originator Renerks:

For approval and release of a new supporting document. This document has been generated to ensure retrievability of the Project W-320 "Civil/Structural Calculations, Vol. 7".

11. Receiver Remarks: 11A. Design Baseline Document? [X] Yes [] No

6. Design Authority/ Design Agent/Cog. Engr.:

JW Bailey, NHC

\section{From: (Originating organization) TWRS Projects/SST Retrieval \\ 4. Related EDT No.:}

7. Purchase Order No.:

$\mathrm{n} / \mathrm{a}$

9. Equip./Component No.:

$n / a$

10. System/Bldg./Facility:

241-C-106

12. Major Assm. Dwg. No.:

$$
n / a
$$

13. Permit/Permit Application No.: $n / a$

14. Required Response Date:

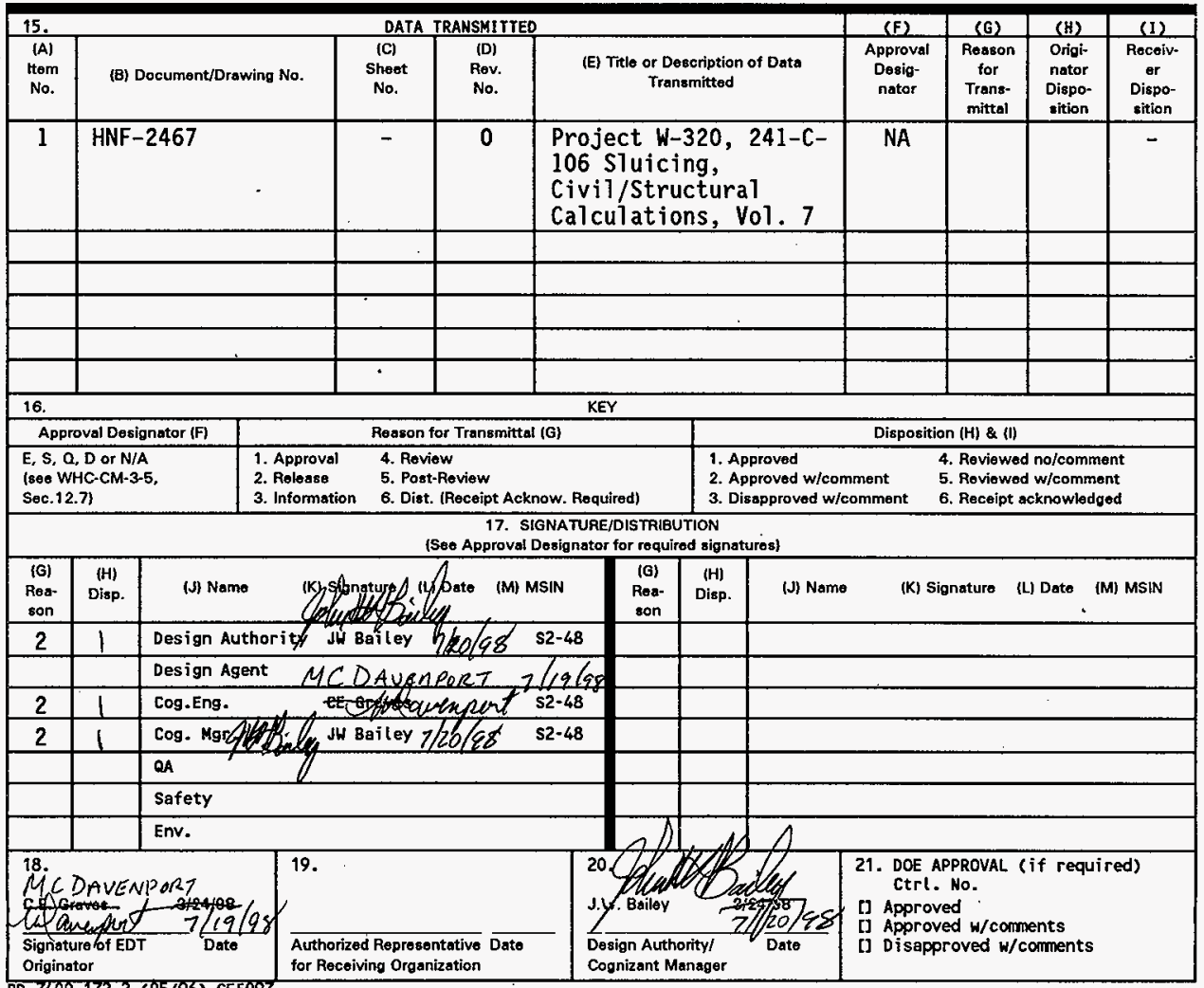




\title{
Project W-320, 241-C-106 Sluicing Civil/Structural Calculations, Vol. 7
}

John W. Bailey

Numatec Hanford Co., Richland, WA 99352

U.S. Department of Energy Contract DE-AC09-96RL13200

\author{
EDT/ECN: 622225 UC: 506 \\ Org Code: 8C452 Charge Code: D2991/HANA0600 \\ B\&R Code: EW3130010 Tota1 Pages: 265
}

Key Words: W-320, Sluicing, Tank 241-C-106, Tank 241-AY-102, WRSS, calculations, civil/structural.

Abstract: This supporting document has been prepared to make the FDNW calculations for Project $W-320$, readily retrievable.

IRADEMARK DISCLAIMER. Reference herein to any specific commercial product, process, or service by trade name, trademark, manufacturer, or otherwise, does not necessarily constitute or imply its endorsement, recommendation, or favoring by the United states Government or any agency thereof or its contractors or subcontractors.

Printed in the United States of Anerica. To obtain copies of this document, contact: Document Control Services, P.O. Box 950, Mailstop H6-08, Richland WA 99352 , Phone (509) 372-2420;

Fax (509) 376-4989.
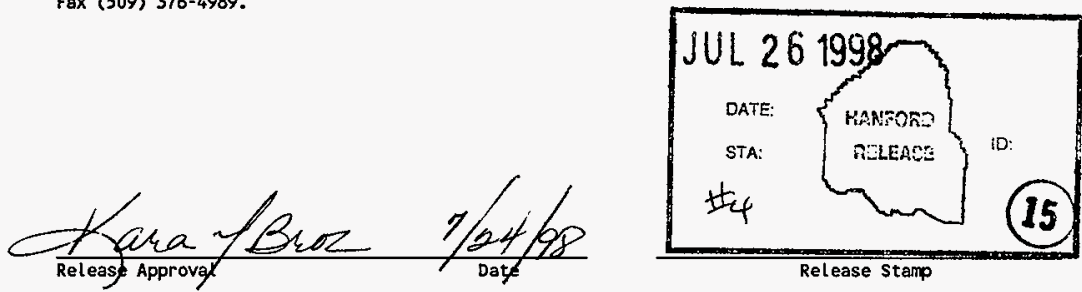

Approved for Public Release 
HNF-2467, Rev. 0

\title{
Project W-320, 241-C-106 Sluicing Civil/Structural Calculations, Vol. 7
}

\author{
TABLE OF CONTENTS
}

\begin{tabular}{|c|c|c|}
\hline Calc. No. & Title & Page \\
\hline W320-24-019 & $\begin{array}{l}\text { Prefiltration/Recirculation Skid, } \\
\text { Structural Design (Process Control Buildir }\end{array}$ & $\begin{array}{c}A-i \\
n g)\end{array}$ \\
\hline$W 320-24-020$ & $\begin{array}{l}\text { Intake Air Filter Pad and Cooling } \\
\text { Coil Support Frame Analysis }\end{array}$ & $B-i$ \\
\hline W320-24-021 & $\begin{array}{l}\text { Misc. Mechanical (HVAC) Equipment } \\
\text { Foundation - Exhaust Air Cleanup Train Sk }\end{array}$ & $i d-i$ \\
\hline$W 320-24-022$ & Service Building Foundation & $D-\mathbf{i}$ \\
\hline$W 320-24-023$ & $\begin{array}{l}\text { Misc. Mechanical (HVAC) Equipment } \\
\text { Foundation - Chiller and Pumps }\end{array}$ & $E-\mathbf{i}$ \\
\hline W320-24-024 & Electrical Equipment Pad (241-C-51) & $\mathbf{F}-\mathbf{i}$ \\
\hline
\end{tabular}




\section{W320-24-019}

Prefiltration/Recirculation Skid, Structural Design (Process Control Building) 
D. L. Evans

M. S. Ruben

SUBJECT INDEPENDENT SEISMIC REVIEW - PROJECT W-320, TANK 241-C-106, PREFILTRATION/RECIRCULATION SKID

Input design documēnts:

(1) FDC WHC-SD-W320-FDC-001, Rev 2, For Tank 241-C-1-106, Waste Retrieval, Project W-320.

(2) LoI 9360642, Rev 2 - Project W-320, Tank 241-C-106, S7uicing Letter of Instruction, Removal of In-Tank Equipment - Definitive Design, Procurement, Fabrication, Training, and Testing.

(3) LOI KGS-94-013, 3/1 Analysis Requirements for Project W-320 Equipment Removal System.

(4) SDC-4.1 Rev 12, Design Loads for Facilities

(5) WHC-SD-WM-SEL-033 Rev 1 - Safety Classifications, Project W-320

Output design documents:

(1) Calc. No. W320-24-019 - Prefiltration/Recirculation Skid

(2) Drawings $\mathrm{H}-2-18455$, Sht $1-5$

A review of the noted output design documents for compliance with the input design documents was completed on February 24, 1995 relative to the seismic design requirements. The output design documents were correct and no changes have to be made as a result of the review.

GLH: IbW 


\section{CALCULATION IDENTIFICATION AND INDEX}

Page $i$ of $i \mathfrak{i}$

Date

$05 / 16 / 94$

This sheet shows the status and description of the attached Design Analysis sheets. Discipline Civil wo/Job No. W-320/ER4319

Calculation No. W320-24-019

Project No. \& Name PROJECT W-320, TANK 241-C-106

Calculation Item Prefiltration/Recirculation Skid, Structural Design (Process Control Bldg)

These calculations apply to:

Dwg. No. H-2-818455 Sht. $1,2,3,4, \& 5$

Rev. No. $0,0,0,0,0$

Dwg. No.

Rev. No.

other (Study, CDR)

Rev. No.

The status of these calculations is:

[] Prel iminary Calculations

[X] Finat Calculations

[ ] Check Calculations (On Calculation Dated)

[] void Calculation (Reason Voided )

Incorporated in Final Drawings?

[X] Yes [ ] No

This calculation verified by independent "check" calculations? [ ] Yes [X] No

Original and Revised Calculation Approvals:

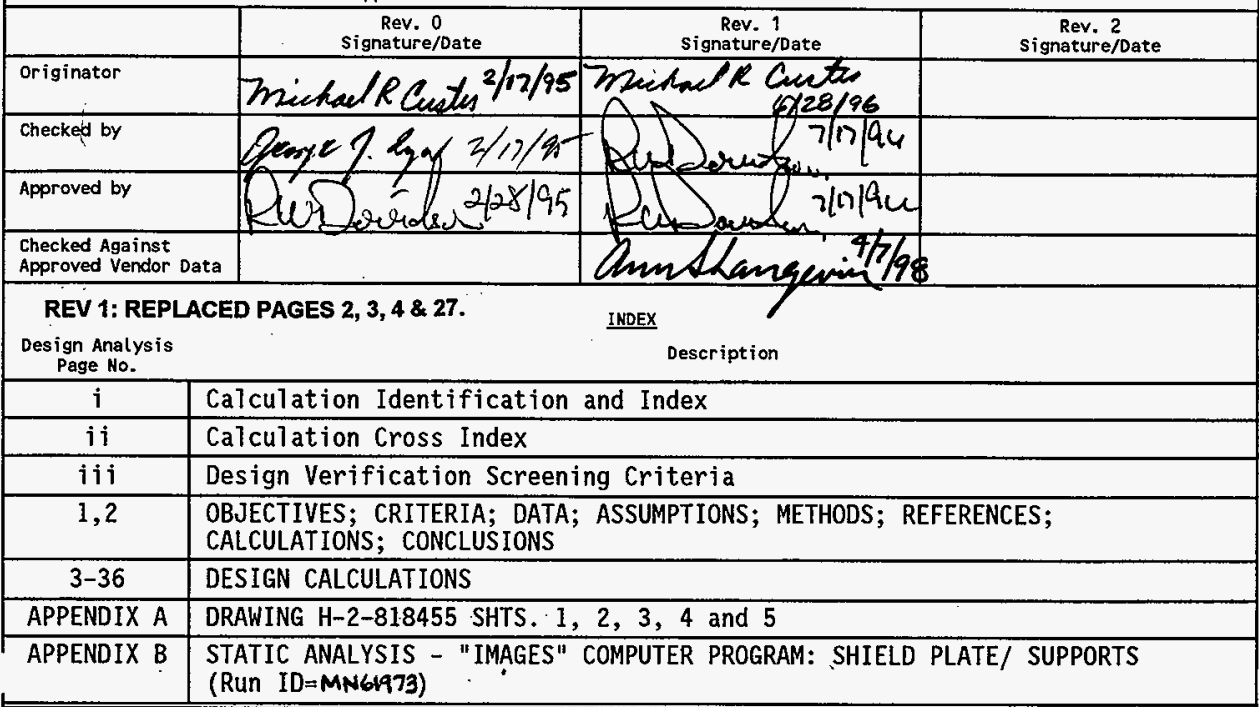


CALCULATION CROSS INDEX,$\overline{\text { iypical) }}$

Subject Calculation No. W320-24-0,9, REV.O

\begin{tabular}{|c|c|c|c|c|c|c|c|c|c|c|}
\hline \multirow{2}{*}{$\begin{array}{l}\text { Subject } \\
\text { Calculation } \\
\text { Revision } \\
\text { No. }\end{array}$} & \multirow{2}{*}{$\begin{array}{l}\text { Superceded } \\
\text { by } \\
\text { Calculation } \\
\text { No. }\end{array}$} & \multicolumn{2}{|c|}{$\begin{array}{l}\text { These interfacing calculation/documents } \\
\text { provide input to the subject calculation, } \\
\text { and if revised may require revision of } \\
\text { the subject calculation. }\end{array}$} & \multicolumn{2}{|c|}{$\begin{array}{l}\text { Results and conclusions of the subject } \\
\text { calculation are used in these interfacing } \\
\text { calculations and/or documents. }\end{array}$} & \multicolumn{2}{|c|}{$\begin{array}{c}\text { Does the output } \\
\text { interface calculation' } \\
\text { documents require } \\
\text { revision? }\end{array}$} & \multicolumn{2}{|c|}{$\begin{array}{c}\text { Has the output } \\
\text { interface calculation } \\
\text { documents been } \\
\text { revised? }\end{array}$} & \multirow{2}{*}{$\begin{array}{l}\text { Discipline manager's signature and } \\
\text { date indicating evaluation } \\
\text { complete. }\end{array}$} \\
\hline & & Calculation/Document No. & $\begin{array}{c}\text { Revision } \\
\text { No. }\end{array}$ & Calculation/Document No. & $\begin{array}{c}\text { Revision } \\
\text { No. }\end{array}$ & Yes & No & Yes & No & \\
\hline \multirow[t]{5}{*}{0} & $N / A$ & $N / A$ & & $\mathrm{H}-2-818455^{\mathrm{SHT} . \mathrm{i}}$ & 0 & $\checkmark$ & & & L & Lerzores 2128195 \\
\hline & & & & $H-2-818455$ & 0 & & $\checkmark$ & & $\checkmark$ & 然 \\
\hline & & & & $\begin{array}{r}14-2-818455 \\
\text { SHT. } 3\end{array}$ & 0 & & $\longleftarrow$ & & $\checkmark$ & \\
\hline & & & & $\begin{array}{r}1-2-818455 \\
\text { SHT, } 4\end{array}$ & 0 & & $\checkmark$ & & $\checkmark$ & \\
\hline & & & & $\begin{array}{r}4-2-818455 \\
\text { SHT.5 }\end{array}$ & 0 & & 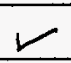 & & $r$ & $\downarrow$ \\
\hline & & & & & & & & & & \\
\hline & & & & & & & & & & \\
\hline & & & & & & & & & & \\
\hline & & & & & & & & & & \\
\hline & & & & . & & & & & & \\
\hline & & & & & & & & & & \\
\hline & & & & & & & & & & \\
\hline- & & & & & & & & & & \\
\hline & & & & & & & & & & \\
\hline & & & & & & & & & & \\
\hline & & & & & & & & & & \\
\hline & & & & & & & & & & \\
\hline & & & & & & & & & & \\
\hline & & & & & & & & & & \\
\hline
\end{tabular}




\section{DESIGN VERIFICATION SCREENING CRITERIA}

$$
\text { Project/Document No. W-320/CALCULATION W320-24-019, }
$$

When the design or design change affects hardware, formal design verification must be performed if one or more of the following questions must be answered affirmatively (YES).

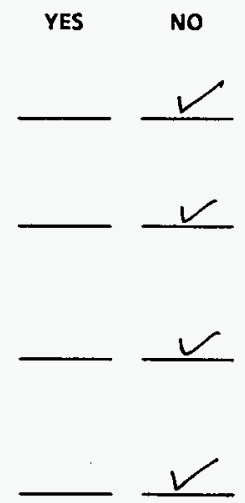

1. Does the design or design change involved meet the established criteria to be considered Safety Class 1 or 2?

2. Does this design or design change cause or permit changes to Safety Class 1 or 2 instrument or alarm setpoints outside of previously approved operational limits?

3. Does this design or design change significantly affect the nuclear safety consequences of a malfunction or failure of the structure, system, or component?

4. Does this design or design change involve or change design that has previously undergone formal design verification?
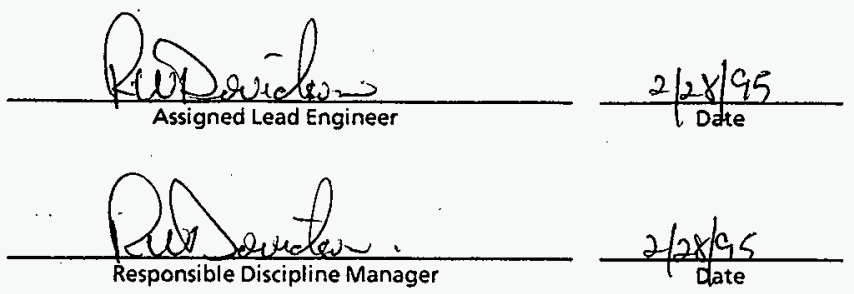

Original Design Package Distribution:

Project Engineer

Chief Design Engineer

Engineering Document Control
Design Change Distribution:

Attach to Engineering Change Notice 


\section{KAISER ENEINEERS}

HANFCRO

\section{DESIGN ANALYSIS}

client WHC

subject Prefiltration/Recirculation Skid

Structural Design (Process Control

Bldg.) calc. Ho. W320-24-019

Revision 0

Page No. 1 of 36

Wo/Job No. W-320/ER-4319

Date $05 / 16 / 94$ By M.R Custer MRC

Checked 02/17/95 8y G.J Zyn

By

OBJECTIVE:

The objective of this calculation is to provide the detailed, structural design of the Prefiltration/ Recirculation skid, including a radiation shield and details for anchorage to a reinforced concrete foundation.

\section{CRITERIA:}

1. DOE ORDER 6430.1A (04/06/89)

2. HANFORD PLANT STANDARDS, STANDARD ARCH./CIVIL DESIGN CRITERIA, SDC-4.1, REV.11

3. FUNCTIONAL DESIGN CRITERIA, WHC-SD-W320-FDC-001, REV.2

4. LETTERS OF INSTRUCTION:

DATA :

1. Equipment supported on the skid is Safety Class 3 per Reference 6 , therefore the skid is Safety Class 3 .

ASSUMPTIONS :

1. None

METHODS:

Hand calculations and "IMAGES 3D" (version 2.0), computer program REFERENCES:

1. AISC Manual of Steel Construction, Nineth Edition

2. Uniform Building Code (UBC), 1991 Edition

3. American Welding Society (AWS), Standard D1.1, Structural Welding Code

4. ASTM A 36-91

5. HANFORD PLANT STANDARDS, STANDARD ARCH./ CIVIL DESIGN CRITERIA, SDC-4.1, REV. 11.

6. WHC-SD-WM-SEL-033, REV.0-C, Interim Safety Equipment List for 241-C-106 WASTE RETRIEVAL

7. DESIGN OF WELDED STRUCTURES, BLODGETT (Eighth Edition)

8. ASCE 7-93, MINIMUM DESIGN LOADS FOR BUILDINGS AND OTHER STRUCTURES 


\section{DESIGN ANALYSIS}

Client WHC

Subject Prefiltration/Recirculation Skid,

Structural Design (Process Control Bldg.)

Location TANK 241-C-106

CALCULATIONS:

\section{REFER TO DESIGN CALCULATIONS SECTION}

\section{CONCLUSIONS:}

The structural skid supporting the Process Building and equipment is designed based on the criteria, codes and standards, referenced in the calculation. The final members and the associated elements satisfy the design requirements of the structure. Refer to drawing $\mathrm{H}-2-818455$ shts. $1,2,3,4$ and 5 for design drawing information.

\section{REVISION 1:}

\section{CONCLUSION:}

Revision 1 incorporates vendor data for the weight of the individual equipment/components. The updated information does not affect the original conclusion of the calculation, since the overall effect is a reduction in the total weight of the equipment and a nominal relocation of the center of gravity for the skid assembly.

Revision 1 also provides an assessment of the current calculation to determine potential impact due to changes, as documented in the following ECNs. No changes to the calculation is required based on the following:

1) ECN W-320-161: Provides location and anchorage details for equipment numbers HME-1361 and HMF-1361 for attachment to the Process Skid. The reactions from these equipment were considered in the original design and the bolting details, as provided exceeds the required capacity indicated on the vendor drawings.

2) ECN W-320-174: The ECN resolves conflicting detail/section numbering and provides minor dimensional changes. These changes do not impact the original design in the calculation. Detail 20 and Section T were added by this ECN to provide support for electrical/ instrumentation equipment. The support detail for the electrical equipment is based on the detail for the lifting lugs on the skid assembly. Since the capacity of the lifting lugs greatly exceeds the loads applied by the electrical equipment, the original calculation adequately addresses the design of the equipment supports.

3) ECN W-320-351: The ECN removes the existing 1" diameter anchor bolts and replaces them with a $3 / 16^{\prime \prime}$ fillet weld, 2 "long at each existing embed plate location. The combined shear and tension which exists at the equipment anchorage is distributed to the embedded plates. The maximum resultant force due to combined shear and tension on the weld is:

$R=\left[(2.5)^{2}+(2.85)^{2}\right]^{1 / 2}=3.79$ kips

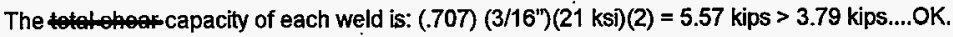




\section{DESIGN ANALYSIS}

Client WHC

Subject Prefiltration/Recirculation Skid,

Structural Design (Process Building)

Page No. 3 of 36

WO/Jos No. W-320/ER4319

Date 06/28/96

By M.R Custer MRC

Checked $7|i n| q u$

By

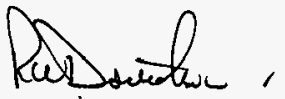

Location TANK 241-C-106

Revised

By

\section{DESIGN CALCULATIONS:}

The design and analysis of the skid, consists of developing conservative weight estimates for the major equipment, design of the skid support system to integrate the equipment as a module, design of a 1" plate, shielding wall, evaluate the equipment anchorage to the skid and design of the skid anchorage system to a reinforced concrete foundation. The equipment module will be constructed outside the $\mathrm{C}$-Farm area, transported and placed on a concrete foundation at the project site. A prefabricated metal building enclosure will be assembled outside the C-farm area and placed on the foundation, following the skid installation. All the equipment supported on the skid is Safety Class 3 per Reference 6, therefore the design of the skid is Safety Class 3.

\section{DESIGN LOADS:}

\section{DEAD LOADS}

- Mechanical equipment, piping/supports and instrumentation cabinet, tubing and supports:

- Recirculation Fan,

- Heat Exchanger,

- Heating Coil,

- Air Receiver Tank,

- (1) Metal Filter, $1430 \mathrm{lbs}+3360 \mathrm{lb}$

- Piping and pipe supports,

- (1) Instrumentation cabinets/ tubing/ supports

- HEME, Mist Eliminator,

- Moisture Separator,
$642 \mathrm{lbs}$

$2740 \mathrm{lbs}$ (Wt dry = $1976 \mathrm{lbs})$

180 lbs

$150 \mathrm{lbs}$

$4790 \mathrm{lbs}$

$1300 \mathrm{lbs}$

1657 lbs (Installed after transport)

21920 (Wt dry $=21540 \mathrm{lbs}$ )

$264 \mathrm{lbs}$

Total Equipment Weight(dry) $=\mathbf{3 0 , 8 4 2}$ lbs or $30.8 \mathrm{Kips}$

Total Equipment Weight(wet) $=33,643 \mathrm{lbs}$ or 33.6 Kips

- Room shielding plate ( 1 " thick):

Wt. of plate $=11 \mathrm{ft} . \times 21.28 \mathrm{ft} . \times 40.8 \mathrm{lbs} / \mathrm{sq} . \mathrm{ft} .=9551 \mathrm{lbs}$ or $9.60 \mathrm{Kips}$

- Estimated weight of structural steel on skid:

Wt.skid $=(21.0 \mathrm{ft} \times 2 \times 40 \mathrm{lb} / \mathrm{ft})+(11.0 \mathrm{ft} \times 6 \times 40 \mathrm{lb} / \mathrm{ft})+(23.6 \times 40 \mathrm{lb} / \mathrm{ft})$

$+(4.83 \mathrm{ft} \times 40 \mathrm{lb} / \mathrm{ft})+(2.41 \mathrm{ft} \times 20 \mathrm{lb} / \mathrm{ft})+2(4.25 \mathrm{ft} . \times 20 \mathrm{lb} / \mathrm{ft})+2(3.5 \mathrm{ft} \times 40 \mathrm{lb} / \mathrm{ft})$

$+2(1.67 \times 40 \mathrm{lb} / \mathrm{ft})=$

Wt.skid = $6089 \mathrm{lbs}$, say $\underline{6.1}$ Kips 


\section{DESIGN ANALYSIS}

Client WHC

Subject Prefiltration/Recirculation Skid,

Structural Design (Process Building)
Revision 1

Page No. 4 of 36
WO/Job No. W-320/ER4319

Date 06/28/96

By M.R Custer

$M R C$

Checked

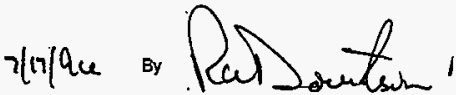

Location TANK 241-C-106

Revised

By

- Estimated weight TS shield frame:

$$
\begin{aligned}
\text { Wt Frame } & =\left(11.0 \mathrm{ft}-4^{\prime \prime} / 12\right)[5(12.21)+(19.02)]+[1.67 * 2+3.42 \\
& +7.75+7.58+\left(11^{2}+4.67^{2}\right)^{1 / 2}+\left(11^{2}+3.42^{2}\right)^{1 / 2}+\left(3.21^{2}\right. \\
& \left.\left.+7.75^{2}\right)^{1 / 2}+\left(7.75^{2}+7.79^{2}\right)^{1 / 2}\right](12.21)=1646 \mathrm{lbs}=1.7 \mathrm{Kips}
\end{aligned}
$$

- Estimated weight of $1 / 2$ " skid cover plate:

Wt. $1 / 2^{\prime \prime}$ cover plate $=21.0 \mathrm{ft} \times 11.7 \mathrm{ft} \times 20.4 \mathrm{lbs} / \mathrm{ft}^{2}=\mathbf{5 0 1 2} \mathrm{lbs}$ or $\underline{\mathbf{5 . 0} \mathrm{Kips}}$

TOTAL DEAD LOADS (dry) $=\mathbf{3 0 . 8}+9.6+6.1+1.7+5.0=53.2$ Kips. (Transport Weight)

NOTE: Actual as-built transport weight $=55 \mathrm{kip}$, (See ECN W-320-351)

TOTAL DEAD LOADS (max) $=33.6+9.6+6.1+1.7+5.0=56.0$ Kips (Design Weight)

\section{LIVE LOADS:}

- Live Load, $\mathrm{LL}=0$

- Equipment Live Load, LL, = Equip. Live Load x 1.5

$\mathrm{LL}_{\mathrm{o}}=213 \mathrm{lb} \times 1.5 / 13.5=23.7 \mathrm{lbs}$ force on bolt (where Torque is $213 \mathrm{lbs}-\mathrm{in}$ )

\section{Equipment live load is negligible}

\section{EARTHQUAKE LOADS:}

- Lateral Seismic Force (Equipment Skid), $F_{p}=Z Z_{1} C_{p} W_{p}$, where:

$$
\begin{aligned}
& Z=0.20(\text { Zone } 2 B) \\
& I=1.25 \\
& C_{p}=.75 \times 2=1.5 \\
& F_{p}=(0.20)(1.25)(1.5) W_{p}=0.375 W_{p} \\
& E_{n-s}=V_{n-s}(100 \% \text { max. }) \\
& E_{o-w}=V_{o-w}(30 \% \text { max. })
\end{aligned}
$$

(Reference 2)

(Reference 5)

(Reference 2)

(Reference 2)

(Reference 2)

$E_{n-s}=V_{n-s}=0.375 W_{p}$ skid $=0.375(61.3)=23.0$ Kips

$E_{a-w}=V_{n-s}=0.375 W_{p}$ skid $(0.3)=0.375(61.3)(0.3)=\underline{6.9 ~ K i p s}$

$E_{(S R S S)}=\left[23.0^{2}+6.9^{2}\right]^{1 / 2}=24.0 \mathrm{Kips}$ (Apply force in direction providing maximum load condition)

$O R E=0.375(1.3) \mathrm{Wp}=0.488(61.3)=29.9 \mathrm{Kips}$ (conservative)

$\underline{U}_{\text {se }} E_{\text {max }}=30.0$ Kips (conservative) 


\section{KAISER ENGINEERS}

HANFDRD

\section{DESIGN ANALYSIS}

client WHC

subject Prefiltration/Recirculation Skid,

Structural Design (Process Building)
Calc. Ho. W320-24-019

Revision 0

Page No. 5 of 36

wo/Job No. W-320/ER4319

Date $05 / 17 / 94$ By M.R Custer MRC

checked $02 / 17 / 95$ By G.J Zyn

Revised

By

- Distribution of Seismic forces by Component:

Total weight of skid and components

Total lateral seismic load

$=61.3 \mathrm{Kips}$

$=\underline{30.0 \mathrm{Kips}}$
LOAD IDENTIFIER

a) Recirculation Fan,

b) Heat Exchanger, Wet

Dry

d) Air Receiver Tank,

e) HEME (Mist Eliminator), Wet

g) Moisture Separator

h) Piping and supports,

i) Instrumentation cabinet,

j) I "Shield Plate,

k) Weight of Skid,

1) Weight of $1 / 2^{\prime \prime}$ cover Pl

m) Weight of TS Frame c) Heating Coil,

f) Metal Filter,

TOTAL WEIGHT =

TOTAL WEIGHT =
VERTICAL COMP. / RATIO

(350) (30000)/61300

(5000) $(30000) / 61300$

(1200)

(150) $(30000) / 61300$

(150) $(30000) / 61300$

$(30000)(30000) / 61300$

(27000)

(1135) $(30000) / 61300$

(400) $(30000) / 61300$

(1000) $(30000) / 61300$

(700) $(30000) / 61300$

(9551) $(30000) / 61300$

(6089) $(30000) / 61300$

$(5000)(30000) / 61300$

(1646) $(30000) / 61300$

61171 lbs/ 61.3 Kips

54371 LBS/ 54.4 Kips
171 Tbs

2447 lbs

586

73 Tbs

73 ibs

14682 lbs

13176

553 lbs

195 1bs

341 1bs

4674 1bs

2965 Tbs

2435 Tbs

802 Jbs

$29898 \mathrm{Tbs} / 30.0 \mathrm{Kips}$
HORIZ. COMPONENT

487 1bs

WIND LOAD: (Maximum affects in the North-South direction) (Reference 8)

$F$ (due to wind Toading) $=q_{z} G_{z} C_{f} A_{f}$

where: $\quad q_{z}=0.00256 K_{z}(I V)^{2}=0.00256(0.80)(1.02 \times 70)^{2}=10.4$ psf

$$
\begin{aligned}
& G_{z}=1.32 \\
& C_{f}=1.2
\end{aligned}
$$

$F=10.4(1.32)(1.2)(11 \mathrm{ft})^{2}=1.99 \mathrm{Kips}<30.0 \mathrm{Kips}$ (seismic load governs)

Note: Equipment exposed to wind loading only during the rigging operation. The equipment is to be housed in a building enclosure therefore, there is no wind loading during normal operations. 


\section{KAISER ENGINEERS \\ HANFERG}

DESIGN ANALYSIS

Client WHC

subject Prefiltration/Recirculation Skid, Structural Design (Process Building)
Calc. No. W320-24-019

Revision 0

Page No. 6 of 36

Wo/Job No. W-320/ER4319

Date $05 / 17 / 94 \quad$ By M.R Custer

Checked 02/17/95 8y G.J Zyn

Revised

By

\section{LOCATE THE CENTER OF GRAVITY OF THE SKID ASSEMBLY:}

COMPONENT WEIGHTS AND LOCATIONS:
Component Description
Weight
X-distance,
Z-Distance,
(ft.)
(ft.)
H-Distance
(1bs)
(ft.)

0.92

9.25

a) Recirculation Fan

350

(FN-1361)

b) Heat Exchanger

(HX-1361)

1200 Dry

5000 Wet

4.17

6.70

2.71

c) Heating Coil

150

4.08

0.75

2.76

(HC-1361)

d) Air Receiver Tank

150

7.75

9.50

2.25

(TK-1361)

e) HEME (Mist Eliminator)

(HME-1361)

27000 Dry

30000 Wet

10.75

5.25

6.45

f) Metal Filter

(HMF-1361)

g) Moisture Separator 400

1135

14.09

4.75

3.29

7.25

4.47

4.42

h) Piping/supports

1000

10.20

3.18

3.50

i) Instrumentation/

700

18.34

3.79

3.50

cabinet/supports

j) I" Shield Plate

9874

13.39

6.22

6.04

k) Skid Frame

6089

10.41

5.42

0.00

1) $1 / 2^{\prime \prime}$ Cover Plate

5000

10.17

5.50

0.52

m) TS support frame

1646

6.17

7.16

Reference points for the $x, z$, and $h$ distances are as follows:

1) $x$-distance, dimension from centerline of W12x40 at the west end of the skid to the center of the component.

2) z-distance, dimension from centerline of W12x40 at the south end of the skid to the center of the component.

3) h-distance, dimension from centerline of W12×40 skid to centerline of component. 


\section{KAISER ENGINEERS}

HANFORD

\section{DESIGN ANALYSIS}

Client WHC

subject Prefiltration/Recirculation Skid, Structural Design (Process Building)
Calc. No. W320-24-019

Revision 0

Page No. 7 of 36

Ho/Job No. W-320/ER4319

Date $05 / 17 / 94 \quad$ By M.R Custer

Checked $02 / 17 / 95$ By G.J Zyn

Revised

By

Location TANK 24I-C-106

CENTER OF GRAVITY OF THE SKID FRAME: (Reference Figure 1)

- Component weights:

- (2) Primary framing beams, (W12 X 40):
$(21.00 \mathrm{ft})(40)=840 \mathrm{lbs}$ each
Tota] $=16801 \mathrm{bs}$

- (6) Secondary framing beams (W12 x 40):

$(11.0 \mathrm{ft})(40)=440$ 1bs each

$\underline{T o t a 1}=26401 \mathrm{bs}$

- (4) Diagonal beams (W12 x 40):

Tota] $=944$ Tbs
a) 251 1bs
b) $2341 \mathrm{bs}$
c) $2391 \mathrm{bs}$
d) $2201 \mathrm{bs}$

- (1) Moisture Separator beam, (W12 x 40):
$4.83 \mathrm{ft} \times 401 \mathrm{~b} / \mathrm{ft}$
Total $=1931 \mathrm{bs}$

- (1) Moisture Separator support beam, (W12 x 16):

$2.41 \mathrm{ft} \times 20 \mathrm{1b} / \mathrm{ft}$

TotaT $=48$ lbs

- (2) Metal Filter support beams, (W12 x 16):

$4.25 \mathrm{ft} \times 201 \mathrm{~b} / \mathrm{ft}=851 \mathrm{bs} / \mathrm{each}$

Total $=170 \mathrm{lbs}$

- (2) HEME support beams (W12 × 40),

$3.5 \mathrm{ft} \times 40=.140 \mathrm{lbs} / \mathrm{each}$

Total $=280$ lbs

- (2) Shield wall support beams (W12 x 40)

$1.1 \mathrm{ft} \times 40 \mathrm{lb} / \mathrm{ft}=44 \mathrm{lbs} / \mathrm{each}$

TotaT $=88$ Tbs 
KAISER ENEINEERS

HANFDRD

DESIGN ANALYSIS

client WHC

subject Prefiltration/Recirculation Skid, Structural Design (Process Building)
Calc. No. W320-24-019

Revision 0

Page No. 8 of 36

WO/Job No. W-320/ER4319

Date 05/17/94 By M.R Custer

MRC

Checked 02/17/95 By G.J Zyn

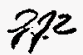

Location TANK 241-C-106

Revised

By

- Center Gravity, East-West direction

$E P x=[4401 b s(4.17 \mathrm{ft})+4401 \mathrm{bs}(9.00 \mathrm{ft})+4401 \mathrm{bs}(12.50 \mathrm{ft})$

+440 lbs $(16.75 \mathrm{ft})+440$ lbs $(20.33 \mathrm{ft})+251$ lbs $(6.58 \mathrm{ft})$

+234 lbs $(14.63 \mathrm{ft})+239$ lbs $(6.58 \mathrm{ft})+2201 \mathrm{bs}(14.63 \mathrm{ft})$

$+1401 \mathrm{bs}(10.75 \mathrm{ft})(2)+8401 \mathrm{bs}(10.17 \mathrm{ft})(2)+85$ lbs $(14.63 \mathrm{ft})(2)$

$+1931 \mathrm{bs}(6.58 \mathrm{ft})+481 \mathrm{bs}(7.25 \mathrm{ft})+441 \mathrm{bs}(16.75 \mathrm{ft}+9.00 \mathrm{ft}] / 6089$ l.bs

$\underline{x}=10.32 \mathrm{ft}$, approx. $13 / 4^{\prime \prime}$ east of the centerline of the skid

- Center of Gravity, North-South direction

$E P Z=[4401 b s(5.50 \mathrm{ft})(6)+251] \mathrm{bs}(9.00 \mathrm{ft})+2341 \mathrm{bs}(9.00 \mathrm{ft})$

+239 lbs $(1.75 \mathrm{ft})+220$ lbs $(1.75 \mathrm{ft})+140$ lbs $(3.50 \mathrm{ft})$

$+1401 \mathrm{bs}(7.00 \mathrm{ft})+8401 \mathrm{bs}(11.00 \mathrm{ft})+851 \mathrm{bs}(5.50 \mathrm{ft})+851 \mathrm{bs}(4.08 \mathrm{ft})$

+193 1bs $(4.5 \mathrm{ft})+48$ 1bs $(3.36 \mathrm{ft})+441 \mathrm{bs}(11.92 \mathrm{ft}-0.58 \mathrm{ft})] / 60891 \mathrm{bs}=$

$z=5.38 \mathrm{ft}$, approx. $11 / 2$ " south of the centerline of the skid

CENTER OF GRAVITY OF THE SHIELD PLATE: (Reference Figures $3,4 \& 5$ )

- Component Weights:

- Shield Plate 1, Wt $=40.8 \mathrm{lbs} / \mathrm{ft}^{2} \quad(11 \mathrm{ft})(8.79 \mathrm{ft})=3946 \mathrm{lbs}$

- Shield Plate 2, Wt $=40.81 \mathrm{bs} / \mathrm{ft}^{2}$ (11 ft) $(7.83 \mathrm{ft})=3514 \mathrm{lbs}$

- Shield Plate 3 , $W t=40.8 \mathrm{lbs} / \mathrm{ft}^{2} \quad(11 \mathrm{ft})(4.66 \mathrm{ft})=2091 \mathrm{lbs}$

- Center of Gravity, East-West

TOTAL $=9551$ 1bs

$x=3946$ lbs $(16.54 \mathrm{ft})+35141 \mathrm{bs}(12.67 \mathrm{ft})+20911 \mathrm{bs}(8.79 \mathrm{ft}) / 9551$ Tbs $=$

$\underline{x}=13.42 \mathrm{ft}$

- Center of Gravity, North-South

$z=3946$ lbs $(3.50 \mathrm{ft})+35141 \mathrm{bs}(7.59 \mathrm{ft})+20911 \mathrm{bs}(9.94 \mathrm{ft}) / 95511 \mathrm{bs}=$ $\underline{z}=6.42 \mathrm{ft}$

- Center of Gravity, Height

$h=11.0 \mathrm{ft} / 2+\left(12^{\prime \prime} / 2+0.50^{\prime \prime}\right) / 12$

$\underline{h}=6.04 \mathrm{ft}$ 


\section{KAISER ENGINEERS}

HANFORD

DESIGN ANALYSIS

Client WHC

subject Prefiltration/Recirculation Skid, Structural Design (Process Building)
Calc. No. W320-24-019

Revision 0

Page No. 9 of 36

HO/Job No. W-320/ER4319

Date $05 / 17 / 94$ By M.R Custer

NQC

Checked $02 / 17 / 95$ By G.J Zyn

Revised

By

CENTER OF GRAVITY OF THE SKID ASSSEMBLY: (Dry) (Ref. Figures $1,3,4$ \& 5)

- Center of Gravity, East-West direction:

$E P x=$ Sum vertical loads about the centerline of the $W 12 \times 40$ at west end of the skid.

$E P X=[0.92 \mathrm{ft}(350 \mathrm{lbs})+4.17 \mathrm{ft}(1200 \mathrm{Tbs})+4.08 \mathrm{ft}$ (150 lbs)

$+7.75 \mathrm{ft}(150 \mathrm{lbs})+10.75 \mathrm{ft}(27000 \mathrm{lbs})+14.09 \mathrm{ft}$ (1135 lbs)

$+7.25 \mathrm{ft}(400)+10.20 \mathrm{ft}(1000 \mathrm{lbs})+18.34 \mathrm{ft}(700 \mathrm{lbs})$

$+13.39 \mathrm{ft}(9551 \mathrm{lbs})+10.41 \mathrm{ft}(6089 \mathrm{lbs})+10.17 \mathrm{ft}$ (5000 1bs)

$+12.38 \mathrm{ft}(1646 \mathrm{lbs})] / 54371 \mathrm{lbs}=$

Use $x=11.08 \mathrm{ft}$. , east of the reference 1 ine $(11.0$ inches east of skid centerline)

- Center of Gravity, North - South direction:

$E P z=$ Sum vertical loads about the centerline of W12x40 at south end of skid.

$E P z=[9.25 \mathrm{ft}(350 \mathrm{lbs})+6.70 \mathrm{ft}(12007 \mathrm{bs})+0.75 \mathrm{ft}(1501 \mathrm{bs})$

$+9.50 \mathrm{ft}(150 \mathrm{lbs})+5.25 \mathrm{ft}(27000 \mathrm{lbs})+4.75 \mathrm{ft}$ (1135 1bs)

$+4.47 \mathrm{ft}(400)+3.18 \mathrm{ft}(1000 \mathrm{Tbs})+3.79 \mathrm{ft}(700 \mathrm{lbs})+6.22 \mathrm{ft}$ (955I 1bs)

$+5.42 \mathrm{ft}(6089 \mathrm{lbs})+5.50 \mathrm{ft}(5000 \mathrm{lbs})+6.17 \mathrm{ft}(1646 \mathrm{lbs})] / 543711 \mathrm{bs}=$

Use $z=5.47 \mathrm{ft}$. . north of the reference line (approx. $\theta$ the skid center 1 ine)

CENTER OF GRAVITY OF THE SKID ASSEMBLY: Design Location (Wet) (Ref. Figures $1,3,4 \& 5$ )

- Center of Gravity, East- West direction:

$$
\begin{aligned}
x= & 11.08 \mathrm{ft}+[300001 \mathrm{bs}(10.75 \mathrm{ft})+50001 \mathrm{bs}(4.17 \mathrm{ft})] / 61171 \text { lbs } \\
& -[27000 \text { lbs }(10.75 \mathrm{ft})+1200(4.17 \mathrm{ft}))] / 54371 \mathrm{lbs}= \\
x= & 11.08 \mathrm{ft}+5.61 \mathrm{ft}-5.4 \dot{\mathrm{ft}}
\end{aligned}
$$

Use $x=11.26 \mathrm{ft}$, or approx. $131 / 8^{\circ}$ east of the skid centerline 


\section{KAISER ENGINEERS}

HANFDRD

DESIGN ANALYSIS

Client WHC

subject Prefiltration/Recirculation Skid, Structural Design (Process Building)

Calc. No. W320-24-019

Revision 0

Page No. 10 of 36

Wo/Job No. W-320/ER4319

Date $05 / 17 / 94$ By M.R Custer

Checked 02/17/95 By G.J Zyn

Revised

By

- Center of Gravity, North-South direction:

$z=5.48 \mathrm{ft}+[30000$ Tbs $(5.25 \mathrm{ft})+5000$ 1bs $(6.70 \mathrm{ft})] / 61171$ 1bs

- $[27000$ lbs $(5.25 \mathrm{ft})+12001 \mathrm{bs}(6.70 \mathrm{ft})] / 54371$ 1bs

$z=5.48 \mathrm{ft}+3.12 \mathrm{ft}-2.75 \mathrm{ft}$

Use $z=5.85 \mathrm{ft}$, or approx. $41 / 8^{\prime \prime}$ north of the skid centerline

- Eccentricity, East - West direction: (Seismic Loads)

EPX = Sum horizontal loads about centerline of W12x40 at west end of skid.

$E P x=[0.92 \mathrm{ft}(171 \mathrm{lbs})+4.17 \mathrm{ft}(2435 \mathrm{lbs})+4.08 \mathrm{ft}$ (73 lbs)

$+7.75 \mathrm{ft}(73 \mathrm{lbs})+10.75 \mathrm{ft}(14610 \mathrm{lbs})+14.09 \mathrm{ft}$ (553 1bs)

$+7.25 \mathrm{ft}(195 \mathrm{lbs})+10.20 \mathrm{ft}(487 \mathrm{lbs})+18.34 \mathrm{ft}$ (342 1bs)

$+13.39 \mathrm{ft}(4809 \mathrm{lbs})+10.41 \mathrm{ft}(2965 \mathrm{lbs})+10.17 \mathrm{ft}(2435 \mathrm{lbs})$

$+12.38 \mathrm{ft}(802 \mathrm{lbs})] / 29898=$

Use $x=10.64 \mathrm{ft} ; \quad E(x)=0.47 \mathrm{ft}$ or approx. $55 / 8$ " east of the skid centerline

- Eccentricity, North - South direction: (Seismic Loads)

$E P z=$ Sum horizontal loads about centerline of the W12x40 at south end of skid.

$E P z=[9.25 \mathrm{ft}(171 \mathrm{lbs})+6.70 \mathrm{ft}(2435 \mathrm{lbs})+0.75 \mathrm{ft}$ (73 1bs)

$+9.50 \mathrm{ft}(73 \mathrm{lbs})+5.25 \mathrm{ft}(14610 \mathrm{lbs})+4.75 \mathrm{ft}$ (553 1bs)

$+4.47 \mathrm{ft}(195 \mathrm{lbs})+3.18 \mathrm{ft}(487 \mathrm{lbs})+3.79 \mathrm{ft}$ (342 1bs)

$+6.22 \mathrm{ft}(4660 \mathrm{lbs})+5.42 \mathrm{ft}(2965 \mathrm{lbs})+5.50 \mathrm{ft}(2435 \mathrm{Tbs})$

$+6.17 \mathrm{ft}(802 \mathrm{lbs})] / 29898 \mathrm{lbs}=$

Use $z=5.54 \mathrm{ft}, \mathrm{e}(z)=0.04 \mathrm{ft}$, or, 0.50 " north of the skid centerline 


\section{KAISER ENEINEERS}

HANFDRD

DESIGN ANALYSIS

client WHC

subject Prefiltration/Recirculation Skid, Structural Design (Process Building)
Calc. No. W320-24-019

Revision 0

Page No. 11 of 36

Wo/Job Ho. W-320/ER4319

Date $05 / 17 / 94 \quad$ By M.R Custer MRC

checked $02 / 17 / 95$ By G.J Zyn

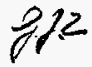

Location TANK 24I-C-106

Revised

By

- Eccentricity, Vertical Direction: (Reference Figures 4,5 \& 6)

$E P h=$ Sum horizontal loads about the centerline (elevation) of the $W 12 \times 40$

$E P h=[2.76 \mathrm{ft}(171$ lbs $)+2.71 \mathrm{ft}(2435$ lbs $)+2.75 \mathrm{ft}$ (73 1bs)

$+2.25 \mathrm{ft}(73 \mathrm{lbs})+6.45(14610)+3.29 \mathrm{ft}(553 \mathrm{lbs})$

$+4.42 \mathrm{ft}(195 \mathrm{lbs})+3.50 \mathrm{ft}(487 \mathrm{lbs})+3.50 \mathrm{ft}(342 \mathrm{Jbs})$

$+6.04 \mathrm{ft}(4660 \mathrm{lbs})+0.00 \mathrm{ft}(2965 \mathrm{lbs})+0.52 \mathrm{ft}(2435 \mathrm{lbs})$

$+7.16 \mathrm{ft}(802 \mathrm{lbs})] / 29898 \mathrm{lbs}=$

Use $h=4.79 \mathrm{ft}$ or approximateiy $571 / 2^{\prime \prime}$, above the center line of the W12 beam

DETERMINE GOVERNING LOAD COMBINATION AND LOADS FOR STRUCTURAL SKID:

Load Combinations:

1) $\mathrm{D}$

2) $\mathrm{D}+\mathrm{L}+(\mathrm{Lr}$ or $\mathrm{S}$ or $\mathrm{R})$; $\mathrm{Lr}, \mathrm{S}$ and $\mathrm{R}=0$, then $\mathrm{D}+\mathrm{L}$

3) $D+(W$ or $E), W=0$, then $D+E$

4) $D+L+(L r$ or $S$ or $R)+(W$ or $E)$; $L r, S, R$ and $W=0$, then $D+L+E$

Load combination governing the design is: $D+L+E$ 


\section{KAISER ENGINEERS \\ HANFORD}

DESIGN ANALYSIS

Client WHC

subject Prefiltration/Recirculation Skid, Structural Design (Process Building)
Calc. No. W320-24-019

Revision 0

Page No. 12 of 36

Wo/sob No. W-320/ER4319

Date $05 / 17 / 94$ By M.R Custer MRC

checked $02 / 17 / 95$ вy G.J Zyn 792

Location TANK 241-C-106

Revised

By

\section{SKID DESIGN:}

\section{- DESIGN OF PRIMARY/ SECONDARY FRAMING BEAMS:}

The structural frame consists of two wide-flange beams with secondary members provided at the major equipment support locations. These members in addition to diagonal braces and the skid cover plate, provides the required support system. The design of the equipment skid considers two basic design conditions. The first condition involves the design of the skid considering the dead load of the equipment during the loading, transport and unloading of the equipment at the site. The second condition involves the design of the skid at the final design location, where it will be subjected to the live and seismic loads associated with the functioning system.

The beams shall be designed and the beam deflections checked, using both the design load conditions at design location and the rigging/transport loads during the transport operations to consider al] load scenarios. The primary differences in the load conditions are (1) the additional seismic load at the design location and (2) the supports and stability concerns for the skid during the rigging operation.

\section{- Beam design - Rigging operation: (Reference Figures 1,2 \& 4)}

The primary/ secondary beams are designed as simply supported with a span of $13.33 \mathrm{ft}$ or $11.0 \mathrm{ft}$ respectively, as shown in Figure 2. The beams supporting the HEME equipment will govern the design, since the maximum loads are supported by these members. The maximum moment for either the primary and secondary beams is determined as shown in Figure 2 is:

$M \max =910 \mathrm{k}-\mathrm{in}$

Sreq $=M \max / \mathrm{fb}$, assume $\mathrm{fb}=24 \mathrm{ksi}$, based on an unbraced length of $5.00 \mathrm{ft}$ Sreq $=910 \mathrm{k}-$ in $/ 24 \mathrm{ksi}=37.9 \mathrm{in}^{3}$

- Determine the section properties of the $1 / 2^{\prime \prime} \mathrm{pl}$ and the W12 $\times 40$ beams:

$\mathrm{Ix}=310 \mathrm{in}^{4}(\mathrm{~W} 12 \times 40), \quad \mathrm{A}=11.8 \mathrm{in}^{2}(\mathrm{W12} \times 40)$

$I_{T}$, includes $1 / 2^{\prime \prime} \mathrm{PL}$ and $W 12 \times 40$ beam:

$I X=I c+A d^{2}, I C=b d^{3} / 12$.

Ic $($ plate $)=24.0(0.5)^{3} / 12=0.25 \mathrm{in}^{4}$

Ic (beam) $=310 \mathrm{in}^{4}$ 


\section{KAISER ENGINEERS}

HANFDRD

\section{DESIGN ANALYSIS}

Client WHC

subject Prefiltration/Recirculation Skid, Structural Design (Process Building)
Calc. No. W320-24-019

Revision 0

Page No. 13 of 36
Wo/Job No. W-320/ER4319

Date $05 / 17 / 94$ By M.R Custer MRC

Checked $02 / 17 / 95$ By G.J Zyn

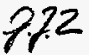

Location TANK 241-C-106

Revised

By

$$
\begin{aligned}
& \bar{x}=[0.50(24)(12.25)+11.8(6.0)] / 11.8+0.5(24)=9.15 \mathrm{in} . \\
& \mathrm{c}_{\mathrm{T}}=9.15^{*}, \mathrm{c}_{\mathrm{c}}=3.35 " \\
& I_{T}=\left[I C+A d^{2}\right]+\left[I C+A d^{2}\right] \\
& I_{T}=\left[0.25+0.5(24)(3.10)^{2}\right]_{1 / 211} \mathrm{Pl}+\left[310+11.8(3.15)^{2}\right]_{W 12 \text { beam }} \\
& I_{T}=543 \mathrm{in}^{4}, \mathrm{c}=59.3 \mathrm{in}^{3}(\mathrm{c}=9.15 \mathrm{in}) \\
& \mathrm{Sx}(\text { total })=543 \mathrm{in}^{4} / 9.15 \mathrm{in.}=59.3 \mathrm{in}^{3}>37.9 \ldots \text { ok }
\end{aligned}
$$

\section{DETERMINE INTERMITTANT WELDS ATTACHING $1 / 2$ " COVER PLATE TO WI2 BEAMS}

- Minimum weld size required per AISC is $3 / 16^{\prime \prime}\left(1 / 2^{\prime \prime}\right.$ cover plate and $1 / 2^{\prime \prime}$ beam flange)

- Minimum length of intermittent weld is $1.5^{\prime \prime}$

- Determine weld requirements for $W 12 \times 40$ and $W 12 \times 16$ :

$$
\begin{array}{ll}
f_{h}=V \text { a y } / I_{x} n & \begin{array}{l}
\text { (Reference 7) } \\
V^{\prime}=16.4 \text { kips }
\end{array} \\
f_{h}=16400 \mathrm{k}(0.5)(24)(3.10) / 543(2)=562 \mathrm{~Tb} / \mathrm{in} & \\
f_{h}^{h}=562 \mathrm{lbs} / \text { in }(8) / 1.5=2997 \mathrm{lbs} / \text { in } & \\
w \text { (required weld size) }=2997 / 14850=0.20^{\prime \prime}, 3 / 16^{\prime \prime}=0.19^{\prime \prime}
\end{array}
$$

Use minimum weld, 3/16", Adequate, based on the conservative design loads used. Use 3/16" fillet weld, 1.5" long $08^{\prime \prime}$ on center with $3^{\prime \prime}$ at each end and each side of the beam flange (minimum). Additional weld length will be provided at each equipment attachment point at the $1 / 2^{*}$ plate, as required.

\section{DESIGN CONNECTIONS FOR THE PRIMARY AND SECONDARY FRAMING MEMBERS}

The connections for the primary and secondary members consist of a coped and welded beam to beam shear and moment resisting connection. The web and beam flanges of the secondary beams will be cut to provide the required fitup for welding the web, using fillet welds each side of the web and full penetration welds connecting the flanges to the primary beams. 
HNF-2467, Rev.0

\section{KAISER ENGINEERS}

HANFORD

DESIGN ANALYSIS

client WHC

subject Prefiltration/Recirculation Skid, Structural Design (Process Building)
Calc. No. W320-24-019

Revision 0

Page No. 14 of 36

Wo/Job No. W-320/ER4319

Date $05 / 17 / 94 \quad$ By M.R Custer

MRL

Checked 02/17/95 By G.J Zyn

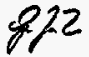

Location TANK 241-C-106

Revised

By

- Check moment capacity of the $W 12 \times 40$ section:

Applied moment, $(M)=910 \mathrm{k}-\mathrm{in}$,

$M=T \times d, d=12^{\prime \prime}, T=P_{\max }=f_{b} A, .66 F_{y}(.515)(8)=97.9 \mathrm{k} \ldots$ ok

$M($ section $)=97.9 \mathrm{k} \times 12^{\prime \prime}=1175 \mathrm{k}-\mathrm{in}>910 \mathrm{k}-\mathrm{in} \ldots . . \mathrm{ok}$

- Check web shear capacity of the W12 $\times 40$ :

$V_{\max }=16.4 \mathrm{k}$,

$A_{w}=d_{w} \times t_{w} ; d_{w}=9.5$ in, $t_{w}=0.295$ in

$F_{v}=.40 F_{y}=14.4 \mathrm{ksi}$, where $F_{Y}=36 \mathrm{ksi}$

$f_{v}=V_{\text {max }} / A_{w}$

$f_{v}=16.4 \mathrm{kips} / 9.5$ in $\times 0.295$ in $=5.85 \mathrm{ksi}<F_{v}=14.4 \mathrm{ksi} \ldots$. ok

Required weld (web to web): Minimum weld required is $3 / 16^{\prime \prime}$ per Reference 1:

- Check shear stress on $3 / 16^{\prime \prime}$ fillet weld each side of the web, 9.5" 1 ong

$$
\begin{aligned}
& F_{v}=0.707 \times 70 \mathrm{ksi} \times 0.30 \times 3 / 16^{\prime \prime}=2.8 \mathrm{k} / \text { in } \\
& f_{v}=V / A, 16.4 \mathrm{kips} /\left(9.5^{\prime \prime}\right)(2)=0.86 \mathrm{k} / \text { in } \\
& f_{v}=0.86 \mathrm{k} / \text { in }<2.8 \mathrm{k} / \text { in } \ldots \text { ok }
\end{aligned}
$$

\section{DESIGN SKID FRAME BRACING SYSTEM AND CONNECTIONS}

The braced, secondary members in addition to the skid cover plate provides the required lateral bracing of the compression flange of the primary framing beams. The skid cover plate is designed to support the smaller equipment and piping supports with direct attachment or with the addition of members as required to transfer the loads to the primary structural frame. The larger equipment is supported directly by secondary framing members, provided at the specific equipment locations. The primary function of the bracing members is to.distribute the horizontal seismic loads from the HEME (mist eliminator) supports to the primary beams and the foundation anchorage. The maximum brace load consists of the 30.0 kip horizontal seismic load, distributed approximately $50 \%$ to each brace, with one pair of braces assumed effective. The critical loading on the braces is the compression load. The load per brace used in the design is $15.0 \mathrm{k}$, with the brace fixed at each end. 
client WHC

subject Prefiltration/Recirculation Skid, Structural Design (Process Building)
Wo/Job No. W-320/ER4319
MRC

checked $02 / 17 / 95$ By G.J Zyn

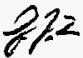

Location TANK 241-C-106

Revised

By

- Check the $112 \times 40$ brace member as a column:

Member W12 x 40, Assume $1 / 2$ seismic load is applied to each brace

$P=15.0 \mathrm{kips}, \mathrm{fa}=P / A=15.0 \mathrm{k} / 11.8 \mathrm{in}^{2}=1.27 \mathrm{ksi}$

Determine $K L / r$, where $K=1.0, L=6.3 \mathrm{ft}, r=1.93 \mathrm{in}$.

$\mathrm{KL} / \mathrm{r}=1.0(6.3)\left(12^{\prime \prime}\right) / 1.93$ in $=39.2<\mathrm{Cc}=126.1, \mathrm{~F}_{\mathrm{a}}=19.27$

$f_{a} / F_{a}=1.27 \mathrm{ksi} / 19.27 \mathrm{ksi}=0.07<0.15 \ldots .0 \mathrm{k}$

The connections of the bracing members to the primary framing members consist of welding the web and flanges of the $W 12 \times 40$ brace to the web and flanges of the W12 $\times 40$ primary and secondary beams. Since the loads supported by the braces are significantly less than the loads on either the primary or secondary beams, the connections are acceptable based on the design of primary/secondary beam connections.

\section{DESIGN THE RADIATION SHIELD/ HEME SUPPORT SYSTEM (Ref. Figures 4 \& 5)}

The radiation shield is analized in conjunction with the HEME support system as an integrated system. The objective in utilizing this approach is to maximize the inherent, structural capacity of the braced, shield plate structure in providing lateral support to the HEME and minimize the overall volume of supporting structures on the skid. This objective was accomplished using Version 2.0 of the "IMAGES" computer program, a finite element analys is program. The input/output data for the analysis is provided in Appendix A, which includes the member stresses, forces, deflections and reactions. The results of the analysis provides the required input for the design of the shield supports and associated connections and the HEME base support, bracing members and connections. Four load cases were analyized based on the $D+L+E$ load combination. Each load case consists of dead loads of the components, accelerated in two directions in addition to the dead load of the HEME and the horizontal seismic components, applied at the center of gravity of the HEME. The load cases are described by the direction of the seismic load, e.g North-South or East-West directions. Load Case 2 consists of the dead loads only. (Note: The live load in this analysis is negligible) 


\section{KAISER ENGINEERS \\ HANFORD}

DESIGN ANALYSIS

client WHC

subject Prefiltration/Recirculation Skid, Structural Design (Process Building)
Calc. Ho. W320-24-019

Revision 0

Page No. 16 of 36

Wo/Job No. W-320/ER4319

Date $05 / 17 / 94$ By M.R Custer

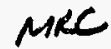

checked 02/17/95 вy G.J Zyn

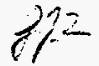

Location TANK 241-C-106

Revised

By

\section{ANALYSIS SUMMARY:}

The following is a summary of the results of the analysis performed, defined by physical location/ structural interface. The summary provides the maximum forces or stresses as shown in the program output. Maximum force components or stresses required at other locations in the structure are developed by extracting and summarizing the information from the program output data. The following are maximum values as provided by the program:

- CONNECTION OF $1^{\prime \prime}$ SHIELD PLATE TO $1^{\prime \prime}$ SHIELD PLATE:

PLATE NODE FX FY FZ MX MY
NO.

NO.

\begin{tabular}{|llllllllll}
$40-55$ & 60 & -146 & 28 & 122 & 101 & -2091 & 0 & 3 & $X$ \\
$39-54$ & 60 & 99 & 12 & -171 & 0 & 1786 & -63 & 3 & $Z$
\end{tabular}

- CONNECTION OF BEAM OR 1" SHIELD PLATE TO 1/2" COVER PLATE:

MEMBER/ NO. NODE $\underset{X}{\text { REACTIONS }} \mathrm{Y} \quad \begin{aligned} & \text { MOMENTS } \\ & M X\end{aligned}$

(GLOBAL)

$\begin{array}{lllllllllll}\text { BEAM } & 23 & 5 & -447 & 2916 & -509 & -429 & -147 & 404 & 1 & \text { TS } 4 \times 4\end{array}$

$\begin{array}{llllllllllll}\text { BEAM } & 29 & 11 & 293 & 752 & -91 & -289 & 4519 & -741 & 4 & \text { TS } 8 \times 4\end{array}$

$\begin{array}{llllllllllll}\text { BEAM } & 1 & 1 & -22 & 316 & -137 & - & - & & \end{array}$

$\begin{array}{lllllllllll}\text { BEAM } 2 & 18 & -20 & 217 & 34 & - & - & & \end{array}$

$\begin{array}{lllllllllll}\text { PLATE } 9 / 10 & 11 & 483 & 2303 & 470 & 415 & -90 & & -561 & 3 & 1 \text { (" PLATE }\end{array}$ 


\section{KAISER ENGINEERS}

HANFERG

DESIGN ANALYSIS

client WHC

subject Prefiltration/Recirculation Skid, Structural Design (Process Building) calc. Ho. W320-24-019

Revision 0

Page No. 17 of 36

Wo/Job No. W-320/ER4319

Date $05 / 17 / 94$ By M.R Custer

ARC

checked $02 / 17 / 95$ вy G.J Zyn

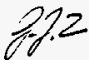

Location TANK 241-C-106

Revised

By

(LOCAL)

MEMBER/ NO. MAX. STRESS FORCES

AXIAL YSHEAR ZSHEAR COMBSHEAR BENDING LOAD CASE

BEAM $56 \quad 1381$

$440 \quad 458 \quad-202$

691

288

$6541,3,4,5$

PLATE $40 \quad 349$

- CONNECTION OF TS TO $1^{11}$ SHIELD PLATE:

\begin{tabular}{lllllll} 
BEAM & NODE & AXIAL SHEAR & TORSION & \multicolumn{2}{l}{ BENDING } & LOAD \\
NO. & LOAD $Y$ & $Z$ & & $y \quad Z$ & CASE
\end{tabular}

\begin{tabular}{|llllllllll}
$31 / 32$ & 60 & 5 & 12 & -16 & 1786 & -32 & 5 & 5 & TS $8 \times 4$ \\
$38 / 39$ & 82 & -21 & 20 & 1.5 & -3806 & 29 & 0 & 4 & TS $4 \times 4$ \\
5 & 98 & -807 & 69 & 949 & -1633 & 1000 & -1180 & 4 & TS $4 \times 4$
\end{tabular}

- CONNECTION OF TS TO TS:

BEAM NODE AXIAL SHEAR

NO. LOAD Y Z

TORSION BENDING

Y

Z

LOAD REMARKS

CASE

$\begin{array}{llllll}44 & 102 & 125 & 30 & -17 & -510\end{array}$

1517

502

4

HORIZONTAL

The analysis of the structure reveals that the initial member sizes and associated, final stress levels are structurally adequate for the design loads. Additional structural design and evaluation is required, primarily for the welds inter-connecting the individual components of the structure.

The required design/ evaluations are as follows:

- Design connection between. $1 "$ the shield wall plates:

Maximum loads node no. 60 (plates 40-55): (Load Case 3)

$$
F_{X}=0.146 k, \quad F_{Y}=0.03 k, \quad F_{Z}=0.122 k
$$




\section{KAISER ENGINEERS}

HANFORD

DESIGN ANALYSIS

client WHC

subject Prefiltration/Recirculation Skid, Structural Design (Process Building)
Calc. No. W320-24-019

Revision 0

Page No. 18 of 36

Wo/Job No. W-320/ER4319

Date $05 / 17 / 94$ By M.R Custer

MRC

Checked $02 / 17 / 95$ By G.J Zyn

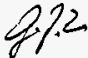

Location TANK 241-C-106

Revised By

$M_{X}=0.10 \mathrm{k}$-in, $\quad M_{Y}=2.09 \mathrm{k}$-in $\quad M_{Z}=0$

$A=1^{13}, \quad S=1^{11} / 3 \mathrm{in}^{2}=0.333 \mathrm{in}^{2}$

$\mathrm{fa}=0.03 / 1+0.1 / 0.33=0.33 \mathrm{ksi}$

$f s=\left[(2.09+0.146)^{2}+(0.122)^{2}\right]^{1 / 2}=2.24 \mathrm{ksi}$

$W_{0}=\left[(0.33)^{2}+(2.24)^{2}\right]^{1 / 2} / 14.85=0.15^{11}$ or $3 / 16$,

Use $5 / 16^{\prime \prime}$ fillet weld (min. weld) $=0.313^{\prime \prime}$

- Design connection between the $1^{13}$ shield plate and the $1 / 2^{11}$ cover plate:

Maximum loads 0 node no. 11 (global reactions of plates 9 and 10): (Load Case 3)

$F_{X}=0.483 \mathrm{k} \quad F_{Y}=2.30 \mathrm{k} \quad F_{Z}=0.470 \mathrm{k}$

$M_{x}=0.415 \mathrm{k}$-in $\quad M_{Y}=-0.09 \mathrm{k}$-in $\quad M_{z}=-0.561 \mathrm{k}$-in

$\mathrm{S}_{\mathrm{x}}=\mathrm{d}^{2} / 3=0.33 \mathrm{in}^{2}, \mathrm{~S}_{\mathrm{y}}=\mathrm{bd}=1^{\prime \prime}\left(1^{\prime \prime}\right)=1 \mathrm{in}^{2}$

$f x=0.483 / 2+0.09 / 1=0.332 k$

$f y=2.30 / 2=1.15 k$

$f z=0.470 / 2=0.235 k$

$W_{0}=\left[(0.332)^{2}+(1.15)^{2}+(0.235)^{2}\right]^{1 / 2} / 14.85=0.08^{4}$

$W=\left(0.08^{\prime \prime} \times 12\right) / 3=0.32^{\prime \prime}$

Use 5/16" fillet weld, (Weld size is conservative, since the loads used to size the welds are located at the corner at the TS $8 \times 4$ column. The column acting as a composite section with the plates will significantly, reduce the applied loads on the plate welds).

- Design connection between: the TS $4 \times 4$ brace and the $1 / 2^{\prime \prime}$ cover plate:

Maximum loads o nodes no. 1 and 18 (Beams 1 and 2): (Load Case 4)

$$
F_{X}=0.02 k \quad F_{Y}=.316 k \quad F_{Z}=-0.137 k
$$




\section{KAISER ENGINEERS}

HANFDRD

\section{DESIGN ANALYSIS}

Client WHC

subject Prefiltration/Recirculation Skid, Structural Design (Process Building)
Calc. No. W320-24-019

Revision 0

Page No. 19 of 36

Wo/Job No. W-320/ER4319

Date $05 / 17 / 94$ By M.R Custer MRC

checked $02 / 17 / 95$ вy G.J Zyn $/ 272$

Location TANK 241-C-106

Revised

By

$\begin{array}{lll}M_{X}=0 & M_{Y}=0 & M_{Z}=0\end{array}$

Area of the weld, $A=1.5^{\prime \prime} \times 2=3^{\prime \prime}$

$W=\left\{\left[(0.316 / 3)^{2}+(0.02 / 3)^{2}+(0.137 / 3)^{2}\right]^{1 / 2}\right\} / 14.85=0.008^{\prime \prime}$

Use $3 / 16^{\prime \prime}$ fillet weld, 1.5" long minimum, each side of TS

- Design connection between horizontal TS to horizontal TS:

Maximum Toads o node no. 102 (Beam 44): (Load Case 4)

$F_{X}=0.125 k \quad F_{Y}=0.03 k \quad F_{Z}=0.017 k$

$M_{x}=0.51 \mathrm{k}$-in $\quad M_{y}=1.52 \mathrm{k}$-in $\quad M_{z}=0.51 \mathrm{k}-$ in

Area, $A=4 \times 1.5^{\prime \prime}=6^{\prime \prime}$

$S=d^{2} / 3+b d=(1.5)^{2} / 3+4(1.5)=6.75 i n^{2}$

$J_{W}=1.5 / 6\left(3 \times 4^{2}+1.5^{2}\right)+4 / 6\left(4^{2}+3 \times 1.5^{2}\right)=27.73 \mathrm{in}^{3}$,

$c=(2)^{1 / 2}(2)=2.828$

$f x=0.125 / 6+(1.52+0.510) / 6.75=0.32 \mathrm{ksi}$

$f h=\left[(0.03 / 3)^{2}+(0.017 / 3)^{2}\right]^{1 / 2}+[0.51(2.828) / 27.73]=0.06 \mathrm{ksi}$

$W=\left\{\left[(0.32)^{2}+(0.06)^{2}\right]^{1 / 23} / 14.85=0.022^{\prime \prime}\right.$

Use $3 / 16^{\prime \prime}$ weld $=0.19^{\prime \prime}>0.022^{\prime \prime}$

- Design connection TS $4 \times 4$ column to $1 / 2^{n}$ plate:

Maximum loads at node no. 5 (Beam B23): (Load Case 1)

$$
\begin{array}{lll}
F_{X}=0.45 k & F_{Y}=2.92 k & F_{z}=-0.51 k \\
M_{X}=-0.43 k & M_{Y}=-0.147 k & M_{Z}=0.404 k
\end{array}
$$

Welds to be provided all around the TS member at attachment to the $1 / 2$ " plate. 


\section{KAISER ENGINEERS}

HANFDRE

\section{DESIGN ANALYSIS}

ctient WHC

subject Prefiltration/Recirculation Skid, Structural Design (Process Building)
Calc. No. W320-24-019

Revision 0

Page No. 20 of 36

wo/Job No. W-320/ER4319

Date $05 / 17 / 94$ By M.R Custer

checked $02 / 17 / 95$ вy G.J Zyn

Loads and moments resolved to the centerline of the TS and the perimeter weld will be designed:

$$
\begin{aligned}
& A=2(4)+2(3)=14^{\prime \prime}, \quad A 1=2(4)=8^{\prime \prime}, \quad A 2=2(3)=6^{\prime \prime} \\
& d=(2)^{1 / 2}(2)=2.83^{\prime \prime} \\
& S X=4^{2} / 3+3 \times 4=17.33 \mathrm{in}^{2}, \quad S y=3^{2} / 3+4 \times 4=10.9 \mathrm{in}^{2} \\
& J W=\left[4 / 6\left(3 \times 4^{2}+4^{2}\right)\right]+\left[3 / 6\left(3^{2}+3 \times 4^{2}\right)=71.17 \mathrm{in}^{3}\right. \\
& a=2.0+0.5=2.50^{\prime \prime} \\
& F_{X}=0.45 \mathrm{k} \quad F_{Y}=2.92 \mathrm{k} \quad F_{Z}=0.51 \mathrm{k} \\
& M_{X}=2.92 \mathrm{k}\left(2.50^{\prime \prime}\right)+0.43=7.73 \mathrm{k}-\mathrm{in} \\
& M_{Y}=0.15 \mathrm{k}-\mathrm{in}+(0.45+0.51) 2.50^{\prime \prime}=2.55 \mathrm{k}-\mathrm{in} \\
& M_{Z}=2.92 \mathrm{k}\left(2.50^{\prime \prime}\right)+0.40=7.70 \mathrm{k}-\mathrm{in} \\
& \mathrm{fa}=2.92 / 14+(7.73 / 17.0+7.70 / 19.0)=1.06 \mathrm{ksi} \\
& \mathrm{fs}=\left[(0.45 / 14)^{2}+(0.51 / 14)^{2}\right]^{1 / 2}+(2.55 \times 2.83) / 71.17=0.15 \mathrm{ksi} \\
& W=\left\{\left[(1.06)^{2}+(0.15)^{2}\right]^{1 / 2}\right\} / 14.85=0.072^{\prime \prime}
\end{aligned}
$$

Use $3 / 16^{\prime \prime}$ weld (typ.)

- Design connection TS $8 \times 4$ column to $1 / 2$ " plate:

Maximum loads o node no. 11 (Beam 29): (Load Case 4)

$F_{X}=0.293 k \quad F_{Y}=0.752 k \quad F_{Z}=0.091 k$

$M_{x}=0.289 \mathrm{k}$-in $\quad M_{Y}=4.519 \mathrm{k}$-in $\quad M_{z}=0.741 \mathrm{k}$-in

Forces applied to centerline of TS $8 \times 4$ same as above except:

$$
\begin{aligned}
& M_{X}=0.29+0.752\left(2.5^{\prime \prime}\right)=2.17 \mathrm{k}-\text { in } \\
& M_{Y}=4.52+0.293\left(2.5^{\prime \prime}\right)=5.25 \mathrm{k}-\text { in }
\end{aligned}
$$


DESIGN ANALYSIS

Client WHC

subject Prefiltration/Recirculation Skid, Structural Design (Process Building)
Calc. No. W320-24-019

Revision 0

Page No. 21 of 36

wo/Job No. W-320/ER4319

Date $05 / 17 / 94 \quad$ By M.R Custer

MRC

checked $02 / 17 / 95$ By G.J Zyn

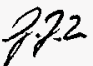

Location TANK 241-C-106

Revised

By

Weld properties:

$A=2(8+4)=24 \quad S_{z}=4(8)+(8)^{2} / 3=53.3 \mathrm{in}^{2}$

$\mathrm{S}_{\mathrm{X}}=4(8)+(4)^{2} / 3=37.3 \mathrm{in}^{2}$

$S_{T}=\left[(4+8)^{3} / 6\right] /\left[(2)^{2}+(4)^{2}\right]^{1 / 2}=64.4$ in

$\mathrm{fa}=0.752 / 24+0.741 / 53.3+2.17 / 37.3=0.10 \mathrm{ksi}$

$f s=\left[(0.293 / 24)^{2}+(0.091 / 24)^{2}\right]^{1 / 2}+5.25 / 64.4=0.094 \mathrm{ksi}$

$W=\left\{\left[(0.10)^{2}+(0.094)^{2}\right]^{1 / 2}\right\} / 14.85=0.009^{\prime \prime}$

Use $3 / 16^{\prime \prime}$ weld (typical)

- Design vertical connection between TS $8 \times 4$ to $1^{\prime \prime}$ shield:

Maximum loads node no. 60 (Beam B31 and B32): (Load Case 5)

$F_{X}=0 \quad F_{Y}=0.012 \quad k \quad F_{Z}=0.016 k$

$M_{X}=1.79 k$-in $\quad M_{Y}=0.032 k$-in $\quad M_{z}=0$

$A=2(1)=2$

$S x=1 \times 4=4 i n^{2}, \quad S z=1 / 3=0.33$

MX $=M_{\text {TORSION }}=1.79+0.016 \times 2=1.82 \mathrm{k}-$ in

$V=1.82 / 4+0.032 / 0.33=0.55 k$

$W_{0}=0.55 / 14.85=0.037,0.037(12) / 3=0.148^{\prime \prime}$

Use $3 / 16^{\prime \prime}$ weld, 3" $12^{\prime \prime}$ on center 
HNF-2467, Rev.0

\section{KAISER ENGINEERS \\ HANFORD}

DESIGN ANALYSIS

client WHC

subject Prefiltration/Recirculation Skid, Structural Design (Process Building)
Calc. No. W320-24-019

Revision 0

Page No. 22 of 36

Ho/Job No. W-320/ER4319

Date $05 / 17 / 94$ By M.R Custer

Checked 02/17/95 вy G.J Zyn D2,2

Location TANK 241-C-106

Revised

By

- Design connection between the HEME support and the $I^{\prime \prime}$ shield plate:

Maximum loads (o node no. 98 (Beam B5): (Load Case 4)

$F_{X}=-0.807 k \quad F_{Y}=0.069 k \quad F_{Z}=0.949 k$

$M_{x}=-1.63 \mathrm{k}$-in $\quad M_{y}=1.00 \mathrm{k}$-in $\quad M_{z}=1.18 \mathrm{k}$-in

Weld section properties:

$\mathrm{Sw}=\mathrm{d}^{2} / 3=6^{2} / 3=12 \mathrm{in}^{2}, \mathrm{Sw}_{\mathrm{w}}=\mathrm{bd}=0.5(6)=3 \mathrm{in}^{2}, \quad \mathrm{c}_{\mathrm{h}}=0.25^{\prime \prime}$

$J w=\left(b^{3}+3 b d^{2}\right) / 6=\left[6.0^{3}+3(6)(0.50)^{2}\right] / 6=36.75 \mathrm{in}^{3}$

$\mathrm{fa}=0.807 / 2+1.0 / 12+1.18 / 3=0.88 \mathrm{ksi}$

$f s=\left\{(0.069 / 2)^{2}+[0.949+1.63(0.25) / 36.75]^{2}\right\}^{1 / 2}=0.96 \mathrm{ksi}$

$W_{0}=\left[\left(0.88^{2}+0.96^{2}\right) 1 / 2\right] / 14.58=0.089^{\prime \prime}$

Use 3/16" weld all-around connection plate and $3 / 16^{\prime \prime}, 4^{\prime \prime}$ Tong between the TS and the connection plate at the (4) sides of the slot (typ.)

CHECK LOCAL STRESSES IN THE W12 X 40 BEAMS: (Reference 1, Sections K1.1 and K1.8)

EQ. KI-1, Stiffeners req'd if $t_{f}<0.4\left(P_{b f} / F_{y c}\right)_{1 / 2}$,

$t_{f}=0.515^{n}, 0.4[12 \mathrm{k}(4 / 3) / 36]^{1 / 2}=0.27 \ldots$ No stiffeners required

EQ. K1-8, Stiffeners req'd if $d_{c}>\left[4100 t_{w c}^{3}\left(F_{y c}\right)^{1 / 2}\right] / P_{b f}$

$d_{c}=12^{\prime \prime}-2\left(1.25^{\prime \prime}\right)=9.5^{\prime \prime}>\left[4100(0.295)^{3}(36)^{1 / 2}\right] 12(4 / 3)=39.5^{\prime \prime}$

.... No stiffeners required

Provide 3/8" stiffener plates, full height between the flanges at each side of W12 beam web at a17 TS columns, except at framing intersections of the W12 beams. Provide 1/4" fillet weld at (3) sides, .each side at each stiffener plate. 
DESIGN ANALYSIS

Client WHC

subject Prefiltration/Recirculation Skid, Structural Design (Process Building)
Calc. Ho. W320-24-019

Revision 0

Page No. 23 of 36

Ho/Job No. W-320/ER4319

Date $05 / 17 / 94$ By M.R Custer MRC

Checked $02 / 17 / 95$ By G.J Zyn

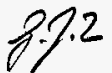

Location TANK 241-C-106

Revised

By

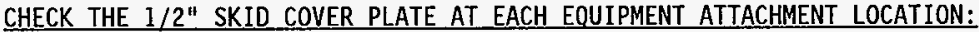

The $1 / 2$ " skid cover plate, provides the structural support for the minor equipment located on the equipment skid. The major equipment, such as the HEME, condenser and the moisture separator are supported directly by the primary/ secondary beams as part of the basic skid structure. The support/ anchorage system for the smaller equipment and miscellaneous supports (i.e pipe, electrical and instrumentation as 1 isted), will be evaluated for direct attachment to the $1 / 2^{\prime \prime}$ plate. Supplemental, structural members will be provided as required. The supplemental, structural steel includes the support of the $1 / 2^{\prime \prime}$ plate to be provided to span the $10^{\prime \prime}$ gap between the equipment skid and the concrete curb.

DESCRIPTION
LOAD (kips)

Vert. /Horiz.
HEIGHT

(ft.)
SUPPORT LOCATION(S)

$\mathrm{X}$ (ft.) $\mathrm{z}$ (ft.)

9.25

3) Air Receiver

Tank (TK-1361)

4) Rad. Monitor (RE-1362)
2.26

2.26

$0.15 / 0.07$

$0.15 / 0.07$

$0.35 / 0.17$

$0.80 / 0.32$
2.00

1.50

9.50

9.71
PLATE SPAN

(ft.)

Support attachments ...ok based on the small magnitude of the applied loads.

- Design supplemental support beams at the perimeter of the skid to support the Mini Power panel/ Instrument Panel at the 10" gap:

The support beams are $W 12 \times 40$ wide-flange beams, cantilevered from the W12 primary and secondary members. The loading on the W12 beams includes the dead weight of the plate and the greater of a $100 \mathrm{lb} / \mathrm{sf}$ live load or the equipment weight (Mini Power Panel or Instrument Pane1). Design of the $W 12 \times 40$ beams is adequate based on the previous calculations for the $W 12$ beams.

NOTE:

1) Mini Power Pane1: (Ref. Section E, drawing H-2-818678), Total weight, Wt.= 275 lbs, area occupied, 32" $\times 32 "$.

2) Instrument Panel: (Reference drawing $\mathrm{H}-2-818588$ ), Total Wt. 300 1bs, area occupied is $24^{\prime \prime} \times 30^{\prime \prime}$. 
HNF-2467, Rev.0

\section{KAISER ENEINEERS \\ HANFDRD}

DESIGN ANALYSIS

Client WHC

subject Prefiltration/Recirculation Skid, Structural Design (Process Building)
Calc. No. W320-24-019

Revision 0

Page No. 24 of 36

wo/Job No. W-320/ER4319

Date $05 / 17 / 94 \quad$ By M.R Custer MRC

Checked $02 / 17 / 95$ By G.J Zyn

Revised

By

CHECK DESIGN OF EQUIPMENT SUPPORTS: (Reference drawing H-2-818480, sht. 3 of 7)

- Recirculation Fan base support (FN-1361):

$P_{v}=3501 \mathrm{bs}, P_{h}$ (seismic) $=1711 \mathrm{bs}$

- Heating Coil base support (HC-1361): Critical check, individual TS $2 \times 2$ as column $P_{v}=150 \mathrm{Tbs}, P_{h}$ (seismic) $=73 \mathrm{Tbs}$

Base supports for the Recirculation fan and the Heating Coil are acceptable by engineering judgment based on the small magnitude of the loads.

- Heat Exchanger (Condenser, HX-1361):

$$
\begin{aligned}
& P_{v}=5000 \text { lbs, } P_{h}(\text { seismic })=2447 \text { lbs } \\
& P_{\max }=5.0 \mathrm{k} / 4+2.45\left(27^{\prime \prime}\right) / 13(2)=3.79 \mathrm{k}(1.5)=5.69 \mathrm{k} \\
& M_{\max }=\left[2.45 \mathrm{k}\left(2.25 \mathrm{ft} \times 12^{\prime \prime}\right)\right] / 4=16.54 \mathrm{k}-\mathrm{in}(1.5)=24.8 \mathrm{k}-\mathrm{in} \\
& \mathrm{k} 1 / r=1.0\left(11^{\prime \prime}\right) / 1.45=7.6 \text {, use } 8, \mathrm{Fa}=21.3 \mathrm{ksi} \\
& \underline{\mathrm{f}}_{a} / \mathrm{F}_{\mathrm{a}}=(5.69 / 5.08) / 21.3=0.053<0.15 \ldots \ldots \mathrm{ok} \\
& \mathrm{f}_{b}=24.8 / 5.35=4.6 \mathrm{ksi} \ldots \text { negligible }
\end{aligned}
$$

Check weld at leg support:

$$
\begin{aligned}
& S x=S y=b d+d^{2} / 3=(4 \times 4)+4^{2} / 3=21.33 \mathrm{in}^{2} \\
& f_{w}=\left[\left(2.45 \mathrm{k} / 4 \times 16^{\prime \prime}\right)^{2}+(24.8 \mathrm{k}-\mathrm{in} / 21.33)^{2}\right]^{1 / 2}=1.16 \mathrm{k} / \mathrm{in} \\
& f_{w}=1.16 \mathrm{k} / \mathrm{in}<f=0.707\left(1 / 4^{\prime \prime}\right) 14.85 \mathrm{ksi}=2.62 \mathrm{k} / \mathrm{in} \ldots \text { weld ok }
\end{aligned}
$$




\section{KAISER ENEINEERS}

HANFDRD

\section{DESIGN ANALYSIS}

client WHC

subject Prefiltration/Recirculation Skid, Structural Design (Process Building)
Calc. No. W320-24-019

Revision 0

Page No. 25 of 36

Wo/Job No. W-320/ER4319

Date $05 / 17 / 94$ By M.R Custer

MRC

Checked $02 / 17 / 95$ sy G.J Zyn

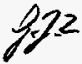

Location TANK 241-C-106

Revised

By

\section{DESIGN LIFTING LUGS:}

The rigging of the skid for transport to the design location will be accomplished using manufactured lifting rings at (4) specific locations on the primary beams of the equipment skid. The lifting rings are designed to accomodate rigging slings used in conjunction with a lifting frame for lifting the equipment skid for transport and placement at the design location. The lifted load at each of the (4) attachment points, as shown on drawing $\mathrm{H}-2-818455$, sht. 1 , is a maximum of 16,500 lbs per location. Pre-manufactured, heavy-duty, safety hoist rings (item HR-125) by the Crosby group, Inc., per page 101, catalog dated April 1994 (stock no. 1016986), with a work load limit of 24,000 lbs will be used. The hoist rings shall be provided each with a heavy hex nut per ASTM A-561 (grade 8) with UNC-3A thread.

Design lifting lug support at the skid:

Check tension on the 1/2" flange plates:

Pmax (maximum load per lug) $=16500$ lbs

$\operatorname{Mmax}\left(\right.$ applied) $=16500$ Tbs $\times 7^{\prime \prime}=116 \mathrm{k}-$ in

d, depth of member $=12^{\prime \prime}$

$T=P \max =M \max / d=116 / 12^{11}=9.7$ kips

$A$, (area of $f$ lange $r e q^{\prime} d$ ) $=P \max / \mathrm{fb}=9.7 / 24 \mathrm{ksi}=0.40 \mathrm{in}^{2}$

A provided $=10^{\prime \prime} \times 1.0^{\prime \prime}=10.0 \mathrm{in}^{2}>0.44 \mathrm{in}^{2} \ldots$ ok

Use flange plates, $1^{\prime \prime} \times 10^{\prime \prime}$, top plate

Check tension on (2), 1/2" web plates:

$\mathrm{P}$ (axial load on each plate) $=16500 \mathrm{lbs} / 2=83001 \mathrm{bs}=8.3 \mathrm{kips}$

$A$, area req' $d=8.3 \mathrm{k} / 24 \mathrm{ksi}=0.35 \mathrm{in}^{2}$

A provided $=0.5^{\mathrm{n}} \times\left(3.75^{\prime \prime}\right)=1.88 \mathrm{in}^{2}>0.35 \mathrm{in}^{2} \ldots$ ok

Use (2)- $1 / 2^{\prime \prime} \times 33 / 4^{\prime \prime}$ web plates

Check bending on built-up section: Note: Built-up section includes (2), $1 / 2^{\text {"l }}$ web plates and (1), $I^{\text {" }}$ top flange plate. 


\section{KAISER ENGINEERS}

HANFERE

DESIGN ANALYSIS

client WHC

subject Prefiltration/Recirculation Skid, Structural Design (Process Building)
Catc. No. W320-24-019

Revision 0

Page No. 26 of 36

Ho/sob No. W-320/ER4319

Date $05 / 17 / 94 \quad$ By M.R Custer MRC

Checked $02 / 17 / 95$ By G.J Zyn

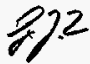

Location TANK 241-C-106

Revised

By

Determine centroid of the area at the critical section, $7^{\text {" from the W12 }}$ centerline:

$$
\begin{aligned}
\bar{x}= & \left([5.25(2)(0.5)(10.5)]_{A}+[1(10)(11.0)]_{A 2}+[3 / 8(6)(10.31)]_{A 3}\right) \\
\bar{x}= & 8.28^{11} \\
I x= & 2\left[b d^{3} / 12+A^{2}\right]_{A 1}+\left[b d^{3} / 12+A d^{2}\right]_{A 2}+\left[b d_{3} / 12+\mathrm{Ad}^{2}\right]_{A 3} \\
I x= & 2\left[(0.5)(10.5)^{3} / 12+10.5(0.5)(3.03)^{2}\right]+\left[(10)(1.0)^{3} / 12+(10)(1.0)(2.72)^{2}\right] \\
& +\left[(6)(3 / 8)^{3} / 12+(6)(3 / 8)(2.03)^{2}\right]= \\
I x= & 192.9+74.8+9.3=277 \mathrm{in}^{4}, \mathrm{C}=8.28^{11} \\
\mathrm{Sx}= & 277 / 8.28=33.5 \mathrm{in}^{3} \\
\mathrm{Fb}= & M \max / \mathrm{Sx}=116 / 33.5=3.47 \mathrm{ksi}<\mathrm{fb}=.6(36 \mathrm{ksi})=21.6 \mathrm{ksi} \ldots . .0 \mathrm{ok}
\end{aligned}
$$

Check critical welds as follows:

1) Web plates to the W12 web:

$$
\begin{aligned}
& \mathrm{Fv}=.707(.25)(70)(0.30)=3.71 \mathrm{k} / \text { in } \times 2=7.42 \mathrm{k} / \mathrm{in} \\
& \mathrm{fv}=8.3 \mathrm{k} / 10^{\prime \prime}(2)=0.42 \mathrm{k} / \text { in }<7.42 \ldots \text { ok (conservative) }
\end{aligned}
$$

2) Web plates to the flange plates:

$$
\begin{aligned}
\mathrm{fh} & =\text { V A y/ Ix n }=16.5 \mathrm{k}\left(1.0^{\mathrm{H}}\right)\left(10^{\mathrm{H}}\right)\left(2.72^{\prime \prime}\right) / 277 \mathrm{in}^{4}(4) \\
\mathrm{fh} & =0.41 \mathrm{k} / \mathrm{in}<3.71 \mathrm{k} / \mathrm{in} \ldots \text { ok }
\end{aligned}
$$

3) Full penetration flange to flange welds adequate, based on the previous design of the flange, since the welds are higher strength than the base material with equal thickness.

Provide built-up lugs at each lift point on the skid as shown per Attachment $A$, per drawing $\mathrm{H}-2-818455$, sheets 1 of 5 ). 


\section{DESIGN ANALYSIS}

Client WHC

Subject Prefiltration/Recirculation Skid,

Structural Design (Process Building)

Location TANK 241-C-106

\section{DESIGN SKID ANCHORAGE:}

The anchorage system for the HVAC skid assembly is provided through the use of $1.0 \mathrm{ft} x 1.0 \mathrm{ft}$ leveling plates and 1" dia. embedded anchor bolts. The design of the embedded anchor bolts are based on the use of (12) anchors and the following:

- Total horizontal shear force (global) on the skid $=30.0 \mathrm{kips}$

Shear force per anchor is $30.0 / 12=2.5 \mathrm{kips}$

- Maximum vertical force (global) on the skid anchors, results from the 30 kip horizontal, seismic component (East-West direction), acting at the center of gravity of the skid assembly, $4.79 \mathrm{ft}$ above the centerline of the W12 skid beams (Figure 5). The tension force/ anchor is determined as follows:

- Forces on anchor bolts:

$$
\begin{aligned}
& F+0.63 F+0.22 F=0.14 F 1+0.59 F 1+F 1 \\
& 1.85 F=1.73 F 1 \\
& F 1=1.069 F
\end{aligned}
$$

Summation of moments about the Center of Gravity: $D L=61.3 \mathrm{k} / 20.33 \mathrm{ft}=3.03 \mathrm{k}$

$30 \mathrm{k}(4.79 \mathrm{ft}) / 2+3.03 \mathrm{k}(12.39 \mathrm{ft}) / 2-3.03 \mathrm{k}(7.94 \mathrm{ft}) / 2=\mathrm{F}(11.26 \mathrm{ft})$

$+0.63 F(7.09 \mathrm{ft})+0.22 \mathrm{~F}(2.43 \mathrm{ft})+0.16 \mathrm{~F}(1.24 \mathrm{ft})+0.63 \mathrm{~F}(5.32 \mathrm{ft})$

$+1.07 \mathrm{~F}(9.07 \mathrm{ft})$

$78.59=11.26 \mathrm{~F}+4.47 \mathrm{~F}+0.54 \mathrm{~F}+0.20 \mathrm{~F}+3.35 \mathrm{~F}+9.71 \mathrm{~F}$

$F=78.59 / 29.53=2.66 k, F 1=1.07(2.66 k)=2.85 k$

- Summary of the applied anchor loads: Shear $=2.50 \mathrm{k}$, Tension $=2.85 \mathrm{k}$

- Allowable anchor loads for 1" dia. A 307 embedded anchors per Reference 2, Table 26-E, page 547 (UBC 1991 Edition)

Shear $=4150 \mathrm{lbs}$

Tension $=3200 \mathrm{lbs} \times 2=6400 \mathrm{lbs}$
Page No. 27 of 36

WO/Job No. W-320/ER4319

By M.R Custer MRC

By

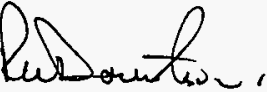

By 
HNF-2467, Rev.0

KAISER ENGINEERS

DESIGN ANALYSIS

client WHC

subject Prefiltration/Recirculation Skid, Structural Design (Process Building)
Talc. Ko. W320-24-019

Revision 0

Page No. 28 of 36

wo/Job No. W-320/ER4319

Date $05 / 17 / 94$ By M.R Custer M/CC

checked $02 / 17 / 95$ By G.J Zyn

Revised

By

- Check Combined Load equation:

$[V / V(a l l o w a b l e)]^{5 / 3}+[T / T(a l l o w a b l e)]^{5 / 3}<$ or $=1.0 \quad$ (Reference 2)

then: $(2.5 / 4.15)^{5 / 3}+(2.85 / 6.4)^{5 / 3}=0.43+0.26=0.69<1.0 \ldots$.ok

Use 12 - 1 " Dis. A 307 embedded anchor bolts w/ minimum 7" embedment, minimum edge distance is $6^{\prime \prime}$ and minimum spacing of $12^{11}$ with special inspection.

- Check pullout capacity of the concrete and compare to the maximum bolt capacity:

$$
\begin{aligned}
& \mathrm{Pu} \text { (concrete) }=\mathrm{Ae}(4)(0.65)(4000)^{1 / 2} \\
& \text { where, Ae }=3.1416\left[7^{2}+1.63(7)\right]=189.8 \mathrm{in}^{2} \\
& \mathrm{Pu} \text { (concrete) }=189.8(4)(0.65)(63.25)=31.2 \mathrm{k} \\
& \mathrm{Pu} \text { (steel) = Fy At, At }=0.606 \mathrm{in}^{2} \\
& \mathrm{Pu}=36 \mathrm{ksi}(0.606)=21.8 \mathrm{k}<31.2 \mathrm{k} \ldots \text { ok, Bolt will yield before concrete }
\end{aligned}
$$

KEF $0037.00(06 / 92)$ KEF055

Page A-32 
HNF-2467, Rev.0

ICF KAISER HANFORD

DESIGN ANALYSIS

Calc. No.: W320-24-019

Revision: 0

Page No.: 29 of 36

Client: WHC

Subject: Prefiltration/Recirculation Skid

Structural Design (Process Building)

Location: TANK 241-C-106
WO/Job No.: W-320/ER4319

Date: $05 / 17 / 94$

Checked: $2 / 17 / 95$

Revised:
By: M.R. Custer MRC

By: Georye ?. Zyn

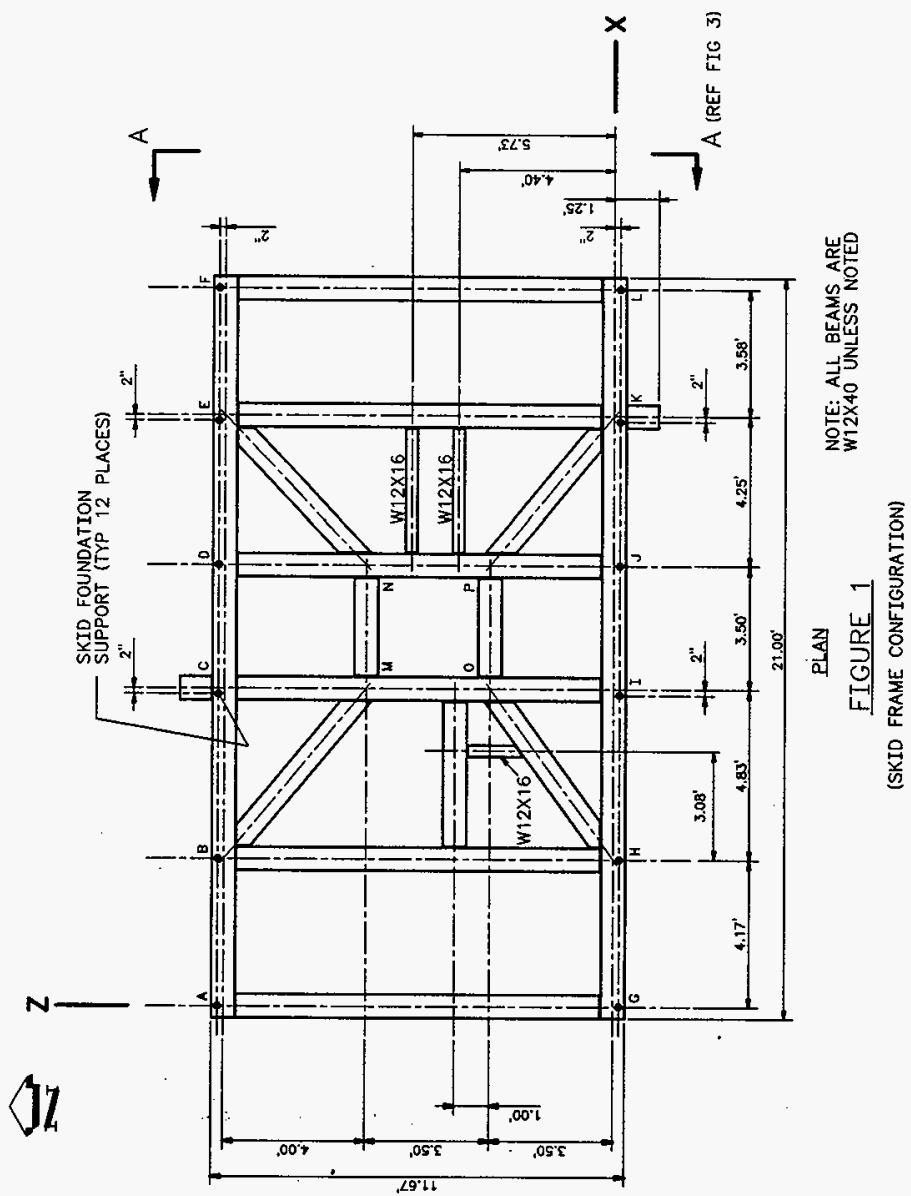


Calc. No.: $\quad$ w320-24-019

Revision: 0

Page No.: 30 of 36
Client: WHC

Subject: Prefiltration/Recirculation Skid Structural Design (Process Building) Location: TANK 241-C-106

\section{DESIGN ANALYSIS}

WO/Job No.: W-320/ER4319

Date: 05/17/94

Checked: $2 / 17 / 95$

Revised:
By: M.R. Custer MRC By: Fourge 7. Zyer

\section{BEAM/LOAD CONFIGURATION FOR PRIMARY /}

SECONDARY BEAMS (RIGGING PHASE).

\section{RIGGING LOAD (D.L.) $=54.8^{\mathrm{K}}$}

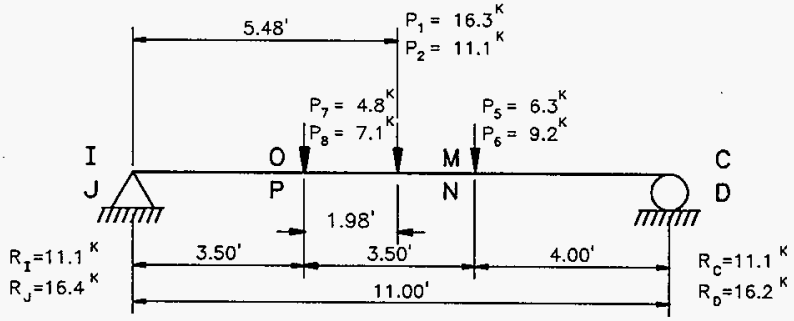

SECONDARY BEAMS

$P=54.8^{k}$

$P_{1}=\left(2.08^{\prime} / 3.5^{\prime}\right)(0.5) 54.8^{K}=16.3^{K}$

$P_{2}=\left(1.42^{1} / 3.5^{\prime}\right)(0.5) 54.8^{K}=11.1^{K}$

$P_{3}=\left(1.98^{\prime} / 3.5^{\prime}\right)(0.5) 54.8^{K}=15.5^{K}$

$P_{7}=\left(1.42^{\prime} / 3.5^{\prime}\right) P_{4}=4.8^{K}$

$P_{8}=\left(2.08^{\prime} / 3.5^{\prime}\right) P_{4}=7.1^{K}$

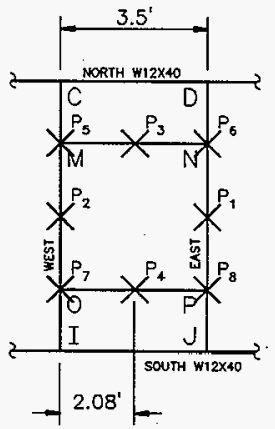

$P_{4}=\left(1.52^{\prime} / 3.5^{\prime}\right)(0.5) 54.8^{K}=11.9^{k}$

$P_{5}=\left(1.42^{\prime} / 3.5^{\prime}\right) P_{3}=6.3^{\mathrm{K}}$

$P_{6}=\left(2.08^{\prime} / 3.5^{\prime}\right) P_{3}=9.2^{K}$

$M_{\text {MAX }(J, P, N, 0)}=16.4^{K}(3.5 \mathrm{FT})+\left(16.4^{K}-7.1^{K}\right)(1.98 \mathrm{FT})$

$M_{\operatorname{MAX}(U, P, N, 0)}=75.8^{K-T}=910^{K-"}$

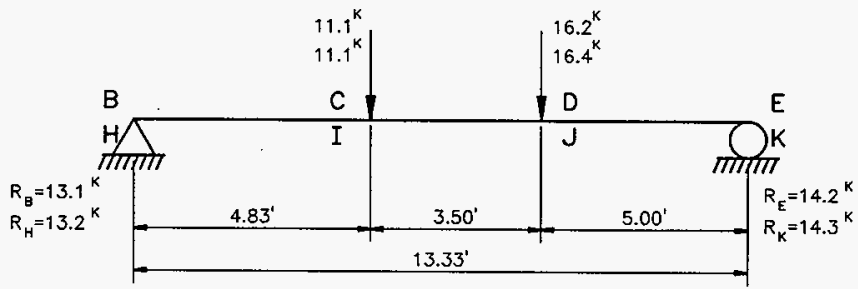

PRIMARY BEAMS

$M_{\text {maX }(H, I, J, K)}=13.2^{K}(4.83 F T)+\left(13.2^{K}-11.1^{K}\right)(3.5 F T)$

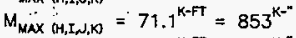

$M_{\text {MAX }(B, C, D, \boxminus}=70.3^{K-F T}=843^{K-N^{*}}$

FIGURE 2 
HNF-2467, Rev.0

\section{ICF KAISER HANFORD}

\section{DESIGN ANALYSIS}

Client: WHC

Subject: Prefiltration/Recirculation Skid

Structural Design (Process Building)

Location: TANK 241-C-106
WO/Job No.: W-320/ER4319

Date: 05/17/94

Checked: $2 / 17 / 95$

Revised:
Calc. No.: W320-24-019

Revision: 0

Page No.: 31 of 36
By: M.R. Custer MRC

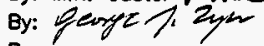

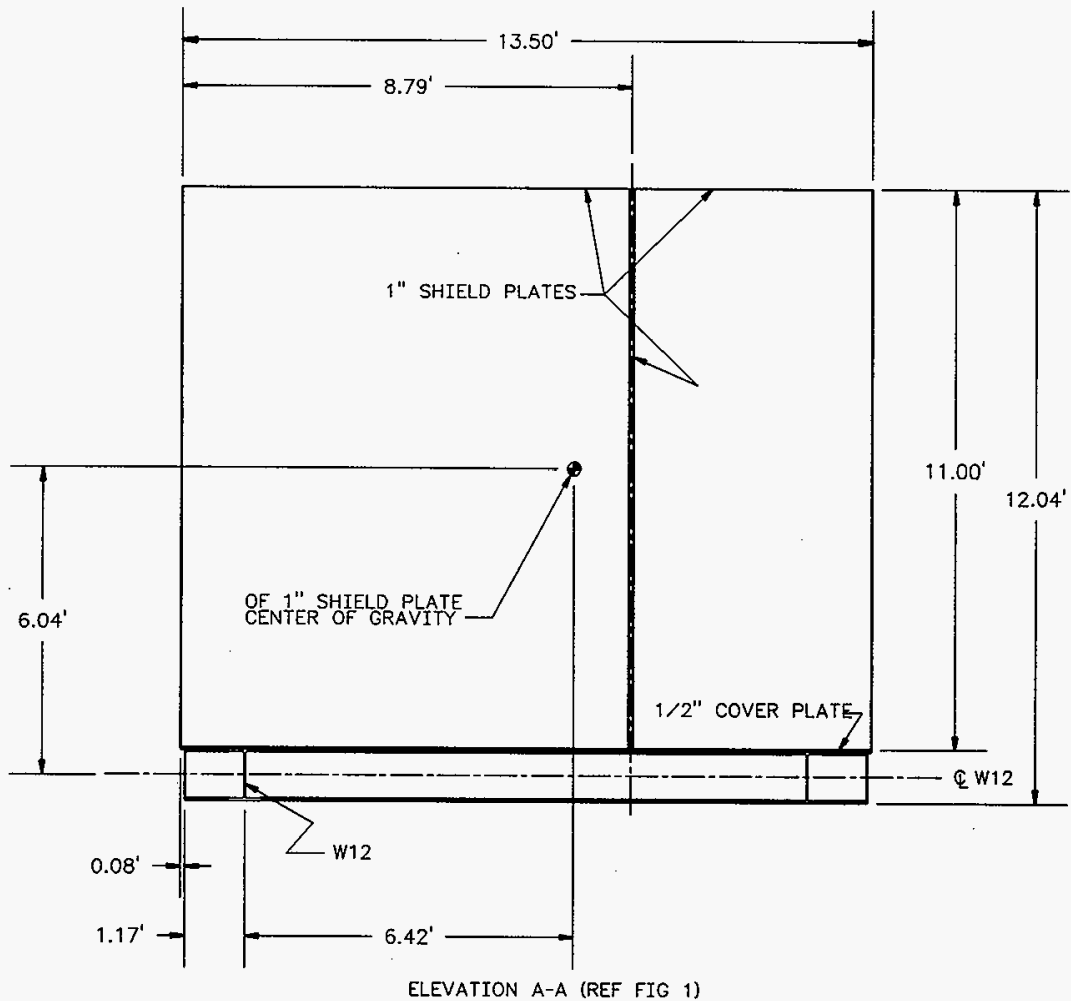

FIGURE 3 
HNF-2467, Rev.0

ICF KAISER HANFORD

DESIGN ANALYSIS

Calc. No.: W320-24-019

Revision: 0

Page No.: 32 of 36

Client: WHC

Subject: Prefiltration/Recirculation Skid

Structural Design (Process Building)

Location: TANK 241-C-106

Wo/Job No.: W-320/ER4319

Date: $05 / 17 / 94$

Checked: $2 / 17 / 95$

Revised:

By: M.R. Custer M/RC

8y: ferye ?. 2yc

By:

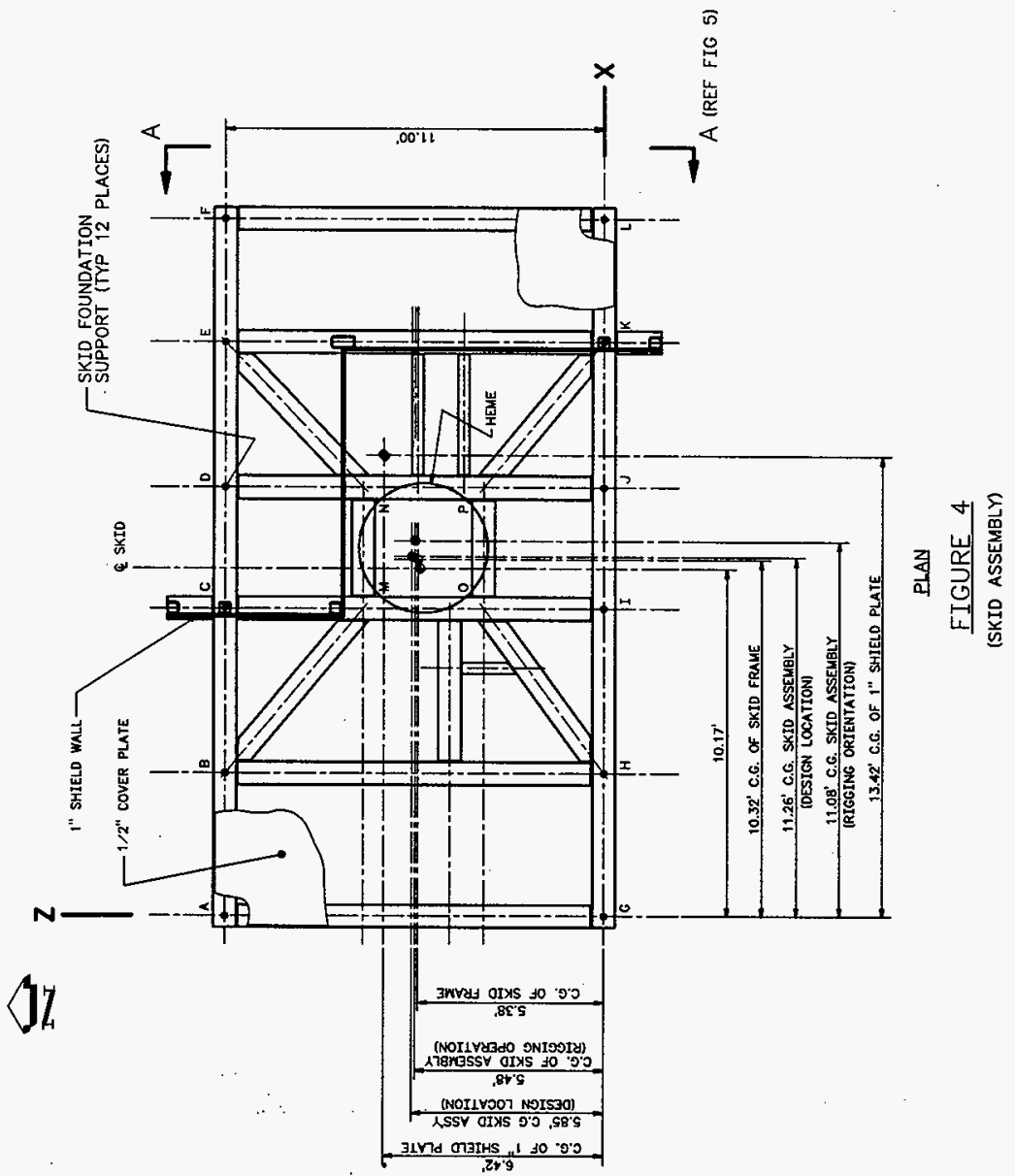


HNF-2467, Rev.0

DESIGN ANALYSIS

Calc. No.: W320-24-019

Revision: 0

Page No.: 33 of 36

Client: WHC

Subject: Prefiltration/Recirculation Skid

Structural Design (Process Building)

Location: TANK 241-C-106
WO/Job No.: W-320/ER4319

Date: $05 / 17 / 94$

Checked: $2 / 17 / 95$

Revised:
By: M.R. Custer $M R C$

By: perye 7. Zur

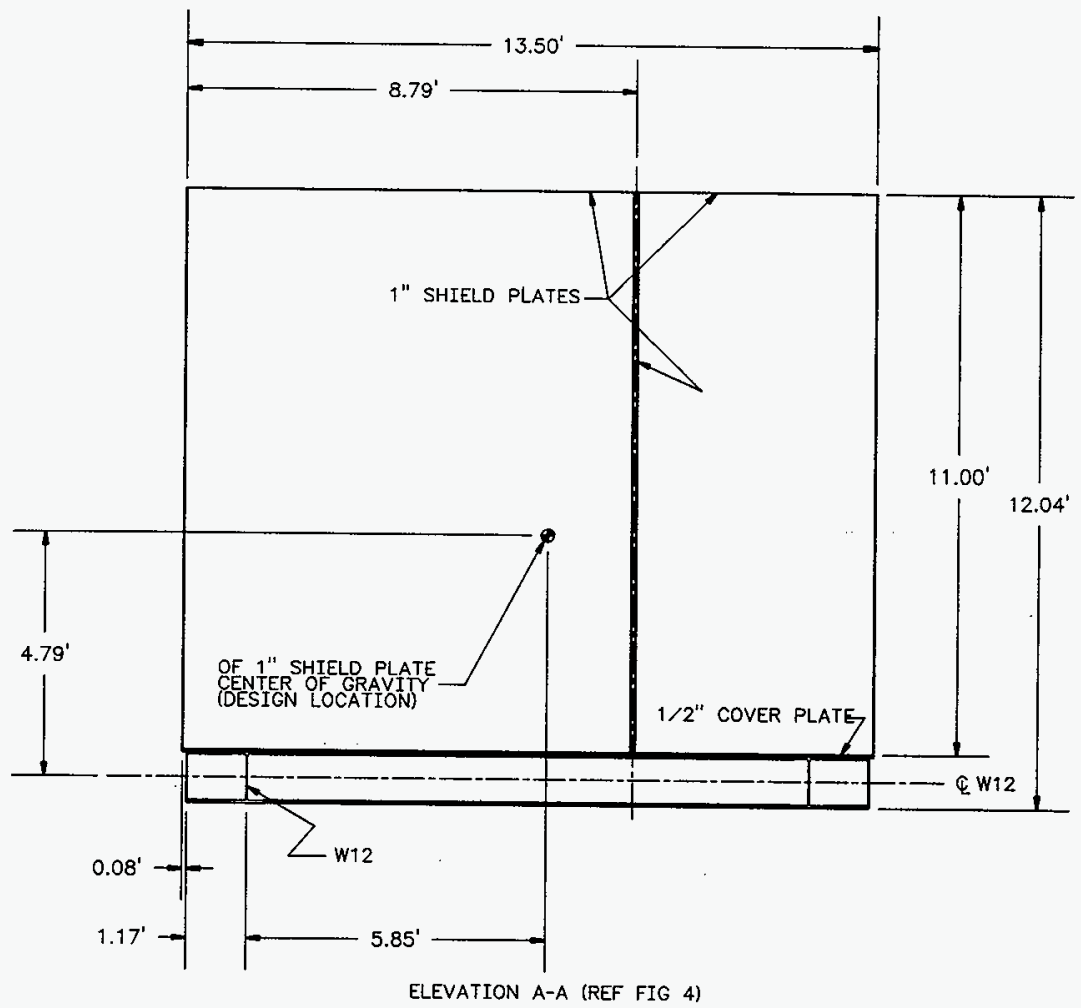

FIGURE 5 
HNF-2467, Rev.0

ICF KAISER HANFORD

DESIGN ANALYSIS

Calc. No.: W320-24-019

Revision: 0

Page No: 34 of 36

Client: WHC

Subject: Prefiltration/Recirculation Skid

Structural Design (Process Building)

Location: TANK 241-C-106
WO/Job No.: W-320/ER4319

Date: 05/17/94

Checked: $3 / 7 / 95$

Revised:
By: M.R. Custer $M / R C$

By: Lerye o. Zye

By:
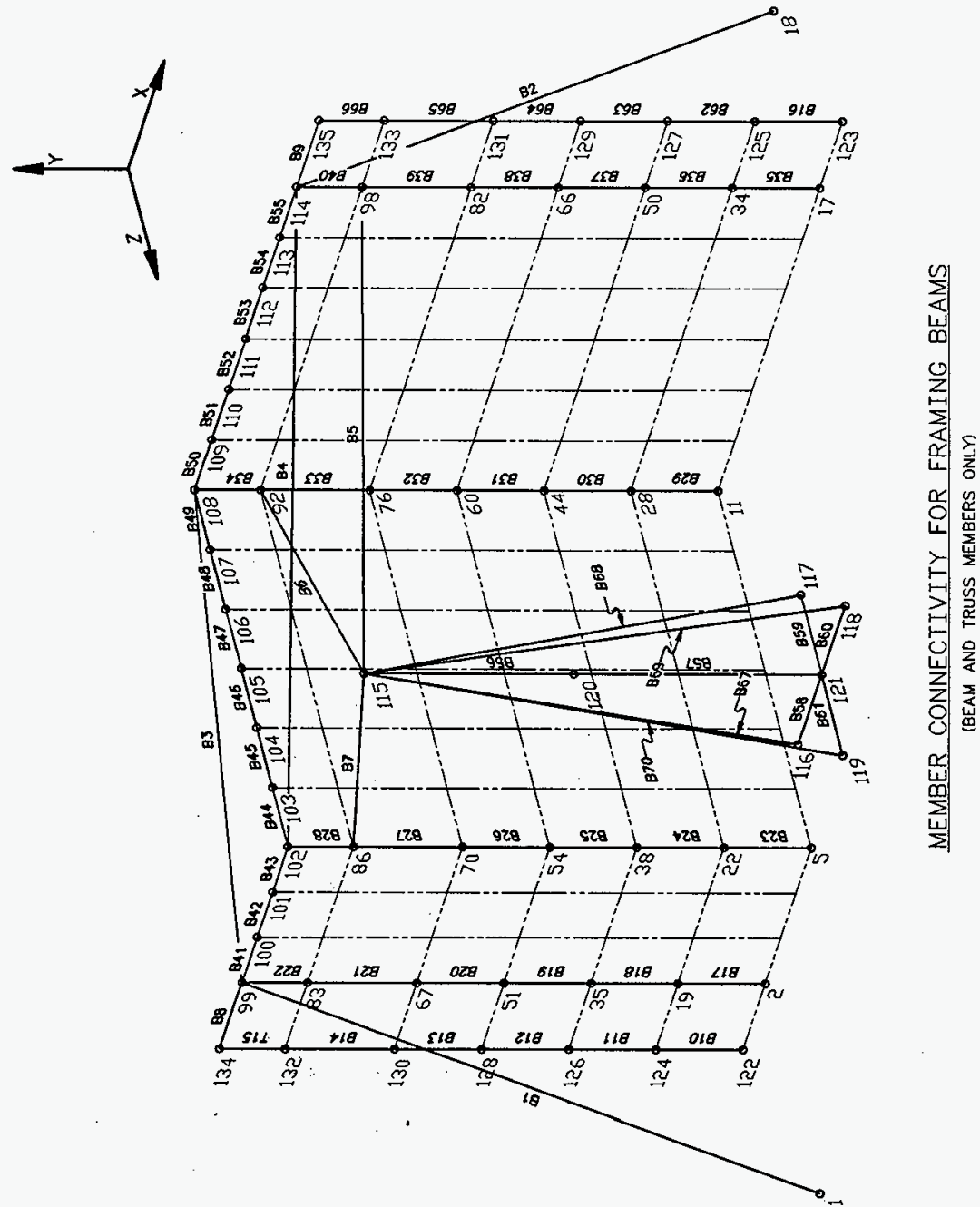


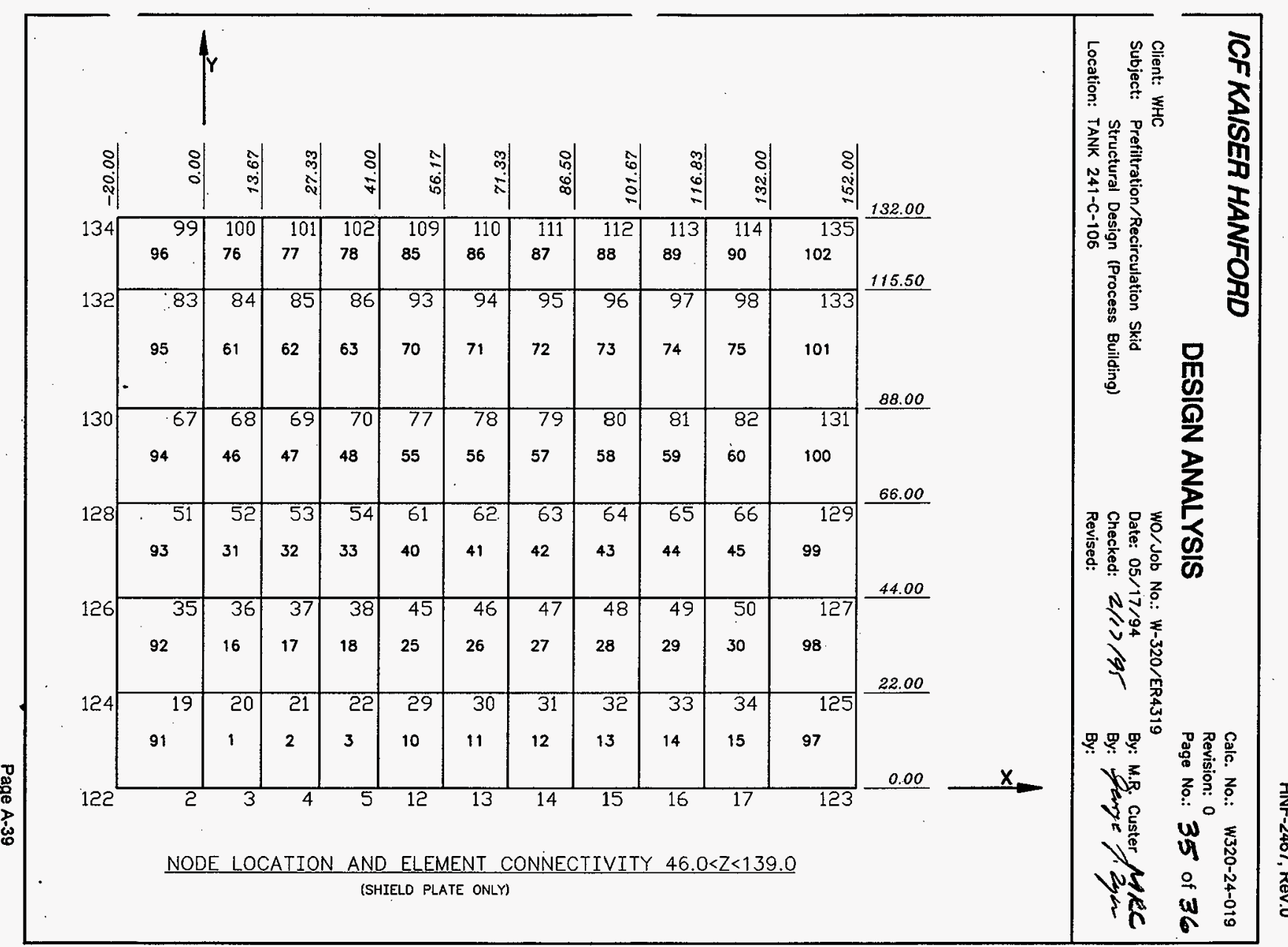


HNF-2467, Rev.0

Calc. No.: W320-24-019

DESIGN ANALYSIS

Revision: 0

Page No.: 36 of 36

Client: WHC

Subject: Prefiltration/Recirculation Skid

Structural Design (Process Building)

Location: TANK 241-C-106
WO/Job No.: $W-320 / E R 4319$

Date: $05 / 17 / 94$ Checked: $2 / 17 / 9$ Revised:
By: M.R. Custer MRC By: Perpi $72 y$ By:
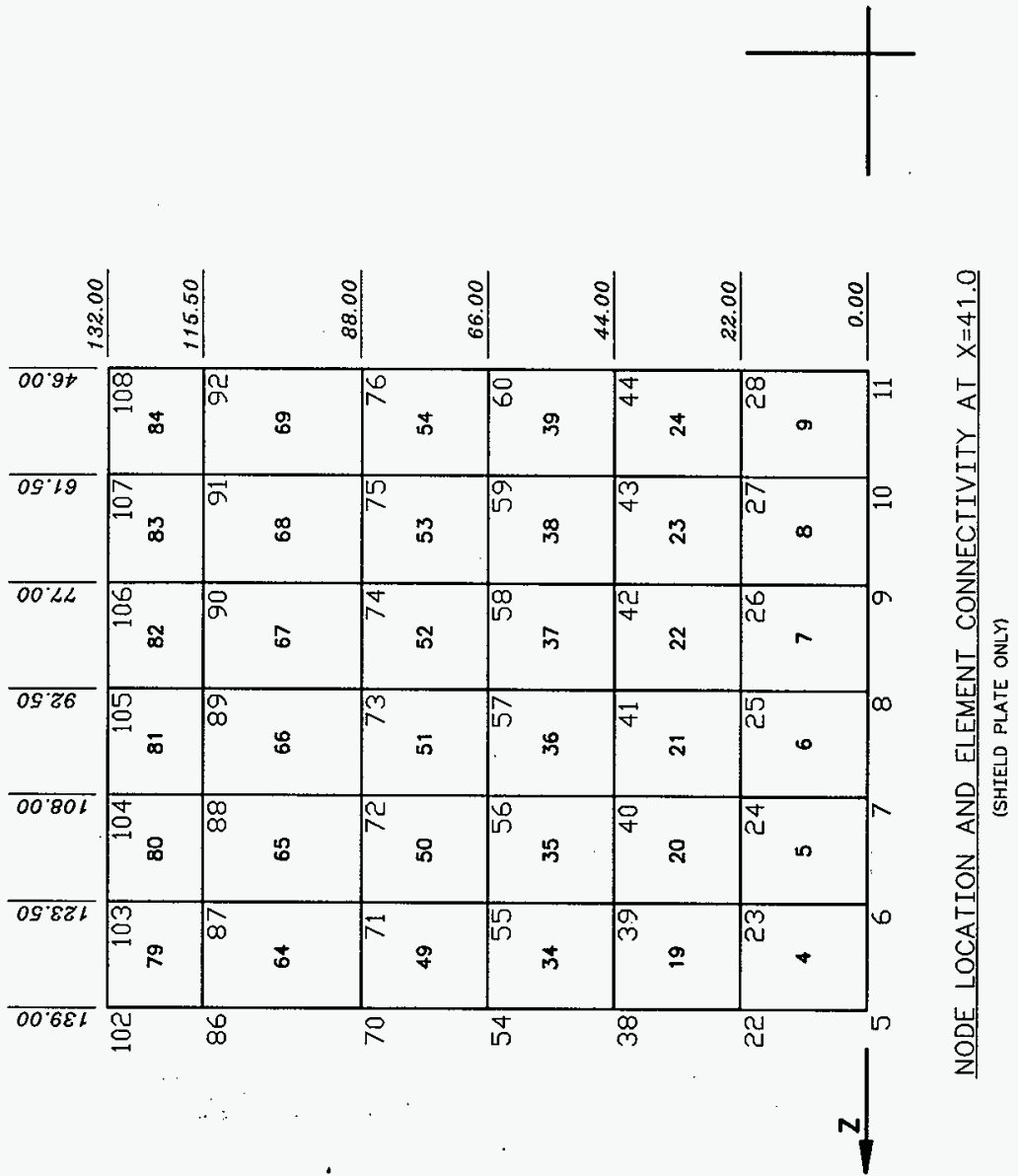


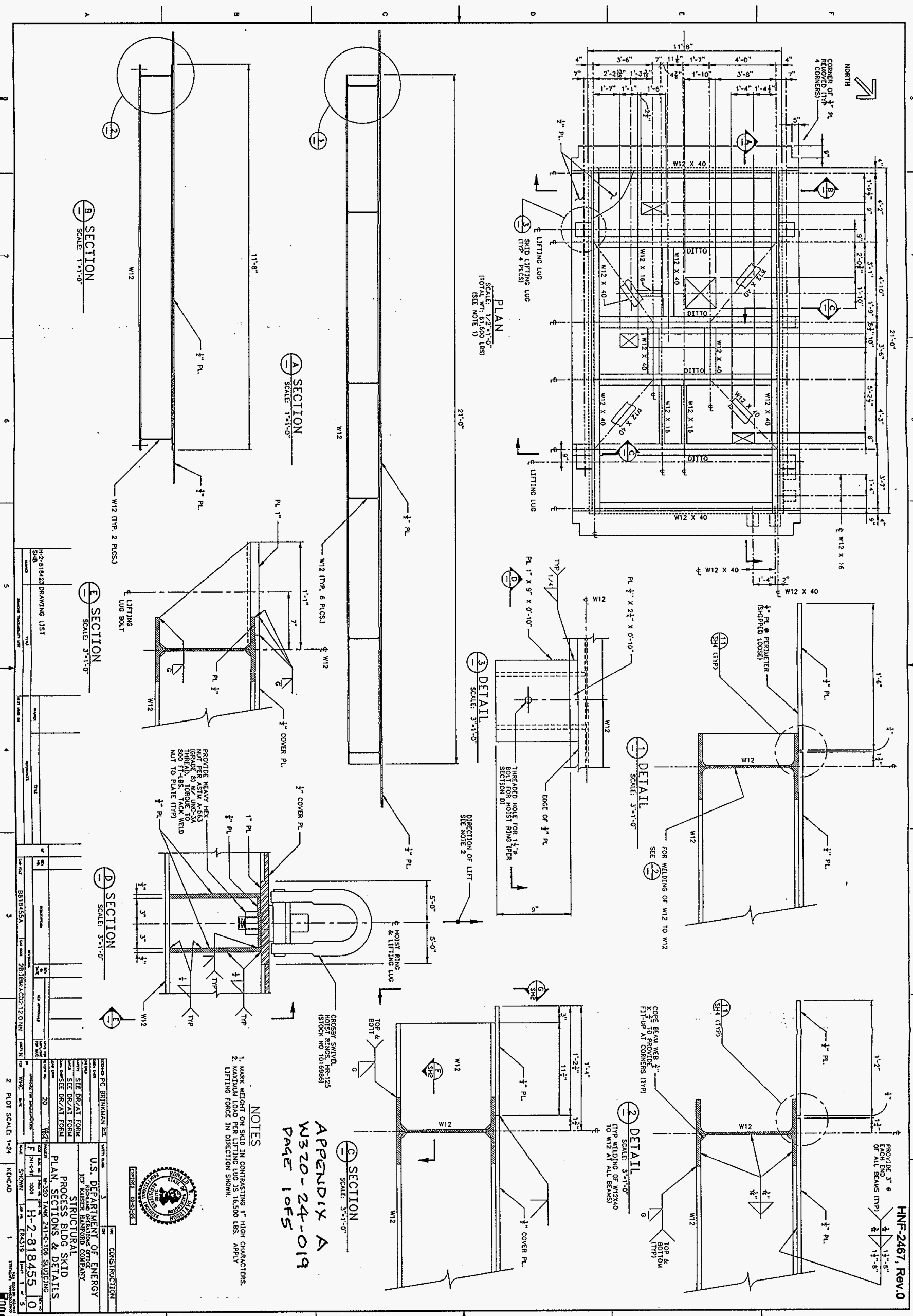




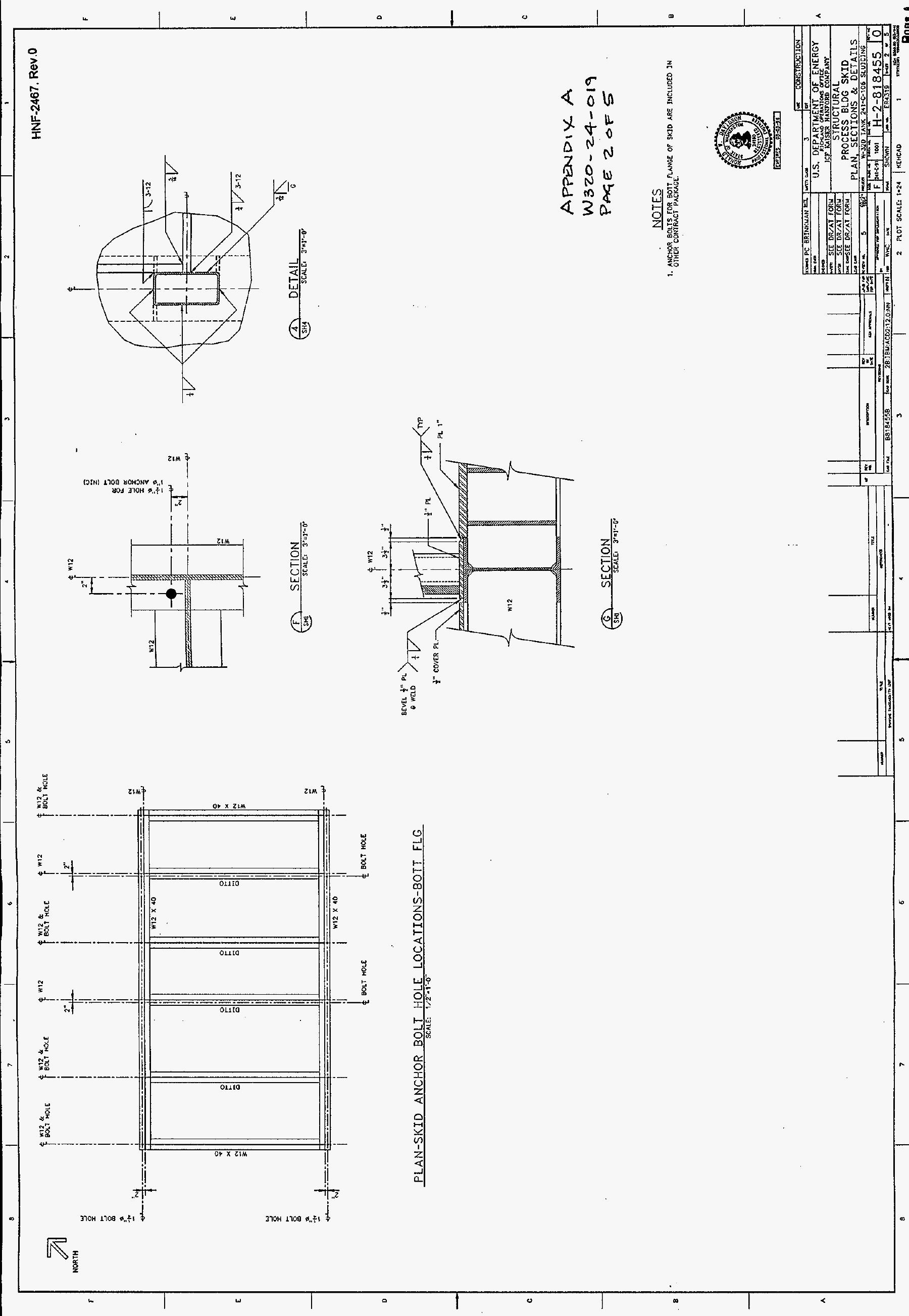




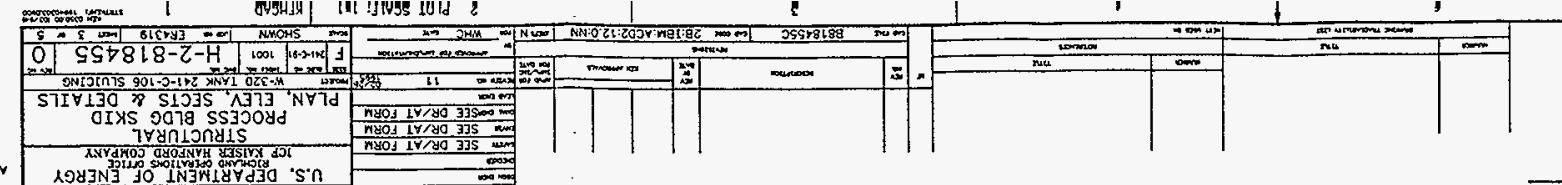

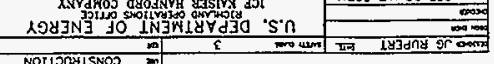

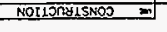

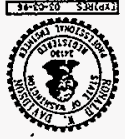

\section{가d}

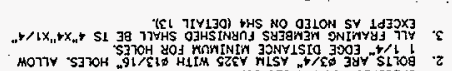

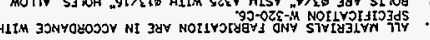

Him a
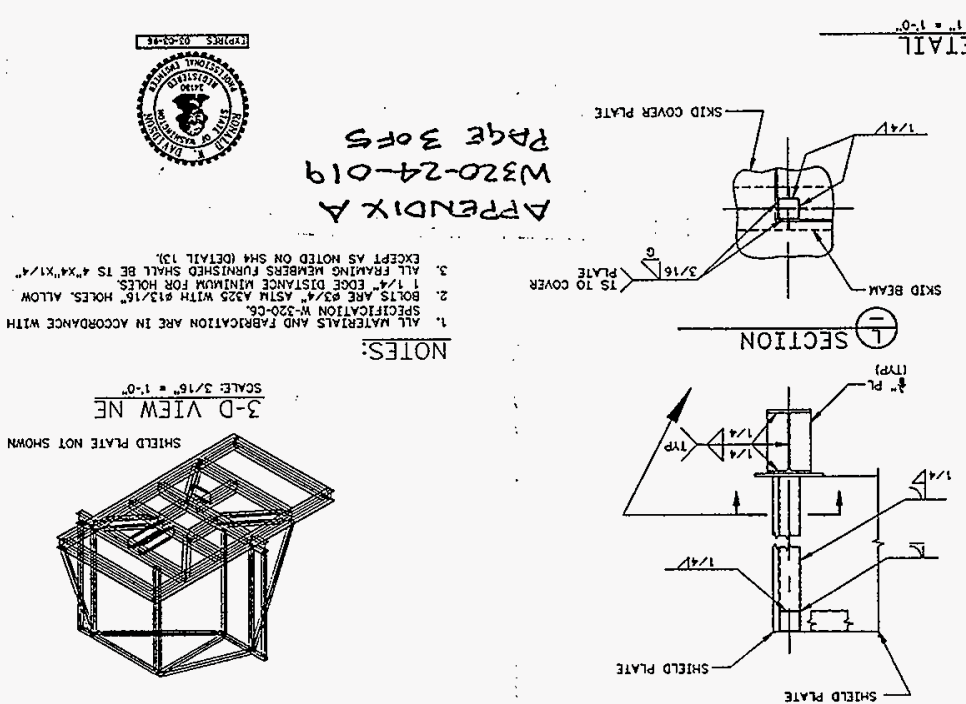

$\frac{0.1 \cdot 1}{7 I \forall 130}(-)$

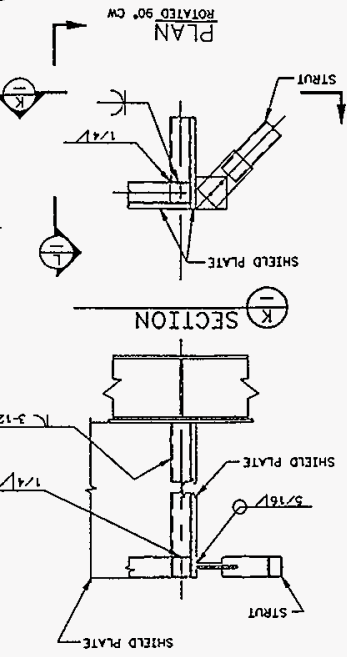

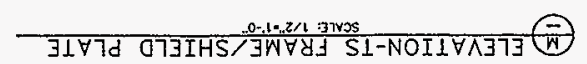

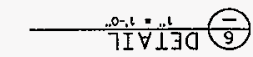

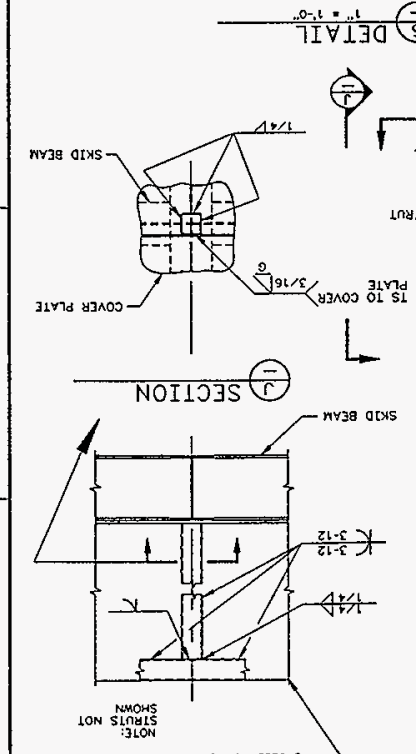

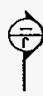
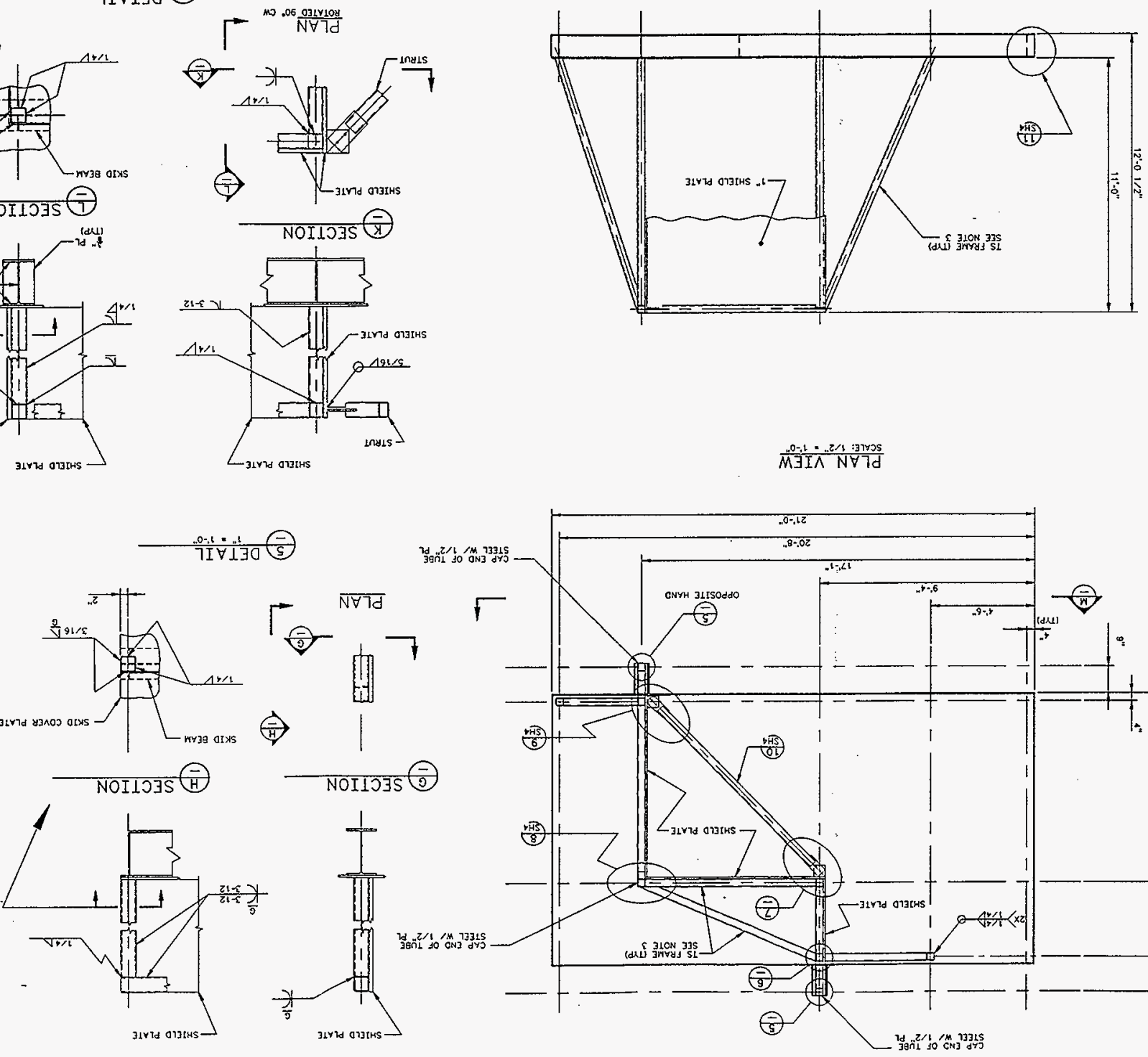

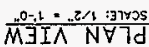

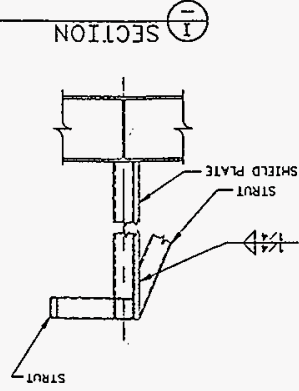




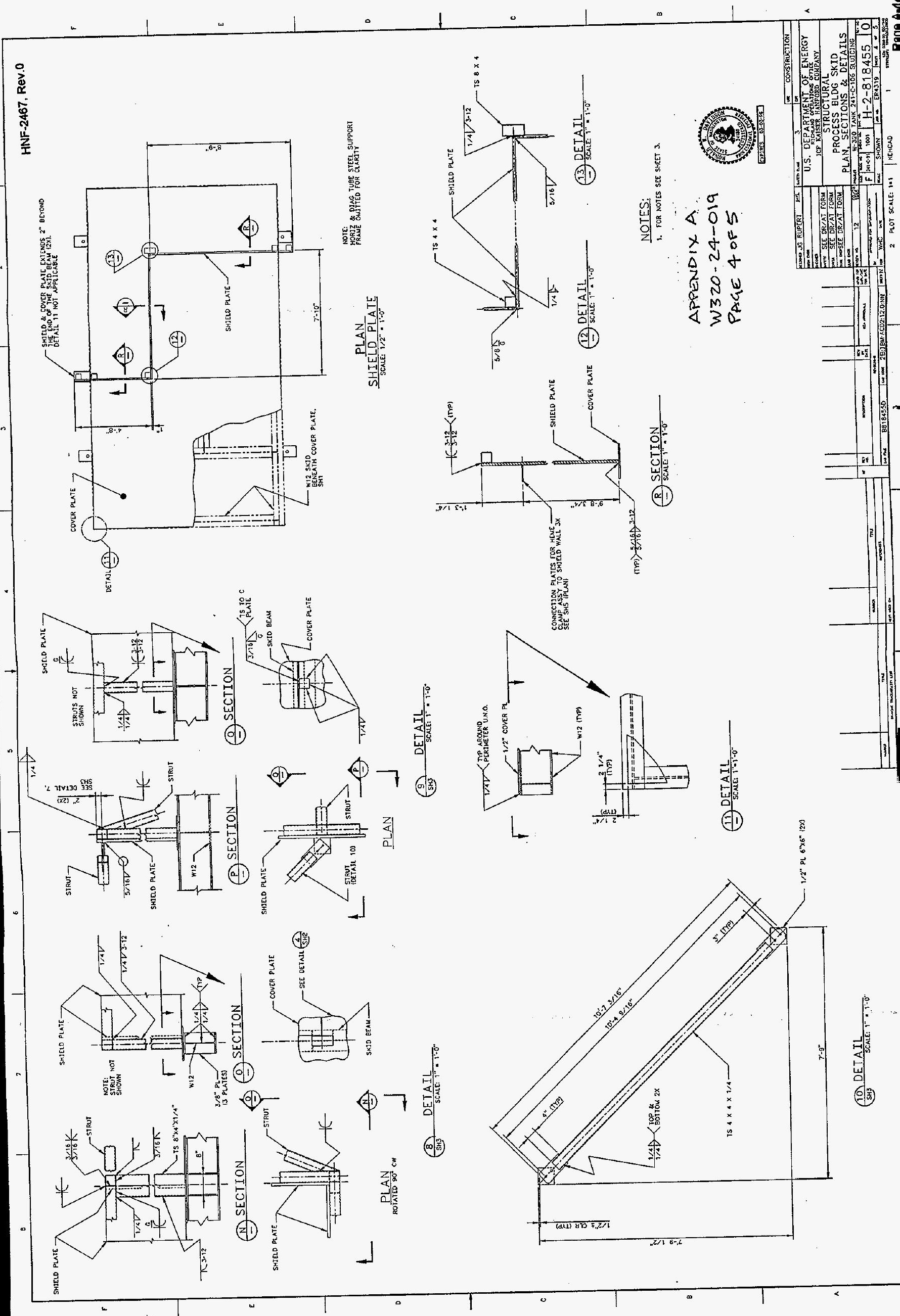



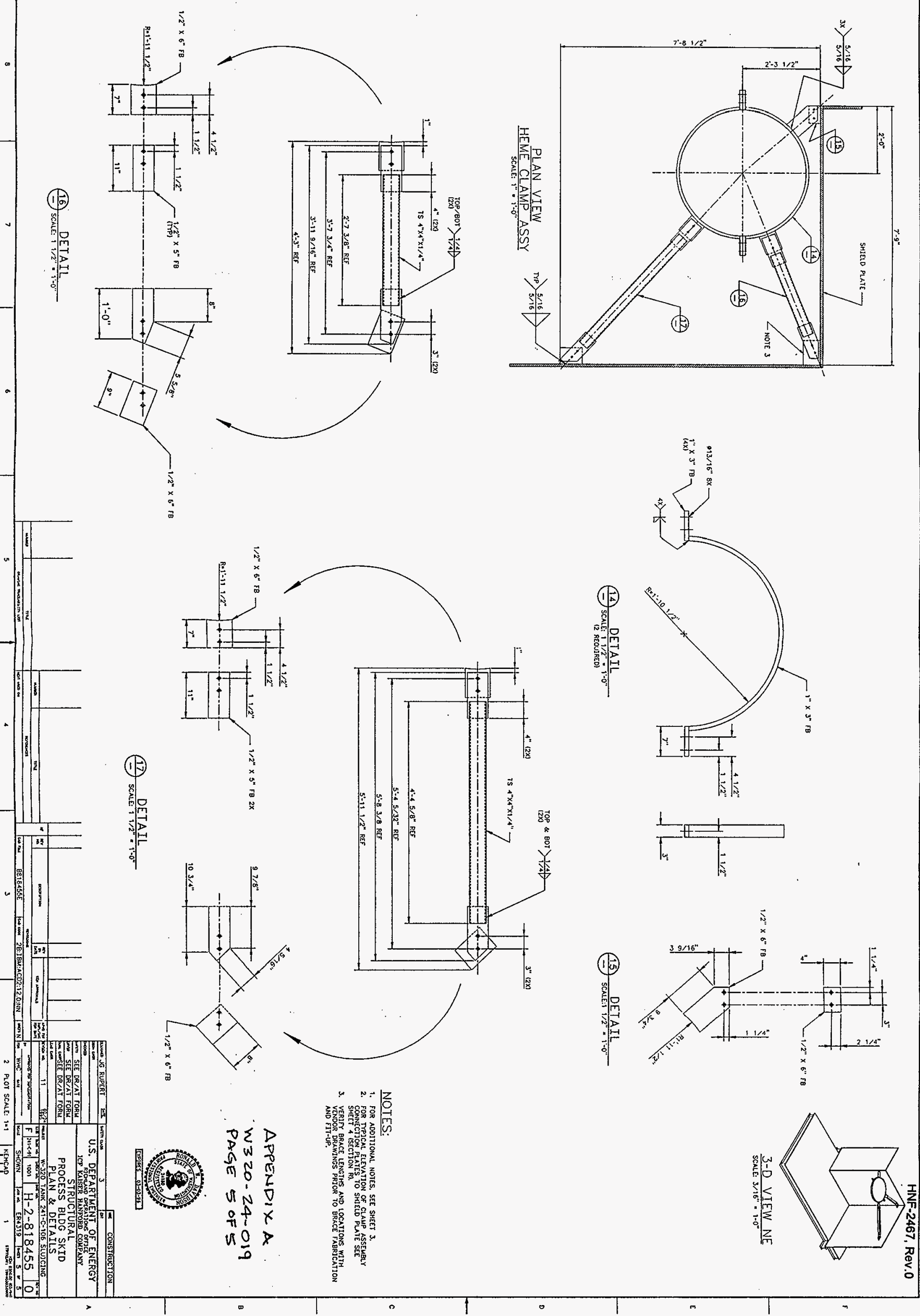
KAISER ENGINEERS HANFORD S/H:801854

PAGE 1

RUN ID =\#N61973

$02 / 22 / 95$

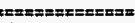
x Copyright (c) 1984 Celestial Software Inc. I

CHECK GEOAETRY

version $2.0 \quad 07 / 01 / 90$

$\mathbf{L}$

\section{MATERIAL PROPERTIES}

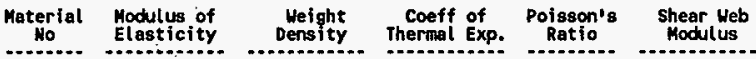
$3.00000 E+07 \quad 2.83000 E-01 \quad 0.00000 E+00 \quad 3.00 E-01 \quad 1.12000 E+07$

NOOE COOROINATES

\begin{tabular}{|c|c|c|c|}
\hline Node & x-coord. & Y-coord. & coord. \\
\hline $\begin{array}{r}1 \\
2 \\
3 \\
4 \\
5 \\
6 \\
7 \\
8 \\
9 \\
10 \\
11 \\
12 \\
13 \\
14 \\
15 \\
16 \\
17 \\
18 \\
19 \\
20 \\
21 \\
22 \\
23 \\
24 \\
25 \\
26 \\
27 \\
28 \\
29\end{array}$ & $\begin{array}{l}0.00000 \mathrm{E}+00 \\
0.00000 \mathrm{E}+00 \\
1.36667 \mathrm{E}+01 \\
2.73333 \mathrm{E}+01 \\
4.10000 \mathrm{E}+01 \\
4.10000 \mathrm{E}+01 \\
4.10000 \mathrm{E}+01 \\
4.10000 \mathrm{E}+01 \\
4.10000 \mathrm{E}+01 \\
4.10000 \mathrm{E}+01 \\
4.10000 \mathrm{E}+01 \\
5.61667 \mathrm{E}+01 \\
7.13333 \mathrm{E}+01 \\
8.65000 \mathrm{E}+01 \\
1.01667 \mathrm{E}+02 \\
1.16833 \mathrm{E}+02 \\
1.32000 \mathrm{E}+02 \\
1.32000 \mathrm{E}+02 \\
0.00000 \mathrm{E}+00 \\
1.36667 \mathrm{E}+01 \\
2.73333 \mathrm{E}+01 \\
4.10000 \mathrm{E}+01 \\
4.10000 \mathrm{E}+01 \\
4.10000 \mathrm{E}+01 \\
4.10000 \mathrm{E}+01 \\
4.10000 \mathrm{E}+01 \\
4.10000 \mathrm{E}+01 \\
4.10000 \mathrm{E}+01 \\
5.61667 \mathrm{E}+01 \\
7.13333 \mathrm{E}+01\end{array}$ & $\begin{array}{l}0.00000 \mathrm{E}+00 \\
0.00000 \mathrm{E}+00 \\
0.00000 \mathrm{E}+00 \\
0.00000 \mathrm{E}+00 \\
0.00000 \mathrm{E}+00 \\
0.00000 \mathrm{E}+00 \\
0.00000 \mathrm{E}+00 \\
0.00000 \mathrm{E}+00 \\
0.00000 \mathrm{E}+00 \\
0.00000 \mathrm{E}+00 \\
0.00000 \mathrm{E}+00 \\
0.00000 \mathrm{E}+00 \\
0.00000 \mathrm{E}+00 \\
0.00000 \mathrm{E}+00 \\
0.00000 \mathrm{E}+00 \\
0.00000 \mathrm{E}+00 \\
0.00000 \mathrm{E}+00 \\
0.00000 \mathrm{E}+00 \\
2.20000 \mathrm{E}+01 \\
2.20000 \mathrm{E}+01 \\
2.20000 \mathrm{E}+01 \\
2.20000 \mathrm{E}+01 \\
2.20000 \mathrm{E}+01 \\
2.20000 \mathrm{E}+01 \\
2.20000 \mathrm{E}+01 \\
2.20000 \mathrm{E}+01 \\
2.20000 \mathrm{E}+01 \\
2.20000 \mathrm{E}+01 \\
2.20000 \mathrm{E}+01 \\
2.20000 \mathrm{E}+01\end{array}$ & $\begin{array}{l}1.94000 \mathrm{E}+02 \\
1.39000 \mathrm{E}+02 \\
1.39000 \mathrm{E}+02 \\
1.39000 \mathrm{E}+02 \\
1.39000 \mathrm{E}+02 \\
1.23500 \mathrm{E}+02 \\
1.08000 \mathrm{E}+02 \\
9.25000 \mathrm{E}+01 \\
7.70000 \mathrm{E}+01 \\
6.15000 \mathrm{E}+01 \\
4.60000 \mathrm{E}+01 \\
4.60000 \mathrm{E}+01 \\
4.60000 \mathrm{E}+01 \\
4.60000 \mathrm{E}+01 \\
4.60000 \mathrm{E}+01 \\
4.60000 \mathrm{E}+01 \\
4.60000 \mathrm{E}+01 \\
0.00000 \mathrm{E}+00 \\
1.39000 \mathrm{E}+02 \\
1.39000 \mathrm{E}+02 \\
1.39000 \mathrm{E}+02 \\
1.39000 \mathrm{E}+02 \\
1.23500 \mathrm{E}+02 \\
1.08000 \mathrm{E}+02 \\
9.25000 \mathrm{E}+01 \\
7.70000 \mathrm{E}+01 \\
6.15000 \mathrm{E}+01 \\
4.60000 \mathrm{E}+01 \\
4.60000 \mathrm{E}+01 \\
4.60000 \mathrm{E}+01\end{array}$ \\
\hline
\end{tabular}

KAISER ENGINEERS HANFORD S/N:801854

PAGE 2 RUN ID=\#N61973 $02 / 22 / 95$
$08: 47: 49$

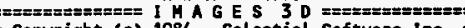
= Copyright (c) 1984 Celestial software Inc. = CHECK GEOMETRY

Version $2.007 / 01 / 90$

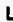

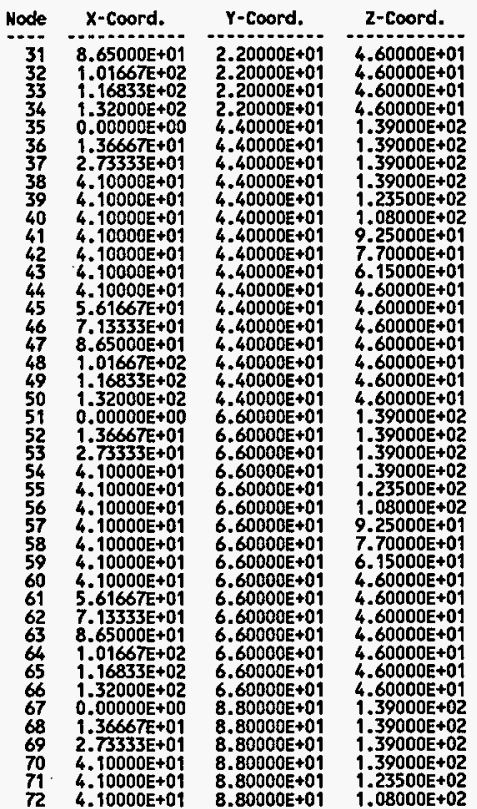




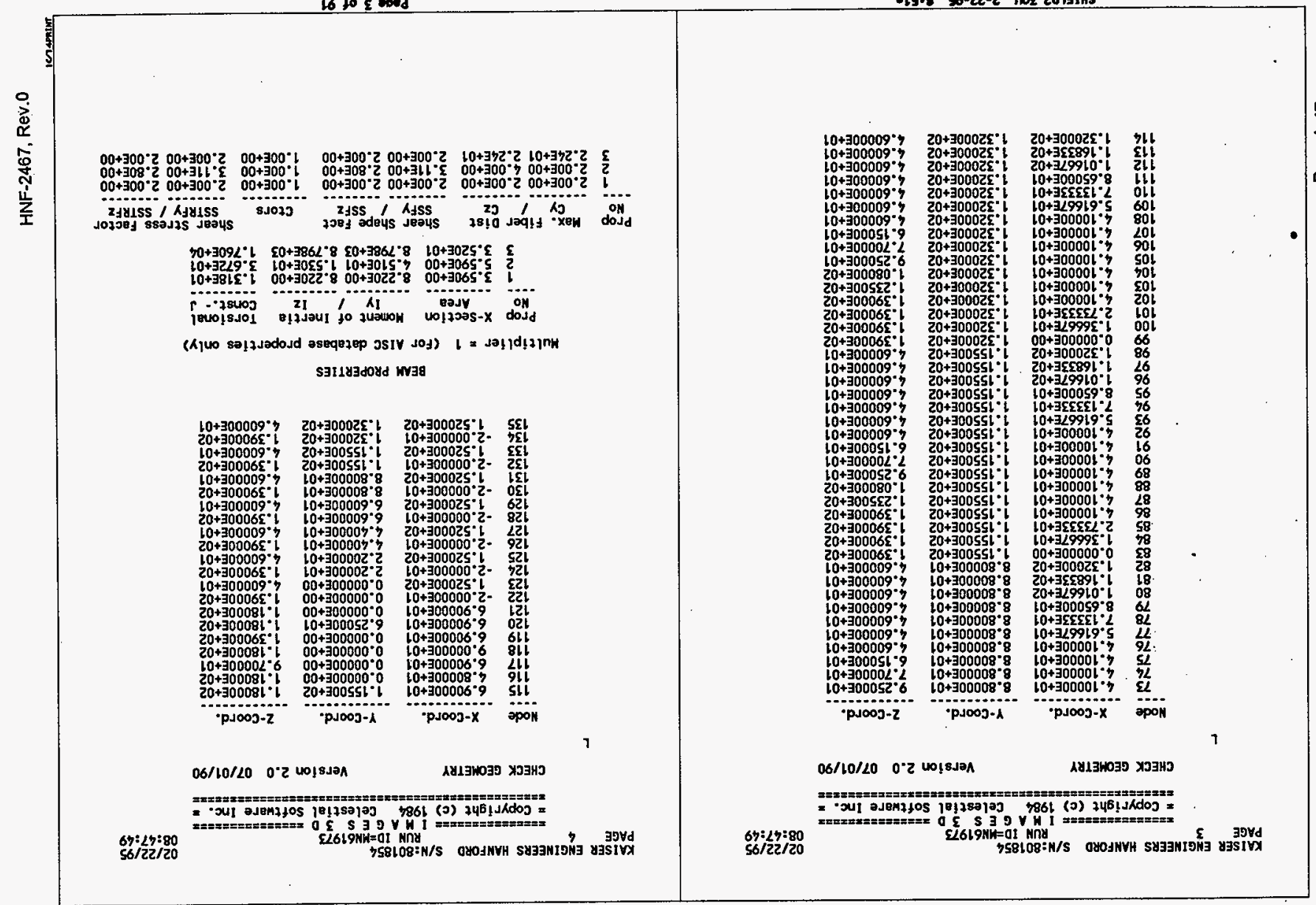


KAISER ENGIMEERS MAHFORD S/N:801854

PAGE 5

RUN ID=N1N61973

$02 / 22 / 95$
$08: 47: 49$

- Copyright (c) 1984 Celestial Sof tware Inc. .

CHECK GEOMETRY

Version $2.007 / 01 / 90$

l

BEAM CONHECTIVITY

\begin{tabular}{rrrrrr} 
Beam & \multicolumn{2}{c}{ Nodes } & \multicolumn{3}{c}{ Prop Mat } \\
Ho & Frow & To & Ref & No & No \\
\hdashline 1 & 1 & 99 & 2 & 1 & 1 \\
2 & 18 & 114 & 17 & 1 & 1 \\
3 & 99 & 108 & 92 & 1 & 1 \\
4 & 114 & 102 & 86 & 1 & 1 \\
5 & 98 & 115 & 120 & 1 & 1 \\
6 & 92 & 115 & 120 & 1 & 1 \\
7 & 86 & 115 & 120 & 1 & 1 \\
8 & 134 & 99 & 12 & 1 & 1 \\
9 & 135 & 114 & 17 & 1 & 1 \\
10 & 122 & 124 & 19 & 1 & 1 \\
11 & 124 & 126 & 35 & 1 & 1 \\
12 & 126 & 128 & 51 & 1 & 1 \\
13 & 128 & 130 & 67 & 1 & 1 \\
14 & 130 & 132 & 83 & 1 & 1 \\
15 & 132 & 134 & 99 & 1 & 1 \\
16 & 123 & 125 & 34 & 1 & 1 \\
17 & 2 & 19 & 20 & 1 & 1 \\
18 & 19 & 35 & 36 & 1 & 1 \\
19 & 35 & 51 & 52 & 1 & 1 \\
20 & 51 & 67 & 68 & 1 & 1 \\
21 & 67 & 83 & 84 & 1 & 1 \\
22 & 83 & 99 & 102 & 1 & 1 \\
23 & 5 & 22 & 21 & 1 & 1 \\
24 & 22 & 38 & 37 & 1 & 1 \\
25 & 38 & 54 & 53 & 1 & 1 \\
26 & 54 & 70 & 69 & 1 & 1 \\
27 & 70 & 86 & 85 & 1 & 1 \\
28 & 86 & 102 & 99 & 1 & 1 \\
29 & 11 & 28 & 27 & 2 & 1 \\
30 & 28 & 44 & 43 & 2 & 1 \\
31 & 44 & 60 & 59 & 2 & 1 \\
32 & 60 & 76 & 75 & 2 & 1 \\
33 & 76 & 92 & 99 & 2 & 1 \\
34 & 92 & 108 & 102 & 2 & 1 \\
35 & 17 & 34 & 33 & 1 & 1 \\
36 & 34 & 50 & 49 & 1 & 1 \\
37 & 50 & 66 & 65 & 1 & 1 \\
38 & 66 & 82 & 81 & 1 & 1 \\
39 & 82 & 98 & 97 & 1 & 1 \\
40 & 98 & 114 & 113 & 1 & 1 \\
41 & 99 & 100 & 84 & 1 & 1 \\
42 & 100 & 101 & 85 & 1 & 1 \\
43 & 101 & 102 & 86 & 1 & 1 \\
& & & &
\end{tabular}

KAISER ENGINEERS HANFORD S/N:801854

PAGE 6 RUN ID=NNG1973

$02 / 22 / 95$

= Copyright (c) 1984 Celestial Software Inc.

CHECK GEOMETRY

Version $2.007 / 01 / 90$

Bean Nodes
No From To / Ref Ho Hat pincodes Rigid End offset Length Beam

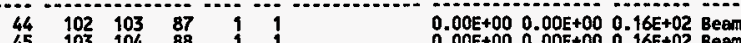
$45103104 \quad 88 \quad 1 \quad 1 \quad 0.00 E+000.00 E+000.16 E+02$ Beam

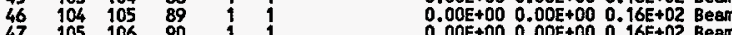
$\begin{array}{lllllllll}47 & 105 & 106 & 90 & 1 & 1 & 0.00 \mathrm{E}+00 & 0.00 \mathrm{E}+00 & 0.16 \mathrm{E}+02 \text { Beam } \\ 48 & 106 & 107 & 91 & 1 & 1 & 0.00 \mathrm{E}+00 & 0.00 \mathrm{E}+000.16 \mathrm{E}+02 \text { Beam }\end{array}$ $\begin{array}{llllllllll}48 & 106 & 107 & 91 & 1 & 1 & 0.00 E+00 & 0.00 E+00 & 0.16 E+02 & \text { Beam } \\ 49 & 107 & 108 & 92 & 1 & 1 & 0.00 E+00 & 0.00 E+00 & 0.16 E+02 & \text { Beam }\end{array}$ $\begin{array}{lllllllll}49 & 107 & 108 & 92 & 1 & 1 & 0.00 E+00 & 0.00 E+C 0 & 0.16 E+02 \\ 50 & 108 & 109 & 93 & 1 & 1 & 0.00 E+00 & 0.00 E+00 & 0.15 E+02 \text { Beann }\end{array}$

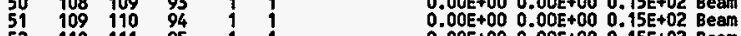
$5211011195 \quad 1 \quad 1 \quad 0.00 E+000.00 E+000.15 E+02$ Beam $0.00 \mathrm{E}+000.00 \mathrm{E}+000.15 \mathrm{E}+02$ Bean $0.00 E+000.00 E+000.15 E+02$ Bean $0.00 E+00 \quad 0.00 E+000.53 E+02$ Bean 0.000 . 0.00 00 O.00E+00 O.2 - 00 + 0 O. $0.00 \% 00$ O. 0.0E+0 0.0E $0.00 \mathrm{O}$ $0.00 E$. $0.00 E+00$ 0.00E+00 0.22E+02 8eam $0.000+00$ 0.00E+00 0.17E 02 Beam $0.00 E+00$ O. $00 E+00$ O.17E+02 Beam 0.000 O O $0.00 E+000.00 E+00$. $0.00 \mathrm{E}+000.00 \mathrm{E}+00 \mathrm{0} 0.12 \mathrm{E}+03$ Beam 作 $0.28 \mathrm{E}+02$ Beam $0.00 E+000.00 E+000.17 E+02$ Bean $0.00 E+00.00 E+000.22 E+02$ Beam Oeam O. . DE $0.00 E+002$ Beam . $0.00 E+000.00 E+00$ 0.22E+02 Beam 0.00E+00 $0.00 E+000.00 E+000.22 E+02$ Beam $0.00 E+00000.22 E+02$ Beam 0.00E+00 0.00E+00 0.28E+02 Beam 0.00E+00 0.00E+00 0.17E+02 Bean $0.00 E+000.00 E+000.22 E+02$ Beam O.0CE+00 0.00E+00 0.22E+02 Bean 0.00E+00 0.00E+00 0.22E+02 Bean $0.00 E+000.00 E+000.22 E+02$ Beam $0.00 E+000.00 E+000.28 E+02$ Bean $0.00 E+000.00 E+00$ 0.17E+02 Beam O. $00 E+00$ O.00E+00 0.14E+02 Bean $0.00 \mathrm{E}+00 \quad 0.00 \mathrm{E}+00$ 0.14E+02 Beam
PLATE ELEMENT COHNECTIVITY

\begin{tabular}{|c|c|c|c|c|c|c|c|c|c|c|}
\hline & & 1 & & & & Mot Thickness & Area & $\begin{array}{l}\text { Shear Neb } \\
\text { Thickness }\end{array}$ & $\begin{array}{l}\text { Aspect } \\
\text { Ratio }\end{array}$ & $\begin{array}{l}\text { Plate } \\
\text { Type }\end{array}$ \\
\hline $\begin{array}{l}\text { QUAD } \\
\text { QUAD } \\
\text { QUUA } \\
\text { QUAD }\end{array}$ & $\begin{array}{l}1 \\
2 \\
3 \\
4 \\
5\end{array}$ & $\begin{array}{l}2 \\
3 \\
4 \\
5 \\
6\end{array}$ & $\begin{array}{l}3 \\
4 \\
5 \\
6 \\
7\end{array}$ & $\begin{array}{l}20 \\
21 \\
22 \\
23 \\
24\end{array}$ & $\begin{array}{l}19 \\
20 \\
21 \\
22 \\
23\end{array}$ & $\begin{array}{ll}1 & 1.000 E+00 \\
1 & 1.000 E+00 \\
1 & 1.000 E+00 \\
1 & 1.000 E+00 \\
1 & 1.000 E+00\end{array}$ & $\begin{array}{l}.007 E+02 \\
3.007 E+02 \\
.007 E+02 \\
3.410 E+02 \\
.410 E+02\end{array}$ & & $\begin{array}{l}6.212 \mathrm{E}-01 \\
6.212 \mathrm{E}-01 \\
6.212 \mathrm{E}-01 \\
7.045 \mathrm{E}-01 \\
7.045 \mathrm{E}-01\end{array}$ & $\begin{array}{l}\mu+B \\
\mu+B \\
\mu+B \\
\mu+B \\
\mu+B\end{array}$ \\
\hline
\end{tabular}


KAISER ENGINEERS HANFORD $S / N: 801854$

$02 / 22 / 95$
$08: 47: 50$

Copright (c) (c) Celestial Software Inc.

CHECK GEOMETRY

Version $2.0 \quad 07 / 01 / 90$

L

Plate Nodes Hat Shear Neb Aspect Plate

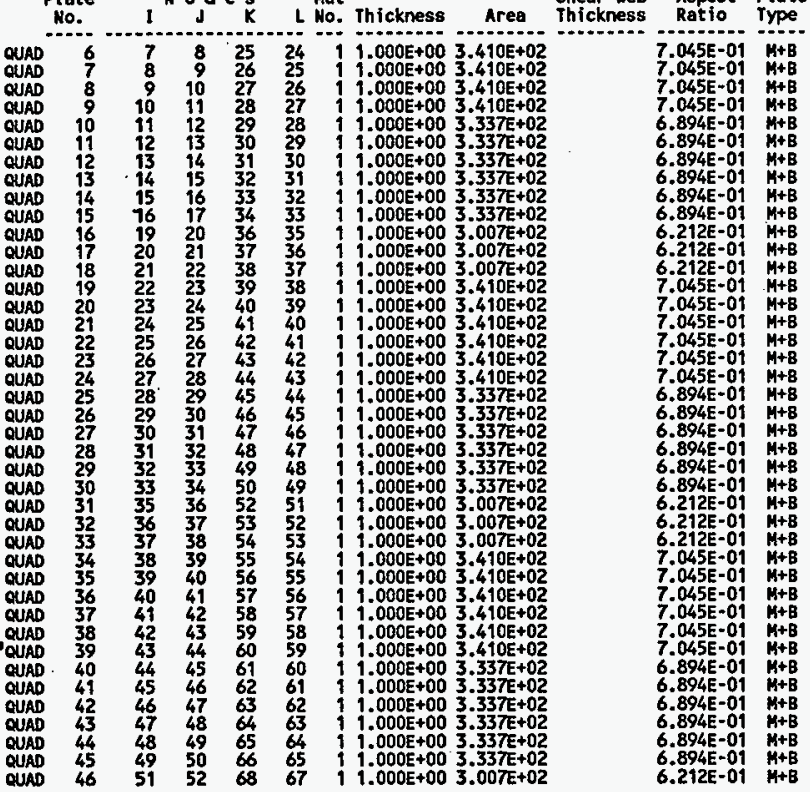

KAISER ENGINEERS HANFORD S/N:801854

PAGE 8 RUN ID=MN61973

$02 / 22 / 95$
$08: 47: 50$

= Copyright (c) 1984 Celestial Software inc. $x$

CHECK GEOHETRY

Version $2.0 \quad 07 / 01 / 90$ $\mathbf{L}$

Plate Nodes Mat Shear Web Aspect Plate No. I J K L No. Thickness Area Thickness Ratio Type $\begin{array}{llllllll}\text { QUAD } & 47 & 52 & 53 & 69 & 68 & 11.000 E+00 & 3.007 E+02\end{array}$

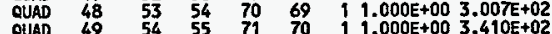

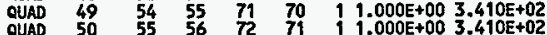
$\begin{array}{llllllll}\text { QUAD } & 50 & 55 & 56 & 72 & 71 & 1.000 \mathrm{E}+00 & 3.410 \mathrm{E}+02 \\ \text { OUAD } & 51 & 56 & 57 & 73 & 72 & 1.000 \mathrm{E}+00 & 3.410 \mathrm{E}+02\end{array}$

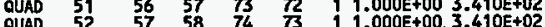
\begin{tabular}{lllllll} 
QUAD & 52 & 57 & 58 & 74 & 73 & 1 \\
QUAD & 53 & 58 & 59 & 75 & 74 & $1.000 E+00.3 .410 E+02$ \\
\hline
\end{tabular} $\begin{array}{llllllll}\text { QUAD } & 53 & 58 & 59 & 75 & 74 & 11.000 E+00 & 3.410 E+02 \\ \text { QUAD } & 54 & 59 & 60 & 76 & 75 & 11.000 E+00 & 3.410 E+02\end{array}$ QUAD 55 5 50 61 77 76 $11.000 E+003.337 \mathrm{E}+02$

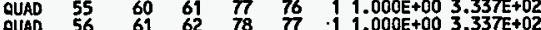

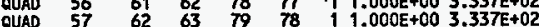
\begin{tabular}{llllllll} 
AUAD & 57 & 62 & 63 & 79 & 78 & $11.000 E+00$ & $3.337 \mathrm{E}+02$ \\
\hline & 58 & 63 & 64 & 80 & 79 & $1.000 \mathrm{~F}+00$ & $3.337 \mathrm{E}+02$
\end{tabular}

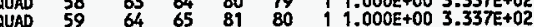
QLAD $59 \quad 64$ 65 81 80, $11.000 E+003.3375+02$ \begin{tabular}{lllllllll} 
& 60 & 65 & 66 & 82 & 81 & 1 & $1.000 E+00$ & $3.337 E+02$ \\
\hline
\end{tabular}

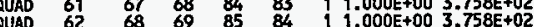

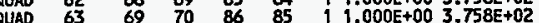
$1.000 E+00 \quad 3.758 E+02$ $1.000 E+004.263 E+02$ $1.0005+004.263 E+02$ $1.000 \mathrm{E}+004.263 \mathrm{E}+02$ $11.000 \mathrm{E}+004.263 \mathrm{E}+02$ $11.000 \mathrm{E}+004.263 \mathrm{E}+02$ $1.000 E+00 \quad 4.263 E+02$ $1.000 \mathrm{E}+004.171 \mathrm{E}+02$ $1.000 \mathrm{E}+004.171 \mathrm{E}+02$ $1.000 E+004.171 E+02$ $1.000 \mathrm{E}+00$ 4.171E+02 $1.000 \mathrm{E}+00$ 4.171 +02 $1,1.000 \mathrm{E}+00002.171 \mathrm{E}+02$ $11.000 \mathrm{E}+0022.255 \mathrm{E}+02$ $11.000 \mathrm{E}+002.558 \mathrm{E}+02$ $11.000 \mathrm{E}+002.558 \mathrm{E}+02$ $11.000 \mathrm{E}+002.558 \mathrm{E}+02$ $11.000 \mathrm{E}+002.558 \mathrm{E}+02$ $11.000 \mathrm{E}+002.558 \mathrm{E}+02$ $11.000 \mathrm{E}+002.558 \mathrm{E}+02$ $11.000 E+002.503 E+02$ $19.000 E+002.503 E+02$ 


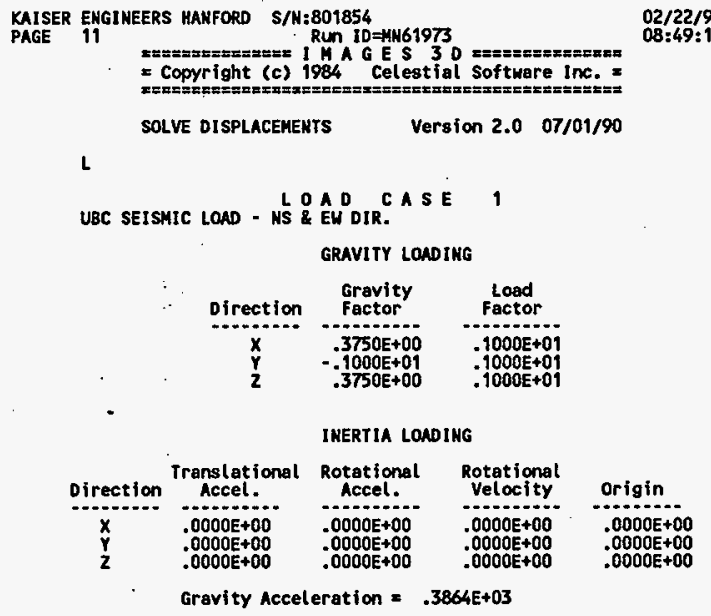

\section{INERTIA LONDING}

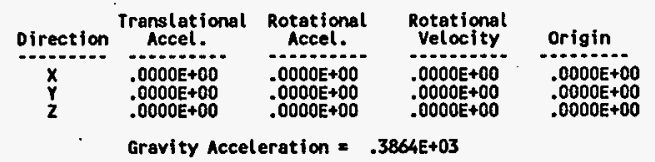

CONCENTRATED LOADS

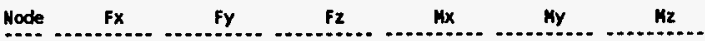
$120 \quad .1125 E+05-.3000 E+05 \quad .1125 E+05 \quad .0000 E+00 \quad .0000 E+00 \quad .0000 E+00$

REFEREMCE TEMPERATURE $=.0000 E+00$
KAISER ENGINEERS HANFORO S/N:801854

PAGE

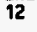

z=:=es=s=es=z=E= RUN ID=MN61973

= Copyright (c) 1984 Celestial Software Inc. =

SOLVE DISPLACEMENTS Version $2.0 \quad 07 / 01 / 90$

L

GRAVITY LOAD

LOAD CASEZ

GRAVITY LOADING

\begin{tabular}{ccc} 
Direction & $\begin{array}{c}\text { Gravity } \\
\text { Factor }\end{array}$ & $\begin{array}{c}\text { Load } \\
\text { Factor }\end{array}$ \\
\hline$X$ & $.0000 E+00$ & $.1000 \mathrm{E}+01$ \\
$\mathbf{Y}$ & $-.1000 \mathrm{E}+01$ & $.1000 \mathrm{01}+01$ \\
$Z$ & $.0000 \mathrm{E}+00$ & $.1000 \mathrm{E}+01$
\end{tabular}

INERTIA LOADING

\begin{tabular}{|c|c|c|c|c|}
\hline Direction & $\begin{array}{c}\text { Translational } \\
\text { Accel. }\end{array}$ & $\begin{array}{l}\text { Rotational } \\
\text { Accel. }\end{array}$ & $\begin{array}{l}\text { Rotational } \\
\text { Velocity }\end{array}$ & Origin \\
\hline $\begin{array}{l}x \\
y \\
z\end{array}$ & $\begin{array}{l}.0000 \mathrm{E}+00 \\
.0000 \mathrm{E}+00 \\
.0000 \mathrm{E}+00\end{array}$ & $\begin{array}{l}.0000 E+00 \\
.0000 E+00 \\
.0000 E+00\end{array}$ & $\begin{array}{l}.0000 E+00 \\
.0000 E+00 \\
.0000 E+00\end{array}$ & $\begin{array}{l}.0000 E+00 \\
.0000 E+00 \\
.0000 E+00\end{array}$ \\
\hline
\end{tabular}

CONCENTRATED LOADS

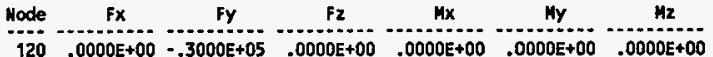

REFERENCE TEMPERATURE $=.0000 E+00$
$02 / 22 / 95$

$08: 49: 22$ 
$\begin{array}{lcc}\text { KAISER ENGLEEERS HANFORO } & S / N: 801854 & 02 / 22 / 95 \\ \text { PAGE } 13 & \text { RUN ID=N1N61973 } & 08: 49: 27\end{array}$

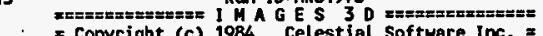
$=$ Copyright (c) 1984 Celestial Software Inc. $=$

SOLVE DISPLACEMENTS Version $2.0 \quad 07 / 01 / 90$

UBC SEISAIC LOAD - SN \& AE D C A S 3

GRAVITY LOADING

\begin{tabular}{|c|c|c|}
\hline Direction & $\begin{array}{l}\text { Gravity } \\
\text { Factor }\end{array}$ & $\begin{array}{l}\text { Load } \\
\text { Factor }\end{array}$ \\
\hline $\begin{array}{l}x \\
y \\
Z\end{array}$ & $\begin{array}{l}-.3750 E+00 \\
-1.1000 E+01 \\
-.3750 E+00\end{array}$ & $\begin{array}{l}1000 \mathrm{E}+01 \\
-1000 \mathrm{E}+01 \\
.1000 \mathrm{E}+01\end{array}$ \\
\hline
\end{tabular}

IHERTIA LOADING

\begin{tabular}{|c|c|c|c|c|}
\hline Direction & $\begin{array}{c}\text { Translational } \\
\text { Accel. }\end{array}$ & $\begin{array}{l}\text { Rotational } \\
\text { Accel. }\end{array}$ & $\begin{array}{l}\text { Rotational } \\
\text { Velocity }\end{array}$ & Origin \\
\hline $\begin{array}{l}\mathbf{X} \\
\mathbf{y} \\
\mathbf{z}\end{array}$ & $\begin{array}{l}.0000 E+00 \\
.0000 E+00 \\
.0000 E+00\end{array}$ & $\begin{array}{l}.0000 E+00 \\
.0000 E+00 \\
.0000 E+00\end{array}$ & $\begin{array}{l}.0000 E+00 \\
.0000 E+00 \\
.0000 E+00\end{array}$ & $\begin{array}{l}.0000 E+00 \\
.0000 E+00 \\
.0000 E+00\end{array}$ \\
\hline
\end{tabular}

CONCENTRATED LOADS

Hode Fx Fy Fu Fu Hy $120=.1125 \mathrm{E}+05-.3000 \mathrm{E}+05-.1125 \mathrm{E}+05 \quad .0000 \mathrm{E}+00 \quad .0000 \mathrm{E}+00 \quad .0000 \mathrm{E}+00$

REFEREMCE TEMPERATURE $=.0000 E+00$
KAISER EKGIHEERS HAHFORD S/H:801854 $02 / 22 / 95$

Run ID=N1N61973 08:49:33

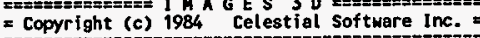

SOLVE DISPLACEMENTS Version 2.0 07/01/90

$\iota$

UBC SEISHIC LOAD - SN O A D CA SE

GRAVITY LOADING

\begin{tabular}{ccc} 
Direction & $\begin{array}{c}\text { Gravity } \\
\text { Factor }\end{array}$ & $\begin{array}{c}\text { Load } \\
\text { Factor }\end{array}$ \\
\hline $\mathbf{X}$ & $-.3750 \mathrm{E}+00$ & $. .1000 \mathrm{E}+01$ \\
$\mathbf{Y}$ & $-.1000 \mathrm{0}+01$ & $.1000 \mathrm{E}+01$ \\
$\mathbf{2}$ & $.3750 \mathrm{01}+00$ & $.1000 \mathrm{E}+01$
\end{tabular}

INERTIA LOADING

\begin{tabular}{ccccc} 
Direction & $\begin{array}{c}\text { Translational } \\
\text { Accel. }\end{array}$ & $\begin{array}{c}\text { Rotational } \\
\text { Accel. }\end{array}$ & $\begin{array}{c}\text { Rotational } \\
\text { Velocity }\end{array}$ & Origin \\
\hline $\mathbf{X}$ & $.0000 \mathrm{E}+00$ & $.0000 \mathrm{E}+00$ & $.0000 \mathrm{E}+00$ & $.0000 \mathrm{E}+00$ \\
$\mathbf{Y}$ & $.0000 \mathrm{E}+00$ & $.0000 \mathrm{E}+00$ & $.0000 \mathrm{E}+00$ & $.0000 \mathrm{E}+00$ \\
$\mathbf{Z}$ & $.0000 \mathrm{E}+00$ & $.0000 \mathrm{E}+00$ & $.0000 \mathrm{E}+00$ & $.0000 \mathrm{E}+00$ \\
& & &
\end{tabular}

CONCENTRATED LOADS

\begin{tabular}{ccccccc} 
Node & Fx & Fy & Fz & Mx & My & Mz \\
\hdashline 120 & $-.1125 \mathrm{E}+05$ & $-.3000 \mathrm{E}+05$ & $.1125 \mathrm{E}+05$ & $.0000 \mathrm{E}+00$ & $.0000 \mathrm{E}+00$ & $.0000 \mathrm{E}+00$
\end{tabular}

REFERENCE TEMPERATURE $\approx .0000 E+00$ 
KAISER ENGINEERS HANFORD S/N:801854

PAGE 15

Run ID =\#1161973

$02 / 22 / 95$
$08 * 49 \cdot 39$

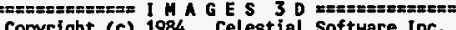

I Copyright (c) 1984 Celestial Software Inc. =

SOLVE DISPLACENENTS

Version $2.0 \quad 07 / 01 / 90$

UBC SEISMIC LOAD - US \& LED C A S E 5

GRAVITY LOADIME

\begin{tabular}{ccc} 
Direction & $\begin{array}{c}\text { Gravity } \\
\text { Factor }\end{array}$ & $\begin{array}{c}\text { Load } \\
\text { Factor }\end{array}$ \\
\hline $\mathbf{X}$ & $. .3750 \mathrm{O}+00$ & $.1000 \mathrm{0}+01$ \\
$\mathbf{Y}$ & $-.1000 \mathrm{0}+01$ & $.1000 \mathrm{01}+01$ \\
$\mathbf{Z}$ & $-.3750 \mathrm{O}+00$ & $.1000 \mathrm{01}+01$
\end{tabular}

INERTIA LOADING

\begin{tabular}{|c|c|c|c|c|}
\hline irection & $\begin{array}{c}\text { Translational } \\
\text { Accel. }\end{array}$ & $\begin{array}{c}\text { Rotational } \\
\text { Accel. }\end{array}$ & $\begin{array}{l}\text { Rotational } \\
\text { Velocity }\end{array}$ & Origin \\
\hline $\begin{array}{l}x \\
y \\
z\end{array}$ & $\begin{array}{l}.0000 E+00 \\
.0000 E+00 \\
.0000 E+00\end{array}$ & $\begin{array}{l}.0000 E+00 \\
.0000 E+00 \\
.0000 E+00\end{array}$ & $\begin{array}{l}.0000 E+00 \\
.0000 E+00 \\
.0000 E+00\end{array}$ & $\begin{array}{l}.0000 E+00 \\
.0000 E+00 \\
.0000 E+00\end{array}$ \\
\hline
\end{tabular}

CONCENTRATED LOADS

Node FX Fy Fz Mx

$120.1125 \mathrm{E}+05-.3000 \mathrm{E}+05-.1125 \mathrm{E}+05 \quad .0000 \mathrm{E}+00 \quad .0000 \mathrm{E}+00 \quad .0000 \mathrm{E}+00$

REFERENCE TEMPERATURE $=.0000 E+00$

KAISER ENGINEERS HANFORD S/N:801854

$02 / 22 / 95$

PAGE 16

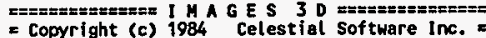
(D)

SOLVE DISPLACEMENTS

Version $2.0 \quad 07 / 01 / 90$

UBC SEISMIC LOAD - WS \& EUDIR.

APPLIED LOAD VECTOR

\begin{tabular}{|c|c|c|c|c|c|}
\hline de & $F x$ & & & & \\
\hline $\begin{array}{l}1 \\
2 \\
3 \\
4 \\
5 \\
6 \\
7 \\
8 \\
9 \\
10 \\
11 \\
12 \\
13 \\
14 \\
15 \\
16 \\
17 \\
18 \\
19 \\
20 \\
21 \\
22 \\
23 \\
24 \\
25 \\
26 \\
27 \\
28 \\
29 \\
30 \\
31 \\
32 \\
33\end{array}$ & 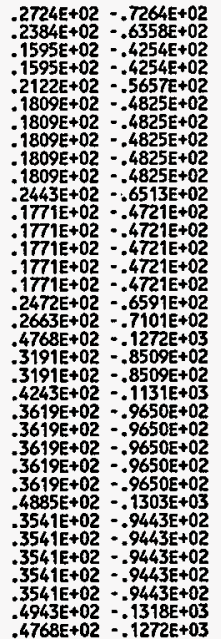 & 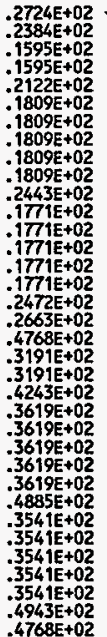 & $\begin{array}{l}9 E+02 \\
7 E+02 \\
0 E+00 \\
0 E+00 \\
7 E+02 \\
0 E+00 \\
O E+00 \\
0 E+00 \\
0 E+00 \\
0 E+00 \\
3 E+02 \\
0 E+00 \\
0 E+00 \\
0 E+00 \\
0 E+00 \\
0 E+00 \\
7 E+02 \\
0 E+04 \\
0 E+00 \\
0 E+00 \\
0 E+00 \\
0 E+00 \\
0 E+00 \\
0 E+00 \\
0 E+00 \\
0 E+00 \\
0 E+00 \\
0 E+00 \\
0 E+00 \\
0 E+00 \\
0 E+00 \\
0 E+00 \\
0 E+00\end{array}$ & & $\begin{array}{l}+03 \\
+02 \\
+00 \\
+00 \\
+02 \\
+00 \\
+00 \\
+00 \\
+00 \\
+00 \\
+02 \\
+00 \\
+00 \\
+00 \\
+00 \\
+00 \\
+02 \\
+03 \\
+00 \\
+00 \\
+00\end{array}$ \\
\hline
\end{tabular}


KAISER EHGIMEERS HAMFORD S/N:801854

PAGE 17

RUn 10=N1161973

$02 / 22 / 95$

= Copyright (c) 1984 Celestial Software Inc.

SOLVE DISPLACEMENTS Version 2.0 07/01/90

I

LOADCASE 1

NS \& EW DIR.

APPLIED LOAD VECTOR

\begin{tabular}{|c|c|c|c|c|c|}
\hline xde & $F \mathbf{x}$ & $F_{2}$ & $M x$ & My & $M_{2}$ \\
\hline $\begin{array}{l}36 \\
37 \\
38 \\
39 \\
40 \\
41 \\
42 \\
43 \\
44 \\
45 \\
46 \\
47 \\
48 \\
49 \\
50 \\
51 \\
52 \\
53 \\
54 \\
55 \\
56 \\
57 \\
58 \\
59 \\
60 \\
61 \\
62 \\
63 \\
64 \\
65 \\
66 \\
67 \\
68 \\
69 \\
70 \\
71 \\
72 \\
73 \\
76\end{array}$ & 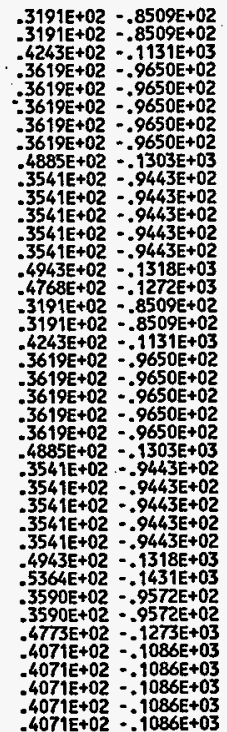 & $\begin{array}{l}.3191 E+02 \\
.3191 E+02 \\
.4243 E+02 \\
.3619 E+02 \\
.3619 E+02 \\
.3619 E+02 \\
.3619 E+02 \\
.3619 E+02 \\
.4885 E+02 \\
.3541 E+02 \\
.3541 E+02 \\
.3541 E+02 \\
.3541 E+02 \\
.3541 E+02 \\
.4943 E+02 \\
.4768 E+02 \\
.3191 E+02 \\
.3191 E+02 \\
.4243 E+02 \\
.3619 E+02 \\
.3619 E+02 \\
.3619 E+02 \\
.3619 E+02 \\
.3619 E+02 \\
.4885 E+02 \\
.3541 E+02 \\
.3541 E+02 \\
.3541 E+02 \\
.3541 E+02 \\
.3541 E+02 \\
.4943 E+02 \\
.5364 E+02 \\
.3590 E+02 \\
.3590 E+02 \\
.4773 E+02 \\
.4071 E+02 \\
.4071 E+02 \\
.4071 E+02 \\
.4071 E+02 \\
.4071 E+02\end{array}$ & $\begin{array}{l}.0000 E+00 \\
.0000 E+00 \\
.0000 E+00 \\
.0000 E+00 \\
.0000 E+00 \\
.0000 E+00 \\
.0000 E+00 \\
.0000 E+00 \\
.0000 E+00 \\
.0000 E+00 \\
.0000 E+00 \\
.0000 E+00 \\
.0000 E+00 \\
.0000 E+00 \\
.0000 E+00 \\
.0000 E+00 \\
.0000 E+00 \\
.0000 E+00 \\
.0000 E+00 \\
.0000 E+00 \\
.0000 E+00 \\
.0000 E+00 \\
.0000 E+00 \\
.0000 E+00 \\
.0000 E+00 \\
.0000 E+00 \\
.0000 E+00 \\
.0000 E+00 \\
.0000 E+00 \\
.0000 E+00 \\
.0000 E+00 \\
.8644 E+01 \\
.0000 E+00 \\
.0000 E+00 \\
.8644 E+01 \\
.0000 E+00 \\
.0000 E+00 \\
.0000 E+00 \\
.0000 E+00 \\
.0000 E+00\end{array}$ & $\begin{array}{l}.0000 E+00 \\
.0000 E+00 \\
.0000 E+00 \\
.0000 E+00 \\
.0000 E+00 \\
.0000 E+00 \\
.0000 E+00 \\
.0000 E+00 \\
.0000 E+00 \\
.0000 E+00 \\
.0000 E+00 \\
.0000 E+00 \\
.0000 E+00 \\
.0000 E+00 \\
.0000 E+00 \\
.0000 E+00 \\
.0000 E+00 \\
.0000 E+00 \\
.0000 E+00 \\
.0000 E+00 \\
.0000 E+00 \\
.0000 E+00 \\
.0000 E+00 \\
.0000 E+00 \\
.0000 E+00 \\
.0000 E+00 \\
.0000 E+00 \\
.0000 E+00 \\
.0000 E+00 \\
.0000 E+00 \\
.0000 E+00 \\
.0000 E+00 \\
.0000 E+00 \\
.0000 E+00 \\
.0000 E+00 \\
.0000 E+00 \\
.0000 E+00 \\
.0000 E+00 \\
.0000 E+00 \\
.0000 E+00\end{array}$ & 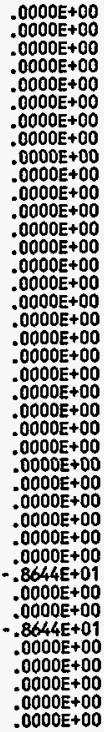 \\
\hline
\end{tabular}

KAISER ENGINEERS HANFORD S/N:801854

PAGE 18 RUN ID=KN61973

$02 / 22 / 95$

= Copyright (c) 1984 Celestial Software Inc. =

SOLVE DISPLACEHENTS

Version $2.0 \quad 07 / 01 / 90$

$\mathbf{L}$

LOAD CASE

\& EW DIR.

APPLIED LOAD VECTOR

\begin{tabular}{|c|c|c|c|c|c|}
\hline & $F$ & Fz & $M x$ & & \\
\hline $\begin{array}{l}76 \\
77 \\
78 \\
79 \\
80 \\
81 \\
82 \\
83 \\
84 \\
85 \\
86 \\
87 \\
88 \\
89 \\
90 \\
91 \\
92 \\
93 \\
94 \\
95 \\
96 \\
97 \\
98 \\
99 \\
100 \\
101 \\
102 \\
103 \\
104 \\
105 \\
106 \\
107 \\
108 \\
109 \\
110 \\
111 \\
112 \\
113 \\
114\end{array}$ & 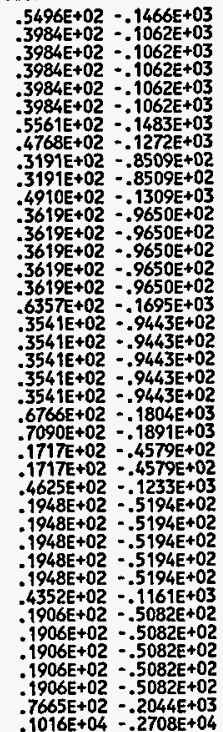 & $\begin{array}{l}.39 \\
.39 \\
.39 \\
.39 \\
.39 \\
.55 \\
.47 \\
.31 \\
.31 \\
.49 \\
.36 \\
.36 \\
.36 \\
.36 \\
.36 \\
.63 \\
.35 \\
.35 \\
.35 \\
.35 \\
.35 \\
.67 \\
.70 \\
.17 \\
.17 \\
.46 \\
.19 \\
.19 \\
.19 \\
.19 \\
.19 \\
.43 \\
.19 \\
.19 \\
.19 \\
.19 \\
.19 \\
.76 \\
.10\end{array}$ & 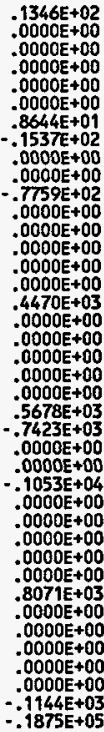 & $\begin{array}{l}0 E+00 \\
0 E+00 \\
0 E+00 \\
0 E+00 \\
0 E+00 \\
0 E+00 \\
0 E+00 \\
0 E+00 \\
0 E+00 \\
10 E+00 \\
5 E+02 \\
10 E+00 \\
10 E+00 \\
10 E+00 \\
10 E+00 \\
10 E+00 \\
9 E+03 \\
10 E+00 \\
10 E+00 \\
10 E+00 \\
10 E+00 \\
10 E+00 \\
1 E+03 \\
19 E+03 \\
6 E=05 \\
16 E-05 \\
18 E+03 \\
30 E+00 \\
30 E+00 \\
30 E+00 \\
30 E+00 \\
10 E+00 \\
27 E+03 \\
10 E+00 \\
70 E-04 \\
39 E-04 \\
29 E-03 \\
29 E-03 \\
06 E+03\end{array}$ & 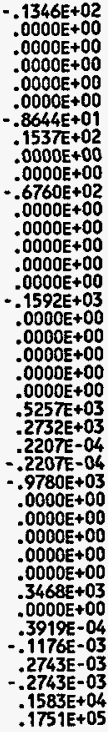 \\
\hline
\end{tabular}


KAISER ENGINEERS HANFORD S/N:801854

PAGE 19

Run ID=AN61975

$02 / 22 / 95$
$08: 49: 45$

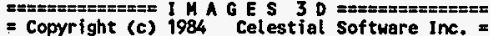

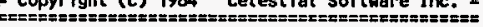

SOLVE DISPLACEMENTS

Version $2.007 / 01 / 90$

L

UBC SEISHIC LOAD - NS \& E EN CAR. A S E 1

APPLIED LOAD VECTOR

\begin{tabular}{|c|c|c|c|c|c|}
\hline de & Fy & $\mathrm{Fz}$ & $M x$ & My & $\mathrm{Mz}$ \\
\hline $\begin{array}{l}116 \\
117 \\
118 \\
119 \\
120 \\
121 \\
122 \\
123 \\
124 \\
125 \\
126 \\
127 \\
128 \\
129 \\
130 \\
131 \\
132 \\
133\end{array}$ & 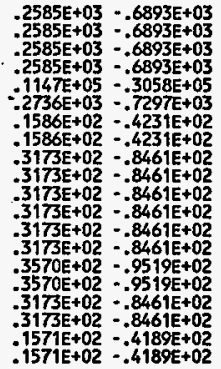 & $\begin{array}{l}.2585 E+03 \\
.2585 E+03 \\
.2585 E+03 \\
.2585 E+03 \\
.1147 \mathrm{E}+05 \\
.2736 \mathrm{E}+03 \\
.1586 \mathrm{E}+02 \\
.1586 \mathrm{E}+02 \\
.3173 \mathrm{E}+02 \\
.3173 \mathrm{E}+02 \\
.3173 \mathrm{E}+02 \\
.3173 \mathrm{E}+02 \\
.3173 \mathrm{E}+02 \\
.3173 \mathrm{E}+02 \\
.3570 \mathrm{E}+02 \\
.3570 \mathrm{E}+02 \\
.3173 \mathrm{E}+02 \\
.3173 \mathrm{E}+02\end{array}$ & $\begin{array}{r}.4221 E+04 \\
.6633 E+04 \\
.4221 E+04 \\
.1808 E+04 \\
-.3416 E+03 \\
.1216 E+04 \\
.1537 E+02 \\
.1537 E+02 \\
.0000 E+00 \\
.0000 E+00 \\
.0000 E+00 \\
.0000 E+00 \\
.0000 E+00 \\
.0000 E+00 \\
.8644 E+01 \\
.8644 E+01 \\
-.2401 E+02 \\
-.1537 E+02 \\
.0000 E+00\end{array}$ & & $\begin{array}{l}E+04 \\
E+04 \\
E+04 \\
E+04 \\
E+03 \\
E+04 \\
E+02 \\
E+02 \\
E+00 \\
E+00 \\
E+00 \\
E+00 \\
E+00 \\
E+00 \\
E+01 \\
E+01 \\
E+02 \\
E+02 \\
E+02 \\
E+02\end{array}$ \\
\hline
\end{tabular}

KAISER ENGINEERS HANFORD S/N:801854
PAGE 20
RUN ID

Run ID = 1461973

$02 / 22 / 95$

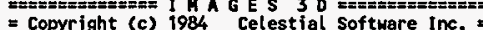

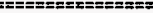

SOLVE DISPLACEMENTS

Version $2.0 \quad 07 / 01 / 90$

$\mathbf{L}$

UBC SEISMIC LOAD - LS O A D C A S E 1

Displacements

\begin{tabular}{|c|c|c|c|c|c|c|}
\hline & T $r a$ & 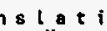 & & & 51 & \\
\hline Node & $x$ & & & $x$ & $\gamma$ & $\mathbf{Z}$ \\
\hline & & $.0000 E+00$ & & .90 & & -.1 \\
\hline & $.0000 E+00$ & $.0000 \mathrm{E}+00$ & & & & \\
\hline & . OUUUE+ $.0000 E+00$ & . $.0000 \mathrm{E}+00$ & & $3 E-04$ & & \\
\hline & $.0000 \mathrm{E}+00$ & $.0000 E+00$ & .0000 & $10 \mathrm{E}+00$ & $.0000 \mathrm{E}+00$ & \\
\hline & .00 & $.0000 E+00$ & .00 & 00 & & \\
\hline & .000 & .0000 & & $+\infty 0$ & & \\
\hline & $0 E+00$ & $.0000 \mathrm{E}+00$ & & $=00$ & & \\
\hline 10 & $.0000 E+00$ & $.0000 E+00$ & $.0000 E$ & $D E+0 D$ & & \\
\hline & & & & & & \\
\hline & $E+00$ & .0000 & .0000 & -03 & .00 & \\
\hline & $\mathrm{E}+00$ & $.0000 \mathrm{E}$ & .0000 & & & \\
\hline & EE+OO & $.0000 \mathrm{E}$ & $00 \%$ & & $E+00$ & \\
\hline & .000 & & & & & \\
\hline & $.0000 \mathrm{E}+00$ & $.0000 E$ & $+00 \%$ & .19 & in & 0 \\
\hline & $E-04$ & .5134 & & & & \\
\hline & & & & & & \\
\hline & 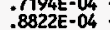 & & 2 & & & \\
\hline & & & & & & \\
\hline & 1 & & & & & \\
\hline & & & & & & \\
\hline & $\frac{4}{3}$ & & 4 & & & \\
\hline & 7 & & & & & \\
\hline & 172 & & & & & \\
\hline & & & & & & 00 \\
\hline & 4 & & & & & \\
\hline & & & & & & \\
\hline & & & & & 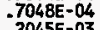 & -.35 \\
\hline & & & 2 & .72 & $\begin{array}{l}2045 E-03 \\
2934 E-03\end{array}$ & .00 \\
\hline & & & & & $-2734 \mathrm{E}-03$ & .00 \\
\hline & & & & 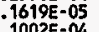 & 04 & .31 \\
\hline & & & & & $E-03$ & \\
\hline
\end{tabular}



KAISER ENGIHEERS HAHFORD $\mathrm{S} / \mathrm{H}: 801854$
PAGE 21

RUn 10=NH61973

$02 / 22 / 95$
$08: 49: 48$

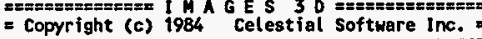

SOLVE DISPLACENENTS Version $2.0 \quad 07 / 01 / 90$

$\mathbf{L}$

UBC SEISHIC LOAD - US \& EH DIR.

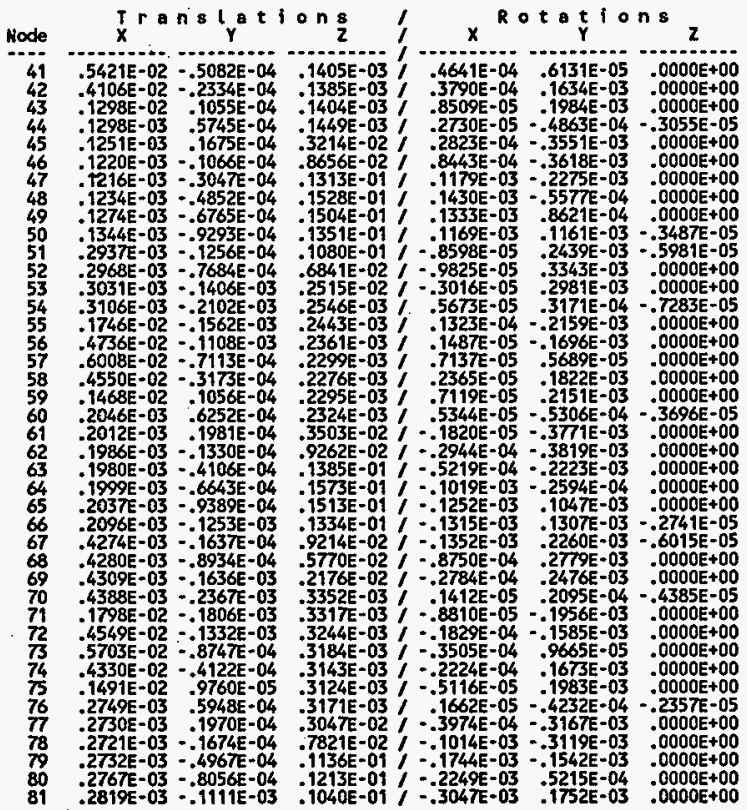

KAISER ENGINEERS HAHFORD S/N:801854

PAGE 22 RUN ID=MN61973

$02 / 22 / 95$

$=$ Copyright (c) 1984 Celestial software Inc. $x$

SOLVE DISPLACEMENTS Version $2.0 \quad 07 / 01 / 90$

$\mathbf{L}$

UBC SEISHIC LOAD - LO AD CASE

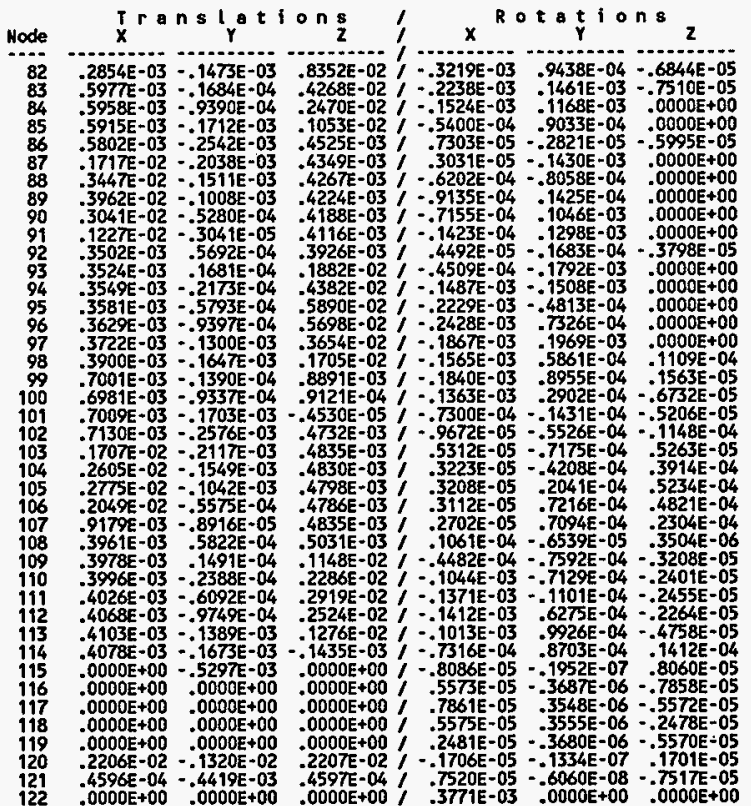


KAISER ENGINEERS HAHFORD S/N:801854

PAGE 23

Run ID =N161973

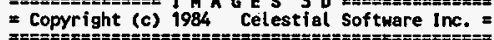

$02 / 22 / 95$

08:49:48

SOLVE DISPLACENENTS

Version $2.007 / 01 / 90$

1

UBC SEISMIC LOAD - NS \& EN DIR.

\begin{tabular}{|c|c|c|c|c|c|c|}
\hline Node & ${ }_{x}^{t} r$ & $8 t_{\gamma}=t$ & $\begin{array}{l}n s \\
z\end{array}$ & $x^{R o}$ & t a t & $\begin{array}{ll}n & \\
& z\end{array}$ \\
\hline $\begin{array}{l}123 \\
124 \\
125 \\
126 \\
127 \\
128 \\
129 \\
130 \\
131 \\
132 \\
133 \\
134 \\
135\end{array}$ & $\begin{array}{l}.0000 \mathrm{E}+00 \\
.6776 \mathrm{E}-04 \\
.7736 \mathrm{E}-04 \\
.1698 \mathrm{E}-03 \\
.1457 \mathrm{E}-03 \\
.2941 \mathrm{E}-03 \\
.2173 \mathrm{E}-03 \\
.4278 \mathrm{E}-03 \\
.2949 \mathrm{E}-03 \\
.5993 \mathrm{E}-03 \\
.3814 \mathrm{E}-03 \\
.6976 \mathrm{E}-03 \\
.4165 \mathrm{E}-03\end{array}$ & $\begin{array}{r}.0000 E+00 \\
.4831 \mathrm{E}-04 \\
-.8661 \mathrm{E}-04 \\
.7691 \mathrm{E}-04 \\
-.1428 \mathrm{E}-03 \\
.9151 \mathrm{E}-04 \\
-.1812 \mathrm{E}-03 \\
.9769 \mathrm{E}-04 \\
-.2060 \mathrm{E}-03 \\
.9931 \mathrm{E}-04 \\
-.2105 \mathrm{E}-03 \\
.9745 \mathrm{E}-04 \\
-.2072 \mathrm{E}-03\end{array}$ & $\begin{array}{l}.0000 \mathrm{E}+00 \\
.7697 \mathrm{E}-02 \\
.7392 \mathrm{E}-02 \\
.1322 \mathrm{E}-01 \\
.1172 \mathrm{E}-01 \\
.1529 \mathrm{E}-01, \\
.1147 \mathrm{E}-01, \\
.1366 \mathrm{E}-01, \\
.7505 \mathrm{E}-02 \\
.7807 \mathrm{E}-02 \\
.1441 \mathrm{E}-02 \\
.3395 \mathrm{E}-02 \\
.1501 \mathrm{E}-02,\end{array}$ & $\begin{array}{l}.3755 E-03 \\
.3208 E-03 \\
.2947 \mathrm{E}-03 \\
.1806 \mathrm{E}-03 \\
.9764 \mathrm{E}-04 \\
.6719 \mathrm{E}-05 \\
-.1200 \mathrm{E}-03 \\
-.1540 \mathrm{E}-03 \\
-.2377 \mathrm{E}-03 \\
-.2706 \mathrm{E}-03 \\
-.2039 \mathrm{E}-03 \\
-.2627 \mathrm{E}-03 \\
-.1512 \mathrm{E}-03\end{array}$ & $\begin{array}{l}.0000 \mathrm{E}+00 \\
.9038 \mathrm{E}-04 \\
.3874 \mathrm{E}-04 \\
.1631 \mathrm{E}-03 \\
.6252 \mathrm{E}-04 \\
.2055 \mathrm{E}-03 \\
.5647 \mathrm{E}-04 \\
.2185 \mathrm{E}-03 \\
-.9127 \mathrm{E}-05 \\
.2083 \mathrm{E}-03 \\
-.3025 \mathrm{E}-04 \\
.1577 \mathrm{E}-03 \\
.4722 \mathrm{E}-04\end{array}$ & $\begin{array}{l}.0000 E+00 \\
-.4368 E-05 \\
-.4036 \mathrm{E}-05 \\
-.5151 \mathrm{E}-05 \\
. .2968 \mathrm{E}-05 \\
-.5882 \mathrm{E}-05 \\
-.3400 \mathrm{E}-05 \\
-.6492 \mathrm{E}-05 \\
-.3775 \mathrm{E}-05 \\
-.5362 \mathrm{E}-05 \\
-.1787 \mathrm{E}-05 \\
-.9112 \mathrm{E}-05 \\
-.4481 \mathrm{E}-05\end{array}$ \\
\hline
\end{tabular}

KAISER ENGINEERS HANFORD S/K:801854

PAGE 24

Run $10=$ HN61973

$02 / 22 / 95$

$=$ Copyright (c) 1984 Celestial Software Inc. =

SOLVE DISPLACEMENTS

Version $2.0 \quad 07 / 01 / 90$

$\mathbf{L}$

GRAVITY LOAD

LOAD CASE 2

APPLIED LOAD VECTON

\begin{tabular}{|c|c|c|c|c|c|}
\hline ocle & Fy & & $M x$ & My & \\
\hline $\begin{array}{l}1 \\
2 \\
3 \\
4 \\
5 \\
6 \\
7 \\
8 \\
9 \\
10 \\
11 \\
12 \\
13 \\
14 \\
15 \\
16 \\
17 \\
18 \\
19 \\
20 \\
21 \\
22 \\
23 \\
24 \\
25 \\
26 \\
27 \\
28 \\
29 \\
30 \\
31 \\
32 \\
33 \\
34\end{array}$ & $\begin{array}{l}.0000 \\
.0000 \\
.0000 \\
.0000 \\
.0000 \\
.0000 \\
.0000 \\
.0000 \\
.0000 \\
.0000 \\
.0000 \\
.0000 \\
.0000 \\
.0000 \\
.0000 \\
.0000 \\
.0000 \\
.0000 \\
.0000 \\
.0000 \\
.0000 \\
.0000 \\
.0000 \\
.0000 \\
.0000 \\
.0000 \\
.0000 \\
.0000 \\
.0000 \\
.0000 \\
.0000\end{array}$ & $\begin{array}{r}.1705 E-14 \\
.0000 E+00 \\
.0000 E+00 \\
.0000 E+00 \\
.0000 E+00 \\
.0000 E+00 \\
.0000 E+00 \\
.0000 E+00 \\
.0000 E+00 \\
.0000 E+00 \\
.0000 E+00 \\
.0000 E+00 \\
.0000 E+00 \\
.0000 E+00 \\
.0000 E+00 \\
.0000 E+00 \\
.0000 E+00 \\
-.4351 E-14 \\
.0000 E+00 \\
.0000 E+00 \\
.0000 E+00 \\
.0000 E+00 \\
.0000 E+00 \\
.0000 E+00 \\
.0000 E+00 \\
.0000 E+00 \\
.0000 E+00 \\
.0000 E+00 \\
.0000 E+00 \\
.0000 E+00 \\
.0000 E+00 \\
.0000 E+00 \\
.0000 E+00 \\
.0000 E+00 \\
.0000 E+00\end{array}$ & 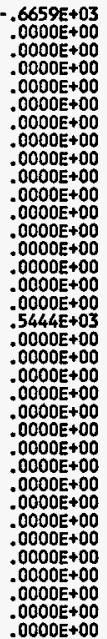 & 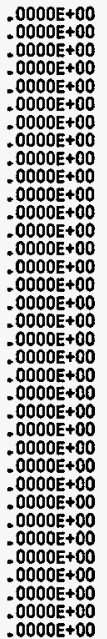 & $\begin{array}{l}.00 \\
.00 \\
.00 \\
.00 \\
.00 \\
.00 \\
.00 \\
.00 \\
.00 \\
.00 \\
.00 \\
.00 \\
.00 \\
.00 \\
.00 \\
.00 \\
.00 \\
.00 \\
.00 \\
.00 \\
.00 \\
.00 \\
.00 \\
.00 \\
.00 \\
.00 \\
.00 \\
.00 \\
.00 \\
.00 \\
.00 \\
.00\end{array}$ \\
\hline
\end{tabular}


KAISER ENGINEERS HAHFORD S/H:801854

PAGE 25

Run ID=N1N61973

$02 / 22 / 95$
$08: 49: 48$

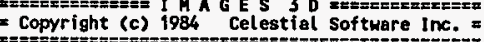

SOLVE DISPLACEMENTS

Version $2.0 \quad 07 / 01 / 90$

$\mathbf{L}$

GRAVITY LOAD

LOAD CASE 2

APPLIED LOAD VECTOR

\begin{tabular}{|c|c|c|c|c|c|}
\hline Node & $\mathbf{F x}$ & $\mathbf{F z}$ & $M x$ & Ny & Mz \\
\hline $\begin{array}{l}36 \\
37 \\
38 \\
39 \\
40 \\
41 \\
42 \\
43 \\
44 \\
45 \\
46 \\
47 \\
48 \\
49 \\
50 \\
51 \\
52 \\
53 \\
54 \\
55 \\
56 \\
57 \\
58 \\
59 \\
60 \\
61 \\
62 \\
63 \\
64 \\
65 \\
66 \\
67 \\
68 \\
69 \\
70 \\
71 \\
72 \\
73 \\
74 \\
75\end{array}$ & 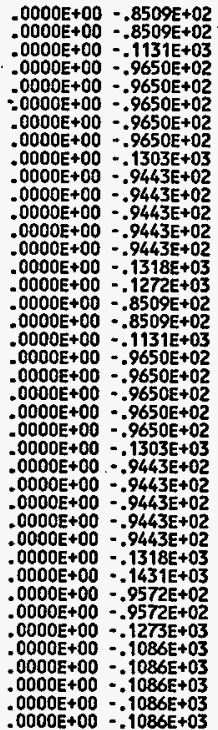 & $\begin{array}{l}.0000 E+00 \\
.0000 E+00 \\
.0000 E+00 \\
.0000 E+00 \\
.0000 E+00 \\
.0000 E+00 \\
.0000 E+00 \\
.0000 E+00 \\
.0000 E+00 \\
.0000 E+00 \\
.0000 E+00 \\
.0000 E+00 \\
.0000 E+00 \\
.0000 E+00 \\
.0000 E+00 \\
.0000 E+00 \\
.0000 E+00 \\
.0000 E+00 \\
.0000 E+00 \\
.0000 E+00 \\
.0000 E+00 \\
.0000 E+00 \\
.0000 E+00 \\
.0000 E+00 \\
.0000 E+00 \\
.0000 E+00 \\
.0000 E+00 \\
.0000 E+00 \\
.0000 E+00 \\
.0000 E+00 \\
.0000 E+00 \\
.0000 E+00 \\
.0000 E+00 \\
.0000 E+00 \\
.0000 E+00 \\
.0000 E+00 \\
.0000 E+00 \\
.0000 E+00 \\
.0000 E+00 \\
.0000 E+00\end{array}$ & $\begin{array}{l}.0000 E+00 \\
.0000 E+00 \\
.0000 E+00 \\
.0000 E+00 \\
.0000 E+00 \\
.0000 E+00 \\
.0000 E+00 \\
.0000 E+00 \\
.0000 E+00 \\
.0000 E+00 \\
.0000 E+00 \\
.0000 E+00 \\
.0000 E+00 \\
.0000 E+00 \\
.0000 E+00 \\
.0000 E+00 \\
.0000 E+00 \\
.0000 E+00 \\
.0000 E+00 \\
.0000 E+00 \\
.0000 E+00 \\
.0000 E+00 \\
.0000 E+00 \\
.0000 E+00 \\
.0000 E+00 \\
.0000 E+00 \\
.0000 E+00 \\
.0000 E+00 \\
.0000 E+00 \\
.0000 E+00 \\
.0000 E+00 \\
.0000 E+00 \\
.0000 E+00 \\
.0000 E+00 \\
.0000 E+00 \\
.0000 E+00 \\
.0000 E+00 \\
.0000 E+00 \\
.0000 E+00 \\
.0000 E+00\end{array}$ & $\begin{array}{l}.0000 E+00 \\
.0000 E+00 \\
.0000 E+00 \\
.0000 E+00 \\
.0000 E+00 \\
.0000 E+00 \\
.0000 E+00 \\
.0000 E+00 \\
.0000 E+00 \\
.0000 E+00 \\
.0000 E+00 \\
.0000 E+00 \\
.0000 E+00 \\
.0000 E+00 \\
.0000 E+00 \\
.0000 E+00 \\
.0000 E+00 \\
.0000 E+00 \\
.0000 E+00 \\
.0000 E+00 \\
.0000 E+00 \\
.0000 E+00 \\
.0000 E+00 \\
.0000 E+00 \\
.0000 E+00 \\
.0000 E+00 \\
.0000 E+00 \\
.0000 E+00 \\
.0000 E+00 \\
.0000 E+00 \\
.0000 E+00 \\
.0000 E+00 \\
.0000 E+00 \\
.0000 E+00 \\
.0000 E+00 \\
.0000 E+00 \\
.0000 E+00 \\
.0000 E+00 \\
.0000 E+00 \\
.0000 E+00\end{array}$ & $\begin{array}{l}.0000 E+00 \\
.0000 E+00 \\
.0000 E+00 \\
.0000 E+00 \\
.0000 E++00 \\
.0000 E++00 \\
.0000 E++00 \\
.0000 E++00 \\
.0000 E+00 \\
.0000 E+00 \\
.0000 E+00 \\
.0000 E+00 \\
.0000 E+00 \\
.0000 E+00 \\
.0000 E+00 \\
.0000 E+00 \\
.0000 E+00 \\
.0000 E+00 \\
.0000 E+00 \\
.0000 E+00 \\
.0000 E+00 \\
.0000 E+00 \\
.0000 E+00 \\
.0000 E+00 \\
.0000 E+00 \\
.0000 E+00 \\
.0000 E+00 \\
.0000 E+00 \\
.0000 E+00 \\
.0000 E+00 \\
.0000 E+00 \\
.0000 E+00 \\
.0000 E+00 \\
.0000 E+00 \\
.0000 E+00 \\
.0000 E+00 \\
.0000 E+00 \\
.0000 E+00 \\
.0000 E+00 \\
.0000 E+00\end{array}$ \\
\hline
\end{tabular}

KAISER ENGINEERS HANFORD S/H:801854

Run 10=4N61973

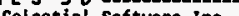
Copyright (c) 1984 Celestial Software Inc.

SOLVE DISPLACEMENTS

Version $2.0 \quad 07 / 01 / 90$

L

GRAVITY LOAD

LOAD CASE 2

APPLIEO LOAD VECTOR

\begin{tabular}{|c|c|c|c|c|c|}
\hline & $F x$ & & & & \\
\hline $\begin{array}{c}76 \\
77 \\
78 \\
79 \\
80 \\
81 \\
82 \\
83 \\
84 \\
85 \\
86 \\
87 \\
88 \\
89 \\
90 \\
91 \\
92 \\
93 \\
94 \\
95 \\
96 \\
97 \\
98 \\
99 \\
100 \\
101 \\
102 \\
103 \\
104 \\
105 \\
106 \\
107 \\
108 \\
109 \\
110 \\
111 \\
112 \\
113 \\
114\end{array}$ & $\begin{array}{l}.00 \\
.00 \\
.00 \\
.00 \\
.00 \\
.00 \\
.00 \\
.00 \\
.00 \\
.00 \\
.00 \\
.00 \\
.00 \\
.00 \\
.00 \\
.00 \\
.00 \\
.00 \\
.00 \\
.00 \\
.00 \\
.00 \\
.00 \\
.00 \\
.00 \\
.00 \\
.00 \\
.00 \\
.00 \\
.00 \\
.00 \\
.00 \\
00\end{array}$ & $\begin{array}{l}.00 \\
.00 \\
.00 \\
.00 \\
.00 \\
.00 \\
.00 \\
.00 \\
.00 \\
.00 \\
.00 \\
.00 \\
.00 \\
.00 \\
.00 \\
.00 \\
.00 \\
.00 \\
.00 \\
.00 \\
.00 \\
.00 \\
.17\end{array}$ & $\begin{array}{l}E+00 \\
E++00 \\
E+00 \\
E+00 \\
E++00 \\
E+00 \\
E+00 \\
E+00 \\
E+00 \\
E+00 \\
E+02 \\
E+00 \\
E+00 \\
E+00 \\
E+00 \\
E+00 \\
E+03 \\
E+00 \\
E+00 \\
E+00 \\
E+00 \\
E+00 \\
E+03 \\
E+03 \\
E+00 \\
E+00 \\
E+04 \\
E+00 \\
E+00 \\
+00 \\
+00 \\
+00 \\
+00 \\
++03 \\
E+00 \\
E+00 \\
E+00 \\
E+00 \\
E+00 \\
E+03 \\
E+03\end{array}$ & $\begin{array}{l}0 \\
0 \\
0 \\
0 \\
0 \\
0 \\
0 \\
0 \\
0 \\
0 \\
0 \\
0 \\
0 \\
0 \\
0 \\
0 \\
0 \\
0 \\
0 \\
0 \\
0 \\
0 \\
0 \\
0 \\
0 \\
0 \\
0 \\
0 \\
0 \\
0 \\
0 \\
0 \\
0 \\
0 \\
0\end{array}$ & 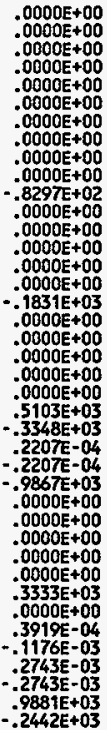 \\
\hline
\end{tabular}


KAISER EMGINEERS HANFORD S/M:801854

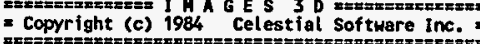
SOLVE DISPLACEMENTS

Version $2.0 \quad 07 / 01 / 90$

GRAVITY LOAD

LOAD CASE 2

APPLIEO LOAD VECTOR

\begin{tabular}{|c|c|c|c|c|c|}
\hline Node & $F x$ & Fz & $M x$ & Hy & $\mathrm{Mz}$ \\
\hline $\begin{array}{l}116 \\
117 \\
118 \\
119 \\
120 \\
121 \\
122 \\
123 \\
124 \\
125 \\
126 \\
127 \\
128 \\
129 \\
130 \\
131 \\
132 \\
133 \\
134 \\
135\end{array}$ & 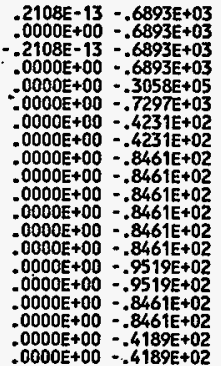 & $\begin{array}{l}.0000 E+00 \\
.2108 E-13 \\
.0000 E+00 \\
.2108 E-13 \\
.0000 E+00 \\
.0000 E+00 \\
.0000 E+00 \\
.0000 E+00 \\
.0000 E+00 \\
.0000 E+00 \\
.0000 E+00 \\
.0000 E+00 \\
.0000 E+00 \\
.0000 E+00 \\
.0000 E+00 \\
.0000 E+00 \\
.0000 E+00 \\
.0000 E+00 \\
.0000 E+00 \\
.0000 E+00\end{array}$ & $\begin{array}{l}.0000 E+00 \\
.2413 \mathrm{E}+04 \\
.0000 \mathrm{E}+00 \\
.2413 \mathrm{E}+04 \\
.0000 \mathrm{E}+00 \\
.0000 \mathrm{E}+00 \\
.0000 \mathrm{E}+00 \\
.0000 \mathrm{E}+00 \\
.0900 \mathrm{0}+00 \\
.0000 \mathrm{E}+00 \\
.0000 \mathrm{E}+00 \\
.0000 \mathrm{E}+00 \\
.0000 \mathrm{E}+00 \\
.0000 \mathrm{E}+00 \\
.0000 \mathrm{E}+00 \\
.0000 \mathrm{E}+00 \\
.0000 \mathrm{E}+00 \\
.0000 \mathrm{E}+00 \\
.0000 \mathrm{E}+00 \\
.0000 \mathrm{E}+00\end{array}$ & $\begin{array}{l}.0000 E+00 \\
.0000 E+00 \\
.0000 E+00 \\
.0000 E+00 \\
.0000 E+00 \\
.0000 E+00 \\
.0000 E+00 \\
.0000 E+00 \\
.0000 E+00 \\
.0000 E+00 \\
.0000 E+00 \\
.0000 E+00 \\
.0000 E+00 \\
.0000 E+00 \\
.0000 E+00 \\
.0000 E+00 \\
.0000 E+00 \\
.0000 E+00 \\
.0000 E+00 \\
.0000 E+00\end{array}$ & $\begin{array}{l}-.2413 E+04 \\
.0000 E+00 \\
.2413 E+04 \\
.0000 E+00 \\
.0000 E+00 \\
.0000 E+00 \\
.0000 E+00 \\
.0000 E+00 \\
.0000 E+00 \\
.0000 E+00 \\
.0000 E+00 \\
.0000 E+00 \\
.0000 E+00 \\
.0000 E+00 \\
.0000 E+00 \\
.0000 E+00 \\
.0000 E+00 \\
.0000 E+00 \\
-.3387 E+02 \\
.3387 E+02\end{array}$ \\
\hline
\end{tabular}

KATSER ENGINEERS HAHFORD S/H:801854

PAGE 28 RUn ID=N1N61973

$=$ Copyright (c) $1984^{\wedge}$ Celestial Software Inc.

SOLVE DISPLACEMENTS Version $2.0 \quad 07 / 01 / 90$

L

GRAVITY LOAD

LOAD CASE 2

DISPLACEMENTS

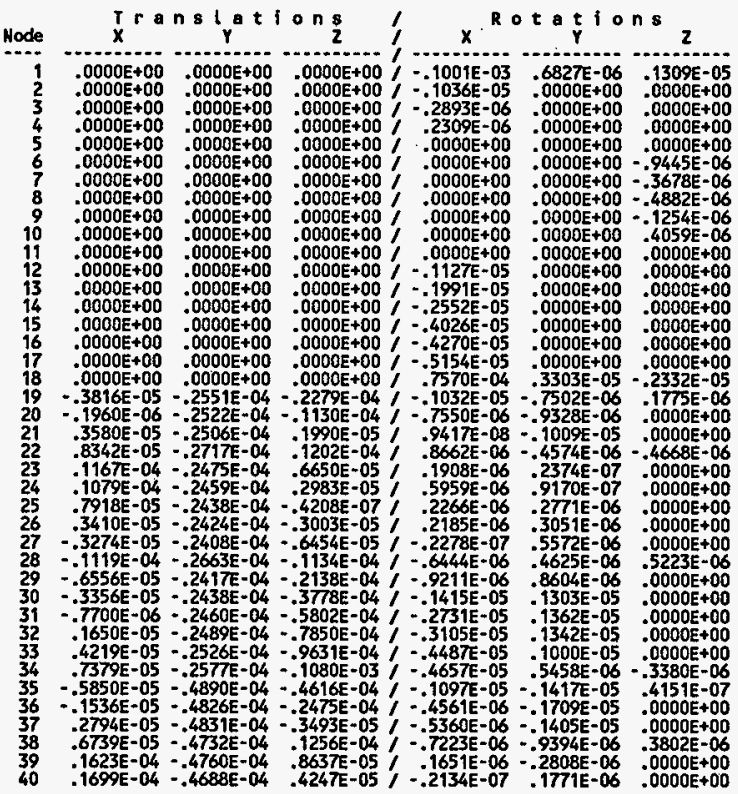


KAISER ENGINEERS HANFORD S/N-801854

PAGE 29

Run $10=11.461973$

$02 / 22 / 95$

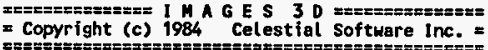
SOLVE DISPLACENENTS

Version $2.0 \quad 07 / 01 / 90$

$\mathbf{L}$

GRAVITY LOAD

LOAD CASE 2

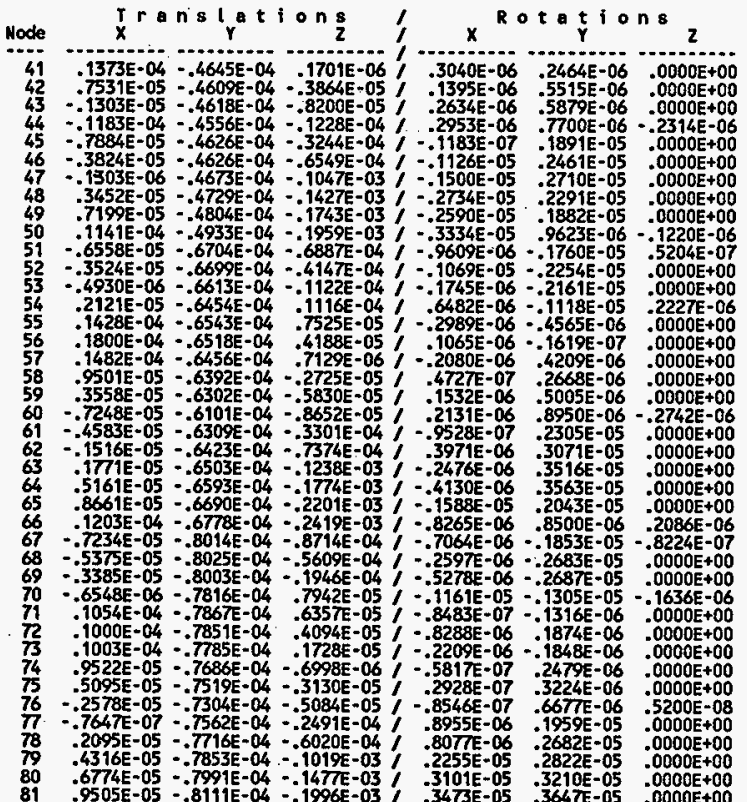

KAISER ENGINEERS HANFORD S/N:801854

PAGE 30 RUN ID=MH61973 $02 / 22 / 95$
$08: 49: 51$ x Copyright (c) 1984 Celestial Softure Inc.
x SOLVE DISPLACEMENTS Version $2.0 \quad 07 / 01 / 90$ $\mathbf{L}$ GRAVIT LOAD LOAD CASE

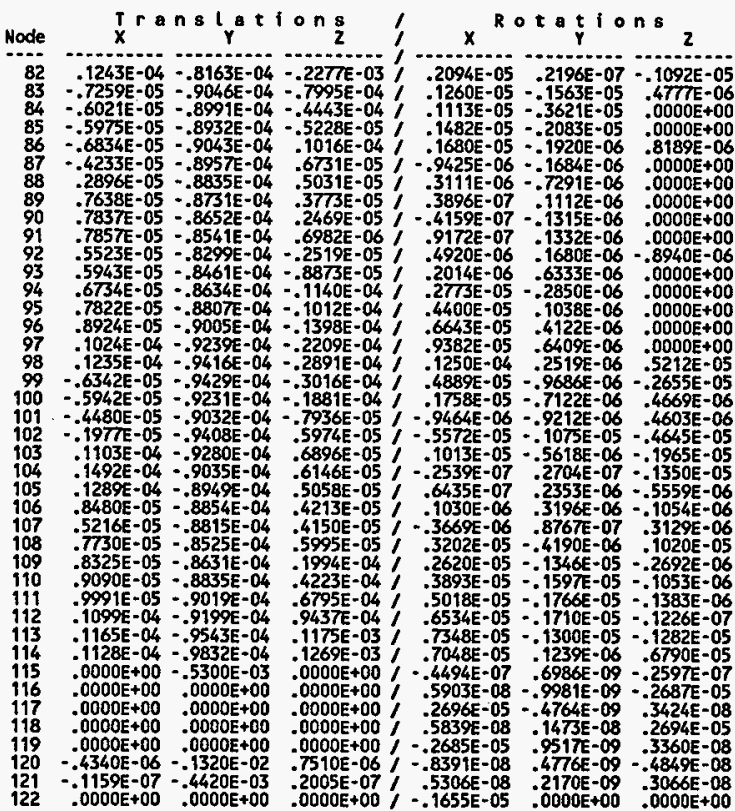


KAISER ENGINEERS HAHFORD S/H:801854

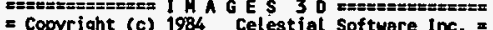
Celestial software inc.

SOLVE DISPLACEMENTS

Version $2.0 \quad 07 / 01 / 90$

L

GRAVITY LOAD

LOAD CASE 2

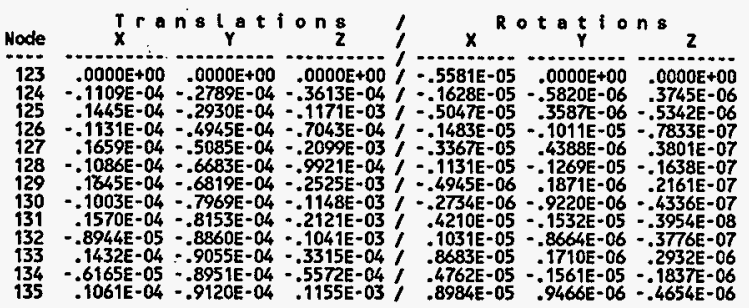

KAISER ENGINEERS HANFORD S/N:801854

PAGE 32 RUN ID=HH61973

$02 / 22 / 95$

× Copyright (c) 1984 Celestial Software inc.

SOLVE DISPLACEMENTS

Version $2.0 \quad 07 / 01 / 90$

$\downarrow$

UBC SEISHIC LOAD - SH \& AE DIR. A S E 3

APPLIED LOAD VECTOR

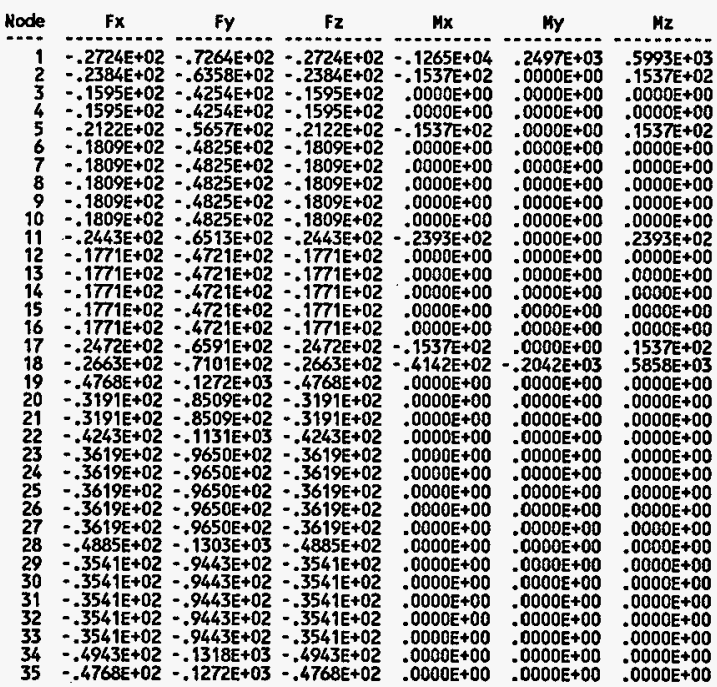


KAISER ENGINEERS HANFORD S/H:801854

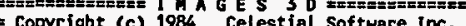
Inc.

SOLVE DISPLACEMENTS

Version $2.0 \quad 07 / 01 / 90$

L

UBC SEISMIC LOAD - SU \& AEDTR: A S E 3

APPLIED LOAD VECTOR

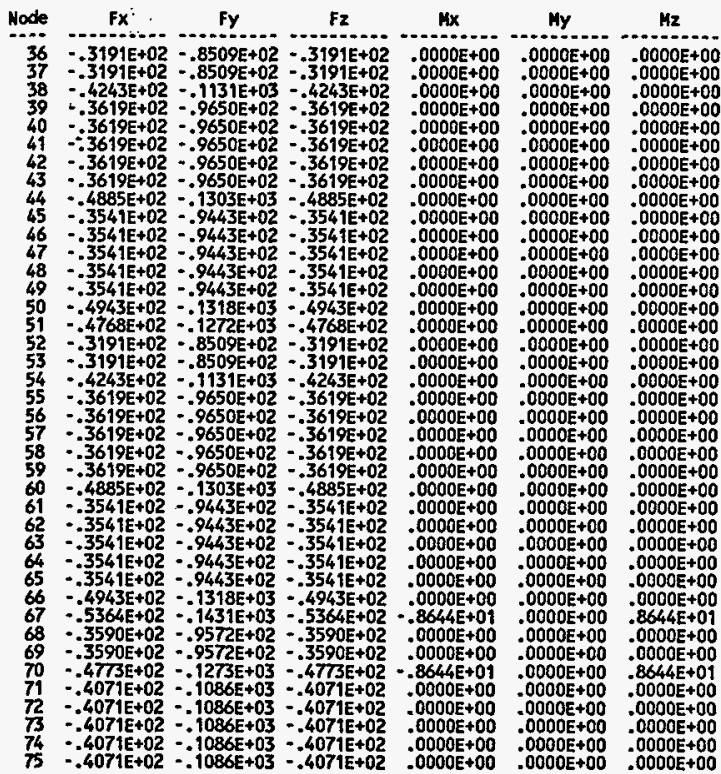

KAISER ENGINEERS HAHFORD S/ $\mathrm{H}: 801854$

RUn ID=NN61973

$02 / 22 / 95$

34

RUn ID=NN61973 $08: 49: 52$

$=$ Copyright (c) $1984^{\circ}$ Celestial Software Inc.

SOLVE DISPLACEMENTS Version $2.0 \quad 07 / 01 / 90$

LOADCASE 3

UBC SEISHIC LOAD - SH \& WE DIR.

APPLIED LOND VECTOR

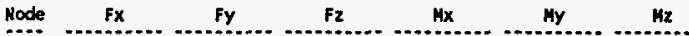
$76-.5496 E+02-.1466 E+03-.5496 E+02-.1346 E+02 \quad .0000 E+00 \quad .1346 E+02$ $\pi \quad=.3984 E+02-.1062 E+03-.3984 E+02 \quad .0000 E+00 \quad .0000 E+00 \quad .0000 E+00$ $78-.3984 E+02-.1062 E+03-.3984 E+02 \quad .0000 E+00 \quad .0000 E+00 \quad .0000 E+00$ $79-.3984 E+02-.1062 E+03-.3984 E+02 \quad .0000 E+00 \quad .0000 E+00 \quad .0000 E+00$ $80-.3984 E+02-.1052 E+03 \quad-.3984 E+02 \quad .0000 E+00 \quad .0000 E+00 \quad .0000 E+00$ $81-.3984 E+02-.1062 E+03-.3984 E+02 \quad .0000 E+00 \quad .0000 E+00 \quad .0000 E+00$ $82-.5561 E+02-.1483 \mathrm{E}+03-.5561 \mathrm{E}+02 \quad-.8644 \mathrm{E}+01 \quad .0000 \mathrm{E}+00 \quad .8644 \mathrm{E}+01$ $83-.4768 \mathrm{E}+02-.1272 \mathrm{E}+03-.4768 \mathrm{E}+02 \quad .1537 \mathrm{E}+02 \quad .0000 \mathrm{E}+00-.1537 \mathrm{E}+02$ $84-.3191 E+02-.8509 E+02-.3191 E+02 \quad .0000 E+00 \quad .0000 E+00 \quad .0000 E+00$ $85-.3191 \mathrm{E}+02-.8509 \mathrm{E}+02-.3191 \mathrm{E}+02 \quad .0000 \mathrm{E}+00 \quad .0000 \mathrm{E}+00 \quad .0000 \mathrm{E}+00$ $86-.4910 E+02-.1309 E+03-.4910 E+02-.4686 E+02 \quad .5445 E+02-.9834 E+02$ $87-.3619 \varepsilon+02-.9650 E+02-.3619 E+02 \quad .0000 E+00 \quad .0000 E+00 \quad .0000 E+00$ $88-.3619 E+02-.9650 E+02 \quad-.3619 E+02 \quad .0000 E+00 \quad .0000 E+00 \quad .0000 E+00$ $89-.3619 E+02 \cdot-9650 E+02-.3619 E+02 \quad .0000 E+00 \quad .0000 E+00 \quad .0000 E+00$ $90-.3619 \mathrm{E}+02-.9650 \mathrm{E}+02-.3619 \mathrm{E}+02 \quad .0000 \mathrm{E}+00 \quad .0000 \mathrm{E}+00 \quad .0000 \mathrm{E}+00$ $91-.3619 \mathrm{E}+02-.9650 \mathrm{E}+02-.3619 \mathrm{E}+02 \quad .0000 \mathrm{E}+00 \quad .0000 \mathrm{E}+00 \quad .0000 \mathrm{E}+00$ $92 \quad-.6357 \mathrm{E}+02 \quad-.1695 \mathrm{E}+03 \quad-.6357 \mathrm{E}+02 \quad .4948 \mathrm{E}+03 \quad-.1079 \mathrm{E}+03 \quad-.2071 \mathrm{E}+03$ $93-.3541 \mathrm{E}+02-.9443 \mathrm{E}+02-.3541 \mathrm{E}+02 \quad .0000 \mathrm{E}+00 \quad .0000 \mathrm{E}+00 \quad .0000 \mathrm{E}+00$ $94-.3541 \mathrm{E}+02 \quad-.9443 \mathrm{E}+02-.3541 \mathrm{E}+02 \quad .0000 \mathrm{E}+00 \quad .0000 \mathrm{E}+00 \quad .0000 \mathrm{E}+00$ $95-.3541 \mathrm{E}+02 \quad-.9443 \mathrm{E}+02-.3541 \mathrm{E}+02 \quad .0000 \mathrm{E}+00 \quad .0000 \mathrm{E}+00 \quad .0000 \mathrm{E}+00$ $96-.3541 \mathrm{E}+02-.9443 \mathrm{E}+02-.3541 \mathrm{E}+02 \quad .0000 \mathrm{E}+00 \quad .0000 \mathrm{E}+00 \quad .0000 \mathrm{E}+00$ $97-.3541 E+02-.9443 E+02-.3541 E+02 \quad .0000 E+00 \quad .0000 E+00 \quad .0000 E+00$ $98-.6766 \mathrm{E}+02-.1804 \mathrm{E}+03-.6766 \mathrm{E}+02 \quad .5986 \mathrm{E}+03+.4101 \mathrm{E}+03 \quad .4949 \mathrm{E}+03$ $99-.7090 \mathrm{E}+02-.1891 \mathrm{E}+03-.7090 \mathrm{E}+02 \quad .4736 \mathrm{E}+03-1759 \mathrm{E}+03-.9427 \mathrm{E}+03$ $100-.1717 \mathrm{E}+02-.4579 \mathrm{E}+02 \quad-.1717 \mathrm{E}+02 \quad .0000 \mathrm{E}+00 \quad-.8276 \mathrm{E}-05 \quad .2207 \mathrm{E}-04$ $101-.1717 E+02-.4579 E+02-.1717 E+02 \quad .0000 E+00 \quad .8276 E-05-.2207 E-04$ $102-.4625 \mathrm{E}+02-.1233 \mathrm{E}+03-.4625 \mathrm{E}+02-.1036 \mathrm{E}+04 \quad .7618 \mathrm{E}+03-.9953 \mathrm{E}+03$ $103-.1948 E+02-.5194 E+02-.1948 E+02 \quad .0000 E+00 \quad .0000 E+00 \quad .0000 E+00$ $104-.1948 \mathrm{E}+02-.5194 \mathrm{E}+02-.1948 \mathrm{E}+02 \quad .0000 \mathrm{E}+00 \quad .0000 \mathrm{O}+00 \quad .0000 \mathrm{E}+00$ $105-.1948 E+02-.5194 E+02-.1948 E+02 \quad .0000 E+00 \quad .0000 E+00 \quad .0000 E+00$ $106-.1948 E+02-.5194 E+02-.1948 E+02 \quad .0000 E+00 \quad .0000 E+00 \quad .0000 E+00$

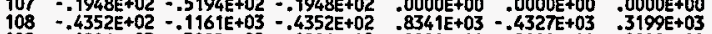

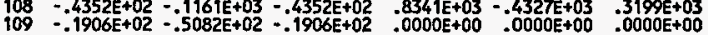
$110=1906 \mathrm{E}+02=.5082 \mathrm{E}+02=.1906 \mathrm{E}+02 \quad .0000 \mathrm{E}+00-.1470 \mathrm{E}-04.04019 \mathrm{E}-04$

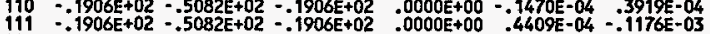
$112=.1906 \mathrm{EE}+02=.5082 \mathrm{E}+02-.1906 \mathrm{E}+02 \quad .0000 \mathrm{E}+00 \quad .4409 \mathrm{E}-04-.1176 \mathrm{E}-03$ $113=.1906 \mathrm{E}+02=02-.5082 \mathrm{E}+02=.1906 \mathrm{E}+02 \quad .0000 \mathrm{E}+00-.1029 \mathrm{E}-03 \quad .2745 \mathrm{E}+02 \quad .0000 \mathrm{E}+00 \quad .1029 \mathrm{E}-03-.2743 \mathrm{E}-03$ $114=.7665 \mathrm{E}+02=.2044 \mathrm{E}+03-.7665 \mathrm{E}+02 \quad .1075 \mathrm{E}+04-.5506 \mathrm{E}+03 \quad .3936 \mathrm{E}+03$ 
KAISER ENGINEERS HAHFORD $S / \mathrm{E}: 801854$
PAGE 35

$===2=-2=2=00 \Omega$ Run $M A$ = Copyright (c) 1984 Celestial Software Inc.

$02 / 22 / 95$
$08: 49: 52$

SOLVE DISPLACEHENTS

Version $2.0 \quad 07 / 01 / 90$

UBC SEISHIC LOAD - SN \& AE DIR: A S E 3

APPLIED LOAD VECTOR

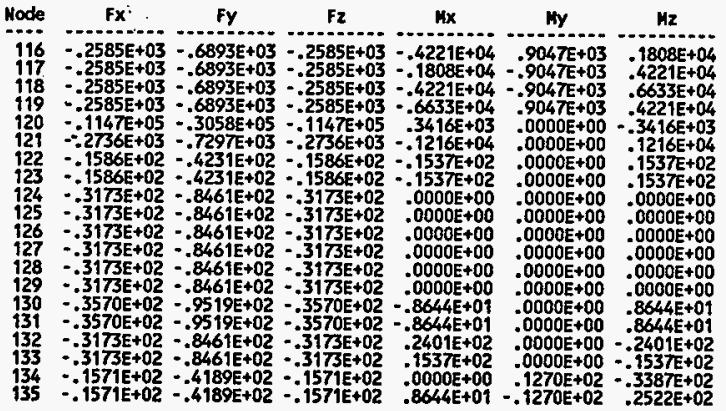

KAISER EHGINEERS HANFORD S/K:801854 36 10 $1222 / 95$ = Copyright (c) 1984 Celestial Software Inc. SOLVE DISPLACEMENTS Version 2.0 07/01/90

L.

UBC SEISHIC LOAD - SH O HE C A S E 3

DISPLACEMENTS

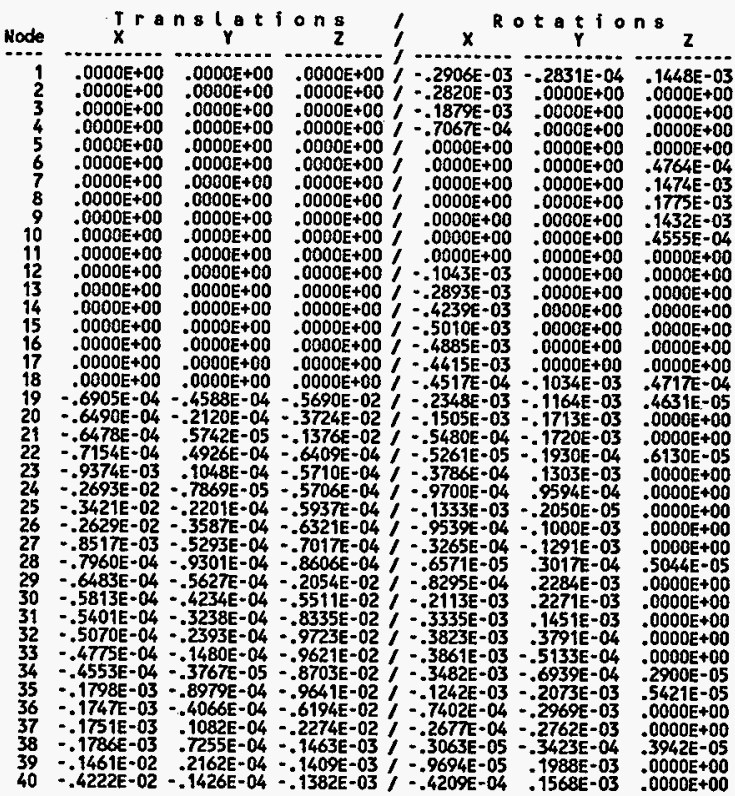


KAISER ENGINEERS HANFORD S/N:801854

Run ID = 4 HN61973

$02 / 22 / 95$

37

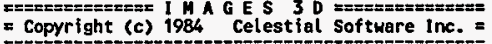

SOLVE DISPLACEMENTS Version $2.0 \quad 07 / 01 / 90$

$\mathbf{L}$

UBC SEISHIC LOAD - SU \& A D C A S E 3

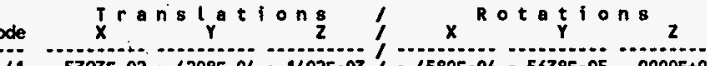

$41-.5393 \mathrm{E}-02-.4208 \mathrm{E}-04-.1402 \mathrm{E}-03 /-.4580 \mathrm{E}-04=.5638 \mathrm{E}-05 \quad .0000 \mathrm{E}+00$

$42-.4091 \mathrm{E}-02-.6885 \mathrm{E}-04 \div .1462 \mathrm{E}-03,-.3762 \mathrm{E}-04=.1623 \mathrm{E}-03 \quad .0000 \mathrm{E}+00$

$44-.1534 \mathrm{E}-03-.1486 \mathrm{E}-03-.1695 \mathrm{E}-03$ / $=.2139 \mathrm{E}-05 \quad .5017 \mathrm{E}-04 \quad .2592 \mathrm{E}-05$

$45-.1409 \mathrm{E}-03-.1093 \mathrm{E}-03-.3279 \mathrm{E}-02 /-.2825 \mathrm{E}-04 \quad .3589 \mathrm{E}-03.0000 \mathrm{C}+00$

$46-.1296 \mathrm{E}-03-.8186 \mathrm{E}-04-.8787 \mathrm{E}-02 /=.8668 \mathrm{E}-04 \quad .3667 \mathrm{E}-03 \quad .0000 \mathrm{E}+00$

$47-. T 219 E-03=.6299 E-04-.1334 E-01 /-.1209 E-03 \quad .2329 E-03 \quad .0000 E+00$

$48-.1165 \mathrm{E}-03-.4607 \mathrm{E}-04-.1556 \mathrm{E}-01 /-.1484 \mathrm{E}-03 \quad .6035 \mathrm{E}-04 \quad .0000 \mathrm{E}+00$

$49-.1130 E-03-.2843 E-04-.1539 E-01 /=.1385 E-03-.8244 E-04 \quad .0000 E+00$

$50-.1116 \mathrm{E}-03-.5743 \mathrm{E}-05-.1390 \mathrm{E}-01 /-.1236 \mathrm{E}-03 \quad-.1142 \mathrm{E}-03 \quad .3243 \mathrm{E}-05$

$51-.3068 \mathrm{E}-03-.1215 \mathrm{E}-03-.1093 \mathrm{E}-01$ / .6676E-05 -.2474E-03 .6086E-05

$=.3039 \mathrm{E}-03=.5713 \mathrm{E}-04 \cdot .6924 \mathrm{E}-02 / .7687 \mathrm{E}-05 \cdot-.3389 \mathrm{E}-03 \quad .0000 \mathrm{E}+00$

$-.3041 \mathrm{E}-03.8302 \mathrm{E}-05-.2538 \mathrm{E}-02 / .2607 \mathrm{E}-05 * .3024 \mathrm{E}-03 \quad .0000 \mathrm{0}+00$

$-.3064 \mathrm{E}-03$.8107E-04 $-.2323 \mathrm{E}-03 /=.437 \mathrm{EE}-05 \cdot-.3395 \mathrm{E}-04$. $04729 \mathrm{E}-05$

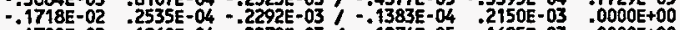

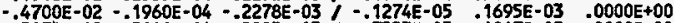

$-.5979 E-02-.5800 E-04-.2285 E-03 / \cdot-.7553 E-05-.4847 E-05 \quad .0000 E+00$

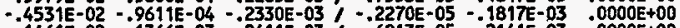

$-.1461 \mathrm{E}-02 \cdot-1366 \mathrm{E}-03 \cdot-2411 \mathrm{E}-03 / \cdot .6813 \mathrm{E}-05 \cdot-2141 \mathrm{E}-03 \quad .0000 \mathrm{E}+00$

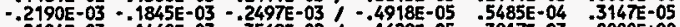

$-.2104 \mathrm{E}-03-.1460 \mathrm{E}-03-.3569 \mathrm{E}-02 / .1629 \mathrm{E}-05 \quad .3817 \mathrm{E}-03 \quad .0000 \mathrm{E}+00$

$-.2016 \mathrm{E}-03=.1152 \mathrm{E}-03-.9410 \mathrm{E}-027.3023 \mathrm{E}-04 \quad .3880 \mathrm{E}-03 \quad .0000 \mathrm{E}+00$

$-.1945 E-03-.8901 E-04-.1409 E-01 / .5169 E-04 \quad .2293 E-03 \quad .0000 E+00$

$=.1896 E-03-.6543 E-04 \cdot-.1608 E-017.1010 E-03 \quad .3306 E-04 \quad .0000 E+00$

$-.1864 \mathrm{E}-03-.3990 \mathrm{E}-04-.1557 \mathrm{E}-01 / .1221 \mathrm{E}-03-.1006 \mathrm{E}-03 \quad .0000 \mathrm{E}+00$

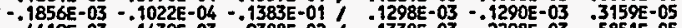

$=.4419 E-03-.1439 E-03-.9388 E-02 / .1338 E-03-.2298 E-03 \quad .5851 E-05$

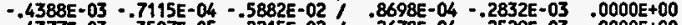

$\begin{array}{lllll}-.4377 E-03 & .3507 E-05 & -.2215 E-02 / .2678 E-04 & -.2529 E-03 & .0000 E+00\end{array}$

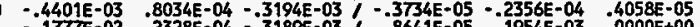

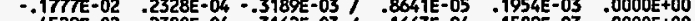

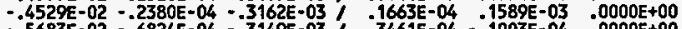

$-.5633 \mathrm{E}-02-.6824 \mathrm{E}-04-.3149 \mathrm{E}-03 / .3461 \mathrm{E}-04 \quad-.1003 \mathrm{E}-04 \quad .0000 \mathrm{E}+00$

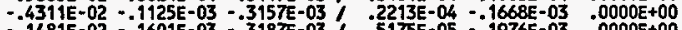

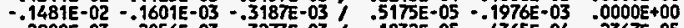

$-.2800 \mathrm{E}-03-.2056 \mathrm{E}-03-.3273 \mathrm{E}-03 /-.1832 \mathrm{E}-05 \quad .4365 \mathrm{E}-04 \quad .2367 \mathrm{E}-05$

$\begin{array}{llllll}-.2731 E-03 & -.1709 E-03 \cdot .3097 E-02 & f & .4153 E-04 & .3206 E-03 & .0000 E+00\end{array}$

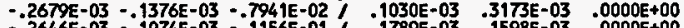

$-.2646 E-03-.1074 E-03-.1156 E-01 / .1789 E-03 \quad .1598 E-03 \quad .0000 E+00$

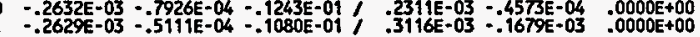

KAISER ENGINEERS HANFORD S/H:801854

$02 / 22 / 95$

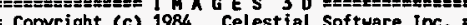
= Copyright (c) 1984 Celestial Sof tware Inc. =

SOLVE DISPLACEMENTS

Version $2.0 \quad 07 / 01 / 90$

$\mathbf{L}$ UBC SEISHIC LOAD - SH Q A DE DIR. A S E 3

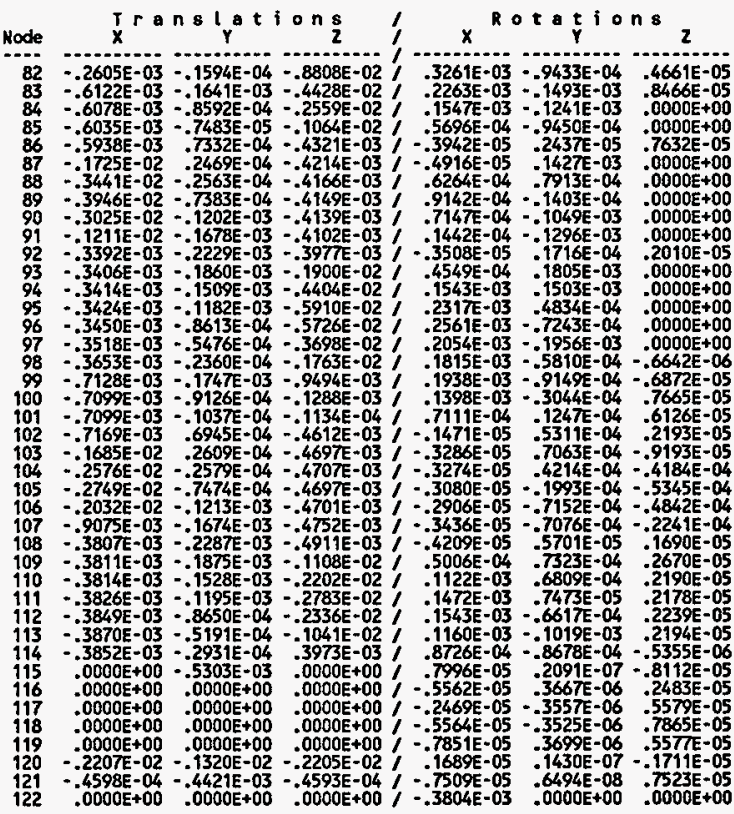


KAISER ENGINEERS HANFORD S/H:801854

PABE 39 Run ID=HN61973

$02 / 22 / 95$

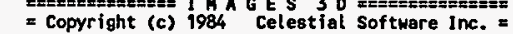

SOLVE DISPLACENENTS

Version $2.0 \quad 07 / 01 / 90$

UBC SEISHIC LOAD - SW \& WE DIR.

\begin{tabular}{|c|c|c|c|c|c|}
\hline & ${ }_{x}^{T} r a$ & $s t_{Y} a t i \circ n_{Z}$ & \multicolumn{3}{|c|}{$R \circ t a t i \circ n s$} \\
\hline 3. & $\begin{array}{r}.0000 E+00 \\
-.8993 \mathrm{E}-04 \\
-.4847 \mathrm{E}-04 \\
-.1924 \mathrm{E}-03 \\
-.1125 \mathrm{E}-03 \\
-.3158 \mathrm{E}-03 \\
-.1844 \mathrm{E}-03 \\
-.4479 \mathrm{E}-03 \\
-.2635 \mathrm{E}-03 \\
-.6471 \mathrm{E}-03 \\
-.3528 \mathrm{E}-03 \\
-.7100 \mathrm{E}-03 \\
-.3953 \mathrm{E}-03\end{array}$ & 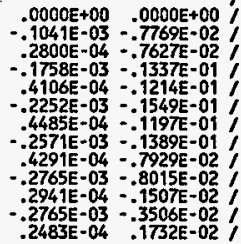 & $\begin{array}{l}366 \mathrm{E}-03 \\
340 \mathrm{E}-03 \\
348 \mathrm{E}-03 \\
336 \mathrm{E}-03 \\
34 \mathrm{E}-03 \\
82 \mathrm{E}-05 \\
190 \mathrm{E}-03 \\
35 \mathrm{E}-03 \\
61 \mathrm{E}-03 \\
27 \mathrm{E}-03 \\
313 \mathrm{E}-03\end{array}$ & $\begin{array}{r}.000 \\
-.915 \\
-.380 \\
-.165 \\
-.616 \\
-.208 \\
-.561 \\
-.220 \\
.606 \\
-.2100 \\
.305 \\
-.160\end{array}$ & $\begin{array}{l}.0000 \\
.51171 \\
.2967 \\
.4995 \\
.3044 \\
.5849 \\
.34431 \\
.6405 \\
.3767 \\
.5286 \\
.23731 \\
.8745\end{array}$ \\
\hline
\end{tabular}

KAISER ENGINEERS HANFORD S/K:801854

PAGE 40 RUN 10=MN61973

$=$ Copyright (c) 1984 Celestial Software Inc.

SOLVE DISPLACEMENTS

Version $2.0 \quad 07 / 01 / 90$

$\mathbf{L}$

UBC SEISHIC LOAD - SN \& EWDIR. A S E 4

APPLIEO LOAD VECTOR

\begin{tabular}{|c|c|c|c|c|c|}
\hline & Fy & $\mathbf{F z}$ & $M x$ & Hy & Mz \\
\hline $\begin{array}{l}1 \\
2 \\
3 \\
4 \\
5 \\
6 \\
7 \\
8 \\
9 \\
10 \\
11 \\
12 \\
13 \\
14 \\
15 \\
16 \\
17 \\
18 \\
19 \\
20 \\
21 \\
22 \\
23 \\
24 \\
25 \\
26 \\
27 \\
28 \\
29 \\
30 \\
31 \\
32 \\
33 \\
34\end{array}$ & $\begin{array}{l}2 \\
2 \\
2 \\
2 \\
2 \\
2 \\
2 \\
2 \\
2 \\
2 \\
2 \\
2 \\
2 \\
2 \\
2 \\
2 \\
2 \\
2 \\
2 \\
2 \\
2 \\
2 \\
2 \\
2 \\
2 \\
2 \\
2 \\
2 \\
2\end{array}$ & $\begin{array}{l}.2724 \mathrm{E}+02 \\
.2384 \mathrm{E}+02 \\
.1595 \mathrm{E}+02 \\
.1595 \mathrm{E}+02 \\
.2122 \mathrm{E}+02 \\
.1809 \mathrm{E}+02 \\
.1809 \mathrm{E}+02 \\
.1809 \mathrm{E}+02 \\
.1809 \mathrm{E}+02 \\
.1809 \mathrm{E}+02 \\
.2443 \mathrm{E}+02 \\
.1771 \mathrm{E}+02 \\
.1771 \mathrm{E}+02 \\
.1771 \mathrm{E}+02 \\
.1771 \mathrm{E}+02 \\
.1771 \mathrm{E}+02 \\
.2472 \mathrm{E}+02 \\
.2663 \mathrm{E}+02 \\
.4768 \mathrm{E}+02 \\
.3191 \mathrm{E}+02 \\
.3191 \mathrm{E}+02 \\
.4243 \mathrm{E}+02 \\
.3619 \mathrm{E}+02 \\
.3619 \mathrm{E}+02 \\
.3619 \mathrm{E}+02 \\
.3619 \mathrm{E}+02 \\
.3619 \mathrm{E}+02 \\
.4885 \mathrm{E}+02 \\
.3541 \mathrm{E}+02 \\
.3541 \mathrm{E}+02 \\
.3541 \mathrm{E}+02 \\
.3541 \mathrm{E}+02 \\
.3541 \mathrm{E}+02 \\
.4943 \mathrm{E}+02\end{array}$ & 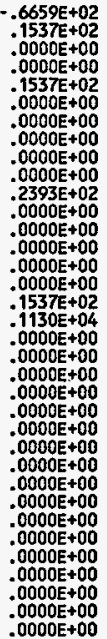 & & \\
\hline
\end{tabular}


KAISER ENGINEERS HAHFORD S/N:801854

PAGE 41

Run ID=HN61973

$02 / 22 / 95$

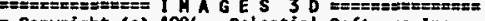
= Copyright (c) 1984 Celestial Software Inc. $=$

SOLVE DISPLACEMENTS

Version $2.0 \quad 07 / 01 / 90$

$\iota$

UBC SEISNIC LOAD - SH \& EHDIR. A S E 4

APPLIED LOAD VECTOR

\begin{tabular}{|c|c|c|c|c|c|}
\hline Node & $\mathbf{F x}:$ & $F z$ & $M x$ & My & $\mathrm{Hz}_{2}$ \\
\hline $\begin{array}{l}36 \\
37 \\
38 \\
39 \\
40 \\
41 \\
42 \\
43 \\
44 \\
45 \\
46 \\
47 \\
48 \\
49 \\
50 \\
51 \\
52 \\
53 \\
54 \\
55 \\
56 \\
57 \\
58 \\
59 \\
60 \\
61 \\
62 \\
63 \\
64 \\
65 \\
66 \\
67 \\
68 \\
69 \\
70 \\
71 \\
72 \\
75 \\
71\end{array}$ & 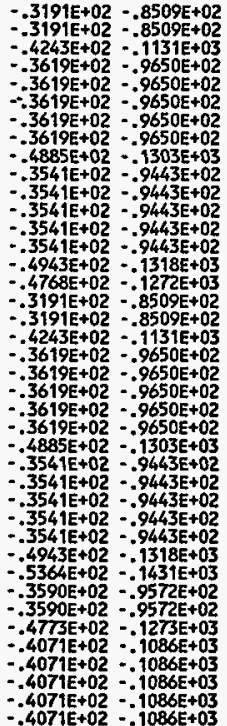 & $\begin{array}{l}.3191 \mathrm{E}+02 \\
.3191 \mathrm{E}+02 \\
.4243 \mathrm{E}+02 \\
.3619 \mathrm{E}+02 \\
.3619 \mathrm{E}+02 \\
.3619 \mathrm{E}+02 \\
.3619 \mathrm{E}+02 \\
.3619 \mathrm{E}+02 \\
.4885 \mathrm{E}+02 \\
.3541 \mathrm{E}+02 \\
.3541 \mathrm{E}+02 \\
.3541 \mathrm{E}+02 \\
.3541 \mathrm{E}+02 \\
.3541 \mathrm{E}+02 \\
.4943 \mathrm{E}+02 \\
.4768 \mathrm{E}+02 \\
.3191 \mathrm{E}+02 \\
.3191 \mathrm{E}+02 \\
.4243 \mathrm{E}+02 \\
.3619 \mathrm{E}+02 \\
.3619 \mathrm{E}+02 \\
.3619 \mathrm{E}+02 \\
.3619 \mathrm{E}+02 \\
.3619 \mathrm{E}+02 \\
.4835 \mathrm{E}+02 \\
.3541 \mathrm{E}+02 \\
.3541 \mathrm{E}+02 \\
.3541 \mathrm{E}+02 \\
.3541 \mathrm{E}+02 \\
.3541 \mathrm{E}+02 \\
.4943 \mathrm{E}+02 \\
.5364 \mathrm{E}+02 \\
.3590 \mathrm{E}+02 \\
.3590 \mathrm{E}+02 \\
.4773 \mathrm{E}+02 \\
.4071 \mathrm{E}+02 \\
.4071 \mathrm{E}+02 \\
.4071 \mathrm{E}+02 \\
.4071 \mathrm{E}+02\end{array}$ & $\begin{array}{l}.0000 E+00 \\
.0000 E+00 \\
.0000 E+00 \\
.0000 E+00 \\
.0000 E+00 \\
.0000 E+00 \\
.0000 E+00 \\
.0000 E+00 \\
.0000 E+00 \\
.0000 E+00 \\
.0000 E+00 \\
.0000 E+00 \\
.0000 E+00 \\
.0000 E+00 \\
.0000 E+00 \\
.0000 E+00 \\
.0000 E+00 \\
.0000 E+00 \\
.0000 E+00 \\
.0000 E+00 \\
.0000 E+00 \\
.0000 E+00 \\
.0000 E+00 \\
.0000 E+00 \\
.0000 E+00 \\
.0000 E+00 \\
.0000 E+00 \\
.0000 E+00 \\
.0000 E+00 \\
.0000 E+00 \\
.0000 E+00 \\
.8644 E+01 \\
.0000 E+00 \\
.0000 E+00 \\
.8644 E+01 \\
.0000 E+00 \\
.0000 E+00 \\
.0000 E+00 \\
.0000 E+00\end{array}$ & $\begin{array}{l}.0000 E+00 \\
.0000 E++00 \\
.0000 E++00 \\
.0000 E+00 \\
.0000 E+00 \\
.0000 E+00 \\
.0000 E+00 \\
.0000 E+00 \\
.0000 E+00 \\
.0000 E+00 \\
.0000 E+00 \\
.0000 E+00 \\
.0000 E+00 \\
.0000 E+00 \\
.0000 E+00 \\
.0000 E+00 \\
.0000 E+00 \\
.0000 E+00 \\
.0000 E+00 \\
.0000 E+00 \\
.0000 E+00 \\
.0000 E+00 \\
.0000 E+00 \\
.0000 E+00 \\
.0000 E+00 \\
.0000 E+00 \\
.0000 E+00 \\
.0000 E+00 \\
.0000 E+00 \\
.0000 E+00 \\
.0000 E+00 \\
.0000 E+00 \\
.0000 E+00 \\
.0000 E+00 \\
.0000 E+00 \\
.0000 E+00 \\
.0000 E+00 \\
.0000 E+00 \\
0.000 E+00\end{array}$ & 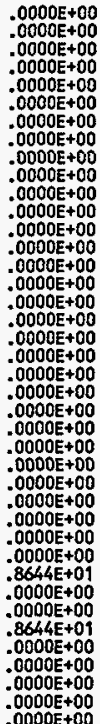 \\
\hline
\end{tabular}

KAISER ENGINEERS HANFORD S/ $\mathrm{H}: 801854$

Run ID = NN61973

= Copyright (c) 1984 Celestial Sof tware Inc. =

SOLVE DISPLACEMENTS Version $2.0 \quad 07 / 01 / 90$

$\mathbf{L}$

UBC SEISHIC LOAD - SH \& ENDIR. A S E 4

APPLIED LOAD VECTOR

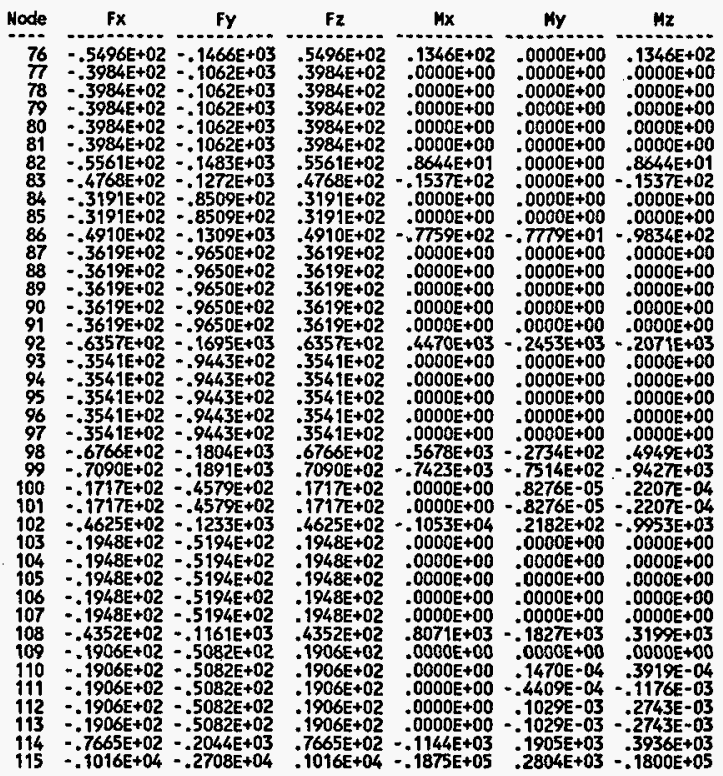


KAISER ENGINEERS HANFORD S/W:801854

PAGE 43

Run ID=N1161973

$02 / 22 / 95$

$08: 49: 56$ x Copyright (c) 1984 Cetestial Software Inc. =

SOLVE DISPLACEMENTS

Version $2.0 \quad 07 / 01 / 90$

$\mathbf{L}$

UBC SEISHIC LOAD - SN \& A DO C A S E 4

APPLIED LOAD VECTOR

\begin{tabular}{|c|c|c|c|c|c|}
\hline Mode & Fy & $\mathbf{F z}$ & $M x$ & My & $\mathrm{Mz}$ \\
\hline $\begin{array}{l}116 \\
117 \\
118 \\
119 \\
120 \\
121 \\
122 \\
123 \\
124 \\
125 \\
126 \\
127 \\
128 \\
129 \\
130 \\
131 \\
132 \\
133 \\
134 \\
135\end{array}$ & 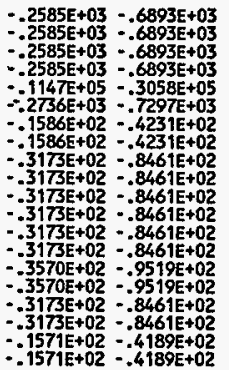 & $\begin{array}{l}.2585 \mathrm{E}+03 \\
.2585 \mathrm{E}+03 \\
.2585 \mathrm{E}+03 \\
.2585 \mathrm{E}+03 \\
.1147 \mathrm{E}+05 \\
.2736 \mathrm{E}+03 \\
.1586 \mathrm{E}+02 \\
.1586 \mathrm{E}+02 \\
.3173 \mathrm{E}+02 \\
.3173 \mathrm{E}+02 \\
.3173 \mathrm{E}+02 \\
.3173 \mathrm{E}+02 \\
.3173 \mathrm{E}+02 \\
.3173 \mathrm{E}+02 \\
.3570 \mathrm{E}+02 \\
.3570 \mathrm{E}+02 \\
.3173 \mathrm{E}+02 \\
.3173 \mathrm{E}+02 \\
.1571 \mathrm{E}+02 \\
1571 \mathrm{E}+02\end{array}$ & $\begin{array}{l}.4221 E+04 \\
.6633 \mathrm{E}+04 \\
.4221 \mathrm{E}+04 \\
.1808 \mathrm{0}+04 \\
-.3416 \mathrm{E}+03 \\
.1216 \mathrm{E}+04 \\
.1537 \mathrm{E}+02 \\
.1537 \mathrm{E}+02 \\
.0000 \mathrm{E}+00 \\
.0000 \mathrm{0}+00 \\
.0000 \mathrm{E}+00 \\
.0000 \mathrm{E}+00 \\
.0000 \mathrm{E}+00 \\
.0000 \mathrm{E}+00 \\
.8644 \mathrm{E}+01 \\
.8644 \mathrm{E}+01 \\
-.2401 \mathrm{E}+02 \\
-.1537 \mathrm{E}+02 \\
.0000 \mathrm{E}+00 \\
. .8644 \mathrm{E}+01\end{array}$ & 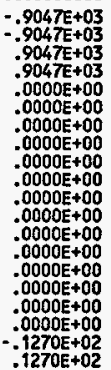 & $\begin{array}{l}.1808 E+04 \\
.4221 E+04 \\
.6633 E+04 \\
.4221 E+04 \\
-.3416 E+03 \\
.1216 E+04 \\
.1537 E+02 \\
.1537 E+02 \\
.0000 E+00 \\
.0000 E+00 \\
.0000 E+00 \\
.0000 E+00 \\
.0000 E+00 \\
.0000 E+00 \\
.8644 E+01 \\
.8644 E+01 \\
-.2401 E+02 \\
.1537 E+02 \\
-.3387 E+02 \\
.2522 E+02\end{array}$ \\
\hline
\end{tabular}

\section{$02 / 22 / 95$
$08: 49: 58$}

KAISER ENGIHEERS HANFORD S/H:801854

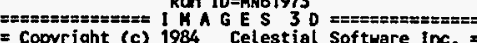

= Copyright (c) 1984 Celestial Software Inc. $x$

SOLVE DISPLACEMENTS

Version $2.0 \quad 07 / 01 / 90$

$\mathbf{L}$

UBC SEISMIC LOND - SN \& A D CASE

DISPLACEMENTS

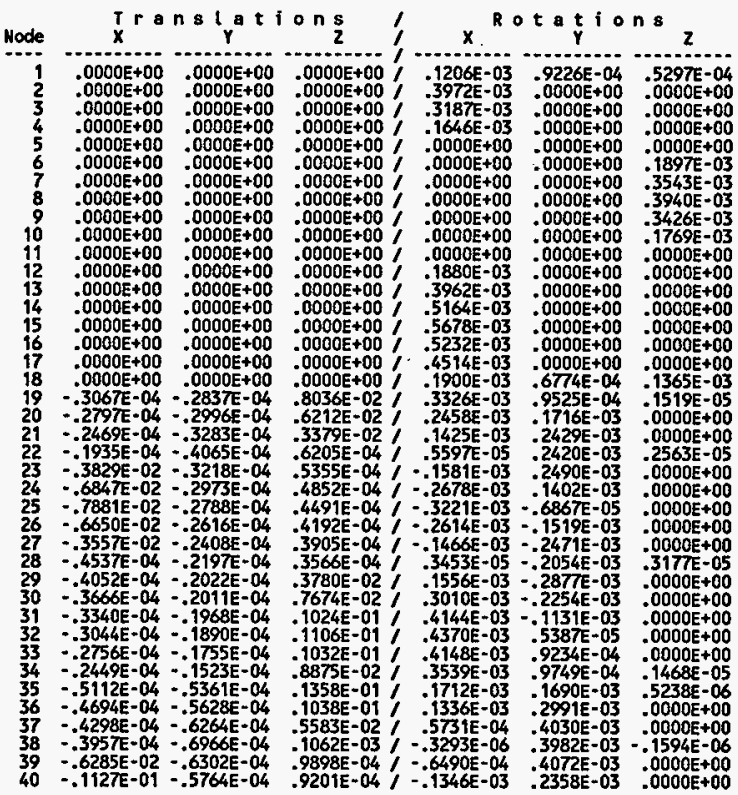


KAISER ENGINEERS HANFORD S/H:801854

PAGE 45

Run $10=$ NN61973

$02 / 22 / 95$
$08: 49: 58$

x Copyright (c) 1984 Celestial Software inc.

SOLVE DISPLACEMENTS

Version $2.0 \quad 07 / 01 / 90$

L

URC SEISHIC LOAD SNOD CASE 4

Node $\quad$ x rans !

$41-.1301 \mathrm{E}-01-.5361 \mathrm{E}-04$.8676E-04/ -.1438E-03 -.1167E-04 .0000E+00

$42-.1098 \mathrm{E}-01 \quad-.4986 \mathrm{E}-04 \quad .8287 \mathrm{E}-04 / \cdot .1319 \mathrm{E}-03-.2508 \mathrm{E}-03 \quad .0000 \mathrm{E}+00$

$43-.5904 E-02-.4566 \mathrm{E}-04 \quad .8009 \mathrm{E}-04,-.6636 \mathrm{E}-04-.4031 \mathrm{E}-03 \quad .0000 \mathrm{E}+00$

$44-.7904 \mathrm{E}-04-.3924 \mathrm{E}-04 \quad .7909 \mathrm{E}-04 / .1377 \mathrm{E}-05-.3478 \mathrm{E}-03$.9984E-06

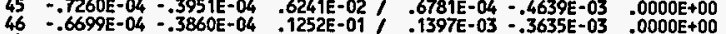

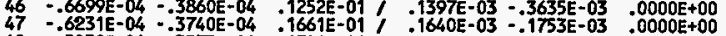

$48-.5839 \mathrm{E}-04 \cdot-3573 \mathrm{E}-04 \quad .1781 \mathrm{E}-01 \%: .1770 \mathrm{E}-03 \quad .1638 \mathrm{E}-04.0000 \mathrm{E}+00$

$49-.5498 \mathrm{E}-04-.3308 \mathrm{E}-04 \quad .1651 \mathrm{E}-01 / .1480 \mathrm{E}-03 \quad .1555 \mathrm{E}-03 \quad .0000 \mathrm{E}+00$

$50-.5252 E-04 \quad-.2841 E-04 \quad .1409 E-01 / .1193 E-03 \quad .1634 E-03 \quad .1006 E-05$

$52=5351 \mathrm{E}-04=.7638 \mathrm{E}-04,1152 \mathrm{E}-01,-.2299 \mathrm{E}-04 \quad .2052 \mathrm{E}-03 \cdot-1008 \mathrm{E}-06$

$52=.5351 E-04=.7638 E-04 \quad .1152 E-01 /-.3045 E-04 \quad .3341 E-03 \quad .0000 E+00$

$54-5066 \mathrm{E}-04-.03435-04$

$54-.5066 \mathrm{E}-04-.9343 \mathrm{E}-04$

$56-.1256 \mathrm{E}-01-.8140 \mathrm{E}-04$

$58.1238 \mathrm{E}-01$.

$58 \cdot-1238 E-01 \cdot-.6955 E-04$

$60 \div .9998 \mathrm{E}-04-.5430 \mathrm{E}-04$

$60-.9998 E-04=.5430 E-04$

$62=.9012 \mathrm{E}-04-.5392 \mathrm{E}-04$

$62=4912 E-04=5392 E-04$

$64=.8146 \mathrm{E}-04=.4917 \mathrm{E}-04$

$\begin{array}{lll}64 & -.8146 \mathrm{E}-04 & -.4917 \mathrm{E}-04 \\ 65 & -.7851 \mathrm{E}-04 & -.4560 \mathrm{E}-04\end{array}$

$66=.7583 \mathrm{E}-04=.3969 \mathrm{E}-04$

$67-.4721 \mathrm{E}-04-.8070 \mathrm{E}-04$

$68 \quad-.4564 \mathrm{E}-04-.8859 \mathrm{E}-04$

$69-.4420 \mathrm{E}-04 \quad-.9830 \mathrm{E}-04$

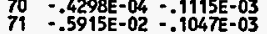

$72 \quad-.5915 \mathrm{E}-02=.1047 \mathrm{E}-03$

$73-\because 1303 \mathrm{E}-01-.9345 \mathrm{E}-04$

$74-.1115 E-01=8582 E-04$

$\begin{array}{llll}.6221 E-02 / .9007 E-06 & -4418 E-03 & .0000 E+00\end{array}$

$\begin{array}{llll}.1405 E-03 & .3531 E-05 & .4471 E-03 & .1576 E-05 \\ 1332 E-03 & .1774 E-05 & .4510 E-03 & .0000 E+00\end{array}$

$1277 \mathrm{E}-03,1795 \mathrm{E}-04$.2640E-03 $0000 \mathrm{00E}+00$

$1228 E-03,1738 E-05-.4721 E-05 \quad 0000 E+00$

$1192 \mathrm{E}-03,4669 \mathrm{E}-05=2779 \mathrm{E}-03$.000 +00

$1176 \mathrm{E}-03 /=.8086 \mathrm{E}-05=.4504 \mathrm{E}-03 \quad .0000 \mathrm{E}+00$

$1170 \mathrm{E}-03, .2179 \mathrm{E}-05=.4043 \mathrm{E}-03 \quad .8421 \mathrm{E}-06$

7030E-02? $3994 \mathrm{E}-05=.5062 \mathrm{E}-03.03000 \mathrm{.000}+00$

$1377 \mathrm{E}-01,-.2633 \mathrm{E}-04-.3823 \mathrm{E}-03 \quad .0000 \mathrm{E}+00$

.

1857E-01/-.1084E-03 .6326E-04 .0000E+00

-1882E-03 $0000 \mathrm{O}+00$

1381E-01 / -.1441E-03 .1871E-03 .1674E-05

$1269 \mathrm{E}-01,-.2064 \mathrm{E}-03$ - $1903 \mathrm{E}-03-.6413 \mathrm{E}-06$

$27 \mathrm{E}-02,-.1596 \mathrm{E}-03 \quad .2874 \mathrm{E}-03 \quad .0000 \mathrm{E}+00$

1610E-03 $\because 1074 E-03$ - $3525 E-03.0000 E+00$

1610E-03,-.2411E-05 $.3636 \mathrm{E}-03 \cdot .3048 \mathrm{E}-05$

1550E-03 103 .

$.1550 \mathrm{E}-03, \quad .1249 \mathrm{E}-03 \quad .2595 \mathrm{E}-03 \quad .0000 \mathrm{E}+00$

$.1495 \mathrm{E}-03 \%: 1066 \mathrm{E}-03-.2476 \mathrm{E}-03 \quad .00000 \mathrm{E}+00$

$\begin{array}{llll}1495 \mathrm{E}-03 & 03 & 1066 \mathrm{E}-03-.2476 \mathrm{E}-03 & .0000 \mathrm{E}+00 \\ 1472 \mathrm{E}-03, .6645 \mathrm{E}-04 & -.4031 \mathrm{E}-03 & .0000 \mathrm{E}+00\end{array}$

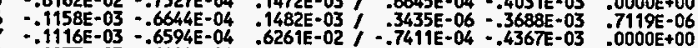

$77-.1116 \mathrm{E}-03-.6594 \mathrm{E}-04$

$.1197 \mathrm{E}-01 \%=.13 \mathrm{T2E}-03=.3152 \mathrm{E}-03 \quad .0000 \mathrm{E}+00$

(1450E-01

$\begin{array}{lll}.1459 E-01,-.2528 E-03 & .1474 E-03 & .0000 E+00 \\ .1149 E-01,-.3366 E-03 & .2609 E-03 & .0000 E+00\end{array}$
KAISER ENGINEERS HANFORD $S / N: 801854$

$\begin{array}{lll}\text { PAGE } 46 & 02 / 22 / 95\end{array}$

$02 / 22 / 95$ $=$ Copyright (c) 1984 Celestial Software Inc.

SOLVE DISPLACEMENTS

Version 2.0 07/01/90

$\mathbf{L}$

UBC SEISHIC LOAD - SN \& AO CASE

Hode $\quad \begin{aligned} & \text { Trans tations } \\ & \text { X }\end{aligned}$

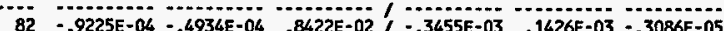

$82-.9225 \mathrm{E}-04-.4934 \mathrm{E}-04 \quad .8422 \mathrm{E}-02 /-.3455 \mathrm{E}-03 \quad .1426 \mathrm{E}-03-.3086 \mathrm{E}-05$

$83-.2328 \mathrm{E}-04=.8548 \mathrm{E}-04 \quad .5432 \mathrm{E}-02 /-.3199 \mathrm{E}-03 \quad .1238 \mathrm{E}-03-.4367 \mathrm{E}-06$

$84 \quad-.2099 \mathrm{E}-04-.9712 \mathrm{E}-04 \quad .3907 \mathrm{E}-02 /-.2423 \mathrm{E}-03 \quad .9829 \mathrm{E}-04 \quad .0000 \mathrm{E}+00$

$85 \quad-.1755 \mathrm{E}-04=1087 \mathrm{E}-03 \quad .2111 \mathrm{E}-02 /-.1057 \mathrm{E}-03 \quad-1643 \mathrm{E}-03 \quad .0000 \mathrm{E}+00$

$86 \quad-.1429 \mathrm{E}-04-.1224 \mathrm{E}-03 \quad .1824 \mathrm{E}-03 / .3927 \mathrm{E}-05 \quad .1157 \mathrm{E}-03 \quad .1081 \mathrm{E}-05$

$87-.3073 \mathrm{E}-02 \div .1209 \mathrm{E}-03 \quad .1763 \mathrm{E}-03 / .1040 \mathrm{E}-03 \quad .2782 \mathrm{E}-03 \quad .0000 \mathrm{E}+00$

$88 \quad-.6571 \mathrm{E}-02-.1133 \mathrm{E}-03 \quad-1765 \mathrm{E}-03 / \quad .1963 \mathrm{E}-03 \quad .1741 \mathrm{E}-03 \quad .0000 \mathrm{E}+00$ $89-.8138 \mathrm{E}-02=.1055 \mathrm{E}-03 \quad .1793 \mathrm{E}-03 / .2170 \mathrm{E}-03 \quad .2813 \mathrm{E}-04 \quad .0000 \mathrm{E}+00$ $90-.7266 \mathrm{E}-02=.9856 \mathrm{E}-04 \quad .1824 \mathrm{E}-03 / .1760 \mathrm{E}-03-.1404 \mathrm{E}-03 \quad .0000 \mathrm{E}+00$ $91 \quad-.4046 \mathrm{E}-02-.9089 \mathrm{E}-04 \quad .1832 \mathrm{E}-03 / .8256 \mathrm{E}-04 \quad-.2756 \mathrm{E}-03 \quad .0000 \mathrm{E}+00$ $92-.1308 E-03-.7422 E-04 \quad .1760 E-03 / .1541 E-05 \quad-.2291 E-03-.2905 E-06$ $93-.1283 E-03-.7299 \mathrm{E}-04 \quad .4073 \mathrm{E}-02 /-.8468 \mathrm{E}-04 \quad-.2842 \mathrm{E}-03 \quad .0000 \mathrm{E}+00$ $94-.1237 \mathrm{E}-03=.7365 \mathrm{E}-04 \quad .7428 \mathrm{E}-02 / \cdot .1932 \mathrm{E}-03 \quad-.1587 \mathrm{E}-03 \quad .0000 \mathrm{E}+00$ $95-.1189 \mathrm{E}-03-.7323 \mathrm{E}-04 \quad .8608 \mathrm{E}-02 / * .2593 \mathrm{E}-03 \quad .3220 \mathrm{E}-05 \quad .0000 \mathrm{E}+00$

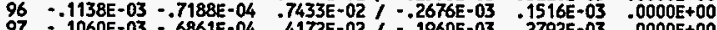
$97-.1060 \mathrm{E}-03-.6861 \mathrm{E}-04 \quad .4172 \mathrm{E}-02,-.1960 \mathrm{E}-03 \quad .2792 \mathrm{E}-03 \quad .0000 \mathrm{E}+00$ $98-.9013 \mathrm{E}-04 \quad-.6012 \mathrm{E}-04 \quad .1422 \mathrm{E}-02,-.1581 \mathrm{E}-03 \quad .8185 \mathrm{E}-04 \quad .1694 \mathrm{E}-04$ $99-.3084 E-05-.8283 E-04 \quad-7193 E-03 ;-.2486 E-03 \quad .6774 E-04=.5866 E-05$ $100-.2801 \mathrm{E}-05-.1010 \mathrm{E}-03 \quad .3662 \mathrm{E}-03 /-.1867 \mathrm{E}-03-.1034 \mathrm{E}-04-.5458 \mathrm{E}-06$ $101-.1196 E-06-.1110 E-03 \quad .5152 E-03 /=.8734 E-04-.8047 E-05 \quad .7050 E-07$ $102 \quad-4789 \mathrm{E}-05-.1229 \mathrm{E}-03 \quad-1581 \mathrm{E}-03 /-.1220 \mathrm{E}-04 \quad .6529 \mathrm{E}-04-.7772 \mathrm{EE}-05$ $103=-1719 \mathrm{E}-02-.1246 \mathrm{E}-03 \quad .1756 \mathrm{E}-03, \quad .2176 \mathrm{E}-05 \quad .1535 \mathrm{E}-03-.5976 \mathrm{E}-04$ $104=-3979 E-02-1143 \mathrm{E}-03 \quad .1845 \mathrm{E}-03, \quad .3516 \mathrm{E}-06 \quad-1338 \mathrm{E}-03-.1170 \mathrm{E}-03$ $105=.5300 \mathrm{E}-02=1065 \mathrm{E}-03.1906 \mathrm{E}-03, \quad .4902 \mathrm{E}-06 \quad .3504 \mathrm{E}-04-.1269 \mathrm{E}-03$ $106-.4924 E-02=.9945 E-04 \quad .1975 E-03 / .5211 E-06-.8364 E-04-.1070 E-03$ $107=.2949 E-02=.9340 E-04 \quad .2097 E-03 /=.2769 E-06-.1690 E-03-.5090 E-04$ 108 - $1450 \mathrm{E}-03 \cdots .7476 \mathrm{E}-04 \quad .2344 \mathrm{E}-03 / .8090 \mathrm{E}-05-.1906 \mathrm{E}-03 \quad .2055 \mathrm{E}-05$ $109=-1346 \mathrm{E}-03-.7598 E-04 \quad .2946 \mathrm{E}-02 / \cdot .5311 \mathrm{E}-04-.1651 \mathrm{E}-03-.3923 \mathrm{E}-06$ $110 \quad .1281 E-03=.7063 E-04 \quad .4841 \mathrm{E}-02 /=.1193 \mathrm{E}-03-.8269 \mathrm{E}-04 \quad .3731 \mathrm{E}-07$

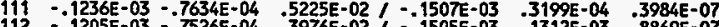
$112=-1205 \mathrm{E}+03-.7526 \mathrm{E}-04 \quad .3976 \mathrm{E}-02 /-.1505 \mathrm{E}-03 \quad .1312 \mathrm{E}-03 \quad .8869 \mathrm{E}-07$ $113=.1205 E-03-.74222-04 \quad .1729 \mathrm{E}-02 /-.9988 \mathrm{E}-04 \quad .1610 \mathrm{E}-03-.2696 \mathrm{E}-07$ $114 \quad-.1290 \mathrm{E}-03 \quad-.6218 \mathrm{E}-04 \cdot .3695 \mathrm{E}-03 / \cdot .6531 \mathrm{E}-04 \quad .1140 \mathrm{E}-03 \quad .3845 \mathrm{E}-05$ $115 \quad .0000 E+00-.5298 \mathrm{E}-03 \quad .0000 \mathrm{E}+00 /-.8085 \mathrm{E}-05-.6391 \mathrm{E}-08-.8059 \mathrm{E}-05$ $116 \quad .0000 E+00 \quad .0000 E+00 \quad .0000 E+00 ， .5574 E-05-.3642 E-06 \quad .2477 \mathrm{E}-05$ $117 \quad .0000 E+00 \quad-.0000 E+00 \quad .0000 E+00 ; \quad .7861 E-05-.3635 E-06 \quad .5570 E-05$ $118 \quad .0000 \mathrm{E}+00 \quad .0000 \mathrm{E}+00 \quad .0000 \mathrm{E}+00 / \quad .5574 \mathrm{E}-05 \quad .3599 \mathrm{E}-06 \quad .7858 \mathrm{E}-05$ $119.0000 E+00 \quad .0000 E+00 \quad .0000 E+00 / .2481 E-05 \quad .3592 E-06 \quad .5571 E-05$ $121-.2206 \mathrm{E}-02-.1320 \mathrm{E}-02 \quad .2207 \mathrm{E}-02 /-.1705 \mathrm{E}-05-.4369 \mathrm{E}-08+.1701 \mathrm{E}-05$ $121-.4596 \mathrm{E}-04-.4420 \mathrm{E}-03 \quad .4597 \mathrm{E}-04 / .7520 \mathrm{E}-05-.1985 \mathrm{E}-08$ - $7516 \mathrm{E}-05$ 
KAISER ENGINEERS HANFORD $S / N: 801854$

PAGE 47

$$
\text { Run ID=\#N61973 }
$$

$02 / 22 / 95$

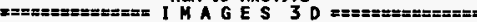
= Copyright (c) 1984 celestial Software Inc.

SOLVE DISPLACEMENTS Version 2.0 07/01/90

UBC SEISHIC LOAD - SN \& EU DIR.

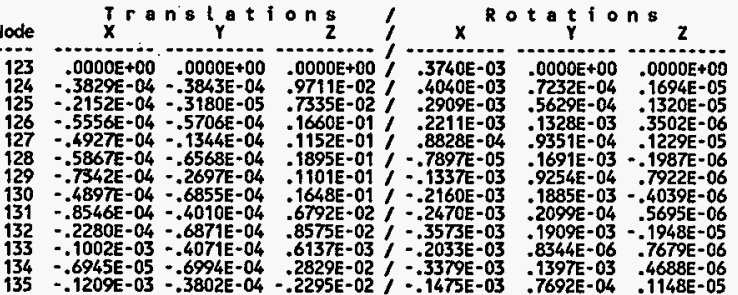

KAISER ENGINEERS HANFORD S/N.801854

PAGE 48

Run ID=1N61973

$02 / 22 / 95$

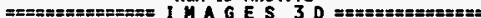
= Copyright (c) 1984 Celestial Software Inc. = SOLVE DISPLACEMENTS Version 2.0 07/01/90
UBC SEISNIC LOAD - NS \& AE
CASE
APPLIED LOAD VECTOR

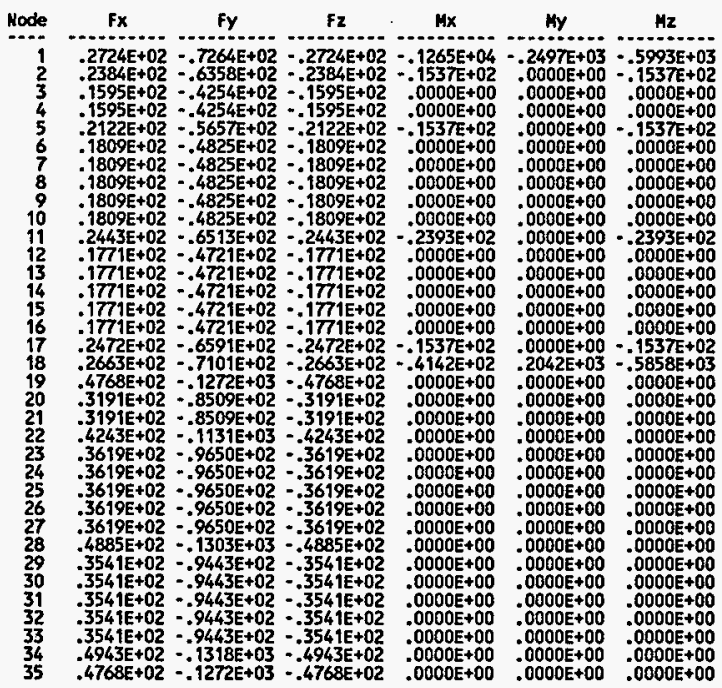


KAISER ENGINEERS HANFORD S/W:801854

z Copyright (c) 1984 G S 3 D =z=z=5E=z=z=s= $=$ Copyright (c) 1984 Celestial Software Inc. = SOLVE DISPLACEMENTS

Version $2.0 \quad 07 / 01 / 90$

$\mathbf{L}$

UBC SEISMIC LOAD - NS \& AE C A SE 5

APPLIED LOAD VECTOR

\begin{tabular}{|c|c|c|c|c|}
\hline Mode & Fx & $M x$ & Hy & Mz \\
\hline $\begin{array}{l}36 \\
37 \\
38 \\
39 \\
40 \\
41 \\
42 \\
43 \\
44 \\
45 \\
46 \\
47 \\
48 \\
49 \\
50 \\
51 \\
52 \\
53 \\
54 \\
55 \\
56 \\
57 \\
58 \\
59 \\
60 \\
61 \\
62 \\
63 \\
64 \\
65 \\
66 \\
67 \\
68 \\
69 \\
70 \\
71 \\
72 \\
73 \\
74 \\
75\end{array}$ & 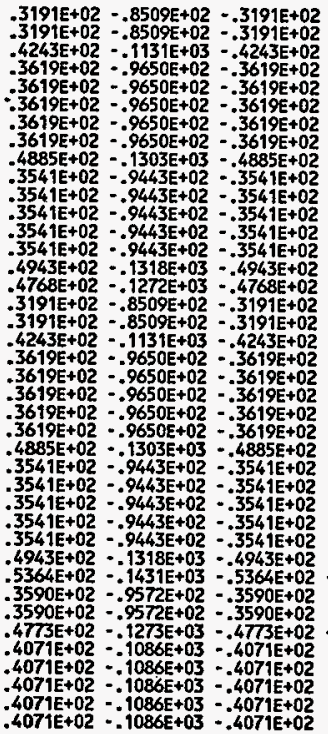 & $\begin{array}{l}.0000 E+00 \\
.0000 E+00 \\
.0000 E+00 \\
.0000 E+00 \\
.0000 E+00 \\
.0000 E+00 \\
.0000 E+00 \\
.0000 E+00 \\
.0000 E+00 \\
.0000 E+00 \\
.0000 E+00 \\
.0000 E+00 \\
.0000 E+00 \\
.0000 E+00 \\
.0000 E+00 \\
.0000 E+00 \\
.0000 E+00 \\
.0000 E+00 \\
.0000 E+00 \\
.0000 E+00 \\
.0000 E+00 \\
.0000 E+00 \\
.0000 E+00 \\
.0000 E+00 \\
.0000 E+00 \\
.0000 E+00 \\
.0000 E+00 \\
.0000 E+00 \\
.0000 E+00 \\
.0000 E+00 \\
.0000 E+00 \\
-8644 E+01 \\
.0000 E+00 \\
.0000 E+00 \\
.8644 E+01 \\
.0000 E+00 \\
.0000 E+00 \\
.0000 E+00 \\
.0000 E+00 \\
.0000 E+00\end{array}$ & $\begin{array}{l}.0000 \mathrm{E}+00 \\
.0000 \mathrm{E}+00 \\
.0000 \mathrm{E}+00 \\
.0000 \mathrm{E}+00 \\
.0000 \mathrm{E}+00 \\
.0000 \mathrm{E}+00 \\
.0000 \mathrm{0}+00 \\
.0000 \mathrm{E}+00 \\
.0000 \mathrm{E}+00 \\
.0000 \mathrm{E}+00 \\
.0000 \mathrm{E}+00 \\
.0000 \mathrm{E}+00 \\
.0000 \mathrm{E}+00 \\
.0000 \mathrm{E}+00 \\
.0000 \mathrm{E}+00 \\
.0000 \mathrm{E}+00 \\
.0000 \mathrm{E}+00 \\
.0000 \mathrm{E}+00 \\
.0000 \mathrm{E}+00 \\
.0000 \mathrm{E}+00 \\
.0000 \mathrm{E}+00 \\
.0000 \mathrm{E}+00 \\
.0000 \mathrm{E}+00 \\
.0000 \mathrm{E}+00 \\
.0000 \mathrm{E}+00 \\
.0000 \mathrm{E}+00 \\
.0000 \mathrm{E}+00 \\
.0000 \mathrm{E}+00 \\
.0000 \mathrm{E}+00 \\
.0000 \mathrm{E}+00 \\
.0000 \mathrm{E}+00 \\
.0000 \mathrm{E}+00 \\
.0000 \mathrm{E}+00 \\
.0000 \mathrm{E}+00 \\
.0000 \mathrm{E}+00 \\
.0000 \mathrm{E}+00 \\
.0000 \mathrm{E}+00 \\
.0000 \mathrm{E}+00 \\
.0000 \mathrm{E}+00 \\
.0000 \mathrm{E}+00\end{array}$ & $\begin{array}{l}.0000 E+00 \\
.0000 E+00 \\
.0000 E+00 \\
.0000 E+00 \\
.0000 E+00 \\
.0000 E+00 \\
.0000 E+00 \\
.0000 E+00 \\
.0000 E+00 \\
.0000 E+00 \\
.0000 E+00 \\
.0000 E+00 \\
.0000 E+00 \\
.0000 E+00 \\
.0000 E+00 \\
.0000 E+00 \\
.0000 E+00 \\
.0000 E+00 \\
.0000 E+00 \\
.0000 E+00 \\
.0000 E+00 \\
.0000 E+00 \\
.0000 E+00 \\
.0000 E+00 \\
.0000 E+00 \\
.0000 E+00 \\
.0000 E+00 \\
.0000 E+00 \\
.0000 E+00 \\
.0000 E+00 \\
.0000 E+00 \\
.8644 E+01 \\
.0000 E+00 \\
.0000 E+00 \\
.8644 E+01 \\
.0000 E+00 \\
.0000 E+00 \\
.0000 E+00 \\
.0000 E+00 \\
.0000 E+00\end{array}$ \\
\hline
\end{tabular}

KAISER ENGINEERS HAHFORD S/N:801854

PAGE 50 RUN 10=MN61973

$02 / 22 / 95$ $08: 49: 59$

= Copyright (c) 1984 Celestial software Inc. Copyright (c) igon

SOLVE DISPLACEMENTS

Version $2.0 \quad 07 / 01 / 90$

L.

UBC SEISUIC LOAD LOAD CASE 5

\section{APPLIED LOAD VECTOR}

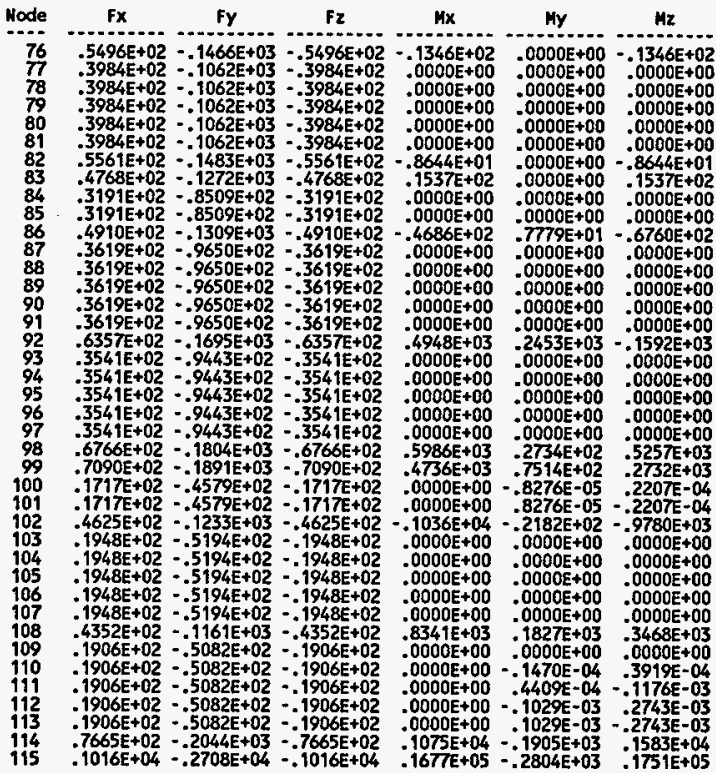


KAISER ENGINEERS HAMFORD S/N:801854

PAGE 51

Run ID=NH61973

$02 / 22 / 95$
$08: 49: 59$

= Copyright (c) 1984 Celestial Software Inc.

SOLVE DISPLACEMEMTS

Version $2.0 \quad 07 / 01 / 90$

UBC SEISHIC LOAD - US: HE CASE 5

APPLIED LOAD VECTOR

\begin{tabular}{|c|c|c|c|c|c|}
\hline $\mathbf{d}$ & $F \mathbf{F}$ & Fy & $M x$ & My & Mz \\
\hline $\begin{array}{l}116 \\
117 \\
118 \\
119 \\
120 \\
121 \\
122 \\
123 \\
124 \\
125 \\
126 \\
127 \\
128 \\
129 \\
130 \\
131 \\
132 \\
133 \\
134\end{array}$ & $\begin{array}{r}-2585 \mathrm{E}+03 \\
.2585 \mathrm{E}+03 \\
.2585 \mathrm{E}+03 \\
-1147 \mathrm{E}+05 \\
-.2736 \mathrm{E}+03 \\
.1586 \mathrm{E}+02 \\
.1586 \mathrm{E}+02 \\
.3173 \mathrm{E}+02 \\
.3173 \mathrm{E}+02 \\
-3173 \mathrm{E}+02 \\
.3173 \mathrm{E}+02 \\
.3173 \mathrm{E}+02 \\
-3173 \mathrm{E}+02 \\
.3570 \mathrm{E}+02 \\
.3570 \mathrm{E}+02 \\
.3173 \mathrm{E}+02 \\
.3173 \mathrm{E}+02 \\
.1571 \mathrm{E}\end{array}$ & 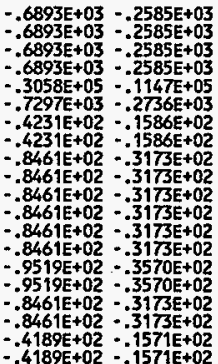 & $\begin{array}{r}-.4221 \mathrm{E}+04 \\
-.1808 \mathrm{E}+04 \\
-.4221 \mathrm{E}+04 \\
-.6633 \mathrm{E}+04 \\
.3416 \mathrm{E}+03 \\
-.1216 \mathrm{E}+04 \\
-.1537 \mathrm{E}+02 \\
-.1537 \mathrm{E}+02 \\
.0000 \mathrm{E}+00 \\
.0000 \mathrm{E}+00 \\
.0000 \mathrm{E}+00 \\
.0000 \mathrm{E}+00 \\
.0000 \mathrm{E}+00 \\
.0000 \mathrm{E}+00 \\
-.8644 \mathrm{E}+01 \\
-.8644 \mathrm{E}+01 \\
.2401 \mathrm{E}+02 \\
.1537 \mathrm{E}+02 \\
.0000 \mathrm{E}+00\end{array}$ & $\begin{array}{l}+03 \\
+03 \\
+\infty 0 \\
+\infty 0 \\
+\infty 0 \\
+\infty 0 \\
+\infty 0 \\
+\infty 0 \\
+\infty 0 \\
+00 \\
+\infty 0 \\
+00 \\
+\infty 0 \\
+\infty 0 \\
+\infty 0 \\
+\infty 0 \\
+\infty 2\end{array}$ & $\begin{array}{l}04 \\
04 \\
04 \\
04 \\
03 \\
04 \\
02 \\
02 \\
00 \\
00 \\
00 \\
00 \\
00 \\
00 \\
01 \\
01 \\
02 \\
02 \\
02\end{array}$ \\
\hline
\end{tabular}

KAISER ENGINEERS HANFORD S/N:801854

52

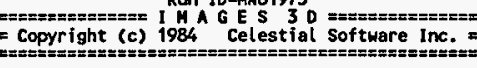

SOLVE OISPLACEMENTS

Version $2.0 \quad 07 / 01 / 90$

L

UBC SEISHIC LOAD - NS \& WE

DISPLACEMENTS

\begin{tabular}{|c|c|c|c|c|c|c|}
\hline \multicolumn{2}{|c|}{$R \circ t a t i \circ n s$} & & \multicolumn{3}{|c|}{ R०tations } \\
\hline $\begin{array}{l}1 \\
2 \\
3 \\
4 \\
5 \\
6 \\
7 \\
8 \\
9 \\
10 \\
11 \\
12 \\
13 \\
14 \\
15 \\
16 \\
17 \\
18 \\
19 \\
20 \\
21 \\
22 \\
23 \\
24 \\
25 \\
26 \\
27 \\
28 \\
29 \\
30 \\
31 \\
32 \\
33 \\
34 \\
35 \\
36 \\
37 \\
38 \\
39 \\
40\end{array}$ & $\begin{array}{l}.0000 E+00 \\
.0000 E+00 \\
.0000 E+00 \\
.0000 E+00 \\
.0000 E+00 \\
.0000 E+00 \\
.0000 E+00 \\
.0000 E+00 \\
.0000 E+00 \\
.0000 E+00 \\
.0000 E+00 \\
.0000 E+00 \\
.0000 E+00 \\
.0000 E+00 \\
.0000 E+00 \\
.0000 E+00 \\
.0000 E+00 \\
.0000 E+00 \\
.2304 E-04 \\
.2758 E-04 \\
.3185 E-04 \\
.3604 E-04 \\
.3853 E-02 \\
.6868 E-02 \\
.7897 E-02 \\
.6657 E-02 \\
.3550 E+02 \\
.2299 E-04 \\
.2741 E-04 \\
.2995 E-04 \\
.3186 E-04 \\
.3374 E-04 \\
.3599 E-04 \\
.3925 E-04 \\
.3942 E-04 \\
.4387 E-04 \\
.4857 E-04 \\
.5304 E-04 \\
.6318 E-02 \\
.1131 E-02\end{array}$ & 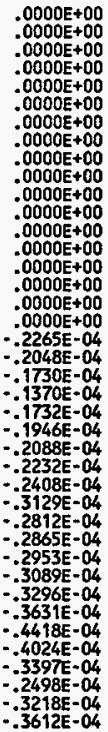 & $\begin{array}{l}.0000 \\
.0000 \\
.0000 \\
.0000 \\
.0000 \\
.0000 \\
.0000 \\
.0000 \\
.0000 \\
.0000 \\
.0000 \\
.0000 \\
.0000 \\
.0000 \\
.0000 \\
.0000 \\
.0000 \\
.0000 \\
-.8082 \\
-.6235\end{array}$ & $\begin{array}{r}-.3209 E-03 \\
-.3992 \mathrm{E}-03 \\
-.3193 \mathrm{E}-03 \\
-.1641 \mathrm{E}-03 \\
.0000 \mathrm{E}+00 \\
.0000 \mathrm{E}+00 \\
.0000 \mathrm{E}+00 \\
.0000 \mathrm{E}+00 \\
.0000 \mathrm{E}+00 \\
.0000 \mathrm{0}+00 \\
.0000 \mathrm{E}+00 \\
-.1903 \mathrm{E}-03 \\
-.4002 \mathrm{E}-03 \\
-.5215 \mathrm{E}-03 \\
-.5759 \mathrm{E}-03 \\
-.5317 \mathrm{E}-03 \\
-.4617 \mathrm{E}-03 \\
-.3861 \mathrm{E}-04 \\
-.3346 \mathrm{E}-03 \\
-.2473 \mathrm{E}-03 \\
-.1425 \mathrm{E}-03 \\
-.3865 \mathrm{E}-05 \\
.1585 \mathrm{E}-03 \\
.2690 \mathrm{E}-03 \\
.3225 \mathrm{E}-03 \\
.2618 \mathrm{E}-03 \\
.1466 \mathrm{E}-03 \\
-.4742 \mathrm{E}-05 \\
-.1574 \mathrm{E}-03 \\
-.3038 \mathrm{E}-03 \\
-.4198 \mathrm{E}-03 \\
-.4432 \mathrm{E}-03 \\
-.4238 \mathrm{E}-03 \\
-.3632 \mathrm{E}-03 \\
-.1734 \mathrm{E}-03 \\
-.1346 \mathrm{E}-03 \\
-.5839 \mathrm{E}-04 \\
-.1115 \mathrm{E}-05 \\
.6523 \mathrm{E}-04\end{array}$ & $\begin{array}{l}. .9089 \mathrm{E}-04 \\
.0000 \mathrm{E}+00 \\
.0000 \mathrm{0}+00 \\
.0000 \mathrm{E}+00 \\
.0000 \mathrm{E}+00 \\
.0000 \mathrm{0}+00 \\
.0000 \mathrm{0}+00 \\
.0000 \mathrm{0}+00 \\
.0000 \mathrm{0}+00 \\
.0000 \mathrm{E}+00 \\
.0000 \mathrm{E}+00 \\
.0000 \mathrm{E}+00 \\
.0000 \mathrm{E}+00 \\
.0000 \mathrm{E}+00 \\
.0000 \mathrm{E}+00 \\
.0000 \mathrm{E}+00 \\
.0000 \mathrm{E}+00 \\
.6114 \mathrm{E}-04 \\
.9675 \mathrm{E}-04 \\
.1734 \mathrm{E}-03\end{array}$ & $\begin{array}{r}-.5035 E-04 \\
.0000 E+00 \\
.0000 E+00 \\
.0000 E+00 \\
.0000 E+00 \\
-.1916 E-03 \\
-.3550 E-03 \\
. .3950 E-03 \\
-.3429 E-03 \\
-.1761 E-03 \\
.0000 E+00 \\
.0000 E+00 \\
.0000 E+00 \\
.0000 E+00 \\
.0000 E+00 \\
.0000 E+00 \\
.0000 E+00 \\
-.1412 E-03 \\
-.1164 E=05 \\
.0000 E+00 \\
.0000 E+00 \\
-.3496 E+05 \\
.0000 E+00 \\
.0000 E+00 \\
.0000 E+00 \\
.0000 E+00 \\
.0000 E+00 \\
-.2133 E-05 \\
.0000 E+00 \\
.0000 E+00 \\
.0000 E+00 \\
.0000 E+00 \\
.0000 E+00 \\
. .2144 E-05 \\
. .4407 E-06 \\
.0000 E+00 \\
.0000 E+00 \\
.9198 E-06 \\
.0000 E+00 \\
.0000 E+00\end{array}$ \\
\hline
\end{tabular}




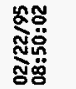

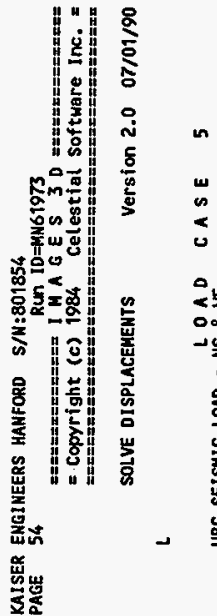

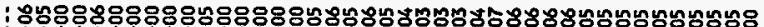

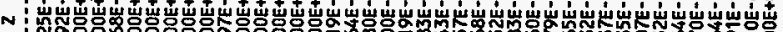
त्र̃

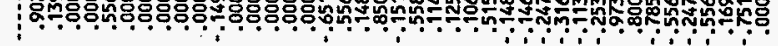

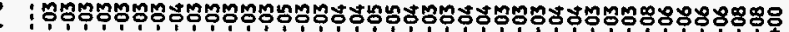

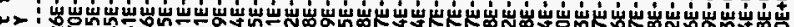

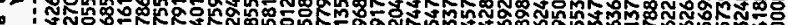
4 $\propto$

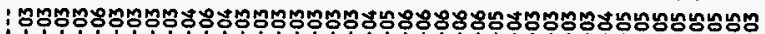

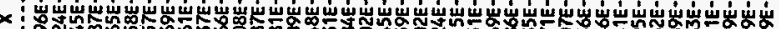
miñ

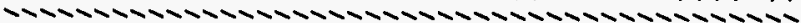

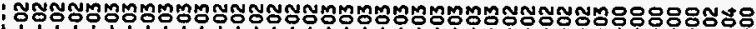

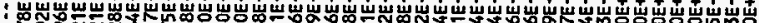
c -

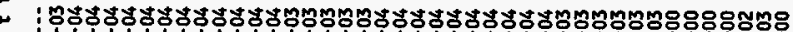

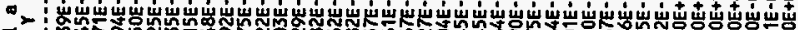

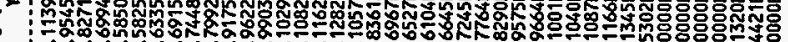

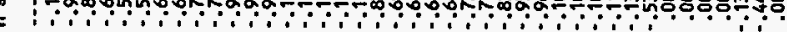

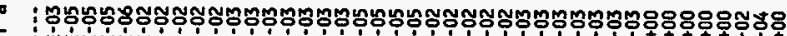

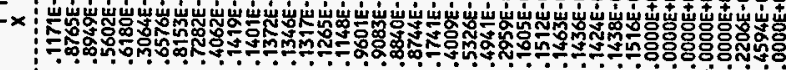

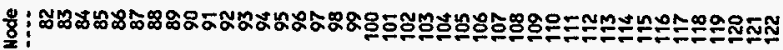

20.

กู่นุ

ชิㅇㅇㅇ

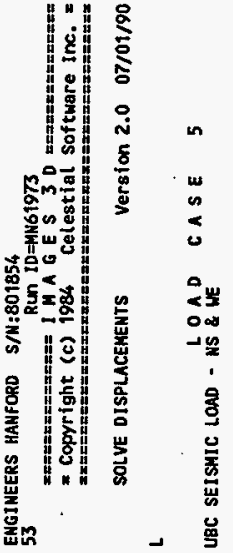

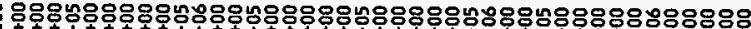

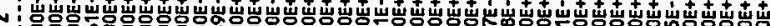

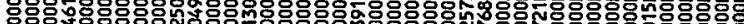

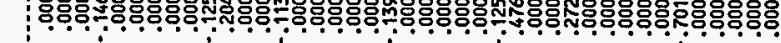

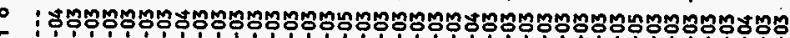
-

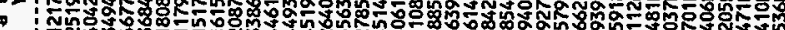

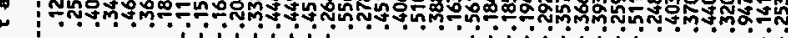

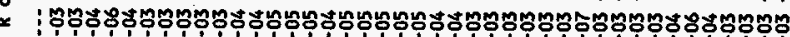
$\times$ นُ

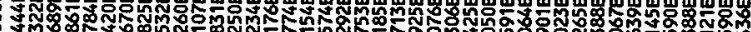
ง

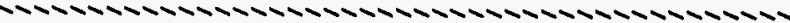

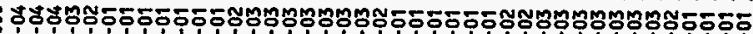

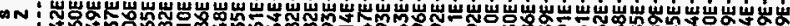

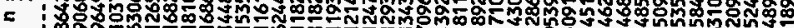

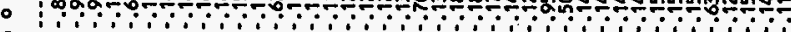

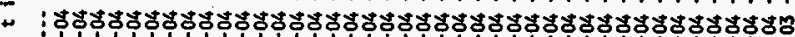

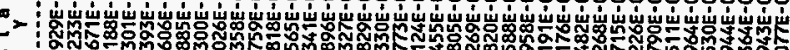
\%

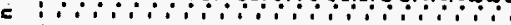

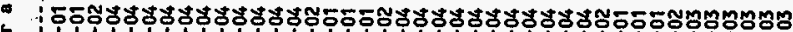

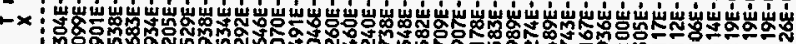
mơñ

苛岩

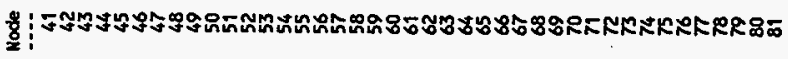


KAISER ENGINEERS HANFORD S/N:801854

PAGE 55 Run $10= \pm 1161973$

02/22/95

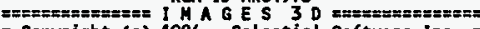
$=$ Copyright (c) 1984 Celestial Software Inc. $== \pm==$

SOLVE DISPLACEMENTS Version 2.0 07/01/90

1

UBC SEISHIC LOAD - LS \& WE CASE 5

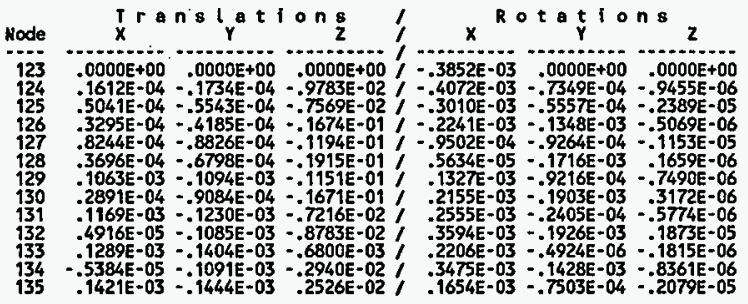

KAISER ENGINEERS HAHFORO S/N:801854

PAGE 56 RUn ID=NN61973

$02 / 22 / 95$
$08: 50: 03$

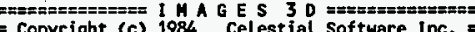

$=$ Copyright (c) 1984 Celestial Software Inc. =

SOLVE BEAM LOADS/STRESSES Version $2.0 \quad 07 / 01 / 90$

L

Load CaSe 1:UBC SEISMIC LOAD - NS \& EH DIR.

BEAM LCADS AND/OR STRESSES

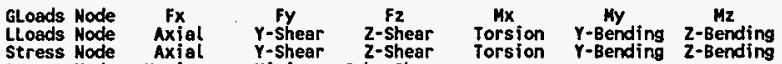

Stress Node Maximum Minimum cmb. Shear **BEAM NO. i***

GLoads $\quad 1-.2192 E+02 \quad .3164 E+03-.1370 \mathrm{EE}+03^{2 * * 1279 E-12} \quad .5684 \mathrm{E}-13-.1137 \mathrm{E}-12$

GLoads $99-.3257 \mathrm{E}+02-.1711 \mathrm{E}+03 \quad .8250 \mathrm{E}+02-.1079 \mathrm{E}+04-.2929 \mathrm{E}+03-.7030 \mathrm{E}+03$

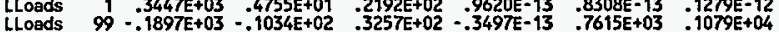

GLoads $18-.1952 E+02 \quad 2173 E+03 \quad .3425 E+02-.4547 E-12-.2842 E-13 \quad .1137 E+12$

GLads $18=.1952 E+02 \quad-2173 E+03 \quad-3425 E+02-.4547 E-12-.2842 E-13-.1137 E+12$

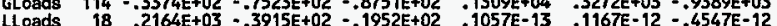

LLoads $114-.9984 E+02 \quad-.5788 E+02-.3374 E+02: 1629 E-13-.9943 E+03 \quad .1309 E+04$

114 -

GLoads $99 \cdot-.1276 E+03 \quad .2912 E+02 \quad .2000 E+03-.6887 E+03 \quad .1200 E+04=.1734 E+03$

GLoads $108 \quad .8883 E+02 \quad .7414 E+02-.2387 E+03-.1405 E+04-.1309 E+03-.7494 E+03$

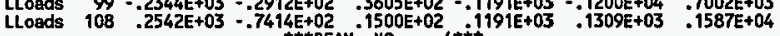

GLoads H**BEAM NO.

(1350E+04-3676E+03- $-1218 E+04$

GLoads $102-.1578 E+03 \quad .6101 \mathrm{E}+02 \quad .1084 \mathrm{E}+03 \quad .8762 \mathrm{E}+03 \quad .6133 \mathrm{E}+03 \quad .7548 \mathrm{E}+03$

Lloads $114-1886 \mathrm{E}+03-7119 \mathrm{E}+02-3316 \mathrm{E}+02 \quad .7328 \mathrm{E}$

$102-1878 E+03-.6101 E+02-.3694 E+02-.7328 E+02-.6133 E+03-1154 E+04$

GLoads $\quad 98-.7686 E+03 \quad .6752 E+02 \quad .8607 \mathrm{E}+03-.1582 E+04 \quad .4126 E+03=.1173 E+04$ **BEAM HO.

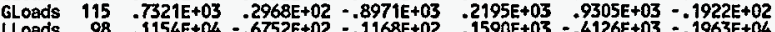

Lloads $115-1157 \mathrm{E}+04-.2968 \mathrm{E}+02-3975 \mathrm{E}+02-.1590 \mathrm{E}+03-.9305 \mathrm{E}+03 \quad .1525 \mathrm{E}+03$

Clocds 115 .

GLoads $92.2316 \mathrm{E}+03 \quad .4411 \mathrm{E}+02.6268 \mathrm{E}+03-.6029 \mathrm{E}+03-.2745 \mathrm{E}+03 \quad .2208 \mathrm{E}+03$

GLoads $115-.2610 \mathrm{E}+03 \quad .3438 \mathrm{E}+02-.6562 \mathrm{E}+03 \quad .2528 \mathrm{E}+03 \quad-.4860 \mathrm{E}+02-.8461 \mathrm{E}+02$

GLoads $115-.2610 \mathrm{E}+03 . .3430 \mathrm{E}+02-.6562 \mathrm{E}+03-.2528 \mathrm{E}+03-.4605 \mathrm{E}+02-.8461 \mathrm{E}+02$

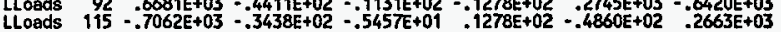

LLoads 115 .

GLoads $\quad 86 \quad .4958 E+03 \quad .3609 E+02-.3248 E+03 \quad .3181 E+03-.7871 E+03 \quad .2739 E+03$

GLoads $115 \cdot-.5091 \mathrm{E}+03 \quad-.5310 \mathrm{E}+00 \quad .3115 \mathrm{E}+03 \quad .6643 \mathrm{E}+02-.8566 \mathrm{E}+03 \quad .2388 \mathrm{E}+03$

LLoads $86 \quad .5915 E+03-.3609 E+02+.3763 E+02 \quad .9013 E+02 \quad .7871 E+03-.4100 E+03$

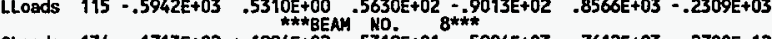

GLoads $134-.1713 E+02 \cdot .1994 E+02 \quad .5319 E+01-.5984 E+03 \quad .7612 E+03 \quad .2700 E-12$ 
KAISER ENGINEERS HANFORD S/N:801854

PAGE 57 Run ID=MN61973

$02 / 22 / 95$ 08:50:04

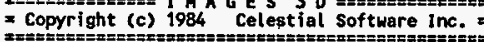

SOLVE BEAM LOADS/STRESSES Version 2.0 07/01/90

Load Case 1:UBC SEISHIC LOAD - NS \& EH DIR.

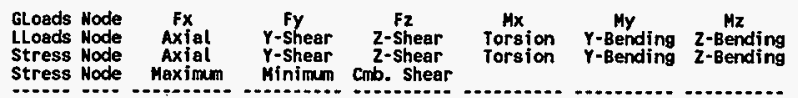

GLoads $99 \quad .9513 E+01 \quad .3843 \mathrm{E}+00-.1294 \mathrm{E}+02 \quad .5984 \mathrm{E}+03 \quad-.9438 \mathrm{E}+03 \quad .1955 \mathrm{E}+03$ LLoads $134-.1713 E+02-.1994 E+02-.5319 E+01-.5984 E+03-.7612 E+03-.2700 E-12$ $99.9513 \mathrm{E}+01-.3843 \mathrm{E}+00 \quad .1294 \mathrm{E}+02 \quad .5984 \mathrm{E}+03 \quad .9438 \mathrm{E}+03-.1955 \mathrm{E}+03$

GLoads $135 \quad .4312 E+02-.2697 \mathrm{E}+02-.7990 \mathrm{E}+01-.5935 \mathrm{E}+03-.5454 \mathrm{E}+03 \quad .1082 \mathrm{E}+03$ $\begin{array}{lllllllll}\text { GLoads } 114 & -.5074 \mathrm{E}+02 & .4729 \mathrm{E}+02 & .3702 \mathrm{E}+00 & .5935 \mathrm{E}+03 & .5618 \mathrm{E}+03 & .6345 \mathrm{E}+03\end{array}$ $\begin{array}{lllllllll}\text { LLoads } & 135 & -.4312 E+02 & .2697 \mathrm{E}+02 & -.7990 \mathrm{E}+01 & .5935 \mathrm{E}+03 & .5454 \mathrm{E}+03 & . .1082 \mathrm{E}+03 \\ \text { LLoads } & 114 & .5074 \mathrm{E}+02 & -.4729 \mathrm{E}+02 & .3702 \mathrm{E}+00 & -.5935 \mathrm{E}+03 & -.4618 \mathrm{E}+03 & .6345 \mathrm{E}+03\end{array}$ LLOds 116 . GLoads $122-.8420 E+01-.2253 E+03-.8650 E+01 \quad .5672 E+03-.6248 E+03 \quad .1109 E+03$ GLoads $124 \quad .3839 E-01 \quad .2477 \mathrm{E}+03 \quad .2682 \mathrm{E}+00-.6653 \mathrm{E}+03 \quad .6248 \mathrm{E}+03-.1781 \mathrm{E}+02$ LLoads $122-.2253 \mathrm{E}+03-.8420 \mathrm{E}+01 \quad .8650 \mathrm{E}+01-.6248 \mathrm{0}-03 \quad .5672 \mathrm{E}+03$ Lloads $124 \quad .2477 \mathrm{E}+03 \quad .3839 \mathrm{E}-01-.2682 \mathrm{E}+00 \quad .6248 \mathrm{E}+03-.6653 \mathrm{E}+03-.1781 \mathrm{E}+02$ GLoads $124-.3615 E+01-.1289 E+03-.6823 E+01 \quad .1526 E+04-.5026 E+03 \quad .1781 E+02$ GLoads $126=.4767 \mathrm{E}+01-1512 \mathrm{E}+03-.1559 \mathrm{E}+01-.1584 \mathrm{E}+04-.5026 \mathrm{E}+03-.3048 \mathrm{E}+02$ LLoads $124=1280 E+03-3615 \mathrm{E}+01-0823 \mathrm{E}+01-5026 \mathrm{E}+03 \quad .1526 \mathrm{E}+04=.1781 \mathrm{E}+02$

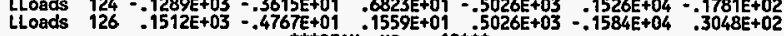

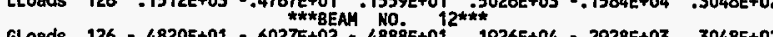
GLoads $126-.4820 E+01-.6027 \mathrm{E}+02-.4888 \mathrm{E}+01.1926 \mathrm{E}+04-.2928 \mathrm{E}+03 \quad .3048 \mathrm{E}+02$ GLoads $128=.3562 E+01 \quad .8262 E+02-.3493 E+01-.1942 E+04 \quad .2928 E+03-.1664 E+02$

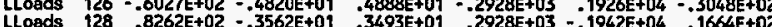

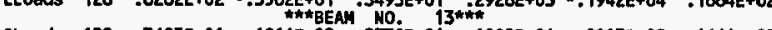
GLoods $128-.3685 E+01-.1911 E+02=.2738 E+01 \quad .1802 E+04-.9003 E+02 \quad .1664 E+02$ GLoads $130-.4696 \mathrm{E}+01 \quad .4146 \mathrm{E}+02-.5644 \mathrm{E}+01-1770 \mathrm{E}+04 \quad .9003 \mathrm{E}+02-.2776 \mathrm{E}+02$ LLoads $128-.1911 E+02-3685 E+01-2738 E+01-9003 E+02-1802 E+04-.1664 E+02$

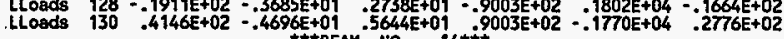

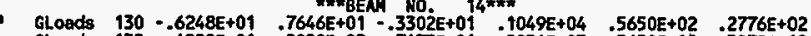
GLoads $132-.4229 E+01 \quad 2029 E+02-7175 E+04-9954 E+03-5650 E+02-3979 E+12$ L $130 \quad 7646 \mathrm{E}+01-6248 \mathrm{E}+01-3302 \mathrm{E}+01-5650 \mathrm{C}+02-1049 \mathrm{E}+04-2776 \mathrm{E}+02$ lloads $132 \quad-2029 E+02-.4229 E+01 \quad .77175 E+01 \quad-5650 E+02-.9954 E+03 \quad .3979 E-12$ (1)*TRUSS NO. 15*k* $\begin{array}{lllllll}\text { GLoods } 132 & .0000 E+00 & -1212 E+02 & .0000 E+00 & .0000 E+00 & .0000 E+00 & .0000 E+00\end{array}$ LLoads $132.1212 E+02.0000 E+00$.000E +00 .0000E+00 $.0000 E+00.000 E+00$

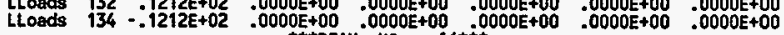
GLoods $123-.1126 E+02.4352 E+03-.8641 E+01^{16 * \star *} .8411 E+03 \cdot .2678 E+03 \quad .1384 E+03$
KAISER ENGINEERS HANFORD $S / N: 801854$

PAGE 58 RUN ID=HN61973

$02 / 22 / 95$

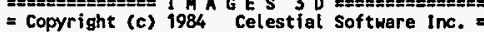

SOLVE GEAM LOADS/STRESSES Version $2.0 \quad 07 / 01 / 90$

ᄂ

Load Case 1:UBC SEISMIC LOAD - MS \& EW DIR.

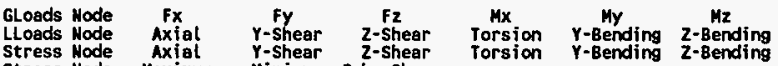
Stress Node Maximum Minimum $\mathrm{cmb}$. Shear

GLOads $125 \quad .2883 E+01-.4128 E+03 \quad .2591 E+00-.9390 E+03 \quad .2678 E+03 \quad .1721 E+02$

LLoads $123.4352 E+03 \quad .1126 E+02-.8641 E+01-.2678 E+03-.8411 E+03 \quad .1384 E+03$

LLoads $125-.4128 E+03-.2883 E+01.2591 E+00.2678 E+03 \quad .9390 E+03 \quad .1721 E+02$

GLoads $\quad 2-.7275 E+01 \quad .3631 \mathrm{E}+02-.5452 \mathrm{E}+01 \quad .4990 \mathrm{E}+03-.7940 \mathrm{E}+03 \quad .9723 \mathrm{E}+02$

GLoads $19-.1107 \mathrm{E}+01-.1396 \mathrm{E}+02-.2930 \mathrm{E}+01-.5267 \mathrm{E}+03 \quad-.7940 \mathrm{E}+03-.2937 \mathrm{E}+02$

LLoads $2 \quad .3631 E+02-.7275 E+01 \quad .5452 E+01-.7940 E+03 \quad .4990 E+03-.9723 E+02$

LLoads $19-.1396 \mathrm{E}+02-.1107 \mathrm{E}+01 \quad .2930 \mathrm{E}+01 \quad .7940 \mathrm{E}+03-.5267 \mathrm{E}+03 \quad .2937 \mathrm{E}+02$

GLoads $19-.4383 E+01 \quad .2523 E+02-.4525 E+01 \quad .1223 E+04-.6193 E+03 \quad .2937 E+02$

GLoads $35-.3999 E+01-.2883 E+01-.3856 E+01-.1230 E+04 \quad .6193 E+03-.2515 E+02$

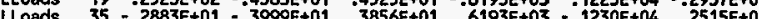

LLoads $35-.2883 E+01-.3999 E+01 \quad .3856 E+010.6193 E+03 \cdot-1230 E+04 \quad .2515 E+02$

GLoads $35-.4425 E+01 \quad .3346 \mathrm{E}+02-.4085 \mathrm{E}+01 \quad .1450 \mathrm{E}+04 \quad-.2727 \mathrm{E}+03 \quad .2515 \mathrm{E}+02$

$51-.3957 \mathrm{E}+01-.11111 \mathrm{E}+02-.4296 \mathrm{E}+01-.1447 \mathrm{E}+04 \quad .2727 \mathrm{E}+03-.2001 \mathrm{E}+02$

$\begin{array}{llllllll}\text { LLoads } & 35 & .3346 \mathrm{E}+02 & -.4425 \mathrm{E}+01 & .4085 \mathrm{E}+01 & -.2727 \mathrm{E}+03 & .1450 \mathrm{E}+04 & -.2515 \mathrm{E}+02 \\ \text { LLoads } & 51 & -.1111 \mathrm{E}+02 & -.3957 \mathrm{E}+01 & .4296 \mathrm{E}+01 & .2727 \mathrm{E}+03 & -1447 \mathrm{E}+04 & .2001 \mathrm{E}+02\end{array}$

GLoads $51-.4579 E+01 \quad .2984 E+02 \div .4115 E+01 \quad .1404 E+04 \quad .1235 E+03 \quad .2001 E+02$

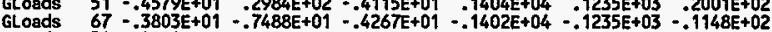

GLoads $67-.380 \mathrm{E}+01-748 \mathrm{C}+01-.4267 E+01-1402 \mathrm{C}+04-1235 E+03-1148 E+02$

LLoads $67-.7488 \mathrm{E}+01-.3803 \mathrm{E}+01 \quad .4267 \mathrm{E}+01-1235 \mathrm{E}+03-.1402 \mathrm{E}+04 \quad .1148 \mathrm{E}+02$

GLoads $67-.3352 E+01 \quad .1581 E+02 \cdot-4105 E+01 \quad .7864 E+03 \quad .4420 E+03 \quad .1148 E+02$

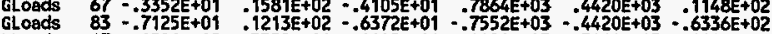

GLoads $83-7125 E+101-6330 E+02$

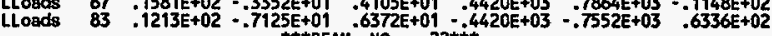

GLoads $83-.2621 E+02-.1078 E+02 \quad .3305 E+01 * .5499 E+03 \quad .5214 E+03 \quad .6336 E+02$

$\begin{array}{llllllll}\text { GLoads } & 83 & -.2621 E+02 & -.1078 E+02 & .3305 E+01 & -.5499 E+03 & -5214 E+03 & .6336 E+02 \\ \text { GLoads } & 99 & .1993 E+02 & -.2754 E+02 & -.9591 E+01 & .6563 E+03 & -.5214 E+03 & .3173 E+03\end{array}$

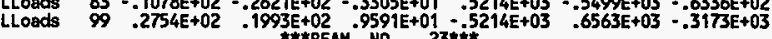

GLoods $\quad 5=.6450 E+01 \quad .5184 E+03-.6594 E+01-.1202 E+03-.1271 E+03 \quad .1194 E+03$

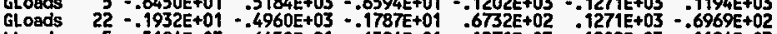

LLoads 5 . $\quad .5184 \mathrm{E}+03 \quad .6450 \mathrm{E}+01-.6594 \mathrm{E}+01-.1271 \mathrm{E}+03 \quad .1202 \mathrm{E}+03 \quad .1194 \mathrm{EE}+03$

Lloods $22-.4960 \mathrm{E}+03 \quad: 1932 \mathrm{E}+01-.1787 \mathrm{E}+01 \quad-1271 \mathrm{E}+03-.6732 \mathrm{E}+02-.6969 \mathrm{E}+02$

GLoads $22-.2303 E+01 \quad .3225 E+03-.1739 E+01.7186 E+02-.9654 E+02-.4892 E+02$ 
KAISER ENGINEERS HANFORD S/ $\mathrm{H}: 801854$

Run ID=NN61973

$02 / 22 / 95$

PAGE 59

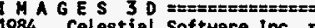
I Copyright (c) 1984 Celestial Software Inc. =

SOLVE BÉAR LOADS/STRESSES Version $2.0 \quad 07 / 01 / 90$

LOAd CASE 1:UBC SEISHIC LOAD - NS \& EH DIR.

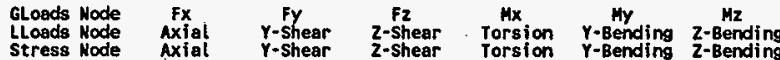
Stress Node Maximum Minimum $\mathrm{Cmb}$. Shear

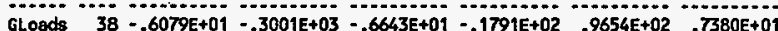

$38 \cdot .6079 E+01 \cdot-3001 E+03 \quad-.6643 E+01-.1791 E+02 \quad-9654 E+02 \quad-7380 E+01$

LLoads $38-.3001 \mathrm{E}+03 \quad .6079 \mathrm{E}+01 \quad-.6643 \mathrm{E}+01 \quad .9654 \mathrm{E}+02 \quad .1791 \mathrm{E}+02 \quad .7380 \mathrm{E}+01$

GLoads $38-.4916 \mathrm{E}+01.2214 \mathrm{k}+03-.4830 \mathrm{E}+01-.6784 \mathrm{EE}+02 \quad .4413 \mathrm{E}+01 \quad .6932 \mathrm{E}+02$

GLoods $54-.3466 E+01-.1991 E+03-.3552 E+01 \quad .5378 E+02-.4413 E+01-.5338 E+02$

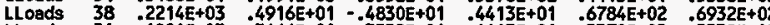

LLoads $54-.1991 \mathrm{E}+03 \quad .3466 \mathrm{E}+01-.3552 \mathrm{E}+01-.4413 \mathrm{E}+01-.5378 \mathrm{E}+02-.5338 \mathrm{E}+02$

GLoads $54-.4162 E+01.1409 E+03-.4764 E+01 \quad .2609 E+02 \quad .7442 E+02 \cdot .1744 E+02$

GLoads $70-.4220 E+01-.1186 E+03-.3618 E+01-.3869 E+02-.7442 E+02-.1680 E+02$

Lloads $54 \quad .1409 E+03 \quad .4162 E+01-.4764 E+01 \quad .7442 E+02-.2609 E+02-.1744 E+02$

LLosds $70-.1186 \mathrm{E}+03 \quad .4220 \mathrm{E}+01-3618 \mathrm{E}+01-.7442 \mathrm{EE}+02 \quad .3869 \mathrm{E}+02 \quad .1680 \mathrm{E}+02$

GLoads $70=-5075 E+01 \quad .8262 E+02-.4926 E+01-.7252 E+02 \quad .1314 E+03 \quad .3619 E+02$

GLoads $86=.5402 E+01-.5468 E+02-.5552 E+01 \quad .8113 E+02-.1314 E+03-.4069 E+02$

LLoods $70 \quad .8262 \mathrm{E}+02 \quad .5075 \mathrm{E}+01-.4926 \mathrm{E}+01 \quad .1314 \mathrm{E}+03 \quad .7252 \mathrm{E}+02 \quad .3619 \mathrm{E}+02$

LLoads $86-.5468 \mathrm{E}+02 \quad .5402 \mathrm{E}+01-.5552 \mathrm{E}+01-1314 \mathrm{E}+03-.8113 \mathrm{E}+02-.4069 \mathrm{E}+02$

GLoads $86 \quad .1790 \mathrm{E}+01 \quad .3074 \mathrm{E}+02-.2055 \mathrm{E}+02 \quad .1015 \mathrm{E}+03 \quad .4833 \mathrm{E}+03 \quad .4998 \mathrm{E}+02$

GLoads $102-.8076 \mathrm{E}+01-.1398 \mathrm{E}+02 \quad .1426 \mathrm{E}+02-.3887 \mathrm{E}+03-.4833 \mathrm{E}+03-.1314 \mathrm{E}+03$

LLoads $86 \quad .3074 E+02-.1790 E+01-.2055 E+02 \quad .4833 E+03-.1015 E+03 \quad .4998 E+02$

Llaods $102-.1398 \mathrm{E}+02 \quad .8076 \mathrm{E}+01 \quad .1426 \mathrm{E}+02-.4833 \mathrm{E}+03 \quad .3887 \mathrm{E}+03-.1314 \mathrm{E}+03$

GLoads $11-.1475 E+02-.2855 E+03-.8286 E+01-.1535 E+03 \quad .5633 E+03 \quad .3603 E+03$

GLoads $28 \quad .1697 \mathrm{E}+01 \quad .3204 \mathrm{E}+03-.4765 \mathrm{E}+01-1148 \mathrm{E}+03-.5633 \mathrm{E}+03-.1794 \mathrm{E}+03$

LLoads $11-.2855 \mathrm{E}+03-.8286 \mathrm{E}+01-.1475 \mathrm{E}+02 \quad .5633 \mathrm{E}+03 \quad .3603 \mathrm{E}+03-.1535 \mathrm{E}+03$

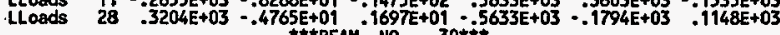
ThtBEAM NO. 30**it

- GLaads $28-.3384 E+01-.1175 E+03-.4314 E+01 \quad .5366 E+02 \quad .3734 E+03-.6870 E+02$

GL oads $44-.9667 \mathrm{E}+01 \quad .1523 \mathrm{E}+03-.8737 \mathrm{E}+01-.5007 \mathrm{E}+01-.3734 \mathrm{E}+03-.4122 \mathrm{E}+00$

$28-.1175 \mathrm{E}+03-.4314 \mathrm{E}+01-.3384 \mathrm{E}+01-.3734 \mathrm{E}+03-.6870 \mathrm{E}+02-.5366 \mathrm{E}+02$

LLoeds $44.1523 \mathrm{E}+03-.8737 \mathrm{E}+01-.9667 \mathrm{E}+01-.3734 \mathrm{EE}+03-.4122 \mathrm{E}+00-.5007 \mathrm{E}+01$

GLoads $44-.6864 E+01-.2127 \mathrm{E}+02-.6098 \mathrm{E}+01+.7378 E+02 \quad .8524 \mathrm{~B}+02 \quad .6708 E+02$

GLoads $\quad 60 \div .6188 \mathrm{E}+01-.5607 \mathrm{E}+02-.6953 \mathrm{E}+01-.8319 \mathrm{E}+02-.8524 \mathrm{E}+02-.5965 \mathrm{E}+02$

$44-.2127 \mathrm{E}+02=.6098 \mathrm{E}+01-.6864 \mathrm{E}+01-.8524 \mathrm{E}+02-.6708 \mathrm{E}+02-.7378 \mathrm{E}+02$

LLosds $60 \quad-5607 \mathrm{E}+02-.6953 \mathrm{E}+01-.6188 \mathrm{E}+01-.8524 \mathrm{E}+02-.5965 \mathrm{E}+02 \quad-.6319 \mathrm{E}+02$

GLaads

$60-.8834 E+01.4057 E+02-.9079 E+01 \quad .2483 E+02-.2069 E+03-.3302 E+02$
KAISER ENGINEERS HANFORD S/N:801854

$02 / 22 / 95$

RUn ID=MH61973 08:50:05 = Copyright (c) 1984 Celestial Software Inc. A

SOLVE BEAM LOADS/STRESSES Version 2.0 07/01/90

Load Case 1:UBC SEISMIC LOAD - NS \& EN DIR.

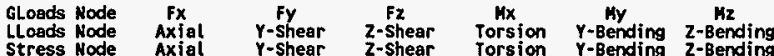
Stress Hode Maximum Minimum Cmb. Shear

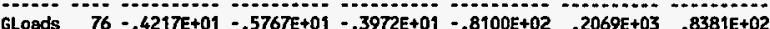

LLoads $60-4057 \mathrm{E}+02-.9079 \mathrm{E}+01-.8834 \mathrm{E}+01-.2069 \mathrm{0}+03-.3302 E+02 \quad-2483 E+02$

LLoads $76-.5767 \mathrm{E}+01-.3972 \mathrm{E}+01-.4217 \mathrm{E}+01, .2069 \mathrm{E}+03 \quad .8381 \mathrm{E}+02-.8100 \mathrm{E}+02$

GLoads $76-.4421 E+01 \quad .3735 E+02-.6374 E+01-.6010 E+02-.3928 E+03 \quad .5691 E+02$

GLoads $92-.1189 \mathrm{E}+02 \quad .6156 \mathrm{E}+01-.9940 \mathrm{E}+01 \quad .1091 \mathrm{E}+03 \quad .3928 \mathrm{E}+03-.1597 \mathrm{EE}+03$

LLoads $76 \quad .3735 \mathrm{E}+02-.6374 \mathrm{E}+01-.4421 \mathrm{E}+01-.3928 \mathrm{E}+03 \quad-5691 \mathrm{E}+02-.6010 \mathrm{E}+02$

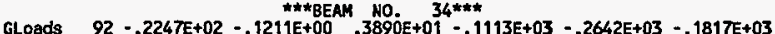

GLoads $108 \quad .1269 \mathrm{E}+02 \quad .2622 \mathrm{E}+02-.1368 \mathrm{E}+02-2562 \mathrm{E}+03-.2642 \mathrm{E}+03 \quad .4718 \mathrm{E}+03$

LLoads $92-.1211 E+00 \quad .3890 E+01-.2247 E+02-.2642 E+03 \quad-.1817 E+03-.1113 E+03$

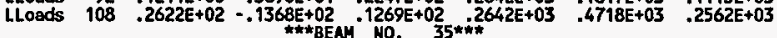

GLoads $\quad 17-.8687 E+01 \quad .2451 E+03-.7520 E+01 \quad .9834 E+03-.4872 E+03 \quad .1049 E+03$

GLoads $34 \quad .3048 E+00 \quad-.2227 \mathrm{E}+03-.8616 \mathrm{E}+00 \quad-.1057 \mathrm{E}+04 \quad .4872 \mathrm{E}+03-.5997 \mathrm{E}+01$

LLoads $\quad 17-2451 E+03 \quad .067 E+01-.7520 E+01-.4872 E+03-.9834 E+03 \quad 1049 E+03$

LLoads. $34-.2227 \mathrm{E}+03-.3048 \mathrm{E}+00-.8616 \mathrm{E}+00 \quad .4872 \mathrm{E}+03 \quad .1057 \mathrm{E}+04-.5997 \mathrm{E}+01$

GLoads $34-.3429 E+01 \quad .2322 E+03-.551 j_{E+01} \quad .2458 E+04-.3156 E+03 \quad .5997 E+01$

GLoads $50=.4952 \mathrm{E}+09-.2099 \mathrm{E}+03-.2871 \mathrm{E}+01-.2487 \mathrm{E}+04 \quad .3156 \mathrm{E}+03-.2275 \mathrm{E}+02$

LLoads $34 \quad .2322 E+03 \quad .3429 E+01-.5511 E+01-.3156 E+03-.2458 E+04 \quad .5997 E+01$

LLoads $50=.2099 \mathrm{E}+03 \quad .4952 \mathrm{E}+01-.2871 \mathrm{E}+01 \quad .3156 \mathrm{E}+03 \quad .2487 \mathrm{E}+04-.2275 \mathrm{EE}+02$

GLoads $50-.5622 E+09.1699 E+03-.3870 \mathrm{E}+01 \quad .2772 \mathrm{E}+04-.1005 \mathrm{E}+03 \quad .2275 \mathrm{E}+02$

GLoads $66-.2760 \mathrm{E}+01-.1475 \mathrm{E}+03-.4512 \mathrm{E}+01-.2765 \mathrm{E}+04 \quad .1005 \mathrm{E}+03 \quad .8738 \mathrm{E}+01$

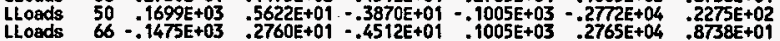

GLoads $66.2181 E+01 \quad .1187 E+03=.3142 E+01 \quad .2130 E+04 \quad .2510 E+03-.8738 E+01$

GLoads $82-.1056 \mathrm{E}+02-.9635 \mathrm{E}+02-.5240 \mathrm{E}+01-.2107 \mathrm{E}+04-.2510 \mathrm{E}+03=.1315 \mathrm{E}+03$

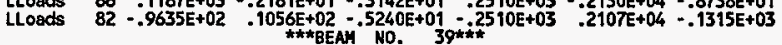

GLoads $82 \cdot-2475 \mathrm{E}+02 \quad .8218 \mathrm{E}+02 \quad .3058 \mathrm{E}+01-.1393 \mathrm{E}+04 \quad .1978 \mathrm{E}+03 \quad .1315 \mathrm{E}+03$

GLoods $98 \quad-1427 \mathrm{E}+02-.5424 \mathrm{E}+02-.1354 \mathrm{E}+02 \quad .1621 \mathrm{E}+04-.1978 \mathrm{E}+03 \quad .4050 \mathrm{E}+03$

$\begin{array}{llllllllll}\text { LLoads } & 82 & .8218 E+02 & .2475 E+02 & .3058 E+01 & .1978 E+03 & .1393 E+04 & .1315 E+03\end{array}$

LLoods $98-.5424 \mathrm{E}+02-.1427 \mathrm{E}+02-.1354 \mathrm{E}+02-.1978 \mathrm{E}+03-.1621 \mathrm{E}+04 \quad .4050 \mathrm{E}+03$

GLoads $98 \cdot-1007 E+03 \quad .2541 E+02-.2302 E+02-.1418 E+04-.2619 E+03 \quad .7679 E+03$ 
KAISER ENGINEERS HANFORD S/N:801854

PAGE 61 Run ID=MN61973

$02 / 22 / 95$ 08:50:05

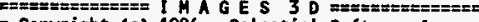
= Copyright (c) 1984 Celestial Sof tware Inc. =

SOLVE BEAM LOAOS/STRESSES Version $2.0 \quad 07 / 01 / 90$ $\mathbf{L}$

Load Case 1:UBC SEISMIC LOAD - NS \& EW DIR.

\begin{tabular}{|c|c|c|c|c|c|c|c|}
\hline $\begin{array}{l}\text { GLoads } \\
\text { LLoads } \\
\text { Stress } \\
\text { Stress }\end{array}$ & $\begin{array}{l}\text { Node } \\
\text { Hode } \\
\text { Node } \\
\text { Hode }\end{array}$ & $\begin{array}{c}\text { Fx } \\
\text { Axial } \\
\text { Axial } \\
\text { Maximm }\end{array}$ & $\begin{array}{c}\text { Fy } \\
\text { Y-Shear } \\
\text { Y-shear } \\
\text { Minimum }\end{array}$ & $\begin{array}{c}\text { Fz } \\
\text { z-Shear } \\
\text { z-Shear } \\
\text { Corb. Shear }\end{array}$ & $\begin{array}{l}M x \\
\text { Torsion } \\
\text { Torsion }\end{array}$ & $\begin{array}{c}\text { My } \\
\gamma-\text { Bending } \\
\gamma \text {-Bending }\end{array}$ & $\begin{array}{l}\text { Mz } \\
\text { 2-Bending } \\
\text { z-Bending }\end{array}$ \\
\hline $\begin{array}{l}\text { GLoads } \\
\text { LLoads } \\
\text { LLoads }\end{array}$ & $\begin{array}{r}114 \\
98 \\
114\end{array}$ & $\begin{array}{l}.9437 \mathrm{E}+02 \\
.2541 \mathrm{E}+02 \\
.8642 \mathrm{E}+01\end{array}$ & $\begin{array}{r}-.8642 E+01 \\
.1007 E+03 \\
-.9437 E+02\end{array}$ & $\begin{array}{r}.1673 \mathrm{E}+02 \\
-.2302 \mathrm{E}+02 \\
-1673 \mathrm{E}+02\end{array}$ & $\begin{array}{l}1090 \mathrm{E}+04 \\
.2619 \mathrm{E}+03 \\
2619 \mathrm{E}+03\end{array}$ & $\begin{array}{r}.2619 \mathrm{E}+03 \\
.1418 \mathrm{E}+04 \\
-.1090 \mathrm{E}+04\end{array}$ & $\begin{array}{l}.8411 E+03 \\
.7679 E+03 \\
.8411 E+03\end{array}$ \\
\hline $\begin{array}{l}\text { GLoads } \\
\text { GLoads } \\
\text { LLoads } \\
\text { LLoads }\end{array}$ & $\begin{array}{r}99 \\
100 \\
99 \\
100\end{array}$ & $\begin{array}{l}1365 E+02 \\
.1886 E+02 \\
1365 E+02 \\
.1886 E+02\end{array}$ & $\begin{array}{r}.3594 \mathrm{E}+02 \\
-.2205 \mathrm{E}+02 \\
-.3594 \mathrm{E}+02 \\
.2205 \mathrm{E}+02 \\
\star \star * \mathrm{BEA}\end{array}$ & $\begin{array}{r}1069 E+02 \\
.5483 E+01 \\
1069 E+02 \\
5483 E+01\end{array}$ & $\begin{array}{l}.5308 E+03 \\
.5308 E+03 \\
5308 E+03 \\
.5308 E+03 \\
. * 5\end{array}$ & $\begin{array}{r}.1153 \mathrm{E}+04 \\
-1043 \mathrm{E}+04 \\
.1153 \mathrm{E}+04 \\
.1043 \mathrm{E}+04\end{array}$ & $\begin{array}{r}.3636 E+03 \\
.3264 E+02 \\
-.3636 E+03 \\
-.3264 E+02\end{array}$ \\
\hline $\begin{array}{l}\text { GLoads } \\
\text { GLoads } \\
\text { LLoads } \\
\text { Lloads }\end{array}$ & $\begin{array}{l}100 \\
101 \\
100 \\
101\end{array}$ & $\begin{array}{r}-.2540 \mathrm{E}+02 \\
.2019 \mathrm{E}+02 \\
-.2540 \mathrm{E}+02 \\
.2019 \mathrm{E}+02\end{array}$ & $\begin{array}{r}.3880 E+01 \\
.1000 E+02 \\
-.3880 E+01 \\
-.1000 \mathrm{E}+02 \\
+4 \mathrm{BEA}\end{array}$ & $\begin{array}{l}.5736 E+01 \\
.5296 E+00 \\
5736 E+01 \\
5296 E+00\end{array}$ & $\begin{array}{l}.7045 \mathrm{E}+03 \\
.7045 \mathrm{E}+03 \\
.7045 \mathrm{E}+03 \\
.7045 \mathrm{E}+03\end{array}$ & $\begin{array}{r}.8092 E+03 \\
-.7664 E+03 \\
-.8092 E+03 \\
.7664 E+03\end{array}$ & $\begin{array}{r}. .3264 E+02 \\
-.9205 E+01 \\
.3264 E+02 \\
.9205 E+01\end{array}$ \\
\hline $\begin{array}{l}\text { GLoads } \\
\text { GLoads } \\
\text { LLoads } \\
\text { LLoads }\end{array}$ & $\begin{array}{l}101 \\
102 \\
101 \\
102\end{array}$ & $\begin{array}{r}-9720 E+02 \\
: 9199 \mathrm{E}+02 \\
-.9720 \mathrm{E}+02 \\
.9199 \mathrm{E}+02\end{array}$ & $\begin{array}{r}-1060 \mathrm{E}+02 \\
.2449 \mathrm{E}+02 \\
.1060 \mathrm{E}+02 \\
-.2449 \mathrm{E}+02 \\
\end{array}$ & & $\begin{array}{l}.7047 \mathrm{E}+03 \\
.7047 \mathrm{E}+03 \\
.7047 \mathrm{E}+03 \\
.7047 \mathrm{E}+03\end{array}$ & $\begin{array}{r}.7553 E+03 \\
-.7342 E+03 \\
-.7553 E+03 \\
.7342 E+03\end{array}$ & $\begin{array}{r}.9205 \mathrm{E}+01 \\
-.2490 \mathrm{E}+03 \\
-.9205 \mathrm{E}+01 \\
.2490 \mathrm{E}+03\end{array}$ \\
\hline $\begin{array}{l}\text { GLoads } \\
\text { GLoads } \\
\text { LLoads } \\
\text { LLoads }\end{array}$ & $\begin{array}{l}102 \\
103 \\
102 \\
103\end{array}$ & $\begin{array}{r}-.7984 E+01 \\
.2078 E+01 \\
.7453 E+02 \\
-.6862 E+02\end{array}$ & $\begin{array}{r}.3185 \mathrm{E}+02 \\
.4759 \mathrm{E}+02 \\
.3185 \mathrm{E}+02 \\
-.4759 \mathrm{E}+02 \\
* * \mathrm{BEA}\end{array}$ & $\begin{array}{l}7453 \mathrm{E}+02 \\
6862 \mathrm{E}+02 \\
7984 \mathrm{E}+01\end{array}$ & $\begin{array}{l}.5259 \mathrm{E}+03 \\
.8978 \mathrm{E}+02 \\
.1643 \mathrm{E}+03 \\
1643 \mathrm{E}+03\end{array}$ & $\begin{array}{r}.3091 E+03 \\
-.2311 E+03 \\
-.3091 E+03 \\
.2311 E+03\end{array}$ & $\begin{array}{r}-.1643 E+03 \\
.1643 E+03 \\
.5259 E+03 \\
.8978 E+02\end{array}$ \\
\hline $\begin{array}{l}\text { GLoads } \\
\text { GLoads } \\
\text { LLoads } \\
\text { LLoads }\end{array}$ & $\begin{array}{l}103 \\
104 \\
103 \\
104\end{array}$ & $\begin{array}{l}-.1086 \mathrm{E}+02 \\
.4955 \mathrm{E}+01 \\
.7805 \mathrm{E}+00 \\
.6686 \mathrm{E}+01\end{array}$ & $\begin{array}{r}.1254 E+02 \\
.3203 E+01 \\
.1254 E+02 \\
.3203 E+01 \\
* A * A E A\end{array}$ & & $\begin{array}{r}.8978 E+02 \\
.1739 E+02 \\
.3324 E+03 \\
.3324 E+03\end{array}$ & $\begin{array}{r}-.4031 \mathrm{E}+03 \\
.5257 \mathrm{E}+03 \\
.4031 \mathrm{E}+03 \\
-.5257 \mathrm{E}+03\end{array}$ & $\begin{array}{r}-.3324 E+03 \\
.3324 E+03 \\
-.8978 E+02 \\
.1739 E+02\end{array}$ \\
\hline $\begin{array}{l}\text { GLoads } \\
\text { GLoads } \\
\text { LLoads } \\
\text { LLoads }\end{array}$ & $\begin{array}{l}104 \\
105 \\
104 \\
105\end{array}$ & $\begin{array}{l}-.3824 E+01 \\
-.2081 E+01 \\
-.1882 E+02 \\
.2472 E+02\end{array}$ & $\begin{array}{r}.7463 E+01 \\
.8284 E+01 \\
. .7463 E+01 \\
.8284 E+01 \\
\end{array}$ & $\begin{array}{l}.1882 E+02 \\
.2472 E+02 \\
.3824 E+01\end{array}$ & $\begin{array}{r}.1739 E+02 \\
.2375 E+02 \\
.1295 E+03 \\
.1295 E+03\end{array}$ & $\begin{array}{r}.9798 E+03 \\
.9933 E+03 \\
.9798 E+03 \\
.9933 E+03\end{array}$ & $\begin{array}{r}-.1295 \mathrm{E}+03 \\
.1295 \mathrm{E}+03 \\
-.1739 \mathrm{E}+02 \\
.2375 \mathrm{E}+02\end{array}$ \\
\hline $\begin{array}{l}\text { SLoeds } \\
\text { SLoads } \\
\text { LLoads } \\
\text { LLoeds }\end{array}$ & $\begin{array}{l}105 \\
106 \\
105 \\
106\end{array}$ & $\begin{array}{l}-1436 E+01 \\
.7341 E+01 \\
.5753 E+01 \\
.1166 E+02\end{array}$ & $\begin{array}{r}.8117 E+01 \\
.7631 E+01 \\
.8117 E+01 \\
.7631 E+01 \\
\end{array}$ & $\begin{array}{l}3 \mathrm{E}+01 \\
6 \mathrm{E}+02 \\
6 \mathrm{E}+01\end{array}$ & $\begin{array}{l}.2375 E+02 \\
-.1999 \mathrm{E}+02 \\
-.4053 \mathrm{E}+02 \\
.4053 \mathrm{E}+02 \\
+4\end{array}$ & $\begin{array}{r}-.8498 \mathrm{E}+03 \\
.7818 \mathrm{E}+03 \\
.8498 \mathrm{E}+03 \\
. .7818 \mathrm{E}+03\end{array}$ & $\begin{array}{r}.4053 \mathrm{E}+02 \\
-.4053 \mathrm{E}+02 \\
-.2375 \mathrm{E}+02 \\
.1999 \mathrm{E}+02\end{array}$ \\
\hline iloads & 106 & $.8036 E+01$ & $.6987 E+01$ & & $.1999 E+02$ & $-.5799 E+02$ & $.2470 E+03$ \\
\hline
\end{tabular}

KAISER ENGINEERS HANFORD S/N:801854

PAGE 62 RUN ID=MH61975 $02 / 22 / 95$

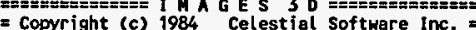

SOLVE BEAM LOADS/STRESSES Version 2.0 07/01/90

L

LOad CASe 1:UBC SEISHIC LOAD - NS \& EW DIR.

GLoads Node Fx $\quad$ Fy $\quad$ Fz $\quad$ Mx $\quad$ My $M$ LLoads Hode Axial Y-Shear Z-Shear Torsion Y-Bending $z$-Bending Stress Node Axial Y-Shear Z-Shear Torsion Y-Bending Z-Bending Stress Node Maximum Minimum Cimb. Shear GLoads $107-.1394 \mathrm{E}+02 \quad .8760 \mathrm{E}+01 \quad .3155 \mathrm{E}+02-.3373 \mathrm{E}+02-.1123 \mathrm{E}+03-.2470 \mathrm{E}+03$

LLoads $106 \quad .3745 \mathrm{E}+02-.6987 \mathrm{E}+01-.8036 \mathrm{E}+01-.2470 \mathrm{E}+03 \quad .5799 \mathrm{E}+02 \quad-.1999 \mathrm{E}+02$

LLoads $107-.3155 \mathrm{E}+02-.8760 \mathrm{E}+01 \quad .1394 \mathrm{E}+02 \quad .2470 \mathrm{E}+03 \quad .1123 \mathrm{E}+03 \quad .3373 \mathrm{E}+02$

GLoBds $107.8381 E+01 \quad .2584 \mathrm{E}+02-.1389 \mathrm{E}+03 \quad .3373 \mathrm{E}+02 \quad .1152 \mathrm{E}+04 \quad .2226 \mathrm{E}+03$

GLoads $108-.1429 \mathrm{E}+02-.1009 \mathrm{E}+02 \quad .1330 \mathrm{E}+03 \quad .2448 \mathrm{E}+03-.1328 \mathrm{E}+04-.2226 \mathrm{E}+03$

LLoads $107-.1389 \mathrm{E}+03-.2584 \mathrm{E}+02-.8381 \mathrm{E}+01-.2226 \mathrm{E}+03-.1152 \mathrm{E}+04-.3373 \mathrm{E}+02$

LLoads $108=.1330 \mathrm{E}+03.1009 \mathrm{E}+02 \quad .1429 \mathrm{E}+02.2226 \mathrm{E}+03 \quad .1328 \mathrm{E}+04-.2448 \mathrm{E}+03$

GLoads $108-.1447 \mathrm{E}+02 \quad .1903 \mathrm{E}+02-.1329 \mathrm{E}+02 \quad .5558 \mathrm{E}+03 \quad .1214 \mathrm{E}+04 \quad .1632 \mathrm{E}+03$

GLoads $109 \quad .8690 \mathrm{E}+01-.3617 \mathrm{E}+01 \quad .7507 \mathrm{E}+01-.5558 \mathrm{E}+03-.1057 \mathrm{E}+04 \quad .8519 \mathrm{E}+01$

LLosds $109.8690 \mathrm{E}+01 \quad .3617 \mathrm{E}+01-.7507 \mathrm{E}+01-5558 \mathrm{E}+03 \quad .1057 \mathrm{E}+04-.8519 \mathrm{E}+01$

GLoads $109-.1618 E+02 \quad .5744 E+01-.1428 E+02 \quad .5977 E+03 \quad .1829 E+02-.8519 E+01$

GLoads $110 \quad .1040 \mathrm{E}+02 \quad .9665 \mathrm{E}+01 \quad .8505 \mathrm{E}+01 \quad-.5977 \mathrm{E}+03 \quad .1545 \mathrm{E}+03 \quad-.2121 \mathrm{E}+02$

LLoads $109-0.1618 E+02 \quad-.5744 E+01 \quad .1428 E+02 \quad .5977 \mathrm{E}+03 \quad-.1829 \mathrm{E}+02 \quad .8519 \mathrm{E}+01$

LLoads $110 \quad .1040 \mathrm{E}+02-.9665 \mathrm{E}+01-.8505 \mathrm{E}+01-.5977 \mathrm{E}+03-.1545 \mathrm{E}+03 \quad .2121 \mathrm{E}+02$

GLoads $110-.2385 E+02 \quad .7818 E+01 * .7282 \mathrm{E}+01.3280 \mathrm{E}+03-.9395 \mathrm{E}+03 \quad .2121 \mathrm{E}+02$

GLoads $111 \quad .1807 E+02 \quad .7591 \mathrm{E}+01 \quad .1504 \mathrm{E}+01-.3280 \mathrm{E}+03 \quad .1006 \mathrm{E}+04-.1950 \mathrm{E}+02$

LLoads $110-.2385 E+02-.7802+01.7282 E+01-3280 E+03-9395 E+03-.2121 E+02$

LLoods $111.1807 E+02-.7591 E+01-.1504 E+01-.3280 E+03-.1006 E+04 \quad .1950 E+02$

GLoads $111-.3305 E+02 \quad .8116 E+01-.1750 E+01 \quad .4089 E+02-.1200 E+04.1950 E+02$

GLoads $112 \quad .2727 \mathrm{E}+02 \quad .7293 \mathrm{E}+01-.4028 \mathrm{E}+01-.4089 \mathrm{E}+02 \quad .1183 \mathrm{E}+04-.1325 \mathrm{E}+02$

LLoods $111-.3305 \mathrm{E}+02-.8116 \mathrm{E}+01 \quad .1750 \mathrm{E}+01 \quad .4089 \mathrm{E}+02 \quad .1200 \mathrm{E}+04-.1950 \mathrm{E}+02$

Lloads $112-.2727 \mathrm{E}+02-.7293 \mathrm{E}+01, .4028 \mathrm{E}+01-.4089 \mathrm{E}+02-.1183 \mathrm{E}+04 \cdot .1325 \mathrm{E}+02$

GLoads $112-.2783 E+02 \quad .1536 \mathrm{E}+01 \quad .7412 \mathrm{E}+01-.4007 \mathrm{E}+03-.6646 \mathrm{E}+03 \quad .1325 \mathrm{E}+02$

GLoads $113 \quad .2205 \mathrm{E}+02 \quad .1387 \mathrm{E}+02-.1319 \mathrm{E}+02 \quad .4007 \mathrm{E}+03 \quad .5083 \mathrm{E}+03-.1068 \mathrm{E}+03$

LLoads $112-.2783 E+02 \quad-.1536 E+01-.7412 E+01-.4007 E+03 \quad .6646 E+03-.1325 E+02$

LLoads $113.2205 E+02-.1387 E+02.1319 E+02 \quad .4007 E+03-.5083 E+03 \quad .1068 E+03$

$\begin{array}{lllllllll}\text { GLods } & 113 & .1515 \mathrm{E}+02 & .5969 \mathrm{E}+02 & .7410 \mathrm{E}+00 & -.2817 \mathrm{E}+03 & .1787 \mathrm{E}+03 & .1068 \mathrm{0}+03\end{array}$

GLoads $114-.2093 \mathrm{E}+02 \quad-.4428 \mathrm{E}+02-.6519 \mathrm{E}+01 \quad .2817 \mathrm{E}+03-.2338 \mathrm{E}+03 \quad .6816 \mathrm{E}+03$

LLosds $113-1515 \mathrm{E}+02-.5969 \mathrm{E}+02-.7410 \mathrm{E}+00-.2817 \mathrm{E}+03-.1787 \mathrm{E}+03-.1068 \mathrm{E}+03$

Lloads $114=.2093 \mathrm{E}+02 \quad .4428 \mathrm{E}+02 \quad .6519 \mathrm{E}+01 \quad .2817 \mathrm{E}+03 \quad .2338 \mathrm{E}+03-.6816 \mathrm{E}+03$

GLoads $115=.6423 E+04 \quad .1601 E+05-.6422 E+04 \quad .1367 E+06-.2365 E+02+.1368 E+06$ 


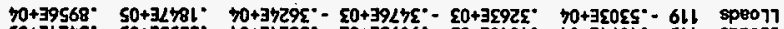

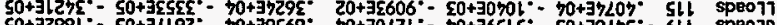

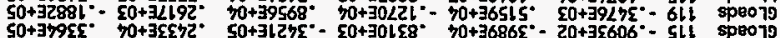

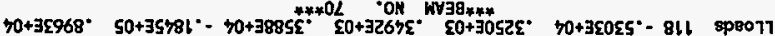

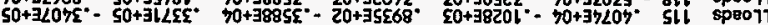
(5)

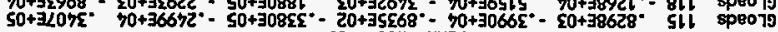

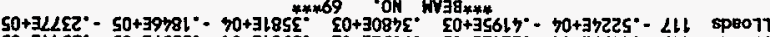

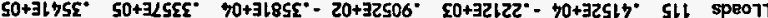

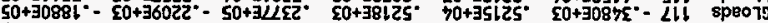

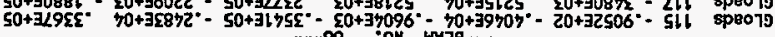

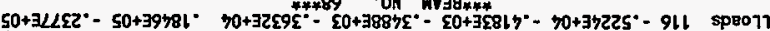

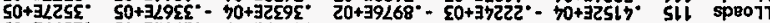

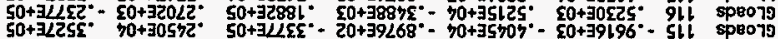

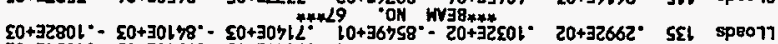

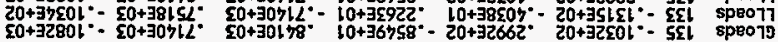

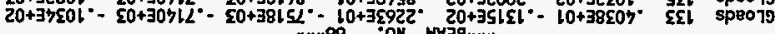

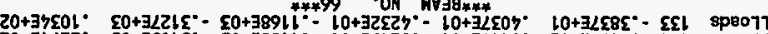

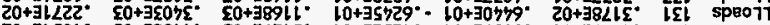

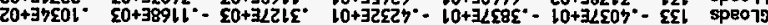

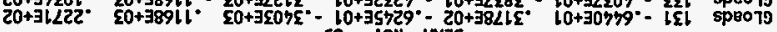

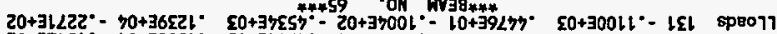

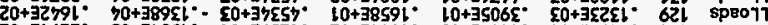

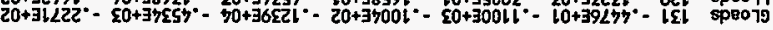

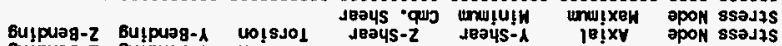

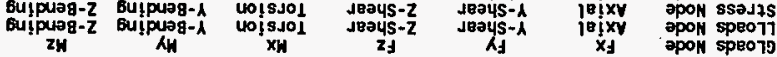

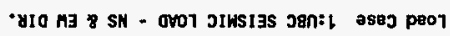

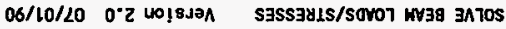

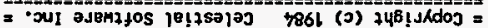
$90: 05: 80$
$56 / 22 / 20$ EL6ínilt=0i uny

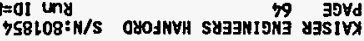

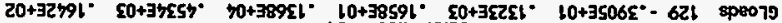

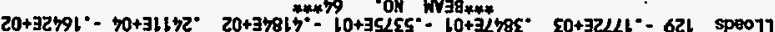

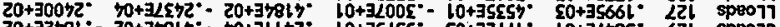

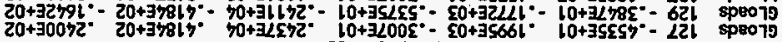

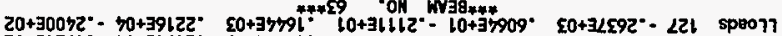

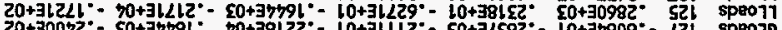

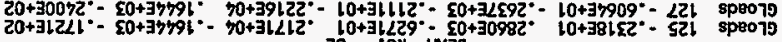

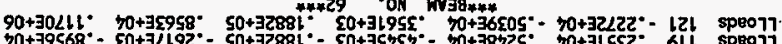

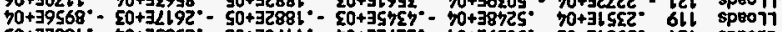

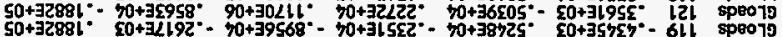

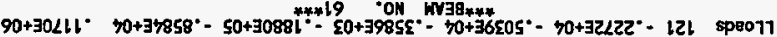

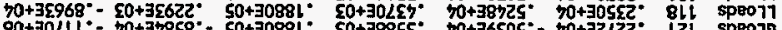

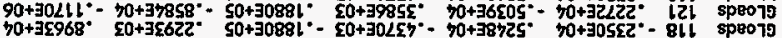

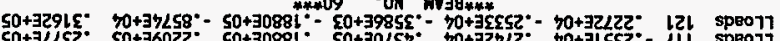

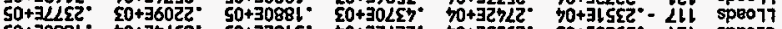

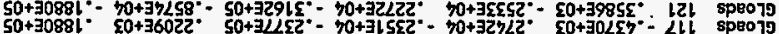

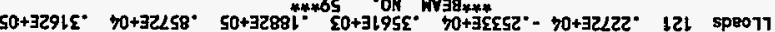

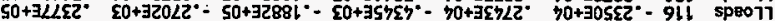

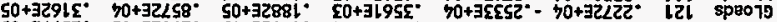

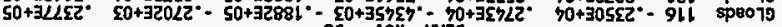

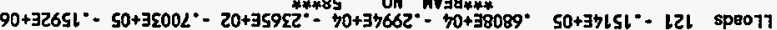

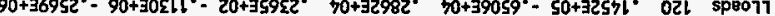

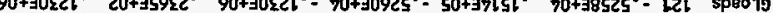
$90+37861^{\circ} 20+35982^{\circ}-90+35861^{\circ}-40+39205^{\circ} 50+3251^{\circ}-70+35205^{\circ} 021$ speoา

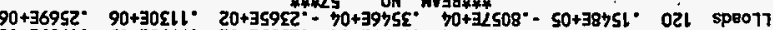

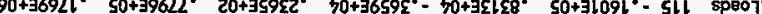
$90+37861^{\circ}-20+35982^{\circ} 90+35861^{\circ} 70+37229^{\circ} 50+38751^{\circ}-70+35229^{\circ}$ 021 sp807

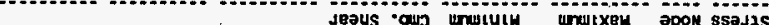

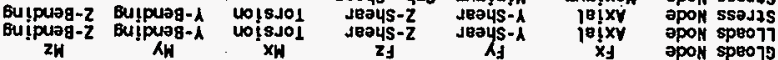

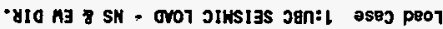
7

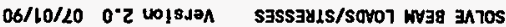

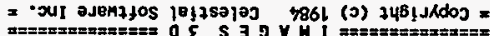
$90: 05: 80$ $56 / 22 / 20$

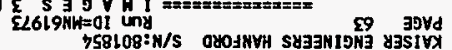


KAISER ENGIHEERS HANFORO S/N:801854

PAGE 65

65 RUn ID=MN61973

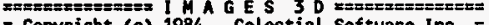
= Copyright (c) 1984. Celestial Software Inc. =

SOLVE BEAM LOADS/STRESSES Version 2.0 07/0i/90

L

Load Case 1:UBC SEISHIC LOAD - HS \& EH DIR.

MAXIMUN STRESS SUMMARY FOR BEAMS/TRUSSES
WITHIN SPECIFIED RANGE

Maxinam (absolute) Stress $=.1381 E+04$ at BEAM 56

Beam Axial Y-Shear Z-Shear Torsion Y-Bending Z-Bending

$\begin{array}{lllllll}56 & .4397 \mathrm{E}+03 & .4578 \mathrm{E}+03 & -.2015 \mathrm{E}+03 & -.1344 \mathrm{E}-02 & .2876 \mathrm{E}+03 & .6540 \mathrm{E}+03\end{array}$ Maximum Minimum Cmb. Shear

$.1381 \mathrm{E}+04=.5019 \mathrm{E}+03 \quad .6907 \mathrm{E}+03$
$02 / 22 / 95$
$08: 50: 07$

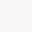

KAISER ENGINEERS HANFORD S/N:801854

PAGE 66 RUN ID=NN61973

$02 / 22 / 95$
$08: 50: 07$

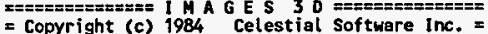

$=== \pm====$

SOLVE PLATE LCADS/STRESSES Version 2.0 07/01/90

L

Load Case 1:UBC SEISHIC LOAD - NS \& EW DIR.

PLATE LOADS AND/OR STRESSES

GLoods Node Fx Fy

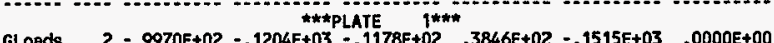

$\begin{array}{lllllll}\text { GLoads } \quad 2 & -.9970 E+02 & -.1204 E+03 & -.1178 E+02 & .3846 E+02 & -.1515 E+03 & .0000 E+00\end{array}$

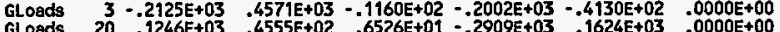

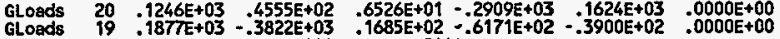

GLoads

GLoads

GLoads

GLoads

GLoods

GLoads

GLoads

GLoads

GLoads

GLoads

GLoads

GLoads

$19.1877 \mathrm{E}+03-.3822 \mathrm{E}+03 \quad 1685 \mathrm{E}+02-$

$3-.2597 E+02, .1060 E+03 \quad .6897 E+01.2002 E+03-.1552 E+03 \quad .0000 E+00$

$4-.3094 E+03 \quad .7286 E+03 \quad .1585 E+01-.6005 E+02-.1318 E+03 \quad .0000 E+00$

$21-8580 \mathrm{E}+02-.1887 \mathrm{E}+03-.2014 \mathrm{E}+02-.9498 \mathrm{E}+02 \quad .2888 \mathrm{E}+02.0000 \mathrm{E}+00$

$20.2496 \mathrm{E}+03-.6459 \mathrm{E}+03 \quad 1166 \mathrm{E}+02 \quad .1414 \mathrm{E}+03 \quad .4559 \mathrm{E}+01 \quad .0000 \mathrm{E}+00$

$4.9448 \mathrm{E}+02 \quad .4618 \mathrm{E}+03 \quad .1165 \mathrm{E}+02 \quad .6005 \mathrm{E}+02-.4049 \mathrm{E}+02$

.

.

$22 \quad .3661 \mathrm{E}+02-.6100 \mathrm{E}+03-.0475 \mathrm{E}+01-.2415 \mathrm{E}+03-.3444 \mathrm{E}+03 \quad .0000 \mathrm{E}+00$

.

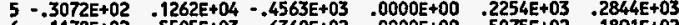

$\begin{array}{lllllll}6 & .1178 E+02 & .5505 E+03 & .6360 E+02 & .0000 E+00 & .5075 E+02 & -.1891 E+02\end{array}$ $23-.2630+02-.1108 E+04 \quad-3304 E+03 \quad .6596 E+02-.3956 E+02 \quad .0000 E+00$

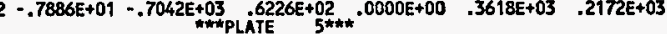

$6.1143 E+01.9113 E+03=.4077 E+03 \quad .0000 E+00 \quad .7913 E+02 \quad .1891 E+02$

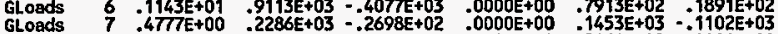

GLoads $24 \quad 1339 \mathrm{E}+02-2455 \mathrm{E}+03 \quad .3554 \mathrm{E}+03.1546 \mathrm{E}+02 \quad .5661 \mathrm{E}+02-0000 \mathrm{O}+00$

$\begin{array}{llllllll}\text { GLoads } & 24 & .1339 E+02 & -.8455 E+03 & .3554 E+03 & .1546 E+02 & .5461 E+02 & .0000 E+00 \\ \text { GLoads } & 23-.1501 E+02 & -.2943 E+03 & .7931 E+02 & -.7113 E+02 & -.6412 E+02 & .0000 E+00\end{array}$

GLoads

GLoads

GLoods

GLoads

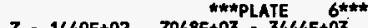

$.0000 E+00-.2831 E+02 \quad .1102 E+03$

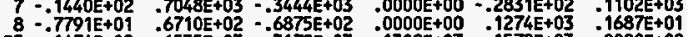

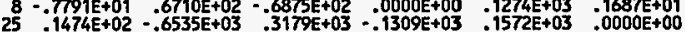

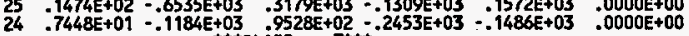

GLoods

GLoads

GLoads

$8-.7880 E+01.5264 E+03-.2763 E+03.0000 E+00-.1301 E+03-.1687 E+01$

$9-.1455 \mathrm{E}+02-.7970 \mathrm{E}+02-.1157 \mathrm{E}+03 \quad .0000 \mathrm{E}+00 \quad .2426 \mathrm{E}+02 \quad .1188 \mathrm{E}+03$

$\begin{array}{lllllll}26 & .7626 \mathrm{E}+01 & -.4766 \mathrm{E}+03 & -2615 \mathrm{E}+03 & -.2501 \mathrm{E}+03 & .1512 \mathrm{E}+03 & .0000 \mathrm{E}+00 \\ 25 & .1481 \mathrm{E}+02 & .2988 \mathrm{E}+02 & .1305 \mathrm{E}+03 & -.1264 \mathrm{E}+03 & -.1527 \mathrm{E}+03 & .0000 \mathrm{E}+00\end{array}$ $\rightarrow \star A P L A T E$

GLoads $9.1762 E+01 \quad 3352 E+03-.2023 E+03 \quad .0000 E+00-.1474 E+03-.1188 E+03$

GLoads $9 \cdot-1762 E+01 \quad .3352 E+03 \quad-.2023 E+03 \quad .0000 E+00-.1474 E+03-.1188 E+03$

GLoads $10.2527 E+01-.2440 E+03-.1627 E+03-0000 E+00-.8768 E+02 \quad .1600 E+01$

$\begin{array}{llllllll}\text { GLoads } & 27 & -.1782 E+02 & -.2740 E+03 & .2027 E+03 & -.5207 E+02 & .5135 E+02 & .0000 E+00 \\ \text { GLoads } & 26 & .1353 E+02 & .1828 E+03 & .1623 E+03 & .2928 E+02 & -.5340 E+02 & .0000 E+00\end{array}$ 


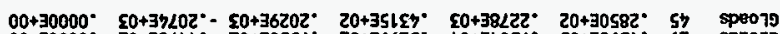

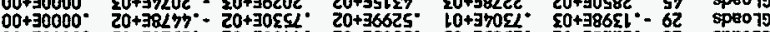

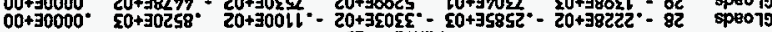

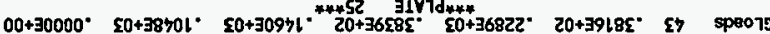

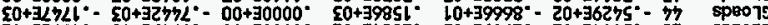
$20+32 \angle 81^{\circ}=\varepsilon 0+35169^{\circ}=00+30000^{\circ} 20+31892^{\circ}-\left[0+36022^{\circ}-20+3700 \Sigma^{\circ}-82\right.$ speop $00+30000^{\circ} 20+3 \angle 22^{\circ} 20+3875 L^{\circ} \quad 50+320 \angle 4^{\circ} 20+36905^{\circ} 20+3 \angle L 97^{\circ} \angle 2$ sp8079

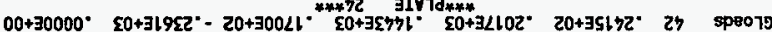

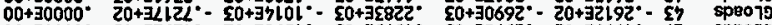

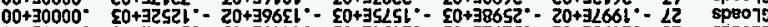

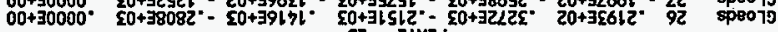

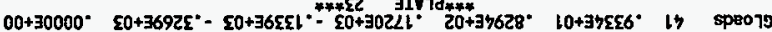

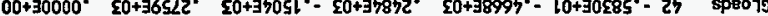

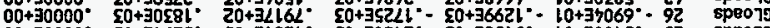

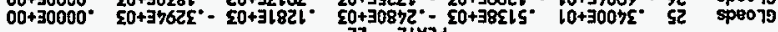

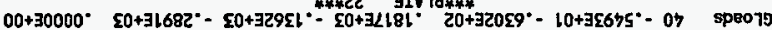

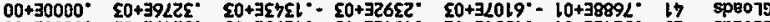

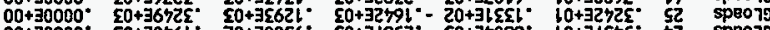

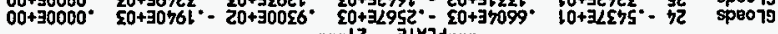

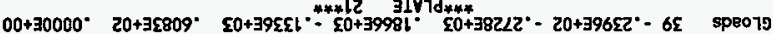

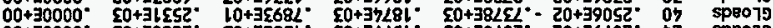

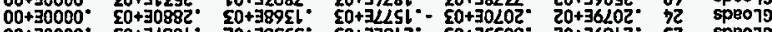

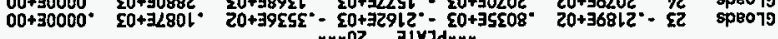

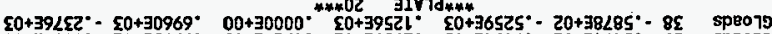

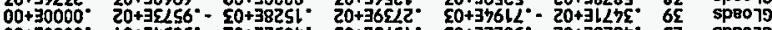

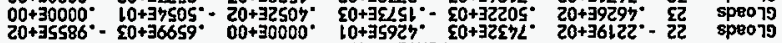

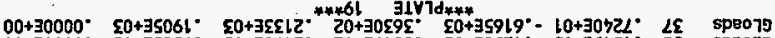

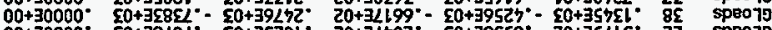

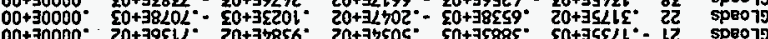

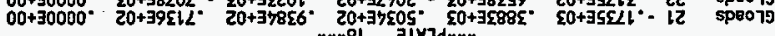

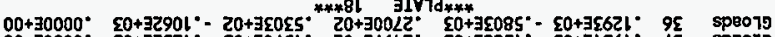

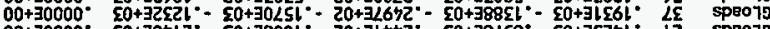

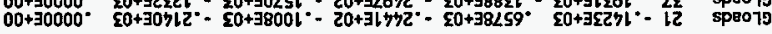

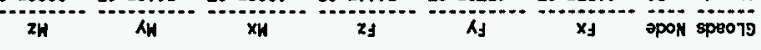

- עIO A3 SN - arot JIWSIJS J8n:b osej peot

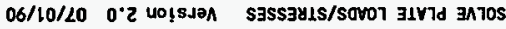

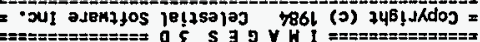

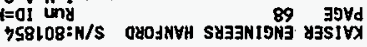

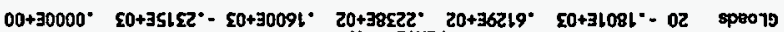

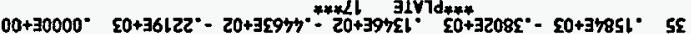
(1) $00+30000^{\circ} 20+31579^{\circ}, 20+30500^{\circ}-10+32598^{\circ}-50+30755^{\circ}-50+31291^{\circ}-02$

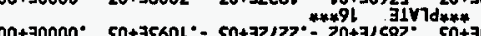

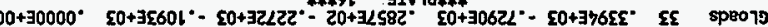

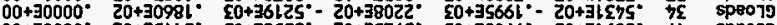

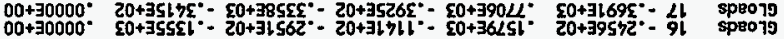

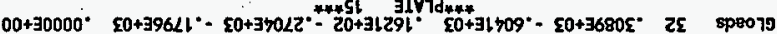

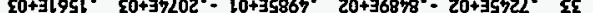

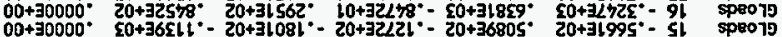

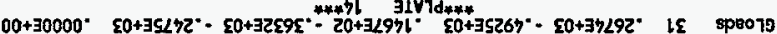

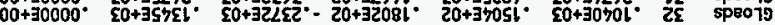

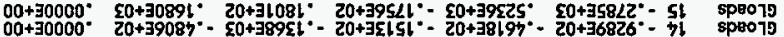

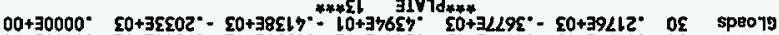

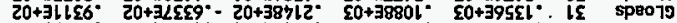

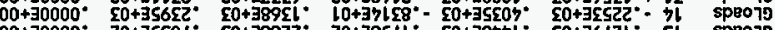

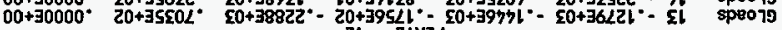

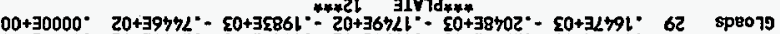

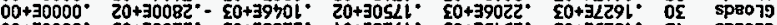

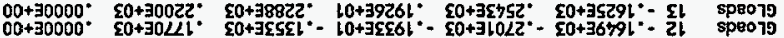

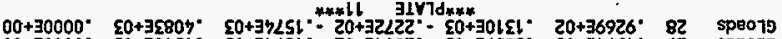

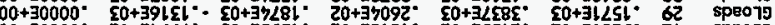

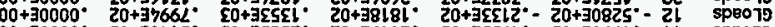

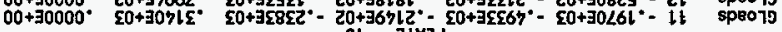

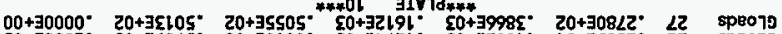

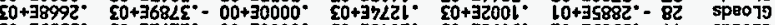

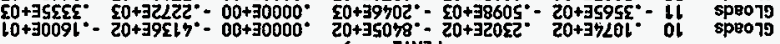

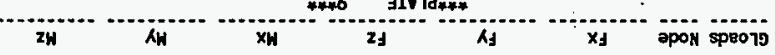

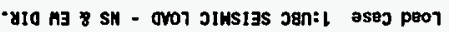
1

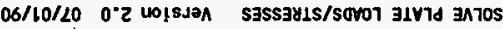
- כuा

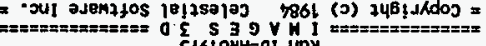
80:05:80

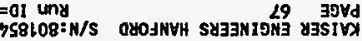




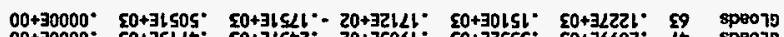

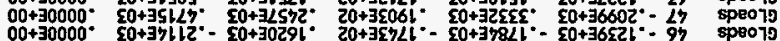

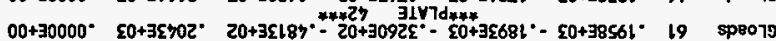

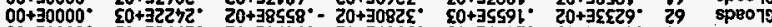

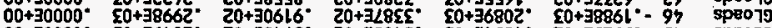

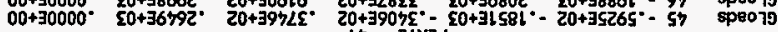

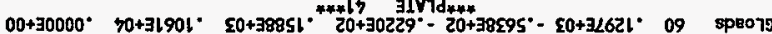

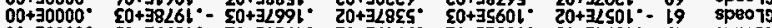

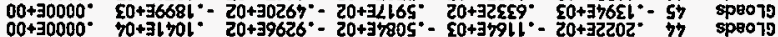

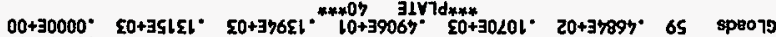
$50+32700^{\circ}-\varepsilon 0+38888^{\circ}-00+30000^{\circ} \quad 20+39271^{\circ} \quad 20+309 \% 8^{\circ}-20+36 \angle 67^{\circ}-09$ speo79

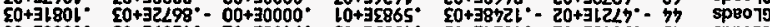

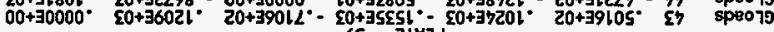

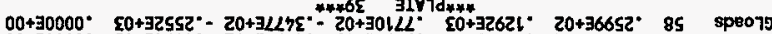

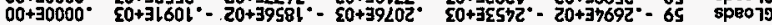

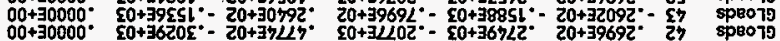

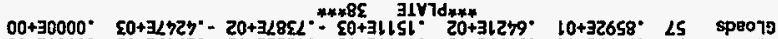

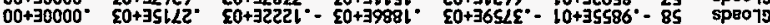

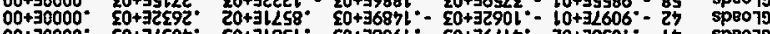

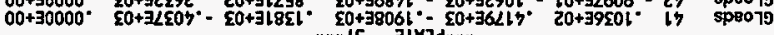

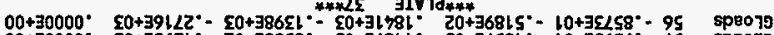

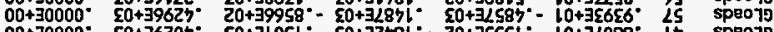

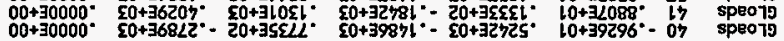

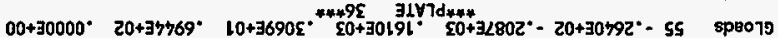

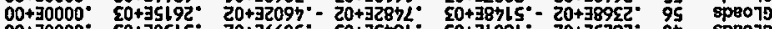

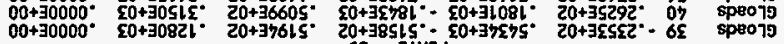

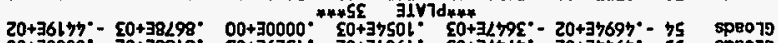

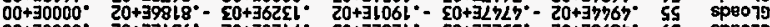

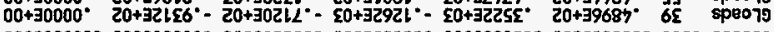

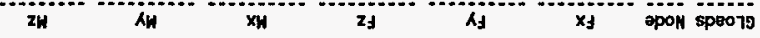

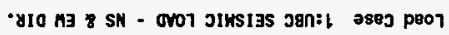

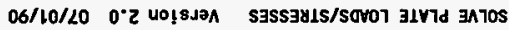

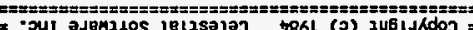

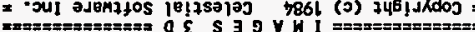
D58108:N/S OYOANYH SyganIONG agsivd

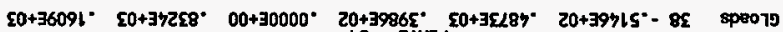

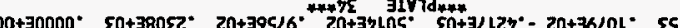

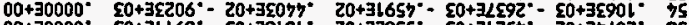

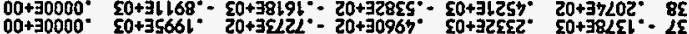

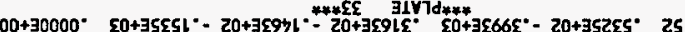

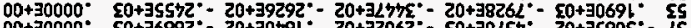
$00+30000^{\circ} \quad 20+36992^{\circ}-20+30491^{\circ}, 20+32062^{\circ}-20+30 \angle 57^{\circ}, 20+3590 \varepsilon^{\circ}-$ LE

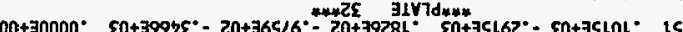

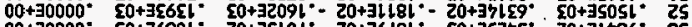
$00+30000^{\circ} \quad 50+36901^{\circ} \quad 20+3510 L^{\circ} \quad 20+36 \angle 91^{\circ}-50+35275^{\circ}-20+3 t 786^{\circ}-9 \varepsilon$

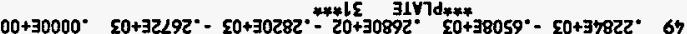
$00+30000^{\circ} \quad 20+36+82^{\circ} \quad \varepsilon 0+30 \varepsilon 17^{\circ}-10+32875^{\circ}-\varepsilon 0+\exists L 0 L 1^{\circ}-\varepsilon 0+357 \varepsilon 1^{\circ}$ oS

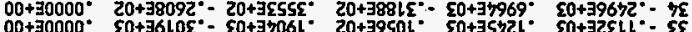

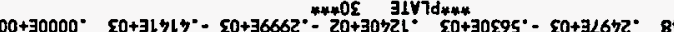
(10)

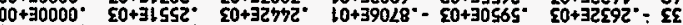

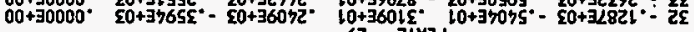

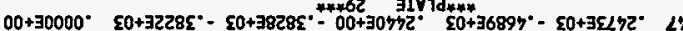

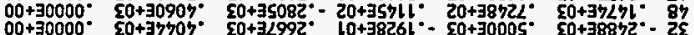

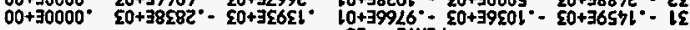
$00+30000^{\circ} \varepsilon 0+3822^{*}-\varepsilon 0+3 \varepsilon 76 Z^{* *}=20+3252 L^{\circ}-\varepsilon 0+3709 \varepsilon^{\circ}-\varepsilon 0+302 \varepsilon Z^{*}$

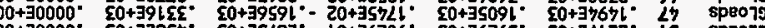

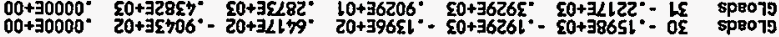

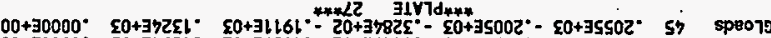

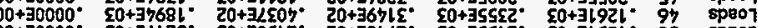

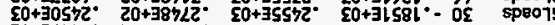

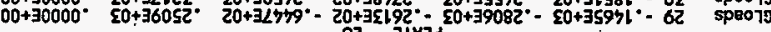

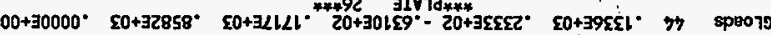
${ }_{2 H} A_{H} X_{H}$ 2

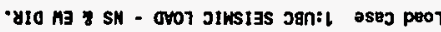

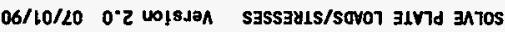
Ol: :05:80
S6/22/20

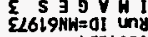

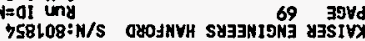

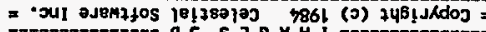


KAISER ENGIHEERS HANFORD $S / N: 801854$

PAGE 71 Run ID=NN61973 $02 / 22 / 95$

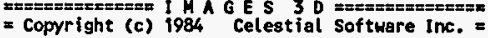

SOLVE PLATE LOADS/STRESSES Version 2.0 07/01/90 $\mathbf{L}$

Load Case 1:UBC SEISHIC LOAD - NS \& EH DIR.

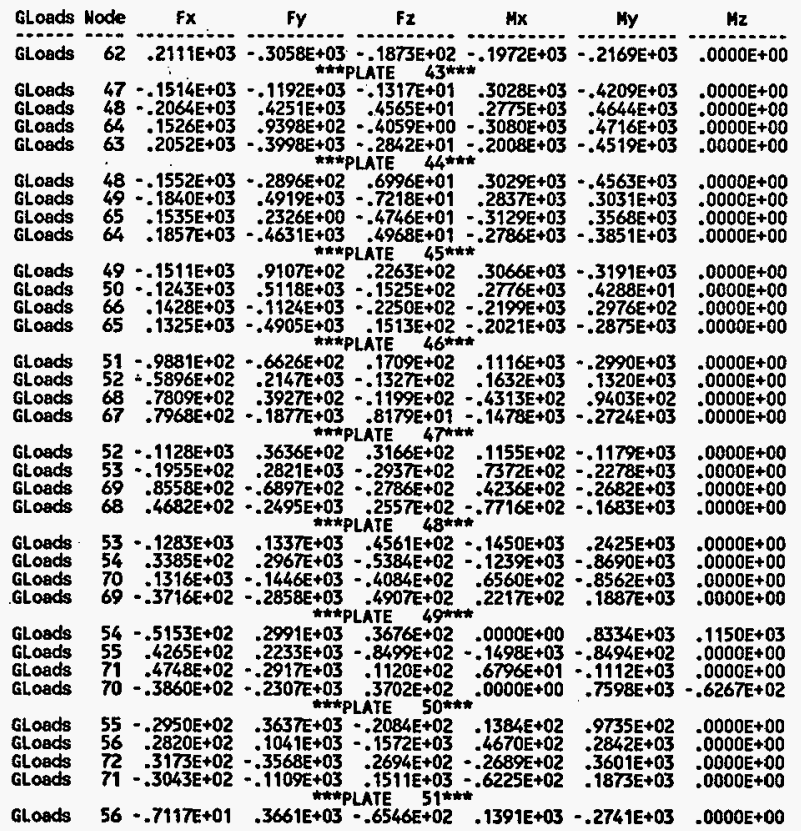

KAISER ENGINEERS HANFORD S/M:801854

PAGE 72 RUN ID=NN61973 $02 / 22 / 95$

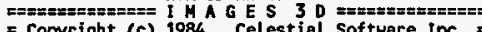
= Copyright (c) 1984 Celestial Software Inc. I SOLVE PLATE LOADS/STRESSES Version $2.0 \quad 07 / 01 / 90$ L

LOad Case 1:UBC SEISHIC LOAD - NS \& EU DIR.

GLoads Node Fx $\quad$ Fy $\quad$ Fz $\quad$ Mx $\quad$ My

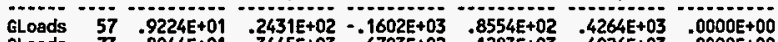
$\begin{array}{lllllllll}\text { GLoods } & 73 & .8044 E+01 & -.3445 E+03 & .6703 E+02 & -.1283 E+03 & .4026 E+03 & .0000 E+00\end{array}$ GLoads $72=.1015 E+02-.4588 E+02 \quad .1586 E+03=.5003 E+02-.2872 E+03 \quad .0000 E+00$

GLoads $57.8979 E+01 \quad .3006 E+03-.1035 E+03 \quad .7399 E+02-.4313 E+03 \quad .0000 E+00$ GLoads $58-.1033 E+02-.3250 E+02 \quad-.1142 E+03 \quad .1135 E+03 \quad .2621 E+03 \quad .0000 E+00$ GLoads $74-.1073 \mathrm{E}+02-.2765 \mathrm{E}+03 \quad .9101 \mathrm{E}+02-.7919 \mathrm{E}+02 \quad .2537 \mathrm{E}+03 \quad .0000 \mathrm{O}+00$

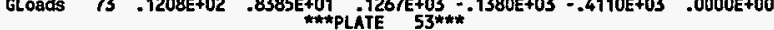

GLoads $58 \quad .3039 E+02 \quad .1828 E+03-.1152 E+03 \quad .4345 E+02-.2784 E+03 \quad .0000 E+00$ GLoads $59-.2837 \mathrm{E}+02-.6282 \mathrm{E}+02-.3622 \mathrm{E}+02 \quad .4072 \mathrm{E}+02 \quad-.1373 \mathrm{E}+03 \quad .0000 \mathrm{E}+00$

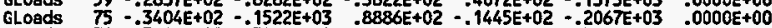
GLoads $74 \quad .3202 E+02 \quad .3220 E+02 \quad .6261 E+02-.2532 E+02-.3449 E+03 \quad .0000 E+00$

GLoads $59.4465 E+02 \quad .1046 E+03-.1401 E+03-.1616 E+03 \quad .1149 E+03 \quad .0000 E+00$

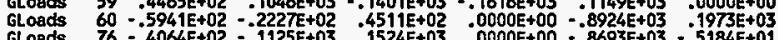

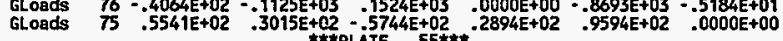

GLoods $60 \quad .3032 E+02-.2836 E+02-.7369 E+02-.2668 E+03 \quad .1013 E+04 \quad .0000 E+00$

$\begin{array}{llllllll}\text { GLoods } & 60 & -3032 E+02 & -.2836 E+02 & -.7369 E+02 & -.2668 E+03 & .1013 E+04 & .0000 E+00 \\ \text { GLoods } & 61 & -.194 \mathrm{E}+03 & .9025 \mathrm{E}+02 & .4705 \mathrm{E}+02 & -.2335 \mathrm{E}+03 & -.2009 \mathrm{E}+03 & .0000 \mathrm{E}+00\end{array}$

GLoods $77-.1978 \mathrm{E}+02 \quad 3894 \mathrm{E}+02 \quad .6169 \mathrm{E}+02-.4506 \mathrm{E}+02-1497 \mathrm{E}+03 \quad .0000 \mathrm{E}+00$

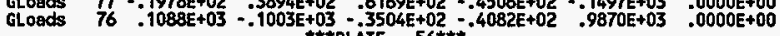

GLoads $61=.3039 E+02=.1049 E+03-.3291 E+02 \quad .1149 E+03 \quad .1943 E+03 \quad .0000 E+00$

$\begin{array}{llllllll}\text { GLosds } & 62 & -.1463 \mathrm{E}+03 & .1629 \mathrm{E}+03 & .3763 \mathrm{E}+02 & .4108 \mathrm{E}+02 & . .2321 \mathrm{E}+03 & .0000 \mathrm{E}+00 \\ \text { GLoads } & 78 & .4353 \mathrm{E}+02 & .0340 \mathrm{E}+02 & .3663 \mathrm{E}+02 & -.8167 \mathrm{E}+02 & .3763 \mathrm{E}+03 & .0000 \mathrm{E}+00\end{array}$

GLoads $77: 1332 \mathrm{E}+03=-.1515 \mathrm{E}+03-.4135 \mathrm{E}+02 \quad \cdot .2951 \mathrm{E}+02 \quad .3236 \mathrm{E}+03 \quad .0000 \mathrm{E}+00$

GLoads $62=.9175 E+02-.1170 E+03-.1629 E+02 \quad .2417 E+03-.2574 E+03 \quad .0000 E+00$

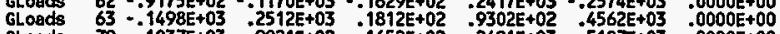

$\begin{array}{lllllllll}\text { GLoads } 79 & .1037 \mathrm{E}+03 & .9921 \mathrm{E}+02 & .1652 \mathrm{E}+02 & -.2491 \mathrm{E}+03 & .5187 \mathrm{E}+03 & .0000 \mathrm{O}+00\end{array}$

$\begin{array}{lllllllll}\text { GLoads } & 78 & -1037 \mathrm{E}+03 & -.9921379+02 & -.2334 \mathrm{E}+03 & -.1835 \mathrm{E}+02 & -.4544 \mathrm{E}+02 & -.1921 \mathrm{E}+03 & .0000 \mathrm{E}+00\end{array}$

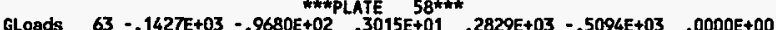

GLoes $64-1373 E+03$ - $3284 E+03$ - $5750 E+01$.

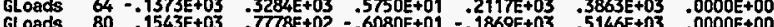

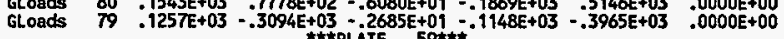

GLoads $64-.1656 E+03-.5366 E+02 \quad .2510 E+02 \quad .3750 E+03-.4728 E+03 \quad .0000 E+00$

GLoads $65-.1160 \mathrm{E}+03 \quad .3654 \mathrm{E}+03-.5201 \mathrm{E}+01 \quad .2348 \mathrm{E}+03 \quad .1632 \mathrm{O}+03 \quad .0000 \mathrm{E}+00$

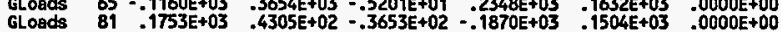


$\begin{array}{lll}\text { KAISER ENGINEERS HAKFORD } & \text { S/N:801854 } & 02 / 22 / 95 \\ \text { PAGE } & 73 & 08: 50: 14\end{array}$ = Copyright (c) 1984 celestial Software Inc. $=$

SOLVE PLATE LOADS/STRESSES Version 2.0 07/01/90

L.

Load CaSe 1:UBC SEISHIC LOAD - NS \& EH DIR.

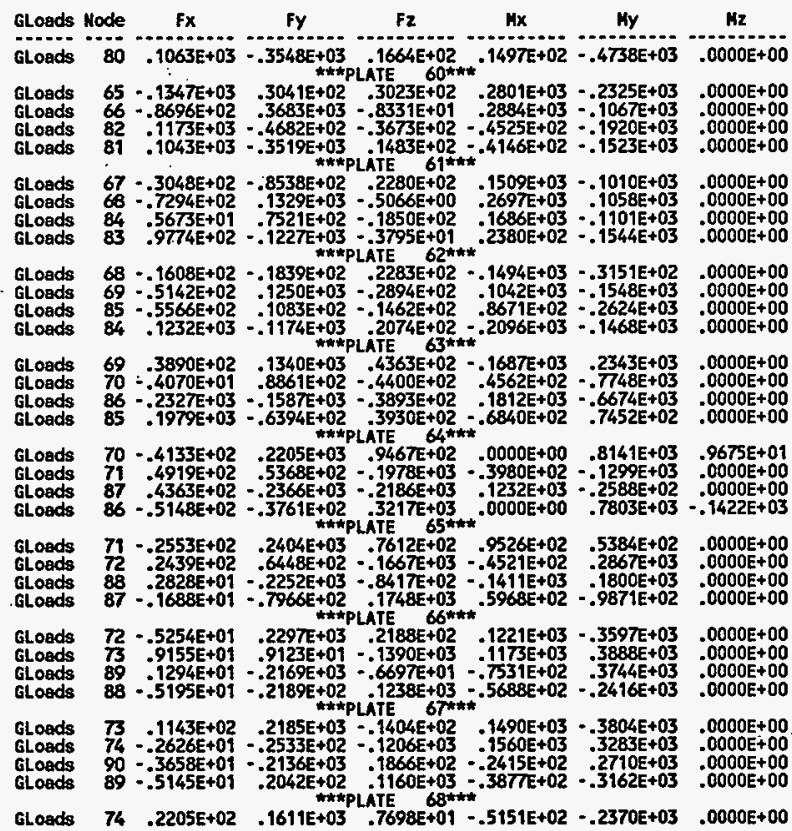

KAISER ENGINEERS HANFORD S/N:801854

PAGE 74 Run ID=KN61973 $02 / 22 / 95$

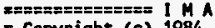
× Copyright (c) 1984 Celestial Software Inc.

SOLVE PLATE LOADS/STRESSES Version 2.0 07/01/90

LOad Case 1:UBC SEISMIC LOAD - NS \& EW DIR

GLoads Mode Fx Fy Fy

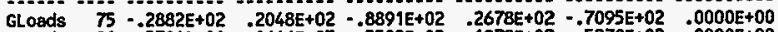

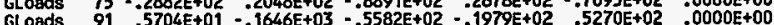

$\begin{array}{llllllll}\text { GLoads } & 91 & -5704 E+01 & -.1646 E+03 & -.5582 E+02 & -.1979 E+02 & .5270 E+02 & .0000 E+00 \\ \text { GLoads } & 90 & .1067 \mathrm{E}+01 & -.1701 \mathrm{E}+02 & .1370 E+03 & -.1417 \mathrm{E}+03 & -.1030 \mathrm{E}+03 & .0000 \mathrm{0}+00\end{array}$

GLoads $75.4816 E+02-.7029 E+01 \quad .9820 E+02-.4127 E+02 \quad .1817 E+03 \quad .0000 E+00$

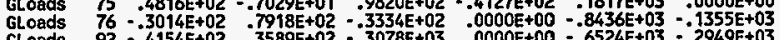

$\begin{array}{llllllll}\text { GLoads } & 92 & -.4154 E+02 & . .3589 E+02 & -.3078 E+03 & .0000 E+00 & -.6524 E+03 & -.2949 E+03 \\ \text { GLoods } & 91 & .2351 E+02 & -.1081 E+03 & .2429 E+03 & .1066 E+03 & .2034 E+03 & .0000 E+00\end{array}$

GLoads $76 \quad .1085 E+02-.5327 E+01-.3339 E+02 \quad .1819 E+03 \quad .9118 E+03 \quad .0000 E+00$

$\begin{array}{llllllll}\text { GLoads } & 76 & .1085 E+02 & -.5327 E+01 & -.3339 E+02 & .1819 E+03 & .91118 E+03 & .0000 E+00 \\ \text { GLoads } & 77 & -.4260 \mathrm{E}+02 & -5315 \mathrm{E}+02 & .5239 \mathrm{E}+02 & -.9881 \mathrm{E}+02 & -.2779 \mathrm{E}+03 & .0000 \mathrm{E}+00\end{array}$

$\begin{array}{llllllll}\text { GLoads } & 93 & .42592 \mathrm{E}+02 & .4410 \mathrm{E}+01 & .3068 \mathrm{E}+02 & .7483 \mathrm{E}+02 & -. .1790 \mathrm{E}+03 & .0000 \mathrm{O}+00\end{array}$

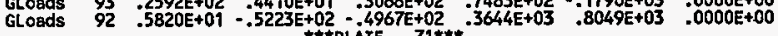

GLoads $77-.3098 E+02-.4686 E+02-.3288 E+02 \quad .1144 E+03 \quad .1040 E+03 \quad .0000 E+00$

$\begin{array}{llllllll}\text { GLoads } & 77 & -.3098 E+02 & -.4686 \mathrm{E}+02 & -.3288 \mathrm{E}+02 & .1144 \mathrm{E}+03 & .1040 \mathrm{E}+03 & .0000 \mathrm{E}+00 \\ \text { GLoads } & 78 & -.5269 \mathrm{E}+02 & .1106 \mathrm{E}+03 & -.2751 \mathrm{E}+02 & -.1347 \mathrm{E}+03 & .1866 \mathrm{E}+03 & .0000 \mathrm{E}+00 \\ \text { GLoads } & 94 & .6130 \mathrm{E}+02 & -4106 \mathrm{E}+02 & .2430 \mathrm{E}+01 & -.2111 \mathrm{E}+03 & .1447 \mathrm{E}+03 & .0000 \mathrm{E}+00\end{array}$

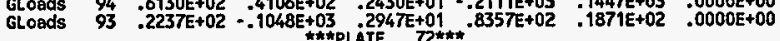

GLoads $78=.8890 E+02-.7689 E+02-5956 E+01 \quad .2618 E+03-.3708 E+03-0000 E+00$

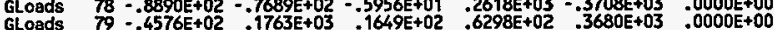

GLoads $95-4575 \mathrm{E}+03 \quad .6788 \mathrm{E}+02-3801 \mathrm{E}+01-1176 \mathrm{E}+03 \quad .4165 \mathrm{E}+03 \quad .0000 \mathrm{E}+00$

$\begin{array}{lllllll}\text { GLoads } 94 & -2714 \mathrm{E}+02 & -.1673 \mathrm{E}+03 & -.6736 \mathrm{E}+01 & .8258 \mathrm{E}+02 & -.2211 \mathrm{E}+03 & .0000 \mathrm{E}+00\end{array}$

GLoads $79 \cdot .1438 E+03-.7236 E+02.9505 E+01.3009 E+03-.4902 E+03 \quad .0000 E+00$

GLoads $80-1418 E+02-2283 E+03 \quad .9248 E+01.1423 E+03-.4314 E+03.0000 E+00$

GLoads $96 \quad 1556 \mathrm{E}+03 \quad 5813 \mathrm{E}+02-1323 \mathrm{E}+02-2642 \mathrm{E}+02 \quad .3820 \mathrm{E}+03 \quad 0000 \mathrm{E}+00$

GLoads $95 \quad .2332 E+01-.2140 E+03-.5525 E+01.9890 E+02-.3836 E+03 \quad .0000 E+00$

GLoads $80-.2066 E+03-.5749 E+02 \quad 20203 E+02 \quad .2962 E+02-.4722 E+03 \quad 0000 E+00$

GLoads $81 \quad .2902 E+02 \quad .2796 E+03 \quad-.3619 \varepsilon+02 \quad-.2260 E+03 \quad .1005 E+03 \quad .0000 E+00$

GLoads $97-2449 \mathrm{E}+03 \quad-4233 \mathrm{E}+02 \quad .1943 \mathrm{E}+02-1780 \mathrm{E}+03 \quad-3456 \mathrm{E}+03 \quad 0000 \mathrm{E}+00$

GLoads $96-.6729 \mathrm{E}+02-.2645 \mathrm{E}+03-.3266 \mathrm{E}+01-.7003 \mathrm{E}+02-.2282 \mathrm{E}+03 \quad .0000 \mathrm{E}+00$

GLads $01.2688 E+03-.7698 E+02$ PLATE 75*** $4545 E+03-.9861 E+02 \quad 0000 E+00$

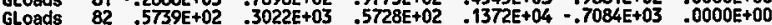

GLads 98 3982E+03 $30211 \mathrm{E}+02-1845 \mathrm{E}+03-1703 \mathrm{E}+04-9188 \mathrm{E}+03.0000 \mathrm{E}+00$

GLoads $97-.1868 \mathrm{E}+03=.3063 \mathrm{E}+03 \quad .2947 \mathrm{E}+02,00 * * 7338 \mathrm{E}+03 \quad-.2034 \mathrm{E}+03 \quad .0000 \mathrm{E}+00$

GLoads $83-.7677 \mathrm{E}+01-.6441 E+02 \quad-1677 \mathrm{E}+02 \quad .1570 E+03 \quad-2528 E+03 \quad .0000 \mathrm{C}+00$

$\begin{array}{lrllllll}\text { GLoads } & 84 & -.6767 \mathrm{E}+02 & .3649 \mathrm{E}+02 & .2857 \mathrm{E}+02 & .1164 \mathrm{E}+03 & .1021 \mathrm{E}+03 & .0000 \mathrm{0}+00 \\ \text { GLoods } & 100 & .6528 \mathrm{E}+01 & .5447 \mathrm{E}+02 & .3248 \mathrm{E}+02 & .2035 \mathrm{E}+03 & .1430 \mathrm{E}+03 & .0000 \mathrm{E}+00\end{array}$ 
KAISER ENGINEERS HAHFORD S/N:801854

Run ID =NN61973

$02 / 22 / 95$

PAGE 75

* Copyright (c) 1984 Celestial Software Inc. =
*

SOLVE PLATE LOADS/STRESSES Version $2.0 \quad 07 / 01 / 90$

L.

Load Case 1:UBC SEISHIC LOAD - NS \& EH DIR.

GLoads Hode FX

Fy F2. Mx My Mz GLoads $99 \quad .6881 E+02-.2655 E+02-.7781 E+02 \quad .2712 E+03 \quad .3364 E+03 \quad .0000 E+00$ GLoads $84-.2927 \mathrm{E}+02 \cdot .7935 \mathrm{E}+02 \quad .1100 \mathrm{E}+01 \cdot .7548 \mathrm{E}+02 \quad .1548 \mathrm{E}+03 \quad .0000 \mathrm{E}+00$ GLoads $85-.9295 \mathrm{E}+02 \quad 6674 E+02 \quad-2599 E+02 \quad-2735 E+03 \quad .5173 E+02 \quad .0000 E+00$ GLoeds $101 \quad 7252 \mathrm{E}+02 \quad .8082 \mathrm{E}+02=.6836 \mathrm{E}+01 \quad .2789 \mathrm{E}+03-.3542 \mathrm{E}+02 \quad .0000 \mathrm{E}+00$ GLoads $100 \quad .4969 E+02-.6821 E+02-.2026 E+02-.2986 E+02 \quad .9072 E+02 \quad .0000 E+00$ GLoads $85-.1735 E+02-.9871 E+02-.1876 E+02-.2918 E+03 \quad .1361 E+03 \quad .0000 E+00$ GLoads $86-.1573 E+03 \quad .1300 E+03-.9752 E+02-.6819 E+03-.1073 E+03-0000 E+00$ GLoads $10201582 E+03 \quad .0000 E+00$ GLoads $101 \quad .1644 \mathrm{E}+02-.1121 \mathrm{E}+03 \quad .2242 \mathrm{E}+02-.2787 \mathrm{E}+03 \quad .4648 \mathrm{E}+02 \quad .0000 \mathrm{E}+00$ GLoads $86-.1667 \mathrm{E}+02-.3664 \mathrm{E}+02 \quad .1997 \mathrm{E}+03 \quad .0000 \mathrm{E}+00 \quad .4296 \mathrm{E}+03-.1410 \mathrm{E}+03$ GLoads $87 \quad 2698 \mathrm{E}+02 \quad .1753 \mathrm{E}+03-.2007 \mathrm{E}+02-.1169 \mathrm{E}+03 \quad .1227 \mathrm{E}+03 \quad .0000 \mathrm{E}+00$ GLoads $103 \quad .5328 \mathrm{E}+02 \quad 0258 \mathrm{E}+02-.5143 \mathrm{E}+02 \quad .0000 \mathrm{E}+00 \quad .2714 \mathrm{E}+03 \quad .6417 \mathrm{E}+02$ GLoads $102-.6360 \mathrm{E}+02-.1546 \mathrm{E}+03-1282 \mathrm{E}+03 \quad .0000 \mathrm{E}+00 \quad .4205 \mathrm{E}+03-.2102 \mathrm{E}+03$ GLoads $\quad 87-.3274 E+02.4440 E+02 \quad .1002 E+03 \cdot .6603 E+02 \quad .1916 E+01 \quad .0000 E+00$ GLoads $88 \cdot .2278 E+02 \quad .9342 E+02-.3647 \mathrm{E}+02 \quad .3034 \mathrm{E}+01 \quad .1515 \mathrm{E}+03 \quad .0000 \mathrm{E}+00$ GLoads $104-4089 E+02-.2562 E+02+.5929 E+02.0000 E+00-.4706 E+03-.2607 E+01$ GLoads $103-.3093 \mathrm{E}+02-.1122 \mathrm{E}+03-.4403 \mathrm{E}+01 \quad .0000 \mathrm{E}+00 \quad .3628 \mathrm{E}+03 \quad .1039 \mathrm{E}+03$ $\begin{array}{llllllll}\text { GLoads } & 88 & .1578 E+02 & .5716 E+02 & .3302 E+02 & .1949 E+03 & -.8988 E+02 & .0000 E+00\end{array}$ GLoads $89-1842 \mathrm{E}+02 \quad .1941 \mathrm{E}+02-.6677 \mathrm{E}+02 \quad .7223 \mathrm{E}+02 \quad .1353 \mathrm{E}+03 \quad .0000 \mathrm{E}+00$ GLoads $105=.5752 \mathrm{E}+01-5534 \mathrm{E}+02-.2697 \mathrm{E}+02 \quad .0000 \mathrm{E}+00 \quad .1674 \mathrm{E}+03-.9693 \mathrm{E}+02$

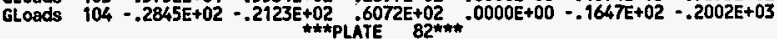
$\begin{array}{llllllllll}\text { GLoods } & 89 & .2162 E+02 & .8057 \mathrm{E}+02 & -.6332 \mathrm{E}+01 & .4186 \mathrm{E}+02 & -.1935 \mathrm{E}+03 & .0000 \mathrm{E}+00\end{array}$ GLoads $90=.5969 \mathrm{E}+00-.5113 \mathrm{E}+01=.7194 \mathrm{E}+02$ GLoads $106-.4099 \mathrm{E}+02-.7820 \mathrm{E}+02 \quad .1875 \mathrm{E}+02 \quad .0000 \mathrm{E}+00-.1534 \mathrm{E}+03-.1221 \mathrm{E}+03$ GLoods $105 \quad .1997 \mathrm{E}+02 \quad .2751 \mathrm{E}+01 \quad .5951 \mathrm{E}+02 \quad .0000 \mathrm{E}+00-.3109 \mathrm{E}+03=.7314 \mathrm{E}+02$ $\begin{array}{lllllllll} & \text { GLoads } 90 & .3938 E+02 & .1392 E+03 & -.4756 E+02 & .5614 E+02 & -.1812 E+03 & .0000 E+00\end{array}$ GLoads $91-.1478 E+02-.1369 E+02-.1089 E+03 \quad .1379 E+03-.1353 E+03 \quad .0000 E+00$ GLoads $107=.7846 E+02-.1529 E+03 \quad .1126 E+03 \quad .0000 E+00-.5582 E+03-.1275 E+03$ GLoads $106 \quad .5386 \mathrm{E}+02 \quad .2740 \mathrm{E}+02 \quad .4393 \mathrm{E}+02 \quad .0000 \mathrm{E}+00-.5704 \mathrm{E}+03-.8433 \mathrm{E}+02$ $\begin{array}{lllllllll}7 & \text { GLoads } & 91 & .2176 E+02 & .1898 E+03 & -.4202 E+02 & -.2247 E+03 & -.1207 E+03 & .0000 E+00 \\ 0 & \text { GLoads } & 92 & -.9019 E+02 & -.1158 E+03 & -.2134 E+03 & .0000 E+00 & -.4873 E+03 & .4155 E+03\end{array}$ GLoads $108-.2916 \mathrm{E}+02-.1561 \mathrm{E}+03 \quad .2470 \mathrm{E}+03 \quad .0000 \mathrm{O}+00-.7601 \mathrm{EE}+03 \quad .3370 \mathrm{E}+03$ GLoads $107 \quad .9759 \mathrm{E}+02 \quad .8210 \mathrm{E}+02 \quad .8400 \mathrm{E}+01 \quad .0000 \mathrm{E}+00 \quad-.4818 \mathrm{E}+03 \quad .1519 \mathrm{E}+03$ GLoads $92-.3554 E+02-.1348 E+02-.1403 E+02 \quad .2406 E+03 \quad .4806 E+03 \quad .0000 E+00$
KAISER ENGIMEERS HANFORO S/N:801854

Run ID=MN61975

$02 / 22 / 95$

PAGE 76

$==1 H A G E S$ c Copyright (c) 1984 Celestial Sof tware Inc. =

SOLVE PLATE LOADS/STRESSES Version $2.0 \quad 07 / 01 / 90$

Load CaSe 1:UBC SEISHIC LOND - NS \& EW DIR.

GLoads Node Fx $\quad$ Fy $\quad$ Fz $\quad$ Mx $\quad$ My

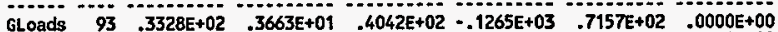
Gloads 109 GLoads $108-.3018 \mathrm{E}+02 \quad .1102 \mathrm{E}+02-.1010 \mathrm{E}+03.03477 \mathrm{E}+03 \quad .7405 \mathrm{E}+03 \quad .0000 \mathrm{E}+00$

$\begin{array}{lrrrrr}\text { GLoads } 93-.4616 \mathrm{E}+02 & .2343 \mathrm{E}+01-.3863 \mathrm{E}+02 \cdot .3190 \mathrm{E}+02 & .8873 \mathrm{E}+02 & .0000 \mathrm{E}+00\end{array}$

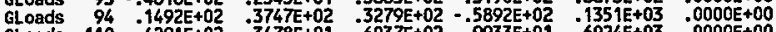
GLoads $110.4291 \mathrm{E}+02-.3478 \mathrm{E}+01 \quad .6037 \mathrm{E}+02 \quad .9933 \mathrm{E}+01 \quad .6024 \mathrm{E}+03 \quad .0000 \mathrm{E}+00$ GLoads $109-.1167 \mathrm{E}+02-.3633 \mathrm{E}+02-.5454 \mathrm{E}+02-.1545 \mathrm{E}+02 \quad .5868 \mathrm{E}+03 \quad .0000 \mathrm{E}+00$

GLoads $94-.6795 E+02-.5685 E+01 \quad .6927 E+01 \quad .1874 E+03-.5864 E+02 \quad .0000 E+00$ $\begin{array}{lllllll}\text { GLoads } 95 & .1730 \mathrm{E}+02 & .5330 \mathrm{E}+02 & .2557 \mathrm{E}+02 & -.1631 \mathrm{E}+02 & .1587 \mathrm{E}+03 & .0000 \mathrm{E}+00\end{array}$ GLoads $111.6683 \mathrm{E}+02.1801 \mathrm{E}+01.1582 \mathrm{E}+02.1054 \mathrm{E}+03.3451 \mathrm{E}+03 \quad .0000 \mathrm{E}+00$ GLoads $110=.1619 \mathrm{E}+02-.4941 \mathrm{E}+02-.4832 \mathrm{E}+02 . .2598 \mathrm{E}+03 \quad .1826 \mathrm{E}+03.0000 \mathrm{E}+00$

GLoads $95-.9174 E+02-.1578 E+01 \quad .1916 E+02 \quad .3503 E+02-.1916 E+03 \quad .0000 E+00$

$\begin{array}{llllllll}\text { GLoads } & 96 & .4165 \mathrm{E}+02 & .5532 \mathrm{E}+02 & .1157 \mathrm{E}+02 & .6249 \mathrm{E}+02 & .5359 \mathrm{E}+02 & .0000 \mathrm{E}+00\end{array}$ GLoads $112.8867 \mathrm{E}+02-.8277 \mathrm{E}+00-.2845 \mathrm{E}+02 \quad .2280 \mathrm{E}+03 \quad .3290 \mathrm{E}+02 \quad .0000 \mathrm{E}+00$ GLoads $111=.3857 \mathrm{E}+02-.5292 \mathrm{E}+02+.2292 \mathrm{E}+01.1817 \mathrm{E}+03-.1508 \mathrm{E}+03 \quad .0000 \mathrm{E}+00$

GLoads $96-.9456 E+02 \quad .5659 E+02 \quad .4033 E+02 \quad .3396 E+02-.2073 E+03 \quad .0000 E+00$ $\begin{array}{llllllll}\text { GLoads } \quad 97 & .1067 E+03 & .6832 E+02 & -.3401 E+01 & .1006 E+03 & .1121 E+02 & .0000 E+00\end{array}$ GLoads $113 \quad .6272 \mathrm{E}+02-.8150 \mathrm{E}+02 \quad-.7527 \mathrm{E}+02 \quad .2611 \mathrm{E}+03-.4455 \mathrm{E}+03 \quad .0000 \mathrm{E}+00$ GLoads $112-.7483 \mathrm{E}+02-.4341 \mathrm{E}+02.3834 \mathrm{E}+02.2136 \mathrm{E}+03-.5515 \mathrm{E}+03 \quad .0000 \mathrm{E}+00$

GLoads $97-.1293 E+03 \quad .1012 E+03-.1009 E+02-.6564 E+03-.1533 E+03 \quad .0000 E+00$

GLoads $98 \quad .1971 E+03-.1609 E+01 \quad-.1770 E+03-.1162 E+04 \quad-.5292 E+03 \quad .0000 E+00$

GLoads $114 \quad .1886 \mathrm{E}+02-.7213 \mathrm{E}+02 \quad .8611 \mathrm{E}+02 \cdot .8885 \mathrm{E}+03 \quad-.4547 \mathrm{E}+03 \quad .0000 \mathrm{E}+00$

GLoads $113-.8664 \mathrm{E}+02-.2746 \mathrm{E}+02 \quad .1010 \mathrm{E}+03 \cdot .3801 \mathrm{E}+03-.2416 \mathrm{E}+03 \quad .0000 \mathrm{E}+00$

GLoads $122 \cdot .2513 E+03-.6323 E+03=.6732 E+02-.5672 E+03-.1189 E+03 \quad .0000 E+00$

GLoads $2=.1179 \mathrm{E}+03 \quad .1669 \mathrm{E}+02-.4577 \mathrm{E}+02-.5375 \mathrm{E}+03-.5515 \mathrm{E}+02 \quad .0000 \mathrm{E}+00$

$\begin{array}{lllllllll}\text { GLoads } & 19 & .2164 E+03 & .3894 E+03 & .4648 E+02 & -.6713 E+03 & .1418 E+03 & .0000 E+00\end{array}$

$\begin{array}{lllllllll}\text { GLoads } & 124 & .1528 E+03 & .2262 E+03 & .6661 E+02 & -.7120 E+03 & .4633 E+02 & .0000 E+00\end{array}$

GLoads $124-.1259 E+03-.4073 E+03=.3671 E+02-.1491 E+03-.1685 E+03 \quad .0000 E+00$

GLoads $19-.1703 \mathrm{E}+03 \quad .6161 \mathrm{E}+02-.2193 \mathrm{E}+02-.1556 \mathrm{E}+03 \quad .2343 \mathrm{E}+02 \quad .0000 \mathrm{E}+00$

$\begin{array}{lllllllll}\text { GLoads } & 35 & .1513 \mathrm{E}+03 & .2642 \mathrm{E}+03 & .2068 \mathrm{E}+02 & -.4786 \mathrm{E}+03 & .1669 \mathrm{E}+03 & .0000 \mathrm{E}+00\end{array}$

GLoads $126 \quad .1449 \mathrm{E}+03 \quad .8146 \mathrm{E}+02 \quad .3796 \mathrm{t}+02-.5070 \mathrm{E}+03-.4688 \mathrm{E}+02 \quad .0000 \mathrm{E}+00$

GLoads $126-.1120 E+03-.2347 \mathrm{E}+03-.8166 \mathrm{E}+01 \quad .1651 \mathrm{E}+03-.1630 \mathrm{E}+03 \quad .0000 \mathrm{E}+00$

GLoads $35-.1084 E+03 \quad .9479 E+02-.3539 E+01 \quad .1192 E+03 \quad .8499 E+02 \quad .0000 E+00$

$\begin{array}{llllllll}\text { GLoads } & 51 & .1191 E+03 & .1476 E+03 & .4495 E+01 & -.2682 E+03 & .1706 E+03 & .0000 E+00\end{array}$ 


\section{KAISER ENGINEERS HANFORD S/H:801854$$
\text { N:801854 }
$$

PAGE $\frac{\text { ENGI }}{7}$

Run ID=N1161973

02/22/95

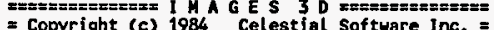
= Copyright (c) 1984 Celestial Software Inc. =

SOLVE PLATE LOAOS/STRESSES Version 2.0 07/01/90

L.

Load Case 1:UBC SEISHIC LOAD - NS \& EW DIR.

Gloads Node Fx $\quad$ Fy $\quad$ Fz $\quad$ Mx Hy GLoads $128 \quad .1012 E+03-.7753 E+01 \quad .7210 E+01-.2737 \mathrm{E}+03-.7355 \mathrm{E}+02-0000 \mathrm{E}+00$ GLoads $128-.7064 \mathrm{E}+02-.1180 \mathrm{E}+03$ PLATE $94 * * *$

$\begin{array}{ll}.4131 E+03 & -.1292 E+03\end{array}$

$\begin{array}{lllllllll}\text { GLoods } 51 & -.7402 \mathrm{E}+02 & .8655 \mathrm{E}+02 & .7867 \mathrm{E}+01 & .2972 \mathrm{E}+03 & .7877 \mathrm{E}+02 & .0000 \mathrm{E}+00\end{array}$

GLoads of .7072E+02 $.7257 E+02-.1892 E+02-.6027 E+02-.1326 E+01 \quad .0000 E+00$

GLoads $130 \quad .7394 \mathrm{E}+02-.4111 \mathrm{E}+02-.1132 \mathrm{E}+02 \quad .1515 \mathrm{E}+02-.1692 \mathrm{E}+03 \quad .0000 \mathrm{E}+00$

GLoads $130-.3673 E+02-.7804 E+02.4653 E+02 \quad .7063 E+03 \quad .2266 E+02 \quad .0000 E+00$

$\begin{array}{llllllll}\text { GLoads } & 67 & -.6854 \mathrm{E}+02 & .7429 \mathrm{E}+02 & .4052 \mathrm{E}+02 & .6733 \mathrm{E}+03 & .5620 \mathrm{E}+02 & .0000 \mathrm{E}+00\end{array}$

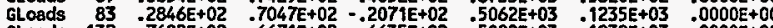

GLoads $132: .7682 E+02-.6671 \mathrm{E}+02-.6635 \mathrm{E}+02 \quad .5082 \mathrm{E}+03 \quad: 9939 \mathrm{E}+03 \quad .0000 \mathrm{E}+00$

GLoads $132-.4610 E+02-.3634 \mathrm{E}+02.10200 \mathrm{PLATE}+03.487 \%$

GLoads $83-.4588 E+02 \quad .1051 E+02 \quad .5010 E+02 \quad .6182 E+03-.3013 E+03 \quad .0000 E+00$

GLoads $99 \quad .6295 E+02 \quad .6538 E+02-.1567 E+03 \quad .7732 E+03-.9319 E+03 \quad .0000 E+00$

GLoads $134 \quad .2903 \mathrm{E}+02-.3955 \mathrm{E}+02 \quad .6580 \mathrm{E}+01 \quad .5984 \mathrm{EE}+03-.7612 \mathrm{E}+03 \quad .0000 \mathrm{E}+00$

GLoods $17=.2450 E+02 \quad .6026 E+03-.7013 E+02-.6476 E+03-.1007 E+03 \quad .0000 E+00$

GLoads $123=.4734 E+03 \quad .1327 E+04-.8368 E+02-.8411 E+03-.5672 E+02 \quad .0000 E+00$

GLoads $125 \quad .1184 E+03 * .791 E+03 \quad .7443 E+02 \quad-.1037 E+04-.1236 E+02$

GLoads $34 \quad .3795 \mathrm{E}+03-.1150 \mathrm{E}+04 \quad .7938 \mathrm{E}+02-.8577 \mathrm{E}+03=.1516 \mathrm{E}+02 \quad .0000 \mathrm{E}+00$

GLoads $34-.1400 E+03 \quad .5344 E+03-.2215 E+02-.5733 E+02-.1489 E+03 \quad .0000 E+00$

GLoads $125-.9561 \mathrm{E}+02 \quad .8436 \mathrm{E}+03-.4507 \mathrm{E}+02-.1941 \mathrm{E}+03-.9105 \mathrm{E}+02 \quad .0000 \mathrm{E}+00$

GLoads $50: 1277 \mathrm{EE}+03-.7936 \mathrm{E}+03 \quad .3977 \mathrm{EE}+02-5601 \mathrm{E}+03-.7097 \mathrm{E}+02 \quad .0000 \mathrm{E}+00$

$\begin{array}{llllllll}\text { GLoads } 50-.8628 E+02 & .3824 E+03 & .2875 E+02 & .4403 E+03 & -.1770 E+03 & .0000 E+00\end{array}$

GLoads $127-.7400 E+02 \quad .5863 E+03 \quad .1015 E+01 \quad .4464 E+03 \quad-.1647 E+03 \quad .0000 E+00$

GLoads $129 \quad .6687 E+02-.4099 E+03-.4278 E+02-.6462 E+02-.2540 E+03 \quad .0000 E+00$

$66 \quad .9341 \mathrm{E}+02-.5587 \mathrm{E}+03 \quad .1301 \mathrm{E}+02-.1372 \mathrm{E}+03-.2395 \mathrm{E}+03 \quad .0000 \mathrm{E}+00$

- GLoads $66-.1076 E+03 \quad .2221 E+03.0652 E+02 \quad .7035 E+03-.3502 E+02 \quad .0000 E+00$ GLoads $129-.3577 \mathrm{E}+02 \quad .3925 \mathrm{E}+03 \quad .6984 \mathrm{E}+02 \quad .1108 \mathrm{E}+04 \quad-.1576 \mathrm{E}+03 \quad .0000 \mathrm{E}+00$ GLosds $131 \quad .1178 E+03-.2348 E+03-.7892 E+02 \quad .8396 E+03-.7481 E+02-0000 E+00$ GLoods $82 \quad .2558 \mathrm{E}+02-.3798 \mathrm{E}+03-5744 \mathrm{E}+02 \quad .3490 \mathrm{E}+03 \quad .8579 \mathrm{E}+02 \quad .0000 \mathrm{E}+00$

$\begin{array}{lrlllllll}\text { GLads } & 82 & -.1188 E+03 & .1552 E+02 & .8526 E+02 & .1825 E+04 & .8677 E+03 & .0000 E+00\end{array}$ $\begin{array}{llllllll}\text { GLoads } 131 & -.8062 E+02 & .2430 E+03 & .1215 E+03 & .7399 E+03 & .4114 E+03 & .0000 E+00\end{array}$ $\begin{array}{lllllll}\text { GLoads } 133 & -.5906 E+01 & .3128 E+02 & -3438 E+02 & .9452 E+03 & .7017 E+03 & .0000 E+00\end{array}$ GLaads $98 \quad .2053 E+03-.2898 E+03-2411 E+03 \quad .2176 E+04 \quad .1136 E+04 \quad .0000 E+00$ $\begin{array}{llllllll}\text { GLonds } 98 & 9540 E+02 & .6209 E+02=.1804 E+03-.1337 E+04 & .3590 E+03 & .0000 E+00\end{array}$
KAISER ENGIHEERS HAMFORD $S / \mathrm{H}: 801854$
PAGE 78 RUn ID=NN61973

$02 / 22 / 95$
$08: 50: 19$

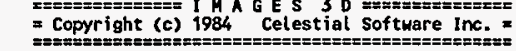

SOLVE PLATE LOADS/STRESSES Version $2.0 \quad 07 / 01 / 90$ $\mathbf{L}$

Load CaSe 1:UBC SEISMIC LOAD - NS \& EH DIR.

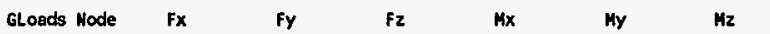

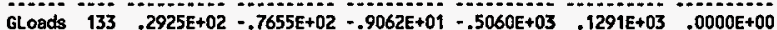
$\begin{array}{llllllll}\text { GLoads } & 135 & =.2404 E+02 & -.2629 E+02 & .2529 E+02 & -.2475 E+03 & -.1686 E+03 & .0000 E+00 \\ \text { GLoads } & 114 & -.1006 E+03 & .4075 E+02 & .1642 E+03 & -.1036 E+04 & .5211 E+01 & .0000 E+00\end{array}$ 
KAISER ENGIHEERS HANFORD S/N:801854

PAGE 79 RUn ID=MN61973

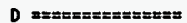
= Copyright (c) 1984 Celestial software Inc. :

SOLVE PLATE LOADS/STRESSES Version $2.0 \quad 07 / 01 / 90$ $\mathbf{L}$

Laed Case 1:UBC SEISMIC LOAD - NS \& EW OIR.

\section{MAXIMUM STRESS SLWHARY FOR PLATES
WITHIN SPECIFIED RANGE}

Maximu (absolute) Stress $=.3463 \mathrm{E}+03$ at Plate 40

Plate sigma $X$ sigma $Y$. Tau $X Y$ Von Mises

$40-.3463 E+03-.8900 E+02 \quad .9047 E+00 \quad .3115 E+03$
KAISER ENGINEERS HAHFORD S/N:801854

PAGE 80

Run ID=HN61973

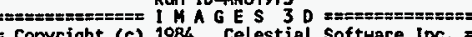
= Copyright (c) 1984 Celestial Software Inc. =

SOLVE REACTIONS

Version $2.0 \quad 07 / 01 / 90$

Load Case 1:UBC SEISHIC LOAD - NS \& EW DIR.

REACTIONS

\begin{tabular}{|c|c|c|c|c|c|}
\hline & & & & & $\mathrm{Mz}$ \\
\hline $\begin{array}{r}1 \\
2 \\
3 \\
6 \\
5 \\
6 \\
7 \\
8 \\
9 \\
10 \\
11 \\
12 \\
13 \\
14 \\
15 \\
16 \\
17 \\
18 \\
115 \\
116 \\
117 \\
118 \\
119 \\
129\end{array}$ & $\begin{array}{l}-.2192 \mathrm{E}+02 \\
-.2445 \mathrm{E}+03 \\
-.2545 \mathrm{E}+03 \\
-.2309 \mathrm{E}+03 \\
-.4472 \mathrm{E}+03 \\
-.5173 \mathrm{E}+01 \\
-.3201 \mathrm{E}+02 \\
-.3377 \mathrm{E}+02 \\
-.3089 \mathrm{E}+02 \\
-.4831 \mathrm{E}+01 \\
-.2653 \mathrm{E}+03 \\
-.2354 \mathrm{E}+03 \\
-.3081 \mathrm{E}+03 \\
-.3359 \mathrm{E}+03 \\
-.3528 \mathrm{E}+03 \\
-.3670 \mathrm{E}+03 \\
-.4229 \mathrm{E}+03 \\
-.1952 \mathrm{E}+02 \\
-.6774 \mathrm{E}+04 \\
-.1827 \mathrm{E}+04 \\
-.7850 \mathrm{E}+03 \\
-.3619 \mathrm{~T}+04 \\
-.7821 \mathrm{E}+03 \\
-.771\end{array}$ & 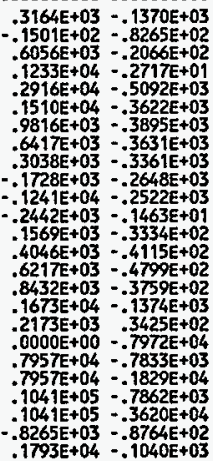 & $\begin{array}{l}.00 \\
.00 \\
.00 \\
.00 \\
. .42 \\
.00 \\
.00 \\
.00 \\
.00 \\
.00 \\
. .30 \\
.00 \\
.00 \\
.00 \\
.00 \\
.00 \\
.00 \\
.00 \\
.00 \\
.00 \\
.00 \\
.00 \\
.00 \\
.00\end{array}$ & $\begin{array}{c}00 \\
04 \\
03 \\
03 \\
03 \\
03 \\
03 \\
01 \\
03 \\
03 \\
03 \\
03 \\
03 \\
03 \\
03 \\
02 \\
02 \\
03 \\
00 \\
00 \\
03 \\
0\end{array}$ & \\
\hline
\end{tabular}


KAISER ENGINEERS HAHFORD S/N:801854

PAGE 81

Run ID=NN61973

$02 / 22 / 95$

GES 3 D $== \pm== \pm===5=$

$=$ Copyright (c) 1984 Celestial Software Inc.

SOLVE BEAM LOADS/STRESSES VErSion 2.0 07/01/90

Load Case 2:GRAVITY LOAD

BEAM LOADS AND/OR STRESSES

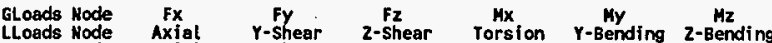

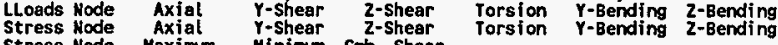
Stress Node Maximum Minimum Cab. Shear

a**Beau

GLoads $1.1036 \mathrm{E}+00 \quad .1225 \mathrm{E}+03-.2811 \mathrm{E}+02 \quad .1137 \mathrm{E}-12-.1779 \mathrm{E}-14=.4814 \mathrm{E}-16$

$99-.1036 \mathrm{E}+00 \quad-2281 \mathrm{E}+02 \quad-2811 \mathrm{E}+02-.9695 \mathrm{E}+03-.5695 \mathrm{E}+01 \quad-.1367 \mathrm{E}+02$

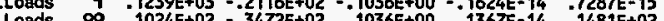

$99.1024 \mathrm{E}+02-.3472 \mathrm{E}+02 \quad \cdot 1036 \mathrm{E}+00 \quad .1367 \mathrm{E}-14 \quad .1481 \mathrm{E}+02 \quad .9695 \mathrm{E}+03$

GLoads $18=.2438 E+00 \quad .1062 E+03 \quad .1867 E+02-.1137 E-12-.3469 E-14 \quad .3140 E-15$

$\begin{array}{lllllllll}\text { GLoods } & 114 & .2438 E+00 & .3583 E+02 & -.1867 \mathrm{E}+02 & .8466 \mathrm{E}+03 & -.1122 \mathrm{E}+02 & .3218 E+02\end{array}$

LLoads $18 \quad .1064 E+03-.1731 E+02-.2438 E+00-.3172 E-14 \quad .1438 E-14-.1137 \mathrm{TE}-1$

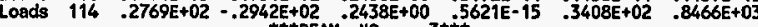

GLoads $99.1182 E+02 \quad .5256 \mathrm{E}+02-.2652 \mathrm{BE}+02 \quad .8460 \mathrm{E}+03-.7417 \mathrm{E}+01 \quad .3663 \mathrm{E}+03$

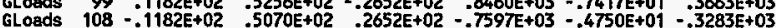

GLods $108=1182 E+02-5070+02-2652 E+02-.797 E+03-.4750 E+01-.3283 E+03$

LLoads $108-.2904 \mathrm{E}+02-.5070 \mathrm{E}+02 \quad .1197 \mathrm{E}+00-.6049 \mathrm{E}+01 \quad .4750 \mathrm{E}+01 \quad .8276 \mathrm{E}+03$

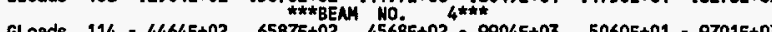

GLoads $114-.4464 E+02 \quad .6587$

GLoads $102-.4464 E+02 \quad .6632 E+02-.4568 E+02 \quad-.99011 E+04 \quad .5164 E+00 \quad .9908 E+03$

LLoads $102-.6387 \mathrm{E}+02=.6632 \mathrm{E}+02-.4286 \mathrm{E}-01 \quad .7628 \mathrm{E}+00-.5164 \mathrm{E}+00 \quad-.1386 \mathrm{E}+0404$

GLoads $98.2216 \mathrm{E}+02 \quad .4801 \mathrm{E}+02 \cdot .2532 \mathrm{E}+02-.5326 \mathrm{E}+03 \cdot .1020 \mathrm{E}+01-.4752 \mathrm{E}+03$

GLods $115-2216 \mathrm{E}+02 \quad 4018 \mathrm{E}+02 \quad-2532 \mathrm{E}+02 \quad 5747 \mathrm{E}+03-2753 \mathrm{E}+00$

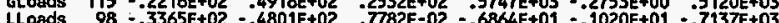

LLoads $115 \quad .3365 \mathrm{E}+02-.4918 \mathrm{E}+02-.7782 \mathrm{E}-02 \quad .6864 \mathrm{E}+01 \quad .2753 \mathrm{E}+00 \quad .7697 \mathrm{EE}+03$

GLoads $92-.1012 E+00 \quad .4186 E+02-.4776 E+00-.5633 E+03 \quad .3580 E+01 \quad .2178 E+03$

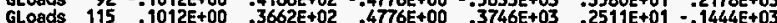

Lloeds $92-6818 E+00-4186 E+02-7884 E-04-1209 E+01-3580 E+01-6030 E+03$

Lloods $115-4818 \mathrm{E}+00-3662 \mathrm{E}+02-7884 \mathrm{E}-01$ 1209E+01-3511E+01 $01015 \mathrm{E}+03$

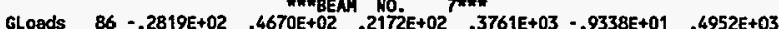

GLoads $115 \quad 2819 E+02-1114 E+02-2172 E+02 \quad 2311 E+03-6622 E+01 \quad 3145 E+03$

L

Lloads $115 \quad 3559 E+02 \quad .1114 E+02-4560 E+00-3794 E+01-6622 E+01-3903 E+03$

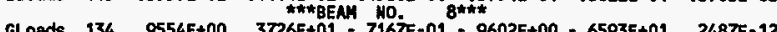

KAISER ENGINEERS HANFORD S/H:801854

PAGE 82 Run ID=HN61973

$02 / 22 / 95$

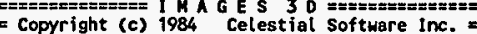
SOLVE BEAM LOADS/STRESSES Version $2.0 \quad 07 / 01 / 90$ L

LOad COSE 2:GRAVITY LOAD

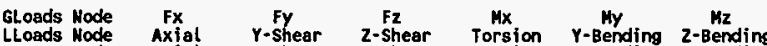
LLoods Node Axial Y-Shear Z-Shear Torsion Y-Bending Z-Bending stress Node Maximum Minimum cmb. Shear

GLoads $99-.9554 \mathrm{E}+00 \quad 1659 \mathrm{E}+02 \quad .7167 \mathrm{E}-01 \quad .9602 \mathrm{E}+00 \quad 8026 \mathrm{E}+01-.1287 \mathrm{O}+03$

GLoads $99-.9554 E+00 \quad .1659 E+02 \quad .7167 E-01 \quad .9602 E+00 \quad .8026 E+01-.1287 E+03$

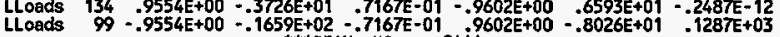

GLoads $135-.3588 \mathrm{E}+01-.5135 \mathrm{E}+01 \quad-.1876 \mathrm{E}+00 \quad .1472 \mathrm{E}+02 \quad .8267 \mathrm{E}+01 \quad .2963 \mathrm{E}+02$

$\begin{array}{lllllll}\text { GLosds } 114 & .3588 E+01 & .2545 E+02 & .1876 E+00 & -.1472 E+02 & -.1202 E+02 & .2763 E+03\end{array}$

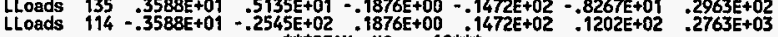

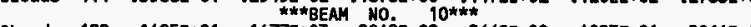

GLoads $122.1495 E+01.1477 E+03 \quad .2842 E-02-.2668 E+00 \quad .4023 E+01-.2064 E+02$

GLoads $124-.1495 \mathrm{E}+01-.1253 \mathrm{E}+03-.2842 \mathrm{E}-02 \quad .3293 \mathrm{E}+00-.4023 \mathrm{E}+01-.1224 \mathrm{E}+02$

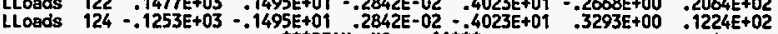

$\begin{array}{lllllllll}\text { GLoads } & 124 & -.6518 E+00 & .1168 E+03 & .1603 \mathrm{E}-01 & -.1449 \mathrm{E}+01 & .2967 \mathrm{E}+01 & .1224 \mathrm{E}+02 \\ \text { GLoads } & 126 & .6518 \mathrm{E}+00 & -.9440 \mathrm{E}+02 & -.1603 \mathrm{E}-01 & .1802 \mathrm{E}+01 & .2967 \mathrm{E}+01 & .2094 \mathrm{E}+01\end{array}$

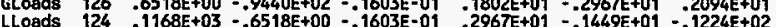

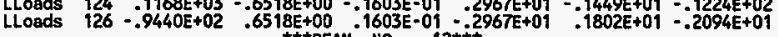

GLoads $126.1273 E+00.9624 E+02 \quad .4966 E-02=.3892 E+01.1779 E+01-.2094 E+01$

GLoads $128-.1273 \mathrm{E}+00 \quad-.7389 \mathrm{EE}+02-.4966 \mathrm{E}-02 \quad .4001 \mathrm{E}+01-.1779 \mathrm{E}+01-.7053 \mathrm{E}+00$

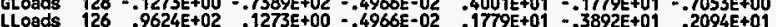

LLoads $128-.7389 \mathrm{E}+02-.1273 \mathrm{E}+00 \quad .4966 \mathrm{E}-02-1779 \mathrm{E}+01 \quad .4001 \mathrm{E}+01 \quad .7053 \mathrm{E}+00$

GLoads $128 \cdot-3663 E-01 \quad .7415 E+02 \quad .3596 E-01-.9219 E+01-.2396 E+01 \quad .7053 E+00$

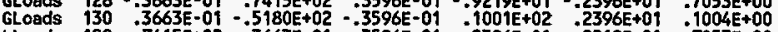

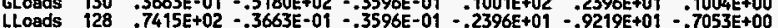

LLoads $130-.5180 \mathrm{E}+02 \quad .3663 \mathrm{E}-01 \quad .3596 \mathrm{E}-01 \quad .2396 \mathrm{E}+01 \quad .1001 \mathrm{E}+02-.1004 \mathrm{E}+00$

GLoads $130.3652 E-02 \quad .4885 E+02-.3157 \mathrm{E}-014-.1213 E+02-.3075 E+00-.1004 E+00$

GLoads $132-.3652 E-02-.2091 E+02 \quad .3157 E-01 \quad .1126 E+02-3075 E+00-.4900 E-13$

LLoads $130-.3885 \mathrm{E}+02-3652 \mathrm{E}-02 \quad 3157 \mathrm{E}-01-3075 \mathrm{E}+00-.313 \mathrm{E}+02-.4902 \mathrm{E}-130$

LLoads $132-.2091 \mathrm{E}+02-.3652 \mathrm{E}-02-.3157 \mathrm{E}-01 \quad .3075 \mathrm{E}+00 \quad .1126 \mathrm{E}+02 \quad .4900 \mathrm{E}-13$

$\begin{array}{lllllllll}\text { GLoads } & 132 & .0000 E+00 & .5923 E+01 & .0000 E+00 & .0000 E+00 & .0000 E+00 & .0000 E+00\end{array}$

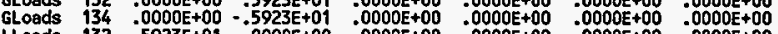

lloads $132 \quad .5923 \mathrm{E}+01.0000 \mathrm{E}+00 \quad .0000 \mathrm{E}+00 \quad .0000 \mathrm{E}+00 \quad .0000 \mathrm{O}+00 \quad .0000 \mathrm{E}+00$

LLoads $134=.5923 \mathrm{E}+01 \quad .0000 \mathrm{E}+00 \quad .0000 \mathrm{E}+00 \quad .0000 \mathrm{E}+00 \quad .0000 \mathrm{E}+00 \quad .0000 \mathrm{E}+00$

GLosds $123-.1839 E+01 \quad .1546 E+03 \quad .3765 E-01-.5571 E+01-.2479 E+01 \quad .2622 E+02$ 
KAISER ENGINEERS HANFORD S/N:801854

PAGE 83 Run ID \#1N61973

$02 / 22 / 95$

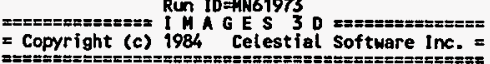

SOLVE BEAM LOADS/STRESSES Version $2.0 \quad 07 / 01 / 90$

$L$

LOad Case 2:GRAVITY LOAD

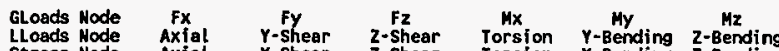

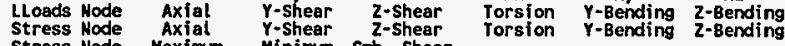
Stress Mode Maximum Minimum Cmb. Shear

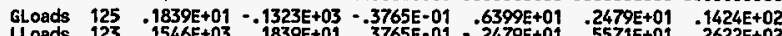

LLoads $123 \quad-1546 E+03 \quad .1839 E+01 \quad .3765 E-01-.2479 E+01 \quad .5571 E+01 \quad .2622 E+02$

Lloads $125-.1323 \mathrm{E}+03-.1839 \mathrm{E}+01-.3765 \mathrm{E}-01 \quad .2479 \mathrm{E}+01-.6399 \mathrm{E}+01 \quad .1424 \mathrm{E}+02$

$\begin{array}{lllllllll}\text { GLoads } 2 & 2.3998 E+00 & .1361 E+03 & .7676 E-02 & .4171 E-01 & .5186 E+01 & -.6387 E+01\end{array}$

GLoads $19-.3998 E+00-.1137 \mathrm{E}+03-.7676 \mathrm{E}-02 \quad .1272 \mathrm{E}+00-.5186 \mathrm{E}+01-.2409 \mathrm{E}+01$

LLoads $2 \quad .1361 \mathrm{E}+03 \quad .3998 \mathrm{E}+00-.7676 \mathrm{E}-02 \quad .5186 \mathrm{E}+01 \quad .4171 \mathrm{E}-01 \quad .6387 \mathrm{E}+01$

LLoads $19-.1137 \mathrm{E}+03-.3998 \mathrm{E}+00 \quad .7676 \mathrm{E}-02-.5186 \mathrm{E}+01 \quad .1272 \mathrm{E}+00 \quad .2409 \mathrm{E}+01$

GLoads $19-.8043 \mathrm{E}-01 \quad .1257 \mathrm{E}+03-.1156 \mathrm{E}-01 \quad .5961 \mathrm{E}+00 \quad .4608 \mathrm{E}+01 \quad .2409 \mathrm{E}+01$

GLoads $35 \quad .8043 \mathrm{E}-01-.1033 \mathrm{E}+03 \quad .1156 \mathrm{E}-01-.8504 \mathrm{E}+00-.4608 \mathrm{E}+01-.6393 \mathrm{E}+00$

LLoads $19.1257 \mathrm{E}+03-.8043 \mathrm{E}-01.1156 \mathrm{E}-01 \quad .4608 \mathrm{E}+01 \quad .5961 \mathrm{E}+00-.2409 \mathrm{E}+01$

Lloads $35-.1033 \mathrm{E}+03 \quad .8043 \mathrm{E}-01-.1156 \mathrm{E}-01-.4608 \mathrm{E}+01 \cdot .8504 \mathrm{E}+00 \quad .6393 \mathrm{E}+00$

GLoads $35 \div-6885 E-01 \quad .9999 E+02 \quad .1409 E-01-.1370 E+01 \quad .2373 E+01 \quad .6393 E+00$

GLoads $51 \cdot .6885 \mathrm{E}-01-.7764 \mathrm{E}+02-.1409 \mathrm{E}-01 \quad .1680 \mathrm{E}+01-.2373 \mathrm{E}+01 \quad .8753 \mathrm{E}+00$

$01-.1409 \mathrm{E}-01 \quad .2373 \mathrm{E}+01-.1370 \mathrm{E}+01-.6393 \mathrm{E}+00$

Lloads $51 \cdot .7764 \mathrm{E}+02 \quad .6885 \mathrm{E}-01.1409 \mathrm{E}-01+.2373 \mathrm{E}+01 \cdot .1680 \mathrm{E}+01-.8753 \mathrm{E}+00$

GLoads $51 \quad .2164 E+00 \quad .7532 E+02-.1328 E-01-.2999 E+01 \quad .6387 E+00-.8753 E+00$

GLoads $67-.2164 \mathrm{E}+00-.5297 \mathrm{E}+02 \quad .1328 \mathrm{E}-01 \quad .2706 \mathrm{E}+01-.6387 \mathrm{E}+00-.3886 \mathrm{E}+01$

$\begin{array}{lllllll}51 & .7532 E+02 & .2164 E+00 & .1328 E-01 & .6387 \mathrm{E}+00 & -.2999 E+01 & .8753 E+00\end{array}$

Lloods $67-.5297 \mathrm{E}+02+.2164 \mathrm{E}+00-1328 \mathrm{E}-01-.6387 \mathrm{E}+00 \quad .2706 \mathrm{E}+01 \quad .3886 \mathrm{E}+01$

GLaAds $\quad 67=.6478 E+00 \quad .5439 E+02 \quad .4936 E-01-.1695 E+02-.1599 E+01 \quad .3886 E+01$

GLoads $83 \quad .6478 E+00-.2645 E+02-.4936 E-01 \quad .1831 E+02 \quad .1599 E+01 \quad .1393 E+02$

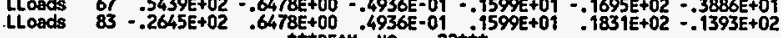

GLoods $83 \quad .7363 E+01 \quad .3332 E+02 \quad .4037 E+00=.5090 E+02-.5482 E+01-.1393 E+02$

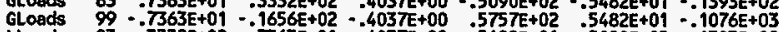

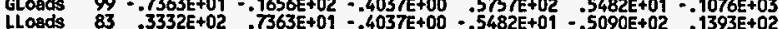

lloeds $99-.1656 \mathrm{E}+02-.7363 \mathrm{E}+01 \quad .4037 \mathrm{E}+00 \quad .5482 \mathrm{E}+01 \quad .5757 \mathrm{E}+02 \quad .1076 \mathrm{E}+03$

Lhos

GLoads $5=.6881 E+00 \quad .1442 E+03-.5354 E+00-.1560 E+02 \quad .3162 E+01 \quad .1280 E+02$

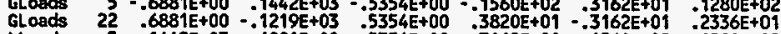

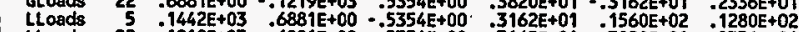

LLoads $22-.1219 \mathrm{E}+03-.6881 \mathrm{E}+00 \quad .5354 \mathrm{E}+00-3162 \mathrm{E}+01-.3820 \mathrm{E}+01 \quad .2336 \mathrm{E}+01$

GLoads $22.5486 E+00.1098 E+03 \quad .2257 E+00 \quad .2029 E+02 \quad .3332 E+01-.1553 E+02$
KAISER ENGINEERS HANFORD S/ $: 801854$

PAGE 84 RUn ID=KN61973

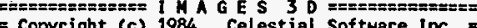
$=$ Copyright (c) 1984 Celestial Software Inc. =

SOLVE BEAM LOADS/STRESSES Version $2.0 \quad 07 / 01 / 90$ L

Load Case 2:GRAVITY LOAD

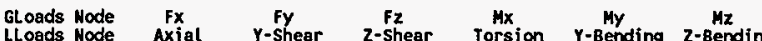
LLoads Node Axial Y-Shear Z-Shear Torsion Y-Bending z-Bending
Stress Node Axial Stress Node Haximum Minimum cmb. Shear

GLoads $38-.5486 \mathrm{E}+00-.8747 \mathrm{E}+02-.2257 \mathrm{E}+00-.1532 \mathrm{E}+02-.3332 \mathrm{E}+01-3460 \mathrm{E}+01$

LLoads $22 \quad .1098 \mathrm{E}+03-.5486 \mathrm{E}+00 \quad .2257 \mathrm{E}+00 \quad: 3332 \mathrm{E}+01-.2029 \mathrm{E}+02-.1553 \mathrm{E}+02$

LLoads $\quad 38-.8747 \mathrm{E}+02 \quad .5486 \mathrm{E}+00 \quad-.2257 \mathrm{E}+00-.3332 \mathrm{E}+01 \quad .1532 \mathrm{E}+02 \quad .3460 \mathrm{E}+01$

GLoads $38-.4321 \mathrm{E}+00 \quad .9545 \mathrm{E}+02 \quad .1246 \mathrm{BE}+00^{25 * *}-.1399 \mathrm{E}+02 \quad .1237 \mathrm{E}+01 \quad .6518 \mathrm{E}+01$

GLoads $54 \quad .4321 \mathrm{E}+00=.7310 \mathrm{E}+02-.1246 \mathrm{E}+00 \quad: 1673 \mathrm{E}+02-.1237 \mathrm{E}+01 \quad .2988 \mathrm{E}+0$

LLoads $38 \quad .9545 \mathrm{E}+02 \quad .4321 \mathrm{E}+00 \quad .1246 \mathrm{E}+00 \quad .1237 \mathrm{E}+01 \quad .1399 \mathrm{E}+02 \quad .6518 \mathrm{E}+01$

LLoads $\quad 54-.7310 \mathrm{E}+02-.4321 \mathrm{E}+00 \quad-1246 \mathrm{E}+00 \quad-1237 \mathrm{E}+01-.1673 \mathrm{E}+02 \quad .2988 \mathrm{E}+0$

GLoads $54.4563 \mathrm{E}+00 \quad .7785 \mathrm{E}+02-.5193 \mathrm{E}+00 \quad .1456 \mathrm{E}+02 \quad .1291 \mathrm{E}+01+.6885 \mathrm{E}+00$

GLoads $\quad 70-.4563 \mathrm{E}+00-.5550 \mathrm{E}+02 \quad .5193 \mathrm{E}+00-.2599 \mathrm{E}+02-.1291 \mathrm{E}+01-.9350 \mathrm{E}+01$

LLoads $54.7785 \mathrm{E}+02-.4563 \mathrm{E}+00-.5193 \mathrm{E}+00-.1291 \mathrm{E}+01-.1456 \mathrm{E}+02-.6885 \mathrm{E}+00$

Lloads $\quad 70-.5550 E+02 \quad .4563 E+00 \quad .5193 E+00-.1291 E+01 \quad .2599 E+02-.9350 E+01$

GLoads $\quad 70-.3387 E+00 \quad .6204 E+02 \quad .5889 E+00-.1738 E+02-.6156 E+01-.4153 E+01$

$\begin{array}{llllllll}\text { GLoads } & 86 & .3387 \mathrm{E}+00 & -.3410 \mathrm{E}+02 & -.5889 \mathrm{E}+00 & .3357 \mathrm{E}+02 & .6156 \mathrm{E}+01 & .1347 \mathrm{E}+02\end{array}$

LLoads $70 \quad .6204 \mathrm{E}+02 \quad .3387 \mathrm{E}+00 \quad .5889 \mathrm{E}+00-.6156 \mathrm{E}+01 \quad .1738 \mathrm{E}+02-.4153 \mathrm{E}+01$

LLoads $86=.3410 \mathrm{E}+02-.3387 \mathrm{E}+00-.5889 \mathrm{E}+00 \quad .6156 \mathrm{E}+01-.3357 \mathrm{E}+02 \quad .1347 \mathrm{E}+02$

GLoads $86.1154 E+02.3216 E+02=.1206 E+02.8880 E+01 \quad .8137 E+01-.1354 E+02$

GLoads $102-.1154 \mathrm{E}+02-1540 \mathrm{E}+02 \quad .1206 \mathrm{E}+02-.2079 \mathrm{E}+03-.8137 \mathrm{E}+01-.1769 \mathrm{E}+03$

LLoads $80.3216 \mathrm{E}+02-.1154 \mathrm{E}+02-.1206 \mathrm{E}+02 \quad .8137 \mathrm{E}+01$

LLads $102-.1540 \mathrm{E}+02 \quad .1154 \mathrm{E}+02 \quad .1206 \mathrm{E}+02-.8137 \mathrm{E}+01 \quad .2079 \mathrm{E}+03-.1769 \mathrm{E}+03$

GLoads $11.3384 E+01 \quad .2204 E+03.1421 E+01 \quad .2908 E+02-.8908 E+01-.6935 E+02$

GLoads $28-.3384 \mathrm{E}+01-.1856 \mathrm{E}+03-.1421 \mathrm{E}+01 \quad .2191 \mathrm{E}+01 \quad .8908 \mathrm{E}+01-.5104 \mathrm{E}+01$

lloads $11-.2204 E+03 \quad .1421 E+01 \quad-3384 E+01-.8908 E+01-.6935 E+02 \quad-2908 E+02$

GLoods

GLoods $44 \quad .1594 \mathrm{E}+01-.1269 \mathrm{E}+03 \quad .9686 \mathrm{E}+00 \quad .8951 \mathrm{E}+01 \quad .5922 \mathrm{E}+01-.2882 \mathrm{E}+02$

$28 \quad .1617 E+03-.9686+03-.1594 E+01-.5922 E+01 \quad .6389 E+02-.3026 E+02$

GLoeds

GLoads

LLoads

$44-.1269 E+03 \quad .9686 E+00 \quad .1594 E+01 \quad .5922 E+01-.2882 E+02 \quad .8851 E+01$

$44.6104 E+00 \quad .1352 E+03 \quad .6550 E+00 \quad .8919 E+01-.2409 E+01-.4078 E+01$

60 -.6104E+00 - $.1004 E+03 \quad-.6550 E+00 \quad .5492 E+01 \quad .2409 E+01-.9350 E+0$

$44-.1352 E+03 \quad .6550 E+00 \quad .6104 E+00-.2409 E+01 \quad-.4078 E+01 \quad .8919 E+0$

$60-.1004 \mathrm{E}+03-.6550 \mathrm{E}+00-.6104 \mathrm{E}+00 \quad .2409 \mathrm{E}+01-.9350 \mathrm{E}+01 \quad .5492 \mathrm{E}+01$

GLoods $60-.1063 E+01.1091 E+03-.7227 E+00=.1720 E+01 \quad .4378 E+01-.5489 E+01$ 
KAISER ENGINEERS HANFORD S/N: 801854

PAGE 85 Run ID=WN61973

$02 / 22 / 95$

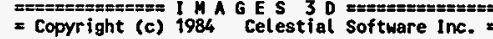

$08: 50: 21$

SOLVE BEAM LOADS/STRESSES Version $2.0 \quad 07 / 01 / 90$ $\mathbf{L}$

Load Case 2:GRAVITY LOAD

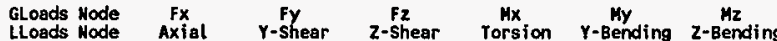

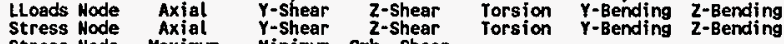
Stress Node Maximum Minimum Comb. Shear

Gloods 76 . $1063 \mathrm{E}+01-7427 \mathrm{E}+02$ -

GLoads $76-1063 E+01-.7427 E+02 \quad .7227 E+00-.1418 E+02-.4378 E+01 \quad .2888 E+02$

LLoads $60-1091 \mathrm{E}+03-.7227 \mathrm{E}+020-.1063 \mathrm{E}+01-.4378 \mathrm{E}+01-.5489 \mathrm{E}+01-.1720 \mathrm{E}+01$

$76-.7427 \mathrm{E}+02 \quad .7227 \mathrm{E}+00 \quad \cdot 1063 \mathrm{E}+01+.4378 \mathrm{E}+01 \quad .2888 \mathrm{E}+02-.1418 \mathrm{E}+02$

$\begin{array}{llllllll}\text { GLoads } & 76 & .1666 \mathrm{E}+01 & .8242 \mathrm{E}+02 & .5929 \mathrm{E}+00 & -.1485 \mathrm{E}+01 & .7699 \mathrm{E}+01 & .2133 \mathrm{E}+02\end{array}$

GLoads $92=.1666 \mathrm{E}+01-.3892 \mathrm{E}+02-.5929 \mathrm{E}+00 \quad: 1779 \mathrm{E}+02-.7699 \mathrm{E}+01-.6715 \mathrm{E}+02$

LLoads $76 \quad 8242 \mathrm{E}+02 \quad 5929 \mathrm{E}+00 \quad 1666 \mathrm{E}+01 \quad 7609 \mathrm{E}+01-2133 \mathrm{E}+02=.1485 \mathrm{E}+01$

LLoads $92-.3892 \mathrm{E}+02-.5929 \mathrm{E}+00-.1666 \mathrm{E}+01-.7699 \mathrm{E}+01-.6715 \mathrm{E}+02 \quad: 1779 \mathrm{E}+02$

GLoads $92-.3275 E+01 \quad .3607 \mathrm{t}+02 \quad .1364 \mathrm{BEAN}+02 \quad .3714 \mathrm{NOE}+02 \quad .1508 \mathrm{E}+02-.1299 \mathrm{E}+03$

$\begin{array}{llllllll}\text { GLoads } & 108 & .3275 \mathrm{E}+01 & -.9967 \mathrm{E}+01 & -.1364 \mathrm{E}+02 & .1879 \mathrm{E}+03 & -.1508 \mathrm{E}+02 & .1840 \mathrm{E}+03\end{array}$

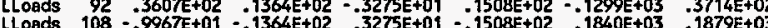

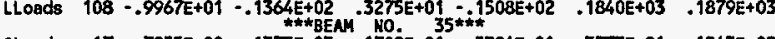

GLoads $\quad 17=.7855 E+00 \quad .1373 E+03 \quad .1708 E-01-.5394 \mathrm{E}+01-.3773 E+01 \quad .1243 E+02$

$\begin{array}{llllllll}\text { GLoads } & 17 & -7855 E+00 & .1373 E+03 & .1708 E-01 & -.5394 E+01 & .3773 E+01 & .1243 E+02\end{array}$

LLoads $17 \quad .1373 E+03 \quad .7855 E+00 \quad .1708 E-01-.3773 E+01 \quad .5394 E+01 \quad .1243 E+02$

LLoads 34 -.1150E+03 -.7855E+00 *1708E-01 .3773E+01 -.5769E+01 .4851E+0

GLoads $34.2209 E+00.1265 E+03 \quad .3068 E-02-.1479 E+02-.2879 E+01-.4851 E+01$

$\begin{array}{llllllll}\text { GLoads } & 34 & .2209 \mathrm{E}+00 & .1265 \mathrm{E}+03 & .3068 \mathrm{E}-02 & -.1479 \mathrm{E}+02 & -.2879 \mathrm{E}+01 & -.4851 \mathrm{E}+01 \\ \text { GLoads } & 50 & -.2209 \mathrm{E}+00 & -.1042 \mathrm{E}+03 & -.3068 \mathrm{E}-02 & .1486 \mathrm{E}+02 & .2879 \mathrm{E}+01 & -.8537 \mathrm{E}-02\end{array}$

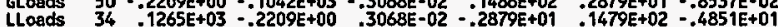

LLoads $50-.1042 \mathrm{E}+03 \quad-2209 \mathrm{E}+00-.3068 \mathrm{E}-02 \quad-2879 \mathrm{E}+01-.1486 \mathrm{E}+02-.8537 \mathrm{E}-02$

GLoads $50-.3377 E+00 \quad .1015 E+03 \quad .4007 E-01-.2767 E+02 \quad .7762 E+00 \quad .8537 E-02$

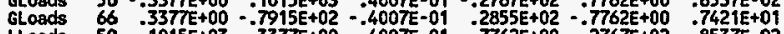

LLoads $50.1015 \mathrm{E}+03.3377 \mathrm{E}+00 \quad .4007 \mathrm{E}-01 . .7762 \mathrm{E}+00.2767 \mathrm{E}+02 \quad .8537 \mathrm{E}-02$

.Loads $66-.7915 \mathrm{E}+02-.3377 \mathrm{E}+00-.4007 \mathrm{E}-01-.7762 \mathrm{E}+00-.2855 \mathrm{E}+02 \quad .7421 \mathrm{E}+01$

GLoads $66 \quad .2000 E+01 \quad .7893 E+02-.5136 E-01-.3330 E+02 \quad .5724 E+01-.7421 E+01$

GLoads $82-.2000 E+01-.5658 E+02 \quad .5136 E-01 \quad .3217 \mathrm{E}+02-.5724 \mathrm{E}+01-.3657 \mathrm{E}+02$

LLoads $66 \quad .7893 \mathrm{E}+02-.2000 \mathrm{E}+01-.5136 \mathrm{E}-01 \quad .5724 \mathrm{E}+01 \quad .3330 \mathrm{E}+02-.7421 \mathrm{E}+01$

LLoads $82+.5658 \mathrm{E}+02 \quad .2000 \mathrm{E}+01 \quad .5136 \mathrm{E}-01-.5724 \mathrm{E}+01-.3217 \mathrm{E}+02-.3657 \mathrm{E}+02$

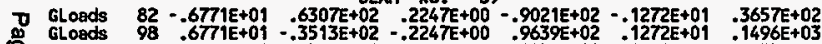

GLoads $98 \quad .6771 \mathrm{E}+01-.3513 \mathrm{E}+02-.2247 \mathrm{E}+00 \quad .9639 \mathrm{E}+02-1272 \mathrm{E}+01 \quad .1496 \mathrm{E}+03$

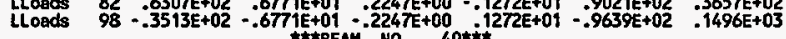

$\rightarrow$ Lloads

$98-.4232 E+02 \quad .3556 E+02 \quad .2354 E+01 \quad .1009 E+03 \quad .1180 E+01 \quad .3255 E+03$
KAISER ENGIMEERS HANFORD S/N:801854

PAGE 86

Run ID=MN61973

$02 / 22 / 95$

$=$ Copyright (c) 1984 Celestial Software Inc. $=$

SOLVE BEAM LOADS/STRESSES Version $2.0 \quad 07 / 01 / 90$

Load Case 2:GRAVITY LOAD

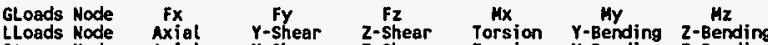

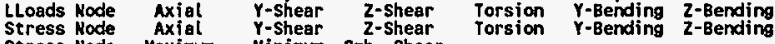
Stress Mode Maximum Minimum Cmb. Shear

GLoads $114 \quad .4232 E+02-.1879 E+02-.2354 E+01-.6204 E+02=.1180 E+01 \quad .3727 E+03$

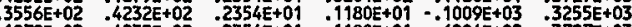

Lloads $114-.1879 E+02-.4232 E+02-.2354 E+01 \quad-1180 E+01 \quad .6204 E+02 \quad .3727 E+03$

GLoads $99-.3153 \mathrm{E}+01-.4171 \mathrm{E}+01.8993 \mathrm{E}-01^{2} .3484 \mathrm{E}+02-.5241 \mathrm{E}+01-.1165 \mathrm{E}+03$

GLoads $100 \quad .3153 \mathrm{E}+01 \quad .1806 \mathrm{E}+02 \quad-.8993 \mathrm{E}-01 \quad-.3484 \mathrm{E}+02 \quad .02 \quad .4012 \mathrm{E}+01-.3543 \mathrm{E}+02$

LLoads $99-.3153 E+01 \quad .4171 E+01-.8993 E-01 \quad-3484 E+02 \quad .5241 E+01 \quad+1165 E+03$

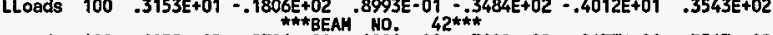

GLoads $100-.1152 E+02 \quad .9796 \mathrm{E}+01.1894 \mathrm{E}+00 \quad .3009 \mathrm{E}+02 \quad .2477 \mathrm{E}+01 \quad .3543 \mathrm{E}+02$

GLoads $101 \quad .1152 \mathrm{E}+02 \quad .4088 \mathrm{E}+01 \quad-.1894 \mathrm{E}+00-.3009 \mathrm{E}+02-.5065 \mathrm{E}+01 \quad .3569 \mathrm{E}+01$

LLoads $101.1152 \mathrm{E}+02-.4088 \mathrm{E}+01-1894 \mathrm{E}+00-3009 \mathrm{E}+02 \quad .5065 \mathrm{E}+01-.3569 \mathrm{E}+01$

LLoads 101 . ITEE+O2 -

GLoads $101-.1972 E+02-.9375 E+01-.1771 E+00 \quad .5147 E+02 \quad .3984 E+01-.3569 E+01$

GLoads $102 \quad .1972 \mathrm{E}+02 \quad .2326 \mathrm{E}+02 \quad .1771 \mathrm{E}+00 \quad-.5147 \mathrm{E}+02 \quad-.1563 \mathrm{E}+01-.2194 \mathrm{EE}+03$

LLoads $102 \quad .1972 \mathrm{E}+02 \quad-.2326 \mathrm{E}+02-1771 \mathrm{E}+00 \quad-.5147 \mathrm{E}+02 \quad .1563 \mathrm{E}+01 \quad .2194 \mathrm{E}+03$

GLoads $102-.1595 \mathrm{E}+00-.1037 \mathrm{*}+02-.6407 \mathrm{BEAK}+01-.2258 \mathrm{NO}+03-.6927 \mathrm{E}+01-.2630 \mathrm{E}+02$

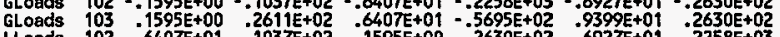

.

LLoads $103-.6407 E+01-.261 * * 02-.1595 E+00-.2630 E+02-.939 \%+01 \quad .5695 E+02$

GLoads $103 \quad .1277 E+00 \quad .1047 E+02 \quad .5208 E+01 \quad .5695 E+02-.1036 E+02-.6033 E+01$

GLoads $104=-1277 \mathrm{E}+00 \quad .5281 \mathrm{E}+01 \quad-.5208 \mathrm{E}+01 \quad-.1677 \mathrm{E}+02 \quad .8379 \mathrm{E}+01 \quad .6033 \mathrm{E}+01$

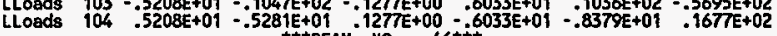

GLoads $104-.2903 E-02, .7597 \mathrm{E}+01.7560 \mathrm{EE}+01.1677 \mathrm{E}+02-.3291 \mathrm{E}+01-.7790 \mathrm{E}+01$

GLoads $104-.2903 \mathrm{E}-02 \quad .7597 \mathrm{E}+01 \quad .7560 \mathrm{E}+01 \quad-1677 \mathrm{E}+02-.3291 \mathrm{E}+01-.7790 \mathrm{E}+01$

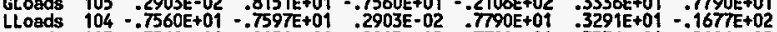

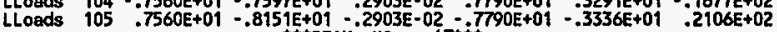

GLoads $105.5381 \mathrm{E}-01.8046 \mathrm{E}+01.5874 \mathrm{E}+01^{4} .2106 \mathrm{E}+02-.1759 \mathrm{E}+01-.4421 \mathrm{E}+01$

$\begin{array}{llllllll}\text { GLoads } & 105 & .5381 \mathrm{E}-01 & .8046 \mathrm{E}+01 & .5874 \mathrm{E}+01 & -2106 \mathrm{E}+02 & * .1759 \mathrm{E}+01 & -.4421 \mathrm{E}+01 \\ \text { GLoads } & 106 & -5381 \mathrm{E}-01 & .7702 \mathrm{E}+01 & -.5874 \mathrm{E}+01 & -.1839 \mathrm{E}+02 & .9247 \mathrm{E}+00 & .4421 \mathrm{E}+01\end{array}$

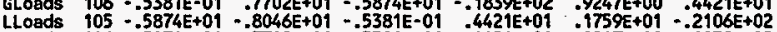

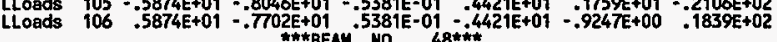

GLoeds $106 \quad .5334 \mathrm{E}-01 \quad .6658 \mathrm{E}+01.4365 \mathrm{E}+00 \quad .1839 \mathrm{E}+02 \quad .327 \mathrm{TE}+01 \quad-.4104 \mathrm{E}+01$ 
KAISER ENGINEERS HANFORD S/N:801854

PAGE 87

Rin ID=MN61973

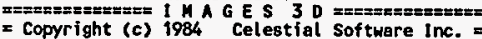

x Copyright (c) 1984 Celestial Software Inc. =

SOLVE BEAH LOADS/STRESSES Version 2.0 07/01/90

LOAd CASE 2:GRAVITY LOAD

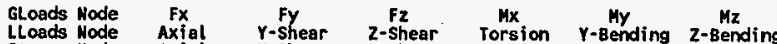

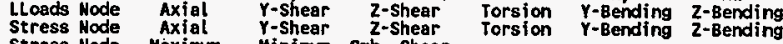
Stress Hode Axial Mode Maximum Minimum cmb. Shear

Gloads 107 - 5334E-01 $90905+01-4365 E+00$ -

GLoads 107 - $5334 E-01 \quad .9090 E+01-.4365 E+00-.3724 E+02-.4104 E+01 \quad .4104 E+01$

$\begin{array}{llllllll}\text { LLoads } & 106 & -.4365 \mathrm{E}+00 & -.6658 \mathrm{E}+01 & -.5334 \mathrm{E}-01 & .4104 \mathrm{E}+01 & -.3277 \mathrm{E}+01 & -.1839 \mathrm{E}+02 \\ \text { LLoads } & 107 & .4365 \mathrm{E}+00 & -.9090 \mathrm{E}+01 & .5334 \mathrm{E}-01 & -.4104 \mathrm{E}+01 & .3104 \mathrm{E}+01 & .3724 \mathrm{E}+02\end{array}$

GLoads $107-.2706 \mathrm{E}-01 \quad .1738 \mathrm{E}+02-.1282 \mathrm{EE}+02 \quad .3724 \mathrm{E}+02 \quad .7852 \mathrm{E}+01-.6940 \mathrm{E}+01$

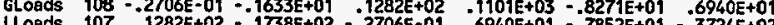

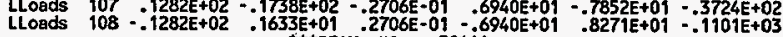

LCads 108 - 0 *

GLoads $108-.4228 E+01 \quad .1124 E+02-.2946 E+00 \quad .5832 E+01 \quad .1731 E+02 \quad .6724 E+02$

GLoads $109 \quad .4228 \mathrm{E}+01 \quad .4171 \mathrm{E}+01 \quad .2946 \mathrm{E}+00-.5832 \mathrm{E}+01-.1284 \mathrm{E}+02-.1364 \mathrm{E}+02$

LLoads $108-.4228 \mathrm{E}+01-.1124 \mathrm{E}+02 \quad .2946 \mathrm{E}+00 \quad .5832 \mathrm{E}+01-.1731 \mathrm{E}+02-.6724 \mathrm{E}+02$

LLoads $109.4228 \mathrm{E}+04-.4171 \mathrm{E}+01 \quad .2946 \mathrm{E}+00-.5832 \mathrm{E}+01 \quad .1284 \mathrm{E}+02 \quad .1364 \mathrm{E}+02$

GLoads $109:=5430 E+01 \quad .7287 \mathrm{E}+01 \quad-2044 \mathrm{E}-01-1276 \mathrm{E}+02 \quad .3927 \mathrm{E}+01 \quad-1364 \mathrm{E}+02$

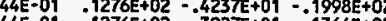

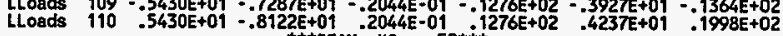

(1)

GLads $119-.6401 E+01-770 E+01-1102 E+00-1128 E+02 \quad .3583 E+01 \quad-1998 E+02$

GLoads $119.6401 E+01-.7709 E+01 \quad .1102 E+00-1128 E+02-.1912 E+01-.2005 E+02$

LLoads $111 \quad .6401 E+01-.7709 E+01 \quad-1102 E+00 \quad-1128 E+02 \quad .1912 E+01 \quad .2005 E+02$

GLoads $111 \cdot-.7059 E+01 \quad .8050 E+01-.2935 \mathrm{E}-01-.1520 \mathrm{E}+02-.6891 \mathrm{E}+00 \quad .2005 \mathrm{E}+02$

GLoads $112.7059 E+01.7359 E+01 \quad .2935 \mathrm{E}-01 \quad .1520 \mathrm{E}+02 \quad .1134 \mathrm{EE}+01 \quad-.1480 \mathrm{E}+02$

LLoads $111-.7059 E+01-.8050 \mathrm{E}+01 \quad .2935 \mathrm{E}-01-1520 \mathrm{E}+02 \quad .6891 \mathrm{E}+00-.2005 \mathrm{E}+02$

LLoads $112 \quad .7059 \mathrm{E}+01-.7359 \mathrm{E}+01-.2935 \mathrm{E}-01 \quad .1520 \mathrm{E}+02-.02 \quad-.1134 \mathrm{E}+01 \quad .1480 \mathrm{E}+02$

- Gloads $112-.4718 E+01 \quad .4366 E+01-.1672 E+00-.8167 E+01-.5408 E+01 \quad .1480 E+02$

GLoads $113 \quad .4718 \mathrm{E}+01 \quad .1104 \mathrm{E}+02 \quad .1672 \mathrm{E}+00 \quad .8167 \mathrm{E}+01 \quad .7944 \mathrm{E}+01-.6544 \mathrm{E}+02$

$113-.4778 E+01-.4366 \mathrm{E}+01-1672 E+00-.8167 \mathrm{E}+1$

7

loods $113 \quad .4718 E+01-.1104 E+02--1672 E+00 \quad .8167 E+01-.7944 E+01 \quad .6544 E+02$

$\begin{array}{llllllll} & \text { GLoads } 113 & .2645 E+01 & .3107 \mathrm{E}+02 & -.2471 \mathrm{E}+00 & .3011 \mathrm{E}+01 & -.2127 \mathrm{E}+02 & .6544 \mathrm{E}+02\end{array}$

GLods $114 \cdot-2645 E+01-.1566 E+02-2471 E+00-.3011 E+01 \quad-2502 E+02 \quad .2890 E+03$

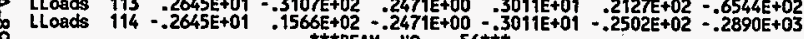

GLoads $115-.1242 E+01.1600 \mathrm{E}+05$. $2150 \mathrm{E}+01^{5 * \pi \hbar}-.2390 \mathrm{E}+03 \quad .8467 \mathrm{E}+00-.1381 \mathrm{E}+03$
KAISER EMGINEERS HANFORO S/N:801854

PAGE 88 RUN ID = MN61973

$02 / 22 / 95$

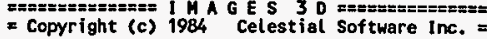

SOLVE BEAH LOADS/STRESSES Version $2.0 \quad 07 / 01 / 90$

L

Load Case 2:GRAVITY LOAD

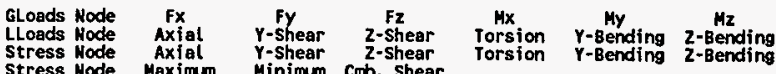
Stress Hode

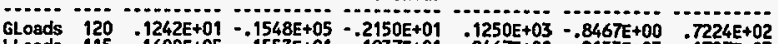

LLoads $115-.1600 E+05-.1553 E+01-.1937 E+01-.8467 \mathrm{E}+00 \quad .2153 \mathrm{E}+03-.1727 \mathrm{E}+03$

Lloads $120.1548 E+05 \quad .1553 E+01 \quad .1937 \mathrm{E}+01 \quad .8467 \mathrm{E}+00-.1126 \mathrm{E}+03.9034 \mathrm{E}+02$

GLoads $120-.1242 E+01-.1452 E+05 \quad .2150 E+01-.1250 E+03 \quad .8467 E+00-.7224 E+02$

GLoads $121.1242 E+01 \quad .1515 E+05-.2150 E+01-.9342 E+01-.8467 E+00-.5399 E+01$

LLoads $120 \quad .1452 E+05-.1553 E+01-.1937 E+01-.8467 E+00 \quad .1126 E+03-.9034 E+02$

LLoads $121-.1515 E+05 \quad .1553 E+01, .1937 E+01 \quad .8467 \mathrm{E}+00 \quad .8418 \mathrm{E}+01-.6751 \mathrm{E}+01$

GLoads $116 \quad .5827 E+00 \quad .3996 E+04-.1114 E+00 \quad .5773 E+01-.1410 E+02 \quad .7412 E+04$

GLoods $121-.5827 \mathrm{E}+00-.3787 \mathrm{E}+04 \quad .1114 \mathrm{E}+00-.5773 \mathrm{E}+01 \quad .1644 \mathrm{E}+02 \quad .7431 \mathrm{E}+05$

LLoads $116 \quad .5827 E+00 \quad .3996 E+04-.1114 E+00 \quad-5773 E+01-.1410 E+02 \quad .7412 E+04$

LLoads $121-.5827 \mathrm{E}+00-.3787 \mathrm{E}+04 \quad .1114 \mathrm{E}+00-.5773 \mathrm{E}+01 \quad .1644 \mathrm{E}+02 \quad .7431 \mathrm{E}+05$

GLoads $117.8335 E-01 \quad .3995 E+04-.1008 E+01-.7394 E+04-.7840 E+01 \quad .3467 E+01$

GLoods $121-.8335 \mathrm{E}-01-.3785 \mathrm{E}+04 \quad .1008 \mathrm{E}+01-.7430 \mathrm{E}+05 \quad .9590 \mathrm{E}+01-.3467 \mathrm{E}+01$

LLords $117-.1008 \mathrm{E}+01 \quad .3995 \mathrm{E}+04-.8335 \mathrm{E}-01 \quad .3467 \mathrm{E}+01-.7840 \mathrm{E}+01 \quad .7394 \mathrm{E}+04$

Lloads $121 \quad .1008 E+01-.3785 E+04 \quad .8335 E-01-.3467 E+01 \quad .9590 E+01 \quad .7430 E+05$

$\begin{array}{llllllll}\text { GLoads } & 118 & .5827 \mathrm{E}+00 & .3995 \mathrm{E}+04 & -.2167 \mathrm{E}-01 & .5153 \mathrm{E}+01 & .1556 \mathrm{E}+02-.7399 \mathrm{E}+04\end{array}$

GLoads $121-.5827 \mathrm{E}+00-.3786 \mathrm{E}+04 \quad .2167 \mathrm{E}-01-.5153 \mathrm{E}+01-.1602 \mathrm{E}+02-.7430 \mathrm{E}+05$

.

Lloads $121 \quad .5827 E+00-.3786 E+04 \quad-.2167 E-01 \quad .5153 E+01 \quad-.1602 E+02 \quad .7430 E+05$

GLoads $119-.6425 E-02 \quad .3997 E+04-.1008 E+01 \quad .7418 E+04 \quad .9302 E+01 \quad .2847 E+01$

GLoads $121 \quad .6425 \mathrm{E}-02-.3788 \mathrm{E}+04 \quad .1008 \mathrm{E}+01 \quad .7432 \mathrm{E}+05-.9167 \mathrm{E}+01-.2847 \mathrm{E}+01$

LLoads $119-1008 E+01 \quad .3997 \mathrm{E}+04-.6425 \mathrm{E}-02-.2847 \mathrm{E}+01 \quad .9302 \mathrm{E}+01 \quad .7418 \mathrm{E}+0$

Llods $121-.1008 E+01 \cdot .3788 E+04 \quad .6425 \mathrm{E}-02 \quad .2847 \mathrm{E}+01-.9167 \mathrm{E}+01 \quad .7432 \mathrm{E}+05$

GLoads, $125 \quad .7116 \mathrm{E}+00 \quad .1166 \mathrm{E}+03 \quad .4874 \mathrm{E}-01-.1829 \mathrm{E}+02-.5538 \mathrm{E}+00-.1424 \mathrm{E}+02$

GLoads $127-.7116 \mathrm{E}+00 \quad-.9428 \mathrm{E}+02-.4874 \mathrm{E}-01 \quad .1936 \mathrm{E}+02 \quad .5538 \mathrm{E}+00 \quad-.1413 \mathrm{E}+0$

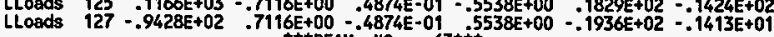

GLoads $127 \cdot .1118 \mathrm{E}+00.9606 \mathrm{E}+02 \quad .2753 \mathrm{E}-01+.3190 \mathrm{E}+02.1740 \mathrm{E}+01.1413 \mathrm{E}+01$

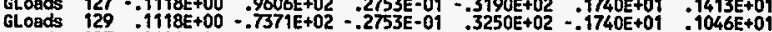

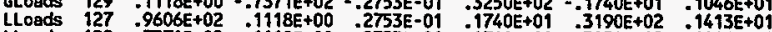

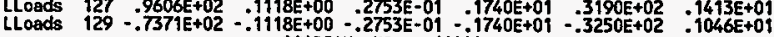

GLoads $129.1211 E+00.7650 E+02.1132 E+00-.5148 E+02 \quad .1188 E+02-.1046 E+01$ 
KAISER ENGINEERS HANFORD S/N:801854

PAGE 89

\section{Rin $10=$ M61973}

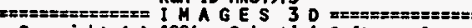

$02 / 22 / 95$

= Copyright (c) 1984 Celestial Software Inc.

SOLVE BEAM LOADS/STRESSES. Version 2.0 07/01/90

LOBd Case 2:GRAYITY LOAD

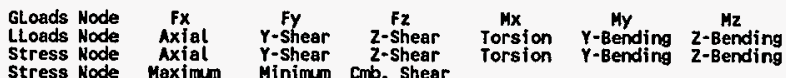
Minimum Cmb. Shear

Cl.

GLoads $131=.1211 E+00-.5415 E+02 \quad-.1132 E+00 \quad-5398 E+02 \quad-.1188 E+02 \quad-.1619 E+0$

LLoads $131-.5415 \mathrm{E}+02 \quad .1211 \mathrm{E}+00-1132 \mathrm{E}+00-.1188 \mathrm{E}+02-.5398 \mathrm{E}+02-.1619 \mathrm{E}+01$

$131-3115 E+00-049318$

.

$\begin{array}{llll}.3731 E+02 & .9416 \mathrm{E}+01 & .6948 \mathrm{E}+0 & \end{array}$

. $0290 \mathrm{E}+02 \quad .621619 \mathrm{E}+0$

LLoads $133-.2137 \mathrm{E}+02-.3115 \mathrm{E}+00 \quad .2032 \mathrm{E}+00 \quad .9416 \mathrm{E}+01-.3731 \mathrm{E}+02 \quad .6948 \mathrm{E}+01$

GLogds $133.2217 \mathrm{E}+01.1259 \mathrm{E}+02-.1258 \mathrm{E}+01-.1488 \mathrm{E}+02-.7148 \mathrm{E}+01-.6948 \mathrm{E}+01$

GLoods $135-.2217 \mathrm{E}+01 \quad .4172 \mathrm{E}+01-.1258 \mathrm{E}+01-.5877 \mathrm{E}+01-.7148 \mathrm{E}+01-.2963 \mathrm{E}+02$

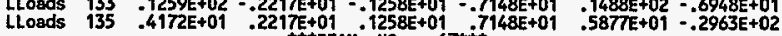

Lhosds

GLoads $115 \quad-.8973 E+03 \quad-.4020 E+04 \quad .2067 E+01-.2330 E+03 \quad .2931 E+02 \quad .4625 E+03$

GLods $116 \cdot 8973 E+03 \quad 5189 E+04-2067 E+01-5773 E+01-1410 E+02-7412 E+04$

LOnd $1150415 E+04-1638 E+03-2067 \mathrm{E}+01-1284 E+02-2345 E+03-4625 E+03$

LLads $116=5266 E+04-4539 E+02-2067 \mathrm{E}+01-1284 E+02-8202 E+01-7412 E+04$

**BEAM NO. $68^{* * *}$ K

GLoads $115 \cdot-.1198 E+01-.4020 E+04-.8940 E+03-.8385 E+03 \quad .1731 E+02-.1349 E+03$

GLosds $117 \quad .1198 \mathrm{E}+01 \quad .5190 \mathrm{E}+04 \quad .8940 \mathrm{E}+03 \quad .7394 \mathrm{E}+04 \quad .7840 \mathrm{E}+01-.3467 \mathrm{E}+01$

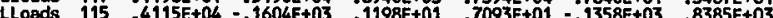

Lloods $117-.5266 \mathrm{E}+04-4876 \mathrm{E}+02-1198 \mathrm{E}+01-7093 \mathrm{E}+01-4813 \mathrm{E}+01-7394 \mathrm{E}+04$

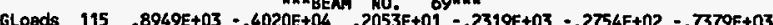

GLoods $118 \cdot .8940 \mathrm{E}+03-5189 \mathrm{E}+04=2053 \mathrm{E}+01=5153 \mathrm{E}+01-.1556 \mathrm{E}+02-.7390 \mathrm{E}+04$

LLoads 115 4145E+04 - 1613E+03 - 2053E+01 - 1439E+02 $3331 E+03-7370 E+03$

LLods $118-.5266 \mathrm{E}+04-.4786 \mathrm{E}+02 \quad .2053 \mathrm{2}+01 \quad .1439 \mathrm{E}+02 \quad .7854 \mathrm{E}+01-.7399 \mathrm{E}+04$

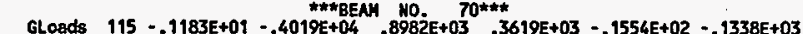

Gloads $119 \quad 1183 E+01-5189 E+04-8982 E+03-7418 E+04-9302 E+01-3875+01$

Lloads $115-4115 E+04-1647 E+03-1183 E+01-8642 E+01-1344 E+03-3619 E+03$

Lloads $119 \cdot-.5266 \mathrm{E}+04=.4449 \mathrm{E}+02 \quad .1183 \mathrm{E}+01 \quad .8642 \mathrm{E}+01 \quad .4465 \mathrm{E}+01 \quad-.7418 \mathrm{E}+04$

0
0
0
0
2
8
KAISER ENGINEERS HANFORO S/M:801854

PAGE 90

Run ID=NH61973

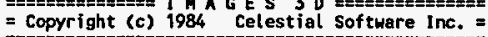

SOLVE BEAM LOADS/STRESSES Version $2.0 \quad 07 / 01 / 90$

L

LOad Case 2:GRAVITY LOAD

\section{MAXIMUN STRESS SUMMARY FOR BEAMS/TRUSSES \\ WITHIN SPECIFIED RANGE 1 - 70}

Maximum (absolute) Stress $=.4557 E+03$ at BEAM 56

Bean Axial Y-Shear 2-Shear Torsion Y-Bending z-Bending

$56 \quad .4547 \mathrm{E}+03-.8826 \mathrm{E}-01-.1101 \mathrm{E}+00 \quad .4812 \mathrm{E}-04-.5482 \mathrm{E}+00 \quad .4396 \mathrm{E}+00$

Maximum Minimum Cmb. Shear

$\begin{array}{lll}.4557 \mathrm{E}+03 & .4537 \mathrm{E}+03 \quad .2278 E+03\end{array}$
$02 / 22 / 95$
$08: 50: 2^{3}$

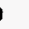


KAISER EHGINEERS HAMFORD S/N:801854

PAGE 91 Run ID=HN61973

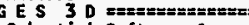
= Copyright (c) 1984 Celestial Software Inc.

SOLVE PLATE LOADS/STRESSES Version $2.0 \quad 07 / 01 / 90$

Load Case 2:Gravity LOAD

PLATE LOADS AMD/OR STRESSES

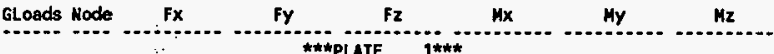
*t心PLATE

$\begin{array}{lllllllll}\text { GLoads } \quad 2 & .9833 E+02 & .2615 E+03 & -.7696 E-01 & -.1955 E+01 & .3168 E+00 & .0000 E+00\end{array}$

$3-.8562 E+02 \quad .2401 E+03-.3503 E+00-.2426 E+01 \quad .2563 E+00 \quad .0000 E+00$

$20-.6920 \mathrm{E}+02-.2606 \mathrm{E}+03 \quad .2576 \mathrm{E}+00-.2999 \mathrm{E}+01-.1103 \mathrm{E}+01 \quad .0000 \mathrm{E}+00$

GLoads $19.5648 E+02-.2410 E+03 \quad 1696 E+00-.2019 E+01-.7367 E+00 \quad .0000 E+00$

$\begin{array}{lllllllll}\text { GLoads } 5 & .8289 E+02 & .2378 E+03 & .2165 E+00 & .2426 E+01 & .9224 E+00 & .0000 E+00\end{array}$

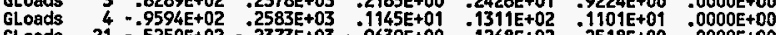

$\begin{array}{llllllll}\text { GLoads } & 21 & -.525 \mathrm{E}+02 & -.2373 \mathrm{E}+03 & -.9639 \mathrm{E}+00 & .1268 \mathrm{E}+02 & .2518 \mathrm{E}+00 & .0000 \mathrm{E}+00 \\ \text { GLoods } & 20 & .6554 \mathrm{E}+02 & -.2588 \mathrm{E}+03 & .3977 \mathrm{E}+00 & .1738 \mathrm{E}+01 & .2014 \mathrm{E}+00 & .0000 \mathrm{E}+00\end{array}$

$\begin{array}{llllllll}\text { GLoads } 4 & .6987 E+02 & .2278 E+03 & -.1179 E+09 & -.1311 E+02 & .3266 E+00 & .0000 E+00\end{array}$

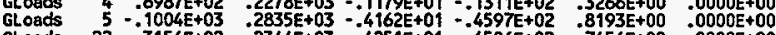

GLoads $22-.3154 \mathrm{E}+02-.2344 \mathrm{2}+03 \quad .4251 \mathrm{E}+01-.4506 \mathrm{E}+02 \quad .7454 \mathrm{E}+00 \quad .0000 \mathrm{E}+00$

GLoads

GLoads

GLoads

GLoeds

GLoads

GLoads

GLoads

GLoads

GLoads

GLoads

- Gloads

GLoods

GLoads

GLoeds

TH

GLoads

GLods

$21.6208 E+02-.2770 E+03 \quad 1090 E+01-.1337 E+02-.6705 E+00 \quad .0000 E+00$ $5=.6061 \mathrm{E}+01 \quad .3364 \mathrm{E}+03-.1155 \mathrm{E}+03 \quad .0000 \mathrm{E}+00-.6701 \mathrm{E}+00 \quad .6790 \mathrm{E}+02$ $6-.1795 \mathrm{E}+01.2400 \mathrm{E}+03 \quad .5363 \mathrm{E}+02 \quad .0000 \mathrm{E}+00 \quad .1916 \mathrm{E}-01 \quad .1804 \mathrm{E}+02$ $\begin{array}{lllllll}23 & .9578 E+01 & -.3279 E+03 & .7745+02 & -.1919 E+02 & -.6477 E+00 & .000 E+00 \\ -.2485 E+03 & .1549 E+02 & -.0000 E+00 & -.2065 E+01 & .670 E+02\end{array}$ $6 \quad .1548 E+01 \quad .3049 E+03-.1112 E+03 \quad .0000 E+00 \quad .6312 E-01-.1804 E+02$ $7 \quad .5131 E+00.2500 E+03 \quad .7299 E+02 \quad .0000 E+00 \quad .3603 E+00-.5170 E+01$ $24-.4077 E+00-.3043 E+03 \quad .8521 E+02 \quad .5173 E+011.8063 E+00 \quad .0000 E+00$ $23-.1654 \mathrm{E}+01-.2506 \mathrm{E}+03-.4696 \mathrm{E}+02 \quad .91696 \mathrm{E}+02 \quad . .4054 \mathrm{E}+00 \quad .0000 \mathrm{E}+00$ $7-.4444 E+00 \quad .2849 E+03-.01018 E+03 \quad .0000 E+00-.5035 E+00 \quad .5170 E+01$ $8-.3637 E+00.2689 E+03 \quad .9106 E+02 \quad .0000 E+00-.2697 E+00 \quad .3610 E+01$ $25 \quad .2614 E+00-.2841 E+03 \quad .8032 E+02-3989 E+01 \quad-.1479 E+00 \quad .0000 E+00$ $24 \quad .5467 E+00-.2696 E+03-.6959 E+02-.5009 E+01-.6646 E+00 \quad .0000 E+00$ $8 \quad .2774 E+00 \quad .2659 E+03-.8947 E+02 \quad .0000 E+00-.1632 E+00-.3610 E+01$ $9.7649 E+00 \quad .2841 E+03: 1027 E+03 \quad .0000 E+00-.6047 E-01-.8030 E+01$ $26-.7355 \mathrm{E}+00-.2654 \mathrm{E}+03 \quad .6846 \mathrm{E}+02 \quad .8050 \mathrm{E}+01 \quad .4125 \mathrm{E}+00 \quad .0000 \mathrm{E}+00$ $25=.3068 E+00-.2846 E+03-.8165 E+02 \quad .3241 E+01 \quad-.2669 E+00 \quad .0000 E+00$

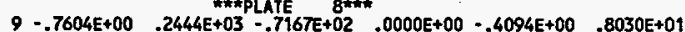
$10-2754 \mathrm{E}+012097 \mathrm{E}+03 \quad 1110 \mathrm{E}+03-000 \mathrm{E}+00-3065 \mathrm{E}+00 \quad 3090 \mathrm{E}+02$

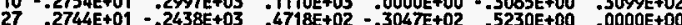

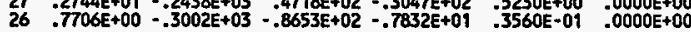

KAISER EMGINEERS HANFORD S/W:801854

PAGE 92

Run ID=MN61973

$02 / 22 / 95$

$=$ Copyright (c) 1984 Celestial software Inc. =

SOLVE PLATE LOADS/STRESSES Version 2.0 07/01/90

LOad Case 2:GRAVITY LOAD

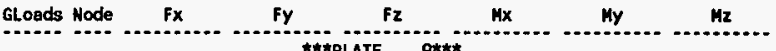

GLoads $10 \quad .2842 E+01 \quad .2370 E+03-.5675 E+02 \quad .0000 E+00-.4176 E+00-.3099 E+02$

$11.1033 E+02 \quad .3278 E+03 \quad .1144 E+0$

GLoads $28-.1042 E+02-.2460 E+03 \quad .22066+02 \quad .0300 E+02-.4044 E+00-.1134 E+03$

$27-.2746 E+01-.3188 E+03-.7966 E+02 \quad .3139 E+02-.2049 E+00 \quad .0000 E+00$

GLoads $11.1152 E+03 \quad .3223 E+03 \quad .7919 E+01 \quad .8818 E+02-.1066 E+09 \quad .0000 E+00$

GLoads $12-.5882 E+02 \quad .2320 E+03 \quad .2083 E+01 \quad .2242 E+02-.4008 E+00 \quad .0000 E+00$

GLoads $29-.8155 E+02-.3138 E+03 \quad-.2153 E+01 \quad .2231 E+02 \quad .8440 E+00 \quad .0000 E+00$

GLoads $28 \quad .2519 E+02-.2405 E+03=-7849 E+01 \quad .22314 E+02-.4377 E+00 \quad .0000 E+00$

GLoads $12 \quad .1137 E+03 \quad-2970 E+03-.2135 E+01 \quad-.2242 E+02-.6202 E+00 \quad-0000 E+00$

GLoads $13-.7305 E+02 \quad .2388 E+03-.5190 E+00-.7334 E+01-.4024 E+00 \quad .0000 E+00$

GLoads $30 \quad-.9049 \mathrm{E}+02 \quad-.2977 \mathrm{E}+03 \quad .5877 \mathrm{E}+00 \quad-.6575 \mathrm{E}+01 \quad .1484 \mathrm{E}+01$

(3)

GLoads $13.1084 E+03 \quad-2844 E+03 \quad .6341 E+00 \quad .7334 E+01 \quad-.1228 E+01 \quad .0000 E+00$

GLoads $14-.9071 E+02 \quad .2594 E+03 \quad .8864 E-01 \quad .2649 E+00-.1321 E+01 \quad .0000 E+00$

GLoads $30.7196 \mathrm{E}+02-.2587 \mathrm{E}+03 \quad-4831 \mathrm{E}+00 \quad .8099 \mathrm{E}+01: .1307 \mathrm{E}+00 \quad .0000 \mathrm{E}+00$

GLoads $14-1013 \mathrm{E}+03 \quad-2734 \mathrm{E}+03-.1721 \mathrm{E}+00-2649 \mathrm{E}+00-.4611 \mathrm{E}+00 \quad 000 \mathrm{E}+00$

GLoads $14-1013 E+03 \quad-2734 E+03-.1721 E+00-26492+00-.4691 E+00 \quad .0000 E+00$

GLods $15-1037 E 3$.27

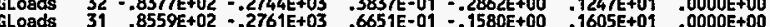

Gloads

GLoads 15 *k*PLATE 14*k*

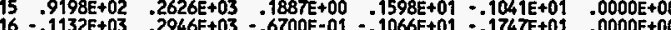

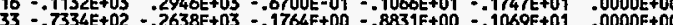
$32.9458 E+02-.2934 E+03 \quad-5474 E-01 \quad .3028 E+01 \quad .1663 E+00 \quad .0000 E+00$ $\begin{array}{llllllll} & .7827 E+02 & .2499 E+03 & -.3677 E-01 & .1066 E+01 & .3508 E+00 & .0000 E+00\end{array}$

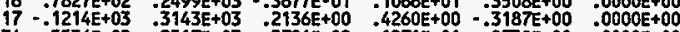
$34-.5536 \mathrm{E}+02-.2517 \mathrm{E}+03-.5721 \mathrm{E}-02 \quad .1271 \mathrm{E}+01 \quad .8738 \mathrm{E}+00 \quad .0000 \mathrm{E}+00$

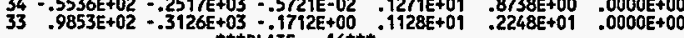

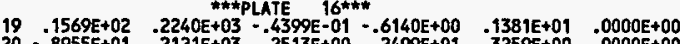

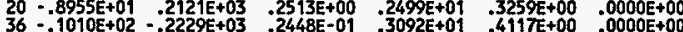

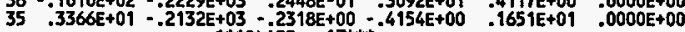
$20.1261 E+02.2222 E+03-1113 E+00-.1238 E+01 \quad .5753 E+00 \quad .0000 E+00$ 
KAISER ENGLNEERS HANFORD S/N:801854

PAGE 93 RUN ID=MN61975

$02 / 22 / 95$

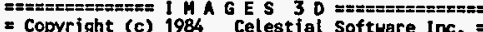

$=$ Copyright (c) 1984 Celestial Software Inc. =

SOLVE PLATE LOADS/STRESSES Version 2.0 07/01/90

L

Lad Case 2:GRAVITY LOAD

GLoads Hode Fx Fy

GLoads $21=.5621 E+01.2116 \mathrm{E}+03-.4196 \mathrm{E}+00-.4298 \mathrm{E}+01-8619 \mathrm{E}+00-0000 \mathrm{E}+00$

GLoads $37-.8152 E+01-.2229 E+03 \quad .4048 E+00-.5060 E+01-.3583 E+00 \quad 0000 E+00$

GLosds $36-1168 \mathrm{E}+01-.2110 \mathrm{E}+03 \quad .1261 \mathrm{E}+00=.1084 \mathrm{E}+01-.1281 \mathrm{E}+01 \quad .0000 \mathrm{E}+00$

$\begin{array}{llllllllll}\text { GLoads } 21-.3963 E+01 & .2176 E+03 & .2934 E+00 & .4987 E+01 & -.4433 E+00 & .0000 E+00\end{array}$

GLoads $22 \quad .1905 \mathrm{E}+02 \quad .1837 \mathrm{E}+03 \quad .1861 \mathrm{E}+01 \quad .2095 \mathrm{E}+02 \quad .2758 \mathrm{E}+01.0000 \mathrm{E}+00$

GLoads $38-.2611 E+01-.2080 E+03-.1571 E+01 \quad .1815 E+02 \quad .2357 E+01.0000 E+00$

GLoads $37-.1248 \mathrm{E}+02-.1933 \mathrm{E}+03-.5839 \mathrm{E}+00 \quad \cdot 3313 \mathrm{E}+01-.7014 \mathrm{E}+00 \quad .0000 \mathrm{E}+00$

$\begin{array}{lllllllll}\text { GLoeds } & 22 & .4976 E+01 & .2204 E+03 & .8618 E+01 & .0000 E+00 & -.1608 E+01 & -.5451 E+02\end{array}$

GLoads $23 \quad .1320 \mathrm{E}+01 \quad .2330 \mathrm{E}+03-.6465 \mathrm{E}+01 \quad-.1606 \mathrm{E}+02 \quad .8382 \mathrm{E}+00 \quad .0000 \mathrm{E}+00$

GLoads $39-.1329 \mathrm{E}+01-.2300 \mathrm{E}+03 \quad .1708 \mathrm{E}+01 \quad .1520 \mathrm{E}+02 \quad .1674 \mathrm{E}+01 \quad .0000 \mathrm{O}+00$

GLoods $38-.4967 \mathrm{E}+01-.2235 \mathrm{E}+03-3862 \mathrm{E}+01.0000 \mathrm{E}+00-.1043 \mathrm{E}+01-.5274 \mathrm{E}+02$

GLoads $23-.1244 E+01 \quad .2489 E+03-.2398 E+02-.1384 E+02-.6019 E+00 \quad .0000 E+00$

GLoads $24 \cdot .4546 E+00 \quad .2354 E+03 \quad .1585 E+02-.4014 E+01 \quad .3910 E+00 \quad .0000 E+00$

GLoads $40 \quad .2736 E+00-.2469 E+03 \quad .1884 E+02-.5179 E+01-.5036 E+00 \quad .0000 E+00$

GLoads $39.1426 E+01-.2374 E+03-1072 E+02-1435 E+02-.2091 E+01 \quad .0000 E+00$

$\begin{array}{lllllllll}\text { GLoads } & 24 & .3156 E+00 & .2420 E+03 & -.3148 E+02 & .3850 E+01 & -.5326 E+00 & .0000 E+00\end{array}$

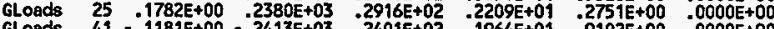

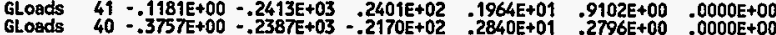

GLosds $40-.3757 E+00 \cdot .2387 E+03 \quad-2170 E+02 \quad .2840 E+01 \quad .2796 E+00 \quad .0000 E+00$

GLoads $25 \cdot-.1329 E+00 \quad .2342 E+03-.2783 E+02-.1461 E+01-.3941 E+00 \quad .0000 E+00$

GLoads $26-.3861 \mathrm{E}+00 . .2413 \mathrm{E}+03 \quad .3333 \mathrm{E}+02-.4108 \mathrm{E}+01 \quad .4477 \mathrm{E}-01 \quad .0000 \mathrm{E}+00$

.

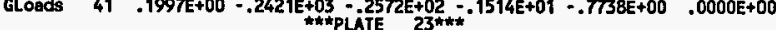

$\begin{array}{lllllllll}\text { GLos } & 26 & .3510 E+00 & .2278 E+03 & -.1526 E+02 & .3890 E+01 & -.4928 E+00 & .0000 E+00\end{array}$

$\begin{array}{llllllll}\text { GLosds } & 27 & -1266 \mathrm{E}+01 & .2443 \mathrm{E}+03 & .2624 \mathrm{E}+02 & .1382 \mathrm{E}+02 & .1486 \mathrm{E}+00 & .0000 \mathrm{E}+00\end{array}$

- GLosds $43-.1294 \mathrm{E}+01-.2287 \mathrm{E}+03 \quad .8974 \mathrm{E}+01$ : $1410 \mathrm{E}+02 \quad .1161 \mathrm{E}+00$.0000E+00

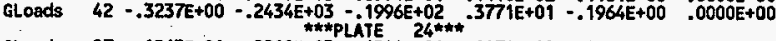

GLoads $27 \cdot .1265 E+01 \quad .2218 E+03 \quad .6244 E+01-.1474 E+02-.4667 E+00 \quad .0000 E+00$

GLoads $28-.4949 \mathrm{E}+01 \quad .2103 \mathrm{E}+03-.6455 \mathrm{E}+01 \quad .0000 \mathrm{E}+00=.9360 \mathrm{E}+00 \quad .5458 \mathrm{E}+02$

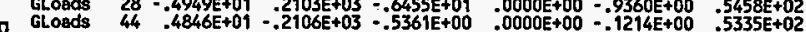

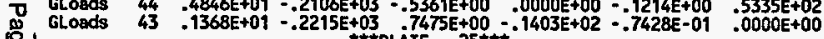

$\begin{array}{lllllllll} & 0 \\ 8\end{array}$

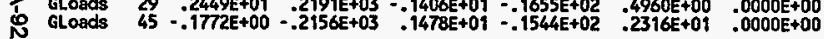

KAISER ENGINEERS HANFORD $S / \mathrm{W}: 801854$

Run ID=NH61973

= Copyright (c) $1984^{\circ}$ Celestial Software Inc,

SOLVE PLATE LOADS/STRESSES Version $2.0 \quad 07 / 01 / 90$

$\mathbf{L}$

Loed Case 2:GRAVITY LOAD

GLoads Hode Fx Fy Fy GLoads $44 \quad .2573 \mathrm{E}+01-.2081 \mathrm{E}+03 \quad .5294 \mathrm{E}+01 \quad-.5793 \mathrm{E}+02-.5075 \mathrm{E}+00 \quad .0000 \mathrm{E}+00$

GLoads $29.2925 E+02.2384 E+03 \quad .1492 E+01.1631 E+02-.1920 E+01 \quad .0000 E+00$

GLoads $30-.2087 \mathrm{E}+02 \quad .2255 \mathrm{E}+03 \quad .2506 \mathrm{E}+00 \quad .2144 \mathrm{E}+01-.4928 \mathrm{E}+00$

GLoads $46-.2301 E+02-.2376 E+03-.5002 E+00 \quad .2521 E+01 \quad .1265 E+00 \quad .0000 E+00$

GLoads $45.1464 E+02-.2262 E+03-.1243 E+01.1737 E+02-.1498 E+01 \quad .0000 E+00$

$\begin{array}{llllllll}\text { GLoads } & 30 & .3941 E+02 & .2365 E+03 & . .3552 E+00 & -3669 E+01 & -.1122 E+01 & .0000 E+00\end{array}$

GLoads $31-.3610 \mathrm{E}+02 \quad .2325 \mathrm{E}+03-.5159 \mathrm{E}-01-.2119 \mathrm{E}+01-1285 \mathrm{E}+01-000 \mathrm{O}+00$

GLoads $\quad 47-.3138 \mathrm{E}+02-.2373 \mathrm{E}+03 \quad .5139 \mathrm{E}-01=.2900 \mathrm{E}+00, .1268 \mathrm{E}+01 \quad .0000 \mathrm{E}+00$

GLoads $46.2807 \mathrm{E}+02-.2317 \mathrm{E}+03 \quad .3554 \mathrm{E}+00-.2872 \mathrm{E}+01: 1136 \mathrm{E}+01 \quad .0000 \mathrm{E}+00$

$\begin{array}{lllllllll}\text { GLoods } & 31 & .4017 E+02 & .2342 E+03 & .2247 E+00 & .2075 E+01 & -.4484 E+00 & .0000 E+00\end{array}$

GLoods $32-.4495 E+02 \quad .2421 E+03-.6981 E-01-.2069 E+01-.1672 E+01 \quad .0000 E+00$

GLosds $48-.3174 E+02-.2352 E+03-.1518 E+00-.8416 E+00-.1542 E+01 \quad .0000 E+00$

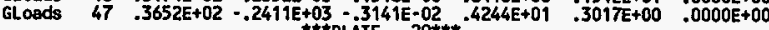

GLoads $32.3415 E+02.2313 E+03-.2330 E-01-.6726 E+00 \quad .2593 E+00 \quad .0000 E+00$

GLoads $33=.4675 E+02 \quad .2509 E+03 \quad .7158 E-01-.1254 E+01-.2119 E+01 \quad .0000 E+00$

GLoads $49-.2560 E+02-.2326 \mathrm{E}+03 \quad .4169 \mathrm{E}-01 \quad .2040 \mathrm{E}+01 \quad .5477 \mathrm{E}+00 \quad .0000 \mathrm{E}+00$

$\begin{array}{lllllllll}\text { GLoads } \quad 48 & .3821 \mathrm{E}+02 & -.2496 \mathrm{E}+03 & -.8996 \mathrm{E}-01 & .9493 \mathrm{E}+00 & .3030 \mathrm{E}+01 & .0000 \mathrm{E}+00\end{array}$

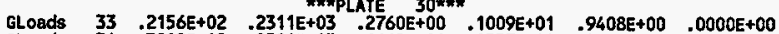

GLoads $34-.3902 E+02 \quad .2591 \mathrm{E}+03 \quad .6662 \mathrm{E}-01-.8557 \mathrm{E}+00-.2764 \mathrm{E}+01 \quad .0000 \mathrm{E}+00$

GLoads $50-.1394 \mathrm{E}+02-.2338 \mathrm{E}+03-.4633 \mathrm{E}+00 \quad .2445 \mathrm{E}+01-.4309 \mathrm{E}+01 \quad .0000 \mathrm{E}+00$

GLoads $49 \quad .3140 \mathrm{E}+02 \quad-.2564 \mathrm{E}+03 \quad .1207 \mathrm{E}+00 \quad .4939 \mathrm{E}+01 \quad .1165 \mathrm{E}+00 \quad .0000 \mathrm{E}+00$

GLoads $35-.8625 E+01 \quad .1743 E+03-.7110 E-01 \quad .2522 E+00 \quad .1952 E+01 \quad .0000 E+00$

$\begin{array}{lllllllll}\text { GLoads } & 36 & .1430 E+02 & .1670 E+03 & .1082 E+00 & .2383 E+00 & .5775 E+00 & .0000 E+00\end{array}$

GLoeds $52=.1683 E+01-.1761 E+03-.3591 E-01-.2507 E+00-.1626 E+01 \quad .0000 E+00$

GLoods $51-.3991 E+01-.1651 E+03-.1168 E-02 \quad .5758 E+00 \quad .8504 E-01 \quad .0000 E+00$

GLaads $36-.5366 E+01 \quad .1819 E+03-.2587 E+00-.2246 E+01 \quad .2921 E+00 \quad .0000 E+00$

GLoads $\quad 37-.1963 \mathrm{E}+02 \quad .1561 \mathrm{E}+03-.1530 \mathrm{E}+00-.1905 \mathrm{E}+01 \quad .1450 \mathrm{E}+01 \quad .0000 \mathrm{E}+00$

GLoads $53-.5083 E+01-.1790 E+03 \quad .5602 E+00=.1755 E+01 \quad .2314 E+01-.0000 E+00$

GLoads $\quad 52-.9178 E+01-.1589 \mathrm{E}+03-.1485 \mathrm{E}+00-3153 \mathrm{E}+01 \quad .1509 \mathrm{E}+01 \quad .0000 \mathrm{E}+00$

$\begin{array}{llllllll}\text { GLoads } & 38 & .1001 E+01 & .1750 E+03 & -3322 E+00 & .3652 E+01 & -.3903 E+00 & .0000 E+00\end{array}$

GLoads $54=.1171 E+02-1514 E+03 \quad .1093 E+01 \quad .1117 E+02 \quad .1750 E+01 \quad .0000 E+00$

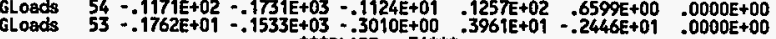

GLoads $38-.3915 E+01 \quad .1813 E+03 \quad .4441 E+01 \quad .0000 E+00-.9682 E+00 \quad .4276 E+02$ 
KAISER ENGINEERS HAHFORD S/H:801854

PAEE 95

$$
\text { Run 1D=MN61973 }
$$

$02 / 22 / 95$

x Copyright (c) 1984 Q E S

(c) igan Celestial Software Inc.

SOLVE PLATE LOADS/STRESSES Version 2.0 07/01/90

Lood Case 2:GRAvitr LOAD

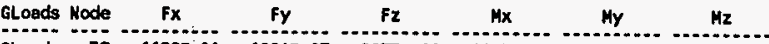

GLoads $39-.1129 \mathrm{E}+01 \quad .1884 \mathrm{E}+03 \quad-.9873 \mathrm{E}+00 \quad-.1187 \mathrm{E}+02 \quad .1730 \mathrm{E}+01 \quad .000 \mathrm{E}+00$

GLoads $55 \quad-1002 \mathrm{E}+01-.1835 \mathrm{E}+03 \quad-.2429 \mathrm{E}+01-.1306 \mathrm{E}+02 \quad-.1395 \mathrm{E}-01 \quad .0000 \mathrm{E}+00$

GLoads $54.4042 \mathrm{E}+01-.1862 \mathrm{E}+03=.1025 \mathrm{E}+01.0000 \mathrm{E}+00=.2717 \mathrm{E}+01 \quad .4326 \mathrm{E}+02$

$\begin{array}{lllllllll}\text { GLods } & 39 & .1032 E+01 & .1825 E+03 & .9996 E+01 & .1103 E+02 & -.1312 E+01 & .0000 E+00\end{array}$

$\begin{array}{llllllll}\text { GLoads } \quad 40 & -3033 \mathrm{E}+00 & -1989 \mathrm{E}+03 & .4079 \mathrm{E}+00 & .4360 \mathrm{E}+01 & .5911 \mathrm{E}+00 & .0000 \mathrm{E}+00\end{array}$

GLoads $56-.2009 E+00-.1841 E+03-.2518 E+01 \quad .3990 E+01 \quad .2092 E+01 \quad .0000 E+00$

$55-.1135 \mathrm{E}+01-.1973 \mathrm{E}+03-.7886 \mathrm{E}+01,01001 \mathrm{E}+02 \quad .2160 \mathrm{E}+00 \quad .0000 \mathrm{E}+00$

GLoads $\quad 40-.2011 E+00 \quad .1903 E+03 \quad .2451 E+01-.2022 E+01-.3671 E+00 \quad .0000 E+00$

GLoads $41-.1377 \mathrm{E}+00 \quad .1953 \mathrm{E}+03 \quad .1576 \mathrm{E}+01+.1125 \mathrm{E}+01 \quad .4979 \mathrm{E}+00 \quad .0000 \mathrm{E}+00$

GLoads $57-.1695 \mathrm{E}-02-.1896 \mathrm{E}+03 \quad .1816 \mathrm{E}+01-.2057 \mathrm{E}+01-.4326 \mathrm{E}+00 \quad .0000 \mathrm{E}+00$

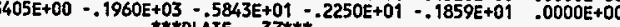

$\begin{array}{lllllllll}\text { GLoads } & 41 & .5608 \mathrm{E}-01 & .1915 \mathrm{E}+03 \quad .1294 \mathrm{E}+00 & .6749 \mathrm{E}+00 & -.6343 \mathrm{E}+00 & .0000 \mathrm{E}+00\end{array}$

GLoads $42-1213 \mathrm{E}+00 \quad .1890 \mathrm{E}+03-.1188 \mathrm{E}+01 \quad .1923 \mathrm{E}+01 \quad .2594 \mathrm{E}+00 \quad .0000 \mathrm{E}+00$

GLoeds $58-.1133 E+00-.1905 E+03 \quad .4105 E+01 \quad .1507 E+01 \quad .3460 E+00 \quad .0000 E+00$

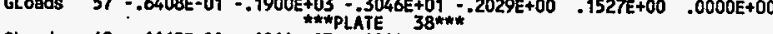

GLoads $42=.1169 E+00 \quad .1914 E+03 \quad .9282 E+00-.1361 E+01-.1508 E+00 \quad .0000 E+00$

GLoads $43-.5237 \mathrm{E}+00 \quad .1743 \mathrm{E}+03-.1052 \mathrm{E}+02-.5378 \mathrm{E}+01 \quad .3086 \mathrm{E}+00 \quad .0000 \mathrm{E}+00$

$\begin{array}{lllllllll}\text { GLoads } 59 & .5257 \mathrm{E}+00 & -.1879 E+03 & .7806 \mathrm{E}+01 & -.5695 E+01 & .3167 E+00 & .0000 E+00\end{array}$

GLoads $58 \cdot 1149 E+00-.1778 E+03 \quad 1790 E+01-.1658 E+01 \quad-.4435 E+00 \quad .0000 E+00$

$\begin{array}{llllllllll}\text { GLoads } 43 & -4496 E+00 & .1794 E+03 & .8033 E+00 & .5301 E+01 & -.3505 E+00 & .0000 E+00\end{array}$

GLoads $44 \quad .1864 E+01 \quad .1614 E+03+.1005 E+02 \quad .0000 E+00 \quad .6394 E+00-.2046 E+02$

GLoads $60-.1808 \mathrm{E}+01-.1745 \mathrm{E}+03 \quad .8155 \mathrm{E}+01 \quad .0000 \mathrm{E}+00 \quad .9503 \mathrm{E}+00-.2024 \mathrm{E}+02$

GLoads $59-.5060 \mathrm{E}+00=.1663 \mathrm{E}+03 \quad .090 \mathrm{E}+01 \quad .4914 \mathrm{E}+01-.3638 \mathrm{E}+00 \quad .0000 \mathrm{E}+00$

GLoods $44 \cdot-1149 E+02 \quad .1536 E+03 \quad .3667 E+01 \quad .4006 E+02 \cdot-3524 E+01 \quad .0000 E+00$

GLoads $45-.3856 \mathrm{E}+01: 1806 \mathrm{E}+03 \quad .6939 \mathrm{E}+00 \quad .9111 \mathrm{E}+01.1363 \mathrm{E}+01 \quad .0000 \mathrm{E}+00$

$61-3215 E+01-1583 E+03-1170 E+01$.

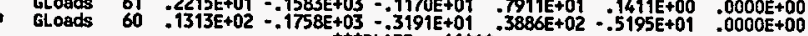

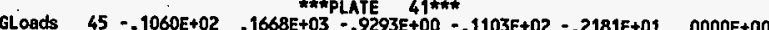

1935E+03-.2701E+00-.2972E+

$62 \quad 3405 \mathrm{E}+01$ - $1707 \mathrm{E}+03 \quad 2313 \mathrm{E}+00$ (.000E+00

0 GLoads $61 \quad .1229 \mathrm{E}+02-.1895 \mathrm{E}+03 \quad .9683 \mathrm{E}+00 \quad-1110 \mathrm{E}+02-.1833 \mathrm{E}+00 \quad .0000 \mathrm{E}+00$

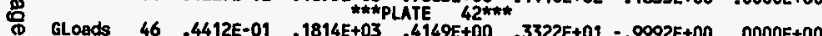

GLoads $46-.4412 E-01 \quad .1814 E+03 \quad .4149 E+00 \quad-3322 E+01-.9992 E+00 \quad .0000 E+00$

6 GLoads $63-.2995 \mathrm{E}+01-.1826 \mathrm{E}+03-.2343 \mathrm{E}+00=.6234 \mathrm{E}-01-.1421 \mathrm{E}+01 \quad .000 \mathrm{E}+000$
KAISER ENGINEERS HANFORD $\$$ /N:801854

Run ID=MN61973

$02 / 22 / 95$

$08: 50: 28$

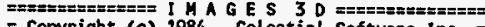
= Copyright (c) 1984 Celestial Software Inc. I

SOLVE PLATE LOADS/STRESSES Version 2.0 07/01/90

Load Case 2:GRAVITY LOAD

Gloads Node Fx $\quad$ Fy $\quad$ Fz $\quad$ Mx $\quad$ Hy $\quad$ Hz GLoads $62 \quad .1243 E+02-.1951 E+03-.7557 E-01 \quad .5814 E+01-.1813 E+01 \quad .0000 E+00$

GLoads $47.4339 E+01.1878 E+03 \quad .5675 E-01=.1698 E+01=.6574 E+00 \quad .0000 E+00$

GLoads $\quad 48-1027 \mathrm{E}+02 \quad .1973 \mathrm{E}+03 \quad .4985 \mathrm{E}-01-.6668 \mathrm{EE}+00-.3232 \mathrm{E}+01.0000 \mathrm{E}+00$

GLoads $64-.5736 E+01-.1889 E+03-.2227 E+00 \quad .3456 E+01-.2928 E+00 \quad .0000 E+00$

GLoads $63.1166 \mathrm{E}+02-.1964 \mathrm{E}+03 \quad .1161 \mathrm{E}+00 \quad \cdot 1254 \mathrm{E}+01 \quad .1560 \mathrm{E}+01 \quad .0000 \mathrm{E}+00$

$\begin{array}{lllllllll}\text { GLoads } \quad 48 & .3797 E+01 & .1928 E+03 & .1919 E+00 & .5592 E+00 & .1743 E+01 & .0000 E+00\end{array}$

GLoads $\quad 49-.6529 E+01: 1975 E+03-.1384 E+00-.3912 E+01-.2609 E+01 \quad .0000 E+00$

GLoads $\quad 65-.5591 E+01-.1935 E+03-.1975 E+00-.6927 E+00-.5151 E+01 \quad .0000 E+00$

GLoads $64 \quad .8323 \mathrm{E}+01-.1967 \mathrm{E}+03 \quad 1440 \mathrm{E}+00 \quad .5224 \mathrm{E}+01 \quad .9229 \mathrm{E}+00 \quad .0000 \mathrm{E}+00$

GLoads $49.7347 E+00 \quad .1971 E+03-.2401 E-01-.3066 E+01 \quad .1945 E+01 \quad .0000 E+00$

$\begin{array}{llllllll}\text { GLoads } \quad 50 & .3571 E+01 & .1894 E+03 & .1353 E+00 & .9516 E-01 & -.3500 E+01 & .0000 E+00\end{array}$

GLoads $66-.6832 E+01-.1957 E+03 \quad .1632 E+00 \quad .5115 E+01 \quad .1067 E+00 \quad .0000 E+00$

GLoads $65.2527 \mathrm{E}+01-.1908 E+03-.2744 \mathrm{E}+00.3039 \mathrm{E}+00 \quad .5975 \mathrm{E}+01 \quad .0000 \mathrm{E}+00$

GLoads $51 \quad .2216 E+00 \quad .1302 E+03-.7403 E-01-.2118 E+01 \quad .1409 E+01 \quad .0000 E+00$

GLoads $52 \quad .9130 E+01 \quad .1156 \mathrm{E}+03-.9715 \mathrm{E}-01-.1218 \mathrm{E}+01-.1676 \mathrm{E}+01 \quad .0000 \mathrm{E}+00$

GLoads $68-.9675 \mathrm{E}+01-.1306 \mathrm{E}+03 \quad .2647 \mathrm{E}+00 \quad .5363 \mathrm{E}+0.00-.5453 \mathrm{E}+00 \quad .0000 \mathrm{E}+00$

GLoods $67 \quad .3237 E+00-.1151 E+03-.9357 \mathrm{E}-01-.9658 \mathrm{E}+00 \quad .3103 \mathrm{E}+01 \quad .0000 \mathrm{O}+00$

$\begin{array}{llllllllll}\text { GLoads } & 52 & .1732 E+01 & .1343 E+03 & .2815 E+00 & .4622 E+01 & .1794 E+01 & .0000 E+00\end{array}$

GLoads $53 \quad .9084 \mathrm{E}+01 \quad .1189 \mathrm{E}+03 \quad .1233 \mathrm{E}+01 \quad .1169 \mathrm{E}+02 \quad .1894 \mathrm{E}+01 \quad .0000 \mathrm{E}+00$

GLoads $69-.1011 E+02-.1363 E+03-.1158 E+01 \quad .1141 E+02-.1360 E+01 \quad .0000 E+00$

GLoads $68+.7051 E+00-.1169 E+03-.3570 E+00 \quad .5605 E+01-.1287 E+01 \quad .0000 E+00$

GLoads $\quad 53-.2239 E+01 \quad .1284 E+03-.1493 E+01-.1390 E+02-.1752 E+01 \quad .0000 E+00$

GLoads $54 \quad .2573 E+01 \quad . \$ 270 E+03-.4017 E+01-.4387 E+02 \quad .4218 E+01 \quad .0000 E+00$

GLoads $70 \quad .3171 E+01-.1275 E+03 \quad .4724 E+01-.4750 E+02 \quad .6866 E+01 \quad .0000 E+00$

GLoads $69-.3505 E+01-.1278 E+03 \quad .7857 E+00=.1595 E+02 \quad .3290 E+00 \quad .0000 E+00$

$\begin{array}{lllllllll}\text { GLoads } & 54 & .4208 E+01 & .1368 E+03 & .6810 E+01 & .0000 E+00 & -.2215 E+01 & -.4556 E+02\end{array}$

GLoads $55.1176 \mathrm{E}+01.1493 \mathrm{E}+03 \quad .2943 \mathrm{E}+01 \quad .1384 \mathrm{E}+02 \quad .7262 \mathrm{E}+00 \quad .0000 \mathrm{E}+00$

GLoads $\quad 71-.1090 E+01-.1354 E+03 \quad .7744 E+01 \quad .1357 E+02 \quad .3270 E+01 \quad .0000 E+00$

GLoads $70-.4294 \mathrm{E}+01-.1506 \mathrm{E}+03-.1750 \mathrm{0}+02,0000 \mathrm{E}+00-.4512 \mathrm{E}+00-.4548 \mathrm{E}+02$

GLoads $55-.1043 E+01 \quad .1349 E+03 \quad .7374 E+01-.1079 E+02-.9282 E+00 \quad .0000 E+00$

GLoads $56-.4105 \mathrm{E}+00 \quad .1459 \mathrm{E}+03 \quad .1251 \mathrm{E}+00-.4189 \mathrm{E}+01 \quad .9418 \mathrm{E}+00 \quad .0000 \mathrm{E}+00$

Gloads $72 \quad .9038 E-01-.1353 \mathrm{E}+03 \quad .2492 \mathrm{E}+00-.5832 \mathrm{E}+01-.1646 \mathrm{E}+01 \quad .0000 \mathrm{E}+00$

GLoads $71.1363 \mathrm{E}+01-.1456 \mathrm{E}+03-.7745 \mathrm{E}+01-.1117 \mathrm{E}+02-.3330 \mathrm{E}+01 \quad .0000 \mathrm{E}+00$

GLaads $56.2709 E+00 \quad .1377 E+03 \quad .8237 E+01.2449 E+01+.1173 E+01 \quad .0000 E+00$ 
KAISER ENGINEERS HANFORD S/N:801854

PAGE 97 Run ID=WN61973

$02 / 22 / 95$

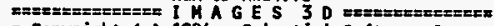
c Copyright (c) 1984 Celestial Sof tware Inc. =

SOLVE PLATE LOADS/STRESSES Version 2.0 07/01/90 L

LoAd Case 2:GRAVITY LOAD

GLoads Hode Fx Fy GLoads $57 \quad .9948 E-01 \quad .1426 E+03-.4664 E+01$. $5570 E+01-.4072 E-01$.0000E+00 GLoads $73=.8671 E-01-.1375 E+03-.3695 E+00 \quad .2132 E+01 \quad .6572 E+00 \quad .0000 E+00$ GLoads $72-.2837 \mathrm{E}+00-.1428 \mathrm{E}+03-.3203 \mathrm{E}+01 \quad .9977 \mathrm{E}+00 \quad .7570 \mathrm{E}+00 \quad .0000 \mathrm{E}+00$

GLoads $57-.3371 E-01.1405 E+03 \quad .5895 E+01-.3096 E+00 \quad .3205 E+00 \quad .0000 E+00$ GLoads $58-.2123 \mathrm{E}+00 \quad .1352 \mathrm{E}+03-.8776 \mathrm{E}+01-.2167 \mathrm{E}+01 \quad .4650 \mathrm{E}+00 \quad .0000 \mathrm{E}+00$ $.0000 \mathrm{E}+00$

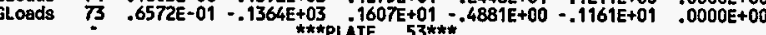
$\begin{array}{llllllllll}\text { GLoads } & 58 & .2107 E+00 & .1366 E+03 & .2881 E+01 & .2319 E+01 & -.3674 E+00 & .0000 E+00\end{array}$ GLoads $59.8694 \mathrm{E}+00 \quad .1277 \mathrm{E}+03-.7252 \mathrm{E}+07 \quad .9912 \mathrm{E}+01 \quad .4492 \mathrm{E}+00 \quad .0000 \mathrm{E}+00$ GLoads $75=.9038 E+00-.1339 E+03 \quad .1909 E+01 \quad .9553 E+01-.2003 E-01 \quad .0000 E+00$ $764 E+00=.1304 E+03 \quad .2462 E+01.1980 E+01-.5939 E+00 \quad .0000 E+00$ GLoads $59+.8891 E+00 \quad .1300 E+03-.1644 E+01-.9131 E+01-.4021 E+00 \quad .0000 E+00$ GLoads $\quad 60-.3193 E+01 \quad .1273 E+03 \quad .1176 E+00 \quad .0000 E+00 \quad .1244 E+01 \quad .3508 E+02$ gLads $76 \quad .3259 \mathrm{E}+01-.1295 \mathrm{E}+03 \quad .7800 \mathrm{0}+01 \quad .0000 \mathrm{0}+00 \quad .8722 \mathrm{0}+00$ $.8236 E+00+.1279 E+03-.6273 E+01-.9741 E+01-.6991 E+00 \quad .0000 E+00$ GLoads $\quad 60-.6453 E+01 \quad .1189 E+03-.3704 E+01-.4263 E+02 \cdot .3787 E+01 \quad .0000 E+00$ GLoads $61=.3179 E+01: 1346 \mathrm{E}+03=.1022 \mathrm{E}+01=.8893 \mathrm{E}+01 \quad .1404 \mathrm{E}+01 \quad .0000 \mathrm{E}+00$

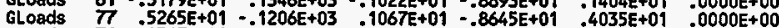

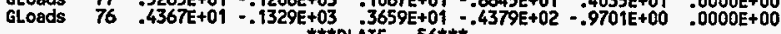
GLoads $61-.1133 E+02-1188 E+03-1223 E+01-1209 E+02-.1361 E+01 \quad 0000 E+00$ GLoads $62-.5467 \mathrm{E}+01 \quad-1446 \mathrm{E}+03 \quad .6776 \mathrm{E}-01 \quad .1402 \mathrm{E}+01 \quad .1060 \mathrm{E}+01 \quad .0000 \mathrm{E}+00$ GLoads $77.1196 \mathrm{E}+02-.1432 \mathrm{E}+03-.7976 \mathrm{E}+00 \quad .1312 \mathrm{E}+02-.4254 \mathrm{E}+01 \quad .000 \mathrm{EE}+00$ GLoads $62-.1037 E+02 \quad .1268 E+03-.2233 E+00-.5938 E+01-.1284 E+01 \quad .0000 E+00$ GLoads $63-.1621 \mathrm{E}+01 \quad .1462 \mathrm{E}+03-.1204 \mathrm{E}+00-.3608 \mathrm{E}+00-.1081 \mathrm{E}+01 \quad .0000 \mathrm{E}+00$ GLoads $79 \quad .2638 \mathrm{E}+01-.1288 \mathrm{E}+03-.4570 \mathrm{E}-02 \quad .3303 \mathrm{E}+01 \quad .9976 \mathrm{E}-01 \quad .0000 \mathrm{E}+00$

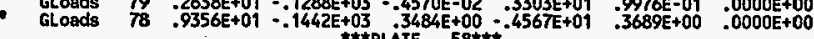
GLoads $63-.7047 E+01 \quad .1384 E+03 \quad .2386 E+00-.8309 E+00 \quad .9419 E+00 \quad 0000 E+00$

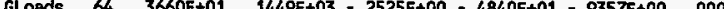
GLoeds 80 2934E+00 - 1400E+03 - $3394 \mathrm{E}-01$ 1233E+01 - $3350 \mathrm{E}+01$.000E+00 O Gloods $79.3094 \mathrm{E}+01-.1433 \mathrm{E}+03 \quad .4786 \mathrm{E}-01 \quad .4133 \mathrm{E}+01-.1501 \mathrm{E}+01 \quad .0000 \mathrm{E}+00$ GLoads GLoads $64-.6247 E+01 \quad .1463 E+03 \quad .3313 E+00-.3839 E+01 \quad .3056 E+00 \quad .0000 E+00$ $65.8950 \mathrm{E}+01 \quad .1432 \mathrm{E}+03 \quad .1275 \mathrm{E}+00 \quad .3197 \mathrm{E}+00-.6902 \mathrm{E}+01 \quad .0000 \mathrm{E}+00$ $81.6688 \mathrm{E}+00-.1471 \mathrm{E}+03-.5816 \mathrm{E}+00 \quad .9734 \mathrm{E}+01-.2233 \mathrm{E}+01 \quad .0000 \mathrm{E}+00$
KAISER ENGINEERS HANFORD S/N:801854

PAGE 98 RUN ID=NH61973 $02 / 22 / 95$ $=$ Copyright (c) 1984 Celestial Software Inc.

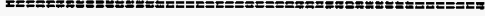

SOLVE PLATE LOADS/STRESSES Version $2.0 \quad 07 / 01 / 90$ L

Load Case 2:GRAVITY LOAD

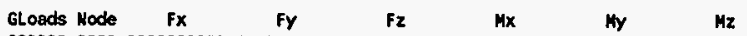
GLoads $80-.3372 E+01-.1424 E+03 \quad .1228 E+00 \quad .3879 E+01 \quad .1942 E+01 \quad .0000 E+60$ GLoads $65-.5886 E+01.1468 E+03 \quad .3444 E+00.6901 E-01 \quad .6078 E+01 \quad .0000 E+00$ GLoads $66 \quad .9071 E+01 \quad .1409 E+03-.4418 \mathrm{E}-01-.6310 \mathrm{E}+01-.4927 \mathrm{E}+01-0000 \mathrm{E}+00$ GLoads $82 \quad .2629 E+01-.1455 E+03-.5215 E+00 \quad .2060 E+01-.1225 E+02 \quad .0000 E+00$ GLoads $81-.5814 \mathrm{E}+01-.1421 E+03 \quad .2213 \mathrm{E}+00 \quad \cdot 1079 \mathrm{E}+02 \quad .2521 \mathrm{E}+01 \quad .0000 \mathrm{E}+00$ GLoeds $67 \cdot .5400 E+01 \quad .7435 E+02 \quad .4224 E-01 \quad .7428 E+01 \quad .8711 E+01 \quad .0000 E+00$ GLoads $68 \quad .4728 \mathrm{E}+01 \quad .7407 \mathrm{E}+02 \quad .1102 \mathrm{E}+01 \quad .4833 \mathrm{E}+01 \quad .5804 \mathrm{E}+00 \quad .0000 \mathrm{E}+00$ GLoads $84-.8437 \mathrm{E}+00 \quad-.7272 \mathrm{E}+02 \quad .2923 \mathrm{E}+00 \quad .7977 \mathrm{E}+01 \quad-.4132 \mathrm{E}+00 \quad .0000 \mathrm{E}+00$ GLoads $83 \quad .1516 E+01 \cdot .7570 E+02-1436 \mathrm{E}+01 \quad \cdot 1122 \mathrm{E}+02 \quad .1018 \mathrm{E}+02 \quad .0000 \mathrm{E}+00$

GLoads $68 \quad .5652 E+01.7776 E+02=1010 E+01-.1097 E+02 \quad .1252 E+01 \quad .0000 E+00$ GLoads $69-.1011 \mathrm{E}+01 \quad .6750 \mathrm{E}+02-.1400 \mathrm{E}+01-.2383 \mathrm{E}+02 \quad .2554 \mathrm{E}+01 \quad .0000 \mathrm{E}+00$ GLoads $85-.2522 E+02-.7684 E+02 \quad .2866 E+01-.2180 E+02 \quad .1032 E+02 \quad .0000 E+00$ GLo8ds $84.2058 E+02 \cdot .6842 E+02-.4573 E+00-.9647 E+01 \quad .5925 E+01 \quad .0000 E+00$

GLoads $69.1463 \mathrm{E}+02.1009 \mathrm{E}+03.1772 \mathrm{E}+01.2837 \mathrm{E}+02-.1523 \mathrm{E}+01 \quad .0000 \mathrm{E}+00$

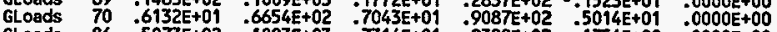
GLoods $86-.5073 E+02-.1083 E+03-.7314 E+01 \quad .9329 E+02 \quad .1754 E+00 \quad .0000 E+00$ $\begin{array}{lllllll} & .0000 E+00\end{array}$

GLoads $70-.4213 E+01 \quad .1029 E+03.4623 E+01.0000 E+00-.3982 E+01.5898 E+02$ GLoads $\quad 71-.1519 \mathrm{E}+01 \quad .8768 \mathrm{E}+02-.1102 \mathrm{E}+02-.1792 \mathrm{E}+02 \quad .7318 \mathrm{E}+00 \quad .0000 \mathrm{O}+000$ GLoads $87.4556 \mathrm{E}+00-.9904 \mathrm{E}+02-.2100 \mathrm{E}+02-.1977 \mathrm{E}+02-.5299 \mathrm{E}+01 \quad .0000 \mathrm{0}+00$

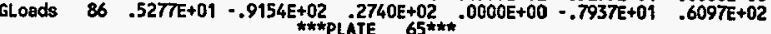

GLoads $71.1246 \mathrm{E}+01.8476 \mathrm{E}+02.1102 \mathrm{E}+02.1551 \mathrm{E}+02-.6714 \mathrm{E}+00.0000 \mathrm{E}+00$ GLoads $72 \quad .4399 \mathrm{E}+00 \quad .8835 \mathrm{E}+02-.7301 \mathrm{E}+01 \quad .7353 \mathrm{E}+01-.5829 \mathrm{E}+00 \quad .0000 \mathrm{E}+00$ GLoads $88-1063 E+00-.8174 E+02-.6034 E+01 \quad .8755 E+01 \quad .4110 E+01 \quad .0000 E+00$ $87-.1792 \mathrm{E}+01-.9137 \mathrm{E}+02 \quad .2311 \mathrm{E}+01,01475 \mathrm{E}+02 \quad .5610 \mathrm{E}+01 \quad .0000 \mathrm{E}+00$

GLoads $72-.2466 E+00 \quad .8113 E+02 \quad .1026 E+02 \div .2519 E+01 \quad .1471 E+01 \quad .0000 E+00$ GLoads $73-.6362 E-01 \quad .8017 E+02-.1019 E+02-.2637 E+01 \quad .9412 E+00 \quad .0000 E+00$ GLoads $89 \quad .7354 \mathrm{E}-01-.8006 \mathrm{E}+02-.4141 \mathrm{E}+00-.2224 \mathrm{E}+01-.3002 \mathrm{E}+00 \quad .0000 \mathrm{E}+00$ Gloads $88 \quad .2367 \mathrm{E}+00-.8125 \mathrm{E}+02 \quad .3491 \mathrm{E}+00=.1151 \mathrm{E}+01-.1959 \mathrm{E}+01 \quad .0000 \mathrm{E}+00$

$\begin{array}{llllllllll}\text { GLoads } & 73 & .8461 E-01 & .8518 E+02 & .8952 E+01 & .9928 E+00 & -.4378 E+00 & .0000 E+00\end{array}$ $\begin{array}{llllllll}\text { GLoads } & 73 & .8461 E-01 & .8518 E+02 & .8952 E+01 & .9928 E+00 & -.4378 E+00 & .0000 E+00 \\ \text { GLoads } & 74 & .4571 E+00 & .7396 E+02 & -.1560 E+02 & .6320 E+01 & .5207 \mathrm{E}+00 & .0000 \mathrm{E}+00\end{array}$

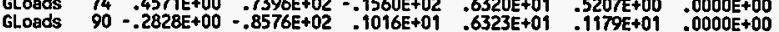

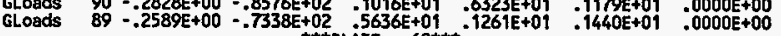
$\begin{array}{llllllll}\text { GLoads } 74-.4610 E+00 & .8710 E+02 \quad .1187 E+02-.5852 E+01 & .1943 E+00 & .0000 E+00\end{array}$ 
KAISER ENGINEERS HANFORD S/N:801854 Run ID=NN61973

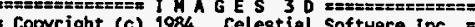
- Copyright (c) 1984 Celestial Software Inc. $=$

SOLVE PLATE LOADS/STRESSES Version $2.0 \quad 07 / 01 / 90$

Load Case 2:GRAVITY LOAD

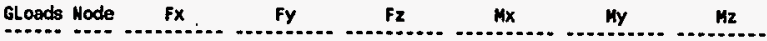
GLoads $75-.1602 \mathrm{E}+01 \quad .7680 \mathrm{E}+02-.1856 \mathrm{E}+02-.2244 \mathrm{E}+02 \quad .8166 \mathrm{E}+00 \quad .0000 \mathrm{E}+00$

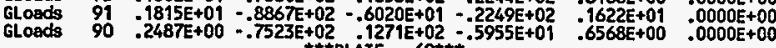
GLoods $75 \quad .1682 E+01 \quad .7636 \mathrm{E}+02 \quad .2292 \mathrm{E}+02 \quad .2263 \mathrm{E}+02-.9745 \mathrm{E}-01 \quad .0000 \mathrm{E}+00$ $\begin{array}{llllllll}\text { GLoods } & 75 & -1682 E+01 & .7636 \mathrm{E}+02 & .2292 \mathrm{E}+02 & -2263 \mathrm{E}+02 & -.9745 \mathrm{E}-01 & .0000 \mathrm{E}+00 \\ \text { GLoads } & 76 & -6265 \mathrm{E}+01 & .8543 \mathrm{E}+02 & -.1738 \mathrm{E}+02 & .0000 \mathrm{E}+00 & .7131 \mathrm{E}+00 & -.8607 \mathrm{E}+02\end{array}$

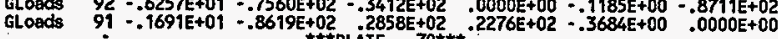

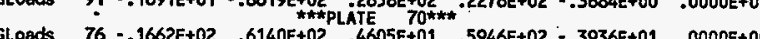
GLoads $76-.1662 E+02 \quad .6140 E+02 \quad .4605 E+01 \quad .5946 E+02-.3936 E+01 \quad .0000 E+00$ GLoods $77 \cdot .4078 \mathrm{E}+01 \quad .9628 \mathrm{E}+02 \quad .7336 \mathrm{E}+00 \quad-1476 \mathrm{E}+02 \quad .1245 \mathrm{E}+01 \quad .0000 \mathrm{E}+00$

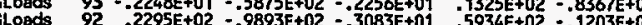

GLoads

GLoads GLoads

GLoeds

GLoads GLoads GLoads GLoads

GLards

GLoads

GLoads

GLoads

GLoads

GLoads

- Gloads -2295E+02 $-.9893 \mathrm{E}+02-30 \mathrm{3033E+01}$

$77-.1315 \mathrm{E}+02 \quad .6134 \mathrm{E}+02-.1003 \mathrm{E}+01-.1923 \mathrm{E}+02-.1026 \mathrm{E}+01 \quad .0000 \mathrm{E}+00$

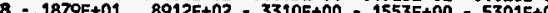
$94-6340 \mathrm{E}+00-6187 \mathrm{E}+02 \quad .3430 \mathrm{E}+00-2333 \mathrm{E}+01-5301 \mathrm{E}+00 \quad-0000 \mathrm{O}+00$ $93.1439 \mathrm{E}+02-.8859 \mathrm{E}+02 \quad .8909 \mathrm{E}+00 \quad-.1963 \mathrm{E}+02 \quad .2887 \mathrm{E}+01 \quad .0000 \mathrm{E}+00$ - $12315+02$ 6910E+02

$\begin{array}{lllllll}78 & -.1231 \mathrm{E}+02 & .6910 \mathrm{E}+02 & .4760 \mathrm{E}+00 & .2925 \mathrm{E}+01 & .2061 \mathrm{E}+01 & .0000 \mathrm{E}+00\end{array}$ $79-4251 \mathrm{E}+01 \quad .8472 \mathrm{E}+02-.5241 \mathrm{E}-01 \quad .4845 \mathrm{E}-02 \quad .1458 \mathrm{E}+01 \quad .0000 \mathrm{E}+00$ $94.039 E+01-.7010 E+02-.2983 E+00 \quad .2998 E+01-.4493 E+01 \quad .0000 E+00$ * $79-.9983 \mathrm{E}+01 \quad .8111 \mathrm{E}+02 \quad .9119 \mathrm{E}-02-.7440 \mathrm{E}+01-.5679 \mathrm{E}-01 \quad .0000 \mathrm{E}+00$ $96-.2309 \mathrm{E}+01-.8277 \mathrm{E}+02-.1538 \mathrm{E}+000.8228 \mathrm{E}+01-1235 \mathrm{E}+01.0000 \mathrm{E}+00$ $95.9009 \mathrm{E}+00-7856 \mathrm{E}+02 \quad 1517 \mathrm{E}+00-4178 \mathrm{E}+01-5525 \mathrm{E}+000.000 \mathrm{E}+00$ $80-.8313 E+01.9596 E+02-8185 E-01-8559 E+01 \quad 1502 E+01-0000 E+00$ $81.1952 \mathrm{E}+02-7879 \mathrm{E}+02-1263 \mathrm{E}+01-.1682 \mathrm{E}+02-.5431 \mathrm{E}+00-0000 \mathrm{E}+00$ $97-.529 E+01-9911 E+02-5543 E+00-8647+01-7068 E+01-0000 E+00$ $96-.6677 \mathrm{E}+01-.7564 \mathrm{E}+02 \quad 7906 \mathrm{E}+00-.2959 \mathrm{E}+01-.4640 \mathrm{E}+01-0000 \mathrm{E}+00$ Mt:TLATE $75^{* * *}$

GLoads $81=.1437 E+02.1042 E+03 \quad .1623 E+01-.3702 E+01 \quad .2553 E+00 \quad .0000 E+00$ GLoads 82 - $2552 \mathrm{E}+02 \quad 8746 \mathrm{E}+02 \quad 1175 \mathrm{E}+01 \quad 2847 \mathrm{E}+02-1944 \mathrm{E}+02$ GLoads $98 \quad .7015 E+01-.1077 E+03-.3444 E+01 \quad .4463 E+02=.1449 E+02 \quad .0000 E+00$ GLoads $97-.1816 \mathrm{E}+02-.8400 \mathrm{E}+02 \quad .6455 \mathrm{E}+00 \quad .7869 \mathrm{E}+01-.7418 \mathrm{E}+00 \quad .0000 \mathrm{E}+00$ GLoods $83-.1310 E+02 \quad 2971 E+02-1551 E+00-.2577 E+01 \quad 2151 E+01 \quad 0000 E+00$ GLoads $84-.6218 E+09 \quad .4716 E+02-.3716 E+01-.3496 E+02-.6184 E+09-0000 E+00$ GLoods $100 \quad .8044 \mathrm{E}+01-.2384 \mathrm{E}+02 \quad .3433 \mathrm{E}+01-.3907 \mathrm{E}+02-.2497 \mathrm{E}+01 \quad .0000 \mathrm{E}+00$
KAISER EHGINEERS HANFORD S/M:801854

PAGE 100 Run ID=NN61973 $02 / 22 / 95$
$08: 50: 32$

in Copyright (c) 1984 Celestial Software Inc.

SOLVE PLATE LOADS/STRESSES Version 2.0 07/01/90

Load Case 2:GRAVITY LOAD

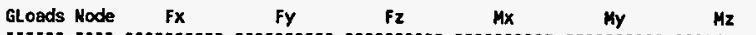

GLoads $99 \quad .1128 E+02-.5304 \mathrm{E}+02 \quad .4378 \mathrm{E}+00.4737 \mathrm{E}+01 \quad .6655 \mathrm{E}+00 \quad .0000 \mathrm{E}+00$

GLoads $84=.1352 E+02.8886 E+01.3881 E+01.3663 E+02-.1328 E+01 \quad .0000 E+00$

GLoads $85-.8880 E+01 \quad .3011 E+02 \quad .1617 \mathrm{E}+02 \quad .1314 \mathrm{E}+03 \quad .2810 \mathrm{E}+01 \quad .0000 \mathrm{O}+00$

GLoads $100.3277 E+00-.3592 E+02+3533 E+01.3582 E+02-.3992 E+01 \quad .0000 E+00$

GLoads $85 \quad .4122 E+01 \quad .2077 E+02=.1754 E+02-.1395 E+03-.5747 E+01 \quad .0000 E+00$

GLoads $86-.6419 \mathrm{E}+01 \quad .3450 \mathrm{E}+02-.6283 \mathrm{E}+02 \quad-.5119 \mathrm{E}+03 \quad .2524 \mathrm{E}+01 \quad .0000 \mathrm{E}+00$

GLoads $102 \quad .1617 \mathrm{E}+02 \quad-.3172 \mathrm{E}+02 \quad .6348 \mathrm{E}+02-.5263 \mathrm{E}+03 \quad .8798 \mathrm{E}+01 \quad .0000 \mathrm{E}+00$

GLoads $101-.1387 \mathrm{E}+02-.2355 \mathrm{E}+02 \quad 1689 \mathrm{E}+02-.1484 \mathrm{E}+03 \quad .3331 \mathrm{E}+01 \quad .0000 \mathrm{E}+00$

$\begin{array}{lllllllll}\text { GLoads } & 86 & .6819 E+02 & .2980 E+02 & .3367 E+02 & .0000 E+00 & .2831 E+00 & -.5561 E+03\end{array}$

GLoads $87.1898 \mathrm{E}+02 \quad .6419 \mathrm{E}+02 \quad .4626 \mathrm{E}+00 \quad .1525 \mathrm{E}+03-.2694 \mathrm{E}+01 \quad .0000 \mathrm{E}+00$

GLoads $103-.1834 E+02-.2785 E+02-.1050 E+02 \quad .0000 E+00 \quad .4961 E+01-.1615 E+03$

GLoads $102-.6863 E+02-.0614 E+02-.2363 E+02,0000 E+00 \quad .7313 E+01-.5682 E+03$

GLoads $\quad 87=.1764 E+02 \quad .2972 E+02 \quad .1823 E+02-.1475 E+03 \quad .2383 E+01 \quad .0000 E+00$

GLoads $88-.4906 E+01 \quad .3918 E+02-.3950 E+01-.4361 E+02 \quad .6486 E-01 \quad .0000 E+00$

GLoads $104 \quad .4493 \mathrm{E}+01-.2398 \mathrm{E}+02-.1317 \mathrm{E}+02 \quad .0000 \mathrm{E}+00-.4854 \mathrm{E}+01 \quad .3965 \mathrm{E}+02$

Gloads $103 \quad-1805 E+02-.4492 E+02-1111 E+01,0000 E+00-.4002 E+01 \quad .1413 E+03$

$\begin{array}{llllllllll}\text { GLoads } & 88 & .4563 E+01 & .2731 E+02 & .9635 E+01 & .3600 E+02 & -.2216 E+01 & .0000 E+00\end{array}$

GLoads $89: 1316 \mathrm{E}+01 \quad .2593 \mathrm{E}+02-.1172 \mathrm{E}+02.0202 \mathrm{.1092}+02 \div .9210 \mathrm{E}+00.0000 \mathrm{E}+00$

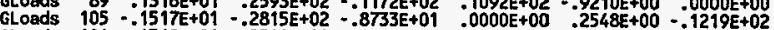

GLoads $104-.4362 \mathrm{E}+01-.2509 \mathrm{E}+02 \quad .1082 \mathrm{E}+02 \quad .0000 \mathrm{E}+00-.2341 \mathrm{E}+00-.3789 \mathrm{E}+02$

GLoads $89-.1130 E+01 \quad .3101 E+02 \quad .6497 E+01-.9961 E+01-.2184 E+00 \quad .0000 E+00$

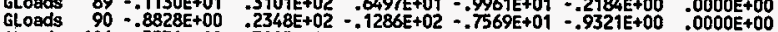

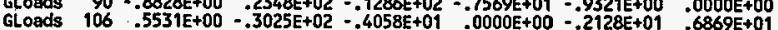

$\begin{array}{llllllll}\text { GLoads } & 105 & .1460 \mathrm{E}+01 & -.2424 \mathrm{E}+02 & -.4058 \mathrm{E}+01 & .0000 \mathrm{104}+00 & -.2128 \mathrm{E}+01 & .6869 \mathrm{E}+01 \\ .0000 \mathrm{E}+00 & -.1832 \mathrm{E}+01 & .8817 \mathrm{E}+01\end{array}$

GLoads $90^{\circ} .9168 E+00 \quad .4101 E+02-.8678 E+00 \quad .7201 E+01-.9036 E+00 \quad .0000 E+00$

GLoads $91 \quad .2149 E+01 \quad .2367 \mathrm{E}+02-.1859 \mathrm{E}+02 \quad .1844 \mathrm{E}+02 \div .4328 \mathrm{EE}+00 \quad .0000 \mathrm{E}+00$

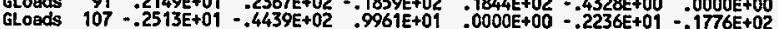

GLoads $106=.5526 \mathrm{E}+00-.2030 \mathrm{E}+02 \quad .9496 \mathrm{E}+01,0000 \mathrm{E}+00=.2073 \mathrm{E}+01-.7186 \mathrm{E}+01$

$\begin{array}{lllllllll}\text { GLoads } & 91 & -.2273 E+01 & .5469 E+02 & -.3972 E+01 & -.1871 E+02 & -.8212 E+00 & .0000 E+00 \\ \text { GLoads } & 92 & -.8388 E+01 & .1604 E+02 & -.3024 E+02 & -.0000 E+00 & -.2102 E+00 & .6645 E+02\end{array}$

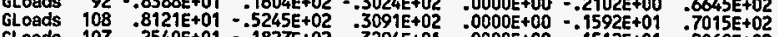

GLoads $107 \quad .2540 \mathrm{E}+01-.1827 \mathrm{E}+02 \quad .3294 \mathrm{E}+01 \quad .0000 \mathrm{E}+00 \quad \because 1512 \mathrm{E}+01 \quad .2060 \mathrm{E}+02$

GLoads $92-.3259 E+01 \quad .2401 E+02 \quad .5487 E+02 \quad .4490 E+03 \quad .1404 E+01 \quad .0000 E+00$ 
KAISER ENGINEERS HAMFORD S/N:801854

PAGE 101

RUn ID=NN61973

$02 / 22 / 95$

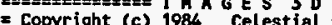

Copyright (c) 1984 Celestial Software Inc. $=$

SOLVE PLATE LOADS/STRESSES Version 2.0 07/01/90

Load Case 2:GRAVITY LOAD

GLoads Node Fx $\quad$ Fy $\quad$ Fz $\quad$ Mx My Mz GLoads $93-.5638 E+01 \quad .3111 E+02 \quad .1558 E+02 \quad .1255 E+03-.5016 E+00 \quad .0000 E+00$ GLoads $109-4214 E+01-.2144 E+02-.1413 E+02-1320 E+03-8675 E+01-0000 E+00$

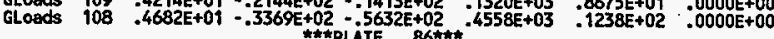

GLoads $93-.6507 E+01 \quad .2179 E+02-.1421 E+02-.1192 E+03 \quad .5981 E+01 \quad .0000 E+00$ GLoads $94 \quad .3159 \mathrm{E}+01 \quad .2685 \mathrm{E}+02=.3673 \mathrm{E}+01=.3333 \mathrm{E}+02 \quad .2345 \mathrm{E}+01 \quad .0000 \mathrm{E}+00$ GLoeds $110.6360 \mathrm{E}+01-.2321 \mathrm{E}+02 \quad .4071 \mathrm{E}+01=.2915 \mathrm{E}+02-.2535 \mathrm{E}+01.000 \mathrm{O}-00 \mathrm{E}+00$ GLoads $109-.3013 \mathrm{E}+01-.2543 \mathrm{E}+02 \quad .1381 \mathrm{E}+02-.1134 \mathrm{E}+03 \quad .2398 \mathrm{E}+00 \quad .0000 \mathrm{E}+00$

GLoads $94-.9816 \mathrm{E}+01 \quad .2431 \mathrm{E}+02 \quad 3356 \mathrm{E}+01.2528 \mathrm{E}+02 \quad 1633 \mathrm{E}+01 \quad 0000 \mathrm{E}+00$ GLoads $95.6400 \mathrm{E}+01.2853 \mathrm{E}+02.0407 \mathrm{E}+00.806 \mathrm{E}+01.1605 \mathrm{E}+01.0000 \mathrm{E}+00$ GLoeds $111.8806 E+01-.2481 E+02-.3560 E+00.9873 E+01.2441 E+01.0000+00$ GLoads $110-.5390 \mathrm{E}+01-.2802 \mathrm{E}+02-3540 \mathrm{E}+01 \quad .98768 \mathrm{EE}+02 \quad .3189 \mathrm{E}+01 \quad .0000 \mathrm{E}+00$

GLoads $95+.9340 E+01 \quad .2570 E+02-.7941 E+00-.6886 E+01 \quad .3441 E+01 \quad .0000 E+00$ Gloods $96 \quad .8735 \mathrm{E}+01 \quad .2554 \mathrm{E}+02 \quad .7396 \mathrm{E}+00 \quad 5943 \mathrm{E}+01 \quad 3938 \mathrm{E}+01.0000 \mathrm{E}+00$ GLoads $112.8751 \mathrm{E}+01-.2488 \mathrm{E}+02-.2207 \mathrm{E}+00 \quad .6004 \mathrm{E}+01-3315 \mathrm{E}+00.0000 \mathrm{E}+00$ GLoods $111=.8147 \mathrm{E}+01+.2636 \mathrm{E}+02 \quad .2752 \mathrm{E}+00-.5960 \mathrm{E}+01 \quad .1599 \mathrm{E}+00 \quad .0000 \mathrm{E}+00$ $\begin{array}{llllllll}\text { GLoods } 96 & .2507 E+00 & -3844 E+02 & -.1376 E+01 & -.1121 E+02 & .1938 E+01 & .0000 E+00\end{array}$ GLoods $113=.3789 E+01-.4349 E+02-.3859 E+01 \quad-.2901 E+02 \quad-3659 E+01 \quad-0000 E+00$

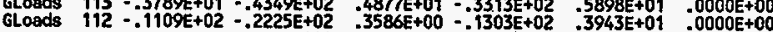
$\begin{array}{llllllllll}\text { GLoeds } & 97 & .8061 E+01 & .6138 E+02 & .2660 E+01 & .2979 E+02 & .4151 E+01 & .0000 E+00\end{array}$ GLaeds $98 \quad .1707 \mathrm{E}+02 \quad .3919 \mathrm{E}+02 \quad .1395 \mathrm{E}+02 \quad .1171 \mathrm{E}+03 \quad .7611 \mathrm{E}+01.0000 \mathrm{O}+00$ GLoads $114-.2156 E+02-.6653 E+02-.1182 E+02, .1052 E+03: 1323 E+02.0000 E+00$ GLoads $113-.3574 E+01-.3404 E+02-.4797 E+01.2195 E+02 \quad .7430 E+01 \quad .0000 E+00$ $\begin{array}{lllllllll}\text { GLoeds } & 122 & -1183 E+03 & .4229 E+03 & .6628 E-01 & .2668 E+00 & .8795 E+00 & .0000 E+00\end{array}$

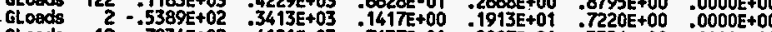

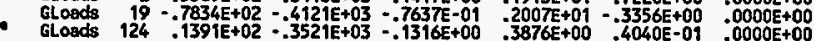
GLoads $124=-1176 E+02.2984 E+03 \quad .1184 E+00.7323 E+00 \quad .1015 E+01 \quad .0000 E+00$ GLoods $19 \quad .6648 E+01 \quad .3123 \mathrm{E}+03-.3003 \mathrm{E}-01-.9710 \mathrm{E}-01 \quad .2693 \mathrm{E}+00 \quad 0000 \mathrm{O}+00$ 7 GLoads $35 \quad-1785 \mathrm{E}+01-.3067 \mathrm{E}+03 \quad .1476 \mathrm{E}-01 \quad .1157 \mathrm{E}+00-.1322 \mathrm{E}+01 \quad .0000 \mathrm{E}+00$ OH Gloods $126 \quad .3331 E+01-.3040 E+03-.1031 E+00 \quad .1194 E+01-.2679 E+00 \quad .0000 E+00$ $\$$ GLoads $126-.4110 E+01 \quad .2399 E+03 \quad-1142 E+00 \quad .8961 E+00 \quad .1456 E+01 \quad .0000 E+00$

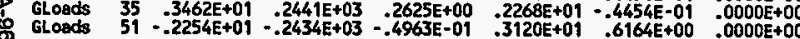

KAISER ENGINEERS HANFORD $S / N: 801854$ PAGE 102 Run ID=*N61973

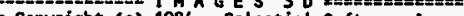
= Copyright (c) 1984 Celestial Software Inc. SOLVE PLATE LOADS/STRESSES Version 2.0 07/01/90

Load Case 2:GRAVITY LOAD

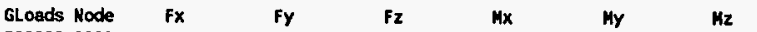
GLoads $128 \quad .2901 E+01-.2406 E+03-.3271 E+00 \quad .2003 E+01 \quad .2229 E+01 \quad .0000 E+00$ GLoads $128-.2737 E+09.1781 E+03 \quad .2961 E+00.3215 E+01.1946 E+01.0000 E+00$

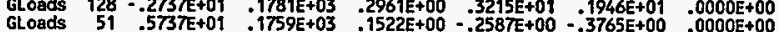

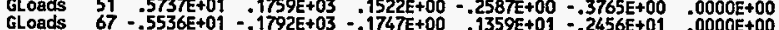

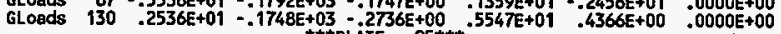

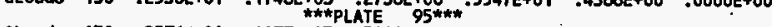

GLoads $130-.2576 E+01 \quad .1077 E+03 \quad .3411 E+00-.3426 E+01 \cdot .2525 E+01 \quad .0000 E+00$

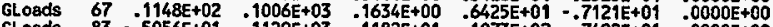
GLoads $133-.5056 E+01-.1129 E+03-.1182 E+01 \quad-1073 E+02=.7492 E+01 \quad .0000 E+00$ .0000E+00

$\begin{array}{lllllll}\text { GLoads } 132 & .3848 E+01 & .3983 E+02 & -.7096 E+00 & -.1141 E+02 & .2937 E+01 & .0000 E+00\end{array}$ GLoads $83.8634 E+01 \quad .4719 E+02 \quad .2420 E+01 \quad .1322 E+02-.9519 E+00 \quad .0000 E+00$

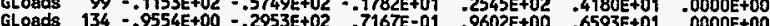

Cloads 134 -.9554E

$\begin{array}{llllllllll}\text { GLoads } & 17 & .4450 \mathrm{E}+02 & .3354 \mathrm{E}+03 & .5200 \mathrm{E}+00 & .4968 \mathrm{E}+01 & -.5668 \mathrm{E}+00 & .0000 \mathrm{E}+00\end{array}$

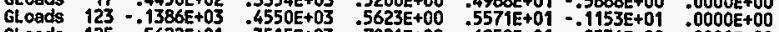
GLoeds $125-.5622 \mathrm{E}+01-.3515 \mathrm{E}+03-.7021 \mathrm{E}+00.0958 \mathrm{E}+01-.9534 \mathrm{E}+00 \quad .0000 \mathrm{0}+00$

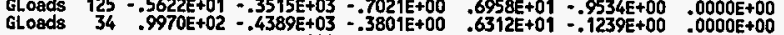

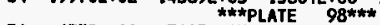

GLosds $34 \cdot-.6332 E+01 \quad .3105 E+03 \quad .3332 E+00 \quad .2294 E+01 \quad .1120 E+01 \quad .0000 E+00$ GLoads $125 \quad .3071 E+01 \quad .3049 E+03 \quad .6911 E+00 \quad .4931 E+01-.9721 E+00 \quad .0000 E+00$ $\begin{array}{llllll}6 & .9184 E+01 & .9129 E+00 & .0000 E+00\end{array}$ $\begin{array}{lllll}.000 & .3442 E+01 & .000 E+00\end{array}$

$\begin{array}{lllllllllll}\text { GLosds } & 50 & .3610 E+01 & .2516 E+03 & .8494 E+00 & .4143 E+01 & .7125 E+00 & .0000 E+00\end{array}$

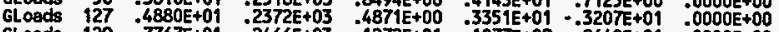
GLoads $129-.7767 E+01-.2466 E+03-.1272 E+01 \quad .1077 E+02-.8649 E+01 \quad .0000 E+00$ GLoads $66-.7231 E+00=.2423 E+03-.6482 E-01 \quad .1113 E+02-.4549 E+01 \quad .0000 E+00$

GLoads 66 - 3854E+01 1878E+03 *ANE 100*k*

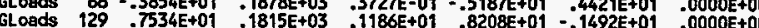

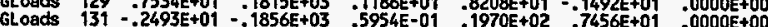
GLads $82-.1188 E+01-.1838 E+03-.1283 E+01.4191 E+01 \quad .1453 E+02 \quad .0000 E+00$

GLoads $132-.1819 E+02 \quad .1122 E+03 \quad-3527 E+00 \quad .2362 E+02 \quad .2416 E+02 \quad-0000 E+00$

GLoads $133 \quad 9280 E+01-9938 E+02 \quad-2673 E+01-1852 E+02-6240 E+01.0000+00$

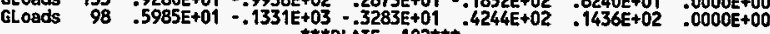

GLoads $98-.1669 E+02, .4371 E+02 \quad .1596 E+02 \quad .1311 E+03-.1095 E+02 \quad .0000 E+00$ 


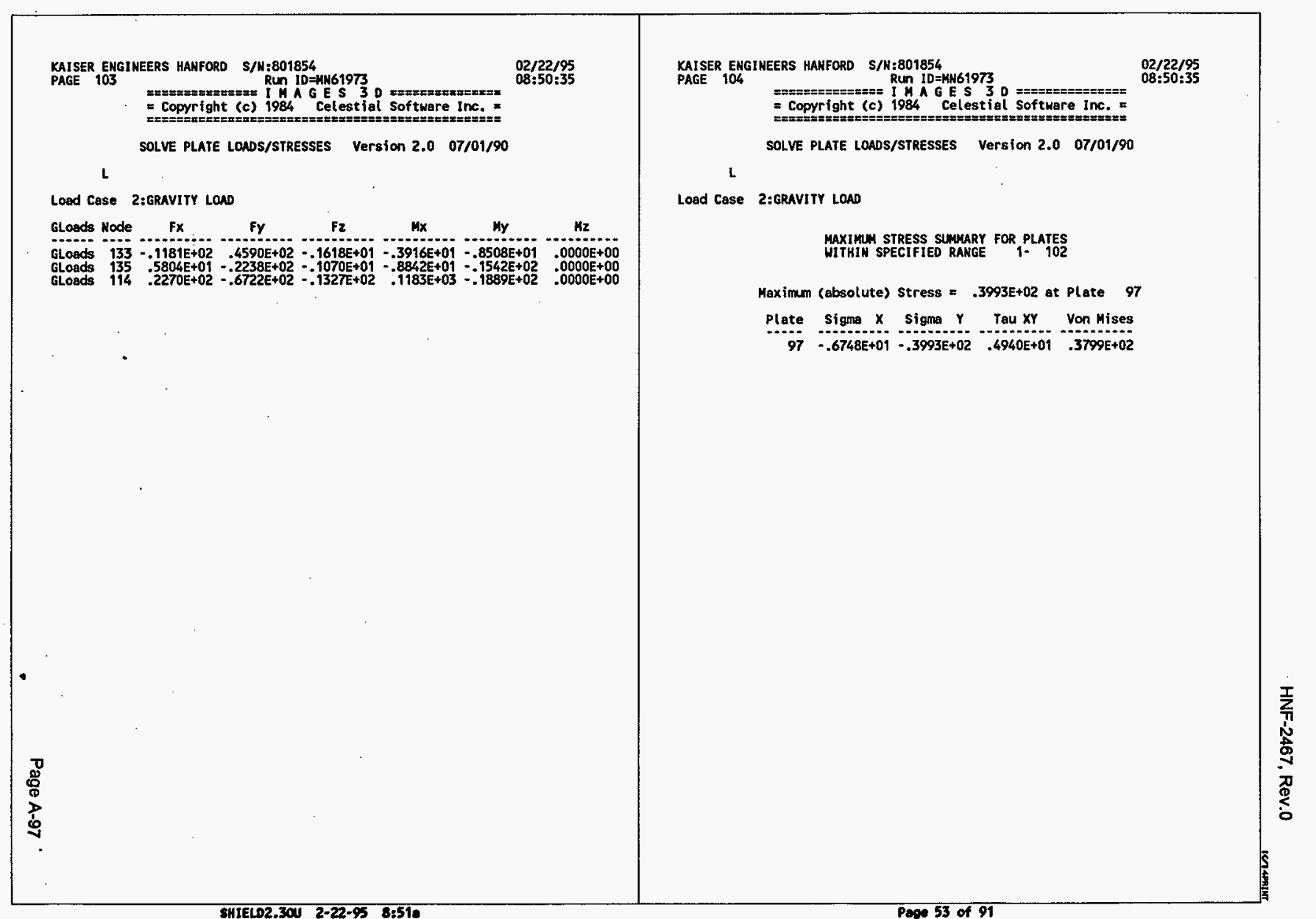


KAISER ENGINEERS HANFORO S/N:801854 Run ID=\#1N61973

$02 / 22 / 95$

PAGE 105

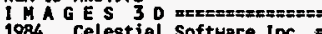
= Copyright (c) 1984 Celestial Software Inc. F Copyright (c) iga celestial Sof tware Inc,

SOLVE REACTIONS

Version $2.0 \quad 07 / 01 / 90$

Load Case 2:GRAVITY LOAD

REACTIOHS

\begin{tabular}{|c|c|c|c|c|c|c|}
\hline & $F x$ & fy & $\mathbf{F z}$ & $M x$ & ну & $\mathrm{Hz}$ \\
\hline $\begin{array}{r}1 \\
2 \\
3 \\
4 \\
5 \\
6 \\
7 \\
8 \\
9 \\
10 \\
11 \\
12 \\
13 \\
14 \\
15 \\
16 \\
17 \\
18 \\
115 \\
116 \\
117 \\
118 \\
119 \\
122 \\
123\end{array}$ & $\begin{array}{r}.1036 \mathrm{E}+00 \\
-4483 \mathrm{E}+02 \\
-.2724 \mathrm{E}+01 \\
-.2607 \mathrm{E}+02 \\
-.1072 \mathrm{0}+03 \\
-.2465 \mathrm{E}+00 \\
-6879 \mathrm{E}-01 \\
-.8635 \mathrm{E}-01 \\
.4517 \mathrm{E}-02 \\
.8825 \mathrm{E}-01 \\
.1289 \mathrm{E}+03 \\
.5488 \mathrm{E}+02 \\
.3537 \mathrm{E}+02 \\
-1061 \mathrm{E}+02 \\
-.1116 \mathrm{E}+02 \\
-.3494 \mathrm{E}+02 \\
-.7774 \mathrm{E}+02 \\
-.2438 \mathrm{E}+00 \\
.8533 \mathrm{E}-01 \\
.8979 \mathrm{E}+03 \\
.1281 \mathrm{E}+01 \\
-.8943 \mathrm{E}+03 \\
.1177 \mathrm{E}+01 \\
.1198 \mathrm{E}+03\end{array}$ & 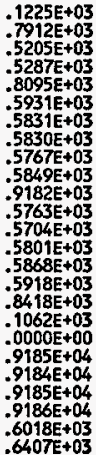 & 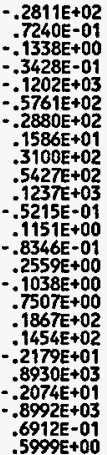 & $\begin{array}{r}.0000 E+00 \\
.0000 E+00 \\
.0000 E+00 \\
.0000 E+00 \\
-.6157 E+02 \\
.0000 E+00 \\
.0000 E+00 \\
.0000 E+00 \\
.0000 E+00 \\
.0000 E+00 \\
.1173 E++03 \\
.0000 E+00 \\
.0000 E+00 \\
.0000 E+00 \\
.0000 E+00 \\
.0000 E+00 \\
.0000 E+00 \\
.0000 E+00 \\
.0000 E+00 \\
.0000 E+00 \\
.0000 E+00 \\
.0000 E+00 \\
.0000 E++00 \\
.0000 E+ \\
.0000 E+00\end{array}$ & $\begin{array}{r}.0000 E+00 \\
.6225 E+01 \\
.1179 E+01 \\
.1427 E+01 \\
.3311 E+01 \\
.8228 E-01 \\
.1432 E+00 \\
.4329 E+00 \\
.4699 E+00 \\
.7242 E+00 \\
.1045 E+02 \\
.1021 E+01 \\
-.1631 E+01 \\
-.1782 E+01 \\
.1831 E+01 \\
.1397 E+01 \\
.4658 E+01 \\
.0000 E+00 \\
.0000 E+00 \\
.0000 E+00 \\
.0000 E+00 \\
.0000 E+00 \\
.0000 E+00 \\
.4903 E+01\end{array}$ & $\begin{array}{r}.0000 E+00 \\
-.6387 E+01 \\
.0000 E+00 \\
.0000 E+00 \\
.8070 E+02 \\
.0000 E+00 \\
.0000 E+00 \\
.0000 E+00 \\
.0000 E+00 \\
.0000 E+00 \\
. .1833 E+03 \\
.0000 E+00 \\
.0000 E+00 \\
.0000 E+00 \\
.0000 E+00 \\
.0000 E+00 \\
.1243 E+02 \\
.0000 E+00 \\
.0000 E+00 \\
.0000 E+00 \\
.0000 E+00 \\
.0000 E+00 \\
.0000 E+00 \\
-.2064 E+02 \\
.2622 E+02\end{array}$ \\
\hline
\end{tabular}

KAISER ENGINEERS HANFORD S/N:801854

Run $10=$ =N61973

$02 / 22 / 95$

PAGE 106

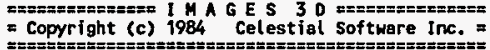

SOLVE BEAM LOADS/STRESSES Version 2.0 07/01/90

Load Case 3:UBC SEISHIC LOAD - SN \& WE DIR.

BEAM LOADS AND/OR STRESSES

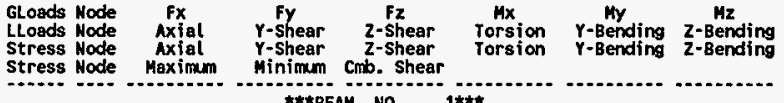
xicea

GLoads $1.2212 E+02-.7145 E+02 \quad .8077 \mathrm{Z} E+02 \quad .2274 E-12-.5684 E-13 \quad .0000 E+00$

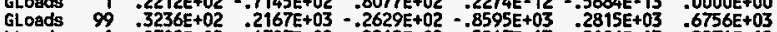

Lloods $1-.9702 E+02-.4707 E+02=.2212 E+02=.5247 E-13 \quad .2186 E-13-.2274 E-12$

LLoads $99 \quad .2102 E+03 \quad-.5910 E+02-.3236 E+02-.1749 E-13-.7319 E+03 \quad .8595 E+03$

GLoads $18 \quad .1903 E+02-.4880 \mathrm{E}+01 \quad .3094 \mathrm{E}+01^{2 * * 2} .2842 \mathrm{E}-13-.2842 \mathrm{E}-13 \quad .0000 \mathrm{E}+00$

GLonds $114 \quad 3423 \mathrm{E}+02 \quad .1469 \mathrm{E}+03 \quad .5016 \mathrm{E}+02 \quad .3844 \mathrm{E}+03-.3496 \mathrm{E}+03 \quad 1003 \mathrm{E}+04$

LLoads $18-.3589 \mathrm{E}+01 \quad .4528 \mathrm{E}+01 \quad .1903 \mathrm{E}+02-.2684 \mathrm{E}-13 \quad .9353 \mathrm{E}-14 \quad .2842 \mathrm{E}-13$

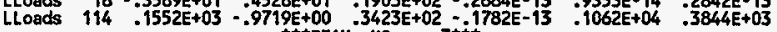

GLoads $99.1512 E+03 \quad .7599 E+02-.2530 E+03 \quad .2381 E+04-.1215 E+04 \quad .9061 E+03$

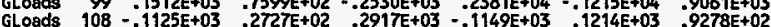

LLoads $99-.2925 \mathrm{E}+03-.7599 \mathrm{E}+02-.3629 \mathrm{E}+02-1312 \mathrm{E}+03 \quad: 1215 \mathrm{E}+04-.92546 \mathrm{E}+02$

LLoads $108-.3123 \mathrm{E}+03-.2727 \mathrm{E}+02-.1477 \mathrm{E}+02-.1312 \mathrm{E}+03-.1214 \mathrm{E}+03 \quad .6768 \mathrm{E}+02$

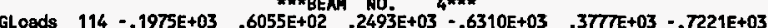

GLoads $102 \quad .2470 \mathrm{E}+03 \quad .7164 \mathrm{E}+02=.1998 \mathrm{E}+03 \quad .1147 \mathrm{E}+04-.6123 \mathrm{E}+03 \quad-1227 \mathrm{E}+04$

LLoads $114 \quad .3163 \mathrm{E}+03-6055 \mathrm{E}+02-3325 \mathrm{E}+02-.7481 \mathrm{E}+02-.3777 \mathrm{E}+03-0560 \mathrm{E}+03$

LLoads $102-.3156 \mathrm{E}+03-.7164 \mathrm{E}+02 \quad .3685 \mathrm{E}+02 \quad .7481 \mathrm{E}+02 \quad .6123 \mathrm{E}+03 \quad .1678 \mathrm{E}+04$

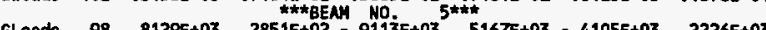

$\begin{array}{llllllll}\text { GLoads } & 98 & .8129 E+03 & .2851 E+02 & -.9113 \mathrm{E}+03 & .5167 \mathrm{E}+03 & -.4105 E+03 & -.2226 \mathrm{E}+03\end{array}$

\begin{tabular}{llllllll} 
GLoads $115=-7765 \mathrm{E}+03$ & $.6869 \mathrm{E}+02$ & $.9477 \mathrm{FE}+03$ & $.9300 \mathrm{E}+03$ & $-.9310 \mathrm{E}+03$ & $.1043 \mathrm{E}+04$ \\
\hline
\end{tabular}

LLoads $115: .1225 \mathrm{E}+04-.6869 \mathrm{E}+02 \quad .3974 \mathrm{E}+02 \quad .1727 \mathrm{E}+03 \quad .9310 \mathrm{E}+03 \quad .9387 \mathrm{E}+04$

GLosds $92-2318 E+03 \quad 3962 E+02-.6277 \mathrm{E}+03-.5236 \mathrm{E}+03 \quad 2817 \mathrm{E}+03 \quad 2147 \mathrm{E}+03$

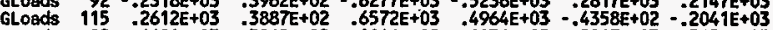

LLoads $92-.6691 \mathrm{E}+03-.3962 \mathrm{E}+02 \quad .1146 \mathrm{E}+02 \quad .1036 \mathrm{E}+02-.2817 \mathrm{~F}+03-.5658 \mathrm{E}+03$

LLoads $115 \quad .7072 E+03-.3887 E+02 \quad .5300 E+01-.1036 E+02 \quad .4358 E+02 \quad .5366 E+03$

GLoods $86-.5522 E+03 \quad .5730 E+02 \quad .3683 E+03 \quad .4341 E+03 \quad .7685 E+03 \quad .7964 E+03$

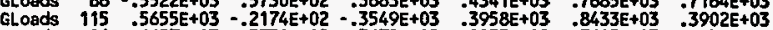

LLoads $86-.6627 \mathrm{E}+03-.5730 \mathrm{E}+02 \quad .3672 \mathrm{E}+02-.8255 \mathrm{E}+02-.7685 \mathrm{E}+03-.8336 \mathrm{E}+03$

LLoods $115 \quad .6654 \mathrm{E}+03 \quad .2174 \mathrm{E}+02-.5538 \mathrm{E}+02 \quad .8255 \mathrm{E}+02-.8433 \mathrm{E}+03-.5496 \mathrm{E}+03$

GLoads $134.1904 \mathrm{E}+02-.1248 \mathrm{E}+02-.5462 \mathrm{E}+01 \quad .5965 \mathrm{E}+03-.7744 \mathrm{E}+03 \quad .1990 \mathrm{E}-12$ 
KAISER ENGINEERS HANFORD S/N:801854

PAGE 107

Run ID=NN61973

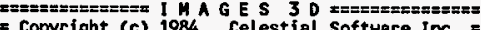
= Copyright (c) 1984 Celestial Software Inc.

SOLVE BEAM LOADS/STRESSES Version $2.0 \quad 07 / 01 / 90$

Load Case 3:UBC SEISHIC LOAD - SH \& WE DIR.

\begin{tabular}{|c|c|c|c|c|c|c|c|}
\hline $\begin{array}{l}\text { CLoads } \\
\text { LLoads } \\
\text { Stress } \\
\text { Stress }\end{array}$ & $\begin{array}{l}\text { Node } \\
\text { Node } \\
\text { Hode } \\
\text { Node }\end{array}$ & $\begin{array}{c}\text { Fx } \\
\text { Axiat } \\
\text { Axial } \\
\text { Maximum }\end{array}$ & $\begin{array}{l}\text { Fy } \\
y \text {-shear } \\
\text { Y-Shear } \\
\text { Minimun }\end{array}$ & $\begin{array}{c}\text { Fz } \\
\text { Z-Shear } \\
\text { Z-Shear } \\
\text { Comb. Shear }\end{array}$ & $\begin{array}{l}\text { Hx } \\
\text { Torsion } \\
\text { Torsion }\end{array}$ & $\begin{array}{l}\text { My } \\
\text { Y-Bending } \\
\text { Y-Bending }\end{array}$ & $\begin{array}{l}\text { Mz } \\
\text { Z-Bending } \\
\text { Z-Bending }\end{array}$ \\
\hline $\begin{array}{l}\text { GLoads } \\
\text { LLoads } \\
\text { LLoads }\end{array}$ & $\begin{array}{r}99 \\
139\end{array}$ & $\begin{array}{l}-1142 E+02 \\
.1904 E+02 \\
-.1142 E+02\end{array}$ & $\begin{array}{r}.3280 E+02 \\
.1248 E+02 \\
-3280 E+02\end{array}$ & $\begin{array}{r}.1308 E+02 \\
.5462 E+01 \\
.1308 E+02\end{array}$ & $\begin{array}{r}.5965 E+03 \\
.5965 E+03 \\
.5965 E+03\end{array}$ & $\begin{array}{r}.9598 \mathrm{E}+03 \\
.7744 \mathrm{E}+03 \\
.9598 \mathrm{E}+03\end{array}$ & $\begin{array}{r}-.4528 \mathrm{E}+03 \\
-1990 \mathrm{E}-12 \\
.4528 \mathrm{E}+03\end{array}$ \\
\hline $\begin{array}{l}\text { GLoads } \\
\text { GLoads } \\
\text { LLoads } \\
\text { LLoads }\end{array}$ & $\begin{array}{l}135 \\
114 \\
135 \\
114\end{array}$ & $\begin{array}{r}-.5030 \mathrm{E}+02 \\
.5792 \mathrm{E}+02 \\
.5030 \mathrm{E}+02 \\
-.5792 \mathrm{0}+02\end{array}$ & $\begin{array}{r}.1670 E+02 \\
.3619 E+01 \\
-.1670 E+02 \\
-.3619 E+01\end{array}$ & $\begin{array}{l}.7615 E+01 \\
.5043 E-02 \\
.7615 E+01 \\
.5043 E-02\end{array}$ & $\begin{array}{r}.6230 E+03 \\
-.6230 E+03 \\
-.6230 E+03 \\
.6230 E+03 \\
\star \star \star\end{array}$ & $\begin{array}{r}.5619 \mathrm{E}+03 \\
-.4858 \mathrm{E}+03 \\
-.5619 \mathrm{E}+03 \\
.4858 \mathrm{E}+03\end{array}$ & $\begin{array}{l}-.4890 \mathrm{E}+02 \\
-.8191 \mathrm{E}+02 \\
-.4890 \mathrm{E}+02 \\
-.8191 \mathrm{E}+02\end{array}$ \\
\hline $\begin{array}{l}\text { GLoads } \\
\text { GLoads } \\
\text { LLoads } \\
\text { LLoads }\end{array}$ & $\begin{array}{l}122 \\
124 \\
122 \\
124\end{array}$ & $\begin{aligned} &-1141 E+02 \\
&-.3028 E+01 \\
& .5207 E+03 \\
&-.4984 E+03\end{aligned}$ & $\begin{array}{r}.5207 \mathrm{E}+03 \\
-.4984 \mathrm{E}+03 \\
.1141 \mathrm{E}+02 \\
-.3028 \mathrm{E}+01\end{array}$ & & $\begin{array}{l}.5678 E+03 \\
.6660 E+03 \\
.6328 E+03 \\
.6328 E+03 \\
.6328\end{array}$ & $\begin{array}{r}.6328 \mathrm{E}+03 \\
-.6328 \mathrm{E}+03 \\
-.5678 \mathrm{E}+03 \\
.6660 \mathrm{E}+03\end{array}$ & $\begin{array}{r}-.1521 E+03 \\
-.6683 E+01 \\
.1521 E+03 \\
.6683 E+01\end{array}$ \\
\hline $\begin{array}{l}\text { GLoads } \\
\text { GLoads } \\
\text { LLoads } \\
\text { LLoads }\end{array}$ & $\begin{array}{l}124 \\
126 \\
124 \\
126\end{array}$ & $\begin{array}{r}.2311 E+01 \\
.6071 E+01 \\
.3624 E+03 \\
-.3400 E+03\end{array}$ & $\begin{array}{r}.3624 E+03 \\
-.3400 E+03 \\
.2311 E+01 \\
.6071 E+01 \\
\end{array}$ & & $\begin{array}{l}-.1529 E+04 \\
.1588 E+04 \\
.5086 E+03 \\
-.5086 E+03 \\
2 * * *\end{array}$ & $\begin{array}{r}.5086 \mathrm{E}+03 \\
=.5086 \mathrm{E}+03 \\
=.1529 \mathrm{E}+04 \\
.1588 \mathrm{E}+04\end{array}$ & $\begin{array}{r}.6683 E+01 \\
.3467 E+02 \\
-.6683 E+01 \\
-.3467 E+02\end{array}$ \\
\hline $\begin{array}{l}\text { GLoads } \\
\text { GLoads } \\
\text { LLoads } \\
\text { LLoads }\end{array}$ & $\begin{array}{l}126 \\
128 \\
126 \\
128\end{array}$ & $\begin{array}{r}.5075 E+01 \\
.3307 E+01 \\
.2528 E+03 \\
-.2304 E+03\end{array}$ & $\begin{array}{r}.2528 E+03 \\
-.2304 E+03 \\
.5075 E+01 \\
.3307 E+01\end{array}$ & & $\begin{array}{r}.1934 \mathrm{E}+04 \\
.1950 \mathrm{E}+04 \\
.2963 \mathrm{E}+03 \\
-.2963 \mathrm{E}+03 \\
-.296\end{array}$ & $\begin{array}{r}.2963 \mathrm{E}+03 \\
-.2963 \mathrm{E}+03 \\
-.1934 \mathrm{E}+04 \\
.1950 \mathrm{E}+04\end{array}$ & $\begin{array}{r}-.3467 \mathrm{E}+02 \\
.1523 \mathrm{E}+02 \\
.3467 \mathrm{E}+02 \\
-.1523 \mathrm{E}+02\end{array}$ \\
\hline $\begin{array}{l}\text { GLoads } \\
\text { GLoads } \\
\text { LLoads } \\
\text { LLoads }\end{array}$ & $\begin{array}{l}128 \\
130 \\
128 \\
130\end{array}$ & $\begin{array}{r}.3612 \mathrm{E}+01 \\
.470 \mathrm{~T}+01 \\
.1674 \mathrm{E}+03 \\
-.1451 \mathrm{E}+03\end{array}$ & $\begin{array}{r}.1674 E+03 \\
-.1451 E+03 \\
.3612 E+01 \\
.4770 E+01 \\
\end{array}$ & $\begin{array}{l}.2810 \mathrm{E}+01 \\
.5572 \mathrm{E}+01 \\
.2810 \mathrm{E}+01\end{array}$ & $\begin{array}{r}.1821 E+04 \\
.1790 E+04 \\
.8524 E+02 \\
.8524 E+02\end{array}$ & $\begin{array}{r}.8524 E+02 \\
-.8524 \mathrm{E}+02 \\
-.1821 E+04 \\
.1790 \mathrm{E}+04\end{array}$ & $\begin{array}{r}-.1523 \mathrm{E}+02 \\
.2796 \mathrm{E}+02 \\
.1523 \mathrm{E}+02 \\
-.2796 \mathrm{E}+02\end{array}$ \\
\hline $\begin{array}{l}\text { GLoads } \\
\text { GLoads } \\
\text { LLoads } \\
\text { LLoads }\end{array}$ & $\begin{array}{l}130 \\
132 \\
130 \\
132\end{array}$ & $\begin{array}{r}.6255 \mathrm{E}+01 \\
.4222 \mathrm{E}+01 \\
.9005 \mathrm{E}+02 \\
-.6211 \mathrm{E}+02\end{array}$ & $\begin{array}{r}.9005 E+02 \\
-.6211 E+02 \\
.6255 E+01 \\
.6222 E+01 \\
\text { H.tRU }\end{array}$ & $\begin{aligned} .7238 E+01 \\
.7239 E+01 \\
-.7238 E+01 \\
.7250 .\end{aligned}$ & $\begin{array}{r}-.1073 E+04 \\
.1018 E+04 \\
-.5711 E+02 \\
.5711 E+02 \\
.5710\end{array}$ & $\begin{array}{r}-.5711 E+02 \\
.5711 E+02 \\
. .1073 E+04 \\
.1018 E+04\end{array}$ & $\begin{array}{r}-.2796 \mathrm{E}+02 \\
.5151 \mathrm{E}-12 \\
.2796 \mathrm{E}+02 \\
-.5151 \mathrm{E}-12\end{array}$ \\
\hline $\begin{array}{l}\text { GLoads } \\
\text { GLoads } \\
\text { LLoads } \\
\text { LLaads }\end{array}$ & $\begin{array}{l}132 \\
134 \\
132 \\
134\end{array}$ & $\begin{array}{r}.0000 \mathrm{E}+00 \\
.0000 \mathrm{E}+00 \\
.2710 \mathrm{E}+00 \\
.2710 \mathrm{E}+00\end{array}$ & $\begin{array}{r}-.2710 E+00 \\
.2710 E+00 \\
.0000 E+00 \\
.0000 E+00 \\
\star \pm \neq B E A\end{array}$ & $\begin{array}{l}.0000 \mathrm{E}+00 \\
.0000 \mathrm{E}+00 \\
.0000 \mathrm{E}+00 \\
.0000 \mathrm{E}+00\end{array}$ & $\begin{array}{l}.0000 E+00 \\
.0000 E+00 \\
.0000 E+00 \\
.0000 E+00 \\
=100\end{array}$ & $\begin{array}{l}.0000 E+00 \\
.0000 E+00 \\
.0000 E+00 \\
.0000 E+00\end{array}$ & $\begin{array}{l}.0000 E+00 \\
.0000 E+00 \\
.0000 E+00 \\
.0000 E+00\end{array}$ \\
\hline GLoads & 123 & $.7586 E+01$ & $-.125 \% E+03$ & & $-.8523 E+03$ & $.2629 E+03$ & $98 E+02$ \\
\hline
\end{tabular}
$08: 50: 36$
KAISER ENGINEERS HAHFORD S/H:801854

PAGE 108 Run ID=N161973

$02 / 22 / 95$

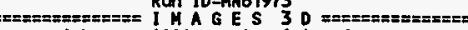
\# Copyright (c) 1984 Celestial Software Inc. = SOLVE BEAM LOADS/STRESSES Version 2.0 07/09/90 L

Load Case 3:UBC SEISHIC LOAD - SN \& WE DIR.

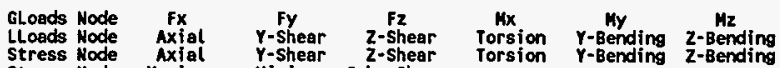
Stress Hode Maximum Minimum cmb. Shear

Geads 125 - $7954 E+00$. Min

GLoads $125 \quad .7954 \mathrm{E}+00 \quad .1482 \mathrm{E}+03 \quad-.3345 \mathrm{E}+00 \quad .9518 \mathrm{E}+03 \quad-.2629 \mathrm{E}+03 \quad-1128 \mathrm{E}+02$

$\begin{array}{llllllll}\text { LLoads } & 123 & -.1259 E+03 & -.7586 E+01 & .8716 E+01 & .2629 E+03 & .8523 E+03 & -.8598 E+02 \\ \text { LLoads } & 125 & .1482 E+03 & -.7954 E+00 & -.3345 E+00 & -.2629 E+03 & -.9518 E+03 & .1128 E+02\end{array}$

GLoads $2 \quad .8075 E+01 \quad .2358 E+03 \quad .5467 \mathrm{E}+01-.4989 \mathrm{E}+03 \quad .8043 \mathrm{E}+03-.1100 \mathrm{E}+03$

$19.3070 \mathrm{E}+00-.2134 \mathrm{E}+03 \quad .2915 \mathrm{E}+01 \quad .5270 \mathrm{E}+03-.8043 \mathrm{E}+03 \quad .2456 \mathrm{E}+02$

LLoads $2.2358 E+03 \quad .8075 \mathrm{E}+01-.5467 \mathrm{E}+01 \quad .8043 \mathrm{E}+03-.4989 \mathrm{E}+03 \quad .1100 \mathrm{E}+03$

LLoads $19-.2134 E+03 \quad .3070 E+00-.2915 E+01 \quad=.8043 E+03 \quad .5270 E+03-.2456 E+02$

GLoads $\quad 19 \quad .4222 E+01 \quad .2261 E+03 \quad-4502 E+01-.1221 E+04 \quad .6285 E+03-.2456 E+02$

GLoads $35 \quad .4160 E+01-.2038 E+03 \quad .3879 E+01 \quad .1228 E+04-.6285 E+03 \quad .23877+02$

LLoads $19.2261 E+03 \quad .4222 E+01-.4502 E+01-6285 E+05-.1221 E+04-.2456 E+02$

LLoads $35-.2038 E+03 \quad .4160 E+01-.3879 E+01-.6285 E+03 \quad .1228 E+04-.2387 E+02$

GLoads $\quad 35 \quad .4287 E+01 \quad .1665 E+03 \quad .4113 E+01-.1452 E+04 \quad .2775 E+03-.2387 E+02$

GLoads $51 \quad .4095 \mathrm{E}+01 \cdot .1442 \mathrm{E}+03 \quad .4268 \mathrm{E}+01 \quad .1451 \mathrm{E}+04-.2775 \mathrm{E}+03 \quad .2176 \mathrm{E}+02$

Lloads $51 \cdot .1442 E+03 \quad .4095 E+01-.4268 E+01-27775 E+03 \quad .1451 E+04-.2176 E+02$

GLoads $51 \quad .5011 E+01 \quad .1208 E+03,4088 E+01-.1410 E+04-.1222 E+03-.2176 E+02$

$\begin{array}{lllllllll}\text { GLoads } & 67 & .3370 E+01 & -.9845 E+02 & .4293 E+01 & .1408 E+04 & .1222 E+03 & .3708 E+01\end{array}$

$51.1208+03-90$

Lloads $67 \cdot .9845 E+02 \quad .3370 E+01-.4293 E+01 \quad .1222 E+03 \quad .1408 E+04=.3708 E+01$

GLoads $67.2057 E+01.9298 E+02 \quad .4204 E+01-.8203 E+03-.4452 E+03-.3708 E+01$

GLoads $\quad 83 \quad .8421 \mathrm{E}+01-.6504 \mathrm{E}+02 \quad .6273 \mathrm{E}+01 \quad .7918 \mathrm{E}+03 \quad .4452 \mathrm{E}+03 \quad .9122 \mathrm{E}+02$

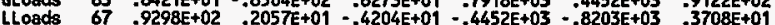

LLoads $83-.6504 \mathrm{E}+02 \quad .8421 \mathrm{E}+01-.6273 \mathrm{E}+01 \quad .4452 \mathrm{E}+03 \quad .7918 \mathrm{E}+03-.9122 \mathrm{E}+02$

GLoads $83.4094 E+02 \quad .7743 E+02-.2498 E+01-4481 E+03-.5324 E+03-.9122 E+02$

GLoads $99-.3465 E+02-.6066 E+02 \quad .8784 E+01-.5412 E+03 \quad .5324 E+03-.5324 E+03$

LLoads $83 \quad .7743 \mathrm{E}+02 \quad .4094 \mathrm{E}+02 \quad .2498 \mathrm{E}+01-.5324 \mathrm{E}+03 \quad .4481 \mathrm{E}+03 \quad .9122 \mathrm{E}+02$

LLoads $99-.6066 \mathrm{E}+02-.3465 \mathrm{E}+02-.8784 \mathrm{E}+01 \quad-5324 \mathrm{E}+03 \quad-.5412 \mathrm{E}+03 \quad .5324 \mathrm{E}+03$

GLoads $5 \quad .5073 E+01-.2300 E+03 \quad .5524 E+01 \quad .8900 E+02 \quad .1334 E+03-.9378 E+02$

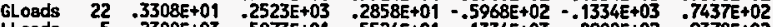

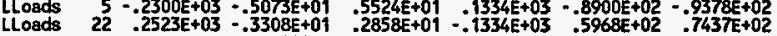

$\begin{array}{lllllll} & .2523 E+03 & -.3308 E+01 & -2858 E+01 & -1.1334 E+03 & .5962+02 & -7437 \mathrm{E}+02\end{array}$

$\begin{array}{lllllll}\text { GLods } 22 & 3400 E+01-.1028 E+03 & -2190 E+01 & -.3128 E+02 & .1032 E+03 & .1786 E+02\end{array}$ 
KAISER ENGINEERS HANFORD S/N:801854

PAGE 109

$$
\text { Run ID }=\$ 1161973
$$

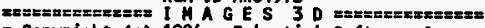

$02 / 22 / 95$

= Copyright (c) 1984 Celestial Software Inc.

SOLVE BEAM LOADS/STRESSES Version 2.0 07/01/90

$L$

Load Case 3:UBC SEISMIC LOAD - SH 2 WE DIR.

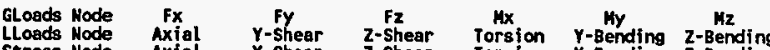
Stress Hode Axial Yx-Shear Z-Shear Torsion Y-Bending Z-Bending Stress Node Maximum Minimum Cmb. Shear

GLoeds $38.4982 \mathrm{E}+01$

GLoeds $38 \quad .4982 E+01 \quad .1252 E+03 \quad .6192 E+01-.1273 E+02-.1032 E+03 \quad-.4604 E+00$

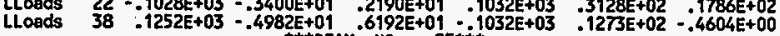

GLoads

Loods

Loads

GLoads

GLoads

toads

Lloads

GLoads

GLoads

LLoads

LLads

GLads

GLoads

LLoads

GLoads

Lloads

LLads

GLoads

GLoads

Loeds

GLoads

$\begin{array}{llllll}.4051 \mathrm{E}+01 & -.3054 \mathrm{E}+02 & .5079 \mathrm{E}+01 & .3986 \mathrm{E}+02 & -.1939 \mathrm{E}+01 & -.5628 \mathrm{E}+02 \\ -5330 \mathrm{E}+01 & .5289 \mathrm{E}+02 & .3303 \mathrm{E}+01 & -.2032 \mathrm{E}+02 & .1939 \mathrm{E}+01 & .5935 \mathrm{E}+02\end{array}$ . $4330 E+01-5209 E+02 \quad .3303 E+01-.2032 E+02-1939 E+01-.5935 E+02$ $54-5289 \mathrm{E}+02-.4330 \mathrm{E}+01 \quad .3303 \mathrm{E}+01 \cdot 1939 \mathrm{E}+01-.3986 \mathrm{E}+02-.5628 \mathrm{E}+02$

$54.5074 \mathrm{E}+01.1477 \mathrm{E}+02 \quad .3725 \mathrm{E}+01 \quad .3039 \mathrm{E}+01-.7184 \mathrm{E}+02 \quad .1606 \mathrm{E}+02$ $70 \quad .3308 \mathrm{E}+01 \quad .7579 \mathrm{E}+01 \quad .4657 \mathrm{E}+01-.1329 \mathrm{E}+02 \quad .7184 \mathrm{EE}+02-.3550 \mathrm{EE}+02$ $54.1477 \mathrm{~F}+02-5074 \mathrm{E}+01 \quad .3725 \mathrm{E}+01-7329 \mathrm{E}+02-.7184 \mathrm{E}+02-.3550 \mathrm{E}+02$ $70.7579 E+01-.3308 E+01 \quad .4657 E+01.7184 E+02 \quad .1329 E+02-.3550 E+02$ $70.4398 \mathrm{E}+01.4147 \mathrm{E}+02.6103 \mathrm{E}+01.37 \mathrm{TTE}+02-.1438 \mathrm{E}+03-.4450 \mathrm{E}+02$ $86 \cdot .6080 \mathrm{E}+01-.1353 \mathrm{E}+02 \quad .4374 \mathrm{E}+01 \quad-.1399 \mathrm{E}+02 \quad .1438 \mathrm{E}+03 \quad .6763 \mathrm{E}+02$ $70 \quad .4147 \mathrm{E}+02-.4398 \mathrm{E}+01 \quad .6103 \mathrm{E}+01-.1438 \mathrm{E}+03-.3777 \mathrm{E}+02-.4450 \mathrm{E}+02$ $86-.1353 \mathrm{E}+02-.6080 \mathrm{E}+01 \quad .4374 \mathrm{E}+01 \quad: 1438 \mathrm{E}+03 \quad .1399 \mathrm{E}+02 \quad .6763 \mathrm{E}+02$ $86 \quad 2129 E+02 \quad .3359 E+02-.3572 E+01-.8369 E+02-.4670 E+03-.7705 E+02$ $02-.1500 \mathrm{E}+02-.1683 \mathrm{E}+02 \quad .9858 \mathrm{E}+01-.2710 \mathrm{E}+02 \quad-.4670 \mathrm{E}+03=.2224 \mathrm{E}+03$ 86 - $350 \mathrm{E}+02-2120 \mathrm{E}+02-3572 \mathrm{E}+01-.2670 \mathrm{E}+03-.4670 \mathrm{E}+03-.2224 \mathrm{E}+03$ $102-.1683 \mathrm{E}+02 \quad-1500 \mathrm{E}+02 \quad .9858 \mathrm{E}+01 \quad .4670 \mathrm{E}+03 \quad .2710 \mathrm{E}+02-.2224 \mathrm{EE}+03$ $11.2152 E+02 \quad .7264 E+03-1113 E+02-2117 E+03-.5811 E+03-.4990 E+03$ $28=.8466 \mathrm{E}+01-.6916 \mathrm{E}+03 \quad .1922 \mathrm{E}+01-.1104 \mathrm{E}+03 \quad .5811 \mathrm{E}+03 \quad .1692 \mathrm{E}+03$ $11 \quad .7264 E+03 \quad .1113 E+02 \quad .2152 E+02-.5811 E+03 \quad-.4990 E+03 \quad .2117 E+03$ $28-.6916 \mathrm{E}+03 \quad .1922 \mathrm{E}+01-.8466 \mathrm{E}+01 \quad .5811 \mathrm{E}+03-.1692 \mathrm{EE}+03-.1104 \mathrm{EE}+03$

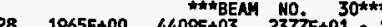

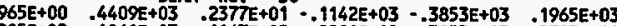

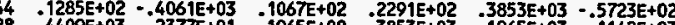

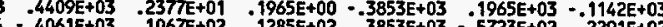

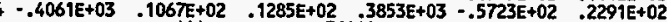
$44.8084 E+01-.2917 \mathrm{E}+03 \quad .7408 \mathrm{E}+01-.9162 \mathrm{E}+02-.9005 \mathrm{E}+02-.7524 \mathrm{E}+02$ $60-.4967 \mathrm{E}+01-.2569 \mathrm{E}+03$ - $.564 \mathrm{EE}+01$

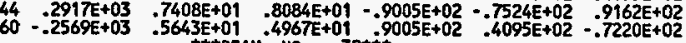
$\begin{array}{llllllll}60 & .6708 E+01 & .1776 E+03 & .7633 E+01 & -.2827 E+02 & .2157 E+03 & .2204 E+02\end{array}$
KAISER ENGINEERS HAHFORD S/N:801854

Run ID $=$ HN61973 $02 / 22 / 95$ = Copyright (c) 1984 Celestial Softure inc.

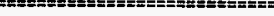

SOLVE BEAN LOADS/STRESSES Version $2.0 \quad 07 / 01 / 90$

L

Load Case 3:UBC SEISMIC LOND - SH \& WE DIR.

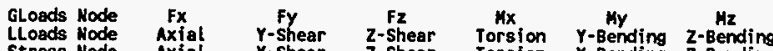
Stress Hode Axial $\quad y$-Shear $z$-Shear Torsion $y$-Bending $z$-Bending Stress Node Maximum Minimum Cmb. Shear

GLoads $76 \quad .6344 \mathrm{E}+01-.1428 \mathrm{E}+03 \quad .5418 \mathrm{E}+01 \quad .5264 \mathrm{E}+02-.2157 \mathrm{E}+03-.2604 \mathrm{E}+02$

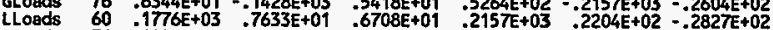

LLoads $76-.1428 E+03 \quad .5418 E+01 \quad .6344 E+01 \quad-.2157 \mathrm{E}+03 \quad-.2604 \mathrm{E}+02 \quad-.5264 \mathrm{E}+02$

$\begin{array}{llllllll}\text { GLoads } & 76 & .7753 E+01 & .1273 E+03 & -7560 E+01 & .5713 E+02 & .4082 E+03 & -.1425 E+02\end{array}$

GLoads $92 \quad .8561 \mathrm{E}+01-.8399 \mathrm{E}+02 \quad .8754 \mathrm{E}+01-.7356 \mathrm{E}+02-.4082 \mathrm{E}+03 \quad .2536 \mathrm{E}+02$

$\begin{array}{llllllll}\text { LLoads } & 76 & .1275 E+03 & .7560 E+01 & .7753 E+01 & .4082 E+03 & -.1425 E+02 & .5713 E+02 \\ \text { LLoeds } & 92 & -.8399 \mathrm{E}+02 & .8754 \mathrm{E}+01 & .8561 \mathrm{E}+01 & -.4082 \mathrm{E}+03 & .2536 \mathrm{E}+02 & -.7356 \mathrm{E}+02\end{array}$

GLoads $92.1592 E+02.7226 E+02 \quad .2339 E+02 \quad .1856 E+03 \quad .2943 E+03-.7819 E+02$

GLoads $108-.6135 \mathrm{E}+01-.4616 \mathrm{E}+02-.1360 \mathrm{E}+02 \quad .1196 \mathrm{E}+03-.2943 \mathrm{E}+03-.1038 \mathrm{E}+03$

Lloads $92-7226 \mathrm{E}+02 \quad .2339 \mathrm{E}+02.1592 \mathrm{E}+02-.2943 \mathrm{E}+03-.7819 \mathrm{E}+02-1856 \mathrm{E}+03$

LLoads $108-.4616 E+02-.1360 E+02-.6135 E+01-.2943 E+03 * .1038 E+03 \quad .1196 E+03$

GLoods $\quad 17.7116 E+01.2962 E+02 \quad .7554 E+01-.9942 E+03 \quad .4797 E+03-.8004 E+02$

GLoods $34 \quad-1266 \mathrm{E}+01-.7264 \mathrm{E}+01 \quad .8274 \mathrm{E}+00 \quad .1068 \mathrm{E}+04,-.4797 \mathrm{E}+03 \quad-1570 \mathrm{E}+02$

LLoads $17 . .2962 E+02=.716 E+01 \quad .7554 E+01 \quad .4797 E+05 \quad .9942 E+03-.8004 E+02$

$\begin{array}{ll} & \\ 0\end{array}$

GLoads $\quad 34 \quad .3871 E+01 \quad .2085 E+02 \quad .5517 \mathrm{E}+01-2488 \mathrm{E}+04 \quad .3099 \mathrm{E}+03-.1570 \mathrm{EE}+02$

$\begin{array}{llllllll}\text { GLoads } 50 & -4510 \mathrm{E}+01 & .1503 \mathrm{E}+01 & .2864 \mathrm{E}+01 & -2517 \mathrm{E}+04 & -.3099 \mathrm{E}+03 & .2273 \mathrm{E}+02\end{array}$

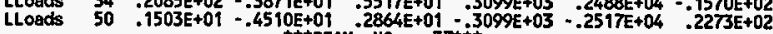

GLoads $50 \quad .4947 E+01 \quad .3311 E+02 \quad .3950 E+01-.2828 E+04 \quad .1020 E+03-.2273 E+02$

GLoads $50 \quad .4947+01 \quad .3311 E+02 \quad .3950 E+01 \quad-.2828 E+04 \quad .1020 E+03-.2273 E+02$

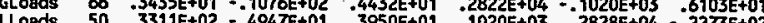

LLoads $66-.1076 \mathrm{E}+02=.3435 \mathrm{E}+01 \quad .4432 \mathrm{E}+01-.1020 \mathrm{E}+03 \quad-.2822 \mathrm{E}+04 \quad-.6103 \mathrm{E}+01$

GLoads $66 \quad .1818 \mathrm{E}+01 \quad .3916 \mathrm{E}+02 \quad-3039 \mathrm{E}+01-.2197 \mathrm{E}+04-.2395 \mathrm{E}+03-.6103 \mathrm{E}+01$

$\begin{array}{llllllll}\text { GLcads } & 82 & .6564 E+01 & -.1681 E+02 & .5342 E+01 & .2172 E+04 & .2395 E+03 & .5831 E+02\end{array}$

LLoads $82-.1681 \mathrm{E}+02-.6564 \mathrm{E}+01 \quad .5342 \mathrm{E}+01 \quad .2395 \mathrm{E}+03 \quad-.2172 \mathrm{E}+04 \quad-.5831 \mathrm{E}+02$

GLoads $82.1121 \mathrm{E}+02 \quad .4395 \mathrm{E}+02-.2609 \mathrm{E}+01 \quad-1212 \mathrm{E}+04-.2003 \mathrm{E}+03-.5831 \mathrm{E}+02$

GLoads $98-.7203 \mathrm{E}+00=-1602 \mathrm{E}+02 \quad .130 \% \mathrm{E}+02-.1428 \mathrm{E}+04 \quad-2003 \mathrm{E}+03-.1058 \mathrm{E}+03$

LLoads $98-.1602 E+02 \quad .7283 E+00 \quad .1309 E+02 \quad .2003 E+03 \quad .1428 E+04=.1058 E+03$

GLosds $98.1603 E+02.4571 E+02.2773 \mathrm{E}+02 \quad .1620 \mathrm{E}+04 \quad .2643 \mathrm{E}+03 \cdot-1168 \mathrm{E}+03$ 


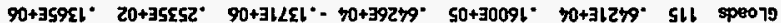

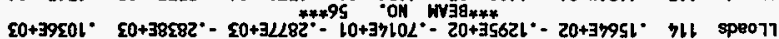

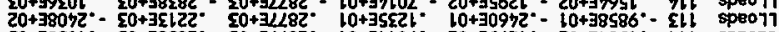

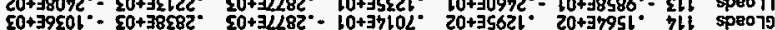

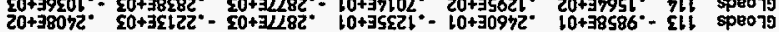

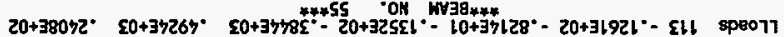

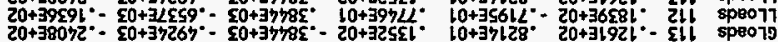

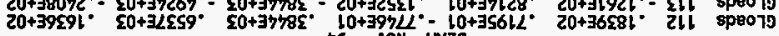

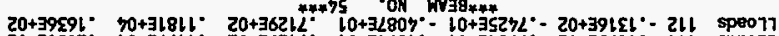

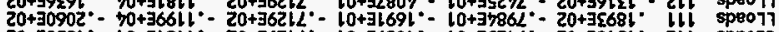

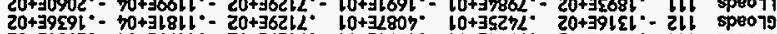

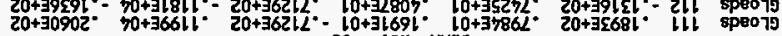

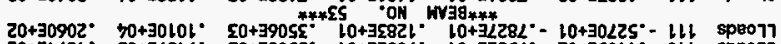

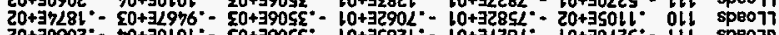

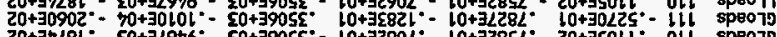

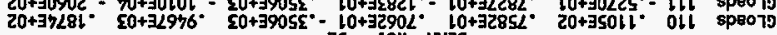

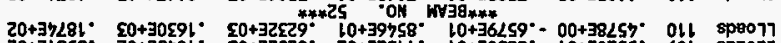

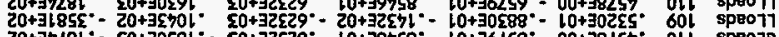

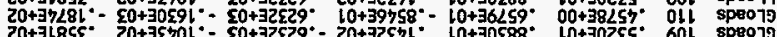

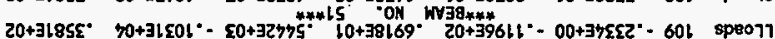

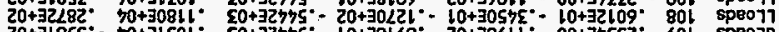

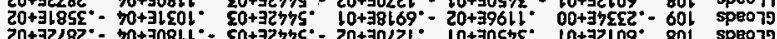

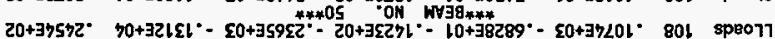

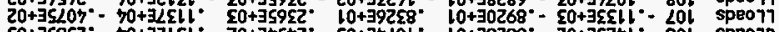

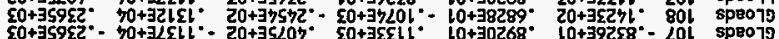

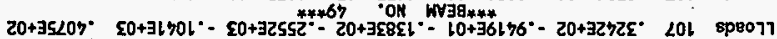

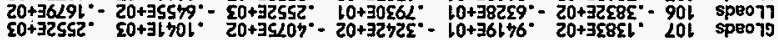

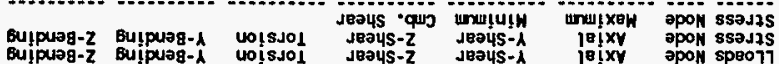

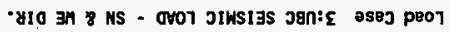

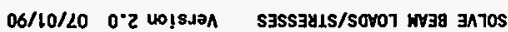

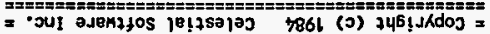
$82: 05: 80$
$56 / 22 / 20$

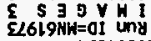

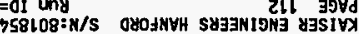

$50+32552^{\circ}-20+355 \% 9^{\circ} 20+36 \angle 91^{\circ} 20+32585^{\circ} \quad 10+38259^{\circ} \quad 10+3056 L^{\circ}-906$ speo70

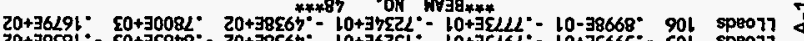

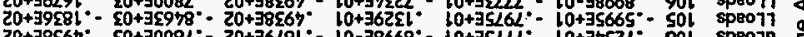

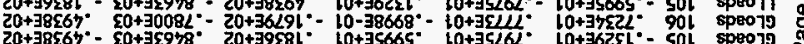

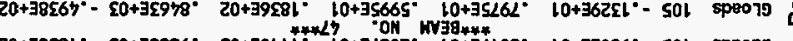

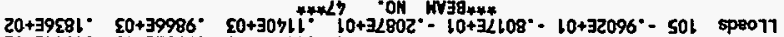

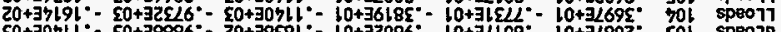

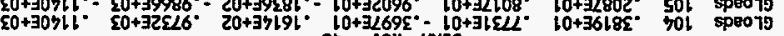

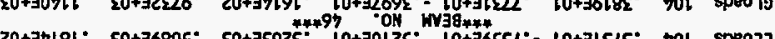

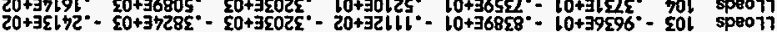

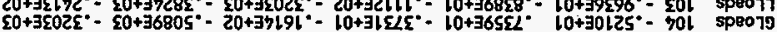

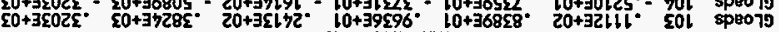

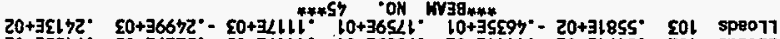

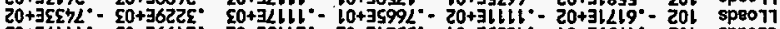

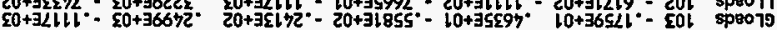

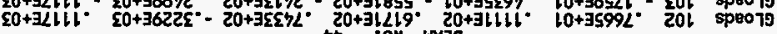

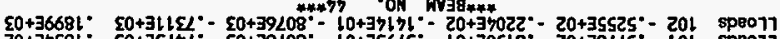

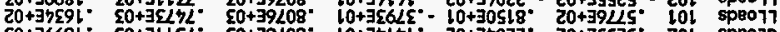

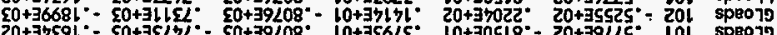

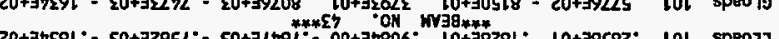

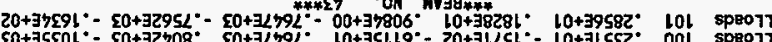

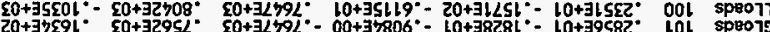

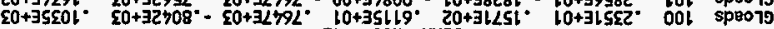

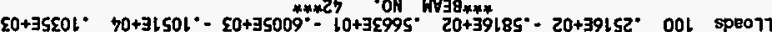

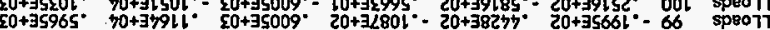

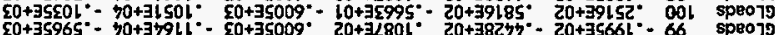

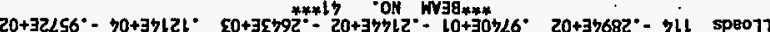

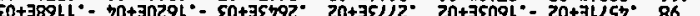

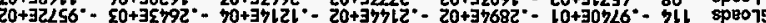

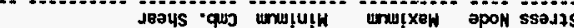

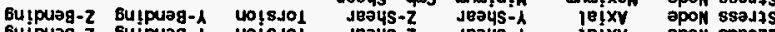

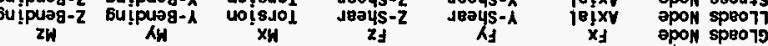

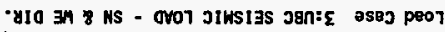

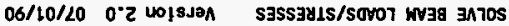

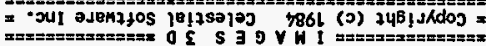
8ร:05:80

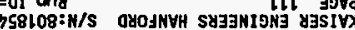


KAISER ENGINEERS HANFORD S/N:801854

PAGE 113

$$
\text { Run iD=NH61973 }
$$

$02 / 22 / 95$
$08: 50: 38$

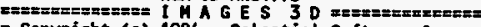
= Copyright (c) 1984 Celestial Software Inc. =

SOLVE BEAH LOADS/STRESSES Version 2.0 07/01/90 L

Load Case 3:UBC SEISHIC LOAD - SH \& WE DIR.

\begin{tabular}{|c|c|c|c|c|c|c|c|}
\hline $\begin{array}{l}\text { SLoads } \\
\text { LLoods } \\
\text { Stress } \\
\text { Stress }\end{array}$ & $\begin{array}{l}\text { Node } \\
\text { Node } \\
\text { Node } \\
\text { Node }\end{array}$ & $\begin{array}{c}\text { Fx } \\
\text { Axial } \\
\text { Axial } \\
\text { Maximum }\end{array}$ & $\begin{array}{c}\text { Fy } \\
\text { Y-shear } \\
\text { Y-Shear } \\
\text { Minimum }\end{array}$ & $\begin{array}{c}\text { Fz } \\
\text { z-Shear } \\
\text { z-shear } \\
\text { mb. Shear }\end{array}$ & $\begin{array}{l}\text { Mx } \\
\text { Torsion } \\
\text { Torsion }\end{array}$ & $\begin{array}{l}\text { My } \\
\text { Y-Bending } \\
Y-\text { Bending }\end{array}$ & $\begin{array}{l}\text { Mz } \\
\text { Z-Bending } \\
\text { Z-Bending }\end{array}$ \\
\hline $\begin{array}{l}\text { GLoads } \\
\text { Loods } \\
\text { Loods }\end{array}$ & $\begin{array}{l}120 \\
115 \\
120\end{array}$ & $\begin{array}{r}.6223 \mathrm{E}+04 \\
.1600 \mathrm{E}+05 \\
.1547 \mathrm{E}+05\end{array}$ & $\begin{array}{r}-.95476+05 \\
-.8316 E+04 \\
.8060 E+04\end{array}$ & & $\frac{-}{2}$ & & \\
\hline $\begin{array}{l}\text { GLoads } \\
\text { GLoads } \\
\text { LLoads } \\
\text { LLoads }\end{array}$ & $\begin{array}{l}120 \\
121 \\
120 \\
121\end{array}$ & $\begin{array}{r}-.5027 \mathrm{E}+04 \\
.5261 \mathrm{E}+04 \\
.1453 \mathrm{E}+05 \\
-.1515 \mathrm{E}+05\end{array}$ & $\begin{array}{r}-.145 \\
.151 \\
.650 \\
. .680\end{array}$ & & $\begin{array}{r}.19 \\
-.12 \\
-.25\end{array}$ & & \\
\hline $\begin{array}{l}\text { GLoads } \\
\text { GLoads } \\
\text { LLoads } \\
\text { LLoads }\end{array}$ & $\begin{array}{l}116 \\
121 \\
116 \\
121\end{array}$ & $\begin{array}{r}.2351 E+04 \\
-.2273 E+04 \\
.2351 E+04 \\
-.2273 E+04\end{array}$ & $\begin{array}{r}.52 \\
-.50 \\
.52 \\
-.50\end{array}$ & & & $\begin{array}{r}.2420 E+03 \\
-.8539 E+04 \\
.2420 E+03 \\
-.8539 E+04\end{array}$ & $\begin{array}{r}-.89 \\
.11 \\
-.89 \\
.11\end{array}$ \\
\hline $\begin{array}{l}\text { GLoads } \\
\text { GLoads } \\
\text { LLoads } \\
\text { LLoads }\end{array}$ & $\begin{array}{l}117 \\
121 \\
117 \\
121\end{array}$ & $\begin{array}{r}.4372 E+03 \\
-.3587 \mathrm{E}+03 \\
.2349 \mathrm{E}+04 \\
-.2270 \mathrm{E}+04\end{array}$ & & & & $\begin{array}{r}-.23 \\
.85 \\
. .23 \\
.85\end{array}$ & $\begin{array}{r}-.18 \\
.18 \\
-.85 \\
.11\end{array}$ \\
\hline $\begin{array}{l}\text { GLoads } \\
\text { GLoads } \\
\text { LLoads } \\
\text { LLoads }\end{array}$ & $\begin{array}{l}118 \\
121 \\
118 \\
121\end{array}$ & $\begin{array}{r}.2351 \mathrm{E}+04 \\
-.2273 \mathrm{O}+04 \\
-.2351 \mathrm{E}+04 \\
.2273 \mathrm{E}+04\end{array}$ & & & & $\begin{array}{r}-.1 \\
.8 \\
-.1 \\
.8\end{array}$ & $\begin{array}{r}-.2 \\
-.3 \\
.2 \\
.3\end{array}$ \\
\hline $\begin{array}{l}\text { Loads } \\
\text { Loads } \\
\text { Loads } \\
\text { Loads }\end{array}$ & $\begin{array}{l}119 \\
121 \\
119 \\
121\end{array}$ & $\begin{array}{r}.4345 E+03 \\
-.3560 E+03 \\
-.2349 E+04 \\
.2270 E+04\end{array}$ & 14 & & $\begin{array}{r}.2379 E+05 \\
.3166 E+05 \\
.1881 E+05 \\
.1881 E+05\end{array}$ & $\begin{array}{r}.280 \\
. .858 \\
.280 \\
-.858\end{array}$ & $\begin{array}{r}-.1881 E+05 \\
.1881 E+05 \\
.2379 E+05 \\
.3166 E+05\end{array}$ \\
\hline $\begin{array}{l}\text { Loads } \\
\text { Loads } \\
\text { Loads } \\
\text { Loads }\end{array}$ & $\begin{array}{l}125 \\
127 \\
125 \\
127\end{array}$ & $\begin{array}{l}.3747 \\
.4649 \\
.5277\end{array}$ & & & $\begin{array}{r}-.2207 \mathrm{E}+04 \\
.2255 \mathrm{E}+04 \\
.1633 \mathrm{E}+03 \\
\therefore 1633 \mathrm{E}+03\end{array}$ & $\begin{array}{r}.11 \\
-.11 \\
-.2\end{array}$ & $\begin{array}{r}-.1 \\
.2 \\
-.1 \\
.2\end{array}$ \\
\hline $\begin{array}{l}\text { Loads } \\
\text { Loads } \\
\text { Loads } \\
\text { Loods }\end{array}$ & $\begin{array}{l}127 \\
129 \\
127 \\
129\end{array}$ & $\begin{array}{r}.431 \\
.407 \\
.739 \\
.297\end{array}$ & & & $\begin{array}{l}-.2501 E \\
.2476 E \\
-.3836 E\end{array}$ & $\begin{array}{r}. .38 \\
.38 \\
.25 \\
. .24\end{array}$ & $\begin{array}{r}-.21 \\
.18 \\
-.2 \\
.18\end{array}$ \\
\hline oeds & 129 & $.4148 E+01$ & & & & & $2 E+C$ \\
\hline
\end{tabular}

KAISER ENGINEERS HANFORD S/H:801854

$\begin{array}{lll}\text { PAGE } 114 \quad 02 / 22 / 95 \\ \text { RUn ID=HN61973 } & 08: 50: 39\end{array}$

$\begin{array}{lll}\text { KAISER ENGINEERS HAHFORD } S / H: 801854 & 02 / 22 / 95 \\ \text { PAGE } 114 \quad & 08: 50: 39\end{array}$ = Copyright (c) 1984 Celestial Software Inc. a

SOLVE BEAM LOADS/STRESSES Version $2.0 \quad 07 / 01 / 90$

$\mathbf{L}$

LaAd Case 3:UBC SEISMIC LOAD - SN \& WE DIR.

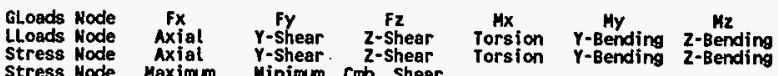
Stress Hode Maximum Minimum Crob. Shear

Cla

$\begin{array}{lllllllll}\text { GLoads } & 131 & .4234 E+01 & .1679 E+01 & .9813 E+01 & .1347 E+04 & .4297 E+03 & .1947 E+02\end{array}$

LLosds $129 \quad .2067 \mathrm{E}+02-.4148 \mathrm{E}+01-.1431 \mathrm{E}+01-.4297 \mathrm{E}+03 \quad .1471 \mathrm{E}+04-.1852 \mathrm{E}+02$

LLoads $131: .1679 E+01-.4234 \mathrm{E}+01.9813 \mathrm{E}+01-.4297 \mathrm{E}+03-.1347 \mathrm{E}+04 \quad .1947 \mathrm{E}+02$

GLoads $131 \quad .5817 E+01.6684 E+02 \quad .5839 E+01 \quad .2545 E+03-.1356 E+03-.1947 E+02$

GLoads $133 \quad .4660 \mathrm{E}+01-.3890 \mathrm{E}+02 \quad .4638 \mathrm{E}+01-.2380 \mathrm{E}+03 \quad .1356 \mathrm{E}+03 \quad .3554 \mathrm{E}+01$

LLo8ds $131 \quad .6684 \mathrm{E}+02-.5817 \mathrm{E}+01 \quad .5839 \mathrm{E}+01 \quad-.1356 \mathrm{E}+03 \quad-.2545 \mathrm{E}+03 \quad-.1947 \mathrm{E}+02$

LLoads $133-.3890 \mathrm{E}+02-.4660 \mathrm{E}+01 \quad .4638 \mathrm{E}+01 \quad .1356 \mathrm{E}+03 \quad .2380 \mathrm{E}+03 \quad .3554 \mathrm{E}+01$

GLoads $133 \quad .3947 E+00 \quad .3833 E+02-.4779 E+01 \quad .7221 E+03 \quad .6997 E+03-.3554 E+01$

GLoads $135 \quad .5892 \mathrm{E}+01-.2157 \mathrm{E}+02 \quad .1107 \mathrm{E}+02-.8528 \mathrm{EE}+03-.6997 \mathrm{E}+03 \quad .4890 \mathrm{E}+02$

LLoads $133 \quad .3833 E+02-.3947 E+00-.4779 E+01 \quad .6997 E+03-.7221 E+03-.3554 E+0$

Lloads $135-.2157 \mathrm{E}+02-.5892 \mathrm{E}+01 \quad 1107 \mathrm{E}+02-.6997 \mathrm{E}+03 \quad .8528 \mathrm{E}+03 \quad .4890 \mathrm{E}+02$

GLoads $115-.8331 E+03-.3994 E+04 \quad .93 \mathrm{BgE}+02 \quad .3331 \mathrm{E}+05-.2391 \mathrm{E}+04-.3435 \mathrm{E}+05$

GLoads $116 \quad .1272 E+04 \quad .5163 \mathrm{E}+04 \quad .3446 \mathrm{E}+03 \quad-.1883 \mathrm{E}+05 \quad-.2420 \mathrm{E}+03 \quad .8949 \mathrm{E}+04$

LLoads $115-.4078 E+04-.1052 E+03 \quad .9389 E+02-.3606 E+04 \quad .3320 E+05-.3435 E+05$

LLoads $116 \cdot .5307 \mathrm{E}+04 \quad .3275 \mathrm{E}+03 \quad .3446 \mathrm{E}+03 \quad .3606 \mathrm{E}+04 \quad-.1848 \mathrm{E}+05 \quad .8949 \mathrm{E}+04$

GLoads $115 \quad .8813 E+02-.3995 E+04-.8276 E+03 \quad .3373 E+05 \quad .2517 E+04-.3394 E+05$

$\begin{array}{llllllll}\text { GLoads } & 117 & .3504 \mathrm{E}+03 & .5164 \mathrm{E}+04 & .1266 \mathrm{E}+04 & -.8981 \mathrm{E}+04 & .2366 \mathrm{E}+03 & -1879 \mathrm{E}+05\end{array}$

LLoads $117-.4076 E+04-.996 \mathrm{E}+02=.8013 E+02-3595+04-.3384 E+05-.3373 E+05$

LLoads $117-.5307 \mathrm{E}+04 \quad .3220 \mathrm{E}+03 \quad-3504 \mathrm{E}+03-3595 \mathrm{E}+04 \quad .1845 \mathrm{E}+05 \quad .8981 \mathrm{E}+04$

GLoads $115.9599 E+03+.4050 E+04 \quad .9345 E+02 \quad .3334 E+05 \quad .2444 E+04-.3555 E+05$

$\begin{array}{lllllll}\text { GLoads } 118 & -.5214 E+03 & .5220 E+04 & .3451 E+03 & -.1831 E+05 & .1982 E+03 & -2376 E+05\end{array}$

LLoads $115 \quad-.4157 E+04-.2199 E+03 \quad-.9345 E+02 \quad .3560 E+04-.3324 E+05 \quad-3555 E+05$

LLoads $118-.5229 E+04-.4208 E+03-.3451 E+03-.3560 E+04 \quad .1847 E+05-.2376 E+05$

$\begin{array}{llllllll}\text { GLoods } & 115 & .8857 E+02 & -.4049 E+04 & .9654 E+03 & .3493 E+05 & -.2464 E+04 & -.3391 E+05\end{array}$

GLoads $119-.3500 E+03 \quad .5219 E+04-.5268 E+03-.2379 E+05-.2803 E+03 \quad .1881 E+05$

$\begin{array}{lllllll}\text { LLoods } 119 & -.4157 \mathrm{E}+04 & -.2254 \mathrm{E}+03 & .8857 \mathrm{E}+02 & -.3641 \mathrm{E}+04 & .3380 \mathrm{E}+05 & .3493 \mathrm{E}+05 \\ -.3229 \mathrm{E}+04 & -.4153 \mathrm{E}+03 & .3500 \mathrm{E}+03 & .3641 \mathrm{E}+04 & -.1846 \mathrm{E}+05 & -.2379 \mathrm{E}+05\end{array}$ 
KAISER ENGINEERS HANFORD S/N:80t854

PAGE 115

N:80t854
Run ID=NH61973

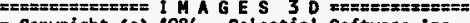

* Copyright (c) 1984 Celestial Software Inc. =

SOLVE BEAH LOADS/STRESSES Version 2.0 07/01/90

Loed Case 3:UBC SEISMIC LOAD - SH \& WE DIR.

\section{MAXIMUA STRESS SUMMARY FOR BEAMS/TRUSSES} HITHIN SPECIFIED RAMGE 1 - 70

Maximum (absolute) stress $=.1381 \mathrm{E}+04$ at BEAH 56

Beam Axial Y-Shear Z-Shear Torsion Y-Bending Z-Bending $56.4396 E+03-.4580 E+03 \quad .2013 E+03 \quad .1440 E-02 \quad-.2882 E+03-.6535 E+03$ $.4396 E+03$
Maximm Minimem comb. Shear

$.1381 E+04-.5021 E+03 \quad .6907 E+03$
$02 / 22 / 95$

$08: 50: 39$

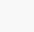

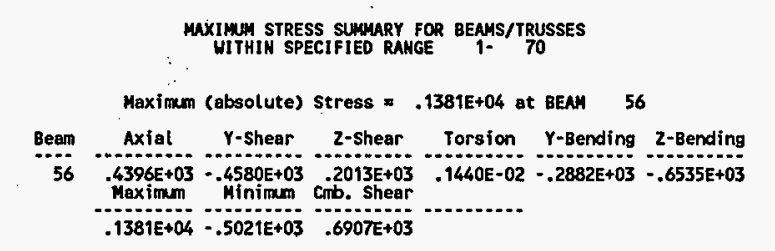

KAISER ENGINEERS HANFORD S/N:801854

PAGE 116 RUN ID=HN61973

c Copyright (c) 1984 Celestial Softuare Inc. =

SOLVE PLATE LOADS/STRESSES Version 2.0 07/01/90

L

Load Case 3:UBC SEISHIC LOND - SH \& WE DIR.

PLATE LOADS AND/OR STRESSES

GLoads Node Fx Fy $\quad$ Fy $\quad$ Fz Mx My

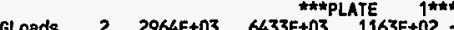

$\begin{array}{llllllll}\text { GLoads } \quad 2 & .2964 E+03 & .6433 E+03 & -1163 E+02 & -.4237 E+02 & .1521 E+03 & .0000 E+00\end{array}$

$\begin{array}{llllllll}\text { GLoads } & 3 & .4130 E+02 & .2312 E+02 & .1090 E+02 & .1954 E+03 & .4181 E+02 & .0000 E+00\end{array}$

$\begin{array}{llllllll}\text { GLoads } & 20 & -.2630 E+03 & -.5667 \mathrm{E}+03 & -.6010 \mathrm{E}+01 & .2849 \mathrm{E}+03 & -.1646 \mathrm{E}+03 & .0000 \mathrm{E}+00 \\ \text { GLoads } & 19 & -.7470 \mathrm{E}+02 & -.9978 \mathrm{E}+02 & -.1651 \mathrm{E}+02 & .5767 \mathrm{E}+02 & .3753 \mathrm{E}+02 & .0000 \mathrm{E}+00\end{array}$

GLoads

GLoads

GLoads

3.

$\begin{array}{lll}.5767 E+02 & .3753 E+02 & .0000 E+00\end{array}$

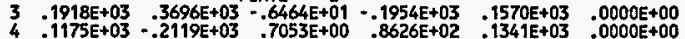

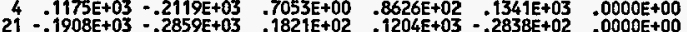

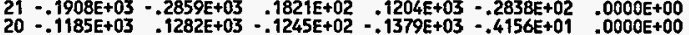

W*:APLATE $3 * \pi \star$

$\begin{array}{llllllll}\text { GLoads } \quad 4 & .4526 E+02 & -.6158 E+01 & -.1401 E+02 & -.8626 E+02 & -4115 E+02 & -0000 E+00\end{array}$

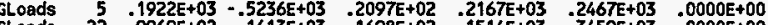
GLoads $22=.9969 E+02 \quad .1413 E+03.1698+02 \quad-1514 E+03 \quad .3459 E+03 \quad .0000 E+00$

GLoads

GLoads $6=.1537 \mathrm{E}+02-.7051 \mathrm{E}+02 \quad .4366 \mathrm{E}+02 \quad .0000 \mathrm{E}+00-.5072 \mathrm{E}+02 \quad .5499 \mathrm{E}+02$

GLoads $23-.2367 \mathrm{E}+02 \quad .4521 \mathrm{EE}+03-.1756 \mathrm{E}+03 \quad-.1043 \mathrm{E}+03 \quad .3827 \mathrm{E}+02 \quad .0000 \mathrm{E}+00$

GLoads $22.2044 E+02 \quad .2071 E+03-.9324 E+02 \quad .0000 E+00-.3659 E+03-.8176 E+02$

GLoads $6.1954 E+01-.3016 E+03 \quad .1852 E+03 \quad .0000 E+00-.7901 E+02-.5499 E+02$

GLoads $\quad 7 \quad .5485 \mathrm{E}+00 \quad-2714 \mathrm{E}+03 \quad 1730 \mathrm{E}+03 \quad .0000 \mathrm{E}+00 \quad-.1446 \mathrm{E}+03 \quad .9988 \mathrm{E}+02$

GLoads $24-1420 \mathrm{E}+02 \quad .2369 \mathrm{E}+03=1849 \mathrm{E}+03=5115 \mathrm{E}+01-5299 \mathrm{E}+02 \quad .0000 \mathrm{E}+00$

GLoads $23=.1170 \mathrm{E}+02-.2068 \mathrm{E}+03-.1732 \mathrm{E}+03 \quad .1051 \mathrm{E}+03 . .6493 \mathrm{E}+02 \quad .0000 \mathrm{E}+00$

GLoads $7.1351 E+02-.1351 E+03 \quad .1408 E+03.0000 E+00 \quad .2730 E+02-.9988 E+02$

$\begin{array}{llllllll}\text { GLoads } & 7 & .1351 \mathrm{E}+02 & -.1351 \mathrm{E}+03 & -1408 \mathrm{E}+03 & .0000 E+00 & .2730 \mathrm{E}+02 & -.9988 E+02\end{array}$

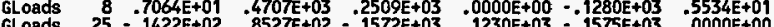

$\begin{array}{llllllll}\text { GLoads } & 25 & -.1422 E+02 & .8527 E+02 & -.1572 E+03 & .1230 E+03 & -.1575 E+03 & .0000 E+00 \\ \text { GLoads } & 24 & -.6354 E+01 & -.4208 E+03 & -.2345 E+03 & .2353 E+03 & .1473 E+03 & .0000 E+00\end{array}$

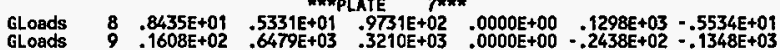

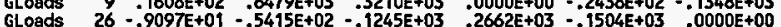

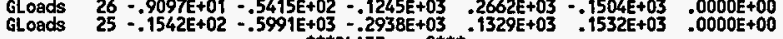

$\begin{array}{lrllllll}\text { GLoeds } & 9=.3283 E+01 & .1535 E+03 & .5899 E+02 & .0000 E+00 & .1465 E+03 & .1348 E+03 \\ \text { GLoads } & 10-.8035 E+01 & .8434 E+03 & .3847 \mathrm{E}+03 & 0000 \mathrm{0}+00 & .8707 \mathrm{E}+02 & .6037 \mathrm{E}+02\end{array}$

$\begin{array}{lllllll}\text { GLoads } 10 & -.8035 E+01 & .8434 E+03 & -3847 \mathrm{E}+03 & -0000 E+00 & -8707 \mathrm{E}+02 & .6037 \mathrm{E}+02\end{array}$

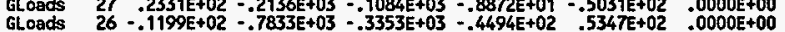


KAISER ENGIHEERS HANFORD S/W:801854

PAGE 117

Run $10=41461973$

$02 / 22 / 95$

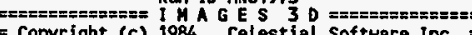
= Copyright (c) 1984 Celestial Software Inc.

SOLVE PLATE LOADS/STRESSES Version 2.0 07/01/90

Load Case 3:UBC SEISHIC LOAD - SN \& WE DIR.

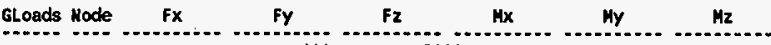
n*:PLATE O*t:

GLoads $10-.5051 \mathrm{E}+01 \quad .4510 \mathrm{E}+03-.2945 \mathrm{E}+02 \quad .0000 \mathrm{E}+00 \quad .4052 \mathrm{E}+02-.6037 \mathrm{E}+02$

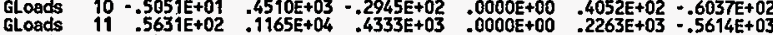

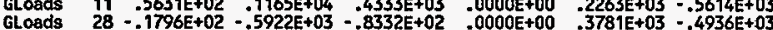

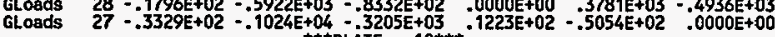

GLoads $\$ 1.4273 E+03 \quad .1138 E+04 \quad .3733 E+02.4147 E+03-.3161 E+03 \quad .0000 E+00$

$\begin{array}{llllllll}\text { GLoads } & 11 & .4273 E+03 & .1138 E+04 & .3733 E+02 & .4147 E+03 & -.3161 E+03 & .0000 E+00 \\ \text { GLods } & 12 & -.6483 E+02 & .4854 E+03 & -.1401 E+02 & -.9048 E+02 & -.8074 E+02 & .0000 E+00\end{array}$

GLoads $29=3202 E+03-1011 E+04=.3034 E+02-.1428 E+03-.8073 E+02 \quad-0000 E+00$

GLoads $28-.4230 E+02-.6121 E+03 \quad .7022 E+01-3317 E+03-.4092 E+03 \quad .0000 E+00$

GLoads

Gloads

GLoads

Clogds

Gloads

GLoads

Gloads

Gloads

GLoads

GLods

GLoads

GLaads

GLoads

GLoads

GLoeds

GLoads

GLoads

GLoads

GLaads

\#**PLATE 11***

$12 \quad-3923 \mathrm{E}+03 \quad-8641 \mathrm{E}+03-.2338 \mathrm{E}+01 \quad .9048 \mathrm{E}+02-.1782 \mathrm{E}+03 \quad .0000 \mathrm{E}+00$

$130.1635 \mathrm{E}+02 \quad .2233 \mathrm{E}+03-.2964 \mathrm{E}+01 \cdot-2435 \mathrm{E}+03 \cdot .2208 \mathrm{E}+03 \quad .0000 \mathrm{E}+00$

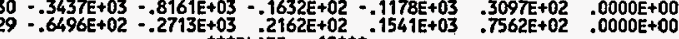

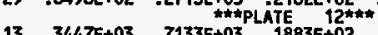

$\begin{array}{llllllll} & .3447 E+03 & .7133 E+03 & .1883 E+02 & .2435 E+03 & -.7281 E+02 & .0000 E+00\end{array}$

$14.4384 \mathrm{E}+02 \quad .1154 \mathrm{E}+03 \quad .8492 \mathrm{E}+01-.1363 \mathrm{E}+03 \quad-.2421 \mathrm{E}+03 \quad .0000 \mathrm{E}+00$

$30=.3149 \mathrm{E}+03 \quad-.6790 \mathrm{E}+03 \quad-.2196 \mathrm{E}+02 \quad .6377 \mathrm{E}+02 \quad-.9285 \mathrm{E}+02 \quad .0000 \mathrm{E}+00$

$14.2055 \mathrm{E}+03 \quad 5030 \mathrm{E}+03$ PLÁTE 13*** 1363E+03 4714E+02 0000E+00

$\begin{array}{lllllll}14 & -2955 E+03 & -5930 E+03 & .1478 E+02 & .1363 E+03 & .4714 E+02 & .0000 E+00\end{array}$

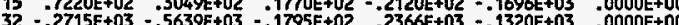

$31=.9620 \mathrm{E}+02-.5963 \mathrm{E}+02=.1454 \mathrm{E}+02 \quad .3629 \mathrm{E}+03 \quad \cdot .2507 \mathrm{E}+03 \quad .0000 \mathrm{E}+00$

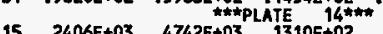

$\begin{array}{lllllll}15 & .2406 \mathrm{E}+03 & .4742 \mathrm{E}+03 & .1310 \mathrm{E}+02 & .2120 \mathrm{E}+02 & .1118 \mathrm{E}+03 & .0000 \mathrm{E}+00\end{array}$

$35=9831 E+02-.4880 E+02-.8338 E+01-.3164 E+02-.8802 E+02 \quad .0000 E+00$

$32=.1197 \mathrm{E}+03 \quad-.1732 \mathrm{E}+02=.5338 \mathrm{1610E}+02 \quad-.2056 \mathrm{E}+03 \quad-.1582 \mathrm{E}+03 \quad .0000 \mathrm{E}+00$

16 **:PLATE 15*t*

$.0000 \mathrm{E}+00$

$\begin{array}{lllllll}16 & .1811 E+03 & .3419 E+03 & -1133 E+02 & -3164 E+02 & -1362 E+03 & .0000 E+00\end{array}$

$34-1650 \mathrm{E}+03-3039 \mathrm{E}+03-2209 \mathrm{E}+02 \quad .5245 \mathrm{E}+03-1685 \mathrm{E}+02.000 \mathrm{0}-00 \mathrm{E}+00$

$33=.1423 \mathrm{E}+03 \cdots 1039 \mathrm{E}+03 \cdot .2891 \mathrm{E}+02 \quad .2294 \mathrm{E}+03 \quad .1138 \mathrm{E}+03 \quad .0000 \mathrm{E}+00$

6320E+03

GLoads $19.2203 E+03 \quad .6329 E+03-5448 E+01-.1939 E+03 \quad .3037 E+03 \quad .0000 E+00$

GLoads

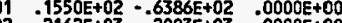

$36-.2128 E+03-.5570 E+03 \quad .1021 E+02 \quad .2162 E+03-.2003 E+03 \quad .0000 E+00$

GLods
KAISER EKGINEERS HANFORD S/N:801854 Run ID=NN61973

$02 / 22 / 95$

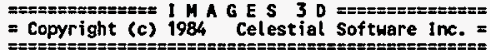

SOLVE PLATE LOADS/STRESSES Version $2.0 \quad 07 / 01 / 90$

Load Case 3:UBC SEISMIC LOAD - SH \& NE DIR.

GLoads Node Fx $F y$ Fy $\quad$ Fz

$\begin{array}{llllllll}\text { GLoads } & 21 & .1311 E+03 & -.2345 E+03 & .2357 \mathrm{E}+02 & .9219 \mathrm{E}+02 & .2157 \mathrm{E}+03 & .0000 \mathrm{E}+00\end{array}$

GLoads $\quad 37=.2094 \mathrm{E}+03-.3069 \mathrm{E}+03 \quad .2578 \mathrm{E}+02 \quad .1469 \mathrm{E}+03 \quad .1225 \mathrm{E}+03 \quad .0000 \mathrm{E}+00$

$\begin{array}{llllll}0 & 0000 & .000+00\end{array}$

$\begin{array}{lllllllll}\text { GLoads } & 21 & .1656 \mathrm{E}+03 & .4695 \mathrm{E}+02 & -.4975 \mathrm{E}+02 & -.8387 \mathrm{E}+02 & -.7225 \mathrm{E}+02 & .0000 \mathrm{E}+00\end{array}$

GLosds $22 \quad .6348 E+01-.2864 E+03 \quad .2420 E+02-.6042 E+02 \quad .7133 E+03 \quad .0000 E+00$

GLoads $38=.1398 E+03 \quad .9625 E+01 \quad .6303 E+02-.2113 E+03 \quad .7430 E+03 \quad .0000 E+00$

$\begin{array}{llllllll} & & .3219 E+02 & .2299 E+03 & -.3747 E+02 & -.2067 E+03 & -.1919 E+03 & .0000 E+00\end{array}$

GLoads $22 \quad .3215 E+02-.3023 E+03 \quad .1297 E+02 \quad .0000 E+00-.6631 E+03-.1047 E+02$

GLoads $23=.4362 \mathrm{E}+02=.3616 \mathrm{E}+02 \quad .1444 \mathrm{E}+03-.8400 \mathrm{E}+01 \quad .6730 \mathrm{E}+01-.0000 \mathrm{E}+00$

GLoads $39-.3737 \mathrm{E}+02 \quad .2595 \mathrm{E}+03-.2397 \mathrm{E}+02-.1224 \mathrm{E}+03 \quad .9908 \mathrm{EE}+02 \quad .0000 \mathrm{E}+00$

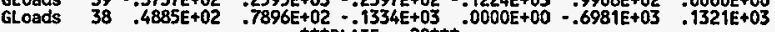

GLoads $23.1940 E+02-.3056 E+03.1683 E+03 \quad .7684 E+01-.1099 E+03 \quad .0000 E+00$

GLoads $24-.2170 E+02 \quad .2638 E+03 \quad .1894 \mathrm{E}+03-.1448 \mathrm{E}+03-.2872 \mathrm{E}+03 \quad .0000 \mathrm{E}+00$

GLoads $\quad 40-.2451 \mathrm{E}+02 \quad .2439 \mathrm{E}+03-.1497 \mathrm{E}+03-.1825 \mathrm{E}+02-.2541 \mathrm{E}+03 \quad-0000 \mathrm{E}+00$

GLoads $39.2681 E+02-.2021 E+03-2080 E+03-.1049 E+03-.6501 E+02 \quad .0000 E+00$

GLoads $24 \quad .6068 E+01-.1764 E+03 \quad-1938 E+03+.8530 E+02 \quad .1929 E+03 \quad .0000 E+00$

GLoads $25-.2886 \mathrm{E}+01 \quad .4626 \mathrm{E}+03 \quad .2225 \mathrm{E}+03-.1248 \mathrm{E}+03-.3243 \mathrm{E}+03 \quad .0000 \mathrm{E}+00$

GLoads $\quad 41-.7925 \mathrm{E}+01.1282 \mathrm{E}+03-.1911 \mathrm{E}+03 \quad .1382 \mathrm{E}+03-.3258 \mathrm{~F}+03 \quad .0000 \mathrm{E}+00$

$\begin{array}{llllll} & -472 E+01-.4144 E+03 & -.2251 E+03 & .1419 E+03 & .2897 E+03 & .0000 E+00\end{array}$

GLcads $25-.3666 \mathrm{E}+01-.4533 \mathrm{E}+02 \quad .1923 \mathrm{E}+03-.1310 \mathrm{E}+03 \quad .3286 \mathrm{E}+03 \quad .0000 \mathrm{E}+00$

GLoads $26 \quad .6132 \mathrm{E}+01 \quad .6125 \mathrm{E}+03 \quad .2391 \mathrm{E}+03 \quad-.8738 \mathrm{E}+02-.1829 \mathrm{E}+03 \quad .0000 \mathrm{E}+00$

GLoads $42.6400+01-61-.8935 E+01-.5670 E+03-2235 E+03 \quad 1309+03-.2757 E+03 \quad-0000 E+00$

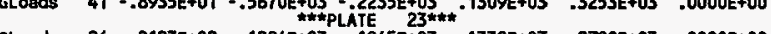

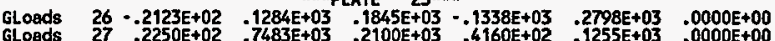

GLods $27.2250+02-7483 E+03-.2100 E+03$ - $4160 E+02-1255+03$ - $0000+00$

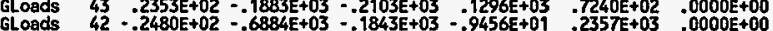

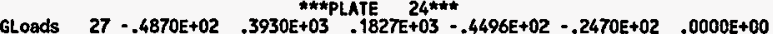

LLads $27-.4870 E+02-3930 E+03 \quad-1827 E+03-.4496 E+02-.2470 E+02 \quad-0000 E+00$

GLoads $28 \quad .2014 E+02 \quad .6916 E+03 \quad-1390 E+02 \quad .0000 E+00 \quad .6897 E+03 \quad-1279 E+03$

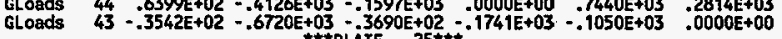

$\begin{array}{llllllll}\text { GLoads } 28 & .1259 E+02 & .6678 E+03 & -2230 E+02 & -.1071 E+03 & -.8544 E+03 & .0000 E+00\end{array}$

GLoads $29 \quad .1447 \mathrm{E}+03 \quad .4308 \mathrm{E}+03 \quad-.5580 \mathrm{E}+02 \quad-.1084 \mathrm{E}+03 \quad . .4577 \mathrm{E}+02 \quad .0000 \mathrm{E}+00$

GLoads $45-.2885 \mathrm{E}+02-.6590 \mathrm{E}+03 \quad-.4019 \mathrm{E}+02=.2338 \mathrm{E}+03 \quad .2120 \mathrm{E}+03 \quad .0000 \mathrm{E}+00$ 
KAISER ENGINEERS HANFORD S/N:801854

PAGE 119 RUn ID=An6197

$02 / 22 / 95$
$08: 50: 42$

$=$ Copyright (c) 1984 Celestial Software Inc.

SOLVE PLATE LOADS/STRESSES Version $2.0 \quad 07 / 01 / 90$

1

Load Case 3:UBC SEISMIC LOND - SN \& WE DIR.

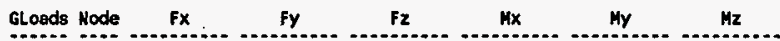

GLoads $44-.1285 E+03-.4396 E+03 \quad .7369 E+02-.2876 E+03-.8592 E+03 \quad .0000 E+00$

$\begin{array}{lllllllll} & \text { GLoods } & 29 & .2650 E+03 & .7573 E+03 & .2911 E+02 & .9709 E+02 & -.2547 E+03 & .0000 E+00\end{array}$

GLOads $30.1434 E+03 \quad .2054 E+03-.2698 E+02-.2407 E+03-.3227 E+03 \quad .0000 E+00$

GLoads $46 \cdot-1722 E+03 \cdot .7108 E+03 \cdot .3249 E+02 \cdot .3533 E+02 \cdot .1891 E+03 \quad .0000 E+00$

GLoads $\quad 45-.1762 \mathrm{E}+03-.2519 \mathrm{E}+03 \quad .3036 \mathrm{E}+02 \quad .2259 \mathrm{E}+03-.1354 \mathrm{E}+03 \quad .0000 \mathrm{E}+00$

$\begin{array}{lllllllll}\text { GLoads } & 30 & .2386 E+03 & .6659 E+03 & .1325 E+02 & -.7151 E+02 & .8819 E+02 & .0000 E+00\end{array}$

GLoads $31 \quad .1495 E+03 \quad .7224 E+02-.9132 E+01-.2915 E+03-.4407 E+03 \quad .0000 E+00$

GLoads $\quad 47-.2122 E+03 \quad-.6352 E+03 \cdots .1735 E+02 \quad .1651 E+03-.3291 E+03 \quad .0000 E+00$

GLoads $46-.1759 \mathrm{E}+03-.1030 \mathrm{E}+03 \quad .1323 \mathrm{E}+02 \quad .2885 \mathrm{E}+03 \quad .2800 \mathrm{E}+03 \quad .0000 \mathrm{E}+00$

$\begin{array}{lllllllll}\text { GLoads } & 31 & .2262 E+03 & .5720 E+03 & .1022 E+02 & -.1352 E+03 & .2829 E+03 & .0000 E+00\end{array}$

GLoads $32 \quad .1589 \mathrm{E}+03-.1583 \mathrm{E}+02 \quad .1788 \mathrm{E}+01-.2708 \mathrm{E}+03-.4078 \mathrm{E}+03 \quad .0000 \mathrm{E}+00$

GLoads $\quad 48-.2108 E+03 \cdot .5428 E+03-.1175 E+02 \quad-2788 E+03-.4091 E+03 \quad .0000 E+00$

$\begin{array}{lllllll}\text { GLoads } \quad 47 * .1743 \mathrm{E}+03 & -.1336 \mathrm{E}+02 & -2502 \mathrm{E}+00 & .3913 \mathrm{E}+03 & .3829 \mathrm{E}+03 & .0000 \mathrm{E}+00\end{array}$

$\begin{array}{lllllllll}\text { GLoads } & 32 & .1970 E+03 & .4680 E+03 & -.3155 E+01 & -.2422 E+03 & .3599 E+03 & .0000 E+00\end{array}$

GLoads $33 \cdot .1697 \mathrm{E}+03 \cdot .9320 \mathrm{0}+02 \quad .8850 \mathrm{E}+01-.2467 \mathrm{E}+03-.2594 \mathrm{E}+03 \quad .0000 \mathrm{E}+00$

GLoads $\quad 49-.1934 E+03 \cdot-.4386 E+03 \quad .6885 E+01 \quad .3124 E+03 \quad-.2820 E+03 \quad .0000 E+00$

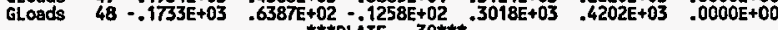

$\begin{array}{llllllll}\text { GLoads } & 33 & .1564 E+03 & .3376 E+03 & -.1001 E+02 & -.1884 E+03 & .3038 E+03 & .0000 E+00\end{array}$

GLaads $34 \quad .1715 \mathrm{E}+03-.1782 \mathrm{E}+03 \quad .3201 \mathrm{E}+02-.3724 \mathrm{E}+02 \quad .2055 \mathrm{E}+02 \quad .0000 \mathrm{O}+00$

Gloads $50=.1623 \mathrm{E}+03-.2975 \mathrm{E}+03 \quad .4555 \mathrm{E}+01 \quad .4178 \mathrm{E}+03-.3710 \mathrm{E}+02 \quad .0000 \mathrm{E}+00$

GLoads $49-.1656 \mathrm{E}+03 \quad .1380 \mathrm{E}+03 \quad-2656 \mathrm{E}+02 \quad .2919 \mathrm{E}+03 \quad .2674 \mathrm{E}+03 \quad .0000 \mathrm{EE}+00$

$\begin{array}{lllllllll}\text { GLads } & 35 & .1363 E+03 & .4628 E+03 & -.1678 E+02 & -.1839 E+03 & .3805 E+03 & .0000 E+00\end{array}$

GLoads $36: 1270 \mathrm{E}+03-.8565 \mathrm{E}+01 \quad .1700 \mathrm{E}+02-.6965 \mathrm{E}+02-.02-.1058 \mathrm{E}+03 \quad .0000 \mathrm{E}+00$

GLoads $52-.1538 \mathrm{E}+03-.4153 \mathrm{E}+03 \quad .1804 \mathrm{E}+02 \quad .1597 \mathrm{E}+03-.1426 \mathrm{E}+03 \quad .0000 \mathrm{E}+00$

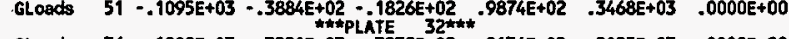

$\begin{array}{llllllll}- \text { GLoads } & 36 & .1808 E+03 & .3221 E+03 & -.3238 E+02 & -.9134 E+02 & .2025 E+03 & .0000 E+00\end{array}$

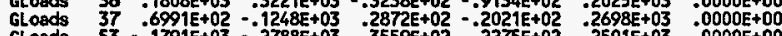

GLoads $\quad 53 \cdot-1791 E+03 \quad-.2788 E+03 \quad .3559 E+02 \quad .2275 E+02 \quad .2501 E+03 \quad .0000 E+00$

GLoods $52-.7161 E+02 \quad .8149 E+02-3193 E+02.0329 E+01 \quad .1565 E+03 \quad .0000 E+00$

$\begin{array}{lllllllll}\text { GLods } & 37 & .1398 E+03 & .1168 E+03 & -.4893 E+02 & .8003 E+02 & -.2003 E+03 & .0000 E+00\end{array}$

GLoads $38 \quad .4208 E+01-.1493 E+03 \quad 45012+02 \quad .1841 E+03 \quad .8946 E+03 \quad .0000 E+00$

$\begin{array}{llllllll}\text { GLoads } & 54 & -.1297 E+03 & -.8251 E+02 & .4367 E+02 & -.1888 E+02 & .9036 E+03 & .0000 E+00 \\ \text { GLoads } & 53 & -.1431 E+02 & .1151 E+03 & -.5074 E+02 & -.8964 E+02 & -.2357 E+03 & .0000 E+00\end{array}$

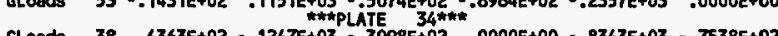

Gloads

$38-4363 E+02-.1247 E+03-.3098 E+02 \quad .0000 E+00-.8343 E+03-.7538 E+02$
KAISER EHGINEERS HANFORD S/N:801854 Run ID=MH61973

$02 / 22 / 95$

PAGE 120

Run $10=4 N 61973$

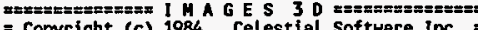

= Copyright (c) 1984 Celestial Software Inc. =

SOLVE PLATE LOADS/STRESSES Version $2.0 \quad 07 / 01 / 90$

L

LOAd Case 3:UBC SEISHIC LOAD - SH 2 VE DIR.

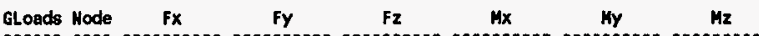

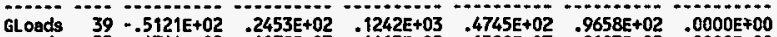

GLoads $\quad 55-.4744 E+02 \quad .1078 E+03 \quad .1415 E+02-.1590 E+03 \quad .8183 E+02 \quad .0000 E+00$

GLoads $54 \quad .5503 \mathrm{E}+02-.7644 \mathrm{E}+01 \div 1074 \mathrm{E}+03 \quad .0000 \mathrm{E}+00-.8732 \mathrm{E}+03 \quad .1307 \mathrm{E}+03$

GLoads $39.2559 E+02-.1785 E+03 \quad .7157 E+02-.2989 E+02-.1306 E+03 \quad .0000 E+00$

GLoads $40=.2565 \mathrm{E}+02 \quad .2178 \mathrm{E}+03 \quad .1851 \mathrm{E}+03-.4227 \mathrm{E}+02-.3138 \mathrm{EE}+03 \quad .0000 \mathrm{0}+000$

GLoeds $56-.2408 E+02 \quad .1465 \mathrm{E}+03-.7986 \mathrm{E}+02 \quad .5400 \mathrm{E}+02-.2573 \mathrm{E}+03 \quad .0000 \mathrm{E}+00$

GLogds $55 \quad .2413 E+02-.1858 E+03-.1768 E+03 \quad .1694 E+02-.6901 E+02 \quad .0000 E+00$

GLoads $40 \quad .9224 E+01-.1437 E+03 \quad .1535 E+03-.8139 E+02 \quad .2782 E+03 \quad .0000 E+00$

GLoads $41-.9082 E+01 \quad .3773 \mathrm{E}+03 \quad .1873 \mathrm{E}+03 \quad-.1324 \mathrm{E}+03-.4019 \mathrm{E}+03 \quad .0000 \mathrm{E}+00$

GLoads $\quad 57 \cdot-.9396 \mathrm{E}+01 \quad .1065 E+03-.1451 \mathrm{E}+03 \quad .8154 \mathrm{E}+02-.4305 E+03 \quad .0000 \mathrm{E}+00$

$\begin{array}{llllllll} & & & \end{array}$

GLoads $\quad 41-.1025 E+02-.3498 E+02 \quad .1911 E+03-.1368 E+03 \quad .4024 E+03 \quad .0000 E+00$

GLoads $42 \quad .9339 \mathrm{E}+01 \quad .4841 \mathrm{E}+03 \quad .1465 \mathrm{E}+03-.8186 \mathrm{E}+02-.2626 \mathrm{E}+03 \quad .0000 \mathrm{E}+00$

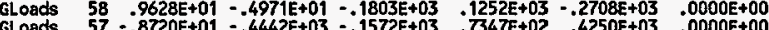

GLoads $57 \cdot .8720 E+01-.4442 E+03-.1572 E+03 \quad * 7347 E+02 \quad .4250 E+03 \quad .0000 E+00$

$\begin{array}{lllllllll}\text { GLoads } & 42 & -.2720 \mathrm{E}+02 & .1079 \mathrm{E}+03 & .2096 \mathrm{E}+03 & -.5047 \mathrm{E}+02 & .3026 \mathrm{E}+03 & .0000 \mathrm{E}+00\end{array}$

$\begin{array}{llllllll}\text { GLads } & 43 & .2497 \mathrm{E}+02 & .5074 \mathrm{E}+03 & .5591 \mathrm{E}+02 & -.3715 \mathrm{E}+02 & .1542 \mathrm{E}+03 & .0000 \mathrm{E}+00\end{array}$

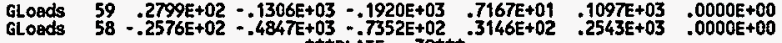

GLoads $43-.4927 E+02 \quad .2564 E+03 \quad .1551 E+03 \quad .8166 E+02-.1217 E+03 \quad .0000 E+00$

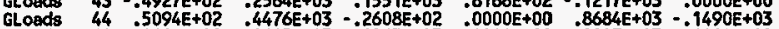

GLOads $44.5094 E+02 \quad .4476 E+03-.2608 E+02 \quad .0000 E+00 \quad .8684 E+03-.1490 E+03$

$\begin{array}{llllllll}\text { GLoads } & 60 & .4618 E+02 & -.2645 E+03 & -.1263 E+03 & .0000 E+00 & .8907 E+03 & .6418 E+02 \\ \text { GLosds } & 59 & -.4785 E+02 & -.4395 E+03 & -.2726 E+01 & -.1296 E+03 & -.1322 E+03 & .0000 E+00\end{array}$

$\begin{array}{lllllllll}\text { GLoads } & 44 & -.4320 E+02 & .4236 E+03 & .5817 E+02 & .1731 E+03 & -.1048 E+04 & .0000 E+00 \\ \text { GLoads } & 45 & .1316 E+03 & .2979 E+03 & -.5778 E+02 & .6742 E+02 & .1926 E+03 & .0000 E+00\end{array}$

$\begin{array}{llllllll}\text { GLoads } & 61 & -1316 E+03 & .29792 E+03 & -.5778 E+02 & .6742 E+02 & .1926 E+03 & .0000 E+00 \\ & 61 & -.1501 E+02 & -.4262 E+03 & -.5621 E+02 & -.1509 E+03 & .1980 E+03 & .0000 E+00\end{array}$

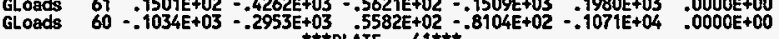

$\begin{array}{llllllllll}\text { GLods } & 45 & .3804 E+02 & .5186 E+03 & .3221 E+02 & -.5953 E+02 & -.2692 E+03 & .0000 E+00\end{array}$

$\begin{array}{llllllll}\text { GLoads } & 45 & .3804 E+02 & .5186 E+03 & .3221 E+02 & -.5953 E+02 & -.2692 E E+03 & .0000 E+00 \\ \text { GLoads } & 46 & .1887 E+03 & .1780 E+03 & -.3441 E+02 & -.9785 E+02 & -.3003 E+03 & .0000 E+00 \\ \text { GLoads } & 62 & -.5552 E+02 & -.5069 E+03 & -.3234 E+02 & . .8302 E+02 & -.2381 E+03 & .0000 E+00\end{array}$

GLoads $61-.1712 \mathrm{E}+03=.1898 \mathrm{E}+03.3454 \mathrm{E}+02 \quad .2593 \mathrm{E}+02-.2047 \mathrm{E}+03 \quad .0000 \mathrm{E}+00$

$\begin{array}{lllllllll}\text { GLoads } & 46 & .1240 E+03 & .5413 E+03 & .1826 E+02 & -.1553 E+03 & .2094 E+03 & .0000 E+00\end{array}$

GLoads $47: .1910 E+03 \quad .5931 E+02-.1924 E+02-.2502 E+03-.4734 E+03 \quad .0000 E+00$

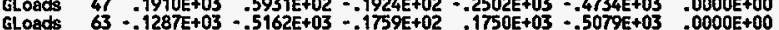


KAISER ENGINEERS HANFORD S/N:801854

PAGE 121

Run 10=41461973

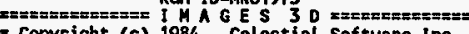

$02 / 22 / 95$

- Copyright (c) 1984 Celestiat Software Inc. =

SOLVE PLATE LOADS/STRESSES Version 2.0 07/01/90

$\mathbf{L}$

Load Case 3:UBC SEISMIC LOAD - SN WE DIR

Gloads Node Fx Fy Fy Fz

GLoads $62-.1863 E+03-.8445 E+02 \quad .1858 E+02 \quad .2088 E+03 \quad .2133 E+03 \quad .0000 E+00$

$\begin{array}{llllllllll}\text { GLoads } \quad 47 & .1607 E+03 & .4948 E+03 & .1431 E+01 & -.3062 E+03 & .4196 E+03 & .0000 E+00\end{array}$

$\begin{array}{llllllll}\text { GLoads } & 47 & -1601 E+03 & .4948 E+03 & .9431 E+01 & -.3062 E+03 & .4196 E+03 & .0000 E+00 \\ \text { GLoads } & 48 & .1859 E+03 & -.2999 E+02 & -.4465 E+01 & -.2788 E+03 & -.4708 E+03 & .0000 E+00\end{array}$

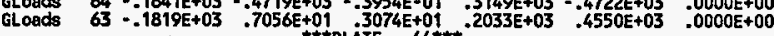

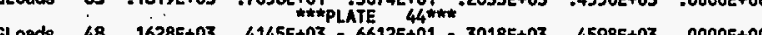

$\begin{array}{llllllll}\text { GLoods } & 48 & .1628 E+03 & .4145 E+03 & -.6612 E+01 & -.3018 E+03 & .4598 E+03 & .0000 E+00\end{array}$

$49-1710 \mathrm{E}+03-.9687 \mathrm{E}+02 \quad .6941 \mathrm{E}+01-.2915 \mathrm{E}+03-.3084 \mathrm{E}+03 \quad .0000 \mathrm{E}+00$

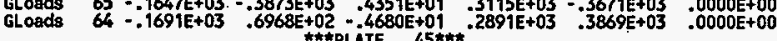

$\begin{array}{llllllllll}\text { CLoads } & 49 & .1526 E+03 & .3031 E+03 & -.2267 E+02 & -.3127 E+03 & .3230 E+03 & .0000 E+00\end{array}$

GLoads $50: 1314 \mathrm{E}+03-.1329 \mathrm{E}+03 \quad .1552 \mathrm{E}+02-.2774 \mathrm{E}+03-.1129 \mathrm{E}+02 \quad .0000 \mathrm{E}+00$

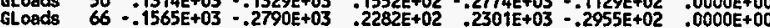

GLoads $65-.1275 \mathrm{E}+03.1088 \mathrm{0}+03-.1567 \mathrm{E}+02.2027 \mathrm{E}+03 \quad .2994 \mathrm{E}+03 \quad .0000 \mathrm{E}+00$

GLoads $51.9925 E+02 \quad .3266 E+03-.1724 E+02-.1159 E+03 \quad .3018 E+03 \quad .0000 E+00$

$52.7722+02-1648 E+02 \quad \cdot 1308 E+02-.1657 \mathrm{E}+03-.1354 \mathrm{E}+03 \quad .0000 \mathrm{E}+00$

$\begin{array}{llllllll}\text { GLoads } & 68 & -.9744 E+02 & -.3006 E+03 & .1252 E+02 & .4421 E+02 & -.9513 E+02 & .0000 E+00 \\ \text { GLoads } & 67 & -.7903 E+02 & -.4250 E+02 & -.8366 E+01 & .1459 E+03 & .2786 E+03 & .0000 E+00\end{array}$

GLoods $52 \quad .1163 E+03 \quad .2323 E+03-.3110 E+02-.2302 E+01 \quad-1215 E+03 \quad .0000 E+00$

GLoads $53.3772 \mathrm{E}+02-.4431 \mathrm{E}+02 \quad .3183 \mathrm{E}+02-.5034 \mathrm{EE}+02 \quad .2315 \mathrm{E}+03 \quad .0000 \mathrm{E}+00$

$\begin{array}{llllllll}\text { GLoads } & 69 & -.1058 E+03 & -.2036 E+03 & .2555 E+02 & -.1953 E+02 & .2655 E+03 & .0000 E+00 \\ \text { GLoads } & 68 & -.4823 E+02 & .1566 E+02 & -.2628 E+02 & .8837 E+02 & .1657 E+03 & .0000 E+00\end{array}$

GLoads $53.1238 E+03 \quad .1230 E+03-.4859 E+02 \quad .1172 E+03 \cdot .2460 E+03 \quad .0000 E+00$

GLoads $56-.2871 \mathrm{E}+02-.4275 \mathrm{E}+02 \quad .4580 \mathrm{E}+02 \quad .3616 \mathrm{E}+02 \quad .8774 \mathrm{E}+03 \quad .0000 \mathrm{E}+00$

GLoads $70-.1253 E+03-.1104 E+03 \quad .5029 E+02-.1606 E+03 \quad .8690 E+03 \quad 0000 E+00$

GLoads 69.3015E+02 $.3014 \mathrm{E}+02=.4750 \mathrm{E}+02-.5407 \mathrm{E}+02-.1880 \mathrm{E}+03 \quad .0000 \mathrm{E}+00$

- GLoads

$54.5995 E+02-.2554 E+02-.2314 E+02 \quad .0000 E+00-.8378 E+03-.2061 E+03$

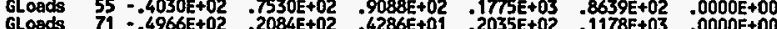

T GLoads

GLosds

GLoads

GLoads

$\overrightarrow{8}$ GLoad

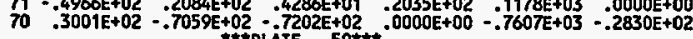

$55.2742 E+02-.9378 E+02 \quad .3558 E+02-.3542 E+02-.9921 E+02 \quad .0000 E+00$

$56-.2902 \mathrm{E}+02 \quad .1878 \mathrm{E}+03 \quad .1575 \mathrm{E}+03-.5508 \mathrm{E}+02-.2824 \mathrm{E}+03 \quad .0000 \mathrm{E}+00$

$72-.3155 E+02 \quad .8627 \mathrm{E}+02-.2644 \mathrm{E}+02 \quad .1523 \mathrm{E}+02-.3634 \mathrm{E}+03 \quad .0000 \mathrm{E}+00$

$71.3316 \mathrm{E}+02-.1803 \mathrm{E}+03-1666 \mathrm{E}+03 \quad .3992 \mathrm{E}+02-.1939 \mathrm{E}+03 \quad .0000 \mathrm{E}+00$

GLoads
KAISER ENGINEERS HANFORD S/N:801B54

PAGE 122 Run ID=NN61973

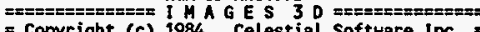
= Copyright (c) 1984 Celestial Software Inc.

SOLVE PLATE LOADS/STRESSES Version 2.0 07/01/90

Load Case 3:UBC SEISHIC LOAD - SH \& WE DIR.

GLoads Hode Fx F Fy . Fy Fy

GLoads $57 \quad-.9025 E+01 \quad .2609 E+03 \quad .1508 E+03 \quad-.8040 E+02-.4265 E+03 \quad .0000 E+00$

GLoads $73-.8217 \mathrm{E}+01 \quad .6947 \mathrm{E}+02 \quad-.6777 \mathrm{E}+02 \quad .1326 \mathrm{E}+03 \quad-.4013 \mathrm{E}+03 \quad .0000 \mathrm{O}+00$

$\begin{array}{llllllll}\text { GLoads } & 72 & .9584 \mathrm{E}+01 & -.2396 \mathrm{E}+03 & -.1650 \mathrm{E}+03 & .5202 \mathrm{E}+02 & .2887 \mathrm{E}+03 & .0000 \mathrm{E}+00\end{array}$

GLoads $57-.9047 \mathrm{E}+01-.1970 \mathrm{E}+02 \quad .1153 \mathrm{E}+03-.7461 \mathrm{E}+02 \quad .4319 \mathrm{E}+03 \quad .0000 \mathrm{E}+00$

GLoads $58 \quad .9906 E+01 \quad .3028 E+03 \quad .9667 E+02-.1178 E+03-.2612 E+03 \quad .0000 E+00$

GLads $74-1109 E+02-1960 E+01-.0046 E+02-7429 E+02-.2539 E+03 \quad .0000 E+00$

Gloads $73-.1195 E+02-.2812 E+03-1235 E+03.1371 E+03 \quad .4086 E+03 \quad .0000 E+00$

GLoads $\quad 58-.2996 E+02 \quad .9038 E+02 \quad .1210 E+03-.3882 E+02 \quad .2777 E+03 \quad .0000 E+00$

GLoads $59.3011 E+02 \quad .3181 E+03 \quad .2171 E+02-.2089 E+02 \quad .1382 E+03 \quad .0000 E+00$

$\begin{array}{llllllll}\text { GLoads } & 75 & -3223 \mathrm{E}+02 & -.1156 \mathrm{E}+03 & -.8504 \mathrm{E}+02 & .3355 \mathrm{E}+02 & .2067 \mathrm{E}+03 & .0000 \mathrm{E}+00\end{array}$

CLoads $74-.3237 E+02-.2930 E+03-.5768 E+02.2928 E+02 \quad .3437 \mathrm{E}+03 \quad .0000 \mathrm{E}+00$

GLoads $59-.4643 E+02 \quad .1554 E+03 \quad .1368 E+03 \quad .1433 E+03-.1157 E+03 \quad .0000 E+00$

GLoads $60 \quad .5303 \mathrm{E}+02 \quad .2770 \mathrm{E}+03-.4488 \mathrm{E}+02 \quad .0000 \mathrm{E}+00 \quad .8949 \mathrm{E}+03-.1272 \mathrm{E}+03$

GLoads $76 \quad-4716 \mathrm{E}+02-1465 \mathrm{E}+03-1368 \mathrm{E}+03$

TS -.5376E+02 $-.2859 E+03 \quad .4490 E+02-.4842 E+02-.9734 E+02-0000 E+00$

GLoads $\quad 60-.4323 \mathrm{E}+02 \quad .2667 \mathrm{E}+03 \quad .6629 \mathrm{E}+02 \quad .1815 \mathrm{E}+03 \quad-.1020 \mathrm{E}+04 \quad .0000 \mathrm{E}+00$

$\begin{array}{llllllll}\text { GLoads } & 61 & .1130 \mathrm{E}+03 & .1790 \mathrm{E}+03 & -.4910 \mathrm{E}+02 & .2157 \mathrm{E}+03 & .2037 \mathrm{E}+03 & .0000 \mathrm{E}+00\end{array}$

$\begin{array}{llllllll}\text { GLoads } & 77 & .3030 E+02 & -.2802 E+03 & -.5955 E+02 & .2777 E+02 & .1577 E+03 & .0000 E+00 \\ \text { GLoads } & 76 & -.1001 E+03 & -.1654 E+03 & .4236 E+02 & -.4677 E+02 & -.9890 E+03 & .0000 E+00\end{array}$

GLoads 61 TB34E+01 $3426 E+03$ PLATE 56**** $0074 E+02-.1970 E+03 \quad 0000 E+00$

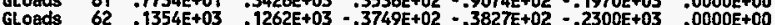

GLoads $78-3385 E+02-3338 E+03-3762 E+02-3826+02=.3801+03 \quad .0000 E+00$

GLoads $77=.1093 \mathrm{E}+03-.1350 \mathrm{E}+03 \quad .3976 \mathrm{E}+02-.3278 \mathrm{E}+0 \mathrm{01}-.3321 \mathrm{E}+03 \quad .0000 \mathrm{E}+00$

$\begin{array}{lllllllll}\text { GLoeds } & 62 & .7100 E+02 & .3707 E+03 & .1584 E+02 & -.2535 E+03 & .2548 E+03 & 0000 E+00\end{array}$

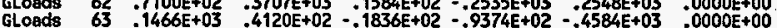

GLoads $79-9838 \mathrm{E}+02-3568 \mathrm{E}+03=.1653 \mathrm{E}+02-2557 \mathrm{E}+03-5185 \mathrm{E}+03-0000 \mathrm{~T}+00$

GLoads $78-.1192 \mathrm{E}+03-.5510 \mathrm{E}+02 \quad .1905 \mathrm{E}+02 \quad .3630 \mathrm{E}+02 \quad . .1929 \mathrm{E}+03 \quad .0000 \mathrm{E}+00$

$\begin{array}{llllllll} & \text { GLoads } & 63 & 1286 E+03 & .3735 E+03-2538 E+01 & -2845 E+03 & .5113 E+03 & 0000 E+00\end{array}$

GLoads $64 \quad 1446 \mathrm{E}+03-.3852 \mathrm{EE}+02-.6255 \mathrm{E}+01-.2213 \mathrm{E}+03-.3882 \mathrm{E}+03 \quad 0000 \mathrm{0}-000 \mathrm{E}+00$

GLoads $80=1537 \mathrm{E}+03-.3578 \mathrm{E}+03.6012 \mathrm{E}+01.1894 \mathrm{E}+03-.5203 \mathrm{E}+03-0000 \mathrm{0}-000$

GLoods $79=.1195 \mathrm{E}+03 \quad .2283 \mathrm{E}+02 \quad .2781 \mathrm{E}+01 \quad .1230 \mathrm{E}+03 \quad .3935 \mathrm{E}+03.0000 \mathrm{.00}+00$

$\begin{array}{lllllllll}\text { GLoads } 64 & .1531 E+03 & .3463 E+03 & -.2444 E+02 & -.3827 E+03 & .4734 E+03 & .0000 E+00\end{array}$

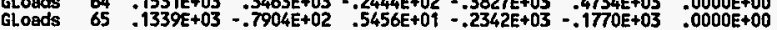

GLoads $\quad 81=1739 E+03-3372 \mathrm{E}+03 \quad 3537 \mathrm{E}+02 \quad 2065 \mathrm{E}+03-1549 \mathrm{E}+03 \quad 0000 \mathrm{E}+00$

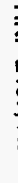

Page 62 of 91 
KAISER ENGIHEERS HANFORD S/H:801854 Run ID=HN61973 $02 / 22 / 95$
$08: 50: 46$

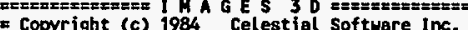
are Inc.

SOLVE PLATE LOADS/STRESSES Version 2.0 07/01/90

LOad CaSe 3:UBC SEISHIC LOAD - SH \& WE OIR.

GLoads Node Fx $\quad$ Fy $\quad$ Fz $\quad$ Mx $\quad$ Hy GLoads $80-.1131 \mathrm{E}+03 \quad .7001 \mathrm{E}+02-1639 \mathrm{E}+02-.7210 \mathrm{E}+01 \quad .4777 \mathrm{E}+03 \quad .0000 \mathrm{E}+00$ GLoads $65 \quad .1229 E+03 \quad .2631 E+03-.2954 E+02-.2800 E+03 \quad .2447 E+03 \quad .0000 E+00$

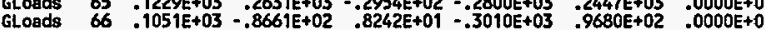

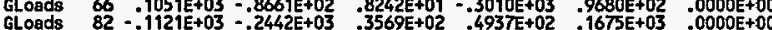
$\begin{array}{lllllll}\text { GLoads } & 81-.1159 \mathrm{E}+03 & .6767 \mathrm{E}+02 & -.1439 \mathrm{E}+02 & -49303 \mathrm{E}+02 & -1574 \mathrm{E}+03 & .0000 \mathrm{E}+00\end{array}$ GLOAds $67.1968 E+02 \quad .2341 E+03-.2272 E+02-1361 E+03 \quad .1184 E+03 \quad 0000 E+00$ GLoads $68 \quad .8239 \mathrm{E}+02 \quad .1526 \mathrm{E}+02 \quad .2710 \mathrm{E}+01=2601 \mathrm{E}+03 \quad .1046 \mathrm{E}+03 \quad 0000 \mathrm{0}+000$ GLoads $84-7361 E+01-2207 E+03-1908 E+02-1527 E+03-1093 E+03-0000+00$ GLoads $83-.9471 \mathrm{E}+02-.2868 \mathrm{E}+02 \quad .9225 \mathrm{E}+00-.1350 \mathrm{E}+01 \quad .1748 \mathrm{E}+03 \quad .0000 \mathrm{E}+00$ GLoads $68 \quad .2738 E+02.1739 E+03-.2485 E+02 \quad .1275 E+03 \quad .3402 E+02 \quad .0000 E+00$ GLoads $69.4940 \mathrm{E}+02.9995 \mathrm{E}+01 \quad .2614 \mathrm{E}+02-.1518 \mathrm{E}+03 \quad .1599 \mathrm{E}+03 \quad .0000 \mathrm{E}+00$ GLoads $85 \quad .5228 E+01-.1645 E+03 \quad .2036 E+02-.1303 E+03 \quad .2830 E+03 \quad .0000 E+00$ GLoads $84-.8201 \mathrm{E}+02-.1940 \mathrm{E}+02-.2165 \mathrm{E}+02 \quad .1903 \mathrm{E}+03 \quad .1586 \mathrm{E}+03 \quad .0000 \mathrm{E}+00$ GLoads $69-.9648 E+01.6779 E+02-.4008 E+02 \quad .2254 E+03-.2373 E+03 \quad .0000 E+00$ GLoads $70 \cdot .1633 \mathrm{E}+02 \quad .4448 \mathrm{E}+02 \quad .5809 \mathrm{E}+02 \quad .1361 \mathrm{E}+03-.7848 \mathrm{E}+03 \quad .0000 \mathrm{E}+00$ $\begin{array}{llllllllll}\text { GLoads } & 86 & .1312 E+03 & -.5793 E+02 & .2430 E+02 & .5394 E+01 & .6677 E+03 & .0000 E+00\end{array}$ GLoads $85-.1379 \mathrm{E}+03-.5433 \mathrm{E}+02-.4230 \mathrm{E}+02 \quad .128 \mathrm{EE}+03-.8929 \mathrm{E}+02 \quad .0000 \mathrm{E}+00$

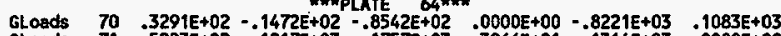
GLosds $71-.5223 \mathrm{E}+02.1217 \mathrm{E}+03 \quad .1757 \mathrm{E}+03 \quad .3966 \mathrm{E}+01 \quad .1314 \mathrm{E}+03 \quad .0000 \mathrm{E}+00$ GLoads $87-.4272 E+02 \quad .3850 E+02 \quad .1766 E+03-.1627 E+03 \quad .1528 E+02 \quad-0000 E+00$

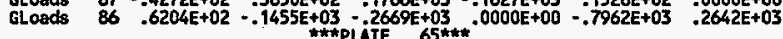

$\begin{array}{llllllll}\text { GLoads } 71 & .2802 E+02 & -.7084 E+02 & -.5407 E+02 & -.6423 E+02 & -.5519 E+02 & .0000 E+00\end{array}$ GLoads $72-.2351 \mathrm{E}+02-.1122 \mathrm{E}+03 \quad .1521 \mathrm{E}+03 \quad .5992 \mathrm{E}+02-.2879 \mathrm{E}+03 \quad .0000 \mathrm{E}+00$ GLoads $88=.2615 E+01 \quad .6170 E+02 \quad .7210 E+02 \quad .1586 E+03-.1717 E+03 \quad .0000 E+00$ GLoads $87=.1897 \mathrm{E}+01-.1031 \mathrm{E}+03-.1701 \mathrm{E}+03-.3019 \mathrm{E}+02 \quad .1099 \mathrm{E}+03 \quad .0000 \mathrm{E}+00$ $\begin{array}{llllllll}\text { - GLoads } 72 & .4761 E+01 & -.6741 E+02 & -.1370 E+01 & -.1272 E+03 & .3626 E+03 & .0000 E+00\end{array}$ GLoads $73-.9283 \mathrm{E}+01 \quad .1512 \mathrm{E}+03 \quad .1186 \mathrm{E}+03-.1226 \mathrm{E}+03-.3869 \mathrm{E}+03 \quad .0000 \mathrm{E}+00$ GLoads $89-.1147 \mathrm{E}+01 \quad .5678 E+02 \quad .5869 E+01 \quad .7087 \mathrm{E}+02-.3750 E+03 \quad .0000 \mathrm{E}+00$

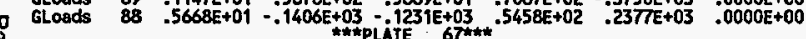
GLoads $73-.1126 E+02-.4811 E+02 \quad .3195 E+02-.1470 E+03 \quad .3796 E+03 \quad .0000 E+00$

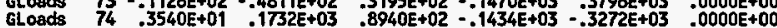
GLoads $90 \quad .3092 E+01 \quad .4204 E+02-.1663 E+02 \quad .3680 E+02-.2686 E+03 \quad .0000 E+00$ $\begin{array}{llllllll}\text { GLoads } 89 & .4627 \mathrm{E}+01-.1672 \mathrm{E}+03-.1047 \mathrm{E}+03 & .3129 \mathrm{E}+02 & .3191 \mathrm{E}+03 & .0000 \mathrm{E}+00\end{array}$ $\begin{array}{lllllllllllll}\text { GLoads } & 74 & -.2297 E+02 & .1311 E+02 & .1604 E+02 & .3981 E+02 & .2374 E+03 & .0000 E+00\end{array}$
KAISER ENGINEERS HANFORD S/H:801854

KAISER ENGINEERS HANFORD S/N:801854
PAGE 124

$02 / 22 / 95$

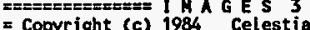
Celestial Software Inc.

SOLVE PLATE LOADS/STRESSES Version $2.0 \quad 07 / 01 / 90$

LOOd CaSe 3:UBC SEISHIC LOAD - SH \& WE DIR.

GLoads Node Fx $\quad$ Fy $\quad$ Fz $\quad$ Mx $\quad$ My $\quad$ Mz $\begin{array}{llllllll}\text { GLLods } & 75 & .2562 \mathrm{E}+02 & .1331 \mathrm{E}+03 & .5179 \mathrm{E}+02 & -.7166 \mathrm{E}+02 & .7259 \mathrm{E}+02 & .0000 \mathrm{E}+00\end{array}$

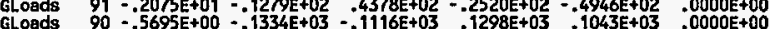

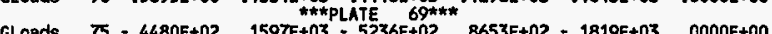

GLoads $\quad 75 \cdot-.4480 E+02 \quad-1597 \mathrm{E}+03-.5236 \mathrm{E}+02 \quad .8653 \mathrm{E}+02-.1819 \mathrm{E}+03 \quad-0000 \mathrm{E}+00$

GLoads $76 \quad-4267 \mathrm{E}+02 \quad .9168 \mathrm{E}+02-.1420 \mathrm{E}+01 \quad .0000 \mathrm{E}+00 \quad .8451 \mathrm{E}+03-.3660 \mathrm{EE}+02$

GLoads $91-.2689 \mathrm{E}+02-.6433 \mathrm{E}+02-.1858 \mathrm{E}+03-.6111 \mathrm{E}+02-.2041 \mathrm{E}+03 \quad .0000 \mathrm{E}+00$

GLoads $76-.4410 E+02.1281 E+03$ * $760270^{* \pi *}-.6300 E+02-.9197 E+03 \quad .0000 E+00$

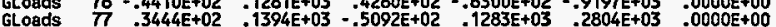

GLoads $93 \quad-.3042 \mathrm{E}+02 \quad-.1219 \mathrm{E}+03 \quad-.3519 \mathrm{E}+02 \quad-.4832 \mathrm{E}+02 \quad .1623 \mathrm{E}+03 \quad .0000 \mathrm{E}+00$

$\begin{array}{lll} & \end{array}$

$\begin{array}{lllllllll}\text { GLoads } & 77 & .4680 E+01 & .1695 E+03 & .308 B E+02 & -.1528 E+03 & -.1060 E+03 & .0000 E+00\end{array}$

GLoads $78.4893 \mathrm{E}+02 \quad .6760 \mathrm{E}+02 \div .2817 \mathrm{E}+02 \quad .1344 \mathrm{E}+03 \div .1877 \mathrm{E}+03 \quad 0000 \mathrm{E}+00$

GLoads $94-.6003 E+02-.1648 E+03-.1543 E+01-2157 E+03-.1440 E+03-0000 E+00$

GLoads $93 \quad .6416 E+01-.7233 E+02-.1165 E+01-.1228 E+03-.1294 E+02 \quad .0000 E+00$

GLoads $78.6427 E+02.2151 E+03.6908 E+01-.2560 E+03 \quad .3749 E+03 \quad .0000 E+00$

GLoads $79 \quad .5427 \mathrm{E}+02-.6848 \mathrm{E}+01-.1660 \mathrm{E}+02 \quad-.6297 \mathrm{E}+02-.3650 \mathrm{EE}+03 \quad .0000 \mathrm{E}+00$

GLoads $95-.1034 E+03-.2081 E+03 \quad .3204 E+01 \quad .1236 E+03-.4254 E+03 \quad 0000 E+00$

GLoads $94-.1510 E+02-.1516 E+00 \quad .6485 E+01-.7113 E+02 \quad .2124 E+03 \quad .0000 E+00$

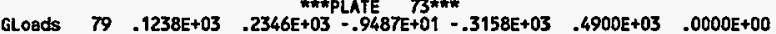

GLoads $80 \quad .3696 \mathrm{E}+02-.6783 \mathrm{E}+02-.9262 \mathrm{E}+01-.1354 \mathrm{E}+03-.4326 \mathrm{E}+03 \quad .0000 \mathrm{E}+00$

GLoads $96-.1602 E+03-.2237 E+03 \quad .1292 E+02 \quad .4287 E+02-.3844 E+03 \quad .0000 E+00$

GLoads $95-.5306 \mathrm{E}+00 \quad .5692 \mathrm{E}+02 \quad .5829 \mathrm{E}+01-.1073 \mathrm{E}+03 \quad .3825 \mathrm{E}+03 \quad .0000 \mathrm{E}+00$

GLoads $80.1900 E+03 \quad .2494 E+03-.2020 E+02-.4674 E+02 \quad .4752 E+03 \quad 0000 E+00$

GLoads $81: 1001 E+02+.1221 E+03 \quad .3367 \mathrm{E}+02 \quad-1924 \mathrm{E}+03 \quad-.1016 \mathrm{EE}+03 \quad .0000 \mathrm{E}+00$

GLoads $97-.2539 E+03-.2405 E+03-.1832 E+02 \quad .1607 E+03-.3597 E+03 \quad .0000 E+00$

GLoads $96 \quad .5393 \mathrm{E}+02 \quad .1132 \mathrm{E}+03 \quad .4847 \mathrm{E}+01 \quad .6411 \mathrm{E}+02 \quad .2189 \mathrm{E}+03 \quad .0000 \mathrm{E}+00$

$\begin{array}{llllllll}\text { GLoads } & 81 & .2400 E+03 & .2854 E+03=03448 E+02 & -.4619 E+03 & .9912 E+02 & .0000 E+00\end{array}$

GLoads $82-.6350 E+01-.1272 E+03-.5493 E+02-.1316 E+04 \quad .6695 E+03 \quad .0000 E+00$

GLoads $98 \cdot .3842 E+03-.2964 E+03-1776 E+03-.1613 E+04 \quad .8998 E+03 \quad .0000 E+00$

GLoads $97.1505 E+03 \quad .1383 E+03-2818 E+02=.7180 E+03 \quad .2020 E+03 \quad .0000 E+00$

GLoads $83-.1853 E+02.1238 E+03-.1708 E+02-.1621 E+03-.2485 E+03 \quad .0000 E+00$

GLoads $84 \quad .5523 \mathrm{E}+02 \quad .5784 \mathrm{E}+02-.3600 \mathrm{E}+02-.1863 \mathrm{E}+03-.1105 \mathrm{E}+03 \quad .0000 \mathrm{E}+00$

GLaads $100.9560 E+01-.1021 E+03-.2561 E+02-.2656 E+03-.1480 E+03 \quad .0000 E+00$ 
KAISER EHGINEERS HAKFORD S/N:801854 Run ID=HN61973

$02 / 22 / 95$
$08: 50: 48$

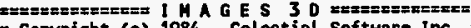

$=$ Copyright (c) 1984 Celestial Software Inc.

SOLVE PLATE LOADS/STRESSES VErsion $2.0 \quad 07 / 01 / 90$

LOod Case 3:UBC SEISHIC LOAD - SN \& WE DIR.

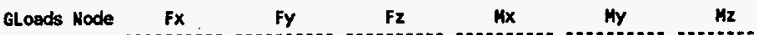

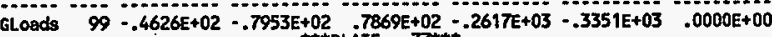

GLoads $84.2235 E+01.9712 E+02 \quad .6662 E+01.1488 E+03-.1574 E+03 \quad .0000 E+00$

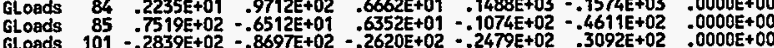

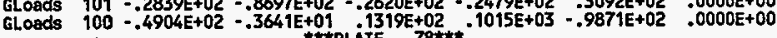

$\begin{array}{llllllllll}\text { GLoads } & 85 & .2560 E+02 & .1403 E+03 & -.1632 E+02 & .1291 E+02 & -.1476 E+03 & .0000 E+00\end{array}$

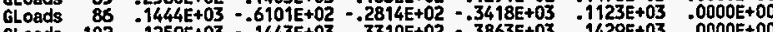

GLoods $102-.154 E+03-.1443 E+03 \quad .3310 E+02-.3863 E+03 \quad-1429 E+03 \quad .0000 E+00$

GLoads $101-.4419 E+02 \quad .6504 E+02$. . 1136E+02 $*-1819 E+02-.3981 E+02 \quad .0000 E+00$

GLoads $86 \quad .1531 E+03 \quad .9625 E+02-.1323 E+03 \quad .0000 E+00-.4291 E+03 \quad-.9712 E+03$

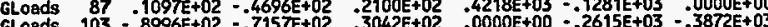

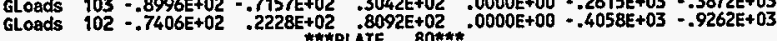

GLoads $\quad 87-.2542 E+01 \quad .1504 E+02-.6370 E+02-.2289 E+03 \quad .2851 E+01 \quad .0000 E+00$

GLoads $83:=.3259 E+02-.1506 E+02 \quad .2855 E+02-.9025 E+02-.1514 E+03 \quad .0000 E+00$

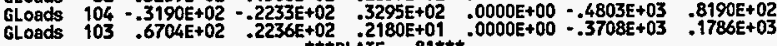

GLoads $88-.6651 E+01-.2543 E+01-.1375 E+02-.1229 E+03 \quad .8545 E+02 \quad .0000 E+00$

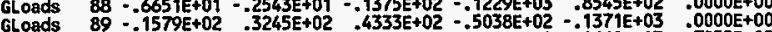

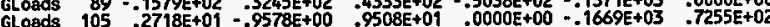

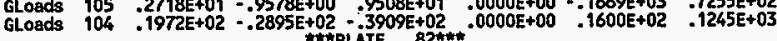

GLOads $89-.2388 E+02-.1855 E+02 \quad .1933 E+02-.6178 E+02 \quad .9931 E+03 \quad .0600 E+00$

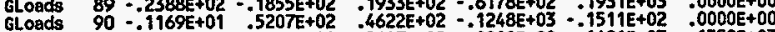

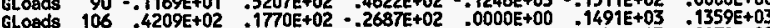

$\begin{array}{lllllll}G & \end{array}$

$\begin{array}{cccccc}0 & 1794 E+03 & .0000 E+00\end{array}$

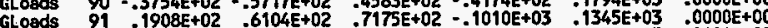

Glods $107 \quad-7343 \mathrm{E}+02 \quad .6412 \mathrm{E}+02-.9264 \mathrm{E}+02 \quad .0000 \mathrm{E}+00 \quad-5538 \mathrm{E}+03 \quad .9194 \mathrm{E}+02$

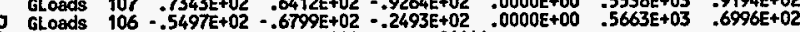

GLoads $91-2630 E+02-.8042 E+02 \quad .3407 E+02.1873 E+03 \quad .1191 E+03 \quad .0000 E+00$

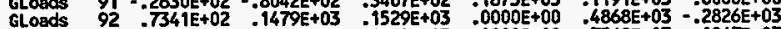

GLoods $108 \quad 4540 E+02 \quad-5121 E+02-.1852 E+03 \quad .0000 E+00 \quad .7569 E+03-.1967 E+03$

GLoods $107-.9251 \mathrm{E}+02-.1187 \mathrm{E}+03-.181 \mathrm{EE}+01 \quad .0000 \mathrm{E}+00 \quad .4788 \mathrm{E}+03-.1107 \mathrm{~F}+03$

GLoads

THP

$.6574 E+03-.4778 E+03 \quad .0000 E+00$
KAISER ENGINEERS HANFORD S/N:801854

Run ID=NN61973

$02 / 22 / 95$

PAGE 126

1984 Celestial Softuare Inc.

$=$ Copyright (c) 1984 Celestial Software Inc. $=$

SOLVE PLATE LOADS/STRESSES Version 2.0 07/01/90

LOAd Case 3:UBC SEISHIC LOAD - SH \& WE DIR.

Gloads Mode Fx

Fy

Fz

Mx

My

$M_{2}$

Cla

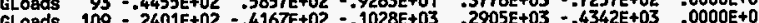

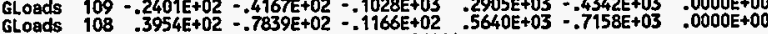

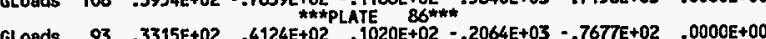

GLads $94-.8507 \mathrm{E}+01 \quad .1623 \mathrm{E}+02-.4014 \mathrm{E}+02-.7740 \mathrm{E}+01-.1304 \mathrm{EE}+03 \quad .0000 \mathrm{E}+00$

GLads $110-3019 \mathrm{E}+02-.4294 \mathrm{E}+02-.5223 \mathrm{E}+02-.6824 \mathrm{E}+02-.6075 \mathrm{E}+03-.0000 \mathrm{E}+00$

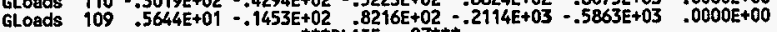

$\begin{array}{llllllll}\text { GLoeds } 94 & .4831 E+02 & .5430 E+02-.2154 E+00 & -.1368 E+03 & .6190 E+02 & .0000 E+00\end{array}$

GLoads $95-.4499 \mathrm{E}+01 \quad .3752 \mathrm{E}+01-.2369 \mathrm{E}+02 \quad .3244 \mathrm{E}+02-.1555 \mathrm{E}+03 \quad .0000 \mathrm{E}+00$

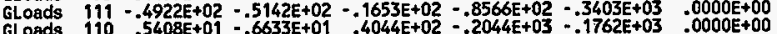

$+.6633 \mathrm{E}+01 \quad .4044 \mathrm{E}+02-.204$

GLosds $95 \quad .7306 E+02 \quad .5298 E+02-.2075 E+02-.4880 E+02 \quad .1985 E+03 \quad .0000 E+00$

GLosds $96-.2417 \mathrm{E}+02-.4248 \mathrm{E}+01-.1010 \mathrm{E}+02-.5060 \mathrm{E}+02-.4572 \mathrm{E}+02 \quad .0000 \mathrm{E}+00$

GLoads $112 \cdot-7116 \mathrm{E}+02-.4893 \mathrm{E}+02-2800 \mathrm{2}+02-.2160 \mathrm{E}+03-.3223 \mathrm{E}+02 \quad .0000 \mathrm{E}+00$

GLoods $114 \quad .2228 E+02 \quad .1975 E+00 \quad .2843 E+01=-1936 E+03 \quad .1511 E+03 \quad .0000 E+00$

GLoads 96 0506E+02 .2029E+02 $-.4308 E+02-.5639 E+02 \quad .2112 E+03 \quad .0000 E+00$

GLoads $97-.7742 E+02-.1372 E+02-.4318 E+01-.1587 E+03 .-3895 E+01 \quad .0000 E+00$

GLoads $113-.7029 E+02=.5474 E+01 \quad .8502 E+02-.3274 E+03 \quad .4573 E+03 \quad .0000 E+00$

GLoads $112 \quad .5265 E+02-.1096 E+01-.3762 E+02-.2397 E+03 \quad .5594 E+03 \quad .0000 E+00$

GLoads $97.1454 E+03 \quad .2156 E+02 \quad .1541 E+02 \quad .7160 E+03 \quad .1616 E+03 \quad .0000 E+00$

GLoads $98-.1629 \mathrm{E}+03 \quad .7999 \mathrm{E}+02 \quad .2049 \mathrm{E}+03 \quad .1397 \mathrm{E}+04 \quad .5444 \mathrm{E}+03 \quad .0000 \mathrm{E}+00$

GLoads $114 \div .6198 \mathrm{E}+02-.6094 \mathrm{E}+02-.1097 \mathrm{E}+03 \quad .1099 \mathrm{E}+04 \quad .4812 \mathrm{E}+03 \quad .0000 \mathrm{E}+00$

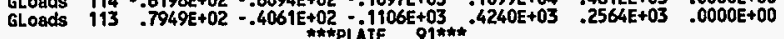

$\begin{array}{lllllllll}\text { GLoads } & 122 & .4879 E+03 & .1478 E+04 & .6745 E+02 & .5678 E+03 & .1206 E+03 & .0000 E+00\end{array}$

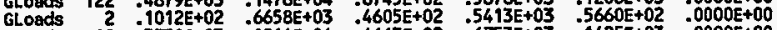

GLoads $19-.3730 \mathrm{E}+03-.1214 \mathrm{E}+04-.4663 \mathrm{E}+02 \quad .6753 \mathrm{E}+03-.1425 \mathrm{E}+03 \quad .0000 \mathrm{E}+00$

GLoads $124=.1250 \mathrm{E}+03-.9304 \mathrm{E}+03-.6688 \mathrm{E}+02 \quad .7128 \mathrm{E}+03-.4625 \mathrm{E}+02 \quad .0000 \mathrm{E}+00$

$\begin{array}{lllllllll}\text { GLoads } & 124 & .1024 E+03 & .1004 E+04 & .3695 E+02 & .1506 E+03 & .1705 E+03 & .0000 E+00\end{array}$

$\begin{array}{llllllll}\text { GLoads } & 19 & .1836 E+03 & .5630 E+03 & .2187 \mathrm{E}+02 & .1554 \mathrm{E}+03 & -.2289 \mathrm{E}+02 & .0000 \mathrm{E}+00\end{array}$

GLoads $35-.1477 \mathrm{E}+03 \quad-.8776 \mathrm{E}+03-.2066 \mathrm{E}+02 \quad-4788 \mathrm{E}+03-.1696 \mathrm{E}+03 \quad .0000 \mathrm{E}+00$

GLoads $126-.1383 \mathrm{E}+03-.6895 \mathrm{E}+03-.3817 \mathrm{E}+02=5093 \mathrm{E}+03 \quad .4635 \mathrm{E}+02 \quad .0000 \mathrm{E}+00$

GLoads $126 \quad .1038 E+03 \quad .7145 E+03 \quad .8394 E+01-.1633 E+03 \quad .1659 E+03 \quad .0000 E+00$

GLoads $35 \quad: 1153 \mathrm{E}+03 \quad .3934 \mathrm{E}+03 \quad .4064 \mathrm{E}+01 \quad-.1147 \mathrm{E}+03 \quad-.8507 \mathrm{E}+02 \quad .0000 \mathrm{E}+00$

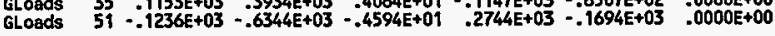


KAISER ENGIHEERS HAKFORD S/N:801854

PAGE 127

Run ID=NN61973

$02 / 22 / 95$

$x$ Copyright (c) 1984 Celest ial software inc. =

SOLVE PLATE LOADS/STRESSES Version 2.0 07/01/90

L

Load Case 3:UBC SEISHIC LOAD - SH \& WE DIR.

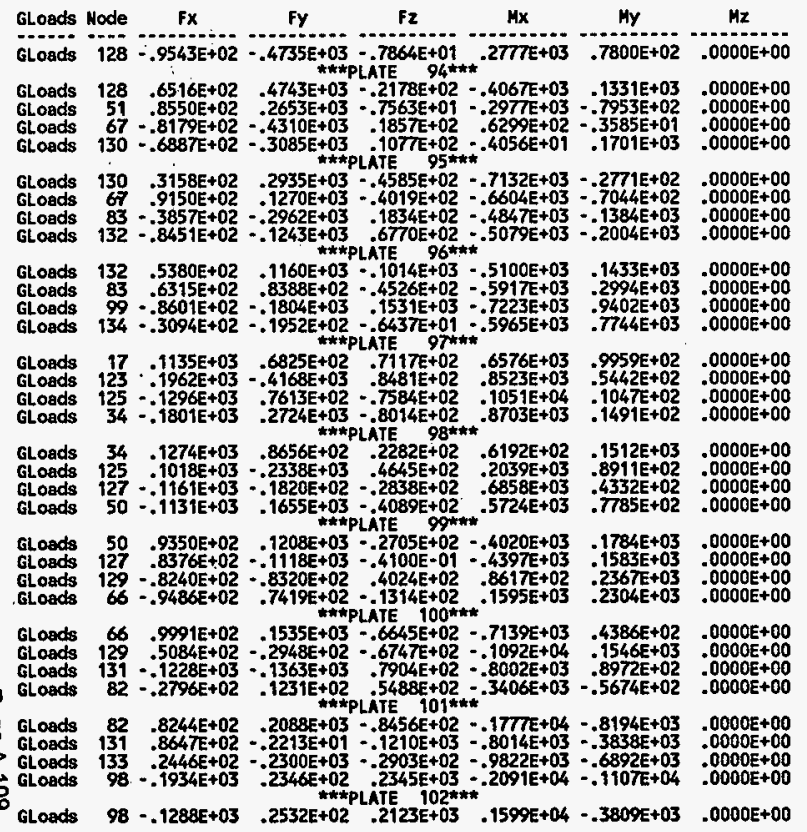

KAISER ENGIHEERS HANFORD S/N:801854

PAGE 128 Run ID=NN61973

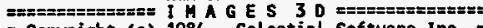
$=$ Copyright (c) 1984 Celestial Software Inc. =

SOLVE PLATE LOADS/STRESSES Version 2.0 07/01/90

Load Case 3:UBC SEISMIC LOAD - SN \& WE DIR.

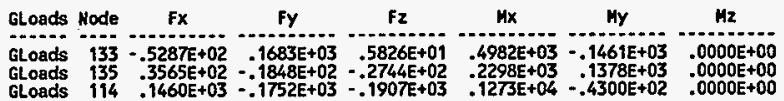


KAISER ENGIMEERS HANFORD S/N:801854 = Copyright (c) 1984 Celestial Software Inc.

SOLVE PLATE LOADS/STRESSES Version 2.0 07/01/90

Lood Case 3:UBC SEISHIC LOAD - SN \& WE DIR.

\section{MAXIMUA STRESS SUAMARY FOR PLATES}

Maximum (absolute) stress $=.3490 E+03$ at Plate 40

Plate Sigma $X$ Sigma $Y$ Tau $X Y$ Von Mises

$40 \quad 3490 E+03 \quad 4588 E+02 \quad 1494 E+01$ $3284 E+03$
KAISER ENGINEERS HAHFORD S/N:B01854

PAGE 130

=ан

$02 / 22 / 95$ $=$ Copyright (c) 1984 Celestiat Software Inc.

SOLVE REACTIONS

Version $2.0 \quad 07 / 01 / 90$

Load Case 3:UBC SEISMIC LOAD - SH \& WE DIR.

REACTIONS

\begin{tabular}{|c|c|c|c|c|c|c|}
\hline & $F x$ & & & & My & 12 \\
\hline $\begin{array}{l}1 \\
2 \\
3 \\
4 \\
5 \\
6 \\
7 \\
8 \\
9 \\
10 \\
11 \\
12 \\
13 \\
14 \\
15 \\
16 \\
17 \\
18 \\
115 \\
116 \\
117 \\
118 \\
119 \\
122\end{array}$ & $\begin{array}{l}.2212 E+02 \\
.3342 E+03 \\
.2490 \mathrm{E}+03 \\
.1787 \mathrm{E}+03 \\
.2329 \mathrm{E}+03 \\
.4680 \mathrm{0}+01 \\
.3215 \mathrm{E}+02 \\
.3359 \mathrm{0}+02 \\
.3089 \mathrm{E}+02 \\
.5008 \mathrm{0}+01 \\
.5230 \mathrm{0}+03 \\
.3452 \mathrm{E}+03 \\
.3788 \mathrm{E}+03 \\
.3571 \mathrm{E}+03 \\
.3305 \mathrm{E}+03 \\
.2971 \mathrm{E}+03 \\
.2674 \mathrm{E}+03 \\
.1903 \mathrm{E}+02 \\
.6775 \mathrm{E}+04 \\
.3623 \mathrm{E}+04 \\
.7876 \mathrm{0}+03 \\
.1830 \mathrm{0}+04 \\
.7845 \mathrm{E}+03 \\
.5110 \mathrm{0}+03\end{array}$ & 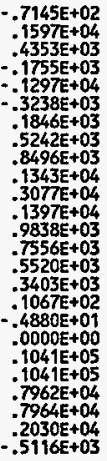 & $\begin{array}{l}.8077 \mathrm{E}+02 \\
.8280 \mathrm{0}+02 \\
.2039 \mathrm{E}+02 \\
.2648 \mathrm{0}+01 \\
.2687 \mathrm{E}+03 \\
.2470 \mathrm{0}+03 \\
.3319 \mathrm{E}+03 \\
.3663 \mathrm{0}+03 \\
.3981 \mathrm{E}+03 \\
.3734 \mathrm{E}+03 \\
.4996 \mathrm{E}+03 \\
.1358 \mathrm{0}+01 \\
.3357 \mathrm{E}+02 \\
.4098 \mathrm{0}+02 \\
.4850 \mathrm{0}+02 \\
.3738 \mathrm{0}+02 \\
.1389 \mathrm{E}+03 \\
.3094 \mathrm{E}+01 \\
.8001 \mathrm{E}+04 \\
.7790 \mathrm{0}+03 \\
.3615 \mathrm{E}+04 \\
.7821 \mathrm{E}+03 \\
.1822 \mathrm{0}+04 \\
.8778 \mathrm{0}+02 \\
.1052 \mathrm{E}+03\end{array}$ & 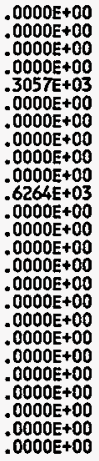 & $\begin{array}{l}.0000 \mathrm{E}+00 \\
-1013 \mathrm{E}+04 \\
.1988 \mathrm{E}+03 \\
-1752 \mathrm{E}+03 \\
-1534 \mathrm{E}+03 \\
.1297 \mathrm{E}+03 \\
.1173 \mathrm{E}+03 \\
.1801 \mathrm{E}+01 \\
.1222 \mathrm{E}+03 \\
.1276 \mathrm{E}+03 \\
.6709 \mathrm{E}+03 \\
-2590 \mathrm{E}+03 \\
.2936 \mathrm{E}+03 \\
.1950 \mathrm{E}+03 \\
.5773 \mathrm{E}+02 \\
.4816 \mathrm{E}+02 \\
.6128 \mathrm{E}+03 \\
.0000 \mathrm{E}+00 \\
-0000 \mathrm{E}+00 \\
.0000 \mathrm{E}+00 \\
.0000 \mathrm{E}+00 \\
.0000 \mathrm{E}+00 \\
.0000 \mathrm{E}+00 \\
.7535 \mathrm{E}+03\end{array}$ & $\begin{array}{l}10 E+00 \\
10 E+03 \\
10 E+00 \\
0 E+00 \\
4 E+03 \\
0 E+00 \\
10 E+00 \\
0 E+00 \\
10 E+00 \\
10 E+00 \\
0 E+04 \\
10 E+00 \\
10 E+00 \\
10 E+00 \\
10 E+00 \\
10 E+00 \\
14 E+02 \\
10 E+00 \\
10 E+00 \\
10 E+00 \\
10 E+00 \\
10 E+00 \\
10 E+00 \\
1 E+03 \\
8 E+02\end{array}$ \\
\hline
\end{tabular}


KAISER ENGINEERS HANFORD $S / N: 801854$

PAGE 131 Run ID=NN61973

$02 / 22 / 95$

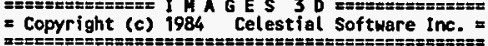

SOLVE BEAH LOADS/STRESSES Version 2.0 07/01/90 $L$

Load Case 4:UBC SEISMIC LOAD - SH \& EH DIR.

\section{BEAM LOADS AND/OR STRESSES}

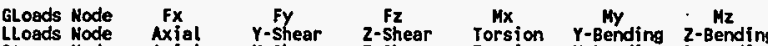

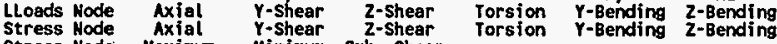

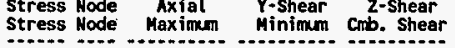

W**BEAM $M 0$,

GLoads $1 \quad .1970 E+02 \quad .3143 \mathrm{E}+03-.1386 \mathrm{E}+03-.1421 \mathrm{E}-12-.2842 \mathrm{E}-13 \quad .0000 \mathrm{E}+00$

$\begin{array}{lllllllll}\text { GLoads } 99 & .3478 \mathrm{E}+02 & -.1690 \mathrm{E}+03 & .8412 \mathrm{E}+02 & -.1407 \mathrm{E}+04 & .4149 \mathrm{E}+03 & .9957 \mathrm{E}+03\end{array}$

Lloads $99=-1884 \mathrm{E}+03 \quad-1263 \mathrm{E}+02-.3478 \mathrm{E}+02 \quad .8743 \mathrm{E}-14 \cdot .1079 \mathrm{E}+04 \quad .1407 \mathrm{E}+0$

GLoads $18 \quad .2130 E+02 \quad .1990 E+03 \quad .2827 E+02 \cdot .2274 E-12-.2842 E-13 \quad .1137 E-12$

GLoads $114+3196 E+02+.5698 E+02-.0153 E+02 \quad .1360 E+04-.2452 E+03 \quad .7035 E+03$

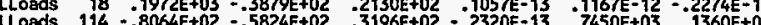

Lloads $114-.8064 E+02-.5824 E+02 \quad 02.3196 E+02-.2320 E-13 \quad .7450 E+03 \quad .1360 E+04$

GLoads $99-.1291 E+03 \quad .2029 E+02 \quad .3627 E+03-.1244 E+04-.4692 E+03-.3908 E+03$

Gloads $108-1678 E+03-8297 \mathrm{E}+02-4014 E+03-1671 E+04-.1388 E+04-.8940 E+03$

Lond 0030

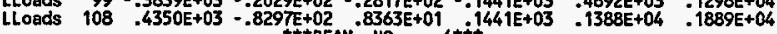

GLoads $114 \quad .1996 \mathrm{E}+03 \quad .7122 \mathrm{E}+02-.1821 \mathrm{E}+03-.1340 \mathrm{E}+04 \quad .1071 \mathrm{E}+04-.1237 \mathrm{E}+04$

GLoads $102=1500 \mathrm{E}+03 \quad .6098 \mathrm{E}+02 \quad .1325 \mathrm{E}+03 \quad .8640 \mathrm{E}+03 \quad .8699 \mathrm{E}+03 \quad .7711 \mathrm{E}+03$

L ords $114=2697 \mathrm{E}+03-7122 \mathrm{E}+02 \quad 1530 \mathrm{E}+02 \quad 5312 \mathrm{E}+02-1071 \mathrm{E}+04-1823 \mathrm{E}+04$

LLoads $102 \quad .1996 \mathrm{E}+03-.6098 \mathrm{E}+02-1454 \mathrm{E}+02-.5312 \mathrm{E}+02-.8699 \mathrm{E}+03 \quad-1157 \mathrm{E}+04$

GLoods $98-.8068 E+03 \quad .6914 E+02.9489 E+03-.1633 E+04 \quad .9997 E+03-.1180 E+0$

GLoads $115 \quad .8433 E+03 \quad .2806 E+02-.9853 E+03 \quad .1539 E+03 \quad .5230 E+03=1138 E+03$

LLoads $98.1245 \mathrm{E}+04-.6914 \mathrm{E}+02-.9763 \mathrm{E}+02 \quad . \$ 870 \mathrm{E}+03-.9997 \mathrm{E}+03=.2006 \mathrm{E}+04$

LLoads $115=.1297 \mathrm{E}+04-.2806 \mathrm{E}+02-.1420 \mathrm{E}+02-.1870 \mathrm{E}+03-.5230 \mathrm{E}+03 \quad .4088 \mathrm{E}+02$

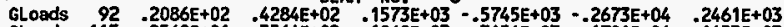

GLoads $115 \quad .8568 \mathrm{E}+01 \quad .3564 \mathrm{E}+02-.1868 \mathrm{E}+03 \quad .3154 \mathrm{E}+03 \quad-.1701 \mathrm{E}+04 \quad-.1453 \mathrm{E}+03$

$92-1542 \mathrm{E}+03-.4284 \mathrm{E}+02-3758 \mathrm{E}$

$115-.1710 \mathrm{E}+03-.3564 \mathrm{E}+02 \quad .7567 \mathrm{E}+02 \cdot 02102$

GLoods $86=.3609 \mathrm{E}+03 \quad .3422 \mathrm{E}+02 \quad .1232 \mathrm{E}+03^{7 * *} .3106 \mathrm{E}+03 \quad .2865 \mathrm{E}+04 \quad .3843 \mathrm{E}+03$

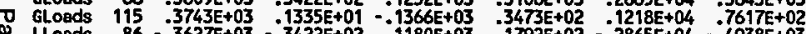

8 LLoads $86-.3627 \mathrm{E}+03-.3422 \mathrm{E}+02 \div 1180 \mathrm{E}+03 \quad .1792 \mathrm{E}+02-.2865 \mathrm{E}+04-.4938 \mathrm{E}+03$

(1) Lloods $115 \quad .3814 \mathrm{E}+03-.1335 \mathrm{E}+01-1153 \mathrm{E}+03-1792 \mathrm{E}+02-.1218 \mathrm{E}+04-.8177 \mathrm{E}+02$

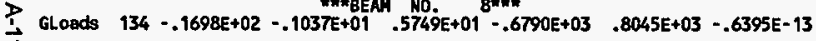

KAISER ENGIHEERS HANFORD S/N:801854

PAGE 132 RUN ID=MN61973

$02 / 22 / 95$

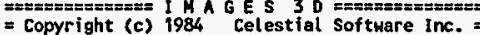

Celestial Soft

SOLVE BEAM LOADS/STRESSES Version $2.0 \quad 07 / 01 / 90$

Load CaSe 4:UBC SEISWIC LOAD - SH \& EN DIR.

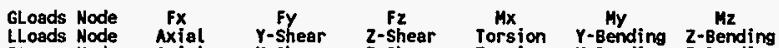

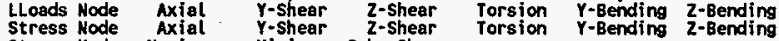
Stress Hode Maximum Minimum cmb. Shear

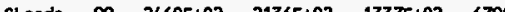

GLoads $99.2460 \mathrm{E}+02 \quad .2136 \mathrm{E}+02-.1337 \mathrm{E}+02 \quad .6790 \mathrm{E}+03-.9957 \mathrm{E}+03-.2239 \mathrm{E}+03$

LLoads $134-.1698 E+02-1037 E+01-.5749 E+01-.6790 E+03-.8045 E+03 \quad .6395 E-13$

$\begin{array}{llllllllll}\text { LLoads } 99 & .2460 E+02 & -.2136 E+02 & .1337 \mathrm{E}+02 & .6790 \mathrm{E}+03 & .9957 \mathrm{E}+03 & .2239 \mathrm{E}+03\end{array}$

$\begin{array}{lllllllll}\text { GLads } 135 & .4738 E+02 & .3137 E+01 & -.8164 E+01 & -.6247 E+03 & -.5132 E+03 & .3116 E+01\end{array}$

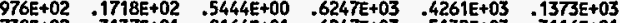

LLoads $135-.4738 E+02-.3137 E+01-.8164 E+01 \quad-6247 E+03 \quad .5132 E+03 \quad-3116 E+01$

LLoads $114.3976+02-.1718 E+02 \quad .5444 E+00-.6247 \mathrm{E}+03-.4261 \mathrm{E}+03 \quad .1373 E+03$

GLoads $122 \quad .8408 E+01.1993 \mathrm{E}+03-.9500 \mathrm{E}+01 \quad .7406 \mathrm{E}+03-.4999 \mathrm{E}+03-.8074 \mathrm{E}+02$

GLoads $124-.2592 \mathrm{E}-01-.1770 \mathrm{E}+03 \quad .1118 \mathrm{EE}+01-.8574 \mathrm{E}+03 \quad .4999 \mathrm{E}+03-.1202 \mathrm{E}+02$

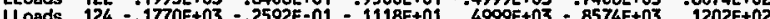

LLoads $124-.1770 E+03 \quad-.2592 E-01-.1118 E+01 \quad 4999 E+03-.8574 E+03 \quad-1202 E+02$

GLoads $124 \quad .3071 E+01 \quad .1024 E+03-.7181 E+09 \quad .2001 E+04 \quad-.4182 E+03 \quad .1202 E+02$

$\begin{array}{lll}-2067 \mathrm{E}+04 & .4182 \mathrm{E}+03 & .1262 \mathrm{E}+02\end{array}$

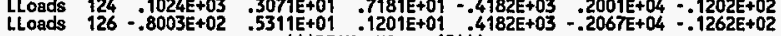

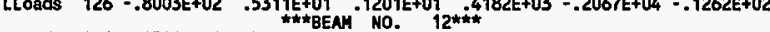

GLoads $126 \quad .4501 E+01 \quad .5337 E+02-.5178 E+01 \quad .2541 E+04-.2506 E+03-.1262 E+02$

GLoads $128 \quad .3881 \mathrm{E}+01 \quad-.3102 \mathrm{E}+02-.3204 \mathrm{E}+01-.2563 \mathrm{E}+04 \quad .2506 \mathrm{E}+03 \quad .5805 \mathrm{E}+01$

$+02-4501 E+01-5178 \mathrm{E}+01-2506 \mathrm{E}+03-254$

Lloads $128-.3102 E+02.3881 \mathrm{E}+01.3204 E+01.2506 \mathrm{E}+03-.2563 \mathrm{E}+04-.5805 \mathrm{E}+01$

GLOads $128 \quad .3531 E+01 \quad .2523 E+02-.1902 E+01 \quad .2343 E+04-.1339 E+03-.5805 E+01$

GLoads $130 \quad .4851 E+01-.2878 E+$

LLoads $128 \quad .2523 E+02 \quad .3531 E+01 \quad .1902 E+01 \quad-.1339 E+03 \quad .2343 E+04 \quad .5805 E+01$

Lloads $130-.2878 E+01 \quad .4851 E+01.0480 E+01 \frac{-1339 E+03-.2293 E+04-.2033 E+02}{* \star *}$

GLoads $130.5978 E+01 \quad .1459 E+02-.3025 E+01 \quad .1274 E+04-.1363 E+02-.2033 E+02$

GLoads $132 \quad .4499 E+01 \quad .1335 E+02-.7452 E+01-.1213 E+04 \quad .1363 E+02 \quad .3908 E-13$

Iloads $130 \quad .1459 \mathrm{E}+02 \quad .5978 \mathrm{E}+01 \quad .3025 \mathrm{E}+01-.1363 \mathrm{E}+02 \quad .1274 \mathrm{E}+04 \quad .2033 \mathrm{E}+02$

LLoads $132.1335 E+02.4499 E+01 \quad .7452 E+01 \quad 1363 E+02=.1213 E+04-.3908 E-13$

GLoads $132 \quad .0000 E+00 \quad .8031 E+01 \quad .0000 E+00 \quad .0000 E+00 \quad .0000 E+00 \quad .0000 E+00$

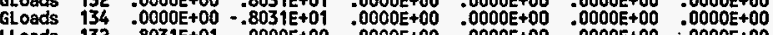

Lloads $132.8031 E+01.0000 E+00 \quad .0000 E+00 \quad .0000 E+00 \quad .0000 E+00 \quad .0000 E+00$

Lloads $134-.8031 \mathrm{E}+01 \quad .0000 \mathrm{E}+00 \quad .0000 \mathrm{E}+00 \quad .0000 \mathrm{E}+00 \quad .0000 \mathrm{E}+00 \quad .0000 \mathrm{E}+00$

GLoods $123.5691 E+01.2674 \mathrm{E}+02=.85 \frac{*}{75} \mathrm{E}+01^{16 * * *} .8680 \mathrm{E}+03-.3891 \mathrm{E}+03-.4667 \mathrm{E}+02$ 


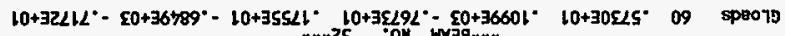

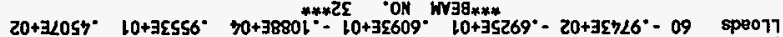
$20+38295^{\circ}-20+78061^{\circ}-40+78801^{\circ} 10+38569^{\circ} 10+39219^{\circ}-50+\exists 2251^{\circ}$ is speo71

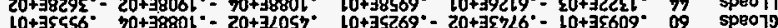

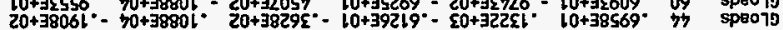
(1)

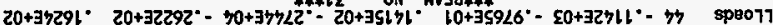

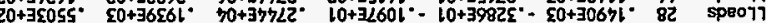

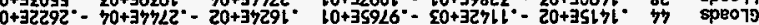

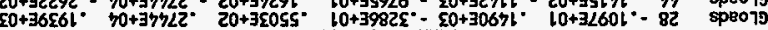

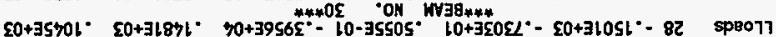

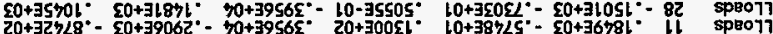

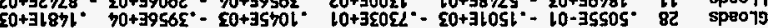

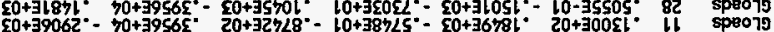

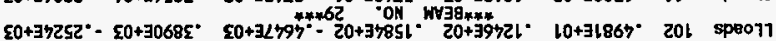

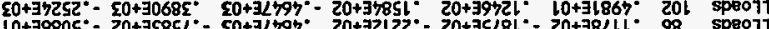

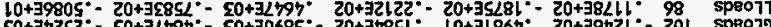

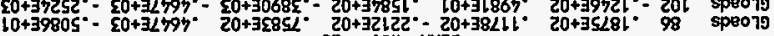
$20+35 \angle 89^{\circ}, 20+38 \angle 6 L^{\circ}-70+3 L \angle \varepsilon^{* * * 82}-10+32915^{\circ}-10+39575^{\circ}-20+36582^{\circ}-98$ speo17

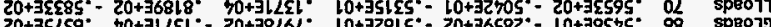

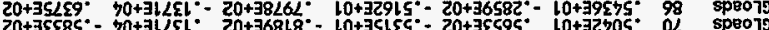

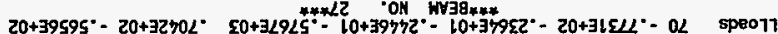

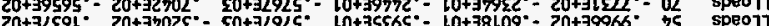

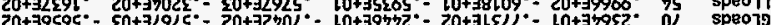

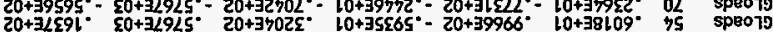
$20+36857^{\circ} 20+32809^{\circ}-50+3185^{* * * 92} 10+3065 \%^{\circ}-10+32515^{\circ}-50+3150 L^{*}-75$ speol1

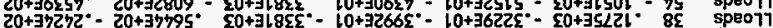

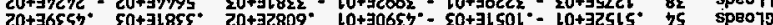

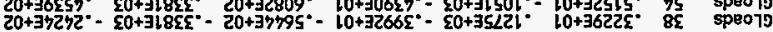

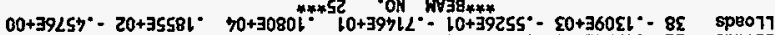
$20+35862^{\circ} \cdot 20+3 \angle 558^{\circ}-70+30801^{\circ}-10+39521^{\circ}-10+39582^{\circ}-50+32551^{\circ}$ Z2 speo71

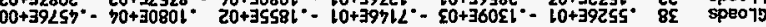

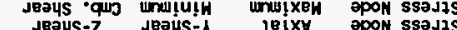

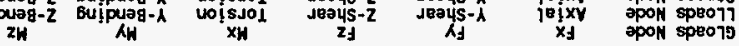

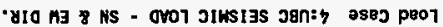

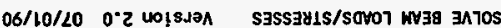

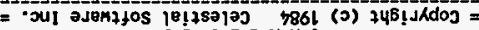
ES:05:80

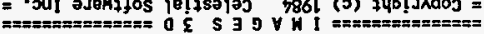

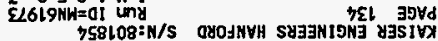

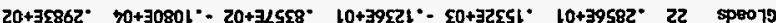
$20+37679^{\circ} 20+3969 L^{\circ}-70+35 \angle 9^{* * 472} 10+31807^{\circ}-10+79809^{\circ}-50+78 \angle 81^{\circ}-22$ $20+3769^{\circ}-20+39692^{\circ}-70+35191^{\circ}-10+3 \angle 807^{\circ}-10+39809^{\circ}-50+38 \angle 81^{\circ}-z 2$

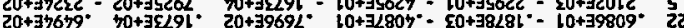

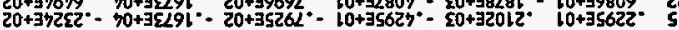

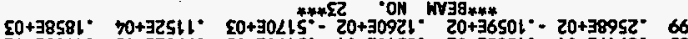

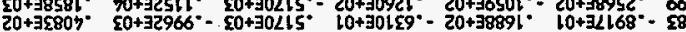

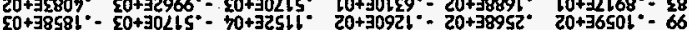

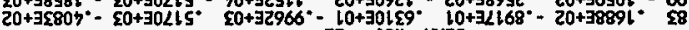
20+52806- 20+302ts CO+92965-

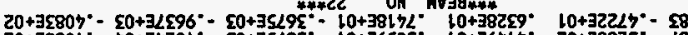

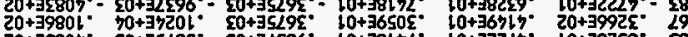

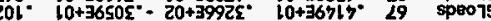

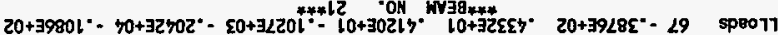
10+37."

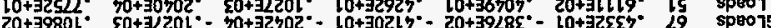

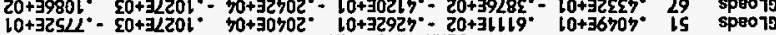

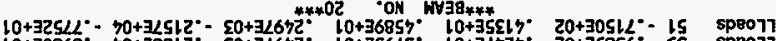

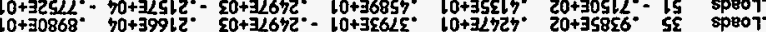

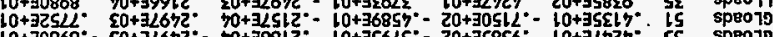

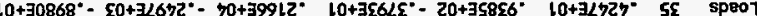

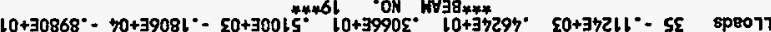

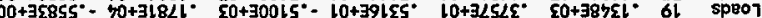

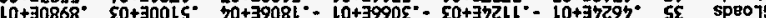

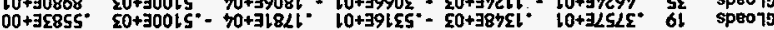

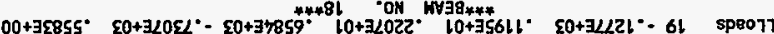

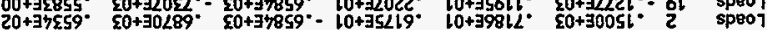

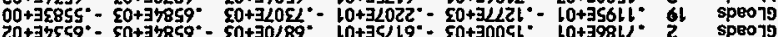

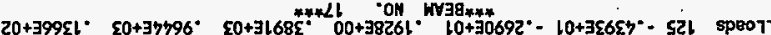

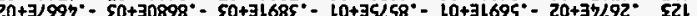

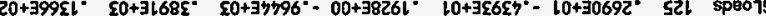

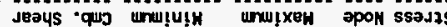

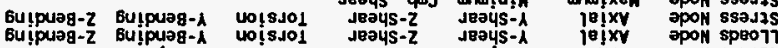

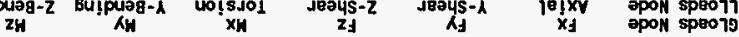

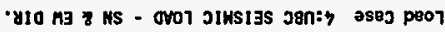

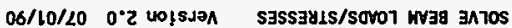

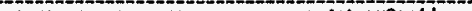

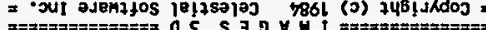
SS:05:80
$56 / 22 / 20$

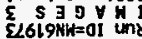

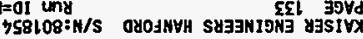


KAISER ENGINEERS HANFORD S/N:801854

PAGE 135 Run 10=\$N61973

$02 / 22 / 95$ $08: 50: 53$

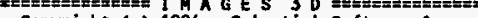
x Copyright (c) 1984 Celestial Software inc. I

SOLVE BEAM LOADS/STRESSES Version $2.0 \quad 07 / 01 / 90$ L

Load Case 4:UBC SEISHIC LOAD - SN \& EW DIR.
GLoads Node
Fx Fy $\quad F z$
Mx My Mz
LLoads Node Axial Y-Shear z-Shear Torsion Y-Bending z-Berndin Stress Node Axial Y-Shear z-Shear Torsion $Y$-Bending $z$-Bending Stress Hode Maximum Minimum Cmb. Shear
GLoads $76 \quad .7321 E+01-.7509 E+02-.5378 E+01-.2700 E+02 \quad .6849 E+03 \quad .2468 E+02$
LLoads $60 \quad-1099 E+03 \quad-.7673 E+01 \quad .5730 E+01 \quad-.6849 E+03 \quad-.7172 E+01 \quad .1755 E+0$
LLoads $\quad 76-.7509 \mathrm{E}+02-.5378 \mathrm{E}+01 \quad .77321 \mathrm{E}+01 \quad .6849 \mathrm{E}+03 \quad .2468 \mathrm{E}+02-.2700 \mathrm{E}+02$
GLoods $76 \quad .1189 E+02 \quad .6924 E+02-.8527 E+01-.6246 E+02-.2153 E+04-.3939 E+02$
GLoads $92-4424 \mathrm{E}+01-.2574 \mathrm{E}+02-.7787 \mathrm{E}+01-.5227 \mathrm{E}+02-.2153 \mathrm{E}+04-.6326 \mathrm{E}+02$
LLoads $76-.6924 \mathrm{E}+02=.8527 \mathrm{E}+01 \quad .1189 \mathrm{E}+02-.2153 \mathrm{E}+04-.3939 \mathrm{E}+02-.6246 \mathrm{E}+02$

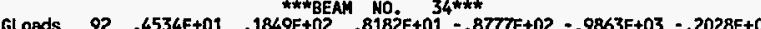

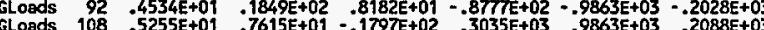
GLoads 108 .5255E+01 $.7615 E+01-.1797 E+02 \quad-3035 E+03 \quad .9863 E+03 \quad .2088 E+03$
LLoads $92 \quad-1849+02 \quad .8182 E+01 \quad .4534 E+01-.9863 E+03 \quad-.2028 E+03-.8777 E+02$
$\begin{array}{llllllllll}\text { LLoads } & 108 & .7615 E+01 & -.1797 E+02 & .5255 E+01 & .9863 E+03 & .2088 E+03 & .3035 E+03\end{array}$
GLoads $17.5983 E+01 \quad .8572 E+02-.7754 E+01 \quad .1039 E+04-.6739 E+03-.5152 E+02$
GLoads $34-2399 \mathrm{E}+01-.6337 \mathrm{E}+02-.6277 \mathrm{E}+00-0117 \mathrm{E}+04-6739 \mathrm{E}+03-1211 \mathrm{E}+02$
$17.8572 E+02-.5983 E+01-.7754 E+01-.6739 E+03-.1039 E+04-.5152 E+02$

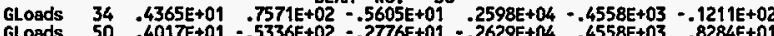 $\begin{array}{lllll}-6337 \mathrm{E}+02-.2399 \mathrm{E}+01 & -.6277 \mathrm{E}+00 \quad .6739 \mathrm{E}+03 & .1117 \mathrm{E}+04 & .1211 \mathrm{E}+02\end{array}$ ***BEAN NO. 36***
GLoads $50.4017 \mathrm{E}+01-.5336 \mathrm{E}+02=.276 \mathrm{E}+01-.2629 \mathrm{E}+04 \quad-4558 \mathrm{E}+03 \quad .8284 \mathrm{E}+0$

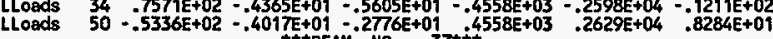
GLoods
$+04-1636 \mathrm{E}+03-3742 \mathrm{E}+02$

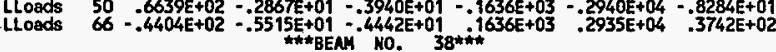
$\begin{array}{llllllll} & \text { GLoads } 66 & -1105 E+02 & .5842 E+02 & -.3002 E+01 & .2254 E+04 & .3072 E+03 & -.3742 E+02\end{array}$
GLoads $82-.2664 \mathrm{E}+01-.3607 \mathrm{E}+02-.5380 \mathrm{E}+01-.2228 \mathrm{E}+04-.3072 \mathrm{EE}+03-.1134 \mathrm{E}+03$
L6 $5842 E+02-1105 E+02-3002 E+01-3072 E+03-2254 E+04-3742 E+02$
LLoads $82 . .3607 \mathrm{E}+02.2664 \mathrm{E}+01-.5380 \mathrm{01}+01-.3072 \mathrm{*}+03 \quad .2228 \mathrm{E}+04-.1134 \mathrm{E}+03$
618E+02 3876E+01-1578E+04 3362E+03
$82-.1782 E+02 \quad .5618 E+02 \quad .3876 E+01-.1578 E+04 \quad-3362 E+03 \quad .1134 E+03$
$98 \quad-2829 E+02-.2824 E+02-.1435 E+02 \quad-1829 E+04-.3362 E+03 \quad .5206 E+03$

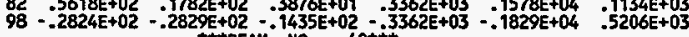
$98-.5415 E+02.2185 E+02-.2581 E+02-.1583 E+04-.2961 E+03 \quad .6598 E+03$

0 ronds
KAISER ENGINEERS HANFORD S/N:801854

RUN ID=*1461975

$02 / 22 / 95$

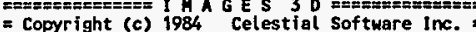

SOLVE BEAM LOADS/STRESSES Version 2.0 07/01/90

Load Case 4:UBC SEISMIC LOAD - SH \& EW DIR.

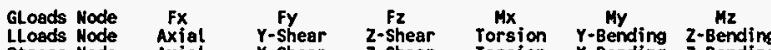
stress Node Axial $Y$-shear $z$-shear Torsion $Y$-Bending Z-Bending Stress Node Maximum Minimum Cnb. Shear

Cloads 114 6044E+02 - $5089 \mathrm{E}+01$. $1953 \mathrm{E}+02$.

$\begin{array}{llllllll}\text { GLosds } 114 & .6044 \mathrm{E}+02 & -.5089 \mathrm{E}+01 & .1953 \mathrm{E}+02 & .1209 \mathrm{E}+04 & .2961 \mathrm{E}+03 & .2856 \mathrm{E}+03\end{array}$

$\begin{array}{llllllll}\text { LLoads } 98 & .2185 E+02 & .5415 E+02 & -.2581 E+02 & -.2961 E+03 & .1583 E+04 & .6598 E+03\end{array}$

LLoads $114-.5089 E+01-.6044 E+02 \quad .1953 E+02 \quad .2961 E+03-.1209 E+04 \quad .2856 E+03$

GLoads $99 \quad .3784 E+00-.9884 E+01-.2831 E+02-.6894 E+03 \quad .1591 E+04-.1952 E+03$

GLoads $100 \quad .4828 E+01 \quad .2377 E+02 \quad .2310 E+02 \quad .6894 \mathrm{E}+03 \quad-.1239 \mathrm{E}+04-.3481 \mathrm{E}+02$

LLoads $99 \quad .3784 E+00 \quad .9884 E+01 \quad .2831 E+02-.6894 E+03-.1591 E+04 \quad-1952 E+03$

LLoads $100.4820 E+01-.2377 E+02-.2310 E+02,0884 E+03 \quad \cdot 1239 E+04 \quad 0.3481 E+02$

GLoads $100-.1853 E+02 \quad .1135 E+02-.1797 E+02-.1105 E+04 \quad .6956 E+02 \quad .3481 E+02$

GLoads $101.2374 \mathrm{E}+02.2536 \mathrm{E}+01.1277 \mathrm{E}+02 \quad .1105 \mathrm{E}+04 \quad .1405 \mathrm{E}+03 \quad .2542 \mathrm{E}+02$

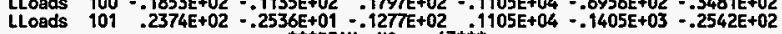

LLods 101 .2374Et02 .2536E+

GLcads $101-.3608 E+02-.1980 E+02-.2497 E+02-.8361 E+03-.1164 E+04-.2542 E+02$

GLoads $102 \quad .4129 \mathrm{E}+02 \quad-3368 \mathrm{E}+02 \quad .1976 \mathrm{E}+02 \quad .8361 \mathrm{E}+03 \quad .1470 \mathrm{E}+04-.3401 \mathrm{E}+03$

LLoads $101-.3608 E+02 \quad .1980 E+02 \quad .2497 E+02-.8361 E+03 \quad .1164 E+04 \quad .2542 E+02$

LLoads 102 . $4129 \mathrm{E}+02-.3368 E+02-.1976 E+02 \quad .8361 \mathrm{E}+03-.1470 \mathrm{E}+04 \quad .3401 \mathrm{E}+03$

GLoads $102.1658 E+02-.3001 E+02-.1246 E+03-.5020 E+03-.1517 E+04 \quad .5100 E+03$

GLoads $103-.1067 \mathrm{E}+02 \quad .4576 \mathrm{E}+02 \quad .1187 \mathrm{E}+03-.8518 \mathrm{E}+02 \quad .1306 \mathrm{E}+04-.5100 \mathrm{E}+03$

.1246E+03 $-3001 E+02-.1658 E+02-.5100 E+03 \quad .1517 E+04-5020 E+$

Lloads $103-.1187 \mathrm{E}+03-.4576 \mathrm{E}+02 \quad .1067 \mathrm{E}+02 \quad .5100 \mathrm{E}+03-.1306 \mathrm{E}+04 \quad .8518 \mathrm{E}+02$

$\begin{array}{llllllllll}\text { GLoads } 103 & .1963 \mathrm{E}+02 & .1250 \mathrm{E}+02 & -.6495 \mathrm{E}+02 & .8518 \mathrm{E}+02 & .1772 \mathrm{E}+03 & .5614 \mathrm{E}+03\end{array}$

Lads $104-.1373 E+02 \quad .3252 E+01 \quad .5905 E+02-.1355 E+02-.4358 E+03-.5614 E+03$

LLoads $103.6495 \mathrm{E}+02-.1250 \mathrm{E}+02-.1963 \mathrm{E}+02-.5614 \mathrm{E}+03-.1772 \mathrm{E}+03-.8518 \mathrm{E}+02$

LLoads $104-.5905 \mathrm{E}+02-.3252 \mathrm{E}+01.1373 \mathrm{E}+02 \quad .5614 \mathrm{E}+03 \quad .4358 \mathrm{E}+03 \quad .1355 \mathrm{E}+02$

GLoads $104 \quad .9112 E+01 \quad .7282 E+01-.4481 E+02 \quad-1355 E+02 \quad-1516 E+04 \quad .9698 E+02$

GLoads $105-.3206 \mathrm{E}+01 \quad .8465 \mathrm{E}+01 \quad .3891 \mathrm{E}+02-.2272 \mathrm{E}+02-.1611 \mathrm{E}+04-.9698 \mathrm{E}+02$

LLoads $105-.3891 E+02-.8465 E+01 \quad .3206 E+01 \quad .9698 E+02 \quad-1611 E+04 \quad .2272 E+02$

GLoads $105 \quad .3258 E+01 \quad .8244 E+01-.5128 E+02 \quad .2272 E+02 \quad .1878 E+04-.1949 E+03$

GLoads $106 \quad: 2647 \mathrm{E}+01 \quad .7503 \mathrm{E}+01 \quad .4538 \mathrm{E}+02-.1698 \mathrm{E}+02-.1883 \mathrm{E}+04 \quad .1949 \mathrm{E}+03$

Lloads $105 \quad .5128 E+02-.8244 E+01-.3258 E+01-1949 E+03-.1878 E+04-.2272 E+02$

Lloads $106-.4538 E+02-.7503 E+01-.2647 \mathrm{E}+01-1949 \mathrm{E}+03 \quad-1883 \mathrm{E}+04 \quad-1698 \mathrm{E}+02$

GLoads $106-.5688 E+01 \quad .5802 E+01=.8729 E+02 \quad .1698 E+02 \quad .1417 E+04=.5504 E+03$ 
KAISER ENGIHEERS HANFORD S/N:801854 Run 10=4N61973

$02 / 22 / 95$

PAGE 137

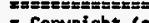
Software Inc.

SOLVE BEAM LOADS/STRESSES Version 2.0 07/01/90

L.

Load Case 4:UBC SEISHIC LOND - SH \& EH DIR.

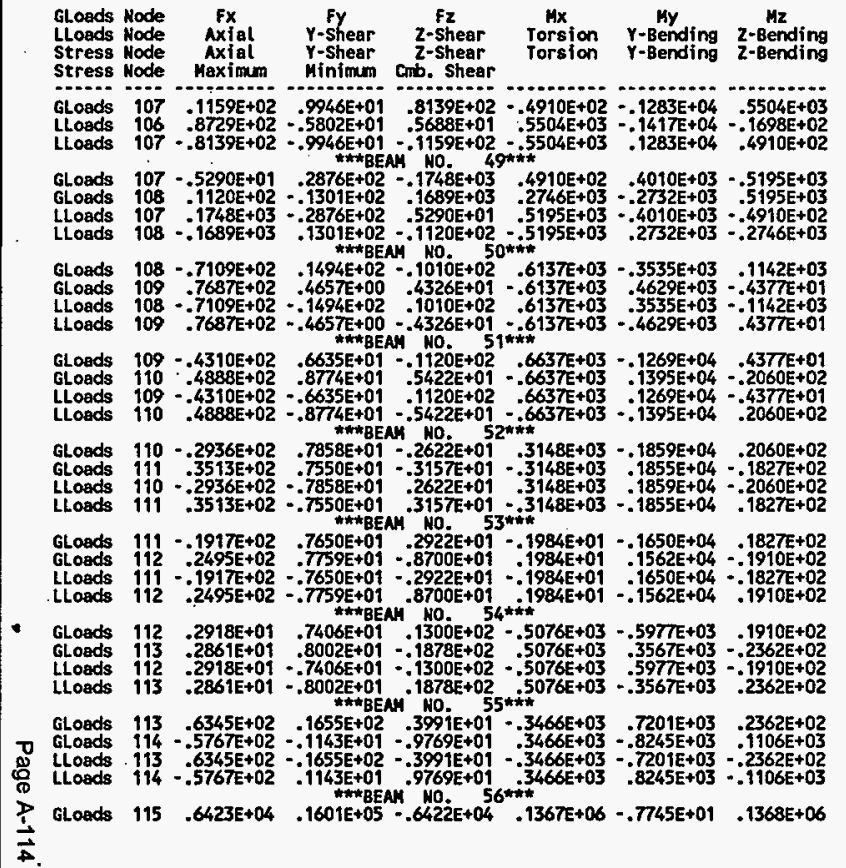
$\overrightarrow{\vec{A}}$

KAISER ENGINEERS HANFORD $S / N: 801854$ $02 / 22 / 95$

PAGE 138

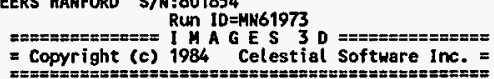
SOLVE BEAM LOADS/STRESSES Version $2.0 \quad 07 / 01 / 90$

$\mathbf{L}$

Load Case 4:UBC SEISMIC LOAD - SH \& EH DIR.

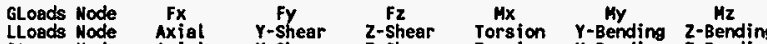
LLoads Mode Axial Y-Shear Z-Shear Torsion Y-Bending z-Bending Stress Mode Maximum Minimim Cmb. Shear

GLoads $120-.6225 \mathrm{E}+04 \quad-.1548 \mathrm{E}+05 \quad .6224 \mathrm{E}+04 \quad .1985 \mathrm{E}+06 \quad .7745 \mathrm{E}+01 \quad .1984 \mathrm{E}+06$ LLoads $120 \quad .1548 \mathrm{E}+05-.3544 \mathrm{E}+04-.8058 \mathrm{E}+04-.7745 \mathrm{E}+01=.2568 \mathrm{E}+06 \quad-1131 \mathrm{EE}+06$ GLoads $120-.5025 \mathrm{E}+04=.1452 \mathrm{E}+05 \quad .5026 \mathrm{E}+04-.1985 \mathrm{E}+06=.7745 \mathrm{E}+01-1984 \mathrm{E}+06$ GLoads $121 \quad .5258 \mathrm{E}+04 \quad .1514 \mathrm{E}+05 \quad-.5260 \mathrm{E}+04-.1230 \mathrm{E}+06 \quad .7745 \mathrm{E}+01-.1230 \mathrm{E}+06$ $\begin{array}{llllllll}\text { LLoads } & 120 & -1452 E+05 & -.2863 E+04 & -.6505 E+04 & .7745 E+01 & .2568 E+06 & -.1131 E+06 \\ \text { LLoads } & 121 & -.1514 E+05 & .2996 E+04 & .6807 \mathrm{E}+04 & -.7745 E+01 & .1592 E+06 & -.7004 E+05\end{array}$ GLoads $116 \quad .2350 E+04 \quad .5248 E+04=.4354 E+03 \cdot-.1881 E+05-.2560 E+03=.8963 E+04$ GLoads $121-.2272 E+04-5039 E+04 \quad 3569 E+03 \quad 1881 E+05-8575 E+04-1170 E+06$ LLoads $121-.2272 E+04-.5039 E+04 \quad .3569 E+03 \quad 1881 E+05 \quad .8575 E+04 \quad .1170 E+06$

GLoads $117.4353 E+03 \quad .2742 E+04=.2351 E+04-.2377 E+05-.2474 E+03-.1881 E+05$

GLoads $121=.3569 \mathrm{E}+03-.2533 \mathrm{E}+04 \quad .2272 \mathrm{E}+04-.3162 \mathrm{E}+05 \quad .8566 \mathrm{E}+04 \quad .1881 \mathrm{E}+05$ LLoads $117-.2351 \mathrm{E}+04 \quad .2742 \mathrm{E}+04-.4353 \mathrm{E}+03-.1881 \mathrm{E}+05 \quad-.2474 \mathrm{E}+03 \quad .2377 \mathrm{E}+05$ LLoads $121 \quad .2272 \mathrm{E}+04-.2533 \mathrm{E}+04 \quad .3569 \mathrm{E}+03 \quad .1881 \mathrm{E}+05 \quad .8566 \mathrm{E}+04 \quad .3162 \mathrm{E}+05$

GLoads $118 \quad .2350 E+04 \quad .2743 E+04-.4362 E+03-.1880 E+05 \quad .2426 E+03-.2377 E+05$

GLoods $121-.2272 \mathrm{E}+04 \quad-.2534 \mathrm{E}+04 \quad .3578 \mathrm{E}+03 \quad .1880 \mathrm{E}+05-.8579 \mathrm{E}+04-.3163 \mathrm{E}+05$ LLoads $118-.2350 \mathrm{E}+04 \quad .2743 \mathrm{E}+04 \quad .4362 \mathrm{E}+03 \quad .1880 \mathrm{E}+05 \quad .2526 \mathrm{E}+03.37 \mathrm{TZE}+05$ lloads $121 \quad .2272 E+04-.2534 E+04 \quad-.3578 E+03 \quad-.1880 E+05-.8579 E+04 \quad .3163 E+05$

GLoads $119.4362 E+03 \quad .5248 E+04-.235 j_{E+04}=.8956 E+04 \quad .2340 E+03-.1881 E+05$ GLoads $121-.3577 E+03-.5039 E+04 \quad .2272 E+04 \quad .1170 E+06-.8570 E+04 \quad .1881 E+05$ LLoads $119 \quad .2351 E+04 \quad .5248 \mathrm{E}+04 \quad .4362 \mathrm{E}+03 \quad .1881 \mathrm{E}+05 \quad .2340 \mathrm{E}+03-.8956 \mathrm{E}+04$ LLoads $121-.2272 E+04-.5039 E+04-.3577 E+03-.1881 E+05-.8570 E+04 \quad .1170 E+06$

GLoads $125.4129 E+01 \quad .6138 E+02-.6192 \mathrm{E}+01 \quad .2234 \mathrm{E}+04-.2573 \mathrm{E}+03-.1366 \mathrm{E}+02$ GLoads $127 \quad .4253 E+01-.3903 E+02-.2190 E+01-.2278 E+04 \quad .2573 E+03 \quad-1502 E+02$ LLoads $125 \quad .6138 \mathrm{E}+02-.4129 \mathrm{E}+01-.6192 \mathrm{E}+01-.2573 \mathrm{E}+03-.2234 \mathrm{E}+04-.1366 \mathrm{E}+02$ LLoads $127=.3903 \mathrm{E}+02-.4253 \mathrm{E}+01-.2190 \mathrm{E}+01 \quad .2573 \mathrm{E}+03 \quad .2278 \mathrm{E}+04 \quad .1502 \mathrm{E}+02$ GLoeds $127.4604 \mathrm{E}+01 \quad .7744 \mathrm{E}+02-.2755 \mathrm{E}+01 \quad .2489 \mathrm{E}+04 \quad .6745 \mathrm{E}+01-.1502 \mathrm{E}+02$ GLoads $129-3778 \mathrm{E}+01-.5509 \mathrm{E}+02-.5627 \mathrm{E}+01-.2457 \mathrm{E}+04-.6745 \mathrm{E}+01 \quad .5932 \mathrm{E}+01$ LLoods $127 \quad .7744 E+02 \quad-.4604 E+01 \quad-.2755 E+01 \quad .6745 E+01 \quad-.2489 E+04-.1502 E+02$ Lloads $129-.5509 E+02-.3778 E+01-.5627 E+01-.6745 E+01 \quad .2457 E+04 \quad .5932 E+01$ GLoads $129.3560 E+01 \quad .7547 E+02, .2060 E+01 \quad .1324 E+04 \quad .4946 E+03-.5932 E+01$ 
KAISER ENGINEERS HANFORD S/N:801854 PAGE 139 Run ID=\#N61973

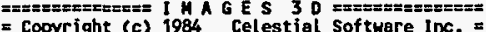
= Copyright (c) 1984 Celestial Software Inc. =

SOLVE BEAM LOADS/STRESSES Version 2.0 07/01/90

L

Load Case 4:UBC SEISHIC LOAD - SN \& EW DIR.

\begin{tabular}{|c|c|c|c|c|c|c|}
\hline $\begin{array}{l}\text { GLoads } \\
\text { LLoads } \\
\text { Stress } \\
\text { Stress }\end{array}$ & $\begin{array}{l}\text { Node } \\
\text { Node } \\
\text { Node } \\
\text { Node }\end{array}$ & $\begin{array}{c}\text { Fx } \\
\text { Axial } \\
\text { Axial } \\
\text { Maximum }\end{array}$ & $\begin{array}{c}\text { Fz } \\
\text { Z-Shear } \\
\text { Z-Shear } \\
\text { Cmb. Shear }\end{array}$ & $\begin{array}{l}\text { Mx } \\
\text { Torsion } \\
\text { Torsion }\end{array}$ & $\begin{array}{l}\text { My } \\
\text { Y-Berding } \\
\text { Y-Bending }\end{array}$ & $\begin{array}{c}\text { Mz } \\
\text { Z-Bending } \\
\text { z-Bending }\end{array}$ \\
\hline $\begin{array}{l}\text { GLoads } \\
\text { LLoads } \\
\text { LLoads }\end{array}$ & $\begin{array}{l}131 \\
129 \\
131\end{array}$ & $\begin{array}{r}.4822 E+01 \\
.7547 \mathrm{E}+02 \\
-.5312 \mathrm{E}+02\end{array}$ & $\begin{array}{l}-.5312 E+02-.1044 E+02 \\
-.3560 E+01 \\
-.4822 E+01-.2000 E+01 \\
1044 E+02\end{array}$ & $\begin{array}{l}-.1186 \mathrm{E}+04 \\
.4946 \mathrm{E}+03 \\
-.4946 \mathrm{E}+03\end{array}$ & $\begin{array}{r}-.4946 E+03 \\
\because 1324 E+04 \\
.1186 E+04\end{array}$ & $\begin{array}{r}.1981 E+02 \\
-.5932 E+01 \\
.1981 E+02\end{array}$ \\
\hline $\begin{array}{l}\text { GLoads } \\
\text { GLoads } \\
\text { LLoads } \\
\text { LLoads }\end{array}$ & $\begin{array}{l}139 \\
133 \\
131 \\
133\end{array}$ & $\begin{array}{l}.4804 E+01 \\
.5674 E+01 \\
.1634 E+02 \\
.1160 E+02\end{array}$ & $\begin{array}{r}.1634 \mathrm{E}+02-.6791 \mathrm{E}+01 \\
.1160 \mathrm{E}+02-.3686 \mathrm{E}+01 \\
-.4804 \mathrm{E}+01-.6791 \mathrm{E}+01 \\
-.5674 \mathrm{E}+01\end{array}$ & $\begin{array}{r}-.4379 E+03 \\
.3952 E+03 \\
.1115 E+03 \\
.1115 E+03\end{array}$ & $\begin{aligned} & .1115 E+03 \\
&-.1115 E+03 \\
& .4379 E+03 \\
&-.3952 E+03\end{aligned}$ & $\begin{array}{r}.1981 \mathrm{E}+02 \\
.3177 \mathrm{E}+02 \\
. .1981 \mathrm{E}+02 \\
.3177 \mathrm{E}+02\end{array}$ \\
\hline $\begin{array}{l}\text { GLoads } \\
\text { GLoads } \\
\text { LLoads } \\
\text { LLoads }\end{array}$ & $\begin{array}{l}133 \\
135 \\
133 \\
135\end{array}$ & $\begin{array}{r}.5257 E+01 \\
.1029 E+01 \\
-.9183 E+01 \\
.2595 E+02\end{array}$ & 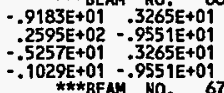 & $\begin{array}{r}-.7896 \mathrm{E}+03 \\
.8953 \mathrm{E}+03 \\
-.7013 \mathrm{E}+03 \\
.7013 \mathrm{E}+03\end{array}$ & $\begin{array}{r}-.7013 E+03 \\
.7013 E+03 \\
.7896 E+03 \\
-.8953 E+03\end{array}$ & $\begin{array}{l}-.3177 \mathrm{E}+02 \\
-.3116 \mathrm{E}+01 \\
-3177 \mathrm{E}+02 \\
-.3116 \mathrm{E}+01\end{array}$ \\
\hline $\begin{array}{l}\text { GLoads } \\
\text { GLoads } \\
\text { LLoads } \\
\text { LLoads }\end{array}$ & $\begin{array}{l}115 \\
116 \\
115 \\
116\end{array}$ & $\begin{array}{r}-.8298 E+03 \\
\because .1268 E+04 \\
.4074 E+04 \\
-.5303 E+04\end{array}$ & $\begin{array}{r}-3990 \mathrm{E}+04-.8968 \mathrm{BE}+02 \\
.5159 \mathrm{E}+04-.3489 \mathrm{E}+03 \\
-.1027 \mathrm{E}+03=.8968 \mathrm{E}+02 \\
.3250 \mathrm{E}+03=.3489 \mathrm{E}+03\end{array}$ & $\begin{array}{r}-.3378 E+05 \\
.1881 E+05 \\
.3617 E+04 \\
-.3617 E+04 \\
+\pi\end{array}$ & $\begin{array}{r}.2465 \mathrm{E}+04 \\
.2560 \mathrm{E}+03 \\
-.3367 \mathrm{E}+05 \\
.1846 \mathrm{E}+05\end{array}$ & $\begin{array}{r}. .3406 E+05 \\
.8963 E+04 \\
.3406 E+05 \\
.8963 E+04\end{array}$ \\
\hline $\begin{array}{l}\text { GLoads } \\
\text { GLoads } \\
\text { LLoads } \\
\text { LLoads }\end{array}$ & $\begin{array}{l}115 \\
117 \\
115 \\
117\end{array}$ & $\begin{array}{r}.9088 E+02 \\
.3477 E+03 \\
.4152 E+04 \\
-.5225 E+04\end{array}$ & 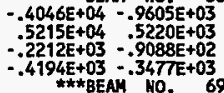 & $\begin{array}{r}-.3540 E+05 \\
.23777 E+05 \\
.3609 E+04 \\
-.3609 E+04\end{array}$ & $\begin{array}{r}.2449 E+04 \\
.2474 E+03 \\
-.3354 E+05 \\
.1847 E+05\end{array}$ & $\begin{array}{r}-.3364 \mathrm{E}+05 \\
.1881 \mathrm{E}+05 \\
.3540 \mathrm{E}+05 \\
. .2377 \mathrm{E}+05\end{array}$ \\
\hline $\begin{array}{l}\text { GLoads } \\
\text { GLoads } \\
\text { LLoods } \\
\text { LLoads }\end{array}$ & $\begin{array}{l}115 \\
118 \\
115 \\
118\end{array}$ & $\begin{array}{r}.9617 E+03 \\
-.5232 E+03 \\
.4152 E+04 \\
-.5225 E+04\end{array}$ & $\begin{array}{rr}-.4046 E+04 & -.8955 E+02 \\
.5215 E+04 & -.3490 E+03 \\
-.2225 E+03 & .8955 E+02 \\
-.4182 E+03 & .3490 E+03\end{array}$ & $\begin{array}{r}-.3379 E+05 \\
.1980 E+05 \\
-.3603 E+04 \\
.3603 E+04\end{array}$ & $\begin{array}{l}-.2482 E+04 \\
-.2426 E+03 \\
-.3369 E+05 \\
-.1846 E+05\end{array}$ & $\begin{array}{r}-.3526 E+05 \\
.2377 E+05 \\
.3526 E+05 \\
. .2377 E+05\end{array}$ \\
\hline $\begin{array}{l}\text { GLoads } \\
\text { GLoads } \\
\text { LLoads } \\
\text { LLoads }\end{array}$ & $\begin{array}{l}115 \\
119 \\
115 \\
119\end{array}$ & $\begin{array}{r}.9075 E+02 \\
.3478 E+03 \\
.4074 E+04 \\
-.5303 E+04\end{array}$ & $\begin{array}{rr}-.3990 \mathrm{E}+04 & .8310 \mathrm{E}+03 \\
.5159 \mathrm{E}+04 & -.1270 \mathrm{E}+04 \\
-.1039 \mathrm{E}+03 & .9075 \mathrm{E}+02 \\
.3262 \mathrm{E}+03 & .3478 \mathrm{E}+03\end{array}$ & $\begin{array}{r}-.3420 E+05 \\
.8956 E+04 \\
-.3595 E+04 \\
.3595 E+04\end{array}$ & $\begin{array}{r}-.2465 E+04 \\
-.2340 E+03 \\
.3355 E+05 \\
-.1846 E+05\end{array}$ & $\begin{array}{r}-.3365 E+05 \\
.1881 E+05 \\
-.3420 E+05 \\
.8956 E+04\end{array}$ \\
\hline
\end{tabular}

KAISER ENGINEERS HANFORO S/N:801854

Run ID=\#N61973

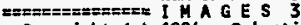

= Copyright (c) 1984 Celestial Software Inc.

SOLVE BEAH LOADS/STRESSES Version 2.0 07/01/90

$\mathbf{L}$

LOAd Case 4:UBC SEISHIC LOAD - SN \& EN DIR.

MAXIMUM STRESS SUAHARY FOR BEAMS/TRUSSES UITHIX SPECIFIED RANGE 1 - 70

Maximum (absolute) stress $=.1381 E+04$ at BEAM

Bean Axial Y-Shear Z-Shear Torsion Y-Bending Z-Bending $\begin{array}{lllllll}56 & .4397 E+03 & .2014 E+03 & .4578 E+03 & -.4402 E-03 & -.6539 E+03 & .2878 E+03\end{array}$ Maximum Minimum crib. Shear

......................................

$.1381 E+04-.5020 E+03 \quad .6907 E+03$ 
KAISER ENGINEERS HANFORO S/ $\mathrm{H:801854}$

PAGE 141 $02 / 22 / 95$
$08: 50: 55$

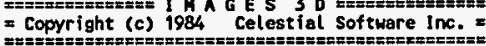

SOLVE PLATE LOADS/STRESSES Version 2.0 07/01/90

L

LaAd Case 4:UBC SEISHIC LOAD - SN \& EH DIR.

PLATE LOADS AND/OR STRESSES

GLoeds Mode Fx

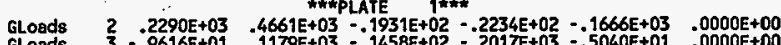

GLoads $\quad 3=.9616 E+01 \quad .1179 E+03-.1458 E+02-.2017 E+03-.5040 E+01 \quad .0000 E+00$

$\begin{array}{lllllll}\text { GLoads } & 20-.2073 \mathrm{E}+03 & -.4710 \mathrm{E}+03 & -1182 \mathrm{E}+02 & -.3549 \mathrm{E}+03 & .2116 \mathrm{E}+03 & .0000 \mathrm{O}+00 \\ \text { GLoads } & 19-.1209 \mathrm{E}+02 & -.1129 \mathrm{E}+03 & .2207 \mathrm{E}+02 & -.1666 \mathrm{E}+03 & -.7765 \mathrm{E}+02 & .0000 \mathrm{O}+00\end{array}$

$-.1209 E+02-.1129 E+03$ ***PLATE $.2207 E+02-.1666 E+03-.7765 E+02 \quad .0000 E+00$

$3.2275 E+03 \quad .4741 E+03-.4334 E+01 \quad .2017 E+03-.2041 E+03 \quad .0000 E+00$

$\begin{array}{llllllll}\text { GLoads } & 3 & -2275 \mathrm{E}+03 & .4741 \mathrm{E}+03 & -.4334 \mathrm{E}+01 & .2017 \mathrm{E}+03 & -.2041 \mathrm{E}+03 & .00002 \\ \text { GLoads } & 4 & -.2227 \mathrm{E}+02 & .1526 \mathrm{E}+03 & -.6742 \mathrm{E}+01 & -.2131 \mathrm{E}+03 & -.6695 \mathrm{E}+02 & .0000 \mathrm{E}+00\end{array}$

$\begin{array}{llllllll}\text { GLoads } & \\ \text { GLoads } & 21 & -.2011 \mathrm{E}+03 & -.4830 \mathrm{E}+03 & .1584 \mathrm{E}+01 & -.2959 \mathrm{E}+03 & .2285 \mathrm{E}+03 & .0000 \mathrm{E}+00\end{array}$

GLoads $20-.4149 \mathrm{E}+01-.1437 \mathrm{E}+03.9492 \mathrm{E}+01 \quad .6369 \mathrm{E}+02-.2797 \mathrm{E}+02 \quad .0000 \mathrm{E}+00$ $4.2338 E+03 \quad .5142 E+03 \quad .6601 E+01 \quad .2131 E+03-.2141 E+03 \quad .0000 E+00$

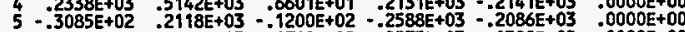
$22=.1909 \mathrm{E}+03-.5385 \mathrm{E}+03-.1719 \mathrm{E}+02 \div .2575 \mathrm{E}+03 \quad .1388 \mathrm{E}+02 \quad .0000 \mathrm{E}+00$

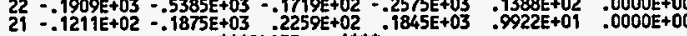
$5=.4562 E+01 \quad .7156 E+03-.3214 E+03 \quad .0000 E+00-.2465 E+03-.6575 E+02$ $6=.1189 E+02 \quad .8818 E+02-.9969 E+02 \quad .0000 E+00-.2501 E+03 \quad .2720 E+03$ $23-.1992 E+02-.6858 E+03 \quad-2611 E+03-.2280 E+03 \quad+5272 E+01 \quad .0000 E+00$ $22.3638 E+02-.1180 E+03$. 1600E+03 $.0000 E+00-.1745 E+01 \cdot .7218 E+02$ $6 \quad .1639 E+02 \quad .6358 E+03-.3124 E+03 \quad .0000 E+00-.6480 E+02-.2720 E+03$ $7.1174 \mathrm{E}+02 \quad .5832 \mathrm{E}+02-.8032 \mathrm{E}+02 \quad .0000 \mathrm{E}+00-.2425 \mathrm{E}+03 \quad-1217 \mathrm{E}+03$ $24=.2018 E+02-.6271 E+03 \quad-2767 E+03 \quad .6760 E+02 \quad-.8363 E+02 \quad .0000 E+00$ $23-.7940 \mathrm{E}+01-.6697 \mathrm{E}+02 \quad 1240 \mathrm{E}+03 \quad 03 \quad .4009 \mathrm{E}+03 \quad .2600 \mathrm{E}+03 \quad .0000 \mathrm{E}+00$ $7.1766 \mathrm{E}+02 \quad .5908 \mathrm{E}+03-.2975 \mathrm{E}+03 \quad .0000 \mathrm{E}+00 \quad .5661 \mathrm{E}+02-.1217 \mathrm{E}+03$

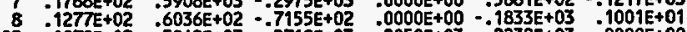
$\begin{array}{llll}-5842 E+03 & .2719 E+03 & .2050 E+03-.2232 E+03 & .0000 E+00\end{array}$ $24-.1064 \mathrm{E}+02-.6688 \mathrm{E}+02 \quad .9718 \mathrm{EE}+02 \quad .3437 \mathrm{E}+03 \quad .2411 \mathrm{E}+03 \quad .0000 \mathrm{E}+00$ $8 \quad .1362 E+02 \quad .5528 E+03-.2808 E+03 \quad .0000 E+00 \quad .1919 E+03-.1001 E+01$

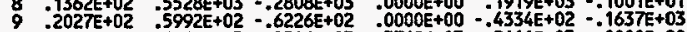
$26-2025 \mathrm{O} 202$ $26-.1235 \mathrm{E}+02-.5468 \mathrm{E}+03 \quad-2596 \mathrm{E}+03 \quad .3769 \mathrm{E}+03-.2411 \mathrm{E}+03 \quad-.0000 \mathrm{E}+00$ $25 \cdot-.2154 E+02 \cdot-.6597 E+02.8346 E+02.2039 E+03 \quad \cdot 2153 E+03 \quad .0000 E+00$ $\begin{array}{lllllll}9 & .6644 E+01 & .5134 E+03 & -.2594 E+03 & .0000 E+00 & .2443 E+03 & .1637 E+03\end{array}$ $10 \quad .5843 \mathrm{E}+01 \quad .5590 \mathrm{E}+02-.5776 \mathrm{E}+02 \quad .0000 \mathrm{E}+00 \quad-.8537 \mathrm{E}+02-.1539 \mathrm{E}+03$ $\begin{array}{lllllll}27 & .5712 E+01 & -.5060 E+03 & .2390 E+03 & .2731 E+03 & -.2274 E+03 & .0000 E+00 \\ 26 & -.1820 E+02 & -.6322 E+02 & .7815 E+02 & .1144 E+02 & .7680 E+02 & .0000 E+00\end{array}$ Run ID=HN61975

KAISER ENGINEERS HANFORD S/N:801854

PAGE 142 Run ID =N1161973

$02 / 22 / 95$

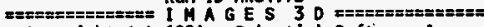
= Copyright (c) 1984 Celestial Software Inc. =

SOLVE PLATE LOADS/STRESSES Version $2.0 \quad 07 / 01 / 90$

Load Case 4:UBC SEISHIC LOAD - SN \& EW DIR.

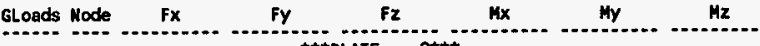

GLoads $10-.4248 E+01 \quad .4696 \mathrm{E}+03-.2320 \mathrm{E}+03 \quad .0000 \mathrm{E}+00 \quad .2223 \mathrm{E}+03 \quad .1539 \mathrm{E}+03$

GLoads $\quad 10-.4248 E+01 \quad .4696 E+03-.2320 E+03 \quad .0000 E+00 \quad-.2223 E+03 \quad-1539 E+03$

GLoads $\quad 11 \quad .3199 \mathrm{E}+02 \quad .4846 \mathrm{E}+02-.5951 \mathrm{E}+02 \quad-0000 \mathrm{E}+00 \quad-2606 \mathrm{C}+03-.4501 \mathrm{E}+03$

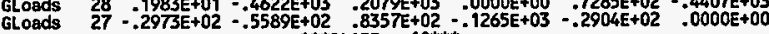

$\begin{array}{llllllll}\text { GLoads } & 11 & .2297 E+03 & -4712 E+03 & -.8093 E+01 & -.2012 E+03 & .3028 E+03 & .0000 E+00 \\ .0000 E+00\end{array}$

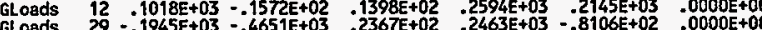

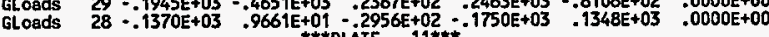

$\begin{array}{llllllll}\text { Gloads } & 12 & .2207 E+03 & .4419 E+03 & -.1096 E+02 & -.2594 E+03 & .1415 E+03 & .0000 E+00\end{array}$

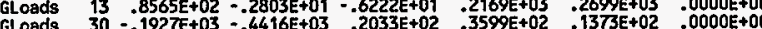

GLoads $30=1927 E+03-.4416 E+03 \quad .2033 E+02 \quad .359 E+02 \quad .1375 E+02 \quad .0000 E+00$

$\begin{array}{llllllll}\text { GLoads } & 13 & .2093 E+03 & .4190 E+03-.1895 E+02 & * .2169 E+03 & .3056 E+02 & .0000 E+00\end{array}$

$\begin{array}{llllllll}\text { GLoads } & 13 & .2093 E+03 & .4190 E+03 & -.1895 E+02 & -.2169 E+03 & -3056 E+02 & .0000 E+00\end{array}$

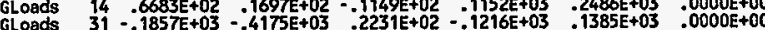

CLads $30=0044 E+02-1846 E+02 \quad .8130 E+01-.4462 E+03-.2536 E+03 \quad 0000 E+00$

GLoads $14.1954 E+03 \quad .3941 E+03-.1637 E+02-.1152 E+03-.9153 E+02 \quad .0000 E+00$

$\begin{array}{llllllll}\text { GLoads } & 14 & -1954 E+03 & -3941 E+03 & -.1637 \mathrm{E}+02 & -.1152 E+03 & -.9153 E+02 & -0000 E+00\end{array}$

GLoads $15-5402 E+02-.2960 E+02-.2067 E+02=-2280+02 \quad .1488 E+03 \quad .0000 E+00$

GLoads $31=.7552 \mathrm{E}+02=.3229 \mathrm{E}+02 \quad .1915 \mathrm{E}+02-.368 \mathrm{E}+03-.2617 \mathrm{E}+03 \quad-0000 \mathrm{E}+00$

$\begin{array}{lllllllll}\text { GLoads } & 15 & .1776 E+03 & .3640 E+03-.1313 E+02 & .2284 E+02 & =.1461 E+03 & .0000 E+00\end{array}$

$\begin{array}{llllllll}\text { GLoads } & 15 & -1776 E+03 & -3640 E+03 & -361313 E+02 & -2284 E+02 & -.14615+03 & -0000 E+00\end{array}$

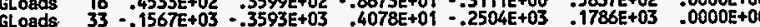

GLoads $32-.6623 E+02-.4062 E+02 \quad .1773 E+02-.2519 E+03 * .1606 E+03 \quad .0000 E+00$

$\begin{array}{lllllllll}\text { GLoads } & 16 & .1523 E+03 & .3231 E+03-.1098 E+02 & .3111 E+00 & -.1588 E+03 & .0000 E+00\end{array}$

$\begin{array}{llllllll}\text { GLoads } & 16 & .1523 E+03 & .3231 E+03 & -.1098 E+02 & .3111 E+00 & -.1588 E+03 & .0000 E+00 \\ \text { GLoads } & 17 & .4125 E+02 & .3427 \mathrm{E}+02 & -.4207 \mathrm{E}+02 & .3867 \mathrm{E}+03 & -.6766 \mathrm{E}+02 & .0000 \mathrm{E}+00\end{array}$

GLoads $34=1301 \mathrm{E}+03=3151 \mathrm{~K}+03 \quad .2234 \mathrm{E}+02=.5779 \mathrm{E}+03 \quad .1317 \mathrm{E}+02 \quad .0000 \mathrm{E}+00$

GLads $33=.6347 \mathrm{E}+02=.4229 \mathrm{E}+02 \quad .3071 \mathrm{E}+02-.2027 \mathrm{E}+03-.8593 \mathrm{E}+02 \quad .0000 \mathrm{E}+00$

$\begin{array}{llllllll}\text { GLoads } 19 & 1252 E+03 & .3788 E+03=.8575 E+01 & .1806 E+03 & -.3177 E+03 & .0000 E+00\end{array}$

$\begin{array}{llllllll}\text { GLoads } & 19 & .1252 E+03 & -378 B E+03 & -.06735+01 & .1806 E+03 & -.3177 E+03 & .0000 E+00 \\ \text { GLoads } & 20 & .4066 E+02 & .1152 E+03 & -.2531 E+00 & .3968 E+02 & .2065 E+03 & .0000 E+00\end{array}$

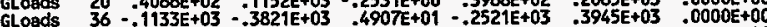

GLoads $35-.5258 E+02-.1119 E+03 \quad .4021 E+01-.4646 E+03-.2197 E+03 \quad .0000 E+00$

$\begin{array}{lllllllll}\text { GLoads } & 20 & .1389 E+03 & .4144 E+03 & .1085 E+02 & .2516 E+03 & -.3901 E+03 & .0000 E+00\end{array}$ 
KAISER ENGINEERS HAHFORD' S/N:801854

PAGE 143 RUn ID=NN61973

$02 / 22 / 95$ $08: 50: 57$ I Copyright (c) 1984 Celestial Software Inc.

SOLVE PLATE LOADS/STRESSES Version 2.0 07/01/90 L

Load Case 4:UBC SEISHIC LOAD - SN \& EH DIR.

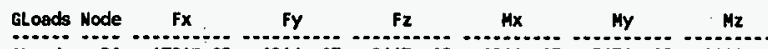

Ge. $\begin{array}{llllllll}\text { GLoads } & 21 & .4794 E+02 & .1246 E+03 & -.2113 E+02 & -.1241 E+03 & .5134 E+02 & .0000 E+00\end{array}$ GLoads $37-.1334 E+03=.4253 E+03-.1027 E+02=.3501 E+03 \quad .2028 E+03 \quad .0000 E+00$ GLoads $36-.5338 \mathrm{E}+02=.1137 \mathrm{E}+03 \quad .2055 \mathrm{E}+02-3700 \mathrm{E}+01 \cdot .2931 \mathrm{E}+03 \quad .0000 \mathrm{E}+00$ $\begin{array}{lllllllll}\text { Gloads } & 21 & .1334 E+03 & .4608 E+03 & .2887 E+02 & .2355 E+03 & -.2898 E+03 & .0000 E+00\end{array}$ GLoads $22 \quad .9024 \mathrm{E}+02 \quad .9833 \mathrm{E}+02-.5498 \mathrm{E}+01 \quad .9697 \mathrm{E}+02-.2617 \mathrm{E}+03 \quad .0000 \mathrm{E}+00$ GLoods $38-.1488 E+03-.4583 E+03-.4510 E+02 \quad .6457 E+02-.5919 E+02$ GLoads

GLoads

GLoods

GLoads

GLoads

GLoads

GLoads

GLoads

GLoads

GLoads

GLaeds

GLoads

GLoads

GLoeds

GLoads

GLoads

$37-.7480 E+02 \cdot .1008 E+03$. $2173 E+02 \quad .1171 E+03-.8082 E+02 \quad .0000 E+00$

$22 \quad-2127 E+02 \quad .5020 E+03-.9793 E+02 \quad .0000 E+00-.3437 E+03-.2259 E+02$ $23=.3081 \mathrm{E}+02 \quad-1153 \mathrm{E}+03-.1791 \mathrm{E}+03-.2138 \mathrm{E}+03-.3535 \mathrm{E}+03 \quad .0000 \mathrm{E}+00$

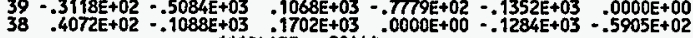
$23.2248 E+02$ S410E+03 $\begin{array}{lllll} & 0202 E+02 & .0000 E+00\end{array}$ $24=.1297 \mathrm{E}+02 \quad .8191 \mathrm{E}+02 \quad-.1464 \mathrm{E}+03 \quad-.2707 \mathrm{E}+03 \quad-.5034 \mathrm{E}+03 \quad .0000 \mathrm{E}+00$ $10-1601 E+03 \quad .8567 E+02-.4349 E+03 \quad .0000 E+00$ 24 ***PLATE $21 * * *$. $24 \quad .7611 E+01 \quad .5156 E+03-.1913 E+03-.1405 E+03 \quad .3459 E+03 \quad .0000 E+00$ 25 $.3487 E+01.6361 E+02-.1217 E+03-.2013 E+03-.4751 E+03 \quad .0000 E+00$

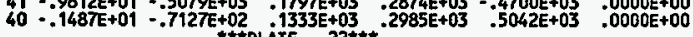
$25.1648 E+01 \quad .4901 E+03-.1975 E+03-.2076 E+03 \quad .4830 E+03 \quad .0000 E+00$ $26.8618 \mathrm{E}+01 \quad .5000 \mathrm{E}+02=.1075 \mathrm{E}+03=.1355 \mathrm{E}+03-.3209 \mathrm{E}+03 \quad .0000 \mathrm{E}+00$ $42.1292 \mathrm{E}+01-.4829 \mathrm{E}+03-1911 \mathrm{E}+03-2938 \mathrm{E}+03=.4780 \mathrm{E}+03 \quad .0000 \mathrm{E}+00$ $41-.1156 \mathrm{E}+02=.5717 \mathrm{E}+02 \quad .1139 \mathrm{E}+03 \quad .2753 \mathrm{E}+03 \quad .4694 \mathrm{E}+03 \quad .0000 \mathrm{E}+00$

$\begin{array}{llllllll}\text { GLoads } 26 & -.1426 E+02 & .4635 E+03 & -.1940 E+03 & -.2528 E+03 & .4852 E+03 & .0000 E+00\end{array}$ $\begin{array}{llllllll}\text { GLoads } 27 & .2634 \mathrm{E}+02 & .3437 \mathrm{E}+02 & -.1031 \mathrm{E}+03 & .8065 \mathrm{E}+02 & -.4028 \mathrm{E}+02 & .0000 \mathrm{E}+00\end{array}$

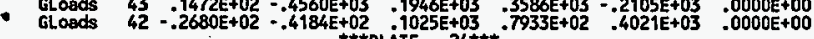
GLoads $27=.385 t E+02 \quad .4311 E+03-.1833 E+03-.2272 E+03 \quad .2967 E+03 \quad .0000 E+00$

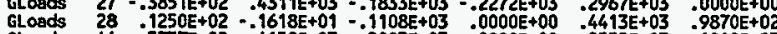

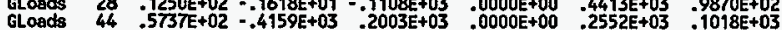

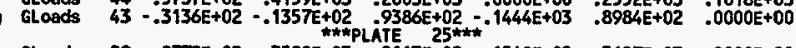
O Gloads GLoads GLoads

$28.8772 E+02 \quad .3598 E+03-.2113 E+02 \quad .1548 E+02 \quad .5627 E+03 \quad .0000 E+00$

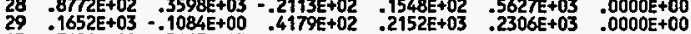
$45-.7621 \mathrm{E}+02-.3667 \mathrm{E}+03 \quad .3579 E+02 \quad .1649 \mathrm{E}+03-.5494 \mathrm{E}+00 \quad .0000 \mathrm{~T}+00$
KAISER ENGIMEERS HANFORD S/N:801854 PAGE 144 Run ID =NN61973

SOLVE PLATE LOADS/STRESSES Version $2.0 \quad 07 / 01 / 90$

L

LOAd Case 4:UBC SEISHIC LOAD - SN \& EW DIR.

GLoads Node Fx $\quad$ Fy $\quad$ Fz $\quad$ MX

$\begin{array}{llllllll}\text { GLosds } \quad 44-.1767 E+03 & .7087 E+09 & -.5646 \mathrm{E}+02 & .5913 \mathrm{E}+02 & .3840 \mathrm{E}+03 & .0000 \mathrm{E}+00\end{array}$

$\begin{array}{lllllllll}\text { GLods } 29 & .1076 \mathrm{E}+03 & .3684 \mathrm{E}+03 & . .2691 \mathrm{E}+02 & -.8986 \mathrm{E}+02 & .6164 \mathrm{E}+02 & .0000 E+00\end{array}$

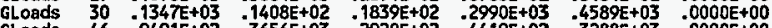

GLoads $46-.9491 E+02-.3656 E+03 \quad .2920 E+02-.4419 E+02 \quad .3288 E+03 \quad .0000 E+00$

GLoads $\quad 45-.1474 E+03-.1685 E+02-2067 E+02-.3525 E+03-.1276 E+03 \quad .0000 E+00$

$\begin{array}{lllllllll}\text { GLoads } \quad 30 & .1130 E+03 & .3515 E+03-.1144 E+02 & .1113 E+03 & -.2190 E+03 & .0000 E+00\end{array}$

$\begin{array}{lllllllll}\text { GLoads } 31 & .1132 \mathrm{E}+03 & .2076 E+02 & .2626 \mathrm{E}+01 & .3054 \mathrm{E}+03 & .4905 \mathrm{E}+03 & .0000 \mathrm{O}+00\end{array}$

GLoads $\quad 47 \cdot .1027 \mathrm{E}+03-.3489 \mathrm{E}+03 \quad .1405 \mathrm{E}+02-.2570 \mathrm{E}+03 \quad .4042 \mathrm{E}+03 \quad .0000 \mathrm{E}+00$

GLoads $46-.1235 E+03=.2340 E+02-.5243 E+01-.3535 E+03-.4228 E+03 \quad .0000 E+00$

GLoads $\quad 31 \quad .1126 E+03 \quad .3346 E+03-.8672 E+01 \quad .1849 E+03-.3673 E+03 \quad .0000 E+00$

$\begin{array}{llllllll}\text { GLoads } & 32 & .9944 E+02 & .2402 E+02 & -.5958 E+01 & .2652 E+03 & .4044 E+03 & .0000 E+00\end{array}$

GLoads $48-1056+03$.

GLoads $\quad 47-.1064 \mathrm{E}+03 \cdot-2707 \mathrm{E}+02 \quad .5310 \mathrm{E}+01 \cdot-4209 \mathrm{E}+03 \cdot-.4356 \mathrm{E}+03 \quad .0000 \mathrm{E}+00$

GLoads $32 \quad .1053 E+03 \quad .3136 E+03 \quad .5757 E+01 \quad .2946 E+03=.4060 E+03 \quad .0000 E+00$

$\begin{array}{llllllll}\text { GLoads } & 33 & .9188 \mathrm{E}+02 & .2304 \mathrm{E}+02 & -.1152 \mathrm{E}+02 & .2339 \mathrm{E}+03 & .2211 \mathrm{E}+03 & .0000 \mathrm{O}+00\end{array}$

GLoads $\quad 49-.1014 \mathrm{E}+03-.3090 \mathrm{E}+03-.1074 \mathrm{E}+02-.3618 \mathrm{E}+03 \quad .2776 \mathrm{E}+03 \quad .0000 \mathrm{E}+00$

GLoads $48 \cdot .9572 E+02-.2757{ }_{* * * P L} \cdot 1651 \mathrm{E}+02-2936 \mathrm{E}+03-.4303 \mathrm{E}+03 \quad .0000 \mathrm{E}+00$

$\begin{array}{llllllllll}\text { GLods } & 33 & .9285 E+02 & .2842 E+03 & .1215 E+02 & .2192 E+03 & -.3137 E+03 & .0000 E+00\end{array}$

$\begin{array}{llllllll}\text { GLoads } & 34 & .8733 E+02 & .1473 E+02 & -.3530 E+02 & .6369 E+01 & -.8318 E+02 & .0000 E+00\end{array}$

GLoads 50 -

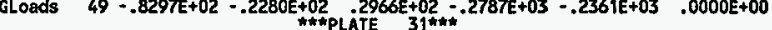

$\begin{array}{lllllllll}\text { GLoads } & 35 & .4112 E+02 & .2406 E+03 & .2145 E+01 & .2493 E+03 & -.3965 E+03 & .0000 E+00\end{array}$

$\begin{array}{llllllll}\text { GLoads } & 36 & .4932 E+02 & .1050 E+03 & -.7427 E+01 & .1051 E+03 & .3248 E+03 & .0000 E+00\end{array}$

GLoads $52-.5442 E+02-.2506 E+03-.6319 E+01-.2910 E+03 \quad .3016 E+03 \quad .0000 E+00$

GLOads $\quad 51 \cdot-.3601 E+02-.9505 E+02 \quad .1160 E+02-.1795 E+03 \cdot-.4178 E+03 \quad .0000 E+00$

$\begin{array}{lllllllllll}\text { GLods } & 36 & .8541 E+02 & .3057 E+03 & .1387 E+02 & .1507 E+03 & -.4262 E+03 & .0000 E+00\end{array}$

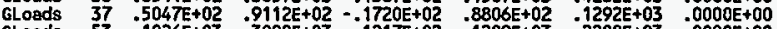

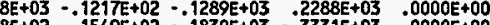

GLoeds $52 \cdot .3326 E+02-.8698 E+02 \quad .1549 E+02-1830 E+03-.3331 E+03 \quad .0000 E+00$

$\begin{array}{lllllllll}\text { GLoads } & 37 & -1259 E+03 & .3499 E+03 & .3765 E+02 & .1449 E+03 & -.2511 E+03 & .0000 E+00\end{array}$

$\begin{array}{lllllllll}\text { GLoads } & 38 & .3952 E+02 & .9093 \mathrm{E}+02 & -.2762 \mathrm{E}+02 & .1043 \mathrm{E}+02 & -.2369 \mathrm{E}+03 & .0000 \mathrm{E}+00\end{array}$

GLoads $54=.1451 E+03-.3571 E+03-.4654 E+02-.1922 E+01-.2472 E+03 \quad .0000 E+00$

GLoods $53-.2024 E+02-.8371 E+02 \quad .3652 E+02 \quad .6719 E+02-.2783 E+03 \quad .0000 E+00$

$\begin{array}{llllllllll}\text { GLoads } & 38 & .2578 E+02 & .3888 E+03 & . .5229 E+02 & .0000 E+00 & -.3170 E+03 & .8375 E+02\end{array}$ 
HNF-2467, Rev.0

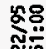

तิㅇㅇㅇ

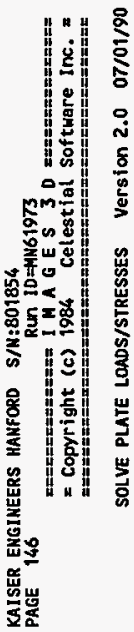

손

学

สํํㅇำ

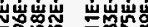

हึํํำ

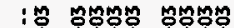

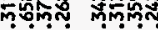

mingm

MMNm

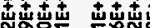

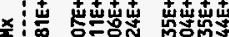

5중

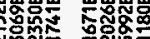

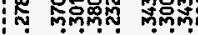

* $\cdots$

\section{? - i $\because \because$}

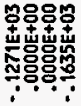

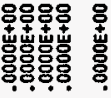

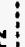

$$
\text { 吾 }
$$

충

s 总:

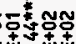

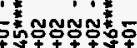

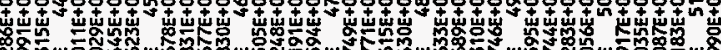

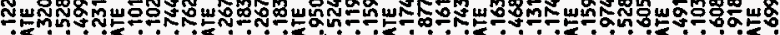

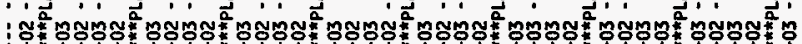

出

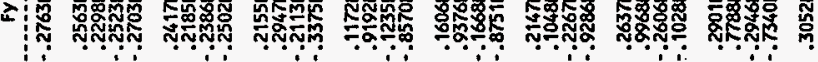

:

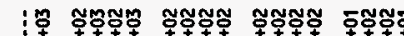

$\because$

กับ?

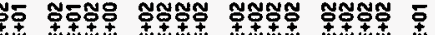

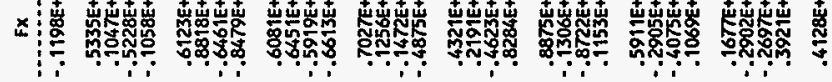

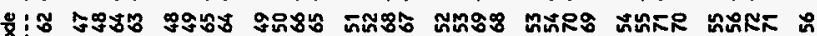

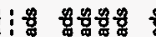

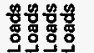

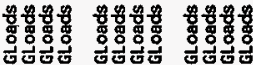

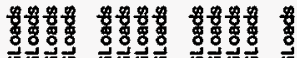

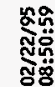

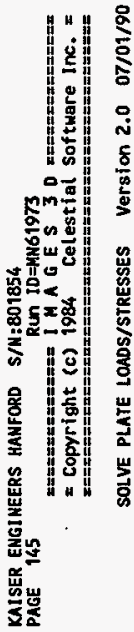

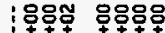

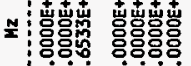

mpm

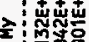

ming

M용

mism

$\times$ 岁岕象

으응

$\because \div$

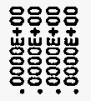

popm

吅嵌。

กุọ?

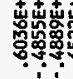

minm

要年

通통

min

点虫虫

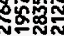

舟

吅婂

胥

$$
\text { N }
$$

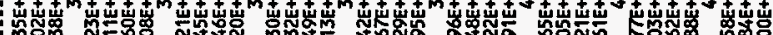

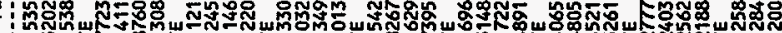

$\because<\cdots$

;

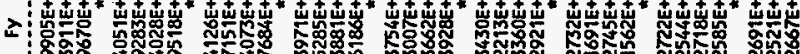

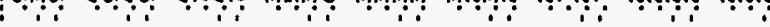

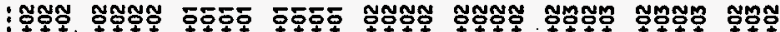

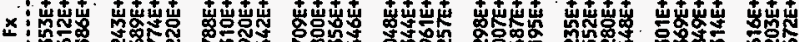

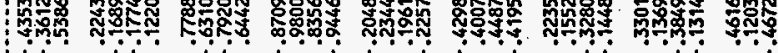

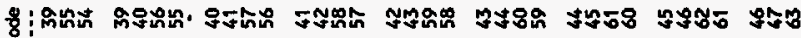

;

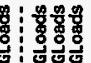

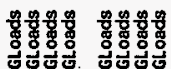

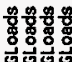

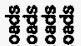

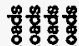

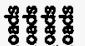

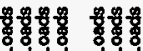

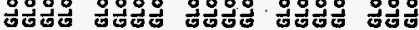


KAISER ENGINEERS HANFORD S/N:801854

PAGE 147

RUn 1D=N1N61973

$02 / 22 / 95$
$08: 51: 01$

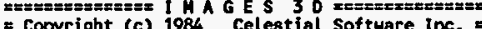

* Copyright (c) 1984 Celestial Software Inc.

SOLVE PLATE LOADS/STRESSES Version 2.0 07/01/90

ᄂ

Load Case 4:UBC SEISHIC LOAD - SN \& EH DIR.

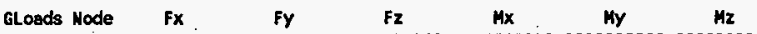

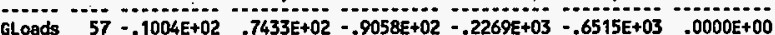

GLoads $73=7441 \mathrm{E}+01-3021 \mathrm{E}+03 \quad 8308 \mathrm{O}+02 \quad .2408 \mathrm{E}+03=.6048 \mathrm{EE}+03.0000 \mathrm{O}+00$

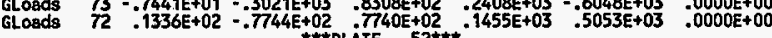

GLoads $57 \cdot .8779 E+01 \quad .2983 E+03-.8905 E+02-.1969 E+03 \quad .6357 E+03 \quad .0000 E+00$

GLoads $58 \quad .5013 \mathrm{E}+01 \quad .6485 \mathrm{E}+02-.7218 \mathrm{E}+02-.2920 \mathrm{E}+03-.5031 \mathrm{E}+03 \quad .0000 \mathrm{E}+00$

GLosds $74 \quad .9788 E+01-.2937 E+03 \quad .9719 E+02 \quad-1363 E+03-.5051 E+03 \quad .0000 E+00$

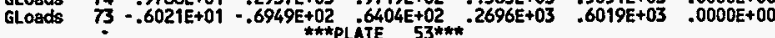

GLogds $58-.2699 \mathrm{E}+02 \quad .2660 \mathrm{E}+03=.1005 \mathrm{E}+03-.1792 \mathrm{E}+03 \quad .5066 \mathrm{E}+03 \quad .0000 \mathrm{E}+00$

GLoads $59 \quad .2400 \mathrm{E}+02 \quad .4957 \mathrm{E}+02 \quad-.4269 \mathrm{E}+02 \quad-.1604 \mathrm{E}+03 \quad-.2186 \mathrm{E}+03 \quad .0000 \mathrm{E}+00$

GLoads $75 \quad .2827 \mathrm{E}+02-.2528 \mathrm{E}+03 \quad .9536 \mathrm{E}+02 \quad .1318 \mathrm{E}+03-.8863 \mathrm{E}+02 \quad .0000 \mathrm{E}+00$

GLoads $74-.2528 \mathrm{E}+02-.6275 \mathrm{E}+02 \quad .4783 \mathrm{E}+02 \quad .1421 \mathrm{E}+03 \quad .6109 \mathrm{E}+03 \quad .0000 \mathrm{E}+00$

GLoads $59-.3785 E+02 \quad .2493 E+03-.1329 E+03 \quad .5119 E+02 \quad .2344 E+03 \quad .0000 E+00$

$\begin{array}{llllllll}\text { GLoads } & 60 & .4282 \mathrm{E}+02 & .3878 \mathrm{E}+02 & -.1446 \mathrm{E}+02 & .0000 \mathrm{E}+00 & .3689 \mathrm{E}+03 & .3658 \mathrm{E}+02\end{array}$

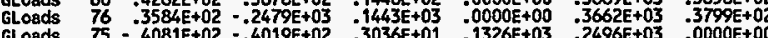

Ghoses

CLoads

GLoads

GLoeds

GLoads

GLoads

GLoads

GLoeds

CLoads

GLoads

GLoads

$75=.4081 E+02-.4019 E+02.3036 E+01 \quad-1326 E+03 \quad .2496 E+03 \quad .0000 E+00$

$60.9444 E+01 \quad .2049 E+03-.5473 E+02-.7332 E+02 \quad .4731 E+03 \quad .0000 E+00$

$61.1204 E+03 \quad-1262 E+02 \quad-3690 E+02-.1170 E+03 \quad .1412 E+03 \quad-0000 E+00$

$77-.1528 E+02-.2010 E+03 \quad .4508 E+02-.1645 E+03 \quad .1749 E+03 \quad .0000 E+00$

$76-.1146 E+03-.1652 E+02-.2726 E+02-.3722 E+02 \quad .4542 E+03 \quad .0000 E+00$

$61.8375 E+01 \quad .1933 E+03-.2481 E+02 \quad .1888 E+03-.1466 E+03 \quad .0000 E+00$ $62.1157 \mathrm{E}+03 \quad .1319 \mathrm{E}+02 \quad .2949 \mathrm{E}+02 \quad .1428 \mathrm{E}+03 \quad .4221 \mathrm{E}+03 \quad .0000 \mathrm{E}+00$ $78-1555 E+02-.1931 E+03 \quad .2668 E+02-1552 E+03 \quad .5708 E+03$ $77-.1085 E+03-.1331 E+02-.3136 E+02-.7334 E+02 \quad .5628 E+01 \quad .0000 E+00$ $62.7168 E+01 \quad-1918 E+03-.7434 E+01 \quad-3297 E+03-.4541 E+03 \quad .0000 E+00$ $.1140 \mathrm{E}+03 \cdot 1755 \mathrm{E}+02 \cdot 1157 \mathrm{~T}$ $79-.8183 E+01-.1933 E+03 \quad .7347 E+01-.3100 E+03 \quad-5980 E+03 \quad .0000 E+00$ $78-.1130 E+03-.1605 E+02-.1148 E+02-.8503 E+02-.4129 E+03$

$\begin{array}{lllllllll}\text { GLods } & 63 & .3065 E+01 & .1818 E+03 & .9531 E+01 & .3251 E+03 & -.6253 E+03 & .0000 E+00\end{array}$ $\begin{array}{lllllllll}\text { GLoads } & 64 & .1007 \mathrm{E}+03 & .3059 \mathrm{E}+02 & .9399 \mathrm{E}+00 & .2644 \mathrm{E}+03 & .3963 \mathrm{E}+03 & .0000 \mathrm{E}+00\end{array}$

$80 \quad .3374 \mathrm{E}+01-.1811 \mathrm{E}+03-.1472 \mathrm{E}+02-.2026 \mathrm{E}+03 \quad .5334 \mathrm{E}+03 \quad .0000 \mathrm{E}+00$ T GLoeds

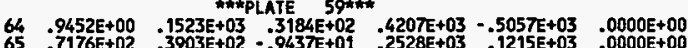

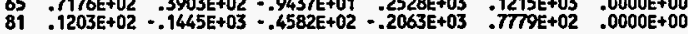

KAISER ENGINEERS HANFORO S/N:801854

PAGE 148 Run ID =1N61973

$02 / 22 / 95$

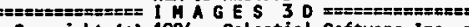

= Copyright (c) 1984 Celestial Software inc.

SOLVE PLATE LOADS/STRESSES Version 2.0 07/01/90

L.

Load Case 4:UBC SEISHIC LOAD - SN \& EW DIR.

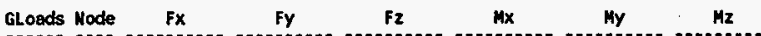

GLoods $80-.8473 E+02-.4681 E+02 \quad .2342 E+02 \quad .2563 E+02-.5317 E+03 \quad .0000 E+00$

$\begin{array}{lllllllll}\text { GLoads } & 65 & .2358 E+02 & .1389 E+03 & .3400 E+02 & .2798 E+03 & -.2084 E+03 & .0000 E+00\end{array}$

$\begin{array}{llllllll}\text { Gloads } & 66 & .3748 \mathrm{E}+02 & .5269 \mathrm{E}+02 & -.3156 \mathrm{E}+02 & -3005 \mathrm{E}+03 & -.1891 \mathrm{E}+03 & .0000 \mathrm{E}+00\end{array}$

$82-3335 \mathrm{E}+02-1413 \mathrm{E}+03=4039 \mathrm{E}+02-3219 \mathrm{E}+02-2763 \mathrm{E}+03-0000 \mathrm{E}+00$

GLoods $81-.2771 \mathrm{E}+02=.5031 \mathrm{E}+02 \quad .1796 \mathrm{E}+02=.5452 \mathrm{E}+02=.1142 \mathrm{E}+03 \quad .0000 \mathrm{E}+00$

$\begin{array}{llllllll}\text { GLogds } \quad 67=.4083 E+02 & .1348 E+02 & .2239 E+02 & .2928 E+03 & -.1321 E+03 & .0000 E+00\end{array}$

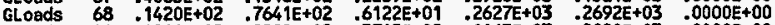

GLoads $84.4811 E+02$

GLoads $83-.2148 \mathrm{E}+02-.6707 \mathrm{E}+02 \quad .8535 \mathrm{E}+01 \quad .1124 \mathrm{E}+03 \quad-.3578 \mathrm{E}+03 \quad .0000 \mathrm{E}+00$

GLoods $68=.3621 E+02 \quad .3883 E+02 \quad .2115 E+02-.1211 E+03-.2601 E+03 \quad .0000 E+00$

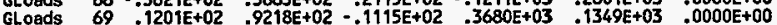

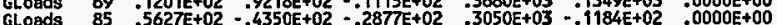

GLoads $84-.3208 E+02 \quad-.8751 E+02 \quad .1878 E+02 \quad-.2769 E+03 \quad-.4087 \mathrm{E}+03 \quad .0000 \mathrm{E}+00$

GLoods $69-.1321 E+02 \quad .7180 E+02 \quad .4574 E+02-.7271 E+01-.8069 E+02 \quad .0000 E+00$

GLoads $70 \quad .9540 \mathrm{E}+01 \quad .8033 \mathrm{E}+02-2351 \mathrm{E}+02 \quad .2920 \mathrm{E}+03-.1278 \mathrm{E}+03 \quad .0000 \mathrm{O}+00$

GLads $86 \quad .3386 \mathrm{E}+02=.7294 \mathrm{E}+02=.9148 \mathrm{E}+02 \quad .3155 \mathrm{E}+03=.7650 \mathrm{E}+03 \quad .0000 \mathrm{E}+00$

GLoods $85-.3019 \mathrm{E}+02=.7919 \mathrm{E}+02 \quad .6925 \mathrm{E}+02 \quad .1112 \mathrm{E}+02-.5982 \mathrm{E}+03 \quad .0000 \mathrm{O}+00$

GLoods $70.2128 E+02 \quad .1678 E+03-.4081 E+01 \quad .0000 E+00-.3243 E+03 \quad .2784 E+03$

GLoads $71=.4885 \mathrm{E}+02 \quad-4351 \mathrm{E}+02-.7449 \mathrm{E}+02-.1960 \mathrm{E}+02 \quad .2617 \mathrm{E}+02 \quad .0000 \mathrm{E}+00$

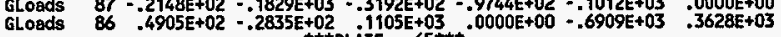

$\begin{array}{llllllll}\text { GLoads } & 71 & .9682 \mathrm{E}+01 & -1819 \mathrm{E}+03 & -.2944 \mathrm{E}+02 & -.3392 \mathrm{E}+03 & .1956 \mathrm{E}+03 & .0000 \mathrm{E}+00 \\ \text { GLoads } & 72-.2643 \mathrm{E}+02 & .7600 \mathrm{E}+02 & -.2584 \mathrm{E}+02 & -.4598 \mathrm{E}+02 & -.4425 \mathrm{E}+03 & .0000 \mathrm{E}+00\end{array}$

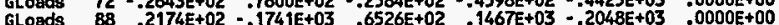

$\begin{array}{llllllll}\text { GLoods } & 83 & .2174 \mathrm{E}+02 & -.1741 \mathrm{E}+03 & .6526 \mathrm{E}+02 & .1467 \mathrm{E}+03 & -.2048 \mathrm{E}+03 & .0000 \mathrm{O}+00 \\ \text { GLoads } & 87 & -.4994 \mathrm{E}+01 & -.8384 \mathrm{E}+02 & -.9977 \mathrm{E}+01 & -.2220 \mathrm{E}+03 & .3791 \mathrm{E}+03 & .0000 \mathrm{E}+00\end{array}$

GLoads $\quad 72-.6704 E+00 \quad-1875 E+03 \quad-.7171 E+02-.2005 E+03 \quad .5719 E+03 \quad .0000 E+00$

GLoads $73-.1287 \mathrm{E}+02 \quad .4816 \mathrm{E}+02-.4504 E+01-.2670 E+03-.5646 \mathrm{E}+03 \quad .0000 \mathrm{E}+00$

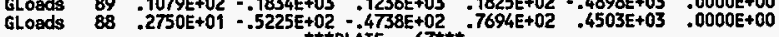

GLod $73-1438 E+02 \quad 2149 E+03-1010 E+03-2435 E+03 \quad 5676 E+03 \quad 0000 E+00$

GLoads $73 \cdot-1438 E+02 \quad .2149 E+03 \cdot-1019 E+03-.2435 E+03 \quad-5676 E+03 \quad .0000 E+00$

GLoads $74=.3642 E+00-.1832 E+02-.1002 E+02-.2474 E+03-.5947 E+03 \quad-0000 E+00$

$\begin{array}{llllllll}\text { GLoads } & 90 & .1044 \mathrm{E}+02 & -.2169 \mathrm{E}+03 & -1515 \mathrm{E}+03 & .3574 \mathrm{E}+02 & -.4138 \mathrm{H}+03 & .0000 \mathrm{C}+00 \\ \text { GLoads } & 89 & .4307 \mathrm{E}+01 & -.1631 \mathrm{E}+02 & -.3962 \mathrm{E}+02 & .4954 \mathrm{E}+02 & .5972 \mathrm{E}+03 & .0000 \mathrm{E}+00\end{array}$

GLonds $74 \cdot .2486 E+02.2295 E+03 \cdot .9429 E+02 \cdot .3114 E+02 \quad .4889 E+03 \quad .0000 E+00$ 
KAISER ENGIHEERS HANFORD S/N:801854 Run ID =\$1161973

$02 / 22 / 95$ PAGE 149

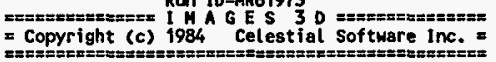
8:51:04 SOLVE PLATE LOADS/STRESSES Version 2.0 07/01/90

Load Case 4:UBC SEISMIC LOAD - SN \& EN DIR.

GLoads Node Fx $\quad$ Fy $\quad$ Fz $\quad$ Mx $\quad$ My Mz

GLoads 75 . $1149 \mathrm{E}+02 \quad .2660 \mathrm{E}+02-.2469 \mathrm{E}+02-.3386 \mathrm{E}+03-.2732 \mathrm{E}+03 \quad .0000 \mathrm{E}+00$

GLoads $91 \quad .6595 \mathrm{E}+01-.2377 \mathrm{E}+03 \quad .216 \mathrm{E}+03-.1815 \mathrm{E}+03-.3304 \mathrm{E}+03 \quad .0000 \mathrm{E}+00$

GLoads $90.6768 \mathrm{E}+01=.1845 \mathrm{E}+02-.2635 \mathrm{E}+01.1838 \mathrm{EE}+03 \quad .3951 \mathrm{E}+03 \quad .0000 \mathrm{E}+00$

$\begin{array}{lllllll}\text { GLoads } 75 \cdot .3966 E+02 & .1578 E+03-.3300 E+02 & .7423 E+02 & .1122 E+03 & .0000 E+00\end{array}$

$\begin{array}{lllllll}\text { GLoads } & 76 & .4529 \mathrm{E}+02 & .2878 \mathrm{E}+02 & -.2729 \mathrm{E}+02 & .0000 \mathrm{E}+00 & .2911 \mathrm{E}+03-.2327 \mathrm{E}+02\end{array}$

GLoads $92.2366 \mathrm{E}+02=.1357 \mathrm{E}+03-.3972 \mathrm{E}+02 \quad .0000 \mathrm{E}+00 \quad .4333 \mathrm{E}+03 \quad .5005 \mathrm{E}+01$

GLoods $91-.2929 E+02-.5087 \mathrm{E}+02.1000 \mathrm{t}+03.0234 \mathrm{E}+02 \quad .2321 \mathrm{E}+03 \quad .0000 \mathrm{E}+00$

$\begin{array}{lllllllll}\text { GLods } & 76 & -.2606 E+02 & .1341 E+03 & -.3560 E+02 & -1267 E+03 & .3562 E+03 & .0000 E+00\end{array}$

GLoads $77 \quad .1169 \mathrm{E}+03 \quad-.3264 \mathrm{E}+02 \quad .4295 \mathrm{E}+02-.6925 \mathrm{E}+02 \quad .4160 \mathrm{E}+02 \quad .0000 \mathrm{E}+00$

GLoads $93-1123 \mathrm{E}+02-.1321 \mathrm{E}+03 \quad .3051 \mathrm{E}+02-.3200 \mathrm{E}+02 \quad .2126 \mathrm{E}+03 \quad .0000 \mathrm{E}+00$

GLoads $92-.1021 \mathrm{E}+03 \quad .3059 \mathrm{E}+02-3786 \mathrm{E}+02 \quad 1767 \mathrm{E}+03 \quad .5037 \mathrm{E}+03 \quad .0000 \mathrm{E}+00$

GLoads $77 \cdot .3295 E+02 \quad .1407 E+03-.1684 E+02 \quad .3071 E+03-.2222 E+03 \quad .0000 E+00$

GLoads $78 \quad-1345 E+03-.3863 E+02 \quad .2519 E+02 \quad-.3992 E+02 \quad .4171 E+03 \quad .0000 E+00$

GLOadS $94 \quad .3913 E+02-.1454 E+03-.419 E+01-.2169 E+03 \quad .3856 E+03 \quad .000 E+00$

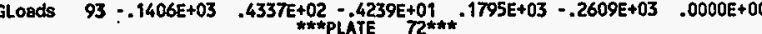

GLoads $78 \cdot .4573 E+02 \quad .1416 E+03-.5539 E+00 \quad .2802 E+03-.5750 E+03 \quad .0000 E+00$

GLoads $79 \quad .1355 \mathrm{E}+03-.1836 \mathrm{E}+02 \quad .1291 \mathrm{E}+02 \quad .1312 \mathrm{E}+03 \quad .4947 \mathrm{E}+03 \quad .0000 \mathrm{E}+00$

$\begin{array}{llllllll}\text { GLoads } \quad 95 & .4869 \mathrm{E}+02 & -.1445 \mathrm{E}+03 & -.2667 \mathrm{E}+01 & -.1080 \mathrm{E}+03 & .5936 \mathrm{E}+03 & .0000 \mathrm{E}+00\end{array}$

GLoads

GLoeds $94-.1385 \mathrm{E}+03 \quad .2126 \mathrm{E}+02-.9686 \mathrm{E}+01$

$79-.6004 E+02 \quad .1367 E+03 \quad .1533 E+02 \quad .3354 E+03-.5793 E+03 \quad .0000 E+00$ $\begin{array}{llllllll}80 & .1340 \mathrm{E}+03 & .7271 \mathrm{E}+01 & .6486 \mathrm{E}+01 & .1737 \mathrm{E}+03 & .4876 \mathrm{E}+03 & .0000 \mathrm{E}+00\end{array}$ $96.6132 E+02-.1413 E+03-.1182 E+02-.1531 E+02 \quad .4580 E+03 \quad .0000 E+00$

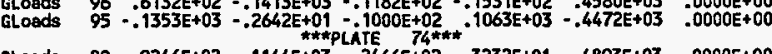

GLoads

***PLATE 74***

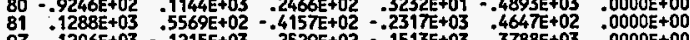

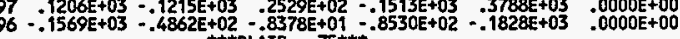

$96-.1569 E+03-.4862 E+02-.8378 E+01-.85$

GLoods $81-.1529 E+03 \quad .3287 E+02 \quad .1093 E+03 \quad .4924 E+03-.1008 E+02 \quad .0000 E+00$

$\begin{array}{llllllll}\text { GLoads } & 82 & .1066 \mathrm{E}+03 & .1079 E+03 & .6422 \mathrm{E}+02 & -1514 \mathrm{E}+04 & -.8587 \mathrm{E}+03 & .0000 \mathrm{O}+00\end{array}$

7 GLoads $98 \quad .2846 E+03-.2382 \mathrm{E}+02-.2048 \mathrm{E}+03 \quad .1918 \mathrm{E}+04-.1129 \mathrm{E}+04 \quad .0000 \mathrm{E}+00$

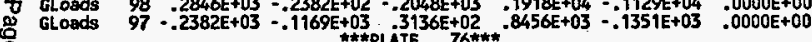

$83-.3426 \mathrm{E}+02-.1822 \mathrm{E}+02 \quad .1149 \mathrm{E}+02 \quad .3071 \mathrm{E}+03 \quad .4838 \mathrm{E}+03 \quad .0000 \mathrm{E}+00$

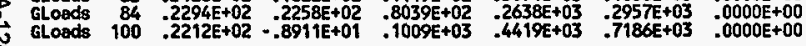

KAISER ENGIMEERS HANFORD S/N:801854

PAGE 150 RUn ID=NNG1973

$02 / 22 / 95$
$08: 51: 05$

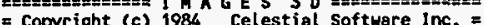
I Copyright (c) 1984 Celestial Software Inc. =

SOLVE PLATE LOADS/STRESSES Version 2.0 07/01/90

L

Load Case 4:UBC SEISMIC LOAD - SN \& EW DIR.

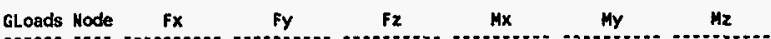

GLcads $99=.1080 E+02 \quad .4556 E+01 \quad-1927 E+03 \quad .5032 E+03 \quad .9788 E+03 \quad .0000 E+00$

GLoads $84-.7088 E+02 \quad .2663 E+01-.3021 E+02-.1032 E+03 \quad .3150 E+03 \quad .0000 E+00$

$\begin{array}{lllllllll}\text { GLoads } & 85 & .2495 \mathrm{E}+02 & .5137 \mathrm{E}+02 & .4890 \mathrm{E}+02 & .2014 \mathrm{E}+03 & .4416 \mathrm{E}+03 & .0000 \mathrm{O}+00\end{array}$

GLoads $101.6632 \mathrm{E}+02 \quad .4086 \mathrm{E}+01 \quad .7532 \mathrm{E}+02 \quad .2361 \mathrm{E}+03 \quad .4902 \mathrm{0}+03 \quad .0000 \mathrm{E}+00$

GLoads $100=.2039 E+02-.5812 E+02-.9402 E+02-.2587 E+02 \quad .4510 E+03 \quad .0000 E+00$

GLoads $85-.8294 E+02-.1377 E+02-.5747 \mathrm{E}+02-.5176 \mathrm{E}+03 \quad .1684 \mathrm{E}+03 \quad .0000 \mathrm{O}+00$

GLoads $\quad 86 \quad .5602 E+02 \quad .1148 E+02-.1009 E+03 \quad-.7817 E+03 \quad .1248 E+03 \quad .0000 E+00$

GLoads $102.9286 \mathrm{E}+02.2102 \mathrm{~T}+02.2096 \mathrm{E}+03-.8091 \mathrm{E}+03 \quad .6575 \mathrm{E}+03 \quad .0000 \mathrm{0}+00$

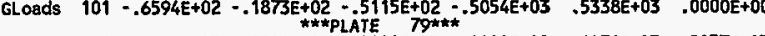

GLoads $86.1638 E+03-.1841 E+02 \quad .2004 E+02 \quad .0000 E+00=.6271 E+03-.8057 E+03$

GLoads $87-.2052 E+02 \quad .1395 E+03 \quad .1144 E+03 \quad .5251 E+03-.1985 E+03 \quad .0000 E+00$

GLoads $102 \div 1013 E+01-1247 E+03=2403 E+03$ O $0000+00-1016 E+04-6896 E+03$

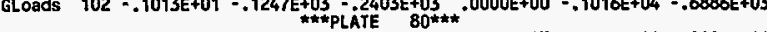

$\begin{array}{llllllll}\text { GLoads } \quad 87 & .1080 E+02 & .3078 E+02 & -.3631 E+02 & -.2056 E+03 & -.7943 E+02 & .0000 E+00\end{array}$

GLoads $88-.5800 E+02 \quad .8513 E+02 \quad .9953 E+02-.7538 E+02-.2635 E+03 \quad-0000 E+00$

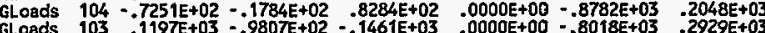

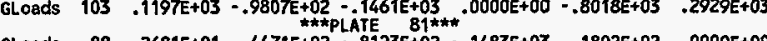

GLoads $88-.2681 E+01 \quad .4471 E+02-.8123 E+02-.1483 E+03 \quad-1802 E+02 \quad .0000 E+00$

GLoads $\quad 89 \cdot-.3363 E+02 \quad .2962 E+02 \quad .6637 E+02-.3345 E+02-.3001 E+03 \quad-0000 E+00$

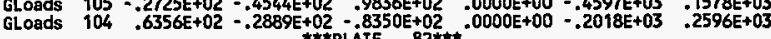

GLoads $\quad 89-.1765 E+02 \quad .7358 E+02-.1141 E+03-.3434 E+02 \quad .1927 E+03 \quad .0000 E+00$

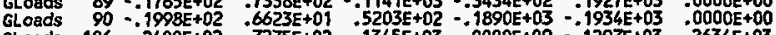

$\begin{array}{lllllll}\text { GLoads } 106 & .2400 \mathrm{E}+02 & -.7275 \mathrm{E}+02 & .1345 \mathrm{E}+03 & .0000 \mathrm{E}+00 & -.1297 \mathrm{E}+03 & .02634 \mathrm{E}+03\end{array}$

GLoads $105.1362 \mathrm{E}+02-.7454 \mathrm{E}+01-.7242 \mathrm{E}+02, .0000 \mathrm{E}+00 \quad \cdot 1928 \mathrm{E}+03 \quad .1341 \mathrm{E}+03$

GLoads $90-.3342 E+02 \quad .1322 E+03-.1647 E+03-.3058 E+02 \quad .2122 E+03 \quad .0000 E+00$

$\begin{array}{llllllll}\text { GLoads } 91 & .1632 \mathrm{E}+02 & -.1569 \mathrm{E}+02 & -1870 \mathrm{E}+02 & -.7147 \mathrm{E}+02 & -.100 \mathrm{E}+03 & .0000 \mathrm{0}+00\end{array}$

$\begin{array}{lllllllll}\text { GLoads } & 106 & -.3453 \mathrm{E}+02 & .2325 \mathrm{E}+02 & -.7905 \mathrm{E}+02 & .0000 \mathrm{E}+00 & .5955 \mathrm{E}+03 & .0207 \mathrm{E}+02\end{array}$

GLoads $91-.2982 E+02.2077+03-.2041 E+03.1907 E+03 \quad .1990 E+03 \quad .0000 E+00$

$\begin{array}{llllllll}\text { GLoads } & 91 & -.2982 E+02 & .2077 \mathrm{E}+03 & -.2041 \mathrm{E}+03 & .1907 \mathrm{E}+03 & .1990 \mathrm{E}+03 & .0000 \mathrm{E}+00 \\ \text { GLoads } & 92 & .4455 \mathrm{E}+02 & -.7413 \mathrm{E}+02 & -.5197 \mathrm{E}+02 & .0000 \mathrm{E}+00 & .2930 \mathrm{E}+03 & .1500 \mathrm{E}+02\end{array}$

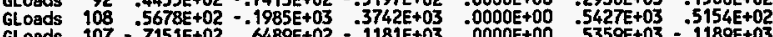

GLoads $107-.7151 \mathrm{E}+02 \quad .6489 \mathrm{E}+02-.1181 \mathrm{E}+03 \quad .0000 \mathrm{E}+00 \quad .5359 \mathrm{E}+03-.1189 \mathrm{E}+03$

$\begin{array}{lllllllllll}\text { GLoads } & 92 & -.3175 E+02 & .4823 E+02 & .7630 E+01 & .4333 E+03 & .2769 E+03 & .0000 E+00\end{array}$ 
KAISER ENGINEERS HANFORO S/H:801854

PAGE 15I RUN ID=MN61973

$02 / 22 / 95$

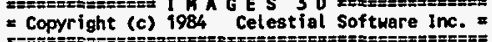

SOLVE PLATE LOADS/STRESSES Version 2.0 07/01/90 L

Load Case 4:UBC SEISMIC LOND - SH \& EN DIR.

\begin{tabular}{|c|c|c|c|c|c|c|c|}
\hline $\operatorname{Lan}$ & de & $F x$ & Fy & Fz & & Hy & $\mathrm{Nz}$ \\
\hline $\begin{array}{l}\text { GLoads } \\
\text { GLoads } \\
\text { GLoads }\end{array}$ & $\begin{array}{r}93 \\
109 \\
108\end{array}$ & $\begin{aligned} & .1406 \mathrm{E}+03 \\
& .7454 \mathrm{E}+02 \\
& \therefore .1834 \mathrm{E}+03\end{aligned}$ & $\begin{array}{r}-.5888 E+02 \\
-.5954 E+02 \\
.7019 E+02 \\
\end{array}$ & $\begin{array}{l}.4010 E+02 \\
.5248 E+02 \\
.1002 E+03\end{array}$ & $\begin{array}{l}37 E+02 \\
6 E+02 \\
38 E+03\end{array}$ & $\begin{array}{l}.1648 E+03 \\
.4773 E+03 \\
.4852 E+03\end{array}$ & $\begin{array}{l}.0000 E+00 \\
.0000 E+00 \\
.0000 E+00\end{array}$ \\
\hline $\begin{array}{l}\text { GLoads } \\
\text { GLoads } \\
\text { GLoads } \\
\text { GLoads }\end{array}$ & $\begin{array}{r}93 \\
94 \\
110 \\
109\end{array}$ & $\begin{array}{r}-.4660 E+02 \\
.1111 E+03 \\
.5707 \mathrm{E}+02 \\
-.1216 \mathrm{E}+03\end{array}$ & $\begin{array}{r}.5317 E \\
-.1706 E \\
-.5313 E \\
.1703 E\end{array}$ & $\begin{array}{r}.265 \\
.366 \\
.323\end{array}$ & $\begin{array}{l}+02 \\
+02 \\
+02 \\
+02\end{array}$ & $\begin{array}{r}. .11 \\
.17 \\
.56 \\
.32\end{array}$ & $\begin{array}{l}.0000 E+00 \\
.0000 E+00 \\
.0000 E+00 \\
.0000 E+00\end{array}$ \\
\hline $\begin{array}{l}\text { GLoads } \\
\text { GLoads } \\
\text { GLoads } \\
\text { GLoads }\end{array}$ & $\begin{array}{r}94 \\
95 \\
111 \\
110\end{array}$ & $\begin{array}{r}-.4717 E+02 \\
.9118 E+02 \\
.4586 E+02 \\
-.8987 E+02\end{array}$ & $\begin{array}{r}.4679 \mathrm{E}+02 \\
.5223 \mathrm{E}+00 \\
.4736 \mathrm{E}+02 \\
.1086 \mathrm{E}+01 \\
k+\mathrm{p}^{2}\end{array}$ & 2 & $\begin{array}{l}.2163 E+03 \\
.7492 E+01 \\
.1296 E+03 \\
.3121 E+03\end{array}$ & $\begin{array}{r}-.2061 E+03 \\
.1624 E+03 \\
.2029 E+03 \\
-.1047 E+03\end{array}$ & $\begin{array}{l}.0000 E+00 \\
.0000 E+00 \\
.0000 E+00 \\
.0000 E+00\end{array}$ \\
\hline $\begin{array}{l}\text { GLoads } \\
\text { GLoads } \\
\text { GLoads } \\
\text { GLoads }\end{array}$ & $\begin{array}{r}95 \\
96 \\
112 \\
111\end{array}$ & $\begin{array}{r}-.4001 E+02 \\
.8594 E+02 \\
.2917 E+02 \\
-.7510 E+02\end{array}$ & $\begin{array}{l}.5322 E+02 \\
.4485 E+01 \\
.5445 E+02 \\
.3254 E+01\end{array}$ & $\begin{array}{l}.3127 E+02 \\
.1724 E+01 \\
.5972 E+02 \\
.2673 E+02\end{array}$ & & & $\begin{array}{l}.0000 E+00 \\
.0000 E+00 \\
.0000 E+00 \\
.0000 E+00\end{array}$ \\
\hline $\begin{array}{l}\text { GLoads } \\
\text { GLoads } \\
\text { GLoads } \\
\text { GLoads }\end{array}$ & $\begin{array}{r}96 \\
97 \\
113 \\
112\end{array}$ & $\begin{array}{r}-.2575 E+02 \\
.1130 E+03 \\
-.1692 E+02 \\
-.7032 E+02\end{array}$ & $\begin{array}{l}.9104 \mathrm{E}+02 \\
.6386 \mathrm{E}+01 \\
.1013 \mathrm{E}+03 \\
.3872 \mathrm{E}+01\end{array}$ & $\begin{array}{l}388 E+02 \\
498 E+02 \\
076 E+03 \\
870 E+02 \\
E \quad 90^{t *}\end{array}$ & & & $\begin{array}{l}.0000 E+00 \\
.0000 E+00 \\
.0000 E+00 \\
.0000 E+00\end{array}$ \\
\hline $\begin{array}{l}\text { GLoads } \\
\text { GLoads } \\
\text { GLoads } \\
\text { GLoads }\end{array}$ & $\begin{array}{r}97 \\
98 \\
114 \\
113\end{array}$ & $\begin{array}{l}-.3082 E+02 \\
.1953 E+03 \\
-.1018 E+03 \\
-.6267 E+02\end{array}$ & $\begin{array}{r}.1376 \mathrm{E}+03 \\
.5763 \mathrm{E}+02 \\
.1213 \mathrm{E}+03 \\
.4133 \mathrm{E}+02 \\
+0 \mathrm{~F}\end{array}$ & & & $\begin{array}{l}-.1790 \mathrm{E}+03 \\
\because .7191 \mathrm{E}+03 \\
=.7053 \mathrm{E}+03 \\
-.3592 \mathrm{E}+03\end{array}$ & $\begin{array}{l}.0000 E+00 \\
.0000 E+00 \\
.0000 E+00 \\
.0000 E+00\end{array}$ \\
\hline $\begin{array}{l}\text { GLoads } \\
\text { GLoads } \\
\text { GLoads } \\
\text { GLoads }\end{array}$ & $\begin{array}{r}122 \\
2 \\
19 \\
126\end{array}$ & $\begin{aligned} & .26898+03 \\
& .3480 E+02 \\
&-.2269 E+03 \\
&-.7671 E+02\end{aligned}$ & $\begin{array}{r}.6715 E+03 \\
.2918 E+03 \\
-.6258 E+03 \\
-.3375 E+03\end{array}$ & $\begin{array}{l}.8468 E+02 \\
. .5952 E+02 \\
.6438 E+02\end{array}$ & $\begin{array}{l}.03 \\
.03 \\
-03 \\
-03\end{array}$ & $\begin{array}{r}-.9483 E+02 \\
-.1851 E+02 \\
.1584 E+03 \\
.5225 E+02\end{array}$ & $\begin{array}{l}.0000 E+00 \\
.0000 E+00 \\
.0000 E+00 \\
.0000 E+00\end{array}$ \\
\hline $\begin{array}{l}\text { GLoads } \\
\text { GLoads } \\
\text { GLoads } \\
\text { GLoads }\end{array}$ & $\begin{array}{r}124 \\
19 \\
35 \\
126\end{array}$ & $\begin{array}{r}.5032 E+02 \\
.6957 E+02 \\
. .6781 E+02 \\
-.5209 E+02\end{array}$ & $\begin{array}{l}03 \\
03 \\
03 \\
03\end{array}$ & $\begin{array}{l}02 \\
02 \\
02\end{array}$ & $\begin{array}{l}-.2173 E+03 \\
-.2235 E+03 \\
-.6650 E+03 \\
-.6841 E+03\end{array}$ & $\begin{array}{r}-.1340 E+03 \\
.8857 E+02 \\
.2014 E+03 \\
-.3843 E+02\end{array}$ & $\begin{array}{l}.0000 E+00 \\
.0000 E+00 \\
.0000 E+00 \\
.0000 E+00\end{array}$ \\
\hline $\begin{array}{l}\text { GLoads } \\
\text { GLoads } \\
\text { GLoads }\end{array}$ & $\begin{array}{r}126 \\
35 \\
51\end{array}$ & $\begin{array}{r}.1893 \mathrm{E}+02 \\
-3110 \mathrm{E}+02 \\
-.2884 \mathrm{E}+02\end{array}$ & $\begin{array}{l}E E+03 \\
E+03 \\
E+03\end{array}$ & $\begin{array}{r}-.1480 E+02 \\
.3164 E+01 \\
.9078 E+01\end{array}$ & $\begin{array}{r}.2098 E+03 \\
.2204 E+03 \\
-.3172 E+03\end{array}$ & $\begin{array}{r}-.1291 E+03 \\
.1546 E+03 \\
.2515 E+03\end{array}$ & $\begin{array}{l}.0000 E+00 \\
.0000 E+00 \\
.0000 E+00\end{array}$ \\
\hline
\end{tabular}

KAISER ENGINEERS HANFORD S/H:801854

PAGE 152 Run ID=HA61973

$02 / 22 / 95$

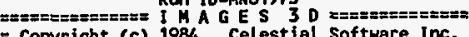
$=$ Copyright (c) 1984 Celestial Software Inc. =

SOLVE PLATE LOADS/STRESSES Version $2.0 \quad 07 / 01 / 90$

LOad Case 4:UBC SEISMIC LOAD - SH \& EH DIR.

GLoads Node Fx Fy $\quad$ Fz $\quad$ Mx $\quad$ My Mz GLoads $128-.2118 E+02-.1273 E+03 \quad .2560 E+01-.3691 E+03=.3208 E+02 \quad .0000 E+00$ $\begin{array}{lllllllll}\text { GLoods } & 128 & -.9575 \mathrm{E}+01 & .7083 \mathrm{E}+02 & .2589 \mathrm{E}+02 & .5889 \mathrm{E}+03 & -.8463 \mathrm{E}+02 & .0000 \mathrm{P}+00\end{array}$ $\begin{array}{lrrrrrrr}\text { GLoads } & 128 & -.9575 E+01 & .7083 E+02 & .2589 E+02 & .5889 E+03 & -.8463 E+02 & .0000 E+00 \\ \text { GLoods } & 51 & .1034 E+02 & .1033 E+03 & .1797 E+02 & .3982 E+03 & .1563 E+03 & .0000 E+00\end{array}$

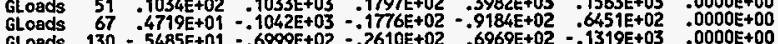
GLoods $130=.5485 \mathrm{E}+01-.6999 \mathrm{E}+02-.2610 \mathrm{E}+02-.0969 \mathrm{E}+02-.1319 \mathrm{E}+03 \quad .0000 \mathrm{E}+00$

GLoads $130-.3161 E+02-.1177 E+02 \quad .6188 E+02 \quad .9493 E+03 \quad .1164 E+02 \quad .0000 E+00$

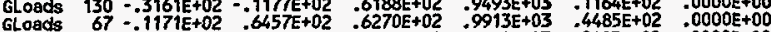

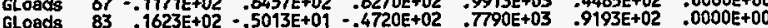

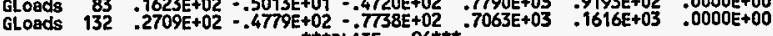
GLoads $132-.5808 E+02 \cdot-.4423 E+02 \quad .1113 E+03 \quad .5064 E+03-.1752 E+03 \quad .0000 E+00$

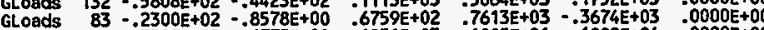
GLoeds $09 \quad .7599 \mathrm{E}+02 \quad .6775 \mathrm{E}+02-.1851 \mathrm{E}+03 \quad .1005 \mathrm{E}+04 \quad-.1002 \mathrm{E}+04 \quad .0000 \mathrm{E}+00$ GLoads $134.5086 E+01-.2266 E+02 \quad .6149 E+01 \quad .6790 E+03 \quad-.8045 E+03 \quad .0000 E+00$ **:PLATE 97***:

GLoads $\quad 17 \quad .1126 E+03 \quad .2524 E+03-.7256 E+02-.6518 E+03-.1198 E+03 \quad .0000 E+00$ GLoads $123 \quad .5919 \mathrm{E}+02 \quad .8692 \mathrm{E}+01-.8457 \mathrm{E}+02-.8680 \mathrm{E}+03-.8090 \mathrm{E}+02 \quad .0000 \mathrm{E}+00$ GLaads $125-.9623 \mathrm{E}+02-.1977 \mathrm{E}+03 \quad .7466 \mathrm{E}+02-.1068 \mathrm{E}+04-.5995 \mathrm{E}+01 \quad .0000 \mathrm{E}+00$ GLoads $34-.7357 \mathrm{E}+02-.6346 \mathrm{E}+02 \quad .8246 \mathrm{E}+02-.8689 \mathrm{E}+03 \quad .8583 \mathrm{E}+01 \quad .0000 \mathrm{E}+00$ GLoads $34 \quad .7055 E+02 \quad .2420 E+03-.22222 E+02-.4096 E+02-.1568 E+03 \quad .0000 E+00$ GLoads $125 \quad .6606 \mathrm{E}+02 \quad .7842 \mathrm{E}+02=.4531 \mathrm{E}+02=.2017 \mathrm{E}+03-.1258 \mathrm{E}+03 \quad .0000 \mathrm{E}+00$ GLoads $127-6905 \mathrm{E}+02-2287 \mathrm{E}+03$ - $2637 \mathrm{E}+02-.6830 \mathrm{E}+03$ GLoads $50=.6756 \mathrm{E}+02-.9173 \mathrm{E}+02 \quad .4116 \mathrm{E}+02-5600 \mathrm{E}+03 \quad-.4492 \mathrm{E}+02 \quad .0000 \mathrm{E}+00$ $\begin{array}{lllllllll}\text { Gloads } & 50 & .5233 E+02 & .2158 E+03 & .3143 E+02 & .4329 E+03 & -.1803 E+03 & .0000 E+00\end{array}$ $\begin{array}{lrllllll}\text { GLoads } & 127 & .3684 \mathrm{E}+02 & .1280 \mathrm{E}+03 & .1918 \mathrm{E}+01 & .4724 \mathrm{E}+03 & -.2128 \mathrm{E}+03 & .0000 \mathrm{E}+00\end{array}$ GLoads $129=.5696 \mathrm{E}+02-.2261 \mathrm{E}+03-.4887 \mathrm{E}+02-.4147 \mathrm{E}+02-.3052 \mathrm{O}+03 \quad .0000 \mathrm{O}+00$ GLoads $66-.3221 \mathrm{E}+02-.1178 \mathrm{E}+03 \quad 1552 \mathrm{E}+02=-1301 \mathrm{E}+03-.2407 \mathrm{E}+03 \quad .0000 \mathrm{E}+00$ GLoads $66-.3686 E+01.1525 E+03 \quad .7131 E+02 \quad .7469 E+03-.3211 E+01 \quad .0000 E+00$

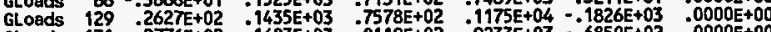
GLoads $131.2776 \mathrm{E}+02-.1683 \mathrm{E}+03-.8119 \mathrm{E}+02 \quad .9233 \mathrm{E}+03-.6850 \mathrm{E}+02 \quad .0000 \mathrm{E}+00$ GLoads $82-.5035 \mathrm{E}+02-.1276 \mathrm{E}+03-.6590 \mathrm{E}+02 \quad .92909 \mathrm{E}+03 \quad .1461 \mathrm{E}+03 \quad .0000 \mathrm{E}+00$

$\begin{array}{lllllllll}\text { GLoads } 82-.4856 E+02 & .1774 E+02 & .8976 E+02 & .1934 E+04 & .9600 E+03 & .0000 E+00\end{array}$

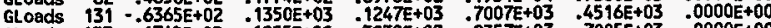
GLoads $133-.6742 \mathrm{E}+02 \quad .1925 \mathrm{E}+02 \quad .3832 \mathrm{E}+02 \quad .9357 \mathrm{E}+03 \quad .7005 \mathrm{E}+03 \quad .0000 \mathrm{E}+00$ $\begin{array}{lllllll}\text { GLoads } 98 & 91796 \mathrm{E}+03 & -.1720 \mathrm{E}+03 & -.2528 \mathrm{E}+03 & -.2327 \mathrm{E}+04 & .1148 \mathrm{E}+04 & .0000 \mathrm{E}+00\end{array}$ $\begin{array}{lllllll}\text { GLoads } & 98 & .1321 \mathrm{E}+03 & .8125 \mathrm{E}+02-.1984 \mathrm{E}+03 * .1511 \mathrm{E}+0.4 & .3325 \mathrm{E}+03 & .0000 \mathrm{E}+00\end{array}$ 


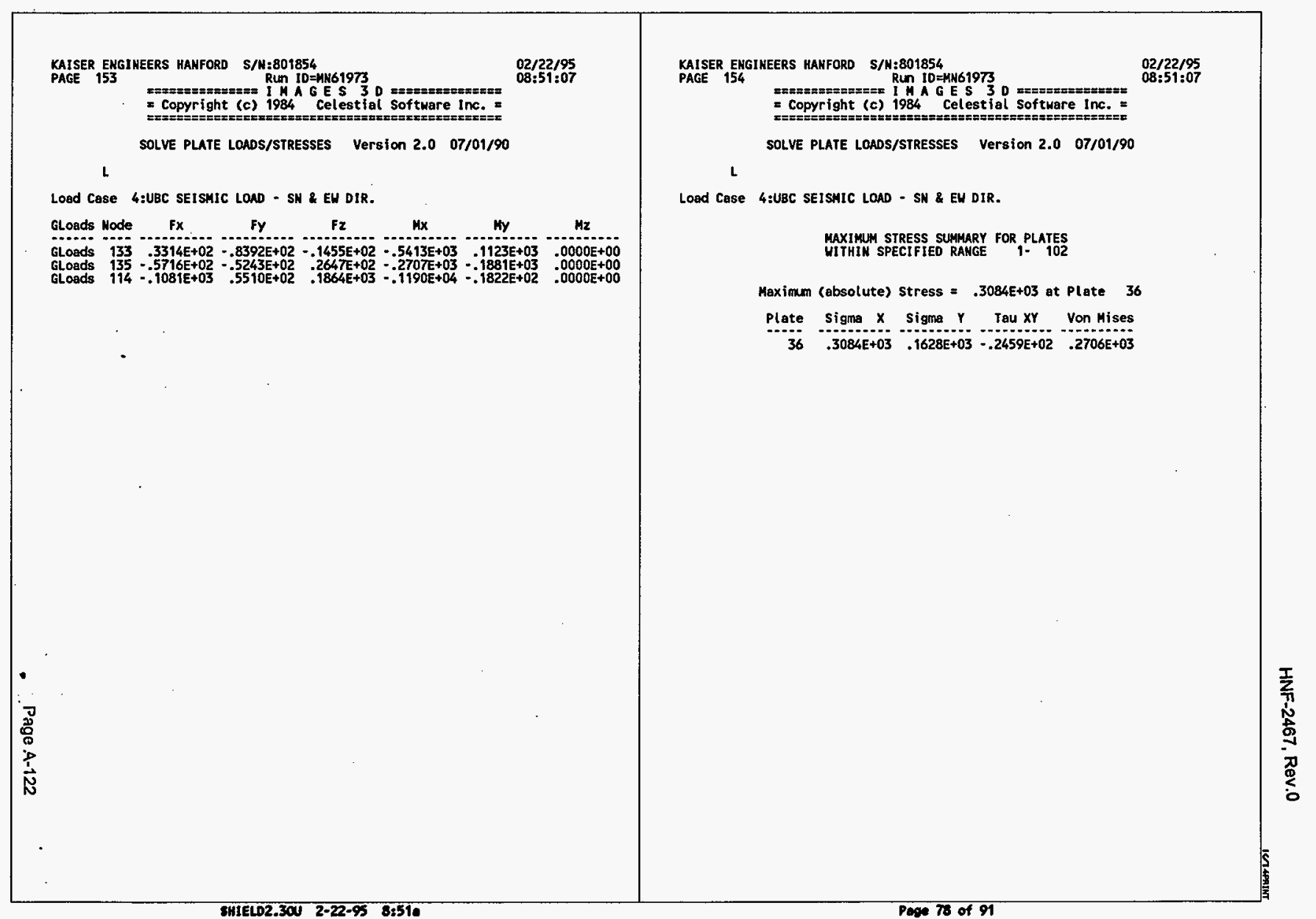


KAISER ENGIMEERS MALFORD S/W:801854

PAGE 155 Run ID=NN61973

$02 / 22 / 95$
$08: 51: 07$ $=$ Copyright (c) 1984 Celestial Software Inc.

SOLVE REACTIONS

Version $2.0 \quad 07 / 01 / 90$

L

Load Case 4:UBC SEISHIC LOND - SH \& EU DIR.

REACTIONS

\begin{tabular}{|c|c|c|c|c|c|}
\hline de & $F x$. & $F_{2}$ & $H x$ & My & $\mathrm{Mz}$ \\
\hline $\begin{array}{r}10 \\
11 \\
12 \\
13 \\
14 \\
15 \\
16 \\
17 \\
18 \\
115 \\
116 \\
117 \\
118 \\
119 \\
122\end{array}$ & $\begin{array}{l}.1970 \mathrm{E}+02 \\
.2906 \mathrm{E}+03 \\
.2339 \mathrm{E}+03 \\
.2275 \mathrm{E}+03 \\
.1609 \mathrm{0}+02 \\
.2259 \mathrm{0}+02 \\
: 4749 \mathrm{0}+02 \\
.4448 \mathrm{E}+02 \\
.4501 \mathrm{0}+02 \\
.1969 \mathrm{E}+02 \\
.2926 \mathrm{E}+03 \\
.3402 \mathrm{E}+03 \\
.3127 \mathrm{E}+03 \\
.2799 \mathrm{E}+03 \\
.2493 \mathrm{E}+03 \\
.2154 \mathrm{E}+03 \\
.1804 \mathrm{E}+03 \\
.2130 \mathrm{0}+02 \\
.7963 \mathrm{E}+04 \\
.3619 \mathrm{E}+04 \\
.7830 \mathrm{0}+03 \\
.1827 \mathrm{E}+04 \\
.7840 \mathrm{E}+03 \\
.2889 \mathrm{E}+03\end{array}$ & 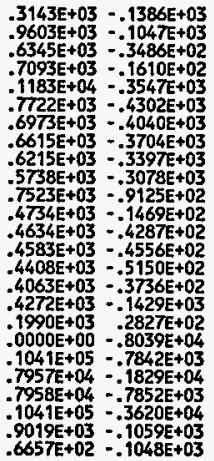 & $\begin{array}{l}00 \\
00 \\
00 \\
00 \\
03 \\
00 \\
00 \\
00 \\
00 \\
00 \\
03 \\
00 \\
00 \\
00 \\
00 \\
00 \\
00 \\
00 \\
00 \\
00 \\
00 \\
00 \\
00 \\
00\end{array}$ & $\begin{array}{l}3 \\
3 \\
4 \\
3 \\
3 \\
3 \\
1 \\
3 \\
3 \\
4 \\
3 \\
3 \\
3 \\
1 \\
3 \\
3 \\
0 \\
0 \\
0 \\
0 \\
0 \\
0 \\
0\end{array}$ & \\
\hline
\end{tabular}

KAISER ENGINEERS HANFORD S/N:801854 Run ID=NN61973

PAGE 156

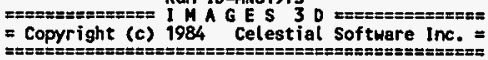
$08: 51: 08$

SOLVE BEAH LOAOS/STRESSES Version 2.0 07/01/90

L

Load Case 5:UBC SEISHIC LOAD - NS \& WE

BEAM LOAOS AND/OR STRESSES

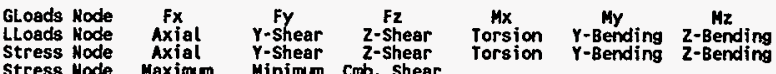
Stress Hode Maximum Minimum Cmb. Shear t**BEEAN NO. 1***

GLoads $\quad 1-.1949 E+02-.6938 E+02 \quad .8238 E+02 \quad .4547 E-12-.2842 E-13-.1137 E-12$

GLoads $99=.3499 \mathrm{E}+02 \quad .2147 \mathrm{E}+03=.2790 \mathrm{E}+02-.5325 \mathrm{E}+03-.4263 \mathrm{E}+03-.1023 \mathrm{E}+04$

Lloads $99.2089 \mathrm{E}+03-.5681 \mathrm{E}+02 \quad .3499 \mathrm{E}+02-.3497 \mathrm{E}-13 \quad: 1108 \mathrm{E}+04 \quad .5325 \mathrm{E}+03$

GLads $18=.2179 E+02.1337 E+02.9071 \mathrm{E}+011^{2} .6395 \mathrm{E}-13 \quad .0000 \mathrm{E}+00 \quad .0000 \mathrm{E}+00$

GLoads $114-.3147 \mathrm{E}+02 \quad .1286 \mathrm{E}+03 \quad .4419 \mathrm{E}+02 \quad .3336 \mathrm{E}+03 \quad .2227 \mathrm{E}+03-.6392 \mathrm{E}+03$

LLoads $18 \quad .1561 \mathrm{E}+02 \quad .4164 \mathrm{E}+01 \quad-.2179 \mathrm{E}+02 \quad .0000 \mathrm{E}+00 \quad .0000 \mathrm{E}+00 \quad .6395 \mathrm{E}-13$

LLoads $114: .1360 \mathrm{E}+03 \quad-.6083 \mathrm{E}+00 \quad-.3147 \mathrm{E}+02 \quad .5426 \mathrm{E}-14 \quad-.6769 \mathrm{E}+03 \quad .3336 \mathrm{E}+03$

GLoads $99.1527 \mathrm{E}+03 \quad .8482 \mathrm{E}+02=.4157 \mathrm{E}+03^{3 *} \quad .2936 \mathrm{E}+04 \quad .4544 \mathrm{E}+03 \quad .1123 \mathrm{E}+04$

GLoads $108-.1915 E+03 \quad .1844 E+02 \quad .4544 E+03 \quad .1512 E+03 \quad .1378 E+04 \quad .2374 E+03$

LLoads $99 \quad .4420 \mathrm{E}+03-.8482 \mathrm{E}+02 \quad .2793 \mathrm{E}+02 \quad .1562 \mathrm{E}+03-.4544 \mathrm{E}+03-.3139 \mathrm{E}+04$

LLoads $108-.4931 \mathrm{E}+03-.1844 \mathrm{E}+02-.8123 \mathrm{E}+01-.1562 \mathrm{E}+03-.1378 \mathrm{E}+04-.2341 \mathrm{E}+03$

GLoads $114-.2889 E+03 \quad .6052 E+02 \quad .2735 E+03-.6405 E+03-.1061 E+04-.7032 E+03$

GLoads $102 \quad .2393 E+03 \quad .7167 E+02-.2239 E+03 \quad-1159 E+04-.8689 E+03 \quad .1211 E+04$

LLods $114-3975 E+03-.6052 E+02-.1521 E+02-.5464 E+02 \quad .1061 E+04-.9496 E+03$

LLoads $102-.3274 \mathrm{E}+03-.7167 \mathrm{E}+02 \quad-1445 \mathrm{E}+02 \quad .5464 \mathrm{E}+02 \quad .8689 \mathrm{E}+03 \quad-1675 \mathrm{E}+04$

GLoads $98 \quad .8512 E+03 \quad .2689 E+02-.9995 E+03 \quad .5678 E+03-.9977 E+03 \quad .2301 E+03$

GLoads $115-.8876 \mathrm{E}+03 \quad .7031 \mathrm{E}+02 \quad .1036 \mathrm{E}+04 \quad .9956 \mathrm{E}+03-.5236 \mathrm{E}+03 \quad .1138 \mathrm{EE}+04$

LLoads $98-.1313 E+04-.2689 E+02-.1762 E+02 \quad-.2007 E+03 \quad .9977 E+03 \quad .5789 E+03$

LLoods $115.1364 \mathrm{E}+04-.7031 \mathrm{E}+02 \quad .1419 \mathrm{E}+02 \quad .2007 \mathrm{E}+03 \quad .5236 \mathrm{E}+03 \quad .1499 \mathrm{E}+04$

GLoads $92-.2107 E+02 \quad .4088 E+02-.1583 E+03-.5520 E+03 \quad .2680 E+04 \quad .1894 E+03$

GLoads $115-.8365 \mathrm{E}+01 \quad .3760 \mathrm{E}+02 \quad .187 \mathrm{FE}+03 \quad .4338 \mathrm{E}+03 \quad .1706 \mathrm{E}+04 \quad-.1435 \mathrm{E}+03$

LLoods $92-.1552 E+03-.4088 E+02 \quad .3773 E+02-.2354 E+02-.2680 E+04-.5631 E+03$

LLoads $115 \quad .1719 E+03 \cdot .3760 E+02-.7583 E+02 \quad .2354 E+02-.1706 E+04 \quad .4563 E+03$

GLoads $86 \quad .3046 E+03 \quad .5917 E+02 \div .7979 E+02 \quad .4416 E+03-.2883 E+04 \quad .6061 E+03$

GLoads $115 \quad-. .3179 E+03 \quad-.2361 E+02 \quad .9312 E+02 \quad .4275 E+03 \quad-.1232 E+04 \quad .5528 E+03$

LLoads $86 \quad .2915 E+03-.5917 \mathrm{E}+02-.1189 \mathrm{E}+03-.1034 \mathrm{E}+02 \quad .2883 \mathrm{E}+04-.7498 \mathrm{E}+03$

LLoads $115-.3102 E+03 \quad .2361 E+02 \quad-1162 E+03 \quad .1034 E+02 \quad .1232 E+04-.6988 E+03$

GLoads $134 \quad .1889 E+02 \quad .8490 E+01-.5893 E+01 \quad .6771 E+03-.8177 E+03-.1208 E-12$ 
KAISER ENGIMEERS HANFORD S/N:B01854 Run ID=FH61973 $02 / 22 / 95$ PAGE 157

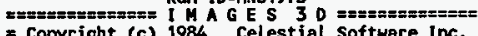
- Copyright (c) 1984 Celestial Software Inc. SOLVE BEAM LOADS/STRESSES Version $2.0 \quad 07 / 01 / 90$

Load Case 5:UBC SEISHIC LOAD - NS \& WE

\begin{tabular}{|c|c|c|c|c|c|c|c|}
\hline $\begin{array}{l}\text { GLoads } \\
\text { LLoads } \\
\text { Stress } \\
\text { Stress }\end{array}$ & $\begin{array}{l}\text { Node } \\
\text { Node } \\
\text { Node } \\
\text { Node }\end{array}$ & $\begin{array}{c}\text { Fx } \\
\text { Axial } \\
\text { Axial } \\
\text { Maximum }\end{array}$ & $\begin{array}{c}\text { Fy } \\
y \text {-shear } \\
y \text {-shear } \\
\text { Minimun }\end{array}$ & $\begin{array}{c}\text { Fz } \\
\text { Z-Shear } \\
\text { Z-Shear } \\
\text { Cmb, Shear }\end{array}$ & $\begin{array}{l}\text { Mx } \\
\text { Torsion } \\
\text { Torsion }\end{array}$ & $\begin{array}{l}\text { My } \\
\mathbf{y} \text {-Berding } \\
\gamma-\text { Berding }\end{array}$ & $\begin{array}{l}\mathrm{Mz} \\
\mathrm{z}-\text {-Bending } \\
\mathrm{z}-\text {-Bending }\end{array}$ \\
\hline $\begin{array}{l}\text { GLoads } \\
\text { LLoads } \\
\text { LLoads }\end{array}$ & $\begin{array}{r}99 \\
134 \\
99\end{array}$ & $\begin{array}{l}-.2651 E+02 \\
.1889 E+02 \\
-.2651 E+02\end{array}$ & $\begin{array}{r}.1183 \mathrm{E}+02 \\
-.8490 \mathrm{E}+01 \\
-.1183 \mathrm{E}+02\end{array}$ & $\begin{array}{l}.1351 E+02 \\
.5893 E+01 \\
.1354 E+02\end{array}$ & $\begin{array}{r}-.6771 E+03 \\
.6771 E+03 \\
-.6771 E+03\end{array}$ & $\begin{array}{r}.1012 E+04 \\
.8177 \mathrm{E}+03 \\
-.1012 E+04\end{array}$ & $\begin{array}{r}-.3339 \mathrm{E}+02 \\
.1208 \mathrm{E}-12 \\
.3339 \mathrm{E}+02\end{array}$ \\
\hline $\begin{array}{l}\text { GLoads } \\
\text { GLoads } \\
\text { LLoads } \\
\text { LLoads }\end{array}$ & $\begin{array}{l}135 \\
114 \\
135 \\
114\end{array}$ & $\begin{array}{r}-.5455 E+02 \\
.4693 \mathrm{E}+02 \\
.5455 \mathrm{E}+02 \\
-.4693 \mathrm{E}+02\end{array}$ & $\begin{array}{r}-.1341 \mathrm{E}+02 \\
.3373 \mathrm{0}+02 \\
.1341 \mathrm{E}+02 \\
-.3373 \mathrm{E}+02 \\
\\
* \mathrm{E}+\mathrm{BEA}\end{array}$ & $\begin{array}{l}.7789 E+01 \\
.1692 E+00 \\
.7789 E+01\end{array}$ & $\begin{array}{r}.6541 E+03 \\
-.6541 E+03 \\
-.6541 E+03 \\
.6541 E+03\end{array}$ & $\begin{array}{r}.5297 \mathrm{E}+03 \\
-.4501 \mathrm{E}+03 \\
-.5297 \mathrm{E}+03 \\
.4501 \mathrm{E}+03\end{array}$ & $\begin{array}{l}.5613 E+02 \\
.4152 E+03 \\
.5613 E+02 \\
.4152 E+03\end{array}$ \\
\hline $\begin{array}{l}\text { GLoads } \\
\text { GLoads } \\
\text { LLoads } \\
\text { LLoads }\end{array}$ & $\begin{array}{l}122 \\
124 \\
122 \\
124\end{array}$ & $\begin{array}{r}-.5418 E+01 \\
-.2964 E+01 \\
.9609 E+02 \\
-.7374 E+02\end{array}$ & $\begin{array}{r}.9609 E+02 \\
-.7374 E+02 \\
-.5418 E+01 \\
-.2964 E+01\end{array}$ & & $\begin{array}{l}.7412 E+03 \\
.8581 E+03 \\
.5080 E+03 \\
.5080 E+03 \\
.5\end{array}$ & $\begin{array}{r}.5080 E+03 \\
-.5080 E+03 \\
-.7412 E+03 \\
.8581 E+03\end{array}$ & $\begin{array}{r}.3947 \mathrm{E}+02 \\
-.1246 \mathrm{E}+02 \\
-.3947 \mathrm{E}+02 \\
.1246 \mathrm{E}+02\end{array}$ \\
\hline $\begin{array}{l}\text { GLoads } \\
\text { GLoads } \\
\text { LLoads } \\
\text { LLoads }\end{array}$ & $\begin{array}{l}124 \\
126 \\
124 \\
126\end{array}$ & $\begin{array}{r}-.4374 E+01 \\
\because .4008 E+01 \\
-1311 E+03 \\
-.4088 E+03\end{array}$ & $\begin{array}{r}.1311 \mathrm{E}+03 \\
-.1088 \mathrm{E}+03 \\
=.4374 \mathrm{E}+01 \\
-.4008 \mathrm{E}+01\end{array}$ & $\begin{array}{l}7213 E+01 \\
1169 E+01 \\
7213 E+01\end{array}$ & $\begin{array}{r}. .2004 \mathrm{E}+04 \\
.2070 \mathrm{E}+04 \\
.4241 \mathrm{E}+03 \\
-.4241 \mathrm{E}+03\end{array}$ & $\begin{array}{r}.4241 E+03 \\
-.4241 E+03 \\
-.2004 E+04 \\
.2070 E+04\end{array}$ & $\begin{array}{r}.1246 \mathrm{E}+02 \\
-.8434 \mathrm{E}+01 \\
-.1246 \mathrm{E}+02 \\
.8434 \mathrm{E}+01\end{array}$ \\
\hline $\begin{array}{l}\text { GLoads } \\
\text { GLoads } \\
\text { LLoads } \\
\text { LLoads }\end{array}$ & $\begin{array}{l}126 \\
128 \\
126 \\
128\end{array}$ & $\begin{array}{l}-.4246 E+01 \\
-.4136 E+01 \\
.1391 E+03 \\
-.1168 E+03\end{array}$ & $\begin{array}{r}.1391 E+03 \\
-.1168 E+03 \\
-.4246 E+01 \\
-.4136 E+01\end{array}$ & & $\begin{array}{r}-.2549 E+04 \\
.2571 E+04 \\
.2542 E+03 \\
-.2542 E+03\end{array}$ & $\begin{array}{r}.2542 E+03 \\
-.2542 E+03 \\
-.2549 E+04 \\
.2571 E+04\end{array}$ & $\begin{array}{r}.8434 E+01 \\
-.7216 E+01 \\
-.8434 E+01 \\
.7216 E+01\end{array}$ \\
\hline $\begin{array}{l}\text { GLoads } \\
\text { GLoads } \\
\text { LLoads } \\
\text { LLoads }\end{array}$ & $\begin{array}{l}128 \\
130 \\
128 \\
130\end{array}$ & $\begin{array}{l}-.3604 \mathrm{E}+01 \\
-.4778 \mathrm{E}+01 \\
.1231 \mathrm{E}+03\end{array}$ & $\begin{array}{r}.1231 E+03 \\
\therefore .1007 E+03 \\
-.3604 E+01 \\
-.4778 E+01\end{array}$ & $\begin{array}{l}.1974 E+01 \\
.6408 E+01 \\
.1974 E+01\end{array}$ & $\begin{array}{r}-.2361 \mathrm{E}+04 \\
.2313 \mathrm{0}+04 \\
-1291 \mathrm{E}+03 \\
-.1291 \mathrm{0}+03 \\
4 * 129\end{array}$ & $\begin{array}{r}.1291 E+03 \\
-.1291 E+03 \\
-.2361 E+04 \\
.2313 E+04\end{array}$ & $\begin{array}{r}.7216 E+01 \\
-.2013 E+02 \\
-.7216 E+01 \\
.2013 E+02\end{array}$ \\
\hline $\begin{array}{l}\text { GLoads } \\
\text { GLoads } \\
\text { LLoads } \\
\text { LLoads }\end{array}$ & $\begin{array}{l}130 \\
132 \\
130 \\
132\end{array}$ & $\begin{array}{r}-.5970 E+01 \\
-.4507 E+01 \\
.8311 E+02 \\
-.5517 \mathrm{E}+02\end{array}$ & $\begin{array}{r}.8311 E+02 \\
-.5517 \mathrm{E}+02 \\
-.5970 \mathrm{E}+01 \\
-.4507 \mathrm{E}+01 \\
\end{array}$ & $\begin{array}{l}52 E+01 \\
5 E+01 \\
52 E+01 \\
5 E+01\end{array}$ & $\begin{array}{l}-1298 \mathrm{E}+04 \\
.1235 \mathrm{E}+04 \\
.1301 \mathrm{E}+02 \\
-1301 \mathrm{E}+02\end{array}$ & $\begin{array}{r}.1301 E+02 \\
\therefore 1301 E+02 \\
-\quad 1298 E+04 \\
.1235 E+04\end{array}$ & $\begin{array}{r}.2013 E+02 \\
-.8882 E-13 \\
-.2013 E+02 \\
.8882 E-13\end{array}$ \\
\hline $\begin{array}{l}\text { GLoods } \\
\text { GLoads } \\
\text { LLoads } \\
\text { LLoads }\end{array}$ & $\begin{array}{l}132 \\
134 \\
132 \\
134\end{array}$ & $\begin{array}{r}.0000 E+00 \\
.0000 E+00 \\
.3814 E+01 \\
.3814 E+01\end{array}$ & $\begin{array}{r}.3814 E+01 \\
-.3814 E+01 \\
.0000 E+00 \\
.0000 E+00 \\
\star *+B E A\end{array}$ & $\begin{array}{l}.0000 E+00 \\
.0000 E+00 \\
.0000 E+00 \\
.0000 E+00 \\
\text { HO. }\end{array}$ & $\begin{array}{l}.0000 E+00 \\
.0000 E+00 \\
.0000 E+00 \\
.0000 E+00 \\
.+ \pm\end{array}$ & $\begin{array}{l}.0000 E+00 \\
.0000 E+00 \\
.0000 E+00 \\
.0000 E+00\end{array}$ & $\begin{array}{l}.0000 E+00 \\
.0000 E+00 \\
.0000 E+00 \\
.0000 E+00\end{array}$ \\
\hline GLoads & 123 & $.93696+01$ & $.2825 E+0$ & & $-.8791 E+03$ & $.3842 E+03$ & $.9910 \mathrm{E}+02$ \\
\hline
\end{tabular}

KAISER ENGINEERS HANFORD S/H:80 854 PAGE 158

Run 10=11.161973

$02 / 22 / 95$

$08: 51: 09$

= Copyright (c) 1984 Celestial Software Inc. $=$

SOLVE BEAM LOADS/STRESSES Version 2.0 07/01/90

Load Case 5:UBC SEISHIC LOAD - NS \& WE

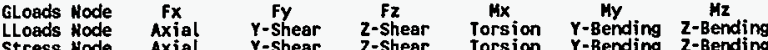
Stress Node Maximum Minimum cmb. Shear Torsion $\begin{array}{llllllll}\text { GLoads } & 125 & .9875 E+00 & -.2602 E+03 & -.2681 E+00 & .9772 E+03 & -.3842 E+03 & .1482 E+02\end{array}$ LLoads $125 \quad-.2602 \mathrm{E}+03 \quad-.9875 \mathrm{E}+00 \quad-.2681 \mathrm{E}+00-.3842 \mathrm{E}+03 \quad-.9772 \mathrm{E}+03 \quad .1482 \mathrm{E}+02$ GLoads $\quad 2-.6387 E+01 \quad .1221 E+03 \quad .6190 E+01-.6869 E+03 \quad .6688 E+03 \quad .5257 E+02$ GLoads $19=.1995 \mathrm{E}+01-.9970 \mathrm{E}+02 \quad .2192 \mathrm{EE}+01-.7309 \mathrm{E}+03-.6688 \mathrm{E}+03-.4259 \mathrm{E}+01$

LLoads $2 \quad .1221 \mathrm{E}+03-.6387 \mathrm{E}+01-.6190 \mathrm{E}+01-.6688 \mathrm{E}+03-.6869 \mathrm{E}+03-.5257 \mathrm{E}+02$

LLoads $19-.9970 \mathrm{E}+02-.1995 \mathrm{E}+01 \quad-.2192 \mathrm{E}+01-.6688 \mathrm{E}+03 \quad .7309 \mathrm{E}+03 \quad .4259 \mathrm{E}+01$

GLoads $19 \cdot 3918 E+01.1166 \mathrm{E}+03 \quad 5203 \mathrm{E}+01-.1780 \mathrm{E}+04 \quad .5192 \mathrm{E}+03 \quad .4259 \mathrm{E}+01$

GLoads $\quad 19 \cdot .3918 E+01 \quad .1166 E+03 \quad .5293 E+01-.1780 E+04-5192 E+03-.4259 E+01$

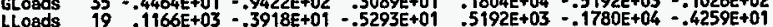

LLoads $35-.9422 \mathrm{E}+02-.4464 \mathrm{E}+01-.3089 \mathrm{E}+01-5192 \mathrm{O}+03 \quad .1804 \mathrm{E}+04 \quad .1026 \mathrm{E}+02$

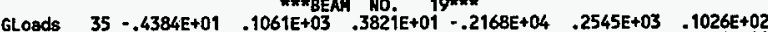

GLosds $51-.3997 \mathrm{E}+01-.8379 \mathrm{E}+02 \quad .3561 \mathrm{E}+01-.2160 \mathrm{E}+04-.2545 \mathrm{E}+03-.6001 \mathrm{E}+01$

Lloods $35.1061 E+03=4384 E+01-3821 E+01-2545 E+03-.2168 E+04-.1026 E+02$

Lloads $51-.8379 \mathrm{E}+02-.3997 \mathrm{E}+01-.4561 \mathrm{E}+01-.2545 \mathrm{E}+03 \quad .2160 \mathrm{E}+04 \quad .6001 \mathrm{E}+01$

GLoods

GLoods

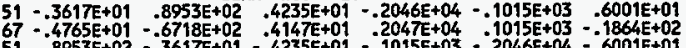
$51 \quad .8953 \mathrm{E}+02-.3617 \mathrm{E}+01-.4235 \mathrm{E}+01-.1015 \mathrm{E}+03-.2046 \mathrm{E}+04-.6001 \mathrm{E}+01$ $67=.6718 \mathrm{E}+02-.4765 \mathrm{E}+01-.4147 \mathrm{E}+01 \quad .1015 \mathrm{E}+03 \quad .2067 \mathrm{E}+04 \quad .1864 \mathrm{E}+02$

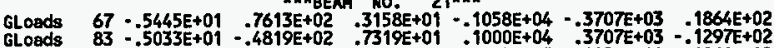

Lloods $67-7613 E+02-.5445 E+01-.3158 E+01-.3707 E+03-.1058 E+04-.1864 E+02$

LLoads $83-.4819 E+02-.5033 \mathrm{E}+01-.7319 \mathrm{E}+01 \quad .3707 \mathrm{E}+03 \quad .1000 \mathrm{E}+04 \quad .1297 \mathrm{E}+02$

GLoods $83-.2154 \mathrm{E}+01.7556 \mathrm{E}+02=.5502 \mathrm{E}+01 \quad .8943 \mathrm{E}+03-.5279 \mathrm{E}+03 \quad .1297 \mathrm{E}+02$

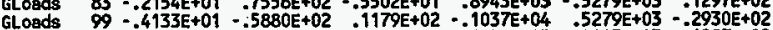

Lloads $83.7556 E+02-.2154 E+01 \quad .5502 E+01-.5279 E+03-.8943 E+03-.1297 E+02$

LLoods $99-.5880 E+02-.4133 \mathrm{E}+01-.1179 \mathrm{E}+02 \quad .5279 \mathrm{E}+03-.1037 \mathrm{E}+04 \quad .2930 \mathrm{E}+02$

GLoads $\quad 5-.3672 E+01.7823 E+02 \quad .3224 E+01.4805 E+02 \quad .1679 E+04 \quad .4884 E+02$

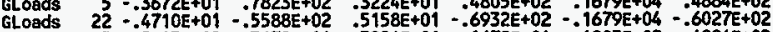

\begin{tabular}{llllllll}
\hline & 5 & $.7823 E+02$ & $3672 E+01$ & $3224 E+01$ & $-1679 E+04$ & $-.4805 E+02$ & $-4884 E+02$
\end{tabular}

LLoods $22-.5588 \mathrm{E}+02 \quad .4710 \mathrm{E}+01 \quad .5158 \mathrm{E}+01-.1679 \mathrm{E}+04 \quad .6932 \mathrm{E}+02-.6027 \mathrm{EE}+02$

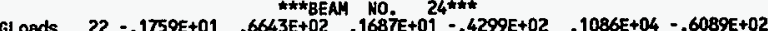


KAISER ENGINEERS HANFORD S/N:801854

PAGE 159

$$
\text { Run ID=NN61973 }
$$

$x==2 x=5 x=580$ = Copyright (c) 1984 Celestial Software Inc.

$02 / 22 / 95$

SOLVE BEAM LOADS/STRESSES Version 2.0 07/01/90

1

Load Case 5:UBC SEISHIC LOAD - NS \& WE

GLoads Node Fx Fy $\quad$ Fy $\quad$ HX Hy LLoads Node Axial Y-Shear z-Shear Torsion Y-Bending z-Bending Stress Node Mxial Y-Shear Z-Shear Torsion Y-Bending Z-Bending Stress Hode Maximum Minimum Cmb. Shear

GLoads $38-.6623 \mathrm{E}+01-.4407 \mathrm{E}+02 \quad .6695 \mathrm{E}+01-.1209 \mathrm{E}+02-02-1086 \mathrm{E}+04 \quad .7377 \mathrm{E}+01$

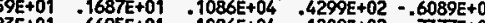
LLoads $\quad 38-.4407 \mathrm{E}+02 \quad .6623 \mathrm{E}+01 \quad .6695 \mathrm{E}+01-1086 \mathrm{E}+04 \quad .1209 \mathrm{E}+02 \quad .7377 \mathrm{E}+01$ GLoads $38-.4093 \mathrm{E}+01 \quad .6341 \mathrm{E}+02 \quad .4241 \mathrm{E}+01 \quad .2846 \mathrm{E}+02 \quad .3405 \mathrm{E}+03 \quad .3728 \mathrm{E}+02$ $54-.4288 \mathrm{E}+01-.4106 \mathrm{E}+02 \quad .4141 \mathrm{E}+01-.2736 \mathrm{E}+02-.3405 \mathrm{E}+03-.3942 \mathrm{E}+02$ Lloads $38.6341 \mathrm{E}+02-4093 \mathrm{E}+01.424 \mathrm{E}+01 \quad .3405 \mathrm{E}+03 \cdot .2846 \mathrm{E}+02-3728 \mathrm{E}+02$

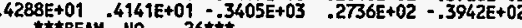

GLoods $54-.5105 E+01 \quad .5604 E+02 \quad .4897 E+01-.2909 E+01-.5741 E+03-.1774 E+02$ GLoods $\quad 70-.3276 \mathrm{E}+01-.3369 \mathrm{E}+02 \quad .3485 \mathrm{E}+01 \quad-1844 \mathrm{E}+02 \quad .5741 \mathrm{E}+03 \quad-3786 \mathrm{E}+02$ - $172 \mathrm{E}+02$ .3786E+02 GLoads $\quad 70-.5719 E+01 \quad .6756 E+02 \quad .6493 E+01 \quad .4714 E+02-.1383 E+04 \quad .5002 E+02$ GLoeds $\quad 86=.4758 E+01-.3962 E+02 \quad .3984 E+01-.1264 E+02-1383 E+04-.3681 E+02$

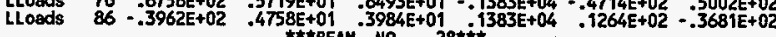
GLoads $86 \quad .4329 E+01 \quad .5255 E+02-.1999 E+01-.5807 E+02-.4484 E+03-.2199 E+02$ GLoads $102-.1062 E+02-.3578 E+02 \quad .8285 E+01-.2677 \mathrm{E}+02 \quad .4484 \mathrm{E}+03-.1013 \mathrm{E}+03$ LLoads $86 \quad .5255 \mathrm{E}+02-.4329 \mathrm{E}+01-1099 \mathrm{E}+01-.4484 \mathrm{E}+03-5807 \mathrm{E}+02-.2199 \mathrm{E}+02$ LLoads $102-.3578 E+02 \quad .1062 \mathrm{E}+02 \quad .8285 \mathrm{E}+01 \quad .4484 \mathrm{E}+03 \quad .2677 \mathrm{E}+02-.1013 \mathrm{E}+03$ GLOods $11 \cdot-.6232 E+01 \quad .2559 E+03 \quad .859 I_{E}+01 \quad .1456 E+03-.3973 E+04 \quad .1519 E+03$ GLOads $28-.6819 E+01-.2211 E+03 \quad .4460 E+01-.1001 E+03 \quad .3973 E+04-.1525 E+03$

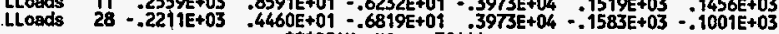

- Gloods GLoads LLoads LLoeds

GLoads Lloads Lloeds GLoods \& $28-.2091 E+01 \quad .1744 E+03 \quad .1349 E+01+.1155 E+03-.2756 E+04-.6615 E+02$ $44-.1096 \mathrm{E}+02-.1395 \mathrm{E}+03 \quad .1170 \mathrm{E}+02 \quad .1665 \mathrm{E}+01 \quad .2756 \mathrm{E}+04-.3142 \mathrm{E}+02$ - $1745+03 \quad-1349 E+01-.2091 E+01-.2756 E+04=.6615 E+02-.1155 E+03$ N"BEAM NO. 31witw $44-.5738 E+01-1382 E+03 \quad-7436 E+01 \quad .5412 E+02-.1093 E+04 \quad-1092 E+02$ $60=.1382 E+03 \quad .7436 E+01-.5738 E+01-.1093 E+04 \quad-1092 E+02 \quad .5412 E+02$ $60-.7856 E+01.1082 E+03 \quad .6228 E+01-.5194 E+01 \quad .6936 E+03-.3807 E+01$ \#*BEAM No. 3 . $60-.7314 E+01-.1034 E+03-.5615 E+01-.34080$
KAISER ENGINEERS HANFORD S/N:80t854

PAGE 160

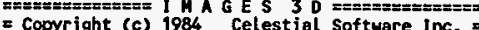
= Copyright (c) 1984 Celestial Software Inc. =

$02 / 22 / 95$

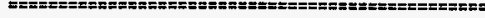

SOLVE BEAM LOADS/STRESSES Version 2.0 07/01/90 L

LOad Case 5:UBC SEISHIC LOAD - HS \& WE

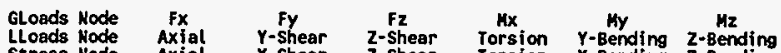
Stress Node $\begin{gathered}\text { Axial } \\ \text { Stress Node Maximum Minimum Cmb. Shear Torsion Y-Bending Z-Bending }\end{gathered}$

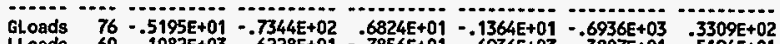

LLoads $60-1082 \mathrm{E}+03 \quad .6228 \mathrm{E}+01-.7856 \mathrm{E}+01 \quad .6936 \mathrm{E}+03-.3807 \mathrm{E}+01-.5194 \mathrm{E}+01$

LLoads $76-.7344 \mathrm{E}+02.0824 \mathrm{E}+01-.5195 \mathrm{E}+01-.6936 \mathrm{E}+03 \quad .3309 \mathrm{E}+02-. .1364 \mathrm{E}+01$

GLoads $76-.8558 E+01.9560 E+02 \quad .9713 E+09.5949 E+02 \quad .2168 E+04 \quad .8205 E+02$

GLoads $92-.7756 \mathrm{E}+01-.5209 \mathrm{E}+02 \quad .6601 \mathrm{E}+01-.1669 \mathrm{E}+02-.2168 \mathrm{E}+04-.7104 \mathrm{E}+02$

LLoads $76 \quad .9560 \mathrm{E}+02 \quad .9713 \mathrm{E}+01-.8558 \mathrm{E}+01 \quad .2168 \mathrm{E}+04 \quad .8205 \mathrm{E}+02 \quad .5949 \mathrm{E}+02$

Lloads $92-.5209 E+02 \quad .6001 E+01-.7756 E+01-.2168 E+04-.7104 E+02-.1669 E+02$

GLoads $92-.1108 E+02.5365 E+02 \quad .1910 E+02 \quad .1621 E+03 \quad .1016 E+04-.5704 E+02$

GLoads $108 \quad-1296 \mathrm{E}+01-.2755 \mathrm{E}+02-.9309 \mathrm{E}+01 \quad .7230 \mathrm{E}+02-.1016 \mathrm{E}+04 \quad .1592 \mathrm{E}+03$

LLoads $92-5365 E+02-1910+02-.1108 E+02 \quad-1016 E+04-.5704 E+02 \quad-1621 E+03$

LLoads $108-.2755 E+02-.9309 E+01 \quad-1296 E+01-1016 E+04 \quad .1592 E+03 \quad .7230 E+02$

$\begin{array}{llllllll}\text { GLoods } \quad 17-.7553 E+01 & .1889 E+03 \quad .7788 E+01-.1049 E+04 & .6664 E+03 & .7638 E+02\end{array}$

GLoads $\quad 34-.8283 \mathrm{E}+00=.1666 \mathrm{E}+03 \quad .5935 \mathrm{E}+00 \quad .1128 \mathrm{E}+04-.6664 \mathrm{E}+03-.2406 \mathrm{E}+01$

$\begin{array}{llllllll}\text { LLoads } & 17 & -1889 E+03 & .7553 E+01 & .7788 E+01 & .6664 E+03 & .1049 E+04 & .7638 E+02\end{array}$

Lloads $34-.1606 E+03 \quad .8283 E+00 \quad .5935 E+00 * .6664 E+03-.1128 E+04-.2406 E+01$

GLoads $34-.3923 E+01 \quad .1773 E+03 \quad .5611 E+01 \quad-.2628 E+04 \quad .4500 E+03 \quad .2406 E+01$

GLoods $50-.4459 E+01-.1550 \mathrm{E}+03 \quad .2770 \mathrm{E}+01 \quad .2659 \mathrm{E}+04,-.4500 \mathrm{E}+03-.8301 \mathrm{E}+01$

$.3923 \mathrm{E}+01 \quad .5611 \mathrm{E}+$

$\begin{array}{llllll} & .459 E+01 & .2770 E+01 & -.4500 E+03 & -.2659 E+04 & -.8301 E+01\end{array}$

GLoads $50-.3542 E+01 \quad .1366 E+03 \quad .4020 E+01-.2996 E+04 \quad .1651 E+03 \quad .8301 E+01$

GLoads $66-.4840 E+01-.1143 \mathrm{E}+03 \quad .4362 \mathrm{E}+01 \quad .2992 \mathrm{E}+04-.1651 \mathrm{E}+03-.2258 \mathrm{E}+02$

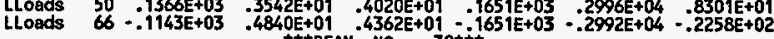

GLoads $66-.7047 \mathrm{E}+01.9944 \mathrm{E}+02 \quad .2899 \mathrm{BE}+01^{38}-.2321 \mathrm{E}+04-.2958 \mathrm{E}+03 \quad .2258 \mathrm{E}+02$

$\begin{array}{llllllll}\text { GLoads } & 66 & -.7047 E+01 & .9944 E+02 & .2899 E+01 & -.2321 E+04 & -.2958 E+03 & .2258 E+02 \\ \text { GLoads } & 82 & -.1335 E+01 & -.7709 E+02 & .5483 E+01 & .2293 E+04 & .2958 E+03 & .4025 E+02\end{array}$

$\begin{array}{llllllll}\text { GLoads } & 82 & -.1335 \mathrm{E}+01 & -.7709 \mathrm{E}+02 & .5483 \mathrm{E}+01 & .2293 \mathrm{E}+04 & .2958 \mathrm{E}+03 & .4025 \mathrm{E}+02 \\ \text { LLoads } & 66 & .9944 \mathrm{E}+02 & . .7047 \mathrm{E}+01 & .2899 \mathrm{E}+01 & -.2958 \mathrm{E}+03 & .2321 \mathrm{E}+04 & .2258 \mathrm{E}+02\end{array}$

$\begin{array}{llllllll}\text { LLoads } & 82 & -.7709 \mathrm{E}+02 & .7047 \mathrm{E}+01 & .2899 \mathrm{E}+01 & -.2958 \mathrm{E}+03 & .2321 \mathrm{E}+04 & .2258 \mathrm{E}+02 \\ & .5483 \mathrm{E}+01 & .2958 \mathrm{E}+03 & -.2293 \mathrm{E}+04 & .4025 \mathrm{E}+02\end{array}$

GLoads $82.4275 E+01 \quad .6996 E+02-.3426 E+01.1398 E+04-.3387 E+03-.4025 E+02$

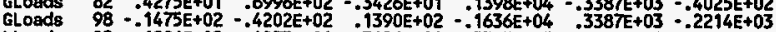

LLoads $82.6996 \mathrm{E}+02-.4275 \mathrm{E}+01-3426 \mathrm{E}+01-3387 \mathrm{E}+03-3398 \mathrm{E}+04-4025 \mathrm{E}+02$

Lloads $98-.4202 E+02.1475 \mathrm{E}+02-1390 \mathrm{E}+02-.3387 \mathrm{E}+03-1636 \mathrm{E}+04-.2214 \mathrm{E}+03$

GLoads $98-.3048 E+02.4926 E+02 \quad .3052 E+02 \quad .1785 E+04 \quad .2985 E+03-.8759 E+01$ 
KAISER ENGINEERS HANFORD $S / N: 801854$

PAGE 161

Run ID=1N61973

$02 / 22 / 95$

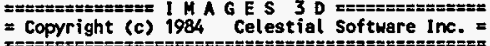

SOLVE BEAM LOADS/STRESSES Version 2.0 07/01/90

L

Load Case 5:UBC SEISMIC LOAD - HS \& WE

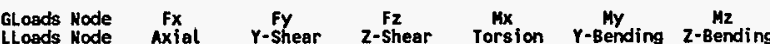

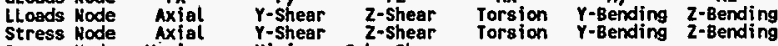
Stress Node Maximum Minimum Cmb. Shear

GLoods $114 \quad .2419 \mathrm{E}+02-.3250 \mathrm{E}+02 \quad-.2423 \mathrm{E}+02-.1333 \mathrm{E}+04 \quad-.2985 \mathrm{E}+03 \quad .4598 \mathrm{E}+03$ LLaeds $98 \quad .4926 E+02 \quad .3048 E+02 \quad .3052 E+02 \quad .2985 E+03-.1785 E+04-.8759 E+01$ Lloads $114-.3250 \mathrm{E}+02-.2419 \mathrm{E}+02-.2423 \mathrm{E}+02-.2985 \mathrm{E}+03 \quad .1333 \mathrm{E}+04 \quad .4598 \mathrm{E}+03$ GLoads $99 \cdot .6684 \mathrm{E}+01.1541 \mathrm{E}+01.2849 \mathrm{E}+02 \quad .7591 \mathrm{E}+03-.1601 \mathrm{E}+04-.3775 \mathrm{E}+02$ GLoads $100 \quad .1477 \mathrm{E}+01: 1234 \mathrm{E}+02-.2328 \mathrm{E}+02-.7591 \mathrm{E}+03 \quad-1247 \mathrm{E}+04-.3606 \mathrm{E}+02$ LLoads $99-.6684 E+01-.1541 E+01-.2849 E+02 \quad .7591 E+03 \quad .1601 E+04 \quad .3775 E+02$ Lloads $100 \quad .1477 \mathrm{E}+01-.1234 \mathrm{E}+02 \quad .2328 \mathrm{E}+02 \quad-.7591 \mathrm{E}+03 \quad-.1247 \mathrm{E}+04 \quad .3606 \mathrm{E}+02$ GLosds $100-.4519 E+01.8244 E+01.1835 E+02.1166 E+04-.6460 E+02 \quad .3606 E+02$ GLoads $101-.6883 E+00 \quad .5641 E+01=.1314 E+02-.1166 E+04-.1506 E+03=1828 E+02$ Lloads $101-.6883 \mathrm{E}+00-.5641 \mathrm{E}+01 \quad .1314 \mathrm{E}+02-.1166 \mathrm{E}+04 \quad .1506 \mathrm{E}+03 \quad .1828 \mathrm{E}+02$ GLoods $101-.3361 E+01.1049 E+01.2462 E+02 \quad .9390 E+03 \quad .1172 E+04 \quad .1828 E+02$ GLoads $102: .1846 E+01: 1284 \mathrm{E}+02-.1941 \mathrm{E}+02-.9390 \mathrm{E}+03-.1473 \mathrm{E}+04-.9882 \mathrm{E}+02$ $61 E+01-1049 E+01-2462 E+02-9390 E+03-1172 E+04-.1828 E+02$ Lloods $102-.1846 E+01-.1284 \mathrm{E}+02 \quad .1941 \mathrm{E}+02-.9390 \mathrm{E}+03 \quad-1473 \mathrm{E}+04 \quad .982 \mathrm{EE}+02$ GLoads $102-.1689 E+02.9275 E+01.1118 E+03 \quad .5044 E+02 \quad .1503 E+04-.5626 E+03$ GLoods $103 \quad .1099 \mathrm{E}+02 \quad .6473 \mathrm{E}+01 \quad-.1059 \mathrm{E}+03 \quad-.2872 \mathrm{E}+02-.1287 \mathrm{EE}+04 \quad .5626 \mathrm{E}+03$ Lloads $103-1059 \mathrm{E}+03-.6473 \mathrm{E}+01 \quad \therefore 1099 \mathrm{E}+02-.5626 \mathrm{E}+03 \quad .1287 \mathrm{E}+04 \quad .2872 \mathrm{E}+02$ LLoads $103 \cdot 1059 E+03-.6473 E+01 * 1099 E+02=.5626 E+03 \quad \cdot 1287 E+04 \quad .2872 E+02$ GLoads $103=.1938 E+02.8438 E+01 \quad .7537 \mathrm{~F}+02 \quad .2872 E+02-.1980 E+03-.5735 E+03$ GLoads $104: .1347 \mathrm{E}+02 \quad .7310 \mathrm{E}+01-.6946 \mathrm{E}+02-.1998 \mathrm{EE}+02 \quad-4526 \mathrm{E}+03 \quad .5735 \mathrm{E}+03$ LLads $104=.7946 \mathrm{E}+02=.7310 \mathrm{E}+01-.1347 \mathrm{EE}+02-.5735 \mathrm{E}+03-.4526 \mathrm{E}+03 \quad .1998 \mathrm{E}+02$ . GLoads $104-.9117 E+01.7912 E+01 \quad .5094 E+02-1998 E+02-.1522 E+04-.1126 E+03$ GLoads $105 \quad-3212 \mathrm{E}+01 \quad .7836 \mathrm{E}+01-.5403 \mathrm{E}+02-.1940 \mathrm{E}+02 \quad .1618 \mathrm{E}+04 \quad .1126 \mathrm{E}+03$ LLoads $105 \quad .5403 \mathrm{E}+02-.7836 \mathrm{E}+01-3212 \mathrm{E}+01-.1126 \mathrm{E}+03 \cdot .1618 \mathrm{E}+04 \quad .1940 \mathrm{E}+02$ 7 GLoods $105-.3150 E+0 t .7847 E+01.6303 E+02 \quad .1940 E+02-.1882 E+04 \quad .1861 E+03$ GLoads $106-.2755 \mathrm{E}+01 \quad .7900 \mathrm{E}+01-.5712 \mathrm{E}+02-.1981 \mathrm{E}+02 \quad .1885 \mathrm{E}+04-.1861 \mathrm{E}+03$

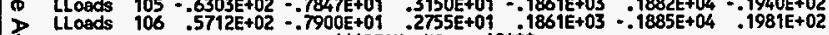

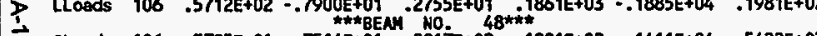
G GLoads $106 \quad .5795 E+01 \quad .7514 E+01 \quad .8817 E+02 \quad .1981 E+02-.1411 E+04 \quad .5422 E+03$
KAISER ENGINEERS HANFORD S/W:801854

PAGE 162 RUN ID=MN61973

$02 / 22 / 95$

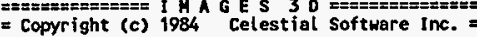

SOLVE BEAM LOADS/STRESSES Version $2.0 \quad 07 / 01 / 90$

L

Load CASe 5:UBC SEISMIC LOAD - NS \& NE

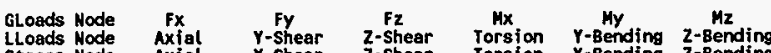
Stress Hode Axial Y-Shear z-shear Torsion Y-Bending Z-Bending Stress Hode Maximm Minimum cmb. Shear

GLoads $107-.1170 E+02 \quad .8233 \mathrm{E}+01-.8226 \mathrm{E}+02-.2538 \mathrm{E}+02 \quad .1275 \mathrm{E}+04-.5422 \mathrm{E}+03$

Lloads $106-.8817 E+02-.7514 E+01-.5795 E+01-.5422 E+03 \quad-1411 E+04-.1981 E+02$

LLoads $107 \quad .8226 E+02-.8233 E+01 \quad 1170 E+02 \quad .5422 E+03-.1275 E+04 \quad .2538 E+02$

GLoads $107.5344 \mathrm{E}+01.0001 \mathrm{E}+01.1492 \mathrm{E}+03 \cdot 2538 \mathrm{E}+02 \cdot .3853 \mathrm{E}+03 \quad .5056 \mathrm{E}+03$

GLads $108-1125 \mathrm{E}+03 \quad .0747 \mathrm{E}+01-1433 \mathrm{E}+03-5441 \mathrm{E}+02-.2567 \mathrm{E}+03-.5056 \mathrm{E}+03$

Loads $107-1402 \mathrm{E}+03.000 \mathrm{E}+01=5344 \mathrm{E}+01-5056 \mathrm{E}+03-3853 \mathrm{E}+03=.2538 \mathrm{E}+02$

LLoads $108: .1433 \mathrm{E}+03-.9747 \mathrm{E}+01 \quad .1125 \mathrm{E}+02 \quad-5056 \mathrm{E}+03-.2567 \mathrm{E}+03-.5441 \mathrm{E}+02$

GLosds $108.6263 E+02.7533 E+01.9515 E+01-.6020 E+03 \quad .38 B 1 E+03 \quad .2031 E+02$

GLoads $109-.6841 \mathrm{E}+02 \quad .7876 \mathrm{E}+01-.3736 \mathrm{E}+01.6020 \mathrm{E}+03-.4886 \mathrm{E}+03-.2291 \mathrm{E}+02$

Loods 108 .6263E+02 - $7533 \mathrm{E}+01-9515 \mathrm{E}+01-.6020 \mathrm{E}+03-.3881 \mathrm{E}+03-.2031 \mathrm{E}+02$

LLoads $109-.6841 \mathrm{E}+02=.7876 \mathrm{E}+01.3736 \mathrm{E}+01.6020 \mathrm{E}+03 \quad .4886 \mathrm{E}+03 \quad .2291 \mathrm{E}+02$

GLoads $109.3224 E+02 \quad .7939 E+01 \quad .1124 E+02-.6892 E+03 \quad .1277 E+04 \quad .2291 E+02$

GLoads $110-.3802 \mathrm{E}+02 \quad .7470 \mathrm{E}+01-.5463 \mathrm{E}+01 \quad .6892 \mathrm{E}+03-.1404 \mathrm{E}+04-.1935 \mathrm{E}+02$

LLoads $109 \quad .3224 E+02-.7939 E+01-.1124 E+02-.6892 E+03-.1277 E+04-.2291 E+02$

LLoads $110=.3802 \mathrm{E}+02-.7470 \mathrm{E}+01 \quad .5463 \mathrm{E}+01_{52 * \pi \hbar}^{.6892 \mathrm{E}+03} \quad .1404 \mathrm{E}+04 \quad .1935 \mathrm{E}+02$

GLoads $110.1656 E+02 \quad .7541 E+01-2401 E+01-.3374 E+03 \quad .1867 E+04 \quad .1935 E+02$

GLoods $111-.2233 E+02 \quad .7868 E+01 \quad .3377 \mathrm{E}+01 \quad .3374 \mathrm{E}+03-.1859 \mathrm{E}+04-.2183 \mathrm{E}+02$

作

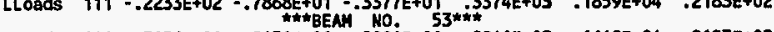

GLoads $111 \quad .5056 E+01 \quad .8451 E+01-.2981 E+01-.2841 E+02 \quad .1649 E+04 \quad .2183 E+02$

GLosds $112-.1083 E+02 \quad .6958 E+01 \quad .8759 E+01 \quad .2841 E+02-.1560 E+04=.1051 E+02$

LLoads $119 \quad .5056 E+01-.8451 E+01 \quad .2981 E+01-.2841 E+02-.1649 E+04-.2183 E+02$

LLoads $112-.1083 \mathrm{E}+02-.6958 \mathrm{E}+01-.8759 \mathrm{E}+01, .2841 \mathrm{E}+02 \quad .1560 \mathrm{E}+04 \quad-1051 \mathrm{E}+02$

GLoods $112-.1235 \mathrm{E}+02 \quad .1325 \mathrm{E}+01-.1334 \mathrm{E}+02 \quad-4913 \mathrm{E}+03 \quad .5869 \mathrm{E}+03 \quad .1051 \mathrm{E}+02$

GLoads $113 \quad .6575 E+01 \quad .1408 E+02 \quad .1912 E+02-.4913 E+03-.3408 E+03-.1073 E+03$

LLoads $113 \quad .6575 \mathrm{E}+01-.1408 \mathrm{E}+02 \quad-1912 \mathrm{E}+02 \quad-.4913 \mathrm{E}+03 \quad .3408 \mathrm{EE}+03 \quad .1073 \mathrm{E}+03$

GLoads $113 \cdot .5816 \mathrm{E}+02 \quad .4559 \mathrm{E}+02-.4485 \mathrm{E}+01 \quad .3526 \mathrm{E}+03-.7627 \mathrm{E}+03 \quad .1073 \mathrm{E}+03$

$\begin{array}{lllllll}\text { GLoads } 113 & -.5816 E+02 & .4559 E+02 & -.4485 E+01 & .3526 E+03 & -.7627 E+03 & .1073 E+03 \\ \text { GLoads } 114 & .5238 E+02 & -.3019 \mathrm{E}+02 & .1026 \mathrm{E}+02 & -.3526 \mathrm{E}+03 & .8745 \mathrm{E}+03 & .4674 \mathrm{E}+03\end{array}$

LLods $113-.5816 \mathrm{E}+02-.4559 \mathrm{E}+02 \quad .4485 \mathrm{E}+01 \quad .3526 \mathrm{E}+03 \quad .7627 \mathrm{E}+03-.1073 \mathrm{E}+03$

LLoads $114 \quad-.5238 E+02 \quad . .3019 E+02-.1026 E+02-.3526 E+03 \quad-.8745 E+03-.4674 E+03$

GLoads $115=.6426 E+04 \quad .1600 E+05 \quad .6426 E+04=.1371 E+06 \quad .9438 E+01-.1371 E+06$ 
KAISER ENGIHEERS HANFORD S/N-801854 PAGE 163 Run ID=MN61973 $02 / 22 / 95$

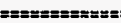
E Copyright (c) 1984 Celestial Software Inc.

SOLVE BEAM LOADS/STRESSES Version 2.0 07/01/90

Load Case 5:UBC SEISNIC LOND - NS \& WE

\begin{tabular}{|c|c|c|c|c|c|c|c|}
\hline $\begin{array}{l}\text { GLoads } \\
\text { LLoads } \\
\text { stress } \\
\text { stress }\end{array}$ & $\begin{array}{l}\text { Node } \\
\text { Node } \\
\text { Node } \\
\text { Node }\end{array}$ & $\begin{array}{c}\text { Fx } \\
\text { Axial } \\
\text { Axial } \\
\text { Maximum }\end{array}$ & $\begin{array}{l}\text { Fy } \\
\text { Y-Shear } \\
\text { y-Shear } \\
\text { Minimum }\end{array}$ & $\begin{array}{c}\text { Fz } \\
\text { 2-Shear } \\
\text { 2-Shear } \\
\text { Cab. Shear }\end{array}$ & $\begin{array}{l}M x \\
\text { Torsion } \\
\text { Torsion }\end{array}$ & $\begin{array}{l}\text { My } \\
\text { Y-Bending } \\
\text { Y-Bending }\end{array}$ & $\begin{array}{c}M z \\
\text { Z-Bending } \\
\text { z-8ending }\end{array}$ \\
\hline $\begin{array}{l}\text { GLoads } \\
\text { LLoads } \\
\text { LLoads }\end{array}$ & $\begin{array}{l}120 \\
115 \\
120\end{array}$ & $\begin{array}{r}.6228 E+04 \\
-.1600 \mathrm{E}+05 \\
.1547 \mathrm{E}+05\end{array}$ & $\begin{array}{r}-.1547 \mathrm{E}+05 \\
-.3660 \mathrm{E}+04 \\
.3548 \mathrm{E}+04 \\
*+\mathrm{BEE}\end{array}$ & $\begin{array}{l}5228 E+04 \\
3318 E+04 \\
3062 E+04\end{array}$ & $\begin{array}{l}1982 E+06 \\
9438 E+01 \\
9438 E+01\end{array}$ & $\begin{array}{l}-.9438 \mathrm{E}+01 \\
.1775 \mathrm{E}+06 \\
.2566 \mathrm{E}+06\end{array}$ & $\begin{array}{l}-.1982 E+06 \\
-.7813 E+05 \\
-.1129 E+06\end{array}$ \\
\hline $\begin{array}{l}\text { GLoads } \\
\text { GLoads } \\
\text { LLoads } \\
\text { LLoads }\end{array}$ & $\begin{array}{l}120 \\
121 \\
120 \\
121\end{array}$ & $\begin{array}{r}.5022 E+04 \\
-.5256 E+04 \\
.1453 E+05 \\
\therefore .1515 E+05\end{array}$ & $\begin{array}{r}-.1453 \mathrm{E}+05 \\
.1515 \mathrm{E}+05 \\
.2860 \mathrm{E}+04 \\
-.2993 \mathrm{E}+04 \\
\end{array}$ & $\begin{array}{l}2 E E+04 \\
25 E+04 \\
1 E+04 \\
3 E+04\end{array}$ & $\begin{array}{l}.1982 E+06 \\
.1230 E+06 \\
.9438 E+01 \\
.0438 E+01\end{array}$ & $\begin{array}{r}.9438 E+01 \\
-.9438 E+01 \\
-.2566 E+06 \\
-.1592 E+06\end{array}$ & $\begin{array}{l}.1982 E+06 \\
.1230 E+06 \\
.1129 E+06 \\
.7003 E+05\end{array}$ \\
\hline $\begin{array}{l}\text { GLoads } \\
\text { GLoads } \\
\text { LLoads } \\
\text { LLoads }\end{array}$ & $\begin{array}{l}116 \\
121 \\
116 \\
121\end{array}$ & $\begin{array}{r}-.2349 E+04 \\
.2271 E+04 \\
-.2349 E+04 \\
.2271 E+04\end{array}$ & $\begin{array}{r}.2745 E+04 \\
-.2536 E+04 \\
-.2745 E+04 \\
-.2536 E+04 \\
\end{array}$ & $\begin{array}{l}.4352 \mathrm{E}+03 \\
.3567 \mathrm{E}+03 \\
.4352 \mathrm{E}+03 \\
.3567 \mathrm{E}+03\end{array}$ & $\begin{array}{l}.1882 E+05 \\
.1882 E+05 \\
.1882 E+05 \\
.1882 E+05\end{array}$ & $\begin{array}{r}.2278 E+03 \\
-.8542 E+04 \\
.2278 E+03 \\
-.8542 E+06\end{array}$ & $\begin{array}{l}.2379 E+05 \\
.3166 E+05 \\
.2379 E+05 \\
.3166 E+05\end{array}$ \\
\hline $\begin{array}{l}\text { GLoads } \\
\text { GLoads } \\
\text { LLoods } \\
\text { LLoads }\end{array}$ & $\begin{array}{l}117 \\
121 \\
117 \\
121\end{array}$ & $\begin{array}{r}-.4352 E+03 \\
.3567 E+03 \\
.2349 E+04 \\
-.2270 E+04\end{array}$ & $\begin{array}{r}.5247 E+04 \\
-.5038 E+04 \\
.5247 \mathrm{0}+04 \\
-.5038 E+04 \\
+\end{array}$ & & $\begin{array}{l}.8981 E+04 \\
.1170 E+06 \\
.1882 E+05 \\
.1882 E+05\end{array}$ & $\begin{array}{r}.2317 E+03 \\
-.8547 E+04 \\
.2317 E+03 \\
-.8547 E+04\end{array}$ & $\begin{array}{r}.1882 E+05 \\
\therefore 1882 E+05 \\
-.8981 E+04 \\
.1170 E+06\end{array}$ \\
\hline $\begin{array}{l}\text { GLoads } \\
\text { GLoads } \\
\text { LLoads } \\
\text { LLoads }\end{array}$ & $\begin{array}{l}118 \\
121 \\
118 \\
121\end{array}$ & $\begin{array}{r}-.2349 E+04 \\
.2271 E+04 \\
.2349 E+04 \\
-.2271 E+04\end{array}$ & $\begin{array}{r}.5247 \mathrm{E}+04 \\
-.5038 \mathrm{E}+04 \\
.5247 \mathrm{E}+04 \\
-.5038 \mathrm{E}+04 \\
\end{array}$ & & $\begin{array}{r}.1881 E+05 \\
.1881 E+05 \\
.1881 E+05 \\
.1881 E+05\end{array}$ & $\begin{array}{r}-.2115 \mathrm{E}+03 \\
.8547 \mathrm{E}+04 \\
. .2115 \mathrm{E}+03 \\
.8547 \mathrm{E}+04\end{array}$ & $\begin{array}{r}.8978 E+04 \\
-1170 E+06 \\
-.8978 E+04 \\
.1170 E+06\end{array}$ \\
\hline $\begin{array}{l}\text { GLoads } \\
\text { GLoads } \\
\text { LLoads } \\
\text { LLoads }\end{array}$ & $\begin{array}{l}119 \\
121 \\
119 \\
121\end{array}$ & $\begin{array}{r}-.4362 E+03 \\
.3577 \mathrm{E}+03 \\
-.2349 \mathrm{E}+04 \\
.2270 \mathrm{E}+04\end{array}$ & $\begin{array}{r}.2745 \mathrm{E}+04 \\
-.2536 \mathrm{E}+04 \\
.2745 \mathrm{E}+04 \\
-.2536 \mathrm{E}+04 \\
\end{array}$ & $\begin{array}{l}.2349 \mathrm{E}+04 \\
.2270 \mathrm{E}+04 \\
.4362 \mathrm{E}+03 \\
.3577 \mathrm{E}+03\end{array}$ & $\begin{array}{l}.2379 \mathrm{E}+05 \\
.3166 \mathrm{E}+05 \\
.1881 \mathrm{E}+05 \\
.1881 \mathrm{E}+05\end{array}$ & $\begin{array}{r}-.2154 E+03 \\
.8551 E+04 \\
-.2154 E+03 \\
.8551 E+04\end{array}$ & $\begin{array}{r}.1881 E+05 \\
-.1881 E+05 \\
.2379 E+05 \\
.3166 E+05\end{array}$ \\
\hline $\begin{array}{l}\text { GLoads } \\
\text { GLoads } \\
\text { LLoads } \\
\text { LLoads }\end{array}$ & $\begin{array}{l}125 \\
127 \\
125 \\
127\end{array}$ & $\begin{array}{r}-.2706 E+01 \\
-.5676 E+01 \\
.1719 E+03 \\
-.1495 E+03\end{array}$ & $\begin{array}{r}.1719 E+03 \\
-.1495 E+03 \\
.2706 E+01 \\
.5676 E+01 \\
\end{array}$ & $\begin{array}{l}.6289 \mathrm{E}+01 \\
.2092 \mathrm{E}+01 \\
.6289 \mathrm{E}+01\end{array}$ & $\begin{array}{r}-.2271 E+04 \\
.2397 E+04 \\
.2562 E+03 \\
-.2562 E+03\end{array}$ & $\begin{array}{r}.2562 E+03 \\
-.2562 E+03 \\
.2271 E+04 \\
-.2317 E+04\end{array}$ & $\begin{array}{l}-.1482 E+02 \\
\because 1784 E+02 \\
-1482 E+02 \\
-1784 E+02\end{array}$ \\
\hline $\begin{array}{l}\text { GLoads } \\
\text { GLoads } \\
\text { LLoads } \\
\text { LLoads }\end{array}$ & $\begin{array}{l}127 \\
129 \\
127 \\
129\end{array}$ & $\begin{array}{r}-.4827 E+01 \\
-.3554 E+01 \\
-1147 E+03 \\
-.9234 E+02\end{array}$ & $\begin{array}{r}.1147 \mathrm{E}+03 \\
-.9234 \mathrm{E}+02 \\
.4827 \mathrm{E}+01 \\
.3554 \mathrm{E}+01 \\
\text { *h*BEA }\end{array}$ & $\begin{array}{l}.2810 \mathrm{E}+01 \\
.5571 \mathrm{E}+01 \\
.2810 \mathrm{0}+04\end{array}$ & $\begin{array}{r}-.2553 \mathrm{E}+04 \\
.2522 \mathrm{E}+04 \\
-.3265 \mathrm{E}+01 \\
.3265 \mathrm{E}+01\end{array}$ & $\begin{array}{r}-.3265 E+01 \\
.3265 E+01 \\
.2553 E+04 \\
-.2522 E+04\end{array}$ & $\begin{array}{r}.1784 \mathrm{E}+02 \\
-.3841 \mathrm{E}+01 \\
.1784 \mathrm{E}+02 \\
-.3841 \mathrm{E}+01\end{array}$ \\
\hline GLonds & 129 & $-.3318 E+01$ & $.7754 E+$ & & $-.1427 E+04$ & $-.4708 E+03$ & $.3841 E+01$ \\
\hline
\end{tabular}

KAISER ENGINEERS HANFORD S/N:801854

PAGE 164 RUn ID=WN61975

$02 / 22 / 95$

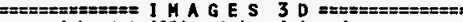
= Copyright (c) 1984 Celestial software Inc. SOLVE BEAM LOADS/STRESSES

Version $2.0 \quad 07 / 01 / 90$

Load Case 5:UBC SEISHIC LOAD - NS \& NE

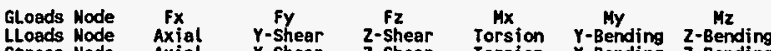
stress Node Axial Y-shear z-shear Torsion Y-Bending z-Bending Stress Node Maximum Minimun Cnb. Shear

GLoads $131-.5064 \mathrm{E}+0 \mathrm{t}-.5519 \mathrm{E}+02 \quad .1021 \mathrm{E}+02 \quad .1294 \mathrm{E}+04 \quad .4708 \mathrm{E}+03-.2305 \mathrm{E}+02$

LLoads $129-.7754 E+02 \quad-3318 E+01-.1833 E+01 \quad-.4708 E+03 \quad .1427 E+04 \quad .3841 E+01$

LLoads $131-.5519 \mathrm{E}+02 \quad .5064 \mathrm{E}+01 \quad .1021 \mathrm{E}+02 \quad .4708 \mathrm{E}+03-.1294 \mathrm{E}+04-.2305 \mathrm{E}+02$

GLoads $131-.5427 \mathrm{E}+01 \quad .8227 \mathrm{E}+02 \quad .6384 \mathrm{E}+01.3521 \mathrm{E}+03-.1303 \mathrm{E}+03 \quad .2305 \mathrm{E}+02$

GLoads $133-.5051 \mathrm{E}+01-.5433 \mathrm{E}+02 \quad .4093 \mathrm{E}+01-.3206 \mathrm{E}+03 \quad .1303 \mathrm{E}+03-.1787 \mathrm{E}+02$ $\begin{array}{llllllll}\text { LLoads } & 131 & .8227 \mathrm{E}+02 & .5427 \mathrm{E}+01 & .6384 \mathrm{E}+01 & -.1303 \mathrm{E}+03 & -.3521 \mathrm{E}+03 & .2305 \mathrm{E}+02 \\ \text { LLoads } & 133 & -.5433 \mathrm{E}+02 & .5051 \mathrm{E}+01 & .4093 \mathrm{E}+01 & .1303 \mathrm{E}+03 & .3206 \mathrm{E}+03 & -.1787 \mathrm{E}+02\end{array}$ $66 * * * * 30302$

GLoads $133-.8243 E+00 \quad 3437 E+02-.5781 E+01 \quad .7598 E+03 \quad .6870 E+03 \quad .1787 E+02$

GLoads $135-.5462 \mathrm{E}+01-.1760 \mathrm{E}+02 \quad .1207 \mathrm{E}+02-.9071 \mathrm{E}+03-.6870 \mathrm{E}+03-.5613 \mathrm{E}+02$

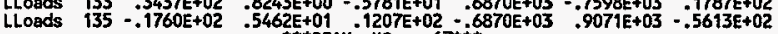

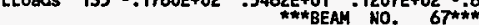

GLoads $115 \cdot .9648 E+03-.4049 E+04.9382 E+02 \quad .3331 E+05-.2407 E+04 \quad .3499 E+05$

GLoeds $116 \quad .5263 \mathrm{E}+03 \quad-.5219 \mathrm{E}+04 \quad: 3447 \mathrm{E}+03-.1882 \mathrm{E}+05-.2278 \mathrm{E}+03 \quad-.2379 \mathrm{E}+05$

LLo8ds $115 \quad .4157 \mathrm{E}+04-.2249 \mathrm{E}+03 \quad .9382 \mathrm{E}+02-.3591 \mathrm{E}+04 \quad .3320 \mathrm{E}+05 \quad .3499 \mathrm{E}+05$

$116-.5229 \mathrm{E}+04-.4157 \mathrm{E}+03 \quad .3447 \mathrm{E}+03 \quad .3591 \mathrm{E}+04-.1848 \mathrm{E}+05-.2379 \mathrm{E}+05$

GLoads $115-.9327 \mathrm{E}+02-.3994 \mathrm{E}+04-.8275 \mathrm{BE}+03 \quad .3372 \mathrm{E}+05-.2414 \mathrm{E}+04 \quad .3337 \mathrm{E}+05$

GLoads $117=.3453 \mathrm{E}+03 \quad .5164 \mathrm{E}+04 \quad .1266 \mathrm{E}+04,-.8981 \mathrm{E}+04 \quad-.2317 \mathrm{E}+03-.1882 \mathrm{E}+05$

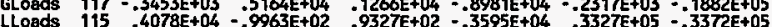

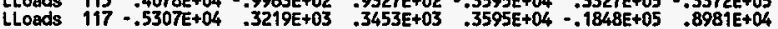

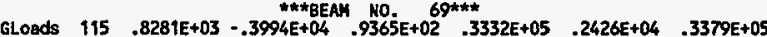

GLoads $115 \quad .8281 E+03 \quad-.3994 E+04 \quad .9365 E+02 \quad .3332 E+05 \quad .2426 E+04 \quad .3379 E+05$

GLoads $118 \cdot-.1267 E+04 \quad .5164 E+04 \quad .3449 E+03-.1881 E+05 \quad .2115 E+03-.8978 E+04$

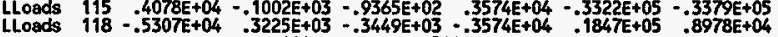

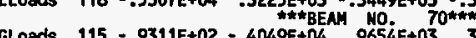

GLoads $115=.9311 E+02-.4049 E+04 \quad .9654 E+03 \quad .3493 E+05 \quad .2434 E+04 \quad .333 B E+05$

GLoads $119-.3454 \mathrm{E}+03 \quad .5219 \mathrm{E}+04-.5268 \mathrm{E}+03-.2379 \mathrm{E}+05 \quad-.2154 \mathrm{E}+03-.18811 \mathrm{E}+05$

Lloads $119=.5229 \mathrm{E}+04=.4452 \mathrm{E}+03-.3454 \mathrm{E}+03-.3577 \mathrm{E}+04 \quad .1847 \mathrm{E}+05=.2379 \mathrm{E}+05$ 
KAISER ENGINEERS HANFORO S/N:801854 PAGE 165

\section{Run ID=*1.161973}

$02 / 22 / 95$

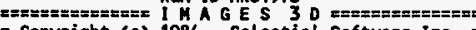
= Copyright (c) 1984 Celestial Software Inc.

SOLVE BEAM LOADS/STRESSES Version $2.0 \quad 07 / 01 / 90$

LOad Case 5:UBC SEISMIC LOAD - WS \& WE

\section{MXXIMUM STRESS SLMMARY FOR BEAMS/TRUSSES} WITHIN SPECIFIED RAMGE $1-70$

Maximum (absolute) Stress $x \quad .1380 E+04$ at BEAM 56

Beam Axial Y-Shear Z-Shear Torsion Y-Bending Z-Bending $56.4396 E+03-.2016 E+03-4580 E+03 \quad .5364 E-03 \quad .6533 E+03-.2874 E+03$ Haximum Minimum Cmb. Shear

Maxinum Minimum Cmb. Shear

$.1380 \mathrm{E}+04-.5011 \mathrm{E}+03 \quad .6902 \mathrm{E}+03$
KAISER ENGIHEERS HANFORD S/N.801854 Run ID =KN61973

$02 / 22 / 95$
$08: 51: 11$

PAGE 166

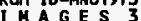

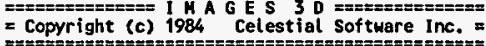

SOLVE PLATE LOADS/STRESSES Version 2.0 07/01/90

Load Case 5:UBC SEISHIC LOAD - NS \& WE

\section{PLATE LOADS AND/OR STRESSES}

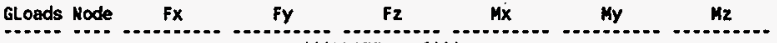
GLo

GLoads $\quad 2=.3233 E+02 \quad .5685 E+02 \quad .1916 E+02 \quad .1843 E+02 \quad .1673 E+03 \quad .0000 E+00$

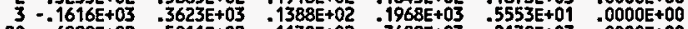
$20 \quad .6889 E+02-.5011 \mathrm{E}+02-.1130 \mathrm{E}+02 \quad .3489 \mathrm{E}+03-.2138 \mathrm{E}+03 \quad .0000 \mathrm{E}+00$ $19.1251 \mathrm{E}+03-.3691 \mathrm{E}+03-.2173 \mathrm{E}+02 \quad .1626 \mathrm{E}+03 \quad .7618 \mathrm{E}+02 \quad .0000 \mathrm{E}+00$

GLoads $3=.6176 E+02 \quad .1558 E+01 \quad .4767 E+01-.1968 E+03 \quad .2060 E+03 \quad .0000 E+00$

GLoads $4 \quad-.1696 \mathrm{E}+03 \quad .3641 \mathrm{E}+03 \quad .9033 \mathrm{E}+01 \quad .2393 \mathrm{E}+03 \quad .6915 \mathrm{E}+02 \quad .0000 \mathrm{E}+00$

$21.9613 \mathrm{E}+02 \quad .8331 \mathrm{E}+01-.35$

GLoads $20 \quad .1352 E+03-.3740 E+03-1029 E+02-.6021 E+02 \quad .2838 E+02 \quad .0000 E+00$

GLoads $\quad 4=.9410 E+02 \quad-.5863 E+02-.8959 E+01-.2393 E+03 \quad-2148 E+03 \quad-0000 E+00$

$\begin{array}{lllllll}5 & -.1700 E+03 & .3553 E+03 & .3672+01 & .1609 E+03 & .2103 E+03 & .0000 E+00\end{array}$

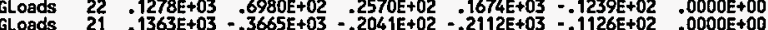

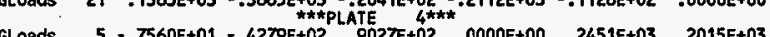

GLoads $\quad 5=.7560 E+01 \quad-.4279 E+02 \quad .9027 \mathrm{E}+02 \quad-0000 \mathrm{E}+00 \quad-2451 \mathrm{E}+03 \quad-2015 \mathrm{E}+03$

$\begin{array}{lllllllll}\text { GLoads } & 6 & .8304 E+01 & .3918 E+03 & .2069 E+03 & .0000 E+00 & .2502 E+03 & -.2359 E+03\end{array}$

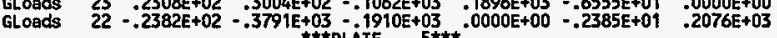

GLoads $6-.1329 E+02-.2605 E+02 \quad .8995 E+02 \quad .0000 E+00 \quad .6492 E+02 \quad .2359 E+03$

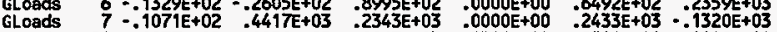

$\begin{array}{llllllll}\text { GLoads } 24 & .1937 \mathrm{E}+02 & .1851 \mathrm{E}+02 & -. .1063 \mathrm{E}+03 & -.5726 \mathrm{E}+02 & .02524 \mathrm{E}+02 & .0000 \mathrm{E}+00\end{array}$

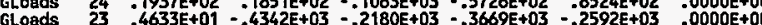

GLoads

GLoads

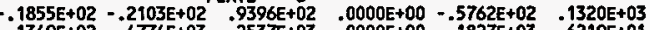

GLoods $24 \quad .1173 \mathrm{E}+02-.4724 \mathrm{E}+03-.2364 \mathrm{E}+03-.3537 \mathrm{E}+03-.2424 \mathrm{E}+03 \quad .0000 \mathrm{EE}+00$

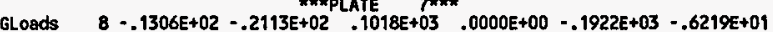

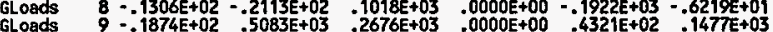

GLoads $26=1874 \mathrm{E}+02 \quad .5083 \mathrm{E}+03 \quad .2676 \mathrm{E}+03 \quad .0000 \mathrm{E}+00 \quad .4321 \mathrm{E}+02 \quad .1477 \mathrm{E}+03$

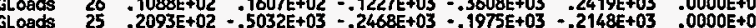

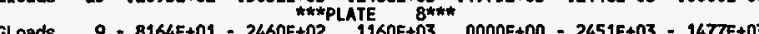

GLoads $\quad 9 \cdot . .8164 \mathrm{E}+01 \cdot .2460 \mathrm{E}+02 \quad .1160 \mathrm{E}+03 \quad .0000 \mathrm{E}+00-.2451 \mathrm{E}+03-.1477 \mathrm{E}+03$

GLoads $10-.1135 E+02 \quad .5434 E+03 \quad-2798 E+03 \quad-0000 E+00-.8598 E+02 \quad .2159 E+03$

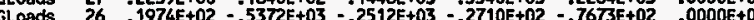


KAISER ENGINEERS HANFORD S/W:801854 PAGE 167 Run ID =11161973 = Copyright (c) 1984 Celestial Software Inc. I

SOLVE PLATE LOADS/STRESSES Version 2.0 07/01/90 L

Load Case 5:UBC SEISMIC LOAD - NS \& ME

GLoads Node Fx $\quad$ Fy $\quad$ Fz $\quad$ Mx $\quad$ My Mz

GLoads $10.9932 E+01.4391 E+01.1185 E+03 \quad .0000 E+00-.2231 E+03 * .2159 E+03$

GLoads $11-.1134 \mathrm{E}+02 \quad .6071 \mathrm{E}+03 \quad .2882 \mathrm{E}+03 \quad .0000 \mathrm{E}+00-.2615 \mathrm{E}+03 \quad .2222 \mathrm{E}+03$

$28-.2283 E+02-.2981 E+02-.1638 E+03 \quad .0000 E+00-.7366 E+02-.2140 E+03$

GLoads

GLoads

GLoads

GLoads

GLoads

GLoads

GLoads

GLoads

CLoads

GLoads

GLoads

GLoads

GLoeds

GLoads

GLoeds

GLoads

GLoads

GLoads

GLosds

- GLoads

GLoads

GLoods

GLoeds

7 GLoeds

GLoads

GLoads

灾 $+03-2429 \mathrm{E}+03 \cdot 1893 \mathrm{E}+03-.2863 \mathrm{E}+02 \quad .0000 \mathrm{E}+00$

$11.6552 E+00.1734 E^{* * * P}+03 \quad .2393 E+02 \quad .3775 E+03-.3049 E+03 \quad .0000 E+00$ $12-.2194 \mathrm{E}+03 \quad .4798 \mathrm{E}+03-.9812 \mathrm{E}+01-.2146 \mathrm{E}+03-.2153 \mathrm{E}+03 \quad .0000 \mathrm{E}+00$ $.3143 \mathrm{E}+02-.1625 \mathrm{E}+03-.2798 \mathrm{E}+02-.2016 \mathrm{E}+03 \quad .8275 \mathrm{E}+02 \quad .0000 \mathrm{E}+00$ $28-1873 E+03-.4908 E+03 \quad .1386 E+02 \quad .3493 E+03-.1357 \mathrm{E}+03 \quad .0000 \mathrm{E}+00$ $12.6701 E+01.1521 E+03.6694 \mathrm{E}+01.2146 \mathrm{E}+03-.1427 \mathrm{E}+03$ $12 \quad .6701 \mathrm{E}+01 \quad .1521 \mathrm{E}+03 \quad .6694 \mathrm{E}+01 \quad .2146 \mathrm{E}+03-.1427 \mathrm{E}+03 \quad .0000 \mathrm{E}+00$

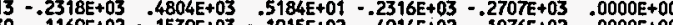
$-1169 E+02-.1539 E+03-.1915 E+02-.4914 E+02-.1076 E+02 \quad .0000 E+00$ . $2123 \mathrm{E}+03$ $13.7509 E+01 \quad .1497 E+03 \quad .2021 \mathrm{E}+02 \quad .2316 \mathrm{E}+03-.3302 \mathrm{E}+02 \quad .0000 \mathrm{E}+00$ $14:-2483 \mathrm{E}+03 \quad .5019 \mathrm{E}+03 \quad .1167 \mathrm{E}+02-.1146 \mathrm{E}+03 \quad-.2513 \mathrm{E}+03 \quad .0000 \mathrm{E}+00$ $-.6389 E+01-.1527 E+03-.2279 E+02-1220 E+03-.1382 E+03$ $.2344 E+03-.4989 E+03-.9097 E+01 * 4624 E+03 \quad .2539 E+03 \quad .0000 E+00$ $14 \quad .7248 E+01 \quad .1528 E+03 \quad \cdot 1602 E+02 \quad .1146 E+03 \quad .9061 E+02 \quad .0000 E+00$

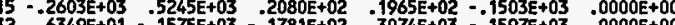
$32-6349 E+01-.1575 E+03=-1781 E+02 \quad-3074 E+03 \quad-.1597 E+03 \quad .0000 E+00$ 15 ***PLATE 14**t $\begin{array}{llllll}.6383 E+01 & .1612 E+03 & -1351 E+02 & -.1965 E+02 & .1440 E+03 & .0000 E+00\end{array}$

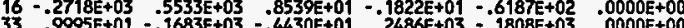
$32.2554+03=5461 E+03-1762 E+02-2570 E+03$. $1608 E+03$. $000 E+00$ (N**PLATE 15**** . $\begin{array}{lllllll}16 & -4197 \mathrm{E}+01 & .1768 \mathrm{E}+03 & -1091 \mathrm{E}+02 & .1822 \mathrm{E}+01 & -1595 \mathrm{E}+03 & -0000 \mathrm{E}+00\end{array}$ $17-.2841 E+03 \quad .5944 E+03 \quad-4249 E+02 \quad .3876 E+03 \quad .6702 E+02 \quad .0000 E+00$ $33 \quad .2605 \mathrm{E}+03=.5828 \mathrm{E}+03=.3105 \mathrm{E}+02 \quad .5805 \mathrm{.} 2050 \mathrm{E}+03 \quad .9043 \mathrm{E}+02 \quad .0000 \mathrm{E}+00$ . $19=.9380 E+02 \quad .6926 E+02 \quad .8587 E+01-.1818 E+03 \quad .3205 E+03 \quad .0000 E+00$ $20 \cdot-.5857 \mathrm{E}+02 \quad .3090 \mathrm{E}+03 \quad .7557 \mathrm{E}+00 \cdot-.3468 \mathrm{E}+02-.2058 \mathrm{E}+03 \quad .0000 \mathrm{E}+00$ $36 \quad .9305 \mathrm{E}+02-.6376 \mathrm{E}+02-.48582+01.2583 \mathrm{E}+03-.3937 \mathrm{E}+03 \quad .0000+00$ $20-.1137 E+03 \quad .3000 E+02-.1107 E+02-.2540 E+03 \quad .3913 E+03 \quad .0000 E+00$
KAISER ENGINEERS HANFORD S/N:801854

Run ID= 4 N61973

$02 / 22 / 95$
$08: 51: 13$

$=$ Copyright (c) 1984 Celestial Softure inc.

Celestial Software inc.

SOLVE PLATE LOADS/STRESSES Version $2.0 \quad 07 / 01 / 90$

L

Load Case 5:UBC SEISMIC LOAD - NS \& WE

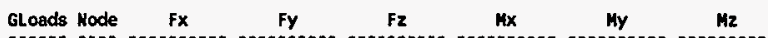

GLoads $21=.5919 \mathrm{E}+02 \quad .2986 \mathrm{E}+03 \quad .2030 \mathrm{E}+02 \quad .1155 \mathrm{E}+03-.4961 \mathrm{E}+02 \quad .0000 \mathrm{E}+00$

GLoads $37 \quad .1171 E+03-.2042 E+02 \quad .1108 E+02 \quad .3399 E+03-.2035 E+03 \quad .0000 E+00$

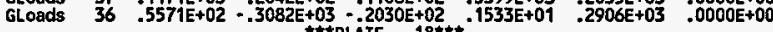

GLoeds $21-.1413 E+03-.2561 E+02-.2828 E+02-.2255 E+03 \quad .2889 E+03 \quad .0000 E+00$

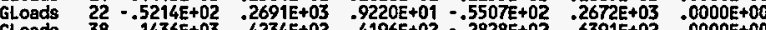

$\begin{array}{llllllll}\text { GLoads } & 38 & .1436 \mathrm{E}+03 & .4234 \mathrm{E}+02 & .4196 \mathrm{E}+02 & -.2828 \mathrm{E}+02 & .6391 \mathrm{E}+02 & .0000 \mathrm{E}+00\end{array}$

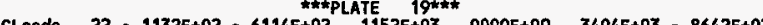

GLads $22 \cdot .1132 E+02 \cdot .6114 E+02 \quad .1152 E+03 \quad .0000 E+00 \quad .3404 E+03-.0042 E+02$

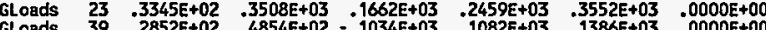

GLoods $39-2852 E+02 \quad .4854 E+02-.1034 E+03 \quad-1082 E+03 \quad .1386 E+03 \quad .0000 E+00$

GLoads $38 \cdot .5066 E+02 \cdot .3382 E+03-1179 E+03 \cdot .0000 E+00.1264 E+03 \cdot .4643 E+02$

GLoads $23-.2497 E+02-.4314 E+02-1219 E+03-.6855 E+02-.8943 E+02 \quad .0000 E+00$

$\begin{array}{llllllll}\text { GLoads } & 24 & .1207 \mathrm{E}+02 & .3889 \mathrm{E}+03 & .1781 \mathrm{E}+03 & .2627 \mathrm{E}+03 & .5042 \mathrm{E}+03 & .0000 \mathrm{E}+00\end{array}$

GLoads $40 \quad .2615 E+02 \quad .3681 E+02-.1184 E+03-.9603 E+02 \quad .4339 E+03 \quad .0000 E+00$

GLoads $39-.1324 E+02-.3826 E+03-1815 E+03-.3820 E+03-.2564 E+03 \quad .0000 E+00$

GLoads $24-.6980 E+01-.3154 \mathrm{E}+02 \quad .1284 \mathrm{E}+03 \quad .1482 \mathrm{E}+03-.3470 \mathrm{E}+03 \quad .0000 \mathrm{E}+00$

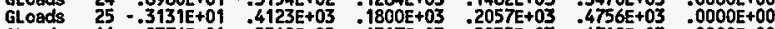

$\begin{array}{llllllll}\text { GLosds } & 41 & .9376 E+01 & .2540 E+02 & -.1317 E+03 & -.2835 E+03 & .4718 E+03 & .0000 E+00\end{array}$

.

GLoads $25-.1913 \mathrm{E}+01-.2162 \mathrm{E}+02 \quad .1418 \mathrm{E}+03 \quad .2047 \mathrm{E}+03+.4838 \mathrm{E}+03 \quad .0000 \mathrm{E}+00$

GLoads $26-.9390 \mathrm{E}+01 \quad .4326 \mathrm{E}+03 \quad .1742 \mathrm{E}+03 \quad .1273 \mathrm{E}+03 \quad .3209 \mathrm{E}+03 \quad .0000 \mathrm{E}+00$

$42-.6534 \mathrm{E}+00 \div 1596 \mathrm{E}+02=1506 \mathrm{E}+03-.3024 \mathrm{E}$

$41.1196 \mathrm{E}+02-.4269 \mathrm{E}+03-1654 \mathrm{E}+03-.2783 \mathrm{E}+03-.4710 \mathrm{E}+03 \quad .0000 \mathrm{E}+00$

GLoads $26.1496 E+02-.7928 E+01.1635 E+03.2606 E+03-.4861 E+03-0000 E+00$

GLoads $27-.2381 \mathrm{E}+02 \quad .4541 \mathrm{E}+03 \quad .1556 \mathrm{E}+03-.5301 \mathrm{E}+02 \quad \div .4058 \mathrm{E}+02 \quad .0000 \mathrm{E}+00$

GLoads $43-.1731 E+02-.1294 E+01-.1767 \mathrm{E}+03-.3304 E+03 \quad .2107 \mathrm{~F}+03 \quad .0000 E+00$

$42.2615 E+02-.4449 E+03-.1424 E+03-.7178 E+02-.4025 E+03-.0000 E+00$

$\begin{array}{llllllll}\text { GLoads } 27 & .3598 E+02 & .1260 E+02 & .1958 E+03 & .1978 E+03 & -.2976 E+03 & .0000 E+00\end{array}$

GLoads $28-2240 E+02 \quad-4223 E+03 \quad 0794 E+02 \quad 0000 E+00=.4432 E+03 \quad .1046 E+02$

GLoads $44=.4768 \mathrm{E}+02-.5414 \mathrm{E}+01 \quad-.2013 \mathrm{E}+03 \quad .0000 \mathrm{E}+00=.2554 \mathrm{E}+03 \quad .4905 \mathrm{E}+01$

GLoads $43 \quad .3410 E+02-.4295 \mathrm{E}+03=.9237 \mathrm{E}+02 \quad .1164 \mathrm{E}+03-.8999 \mathrm{E}+02 \quad .0000 \mathrm{E}+00$

GLoads $28=.9741 E+02 \quad .4959 E+02 \quad .1039 E+02-.1336 E+03-.5651 E+03 \quad .0000 E+00$

GLoads $29-.1603 \mathrm{E}+03 \quad .4382 \mathrm{E}+03-.4460 \mathrm{E}+02-.2483 \mathrm{E}+03-.2296 \mathrm{E}+03 \quad .0000 \mathrm{E}+00$

GLoads $45 \quad .7585 \mathrm{E}+02-.6444 \mathrm{E}+02-.3284 \mathrm{E}+02-.1958 \mathrm{E}+03 \quad-5182 \mathrm{E}+01 \quad .0000 \mathrm{E}+00$ 
KAISER ENGINEERS HAHFORD S/N:801854 PAGE 169 Run ID=ث1461973

$02 / 22 / 95$ $08: 51: 14$ - Copyright (c) 1984 Celestial Software inc. SOLVE PLATE LOADS/STRESSES Version 2.0 07/01/90

Lood Case 5:UBC SEISHIC LOAD - WS \& WE

\begin{tabular}{|c|c|c|c|c|c|c|c|}
\hline Loads & Node & & Fy & Fr & & & \\
\hline GLoads & & $E+03$ & $-.4234 E+03$ & LTE $67056^{* \star 02}$ & $=03$ & -.38 & $.0000 E+C$ \\
\hline $\begin{array}{l}\text { GLoads } \\
\text { GLoads } \\
\text { GLoads } \\
\text { GLoads }\end{array}$ & $\begin{array}{l}29 \\
30 \\
46 \\
45\end{array}$ & $\begin{array}{r}-.4910 \mathrm{E}+02 \\
-.1765 \mathrm{E}+03 \\
.4888 \mathrm{E}+02 \\
.1767 \mathrm{E}+03\end{array}$ & $\begin{array}{r}.1084 E+03 \\
.4369 E+03 \\
-.1097 \mathrm{E}+03 \\
-.4356 \mathrm{E}+03 \\
\\
* 4 \mathrm{~F}\end{array}$ & 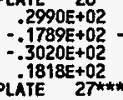 & $\begin{array}{r}.1225 E+03 \\
-.2947 \mathrm{E}+03 \\
.4923 \mathrm{E}+02 \\
.3872 \mathrm{E}+03\end{array}$ & $\begin{array}{r}-.6548 E+02 \\
-.4599 E+03 \\
-.3285 E+03 \\
.1246 E+03\end{array}$ & $\begin{array}{l}.0000 E+0 \\
.0000 E+0 \\
.0000 E+0 \\
.0000 E+0\end{array}$ \\
\hline $\begin{array}{l}\text { GLoads } \\
\text { GLoads } \\
\text { GLoads } \\
\text { GLoads }\end{array}$ & $\begin{array}{l}30 \\
34 \\
47 \\
46\end{array}$ & $\begin{array}{r}-.3416 E+02 \\
-.1854 \mathrm{E}+03 \\
.3994 \mathrm{E}+02 \\
.1796 \mathrm{E}+03\end{array}$ & $\begin{array}{r}.1215 \mathrm{E}+03 \\
.4443 \mathrm{E}+03 \\
-.1258 \mathrm{E}+03 \\
-.4400 \mathrm{E}+03 \\
\\
\star * * \mathrm{~F}\end{array}$ & $\begin{array}{l}.1072 E+02 \\
-.2729 E+01 \\
-.1395 E+02 \\
-5953 E+01 \\
\text { ATE 28* }\end{array}$ & $\begin{array}{r}-.1186 E+03 \\
-.3097 \mathrm{E}+03 \\
.2564 \mathrm{E}+03 \\
.3478 \mathrm{E}+03\end{array}$ & $\begin{array}{r}.2168 E+03 \\
-.4931 E+03 \\
-.4017 E+03 \\
.4251 E+03\end{array}$ & $\begin{array}{l}.0000 \mathrm{E}+0 \\
.0000 \mathrm{E}+0 \\
.0000 \mathrm{E}+0 \\
.0000 \mathrm{E}+0\end{array}$ \\
\hline $\begin{array}{l}\text { GLoads } \\
\text { GLoads } \\
\text { GLoads } \\
\text { GLoads }\end{array}$ & $\begin{array}{l}31 \\
32 \\
48 \\
47\end{array}$ & $\begin{array}{r}-.3225 \mathrm{E}+02 \\
-.1893 \mathrm{E}+03 \\
.4215 \mathrm{E}+02 \\
.1794 \mathrm{E}+03\end{array}$ & $\begin{array}{r}.1338 E+03 \\
.4602 E+03 \\
-.1387 E+03 \\
-.4552 E+03 \\
* \star \star F\end{array}$ & $\begin{array}{r}.9121 \mathrm{E}+01 \\
.5818 \mathrm{E}+01 \\
-.9623 \mathrm{E}+01 \\
-.5316 \mathrm{E}+01 \\
\text { PLATE } 29\end{array}$ & $\begin{array}{r}-.1808 E+03 \\
-.2694 E+03 \\
.3494 E+03 \\
.4294 E+03\end{array}$ & $\begin{array}{r}.3664 E+03 \\
-.4077 E+03 \\
-.4526 E+03 \\
.4362 E+03\end{array}$ & $\begin{array}{l}.0000 E+1 \\
.0000 E+ \\
.0000 E+ \\
.0000 E+\end{array}$ \\
\hline $\begin{array}{l}\text { Gloads } \\
\text { GLoads } \\
\text { GLoads } \\
\text { GLoads }\end{array}$ & $\begin{array}{l}32 \\
33 \\
49 \\
48\end{array}$ & $\begin{array}{r}-.3698 E+02 \\
-.1854 E+03 \\
.5023 E+02 \\
.1721 E+03\end{array}$ & $\begin{array}{r}.1490 E+03 \\
.4787 \mathrm{E}+03 \\
-.1562 E+03 \\
-.4716 E+03 \\
\end{array}$ & & $\begin{array}{r}-.2960 E+03 \\
-.2364 E+03 \\
.3659 E+03 \\
.2955 E+03\end{array}$ & $\begin{array}{r}.4065 E+03 \\
-.2253 E+03 \\
-.2765 E+03 \\
.4363 E+03\end{array}$ & $\begin{array}{l}.0000 E+0 \\
.0000 E+0 \\
.0000 E+0 \\
.0000 E+0\end{array}$ \\
\hline $\begin{array}{l}\text { GLoads } \\
\text { GLosds } \\
\text { GLoads } \\
\text { GLoads }\end{array}$ & $\begin{array}{l}33 \\
34 \\
50 \\
49\end{array}$ & $\begin{array}{r}-.4972 E+02 \\
-.1654 \mathrm{E}+03 \\
.6933 \mathrm{E}+02 \\
.1458 \mathrm{0}+03\end{array}$ & $\begin{aligned} .1780 \mathrm{E}+03 \\
.5035 \mathrm{E}+03 \\
-.1915 \mathrm{E}+03 \\
-.4900 \mathrm{E}+03\end{aligned}$ & 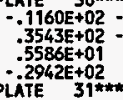 & $\begin{array}{r}-.2172 E+03 \\
-.8081 E+01 \\
.4610 E+03 \\
.2886 E+03\end{array}$ & $\begin{array}{r}.3156 \mathrm{E}+03 \\
.7765 \mathrm{E}+02 \\
.7479 \mathrm{E}+01 \\
.2363 \mathrm{E}+03\end{array}$ & $\begin{array}{l}.0000 E+0 \\
.0000 E+0 \\
.0000 E+0 \\
.0000 E+0\end{array}$ \\
\hline $\begin{array}{l}\text { GLoads } \\
\text { GLaads } \\
\text { GLoads } \\
\text { GLoads }\end{array}$ & $\begin{array}{l}35 \\
36 \\
52 \\
51\end{array}$ & $\begin{array}{r}-.5837 E+02 \\
-.2072 E+02 \\
.5105 E+02 \\
.2803 E+02\end{array}$ & $\begin{array}{r}.1079 E+03 \\
.2289 E+03 \\
-1016 E+03 \\
-.2352 E+03 \\
* * * P\end{array}$ & $\begin{array}{l}.2287 \mathrm{E}+01 \\
.7644 \mathrm{E}+01 \\
.6247 \mathrm{E}+01 \\
.1160 \mathrm{E}+02 \\
\text { ATE } 32 \%\end{array}$ & $\begin{array}{r}-.2488 E+03 \\
-.1046 E+03 \\
.2905 E+03 \\
.1807 E+03\end{array}$ & $\begin{array}{r}.4005 E+03 \\
-.3237 E+03 \\
-.3049 E+03 \\
.4179 E+03\end{array}$ & $\begin{array}{l}.0000 E+0 \\
.0000 E+0 \\
.0000 E+0 \\
.0000 E+0\end{array}$ \\
\hline $\begin{array}{l}\text { GLoads } \\
\text { GLoads } \\
\text { GLoads } \\
\text { GLoads }\end{array}$ & $\begin{array}{l}36 \\
37 \\
53 \\
52\end{array}$ & $\begin{array}{r}-.9614 \mathrm{E}+02 \\
-.1121 \mathrm{E}+02 \\
.9245 \mathrm{E}+02 \\
.1490 \mathrm{E}+02\end{array}$ & $\begin{array}{r}.5800 E+02 \\
.2211 E+03 \\
-.4825 E+02 \\
-.2308 E+03\end{array}$ & $\begin{array}{l}.1439 E+02 \\
.1689 \mathrm{E}+02 \\
.1329 \mathrm{E}+02 \\
.1579 \mathrm{0}+02 \\
\text { LiTe } 33\end{array}$ & $\begin{array}{r}.1552 E+03 \\
-.9187 \mathrm{E}+02 \\
.1254 \mathrm{E}+03 \\
.1767 \mathrm{E}+03\end{array}$ & $\begin{array}{r}.4268 E+03 \\
-.1263 E+03 \\
-.2241 E+03 \\
.3361 E+03\end{array}$ & $\begin{array}{l}.0000 E+0 \\
.0000 E+0 \\
.0000 E+0 \\
.0000 E+0\end{array}$ \\
\hline $\begin{array}{l}\text { GLoads } \\
\text { GLoads } \\
\text { GLoads } \\
\text { GLoads }\end{array}$ & $\begin{array}{l}37 \\
38 \\
54 \\
53\end{array}$ & $\begin{array}{r}-.1238 E+03 \\
-.1457 \mathrm{E}+02 \\
.1217 \mathrm{E}+03 \\
.1671 \mathrm{E}+02\end{array}$ & $\begin{array}{r}.6531 E-01 \\
.2119 E+03 \\
.1095 E+02 \\
-.2229 E+03\end{array}$ & $\begin{array}{r}.3698 \mathrm{E}+02- \\
.2980 \mathrm{0}+02 \\
.4430 \mathrm{E}+02 \\
-.3712 \mathrm{02}+02 \\
-\mathrm{LATE} 34\end{array}$ & $\begin{array}{r}-.1376 \mathrm{E}+03 \\
.1191 \mathrm{E}+02 \\
.2706 \mathrm{E}+02 \\
-.5927 \mathrm{E}+02\end{array}$ & $\begin{array}{l}.2504 \mathrm{E}+03 . \\
.2404 \mathrm{E}+03 \\
.2485 \mathrm{E}+03 \\
.2735 \mathrm{E}+03\end{array}$ & $\begin{array}{l}.0000 E+0 \\
.0000 E+0 \\
.0000 E+0 \\
.0000 E+0\end{array}$ \\
\hline GLoads & 38 & $-.3361 E+02$ & $-.2618 E+02$ & $.6117 E+02$ & $.0000 E+00$ & $.3150 E+03$ & $.1777 \mathrm{E}+$ \\
\hline
\end{tabular}

KAISER ENGINEERS HANFORD S/N:801854

PAGE 170 RUN ID=HN61973

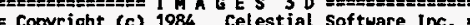
$=$ Copyright (c) 1984 Celestial Software Inc. =

SOLVE PLATE LOADS/STRESSES Version 2.0 07/01/90

L

Load Case 5:UBC SEISMIC LOAD - HS \& WE

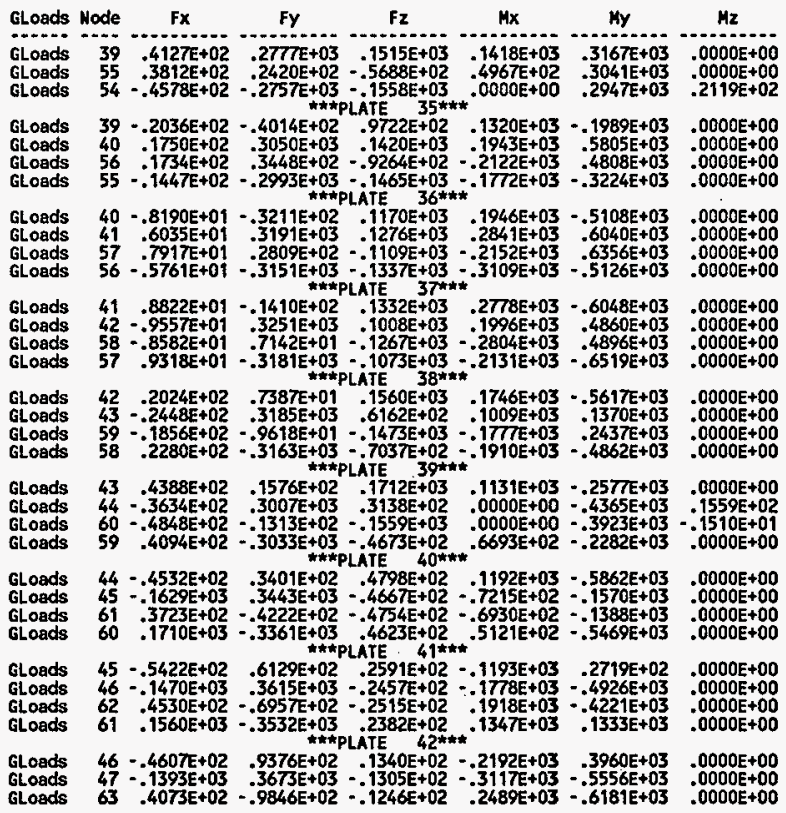


KAISER ENGINEERS HANFORD $S / M: 801854$

PAGE 179

RUn ID=WN61973

$02 / 22 / 95$

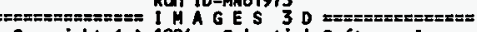
= Copyright (c) 1984 Celestial Software Inc.

SOLVE PLATE LOADS/STRESSES Version 2.0 07/01/90 L

LaAd Case 5:UBC SEISHIC LOAD - NS \& VE

GLoads Node FX $\quad$ Fy $\quad F z \quad M x \quad$ My GLosds $62.1446 E+03-.3626 E+03 \quad 1211 E+02.2897 E+03 \quad .3906 E+03 \quad .0000 E+00$

GLoads $\quad 47-.4467 E+02 \quad .1193 E+03-.3091 E+01-.3741 E+03 \quad .5211 E+03 \quad .0000 E+00$

GLoads $48=.1253 \mathrm{E}+03 \quad .3721 \mathrm{E}+03 \quad .6283 \mathrm{E}+00: 3025 \mathrm{E}+03-.4884 \mathrm{E}+03 \quad .0000 \mathrm{O}+00$

GLoads $64: .4081 \mathrm{E}+02-.1256 \mathrm{E}+03 \quad .4546 \mathrm{E}+01 \cdot .3875 \mathrm{E}+03-.5031 \mathrm{E}+03 \quad .0000 \mathrm{E}+00$

$\begin{array}{lllllllll}\text { GLoads } & 63 & .4081 \mathrm{E}+02 & .1291 \mathrm{E}+03 & -.3657 \mathrm{E}+03 & -.2083 \mathrm{E}+01 & .32349 \mathrm{E}+03 & .5489 \mathrm{E}+03 & .0000 \mathrm{E}+00\end{array}$

GLoads $\quad 48-.5363 E+02 \quad .1438 E+03-.9729 E+01-.3424 E+03 \quad .5047 E+03 \quad .0000 E+00$

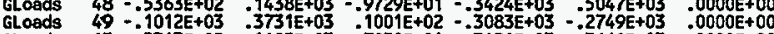

GLoads $65 \quad-5343 \mathrm{E}+02-.1485 \mathrm{E}+03 \quad .7050 \mathrm{E}+01-3421 \mathrm{E}+03-.3661 \mathrm{E}+03 \quad .0000 \mathrm{E}+00$

$\begin{array}{llllllll}\text { GLoads } & 65 & .5343 E+02 & -.1485 E+03 & .7050 E+01 & .3421 E+03 & -.3661 E+03 & .0000 E+00\end{array}$

GLoads $\quad 49-.5934 E+02 \quad .1786 E+03-.2683 E+02-.3462 E+03 \quad .3150 E+03 \quad .0000 E+00$

GLoads $50=.5737 \mathrm{E}+02 \quad .3494 \mathrm{E}+03 \quad .1858 \mathrm{E}+02-.2721 \mathrm{E}+03 \quad .5883 \mathrm{E}+02 \quad .0000 \mathrm{E}+00$

$\begin{array}{llllllll}\text { GLoads } & 66 & -.5737 \mathrm{E}+02 & -3494 \mathrm{E}+03 & .1858 \mathrm{E}+02 & -.2721 \mathrm{E}+03 & .5883 \mathrm{E}+02 & .0000 \mathrm{E}+00\end{array}$

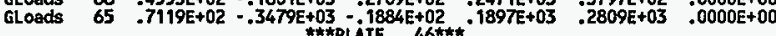

GLoads $51=.6584 E+01 \quad .1431 E+03-.9653 \mathrm{E}+01-.2195 \mathrm{E}+03 \quad .3453 \mathrm{E}+03 \quad .0000 \mathrm{E}+00$

GLoads $52-.5695 \mathrm{E}+01-.1393 \mathrm{E}+03-.5442 \mathrm{E}+01-.3094 \mathrm{E}+03-.3161 \mathrm{E}+03-0000 \mathrm{O}+00$

GLoads $\quad 68-.4633 \mathrm{E}+01-.1378 \mathrm{E}+03-.6612 \mathrm{E}+00 \quad .2457 \mathrm{E}+02-.3609 \mathrm{E}+03$

GLoads

GLoads

GLosds

GLoads

GLoads

GLoads

GLoods

GLoads

- GLoeds

GLoeds

GLoeds

$67-5522 E+01-.1445 E+03 \quad 1576 E+02 \quad .1722 E+03 \quad-2483 E+03$ $.0000 \mathrm{E}+00$

$52-.3974 E+02 \quad .1081 E+03-.1693 E+02-.1579 E+03 \quad-2849 E+03 \quad .0000 E+00$ $63-1598 E+02-1440 E+03 \quad-1124 E+02-.1793 E+03=.2306 E+03 \quad .0000 E+00$ $68-.2239 \mathrm{E}+01-.1463 \mathrm{E}+03-.8144 \mathrm{E}+01.01293 \mathrm{E}+03 \quad .3664 \mathrm{E}+03 \quad .0000 \mathrm{E}+00$

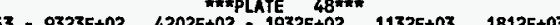

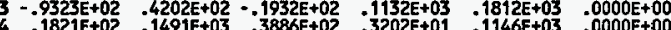
$70.9356 \mathrm{E}+02=2830 \mathrm{E}+02-3657 \mathrm{E}+01 \quad .4468 \mathrm{E}+02 \quad 15497 \mathrm{E}+02 \quad 0000 \mathrm{E}+000$

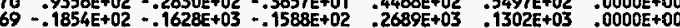
$54-5089 E+02 \quad 0888 E+01$ PLATE $49 * \pi * 2056 E+02.0000 E+00 \quad 2568 E+03$ $\begin{array}{lllll}-3597 E+02 & \end{array}$ $71.3857 E+02-\therefore 1026 E+02-.3734 E+02=.8310 E+03 \quad-2664 E+03 \quad-0000 E+00$ $70-.1928 \mathrm{E}+02-.1985 \mathrm{E}+03-.9555 \mathrm{E}+02 \quad .0000 \mathrm{E}+00 \quad .3002 \mathrm{E}+03 \quad .725 \mathrm{TE}+02$ $55-1886 E+02-2024 E+02$ PL $2101 E+02-2124 E+03-2429 E+03$ $0000 \mathrm{E}+00$ $56-.2810 E+02 \quad-.2024 E+02 \quad .0391 E+02 \quad-2124 E+03 \quad-.2429 E+03 \quad-0000 E+00$ $72 \quad-2715 \mathrm{E}+02-2403 \mathrm{E}+02-6037 \mathrm{E}+02-1127 \mathrm{E}+03 \quad-5314 \mathrm{E}+03 \quad .000 \mathrm{E}+00$ $71-.3649 \mathrm{E}+02 \quad-.2178 \mathrm{E}+03 \quad \because .073 \mathrm{E}+03=.1230 \mathrm{E}+03 \quad-.4473 \mathrm{E}+02 \quad .0000 \mathrm{E}+00$ $56-.3586 E+09-.2984 E+02 \quad .8637 E+02 \quad .2945 E+03-.4824 E+03 \quad .0000 E+00$
KAISER EHGINEERS HANFORD S/N:801854 Run ID=NNS1973

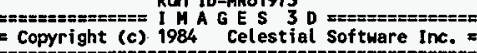

SOLVE PLATE LOADS/STRESSES Version $2.0 \quad 07 / 01 / 90$

Load CaSe 5:UBC SEISMIC LOAD - NS \& WE

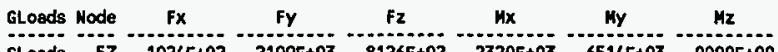
$\begin{array}{lllllll}\text { GLoads } 57 & 51024 E+02 & .2109 E+03 & .8126 E+02 & .2320 E+03 & .6514 E+03 & .0000 E+00\end{array}$ $\begin{array}{llllllll}\text { GLoads } 73 & .7267 E+01 & .2704 E+02 & -.8382 E+02 & -.2366 E+03 & .6062 E+03 & .0000 E+00\end{array}$ Cloads $72 \cdot-.1392 E+02 \cdot .2081 E+03-.8381 E+02-1435 E+03 \cdot .5038 E+03 \quad .0000 E+00$

GL.oads $57.8712 E+01-.1737 E+02 \quad .1008 E+03 \quad .1963 E+03-.635 t E+03 \quad .0000 E+00$

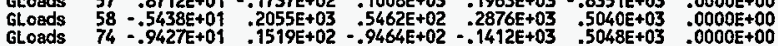

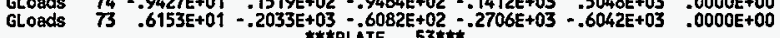

$\begin{array}{lllllllll}\text { GLoads } & 58 & .2741 E+02 & .7180 E+01 & .1063 E+03 & .1838 E+03 & -.5074 E+03 & .0000 E+00\end{array}$

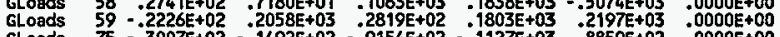

GLoads $75-.3007 \mathrm{E}+02-.1492 \mathrm{E}+02-.9154 \mathrm{EE}+02-1127 \mathrm{E}+03 \quad .8859 \mathrm{E}+02 \quad .0000 \mathrm{O}+000$

Gloads $74 \quad .2493 \mathrm{E}+02-.1980 \mathrm{E}+03-.4291 \mathrm{E}+02-1382 \mathrm{E}+03-.6121 \mathrm{E}+03 \quad .0000 \mathrm{O}+00$

GLoads $59.3607 \mathrm{E}+02 \quad .1070 \mathrm{E}+02 \quad .1296 \mathrm{E}+03-.6945 \mathrm{E}+02 \quad-.2352 \mathrm{E}+03 \quad .0000 \mathrm{E}+00$

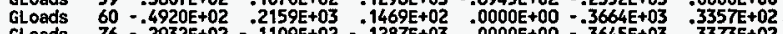

GLoads $76-.2932 \mathrm{E}+02 \quad-.1109 \mathrm{E}+02-1287 \mathrm{E}+03 \quad .0000 \mathrm{E}+00=.3645 \mathrm{E}+03 \quad .3373 \mathrm{E}+02$

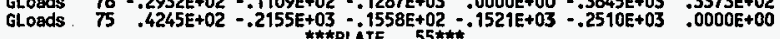

GLoads $60-.2235 E+02 \quad .3293 E+02 \quad .4732 E+02-.1194 E+02-.4806 E+03 \quad .0000 E+09$

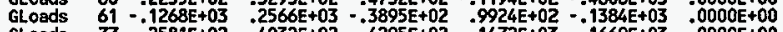

GLoods $77 \quad .2581 \mathrm{E}+02-. .4032 \mathrm{E}+02-.4295 \mathrm{E}+02 \quad .1472 \mathrm{E}+03 \quad-.1669 \mathrm{E}+03 \quad .0000 \mathrm{E}+00$

GLoads $76 \quad .1233 E+03-.2492 E+03 \quad .3458 E+02-.5037 E+02-.4561 E+03$

GLoads $\quad 61-.3104 E+02 \quad .4442 E+02 \quad .2726 E+02-.1646 E+03 \quad .1439 E+03 \quad .0000 E+00$

GLoads $62-.1266 \mathrm{E}+03 \quad 2760 \mathrm{E}+03-.2936 \mathrm{E}+02-1399 \mathrm{E}+03-.4200 \mathrm{E}+03.0000 \mathrm{E}+00$

GLoads $78 \div 2522 \mathrm{E}+02-.4728 \mathrm{E}+02-.2767 \mathrm{E}+02 \div 1588 \mathrm{E}+03-.5746 \mathrm{E}+03.0000 \mathrm{0}+00$

GLoads $77.1324 E+03=.2731 E+03 \quad .2976 E+02 \quad .9958 E+02-.1414 E+02 \quad .0000 E+00$

GLoads $62-.2791 \mathrm{E}+02 \quad .6183 \mathrm{E}+02 \quad .6988 \mathrm{E}+01-.3416 \mathrm{E}+03 \quad .4515 \mathrm{E}+03 \quad .0000 \mathrm{C}+00$

GLoads $63-.1173 \mathrm{E}+03 \quad .2748 \mathrm{E}+03-.1181 \mathrm{E}+02-.1570 \mathrm{E}+03-.5580 \mathrm{E}+03 \quad .0000 \mathrm{E}+00$

GLoods $79-1346 \mathrm{E}+02-.6424 \mathrm{E}+02-.7356 \mathrm{E}+01 \quad .3166 \mathrm{E}+03-.5978 \mathrm{E}+03 \quad .0000 \mathrm{E}+00$

GLoads $78: 1317 \mathrm{E}+03=.2724 \mathrm{E}+03 \quad: 1218 \mathrm{E}+02 \quad .3590 \mathrm{E}+02 \quad .4136 \mathrm{E}+03 \quad .0000 \mathrm{E}+00$

GLoads $63-.1716 E+02.9494 E+02-.9054 E+01-.3268 E+03 \quad .6272 E+03 \quad .0000 E+00$

GLoads $64=.9340 E+02 \quad .2593 E+03-.1445 E+01-.2740 E+03-.3982 E+03-0000 E+00$

GLOads $80-.2787 \mathrm{E}+01-.9891 \mathrm{E}+02 \quad .1465 \mathrm{E}+02-.2050 \mathrm{E}+03+.5391 \mathrm{E}+03.0000 \mathrm{.00}+000$

GLoads $79.1133 \mathrm{E}+03-.2553 \mathrm{E}+03-.4154 \mathrm{E}+01.1648 \mathrm{E}+03 \quad .5105 \mathrm{E}+03 \quad .0000 \mathrm{E}+00$

GLoads $64-.1344 E+02.1403 E+03-.3118 E+02-.4283 E+03 \quad .5063 E+03 \quad .0000 E+00$

GLoads $65-.5386 \mathrm{E}+02 \quad .2473 \mathrm{E}+03 \quad .9692 \mathrm{E}+01-.2522 \mathrm{E}+03-.1353 \mathrm{E}+03 \quad .0000 \mathrm{E}+00$

GLoads $81-.1069 E+02-.1497 E+03 \quad .4465 E+02 \quad .2257 E+03-.8226 E+02 \quad .0000 E+00$ 
KAISER ENGINEERS HANFORD S/N:801854

Run ID=WN61973

$02 / 22 / 95$

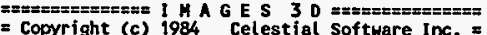

= Copyright (c) 1984 Celestial Software Inc. I

SOLVE PLATE LOADS/STRESSES Version 2.0 07/01/90

L.

Load Case 5:UBC SEISHIC LOAD - NS \& WE

GLoods Hode Fx $\quad$ Fy $\quad$ Fz

GLoads $80 \quad .7999+02-.2380 E+03-.2317 E+02=.1787 \mathrm{E}+02 \quad .5356 \mathrm{E}+03 \quad .0000 \mathrm{E}+00$

GLoads $65-.3535 \mathrm{E}+02,1546 \mathrm{E}+03-.3331 \mathrm{E}+02-.2796 \mathrm{E}+03 \quad .2205 \mathrm{E}+03 \quad .0000 E+00$

GLosds $66=.1934 \mathrm{E}+02 \quad .2290 \mathrm{E}+03 \quad .1147 \mathrm{E}+02=.3131 \mathrm{E}+03 \quad .1792 \mathrm{E}+03 \quad .0000 \mathrm{E}+00$

$\begin{array}{llllllll}\text { GLoads } 82 & .3860 \mathrm{E}+02 & -.1497 \mathrm{E}+03 & .3935 \mathrm{E}+02 & .3631 \mathrm{E}+02 & .2518 \mathrm{E}+03 & .0000 \mathrm{E}+00\end{array}$

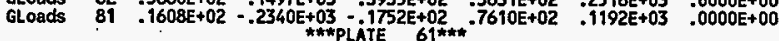

$\begin{array}{lllllllll}\text { GLods } & 67 & .3003 E+02 & .1352 E+03 & -.2231 E+02 & -.2779 E+03 & .1495 E+03 & .0000 E+00\end{array}$

GLoads $68-.4741 \mathrm{E}+01 \quad .7173 \mathrm{E}+02-.3918 \mathrm{E}+01-.2530 \mathrm{E}+03-.2681 \mathrm{E}+03 \quad .0000 \mathrm{E}+00$

GLoads $84-.4980 E+02-.1226 \mathrm{E}+03 \quad .3763 \mathrm{E}+02-.1003 \mathrm{E}+03 \quad .2012 \mathrm{E}+03 \quad .0000 \mathrm{0}+00$

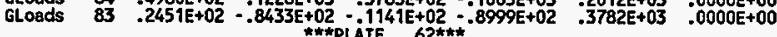

$\begin{array}{lllllllll}\text { GLoads } 68 & .4751 E+02 & .1167 E+03 & * .2317 E+02 & .9919 E+02 & .2626 E+03 & .0000 E+00\end{array}$

GLoads $\quad 69-.1404 E+02 \quad .4282 E+02 \quad .8355 E+01-.4156 E+03-.1298 E+03 \quad .0000 E+00$

GLoads $85-.1067 \mathrm{E}+03-.1102 \mathrm{E}+03 \quad .3451 \mathrm{E}+02-.3486 \mathrm{E}+03 \quad .3247 \mathrm{E}+02 \quad .0000 \mathrm{E}+00$

GLoads

GLoads

GLoads

GLoads

GLoads

GLoads

GLoads

GLoads

Loads

GLoads

GLoads

GLoads

- GLoads

GLoads

GLoads

GLonds

0

$84.7323 \mathrm{E}+02-.4933 \mathrm{E}+02-.1969 \mathrm{E}+02 \quad .3576 \mathrm{E}+03 \quad .4206 \mathrm{E}+03.0000 \mathrm{E}+00$

$69 . .4247 \mathrm{E}+02 \quad .1300 \mathrm{E}+03-.4220 \mathrm{E}+02 \quad .6401 \mathrm{E}+02 \quad .7764 \mathrm{E}+02 \quad .0000 \mathrm{E}+00$

$00.2723 \mathrm{E}+01 \quad .5275 \mathrm{E}+02 \quad .3760 \mathrm{E}+02-.1103 \mathrm{E}+03 \quad .1378 \mathrm{E}+03 \quad .0000 \mathrm{E}+00$

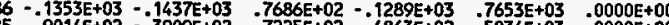

$85.9014 \mathrm{E}+02-.3909 \mathrm{E}+02-.7225 \mathrm{E}+02 \quad .4863 \mathrm{E}+02 \quad .5834 \mathrm{E}+03 \quad .0000 \mathrm{E}+00$

$70-.2971 E+02 \quad .3804 E+02 \quad .1333 E+02 \quad .0000 E+00 \quad .3163 E+03-.1605 E+03$ $71 \quad .4582 \mathrm{E}+02 \quad .1319 \mathrm{E}+03 \quad .5245 \mathrm{E}+02-.1623 \mathrm{E}+02-.2470 \mathrm{E}+02 \quad .0000 \mathrm{E}+00$ $87-.2239 E+02-.1516 E+02-.1009 E+02 \quad .5791 E+02 \quad .9056 E+02 \quad .0000 E+00$ 86-.3850E+02-.1547E+03-.5568E+02 .0000E+00 $.6750 \mathrm{E}+03-.2408 \mathrm{E}+03$ $71-.7189 E+01-.1240 E+02 \quad .5149 E+02 \quad .3703 E+03-.1969 E+03 \quad .0000 E+00$ $72.2731 \mathrm{E}+02 \quad .1007 \mathrm{E}+03 \quad .1124 \mathrm{E}+02 \quad .6069 \mathrm{E}+02 \quad .4414 \mathrm{E}+03 \quad .0000 \mathrm{E}+00$ $88-.2153 E+02-1060 E+02-.7733 E+02-.1292 E+03 \quad .2130 E+03 \quad .0000 E+00$

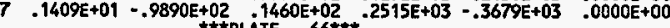
$72 \cdot .1773 E+00-.2522 E+02 \quad .92222 E+02 \quad .1954 E+03-.5689 E+03 \quad .0000 E+00$ $73.1274 \mathrm{E}+02 \quad .1122 \mathrm{E}+03-.1588 \mathrm{E}+02 \quad .2617 \mathrm{E}+03 \quad .5665 \mathrm{E}+03 \quad .0000 \mathrm{E}+00$ $88=1044 E+02 \quad .2327 E+02-1244 E+03-.2270 E+02 \quad .4892 E+03 \quad .0000 E+00$ $\begin{array}{lll} & \end{array}$ $73.1455 E+02-.4451 E+02 \quad .1198 E+03 \quad .2455 E+03-.5685 E+03 \quad .0000 E+00$ $74-1278 E+01 \quad-1296 E+03-.2119 E+02 \quad .2600 E+03 \quad .5957 \mathrm{E}+03 \quad .0000 \mathrm{E}+00$ $90=1101 \mathrm{E}+02 \quad .4537 \mathrm{E}+02-.1495 \mathrm{E}+03-.2309 \mathrm{E}+02 \quad .4162 \mathrm{E}+03 \quad .0000 \mathrm{E}+00$

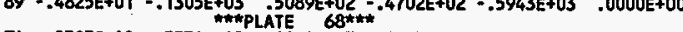
$\begin{array}{ll}74.2393 E+02-.5534 E+02 \quad .1180 E+03 & .1943 E+02-.4885 E+03 \quad .0000 E+00\end{array}$
KAISER ENGIHEERS HANFORD S/N:801854

PAGE 174 RUN ID=MN61973

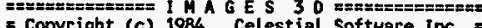

Celestial Software Inc. =

SOLVE PLATE LOADS/STRESSES Version 2.0 07/01/90

$\mathbf{L}$

Load Case 5:UBC SEISHIC LOAD - NS \& WE

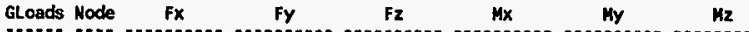

\begin{tabular}{lllllll}
\hline GLoads $\sqrt{3}-.1470 \mathrm{E}+02$ & $.1270 \mathrm{E}+03$ & $-.1243 \mathrm{E}+02$ & $.2938 \mathrm{E}+03$ & $.2748 \mathrm{E}+03$ & $.0000 \mathrm{E}+00$
\end{tabular}

GLoads $91=.6966 \mathrm{E}+01 \quad .6034 \mathrm{E}+02-.1336 \mathrm{E}+03 \quad .1366 \mathrm{E}+03 \quad-3337 \mathrm{E}+03 \quad 0000 \mathrm{O}+00$

GLoads $90-.6270 \mathrm{E}+01-.1320 \mathrm{E}+03 \quad .2805 \mathrm{E}+02-.1958 \mathrm{E}+03-.3938 \mathrm{E}+03 \quad .0000 \mathrm{E}+00$

GLoads $75 \quad .4303 E+02-.5116 E+01 \quad .7884 E+02-.2898 E+02-.1124 E+03 \quad .0000 E+00$

GLoeds $76 \cdot .3276 \mathrm{E}+02 \quad .1421 \mathrm{E}+03-.7465 \mathrm{E}+01 \quad .0000 \mathrm{E}+00-.2897 \mathrm{E}+03-.1489 \mathrm{E}+03$

GLoads $92-.3617 E+02-.1545 E+02-.2853 E+02-0000 E+00+.4335 E+03-.1792 E+03$

GLoads $91.2590 \mathrm{E}+02-.1215 \mathrm{E}+03-.4285 \mathrm{E}+02-.1682 \mathrm{E}+02-.2329 \mathrm{E}+03 \quad .0000 \mathrm{E}+00$

GLoads $\quad 76-.7186 \mathrm{E}+01-.1133 \mathrm{E}+02 \quad .4481 \mathrm{E}+02-.7751 \mathrm{E}+01-.3640 \mathrm{E}+03 \quad .0000 \mathrm{E}+00$

GLoads $77-.1251 E+03 \quad .2252 E+03-.4148 E+02 \quad .9877 E+02-.3911 E+02 \quad .0000 E+00$

Gloads $93-.1572 E+02 \quad .1459 E+02-.3502 E+02 \quad 5851 E+02-.2294 E+03 \quad 0000 E+00$

GLoads $92: .1480 \mathrm{E}+03-.2285 \mathrm{E}+03 \quad .3169 \mathrm{E}+02-.5802 \mathrm{E}+02-.5277 \mathrm{E}+03 \quad .0000 \mathrm{E}+00$

GLoads $\quad 77.6657 \mathrm{E}+01-.1800 \mathrm{E}+02 \quad .1483 \mathrm{E}+02-.3456 \mathrm{E}+03 \quad .2201 \mathrm{E}+03 \quad .0000 \mathrm{E}+00$

GLoads $78=.1382 E+03 \quad .2169 E+03-.2586 E+02 \quad .3961 E+02-.4181 E+03 \quad .0000 E+00$

GLoads $94-.3786 \mathrm{E}+02 \quad .2168 \mathrm{E}+02 \quad .5005 \mathrm{E}+01 \quad .2215 \mathrm{E}+03 \quad-.3849 \mathrm{E}+03 \quad .0000 \mathrm{E}+00$

GLoads $93 \quad .1694 \mathrm{E}+03-.2205 \mathrm{E}+03 \quad .6021 \mathrm{E}+01 \quad-.2188 \mathrm{E}+03 \quad .2667 \mathrm{E}+03 \quad .0000 \mathrm{E}+00$

GLoads $78 \quad .2111 E+02-.3388 E+01 \quad .1506 E+01-.2744 E+03 \quad .5791 E+03 \quad .0000 E+00$

GLoads $79-.1270 \mathrm{E}+03 \quad .1878 \mathrm{E}+03 \quad-.1301 \mathrm{E}+02 \quad-.1311 \mathrm{E}+03 \quad-.4918 \mathrm{E}+03 \quad-0000 \mathrm{E}+00$

GLoads $95-.4461 \mathrm{E}+02 \quad .4283 \mathrm{E}+01 \quad .2070 \mathrm{E}+01 \quad .1140 \mathrm{E}+03-.6026 \mathrm{E}+03 \quad .0000 \mathrm{EE}+00$

GLoads $94 \quad .1505 \mathrm{E}+03 \quad-.1887 \mathrm{E}+03 \quad .9435 \mathrm{E}+01 \quad-2492 \mathrm{E}+02 \quad .3494 \mathrm{E}+03 \quad .0000 \mathrm{E}+00$

GLoads $79.4008 E+02 \quad .2552 E+02-.1532 E+02-.3502 E+03 \quad .5792 E+03 \quad .0000 E+00$

GLoads $80-.1112 E+03 \quad-1532 E+03 \quad-.6500 E+01-.1668 E+03-.4888 E+03 \quad-0000 E+00$

GLoads $96-.6594 \mathrm{E}+02-.2420 \mathrm{E}+02 \quad .1151 \mathrm{E}+02 \quad .3176 \mathrm{E}+02-.4605 \mathrm{E}+03 \quad .0000 \mathrm{E}+00$

GLoads $95 \quad .1371 \mathrm{E}+03=.1545 \mathrm{E}+03 \quad 1031 \mathrm{E}+02=1146 \mathrm{E}+03 \quad .4461 \mathrm{E}+03 \quad .0000 \mathrm{E}+00$

GLoads $\quad 80 \quad .7583 E+02 \quad .7748 E+02-.2482 E+02-.2035 E+02 \quad .4923 E+03 \quad .0000 E+00$

GLoads $81-.8973 \mathrm{E}+02 \quad .1019 \mathrm{E}+03 \quad .3904 \mathrm{E}+02 \quad .1980 \mathrm{E}+03 \quad-.4756 \mathrm{E}+02 \quad .0000 \mathrm{E}+00$

GLoads $97-.1297 \mathrm{E}+03-.7670 \mathrm{E}+02-.2418 \mathrm{E}+02 \quad-1340 \mathrm{E}+03 \quad-.3929 \mathrm{E}+03 \quad .0000 \mathrm{E}+00$

GLoads $96.1436 \mathrm{E}+03-.1027 \mathrm{E}+03.9959 \mathrm{E}+01.7938 \mathrm{E}+02 \quad .1735 \mathrm{E}+03 \quad .0000 \mathrm{E}+00$

$\begin{array}{lllllllll}\text { GLads } & 81 & .1242 E+03 & .1755 E+03-.1060 E+03 & -.4999 E+03 & .1059 E+02 & .0000 E+00\end{array}$

GLo8ds $82-.5551 \mathrm{E}+02 \quad .6702 \mathrm{E}+02-.6187 \mathrm{E}+02 \quad-.1458 \mathrm{E}+04 \quad .8198 \mathrm{E}+03 \quad .0000 \mathrm{E}+00$

GLoads $98-.2705 \mathrm{E}+03-.1915 \mathrm{E}+03 \quad-1980 \mathrm{E}+03-.1829 \mathrm{E}+04 \quad .1100 \mathrm{E}+04 \quad .0000 \mathrm{E}+00$

GLoads $97.2019 E+03-.5106 \mathrm{E}+02-3007 \mathrm{E}+02-.8298 \mathrm{E}+03 \quad: 1336 \mathrm{E}+03 \quad .0000 \mathrm{E}+00$

GLoads $\quad 83 \quad .8052 E+01 \quad .7764 E+02-.1180 E+02-.3122 E+03-.4795 E+03 \quad .0000 E+00$

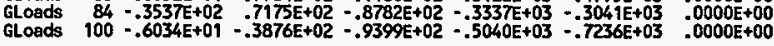


KAISER ENGINEERS HANFORD S/H:801854

PAGE 175 Run ID=\$N61973

$02 / 22 / 95$

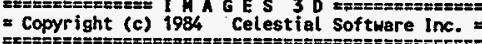

SOLVE PLATE LOADS/STRESSES Version 2.0 07/01/90

LOAd Case 5:UBC SEISHIC LOND - NS \& WE

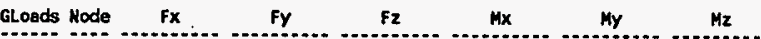
GLoads $99 \quad .3336 E+02-.1106 E+03 \quad .1936 E+03 \quad-.4937 E+03 \quad-.9775 E+03 \quad .0000 E+00$ GLoads $84.4385 E+02.1511 E+02.3797 E+02 \quad .1765 E+03-.3177 E+03 \quad .0000 E+00$ GLoads $85-.4271 \mathrm{E}+02 \quad .8860 \mathrm{E}+01 \quad-.1656 \mathrm{E}+02 \quad .6135 \mathrm{E}+02-.4360 \mathrm{E}+03 \quad .0000 \mathrm{E}+00$ GLoads $101-.2218 E+02 \quad-.1024 E+02-.1084 E+03 \quad .1799 E+02-.4947 E+03 \quad .0000 E+00$ GLoads $100 \quad .2104 \mathrm{E}+02-.1373 \mathrm{E}+02 \quad .8695 \mathrm{E}+02 \quad .9750 \mathrm{E}+02-.4590 \mathrm{E}+03 \quad .0000 \mathrm{E}+00$ $\begin{array}{lllllllll}\text { GLos } & 85 & .9119 E+02 & .5532 E+02 & .2240 E+02 & .2387 E+03 & -.1799 E+03 & .0000 E+00\end{array}$ GLoads $86=.6886 \mathrm{E}+02 \quad .5751 \mathrm{E}+02-.2472 \mathrm{E}+02-.2420 \mathrm{E}+03-.1198 \mathrm{EE}+03 \quad .0000 \mathrm{E}+00$ GLoads $102-.6052 E+02-.8447 \mathrm{E}+02-.8260 \mathrm{E}+02-.2436 \mathrm{E}+03-.6399 \mathrm{E}+03 \quad .0000 \mathrm{0}+00$ GLoads $101 \quad .3820 \mathrm{E}+02-.2836 \mathrm{E}+02 \quad .8493 \mathrm{E}+02 \quad .2086 \mathrm{E}+03-.5272 \mathrm{E}+03 \quad .0000 \mathrm{E}+00$ GLoads $86-.2739 \mathrm{E}+02 \quad .7801 \mathrm{E}+02 \quad .4730 \mathrm{E}+02 \quad .0000 \mathrm{E}+00 \quad .6277 \mathrm{E}+03-.3064 \mathrm{E}+03$

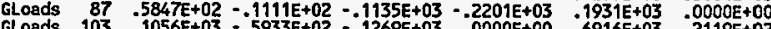
GLoads $103 \quad .1056 \mathrm{E}+03-.5933 \mathrm{E}+02 \quad-.1269 \mathrm{E}+03 \quad .0000 \mathrm{E}+00 \quad .6916 \mathrm{E}+03 \quad .2119 \mathrm{E}+02$ GLoads $102-.1366 \mathrm{E}+03-.7578 \mathrm{E}+01 \quad .1930 \mathrm{E}+03 \quad .0000 \mathrm{E}+00 \quad .1030 \mathrm{E}+04-.4478 \mathrm{E}+03$ $\begin{array}{lllllllll}\text { GLoads } \quad 87=.4608 E+02 & .2866 E+02 & .7277 E+02-.8931 E+02 & .8420 E+02 & .0000 E+00\end{array}$ GLoads $88 \cdot-4819 E+02-.6779 E+01-.1074 E+03-.1183 E+02 \quad .2636 E+03 \quad .0000 E+00$ GL.oads $104 \quad .8150 E+02-.3011 E+02 \quad-.1092 E+03 \quad .0000 E+00 \quad .8685 E+03-.1255 E+03$ CLods

$\begin{array}{llllllllll}\text { GLoads } & 88 & .1181 E+02 & .9913 E+01 & .1005 E+03 & .2203 E+03 & -.2245 E+02 & .0000 E+00\end{array}$ $\begin{array}{lllllllll}\text { GLoads } & 88 & .1181 E+02 & .9913 E+01 & .1003 E+03 & .2203 E+03 & -.2245 E+02 & .0000 E+00\end{array}$ GLoads $105 . .2421 \mathrm{E}+02 \quad-.1085 \mathrm{E}+02-.1158 \mathrm{E}+03 \quad .0000 \mathrm{E}+00 \quad .4602 \mathrm{E}+03-.1822 \mathrm{E}+03$ GLo8ds $104-.7228 E+02-.2130 E+02$. $1051 E+03.0000 E+00 \quad .2014 E+03-.3354 E+03$

GLoods $89.1539 E+02-.1156 E+02 \quad .1271 E+03 \quad .1442 E+02-.1932 E+03 \quad .0000 E+00$

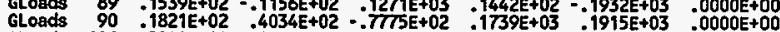

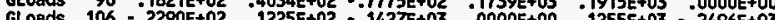

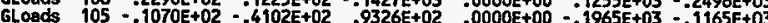
GLods

- GLoads $90 \quad .3526 E+02-.5020 E+02 \quad .1630 E+03 \quad .4498 E+02=.2140 E+03 \quad .0000 E+00$ GLoods $91-.1202 \mathrm{E}+02 \quad .6304 \mathrm{E}+02 \quad-.5588 \mathrm{E}+02 \quad-1084 \mathrm{E}+03 \quad .9986 \mathrm{E}+02 \quad .0000 \mathrm{E}+00$ GLoeds $107=.5666 \mathrm{E}+02 \quad .5101 \mathrm{E}+02=.2052 \mathrm{E}+03 \quad .0000 \mathrm{E}+00-.3508 \mathrm{E}+03-.1236 \mathrm{E}+03$ GLoads $106 \quad .3343 \mathrm{E}+02-.6385 \mathrm{E}+02 \quad .9804 \mathrm{E}+02 \quad-0000 \mathrm{E}+00-.5997 \mathrm{E}+03-.1064 \mathrm{E}+03$

GLoads $91.2527 E+02 \cdot .9836 E+02 \quad .1962 E+03 \cdot .2281 E+03 \cdot .2006 E+03 \quad .0000 E+00$

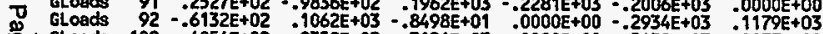
\&. GLads $108-.4054 \mathrm{E}+02 \quad .9359 \mathrm{E}+02-.3124 \mathrm{E}+03 \quad .0000 \mathrm{E}+00=-.5459 \mathrm{E}+03 \quad .8877 \mathrm{E}+02$ GLoads $107 \quad .7659 \mathrm{E}+02-.1014 \mathrm{E}+03 \quad .1247 \mathrm{E}+03 \quad .0000 \mathrm{E}+00-.5389 \mathrm{E}+03 \quad .1601 \mathrm{E}+03$ GLosds $92.2524 E+02-.2088 E+00 \quad .1021 E+03 \quad .4647 E+03-.2741 E+03 \quad .0000 E+00$
KAISER ENGIMEERS HANFORD S/N:801854

Run ID=\#N61973 08:51:22

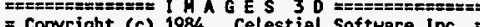
$=$ Copyright (c) 1984 Celestial Software Inc. I

SOLVE PLATE LOADS/STRESSES Version 2.0 07/01/90

Load Case 5:UBC SEISNIC LOAD - NS \& WE

GLoads Hode Fx $\quad$ Fy $\quad$ Fz $\quad$ Mx $\quad$ Hy $\quad$ Mz GLoads $93-1519 \mathrm{E}+03 \quad 1211 \mathrm{E}+03-.8949 \mathrm{E}+01$. $3510 \mathrm{E}+03-1658 \mathrm{E}+03-0000 \mathrm{~B}+00$ $.0000 E+00$

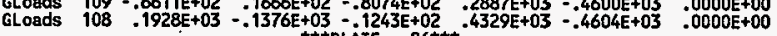
$\begin{array}{lllllllll}\text { GL.oads } & 93 & .3359 E+02 & -.9588 E+01 & .2538 E+01 & -.1907 E+03 & .1285 E+03 & .0000 E+00\end{array}$ GLoads $94-.1048 E+03 \quad .7077 \mathrm{E}+02-.3394 \mathrm{E}+02-.3086 \mathrm{E}+02-.1739 \mathrm{E}+03 \quad .0000 \mathrm{0}+00$ (1) GLoads $109.1156 \mathrm{E}+03-.6789 \mathrm{E}+02 \quad .5996 \mathrm{E}+02-.2015 \mathrm{E}+03-.3287 \mathrm{E}+03 \quad .0000 \mathrm{E}+00$ GLoads $94 \quad .2753 E+02 \quad .1821 E+01-.1591 E+02-.1658 E+03 \quad .2094 E+03 \quad .0000 E+00$ GLoads $95-.7838 \mathrm{E}+02 \quad .5757 \mathrm{E}+02=.1493 \mathrm{E}+02 \quad .2362 \mathrm{EE}+02-.1592 \mathrm{E}+03 \quad .0000 \mathrm{E}+00$ GLoads $111-.2825 \mathrm{E}+02-.2261 \mathrm{E}+01-.1250 \mathrm{E}+02-.1099 \mathrm{E}+03-.1980 \mathrm{E}+03-.0000 \mathrm{E}+00$ GLads $110 \quad .7909 \mathrm{E}+02-.5713 \mathrm{E}+02 \quad 1833 \mathrm{E}+02-.2567 \mathrm{E}+03 \quad .1111 \mathrm{E}+03 \quad .0000 \mathrm{E}+00$ GLoads $95 \quad .2133 E+02-.1810 E+01-.3286 E+02-.2302 E+02 \quad .3158 E+03 \quad .0000 E+00$ GLoads $96-.6847 \mathrm{E}+02 \quad .4659 \mathrm{E}+02-.2445 \mathrm{E}+00-.6809 \mathrm{E}+02 \quad .7957 \mathrm{E}+01 \quad .0000 \mathrm{E}+00$ GLoads $112-.1167 \mathrm{E}+02 \quad .4686 \mathrm{EE}+01 \quad .5928 \mathrm{E}+02-.2560 \mathrm{E}+03 \quad .1633 \mathrm{E}+03 \quad .0000 \mathrm{E}+00$ GLoads $111 \quad .5880 \mathrm{E}+02-.4947 \mathrm{E}+02 \quad-.2618 \mathrm{E}+02 \quad-.1991 \mathrm{E}+03 \quad .4083 \mathrm{E}+03 \quad .0000 \mathrm{E}+00$ $\begin{array}{lllllllll}\text { GLoads } 96 & .2625 E+02 & -.1415 E+02 & -.5663 E+02 & -.4305 E+02 & .2790 E+03 & .0000 E+00\end{array}$ GLoads $97-.8372 E+02 \quad .4821 \mathrm{E}+02 \quad .7261 \mathrm{E}+01 \quad-.1539 \mathrm{E}+03 \quad .7203 \mathrm{E}+02 \quad .0000 \mathrm{~T}+00$ GLoads $113 \quad .9340 E+01 \quad .1432 E+02 \quad .1174 E+03-.3541 E+03 \quad .7294 E+03 \quad .0000 E+00$ GLoads $112 \quad .4813 \mathrm{E}+02-.4838 \mathrm{E}+02-.6798 \mathrm{E}+02-.2636 \mathrm{E}+03 \quad .8096 \mathrm{E}+03 \quad .0000 \mathrm{E}+00$ GLoads $97.4694 \mathrm{E}+02-.1488 E+02 \quad .1158 \mathrm{E}+02 \quad .8497 \mathrm{E}+03 \quad .1873 \mathrm{E}+03 \quad .0000 \mathrm{E}+00$ GLoads $98-.1612 E+03 \quad .1360 E+03 \quad .2396 E+03 \quad .1582 E+04 \quad .7343 E+03 \quad .0000 E+00$ $\begin{array}{lllllll}\text { GLoads } 114 & .5872 E+02 & -.1172 E+02 & -.1059 E+03 & -1221 E+04 & .7318 E+03 & .0000 E+00\end{array}$ GLoads $113 \quad .5553 \mathrm{E}+02-1094 \mathrm{E}+03 \quad-1453 \mathrm{E}+03 \quad .4927 \mathrm{E}+03 \quad .3741 \mathrm{E}+03 \quad .0000 \mathrm{E}+00$ $\begin{array}{llllllll}\text { GLoads } 122=.3219 E+02 & .1744 E+03 & .8481 E+02 & .7412 E+03 & .9659 E+02 & .0000 E+00\end{array}$ GLoads $2=.1426 \mathrm{E}+03 \quad .3907 \mathrm{E}+03 \quad .5980 \mathrm{E}+02 \quad .6685 \mathrm{E}+03 \quad .1995 \mathrm{E}+02 \quad .0000 \mathrm{E}+00$ GLoads $19 \quad .7024 E+02=.1985 E+03 \quad-.6454 E+02 \quad .8448 E+03=.1590 E+03 \quad .0000 E+0$ GLoads $124, .1045 \mathrm{E}+03-.3666 \mathrm{E}+03-.8008 \mathrm{E}+02 \quad .9270 \mathrm{E}+03-.5217 \mathrm{E}+02 \quad .0000 \mathrm{E}+00$ $\begin{array}{lllllllll}\text { GLoads } & 124 & -.7385 E+02 & .2470 E+03 & .5064 E+02 & .2187 E+03 & .1360 E+03 & .0000 E+00\end{array}$ $\begin{array}{llllllll}\text { GLoads } & 19 & -.5628 \mathrm{E}+02 & .3766 \mathrm{E}+03 & .3089 \mathrm{E}+02 & .2233 \mathrm{E}+03 & -.8803 \mathrm{E}+02 & .0000 \mathrm{E}+0\end{array}$ GLOBds $35 \quad .7138 E+02-.2335 \mathrm{E}+03-.3680 \mathrm{E}+02 \quad .6652 \mathrm{E}+03-.2040 \mathrm{E}+03 \quad .0000 \mathrm{E}+00$ $\begin{array}{llllllll}\text { Gloods } 126 & .5875 \mathrm{E}+02 & -.3901 \mathrm{E}+03 & -.4473 \mathrm{E}+02 & .662 & .685 \mathrm{E}+03 & .3790 \mathrm{E}+02 & .0000 \mathrm{E}+00\end{array}$ GLoads $126-.2715 E+02 \quad .2975 E+03 \quad .1503 E+02-.2081 E+03 \quad .1320 E+03 \quad .0000 E+00$ $\begin{array}{llllllll}\text { GLoads } & 35 & -.2417 E+02 & .3233 E+03 & -.2639 E+01 & -.2159 E+03 & -.1547 E+03 & .0000 E+00 \\ \text { GLoads } & 51 & .2433 E+02 & -.2669 E+03 & -.9177 E+01 & .3234 E+03 & -.2502 E+03 & .0000 E+00\end{array}$ 
KAISER EHGINEERS HANFORD S/N:801854

PAGE 977

Run ID=HN61973

$02 / 22 / 95$

$08: 51: 23$

* Copyright (c) 1984 Celest igl Softure Inc.

Celestial Software Inc.

SOLVE PLATE LOADS/STRESSES Version 2.0 07/01/90

L

Lad Case 5:UBC SEISHIC LOAD - NS \& WE

\begin{tabular}{|c|c|c|c|c|c|c|c|}
\hline GLoads & Node & Fx & Fy & $\mathbf{F z}$ & $\mathrm{Mx}$ & My & $\mathrm{Mz}$ \\
\hline GLoads & 128 & $.2699 E+02$ & $.3540 E+0$ & $\begin{array}{l}3214 E+01 \\
\text { LTE } 94 * *\end{array}$ & ${ }_{* \star} \cdot 3731 E+03$ & $.3654 E+02$ & $.0000 E+00$ \\
\hline $\begin{array}{l}\text { GLoads } \\
\text { GLoads } \\
\text { GLoads } \\
\text { GLoads }\end{array}$ & $\begin{array}{r}128 \\
51 \\
67 \\
130\end{array}$ & $\begin{array}{r}.4101 E+01 \\
.1133 E+01 \\
-1579 E+02 \\
.1056 E+02\end{array}$ & $\begin{array}{r}.2854 \mathrm{E}+03 \\
.2485 \mathrm{E}+03 \\
-.2543 \mathrm{E}+03 \\
-.2797 \mathrm{E}+03 \\
* \star \star \mathrm{P}\end{array}$ & $\begin{array}{l}-.2530 E+02 \\
-1766 E+02 \\
.1741 E+02 \\
.2556 E+02 \\
\text { LATE } 95\end{array}$ & $\begin{array}{r}-.5825 E+03 \\
-.3987 \mathrm{E}+03 \\
.9456 \mathrm{E}+02 \\
-.5859 \mathrm{E}+02\end{array}$ & $\begin{array}{r}.8852 E+02 \\
3-.1570 E+03 \\
-.6942 E+02 \\
2-1328 E+03\end{array}$ & $\begin{array}{l}.0000 E+00 \\
.0000 E+00 \\
.0000 E+00 \\
.0000 E+00\end{array}$ \\
\hline $\begin{array}{l}\text { GLoads } \\
\text { GLoads } \\
\text { GLoads } \\
\text { GLoads }\end{array}$ & $\begin{array}{r}130 \\
67 \\
83 \\
132\end{array}$ & $\begin{array}{r}.2646 E+02 \\
.3466 E+02 \\
-.2634 E+02 \\
-.3478 E+02\end{array}$ & $\begin{aligned} & .2272 E+03 \\
&-1367 \mathrm{E}+03 \\
&-.2207 \mathrm{E}+03 \\
&-.1432 \mathrm{E}+03\end{aligned}$ & $\begin{array}{l}-.6119 E+02 \\
-.6237 \mathrm{E}+02 \\
.4484 \mathrm{E}+02 \\
.7873 \mathrm{0}+02 \\
\text { LATE } 96 * \star\end{array}$ & $\begin{array}{l}.9784 \mathrm{E}+03 \\
.7576 \mathrm{E}+03 \\
.7060 \mathrm{E}+03\end{array}$ & $\begin{array}{l}-.1669 E+02 \\
-.5909 E+02 \\
-.1069 E+03 \\
-.1681 E+03\end{array}$ & $\begin{array}{l}.0000 E+00 \\
.0000 E+00 \\
.0000 E+00 \\
.0000 E+00\end{array}$ \\
\hline $\begin{array}{l}\text { GLoads } \\
\text { GLoads } \\
\text { GLoads } \\
\text { GLoads }\end{array}$ & $\begin{array}{r}132 \\
83 \\
99 \\
134\end{array}$ & $\begin{array}{r}.6577 E+02 \\
.4027 E+02 \\
-.9904 E+02 \\
-.6996 E+01\end{array}$ & $\begin{array}{r}.1239 \mathrm{E}+03 \\
.9524 \mathrm{E}+02 \\
-.1827 \mathrm{E}+03 \\
-.3641 \mathrm{E}+02 \\
* * \ldots \mathrm{P}\end{array}$ & $\begin{array}{l}=1127 \mathrm{E}+03 \\
-.6275 \mathrm{E}+02 \\
-1815 \mathrm{E}+03 \\
-.6006 \mathrm{E}+01 \\
\text { LiTE } 97 \mathrm{Th}\end{array}$ & $\begin{array}{l}-.5292 E+03 \\
-.7349 E+03 \\
-.9544 E+03 \\
-.6771 E+03\end{array}$ & $\begin{array}{l}.1811 \mathrm{E}+03 \\
.3655 \mathrm{E}+03 \\
.1011 \mathrm{E}+04 \\
.8177 \mathrm{E}+03\end{array}$ & $\begin{array}{l}.0000 E+00 \\
.0000 E+00 \\
.0000 E+00 \\
.0000 E+00\end{array}$ \\
\hline $\begin{array}{l}\text { GLoads } \\
\text { GLoads } \\
\text { GLoads } \\
\text { GLoads }\end{array}$ & $\begin{array}{r}17 \\
123 \\
125 \\
34\end{array}$ & $\begin{array}{r}-.2362 E+02 \\
\because .3363 \mathrm{E}+03 \\
.8499 \mathrm{E}+02 \\
.2750 \mathrm{E}+03\end{array}$ & $\begin{array}{r}.4184 E+03 \\
.9012 E+03 \\
-.5053 E+03 \\
-.8143 E+03\end{array}$ & $\begin{array}{r}.7360 E+02 \\
.8569 E+02 \\
-.7606 E+02 \\
-.8322 E+02 \\
\text { PLATE } 98 * *\end{array}$ & $\begin{array}{l}.6617 \mathrm{E}+03 \\
.8791 \mathrm{E}+03 \\
.1082 \mathrm{E}+04 \\
.8815 \mathrm{E}+03\end{array}$ & $\begin{array}{r}.1187 \mathrm{E}+03 \\
.7859 \mathrm{E}+02 \\
.4089 \mathrm{E}+01 \\
-.8830 \mathrm{E}+01\end{array}$ & $\begin{array}{l}.0000 E+00 \\
.0000 E+00 \\
.0000 E+00 \\
.0000 E+00\end{array}$ \\
\hline $\begin{array}{l}\text { GLoads } \\
\text { GLoads } \\
\text { GLoads } \\
\text { GLoads }\end{array}$ & $\begin{array}{r}34 \\
125 \\
127 \\
50\end{array}$ & $\begin{array}{r}-.8321 E+02 \\
-.5992 \mathrm{E}+02 \\
.6093 \mathrm{E}+02 \\
.8220 \mathrm{E}+02\end{array}$ & $\begin{array}{r}.3789 E+03 \\
.5313 E+03 \\
-.3739 E+03 \\
. .5364 E+03 \\
* * \star \mathrm{p}\end{array}$ & $\begin{array}{r}.2288 E+02 \\
-4670 E+02 \\
-.2731 E+02 \\
-.4227 E+02 \\
\text { PLATE } 99 \text {. }\end{array}$ & $\begin{array}{r}.4555 \mathrm{E}+02 \\
.2116 \mathrm{E}+03 \\
.7013 \mathrm{E}+03 \\
.5723 \mathrm{E}+03\end{array}$ & $\begin{array}{l}.1590 E+03 \\
.1239 E+03 \\
.5311 E+02 \\
.5180 E+02\end{array}$ & $\begin{array}{l}.0000 E+00 \\
.0000 E+00 \\
.0000 E+00 \\
.0000 E+00\end{array}$ \\
\hline $\begin{array}{l}\text { GLoads } \\
\text { GLoads } \\
\text { GLoads } \\
\text { GLoads }\end{array}$ & $\begin{array}{r}50 \\
127 \\
129 \\
66\end{array}$ & $\begin{array}{r}. .4511 E+02 \\
-.2708 E+02 \\
.4142 E+02 \\
.3077 E+02\end{array}$ & $\begin{array}{r}.2874 \mathrm{E}+03 \\
-3464 \mathrm{E}+03 \\
=.2670 \mathrm{E}+03 \\
-.3668 \mathrm{E}+03 \\
\end{array}$ & $\begin{array}{r}-.2973 \mathrm{E}+02 \\
-.9438 \mathrm{E}+00 \\
.4632 \mathrm{E}+02 \\
.1565 \mathrm{E}+02 \\
\text { PLATE 100* }\end{array}$ & $\begin{array}{r}-.4246 E+03 \\
-.4657 \mathrm{E}+03 \\
.6302 \mathrm{E}+02 \\
.1524 \mathrm{E}+03\end{array}$ & $\begin{array}{l}.1817 E+03 \\
.2063 E+03 \\
.2879 E+03 \\
.2316 E+03\end{array}$ & $\begin{array}{l}.0000 E+00 \\
.0000 E+00 \\
.0000 E+00 \\
.0000 E+00\end{array}$ \\
\hline $\begin{array}{l}\text { GLoads } \\
\text { GLoads } \\
\text { GLoads } \\
\text { GLoads }\end{array}$ & $\begin{array}{r}66 \\
129 \\
131 \\
82\end{array}$ & $\begin{array}{r}-.4021 E+01 \\
-.1120 E+02 \\
-.3275 E+02 \\
.4797 \mathrm{E}+02\end{array}$ & $\begin{array}{r}.2232 E+03 \\
-2196 E+03 \\
-.2028 E+03 \\
-.2399 E+03 \\
\end{array}$ & $\begin{array}{r}-.7123 \mathrm{E}+02 \\
-.7341 \mathrm{E}+02 \\
.8131 \mathrm{E}+02\end{array}$ & $\begin{array}{l}-.7572 E+03 \\
-.1159 \mathrm{E}+04 \\
=.8839 \mathrm{E}+03 \\
-.3825 \mathrm{E}+03\end{array}$ & $\begin{array}{r}.1205 E+02 \\
.1796 E+03 \\
.8341 E+02 \\
-.1171 E+03\end{array}$ & $\begin{array}{l}.0000 E+00 \\
.0000 E+00 \\
.0000 E+00 \\
.0000 E+00\end{array}$ \\
\hline $\begin{array}{l}\text { GLoads } \\
\text { GLoads } \\
\text { GLoads } \\
\text { GLoads }\end{array}$ & $\begin{array}{r}82 \\
131 \\
133 \\
98\end{array}$ & $\begin{array}{r}.1218 E+02 \\
.6950 E+02 \\
.8598 E+02 \\
-.1677 E+03\end{array}$ & $\begin{array}{r}.2066 \mathrm{E}+03 \\
.1057 \mathrm{E}+03 \\
-.2180 \mathrm{E}+03 \\
-.9426 \mathrm{E}+02 \\
\text { ***P }\end{array}$ & $\begin{array}{l}-.8905 E+02 \\
-.1242 E+03 \\
=.3297 E+02 \\
.2462 E+03 \\
\text { LATE 102 }\end{array}$ & $\begin{array}{l}-.1886 E+04 \\
-.7623 \mathrm{E}+03 \\
-.9727 \mathrm{E}+03 \\
-.2243 \mathrm{E}+04 \\
-.04\end{array}$ & $\begin{array}{l}-.9116 E+03 \\
-.4239 E+03 \\
-.6880 E+03 \\
-.1119 E+04\end{array}$ & $\begin{array}{l}.0000 E+00 \\
.0000 E+00 \\
.0000 E+00 \\
.0000 E+00\end{array}$ \\
\hline GLoeds & 98 & $-.1655 E+03$ & $.6165 E+01$ & $.2303 E+03$ & $.1774 \mathrm{E}+04$ & $-.3544 E+03$ & $.0000 E+00$ \\
\hline
\end{tabular}

KAISER ENGINEERS HANFORD S/H:801854

PAGE 178 RUN ID=MN61973

$02 / 22 / 95$

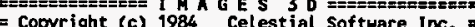

Copyright (c) $19 a 4$ Celestial Software Inc. :

SOLVE PLATE LOADS/STRESSES Version $2.0 \quad 07 / 01 / 90$

$\mathbf{L}$

LOad Case 5:UBC SEISHIC LOAD - HS \& WE

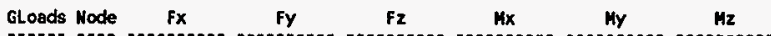

GLoads $133-.5676 \mathrm{E}+02 \quad .1757 \mathrm{E}+03 \quad .1132 \mathrm{E}+02 \quad .5335 \mathrm{E}+03 \quad-.1293 \mathrm{E}+03 \quad .0000 \mathrm{O}+00$

$\begin{array}{llllllll}\text { GLoads } & 135 & .6877 \mathrm{E}+02 & .7663 \mathrm{E}+01 & -.2861 \mathrm{E}+02 & .2530 \mathrm{E}+03 & .1573 \mathrm{E}+03 & .0000 \mathrm{O}+00 \\ \text { GLoads } & 114 & .1535 \mathrm{E}+03 & -.1895 \mathrm{E}+03 & -.2130 \mathrm{E}+03 & .1426 \mathrm{E}+04 & -.1957 \mathrm{E}+02 & .0000 \mathrm{E}+00\end{array}$ 
KAISER ENGINEERS HANFORD S/N:801854

PAGE 179

\section{Run ID=XN61973}

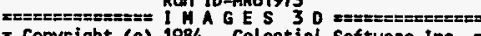

$02 / 22 / 95$ x Copyright (c) 1984 Celestial software Inc.

SOLVE PLATE LOADS/STRESSES Version 2.0 07/01/90

LOAd Case 5:UBC SEISHIC LOAD - NS \& ME

MAXIMUM STRESS SUMMARY FOR PLATES

Maximum (absolute) Stress $=.3094 E+03$ at Plate 36

Plate Sigma $X$ Sigma $Y$ Tau $X Y$ Von Mises

$36-.3094 \mathrm{E}+03-.2130 \mathrm{E}+03 \quad .2512 \mathrm{E}+02 \quad .2776 \mathrm{E}+03$
KAISER ENGIHEERS HAHFORD S/N:80:1854

Run 10=WN61973

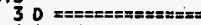
a Copyright (c) 1984 Celestial Software Inc.

SOLVE REACTIONS

Version $2.0 \quad 07 / 01 / 90$

Load Case 5:UBC SEISMIC LOAD - NS \& WE

REACTIOHS

\begin{tabular}{|c|c|c|c|c|c|c|}
\hline de & & Fy & $F_{2}$ & $\mu_{x}$ & v & $z$ \\
\hline $\begin{array}{r}1 \\
2 \\
3 \\
4 \\
5 \\
6 \\
7 \\
8 \\
9 \\
10 \\
11 \\
12 \\
13 \\
14 \\
15 \\
16 \\
17\end{array}$ & $\begin{array}{l}49 E+02 \\
09 E+03 \\
93 E+03 \\
97 E+03 \\
32 E+03 \\
08 E+02 \\
35 E+02 \\
55 E+02 \\
00 E+02 \\
31 E+02 \\
32 E+02 \\
04 E+03 \\
20 E+03 \\
37 E+03 \\
16 E+03 \\
33 E+03 \\
38 E+03 \\
79 E+02 \\
53 E+04 \\
33 E+04 \\
34 E+03 \\
16 E+04 \\
16 E+03\end{array}$ & $\begin{array}{l}.6938 \mathrm{E}+02 \\
.6220 \mathrm{E}+03 \\
.4064 \mathrm{E}+03 \\
.3480 \mathrm{0}+03 \\
.4361 \mathrm{E}+03 \\
.4140 \mathrm{E}+03 \\
.4689 \mathrm{E}+03 \\
.5045 \mathrm{E}+03 \\
.5319 \mathrm{E}+03 \\
.5961 \mathrm{E}+03 \\
.1084 \mathrm{E}+04 \\
.6791 \mathrm{E}+03 \\
.6773 \mathrm{E}+03 \\
.7019 \mathrm{E}+03 \\
.7329 \mathrm{E}+03 \\
.7772 \mathrm{E}+03 \\
.1256 \mathrm{E}+04 \\
.1337 \mathrm{E}+02 \\
.0000 \mathrm{E}+00 \\
.7963 \mathrm{E}+04 \\
.1041 \mathrm{E}+05 \\
.1041 \mathrm{E}+05 \\
.7964 \mathrm{E}+04\end{array}$ & $\begin{array}{l}.8238 \mathrm{E}+02 \\
.1048 \mathrm{E}+03 \\
.3460 \mathrm{E}+02 \\
.1603 \mathrm{E}+02 \\
.1142 \mathrm{E}+03 \\
.3150 \mathrm{E}+03 \\
.3463 \mathrm{E}+03 \\
.3736 \mathrm{E}+03 \\
.4017 \mathrm{E}+03 \\
.4164 \mathrm{E}+03 \\
.3386 \mathrm{E}+03 \\
.1459 \mathrm{E}+02 \\
.4310 \mathrm{E}+02 \\
.4540 \mathrm{E}+02 \\
.5201 \mathrm{E}+02 \\
.3715 \mathrm{E}+02 \\
.1444 \mathrm{E}+03 \\
.9071 \mathrm{E}+01 \\
.8068 \mathrm{E}+04 \\
.7799 \mathrm{E}+03 \\
.3615 \mathrm{E}+04 \\
.7810 \mathrm{E}+03 \\
.1822 \mathrm{E}+04 \\
.1060 \mathrm{E}+03 \\
.1060 \mathrm{E}+03\end{array}$ & $\begin{array}{l}.0000 E+00 \\
.0000 E+00 \\
.0000 E+00 \\
.0000 E+00 \\
.2149 E+00 \\
.0000 E+00 \\
.0000 E+00 \\
.0000 E+00 \\
.0000 E+00 \\
.0000 E+00 \\
.5231 E+03 \\
.0000 E+00 \\
.0000 E+00 \\
.0000 E+00 \\
.0000 E+00 \\
.0000 E+00 \\
.0000 E+00 \\
.0000 E+00 \\
.0000 E+00 \\
.0000 E+00 \\
.0000 E+00 \\
.0000 E+00\end{array}$ & 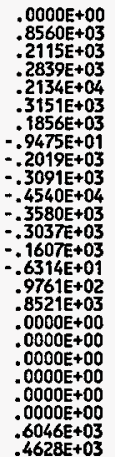 & 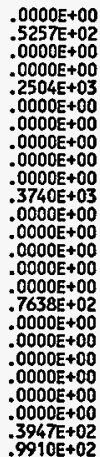 \\
\hline
\end{tabular}


W320-24-020

Intake Air Filter Pad and

Cooling Coil Support Frame Analysis

$$
\text { B-i }
$$


HNF-2467, Rev.0

\section{KAISER ENGINEERS HANFERE}

\section{CALCULATION IDENTIFICATION AND INDEX}

Page $\mathrm{i}$ of $\mathrm{iii}$

$9-2-94$

This sheet shows the status and description of the attached Design Analysis sheets.
Discipline Structural
Wo/sob No. ER4319/W-320
Catcutation No. W320-24-020

Project No. \& Name Project $W-320$, Tank 241-C-106 Sluicing

calculation item INTAKE AIR FILTER PAD AND COOLING COIL SUPPORT FRAME ANALYSIS

These calculations apply to:

Dwg. No. H-2-818458 sh. 3

Rev. No. 0

Dwg. No. H-2-818473 sh. 1

Rev. No. 0

other (Study, CDR) N/A

Rev. No. NA

The status of these calculations is:

[] Preliminary Calculations

[X] Final calculations

[] Check Calculations (On Calculation Dated)

[] Void Calculation (Reason voided )

$\begin{array}{lllll}\text { Incorporated in Final Drawings? } & {[X]} & \text { Yes } & {[]} & \text { No } \\ \text { This calculation verified by independent "check" calculations? } & {[]} & \text { Yes } & {[X]} & \text { No }\end{array}$

Original and Revised Catculation Approvals:

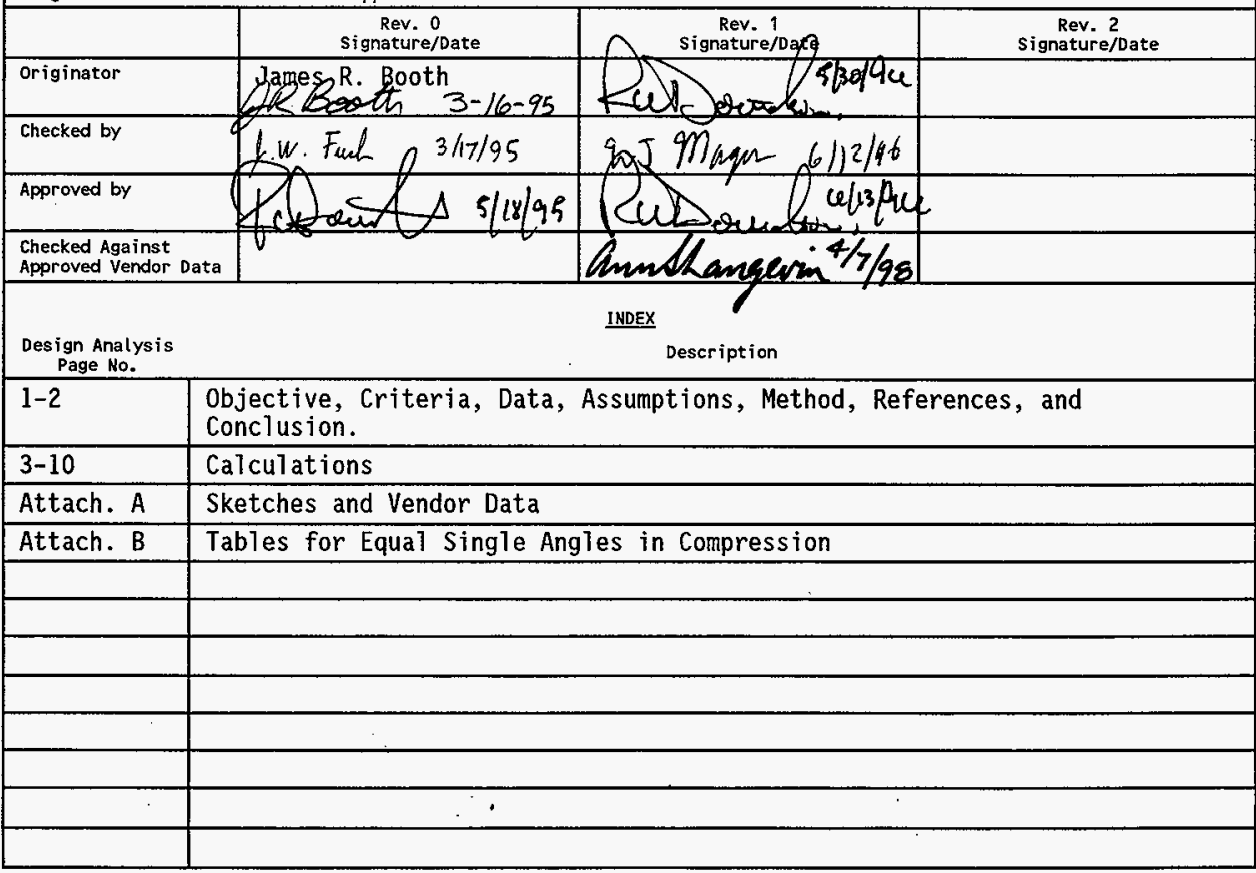

REV 1: Revjsed Page $2 \& 9$ \& Attach A-4, A-5. 


\section{DESIGN VERIFICATION SCREENING CRITERIA}

Project/Document No. $w-320 / W 320-24-020$

When the design or design change affects hardware, formal design verification must be performed if one or more of the following questions must be answered affirmatively (YES).

YES NO

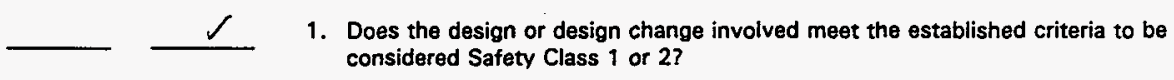
considered Safety Class 1 or 2 ?

2. Does this design or design change cause or permit changes to Safety Class 1 or $\mathbf{2}$ instrument or alarm setpoints outside of previously approved operational limits?

3. Does this design or design change significantly affect the nuclear safety consequences of a malfunction or failure of the structure, system, or component?

4. Does this design or design change involve or change design that has previously undergone formal design verification?
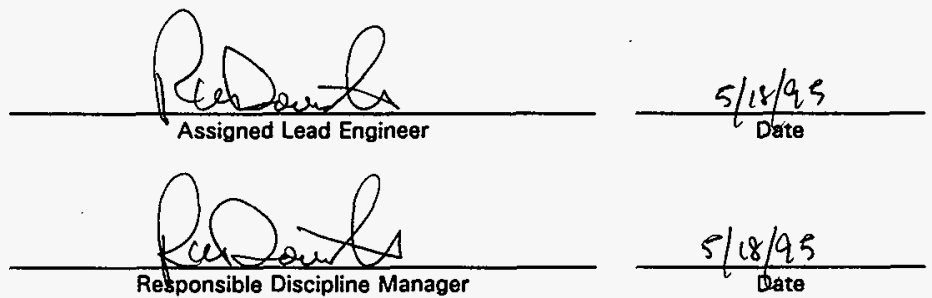

Original Design Package Distribution:

Project Manager

Design Verification Officer

Engineering Document Control
Design Change Distribution:

Attach to Engineering Change Notice 
HNF-2467, Rev.0

\section{KAISER ENGINEERS}

HANFGRE

\section{DESIGN ANALYSIS}

Client WHC

subject Intake Air Filter Pad and Cooling Coil

Support Frame Analys is

Location C-Farm, Tank 24I-C-106
Calc. No. W320-24-020

Revision 0

Page No. 1 of 10

Wo/Job No. ER4319/W-320

Date $9-2-94$

Checked $3 / 77 / 95$

By J.R. Booth $Q R B$

By G.W. Fuak

Revised

OBJECTIVE:

The objective of this calculation is to design a SC3 structural concrete pad and analyze the expansion anchor bolt requirements to support and secure the Intake Air Filter and Cooling Coil located at riser 15 of $\mathrm{C}-106$. Additionally, this calculation will size the support frame members required to support the Cooling Coil.

CRITERIA: $\quad$ 1. SDC 4.1 , Rev. 11 .

2. UCRL 15910, June 1990.

3. DOE order $6430.1 \mathrm{a}$

4. Functional Design Criteria (FDC) for Tank 241-C-106 Waste Retrieval, Project W-320, DOC. NO. WHC-SD-W320-FDC-001, REV.2.

DATA: $\quad$ 1. $f^{*} \mathrm{C}=4000 \mathrm{psi}$

2. $f y=60 \mathrm{ksi}$ for rebar

3. $3 / 8^{\prime \prime}$ dia. expansion anchor bolts with $15 / 8^{\prime \prime}$ minimum embedment.

4. A-36 Structural Steel.

ASSUMPTIONS: $\quad$ See body of calc.

METHOD :

MATHCAD 4.0

REFERENCES: $\quad$ 1. American Concrete Institute (ACI). ACI 318-89 (Revised 1992).

2. American Concrete Institute (ACI). ACI SP-17(91).

3. American Society of Civil Engineers (ASCE). ASCE 7-88.

4. Standard Arch-Civil Design Criteria, SDC-4.1.Design Loads for Facilities. Rev. 11 .

5. International Conference of Building Officials (ICBO). Report No. 4627. March 1989.

6. Uniform Building Code (UBC-91).

7. Design and Evaluation Guidelines for Department of Energy

Facilities Subjected to Natural Phenomena Hazards. UCRL 15910, June 1990.

8. American Institute of Steel Construction (AISC). Manual of steel Construction, Allowable Stress Design. 9th Edition. 
KAISER ENGINEERS

HANFERD

\section{DESIGN ANALYSIS}

client WHC

subject Intake Air Filter Pad and Cooling Coil

Support Frame Analys is

Location C-Farm, Tank 241-C-106
Calc. No. W320-24-020

Revision $\varnothing 1$

Page No. 2 of 10

wo/sob No. ER4319/W-320

Date 9-2-94

checked $3 / 17 / 95$

Revised $5 / 30196$
By J.R. Booth $Q Q B$

By $f$ te. Fel

By

\section{CONCLUSION:}

Intake Air Filter pad \& anchorage for cooling coil frame are adequate as detailed on Dwg H-2-818458, SH 3 and as modified by ECN's W-320-288, 351. For frame support details see Dwg H-2-818473, SH 1 (PS-205) and modifications provided in ECN W-320-225. 
DESIGN ANALYSIS

Wo/Job No.

Date 9/2/94

Checked $3 / 17 / 95$

Revised
Revision 0

Page No. 3 of 10

Client: Westinghouse Hanford Company

Subject: Intake Air Filter Pad and Cooling Coil

Support Frame Analysis

Location: C-Farm, Tank 241-C-106

ER4319N-320

\section{SEE H-2-95451 SH. 5 FOR DETAIL IV OF AIR INTAKE FILTER}

Determine Design Loads:

1. Snow (REF. ASCE $7-88$, pg. 23-27)

$$
\begin{aligned}
& \mathrm{C}_{\mathrm{e}}:=0.9 \\
& \mathrm{C}_{\mathrm{t}}:=1.1 \\
& \mathrm{I}:=1.2 \\
& \mathrm{p}_{\mathrm{g}}:=15 \cdot \frac{\mathrm{lb}}{\mathrm{ft}^{2}} \\
& \mathrm{p}_{\mathrm{f}}:=0.7 \cdot \mathrm{C}_{\mathrm{e}} \cdot \mathrm{C}_{\mathrm{t}} \mathrm{I} \cdot \mathrm{p}_{\mathrm{g}} \\
& \mathrm{p}_{\mathrm{f}}=12.474 \cdot \frac{\mathrm{lb}}{\mathrm{ft}^{2}} \quad \text { Use } \mathrm{p}_{\mathrm{f}}:=20 \cdot \frac{\mathrm{lb}}{\mathrm{ft}^{2}} \\
& \mathrm{~S}:=\mathrm{p}_{\mathrm{f}}(9 \cdot \mathrm{ft} \cdot 9 \cdot \mathrm{ft}) \\
& \mathrm{S}=1.62 \cdot 10^{3} \cdot \mathrm{lb}
\end{aligned}
$$$$
\text { Ref. SDC } 4.1
$$

2. Wind: (Ref. SDC 4.1 and ASCE 7-88, section 6)

Dimensions: 9'-0" X 9'-0" X 6'-0" high, envelope dimensions (See H-2-95451, sh.5)

Exposure Class $=C$ (SDC 4.1,pg.13)

Wind Speed $=70 \mathrm{mph}$ (SDC $4.1, \mathrm{pg} .13$ )

Importance Factor=1.07 (SDC 4.1, pg.13)

$\mathrm{I}:=1.07$

$\mathrm{V}:=70$

$\mathrm{K}_{\mathrm{z}}:=0.80$

(Table 6 of ASCE 7-88)

$q_{z}:=0.00256 \cdot K_{z} \cdot(I \cdot V)^{2} \cdot \frac{\mathrm{lb}}{\mathrm{ft}^{2}}$

(Velocity pressure, Eq.3 of ASCE 7-88)

$\mathrm{q}_{\mathrm{z}}=11.489 \cdot \frac{\mathrm{lb}}{\mathrm{ft}^{2}}$

$\mathrm{G}_{\mathrm{h}}:=1.32$

(Gust response factor, Table 8 of ASCE 7-88)

$\mathrm{C}_{\mathrm{f}}:=1.2$

(Force Coeff. for Solid Signs, Table 13 of ASCE 7-88)

$A_{f}:=9 \cdot \mathrm{ft} \cdot 6 \cdot \mathrm{ft}$

(Projected area normal to wind in sq. $\mathrm{ft}$ )

$\mathrm{W}:=\mathrm{q}_{\mathrm{z}} \cdot \mathrm{G}_{\mathrm{h}} \cdot \mathrm{C}_{\mathrm{f}} \mathrm{A}_{\mathbf{f}}$

(Design Wind Force from Table 4, ASCE 7-88)

$\mathrm{W}=982.749 \cdot \mathrm{lb}$

Note: Wind force acting on filter will be the same in both the N-S and E-W directions.

3. Live Floor Load. Use snow load.
$\mathrm{L}:=\mathrm{S}$
$\mathrm{L}=1.62 \cdot 10^{3} \cdot 1 \mathrm{~b}$ 
DESIGN ANALYSIS

WO/Job No. ER4319NW-320

Client: Westinghouse Hanford Company

Subject: Intake Air Filter Pad and Cooling Coil

Support Frame

Location: C-Farm, Tank 241-C-106
Date 9/2/94

Checked $3 / 17 / 95$

Revised
Calc. No. W320-24-020

Revision 0

Page No. 4 of 10

4. Dead Loads: See H-2-95451 sh. 5, Detail IV and Section A-A of sh. 4.

Intake Ductwork

$$
\begin{aligned}
& \mathrm{D}_{1}:=(((5 \cdot \mathrm{ft} \cdot 8 \cdot \mathrm{ft})+(5 \cdot \mathrm{f} \cdot 2.17 \cdot \mathrm{ft})) \cdot 2+(2.17 \cdot \mathrm{f} \cdot 8 \cdot \mathrm{ft})) \cdot 2.66 \cdot \frac{\mathrm{lb}}{\mathrm{ft}^{2}} \\
& \mathrm{D}_{1}=316.7 \cdot \mathrm{lb}
\end{aligned}
$$

\section{Transition Piece}

$$
\begin{aligned}
& \mathrm{D}_{2}:=2 \cdot(2 \cdot \mathrm{ft} \cdot 8 \cdot \mathrm{ft}+2 \cdot \mathrm{ft} \cdot 2 \cdot \mathrm{ft}) \cdot 3.28 \cdot \frac{\mathrm{lb}}{\mathrm{ft}^{2}} \\
& \mathrm{D}_{2}=131.2 \cdot \mathrm{lb}
\end{aligned}
$$

Round Duct (Estimated Weight)

$\mathrm{D}_{3}:=4 \cdot \mathrm{ft} \cdot 9.6 \cdot \frac{\mathrm{lb}}{\mathrm{ft}}+10 \cdot \mathrm{lb}$

Add $10 \mathrm{lb}$ for Damper

$\mathrm{D}_{3}=48.4 \cdot \mathrm{lb}$

Pre-Filter and Filter Housing Weight from vendor data and P. Langowski, see.page A-3.

$\mathrm{D}_{4}:=600 \cdot \mathrm{lb}$

Angle Support Frame

$L_{l}:=(2 \cdot 3.25 \cdot f t+4 \cdot 3 \cdot f t+8.4 \cdot f t+3.4 \cdot f t+2 \cdot 3.53 \cdot f t) \cdot 4.9 \cdot \frac{\mathrm{lb}}{\mathrm{ft}}$

$\mathrm{L}_{2}:=(3 \cdot 8 \cdot \mathrm{ft}+2 \cdot 3 \cdot \mathrm{ft}) \cdot 6 \cdot 6 \cdot \frac{\mathrm{lb}}{\mathrm{ft}}$

$\mathrm{L}_{3}:=(2 \cdot 15.8 \cdot \mathrm{ft}) \cdot 2.75 \cdot \frac{\mathrm{lb}}{\mathrm{ft}}$

$\mathrm{L}_{4}:=(4 \cdot 15.8 \cdot \mathrm{ft}) \cdot 1.863 \cdot \frac{\mathrm{lb}}{\mathrm{ft}}$

$\mathrm{D}_{5}:=\mathrm{L}_{1}+\mathrm{L}_{2}+\mathrm{L}_{3}+\mathrm{L}_{4}$

$\mathrm{D}_{5}=585.706 \cdot \mathrm{lb}$

\section{Steel Plates (1/4" Steel Plate)}

$\mathrm{D}_{6}:=6 \cdot 0.34 \cdot \mathrm{ft}^{2} \cdot 10.21 \cdot \frac{\mathrm{lb}}{\mathrm{ft}^{2}}$

$\mathrm{D}_{6}=20.828 \cdot \mathrm{lb}$

\section{Weight of Cooling Coil}

$D_{c c}:=1500 \cdot 1 b$

Total estimated weight of Air Inlet Filter \& Cooling Coil

$\mathrm{D}:=\mathrm{D}_{1}+\mathrm{D}_{2}+\mathrm{D}_{3}+\mathrm{D}_{4}+\mathrm{D}_{5}+\mathrm{D}_{6}+\mathrm{D}_{\mathrm{cc}}$

$\mathrm{D}=3.203 \cdot 10^{3} \cdot \mathrm{lb}$ 
KAISER ENGINEERS HANFORD

Client: Westinghouse Hanford Company

Subject Intake Air Filter Pad and Cooling Coil

Support Frame Analysis

Location: C-Farm, Tank 241-C-106
Calc. No. W320-24-020

Revision 0

Page No. 5 of 10

WO/Job No. ER4319M-320

Date 9/2/94

Checked $3 / 17 / 95$

Revised

By JR Booth $2 / 2 B$

By

By

5. Seismic: Ref. SDC 4.1, UCRL 15910, and UBC-91.

Ground motion shall be taken $100 \%$ in one perpendicular direction plus $30 \%$ in the other direction combined in a SRSS basis.

Critical Damping $=5 \%$ for Safety class 3 per SDC 4.1 .

$$
\begin{array}{ll}
\mathrm{Z}:=0.2 & \begin{array}{l}
\text { Peak Ground Acceleration (UBC 91, Zone 2b) } \\
\mathrm{I}:=1.25
\end{array} \\
\mathrm{C}_{\mathrm{p}}:=2 \cdot(0.75) & \begin{array}{l}
\text { Importance Factor (SDC 4.1) } \\
\text { multiplied by 2 per UBC sec. 2336(b). }
\end{array} \\
\mathrm{W}_{\mathrm{p}}:=\mathrm{D} & \text { Weight of filter acting on foundations. } \\
\mathrm{F}_{\mathrm{p}}:=\mathrm{Z} \cdot \mathrm{I} \cdot \mathrm{C}_{\mathrm{p}} \cdot \mathrm{W}_{\mathrm{p}} & \text { Lateral Force per UBC sec.2336(b). } \\
\mathrm{F}_{\mathrm{p}}=1.201 \cdot 10^{3} \cdot \mathrm{N}-\mathrm{S} & \\
\mathrm{F}_{\mathrm{p} 2}:=0.3 \cdot \mathrm{F} p & \\
\mathrm{~F}_{\mathrm{p} 2}=360.319 \cdot \mathrm{lb} & \\
\mathrm{E}:=\sqrt{\mathrm{F}}{ }_{\mathrm{P}}^{2}+\mathrm{F} p 2 & \\
\mathrm{E}=1.254 \cdot 10^{3} \cdot \mathrm{lb} &
\end{array}
$$

6. Load Combinations

a. UBC 91 load combinations, sec. 2609(c) and sec. 2625(c)4. The zero loads below are to make the MathCAD equations work.

$\mathrm{W}_{\mathbf{v}}:=0 \cdot \mathrm{lb}$

$\mathrm{E}_{\mathrm{v}}:=0 \cdot \mathrm{lb}$

1. $\mathrm{U}_{1}:=1.4 \cdot \mathrm{D}+1.7 \cdot \mathrm{L}$

$$
\mathrm{U}_{1}=7.238 \cdot 10^{3} \cdot 1 \mathrm{~b}
$$

\section{Controlling Load Case}

2. $\mathrm{U}_{2}:=0.75 \cdot\left(1.4 \cdot \mathrm{D}+1.7 \cdot \mathrm{L}+1.7 \cdot \mathrm{W}_{\mathrm{v}}\right)$

$$
\mathrm{U}_{2}=5.428 \cdot 10^{3} \cdot \mathrm{lb}
$$

3. $\mathrm{U}_{3}:=0.9 \cdot \mathrm{D}+1.3 \cdot \mathrm{W}_{\mathrm{v}}$

$\mathrm{U}_{3}=2.883 \cdot 10^{3} \cdot 1 \mathrm{~b}$

4. $\mathrm{U}_{4}:=0.75 \cdot\left(1.4 \cdot \mathrm{D}+1.7 \cdot \mathrm{L}+1.87 \cdot \mathrm{E}_{\mathrm{v}}\right)$

$\mathrm{U}_{4}=5.428 \cdot 10^{3} \cdot \mathrm{lb}$

5. $U_{5}:=0.9 \cdot D+1.43 \cdot E_{v}$

$U_{5}=2.883 \cdot 10^{3} \cdot \mathrm{lb}$

6. $\mathrm{U}_{6}:=1 \cdot 4 \cdot\left(\mathrm{D}+\mathrm{L}+\mathrm{E}_{\mathrm{v}}\right)$

$\mathrm{U}_{6}=6.752 \cdot 10^{3} \cdot \mathrm{lb}$ 
Client: Westinghouse Hanford Company

Subject: Intake Air Filter Pad and Cooling Coil

Support Frame Analysis

Location: C-Farm, Tank 241-C-106

WO/Job No. ER4319/N-320

Revision 0

Page No. 6 of 10

Date 9/2/94

Checked 51019

7. $\mathrm{U}_{7}:=0.9 \cdot \mathrm{D}+1 \cdot 4 \cdot \mathrm{E}_{\mathrm{v}}$

$\mathrm{U}_{7}=2.883 \cdot 10^{3} \cdot \mathrm{lb}$

7. Check Soil Loading.

$$
\begin{array}{ll}
\mathrm{q}_{\mathrm{a}}:=2000 \cdot \frac{\mathrm{lb}}{\mathrm{ft}^{2}} & \text { UBC-91, Table No. 29-B } \\
\mathrm{D}_{\mathrm{c}}:=150 \cdot \frac{\mathrm{lb}}{\mathrm{ft}^{3}} \cdot(9 \cdot \mathrm{ft} \cdot 9 \cdot \mathrm{f} \cdot 0.5 \cdot \mathrm{ft}) & \text { Weight of } 9^{\prime}-0^{\prime \prime} \times 9^{\prime}-0^{\prime \prime} \times 0^{\prime}-6^{\prime \prime} \text { THK Concrete Pad } \\
\mathrm{D}_{\mathrm{c}}=6.075 \cdot 10^{3} \cdot \mathrm{lb} & \text { Total Dead Load on Soil } \\
\mathrm{D}_{\mathrm{t}}:=\mathrm{D}+\mathrm{D}_{\mathrm{c}} & \\
\mathrm{D}_{\mathrm{t}}=9.278 \cdot 10^{3} \cdot \mathrm{lb} & \mathrm{D} \mathrm{t} \\
\mathrm{q}_{\text {applied }}:=\frac{-7}{9 \cdot \mathrm{ft}} \cdot 9 \cdot \frac{\mathrm{ft}}{} & \text { Soil loading not a concern } \\
\mathrm{q}_{\text {applied }}=114.541 \cdot \frac{\mathrm{lb}}{\mathrm{ft}^{2}} &
\end{array}
$$

8. Check overturning.

$$
\begin{array}{ll}
M_{\text {ot }}:=E \cdot(4.71 \cdot f t) & \text { Conservative. See page A1. } \\
M_{\text {ot }}=5.906 \cdot 10^{3} \cdot \mathrm{lb} \cdot \mathrm{ft} & \\
M_{\mathrm{r}}:=D_{\mathrm{f}}(3.79 \cdot \mathrm{ft}) & \text { See page A5. } \\
M_{\mathrm{r}}=3.516 \cdot 10^{4} \cdot \mathrm{lb} \cdot \mathrm{ft} & \\
\frac{M_{\text {ot }}}{M_{\mathrm{r}}}=0.168 & \text { Resistance to overturning OK. }
\end{array}
$$

9. Determine factored upward soil pressure.

$$
\begin{array}{ll}
\mathrm{q}_{\mathbf{u}}:=1.7 \cdot \mathrm{q}_{\mathrm{a}} \cdot \mathbf{1} \cdot \mathrm{ft} & \text { Conservative } \\
\mathrm{q}_{\mathbf{u}}=3.4 \cdot 10^{3} \cdot \frac{\mathrm{lb}}{\mathrm{ft}} & \begin{array}{l}
\text { Net factored upward pressure } \\
\text { per lineal foot }
\end{array}
\end{array}
$$


DESIGN ANALYSIS

WO/Job No. ER4319/N-320

Client: Westinghouse Hanford Company

Subject Intake Air Filter Pad and Cooling Coil

Support Frame Analysis

Location: C-Farm, Tank 241-C-106
Date $9 / 2 / 94$ Checked $5 / 1899$ Revised
Calc. No. W320-24-020

Revision 0

Page No. 7 of 10

10. Determine Flexural Steel Requirements. Assume the distance from the outside edge of slab to the HVAC support will act as a cantilever beam. See page A-5.

$1:=1 \cdot \mathrm{ft}$

$\mathrm{M}_{\mathrm{u}}:=\frac{\frac{\mathrm{qu}_{\mathrm{u}} \cdot 1^{2}}{2}}{1000 \cdot \frac{2}{\mathrm{~b}} \cdot \frac{1}{\mathrm{ft}}}$

$\mathrm{M}_{\mathrm{u}}=1.7 \quad \mathrm{ft}-\mathrm{kips}$

Using the design proceedures of ACI SP-17(91), Vol. 1, pg.29.

$f_{\mathrm{c}}:=4000 \mathrm{psi} \quad \mathrm{f}_{\mathrm{y}}:=60000 \mathrm{psi}$

$\mathbf{b}:=12$

Width of beam

$\mathbf{h}:=6$

$\mathrm{d}:=\mathrm{h}-3-0.25$

Effective depth. Try \#4 bars

$\mathrm{d}=2.75$

$F:=\frac{d^{2}}{1000} \quad F=0.008$

$\mathrm{K}_{\mathrm{n}}:=\frac{\mathrm{M}_{\mathrm{u}}}{\mathrm{F}} \quad \mathrm{K}_{\mathrm{n}}=224.793$

$\mathrm{a}_{\mathrm{n}}:=4.31$

$A_{s}:=\left(\frac{M_{u}}{d \cdot a_{n}}\right) \cdot i n^{2}$

$A_{s}=0.143 \cdot$ in $^{2}$

Area of steel required, sq. in/ft. CONTROLS.

$\rho_{\min }:=0.0018$

For grade 60 steel, per Ref. 1, sec. 7.12.2.1

$A_{\text {smin }}:=\rho_{\min } \cdot b \cdot h$

$A_{\text {smin }}=0.13$ in $^{2}$

$S_{\max } \leq 18 \cdot$ in

Max spacing per Ref. 1, sec. 7.12.2.2.

$S:=0.2 \cdot \mathrm{in}^{2} \cdot\left(\frac{12}{\mathrm{~A}_{\mathrm{s}}}\right)$

$S=16.733$ 
HNF-2467, Rev.0

KAISER ENGINEERS

HANFORD

Client: Westinghouse Hanford Company

Subject: Intake Air Filter Pad and Cooling Coil

Support Frame Analysis

Location: C-Farm, Tank 241-C-106

Check Flexural Shear

$$
\begin{aligned}
& \mathrm{v}_{\mathrm{u}}:=\mathrm{q}_{\mathrm{u}^{\cdot d}} \\
& \mathrm{v}_{\mathrm{u}}=3.4 \cdot 10^{3} \cdot \mathrm{lb} \\
& \mathrm{b}_{\mathrm{w}}:=12 \text { in } \phi:=0.85 \\
& \mathrm{~V}_{\mathrm{c}}:=\phi \cdot\left(2 \cdot \sqrt{\mathrm{f}_{\mathrm{c}}}\right) \cdot \mathrm{b}_{\mathrm{w}} \cdot \mathrm{d} \\
& \mathrm{v}_{\mathrm{c}}=3.548 \cdot 10^{3} \mathrm{lb}
\end{aligned}
$$

DESIGN ANALYSIS

WO/Job No. ER 4319N-320

Date 9/2/94

Checked 3/17/95

Revised

By

Ok
Talc. No. W320-24-020

Revision 0

Page No. 8 of 10

By JR Booth QQRB

Check Punching Shear

$\mathrm{V}_{\mathrm{u}}:=\frac{\mathrm{U}_{1}}{4}$

Conservative

$\mathrm{V}_{\mathrm{u}}=1.809 \cdot 10^{3} \cdot \mathrm{lb}$

$b_{0}:=4 \cdot\left(7+\frac{d}{2}\right)$

$\mathrm{b}_{\mathrm{o}}=33.5$

$\mathrm{V}_{\mathrm{c}}:=\phi \cdot 4 \cdot \sqrt{4000} \cdot \mathrm{b}_{\mathrm{o}} \cdot \mathrm{d}$

$\mathrm{V}_{\mathrm{c}}=1.981 \cdot 10^{4} \mathrm{lb}$

OK

Provide \#4 bars@ 12" O/C E.W.

54-4300-037 KEH-0037.00 (06/92)

Page B-11 


\section{HANFORD}

Client: Westinghouse Hanford Company

Subject Intake Air Filter Pad and Cooling Coil

Support Frame Analysis

Location: C-Farm, Tank 241-C-106
Calc. No. W320-24-020

Revision 1

Page No. 9 of 10

10. Expansion Anchor Design: Check expansion anchors for seismic load perpendicular to cooling coil frame. Due to fit-up problems in field, the seismic loading is assumed to be resisted by only (2) expansion anchors in each baseplate. WO/Job No. ER4319N-320

Shear per bolt $=E /(4$ legs $) * 2$ bolts per plate

$=1254 \mathrm{lbs} / 8=157 \mathrm{lbs}$ per exp anchor

Torsional shear $=157 \mathrm{Tbs} * 2\left(3.25^{\prime \prime} /\left(6.5^{\prime \prime}\right)\right.$

$$
=157 \mathrm{lbs}
$$

Resultant shear per bolt $=\sqrt{\left(157^{2}+157^{2}\right)}$

$$
=222 \mathrm{lbs}
$$

UpTift $=E\left(4.71^{\prime}\right) / 2\left(3.58^{\prime}\right) 2-D(.85) / 4(2)$

$$
\begin{aligned}
& =1254 \text { lbs }\left(4.71^{\prime}\right) /\left(3.58^{\prime}\right) 4-3203 \text { Tbs }(.85) / 8 \\
& =72 \text { lbs uplift per bolt }
\end{aligned}
$$

Tension due to prying action $=72$ lbs $* 3.25^{\prime \prime} /$ say $l^{\prime \prime}$

$$
=234 \mathrm{lbs} \text { per bolt }
$$

Total tension per bolt $=72 \mathrm{lbs}+234 \mathrm{lbs}=306 \mathrm{lbs}$

Try: 3/8" dia exp anchors w/ 1-5/8" min emb

$$
\begin{aligned}
& \text { Shear allow }=710 \mathrm{lbs} \\
& \text { Tension allow }=875 \mathrm{lbs} \\
& \mathrm{IF}=222 / 710+306 / 875=.66<1.0 \text { OK }
\end{aligned}
$$

11. Check $L 3 \times 3 \times 3 / 8^{\prime \prime}$ angles for use on Cooling Coil Support Frame. See page $A-4$.

\section{Assumptions}

1. The support frame for the Air Inlet Filter will resist the seismic force in the direction parallel to the ducting.

2. The dimensions shown are approximate and are intended to envelope the dimensions of the actual support frame.

a. Check angle for bending. Assume simply supported beam with concentrated load at center. See page $A-4$, Section $A$.

$$
\text { kip }:=1000 \cdot 1 \mathrm{~b} \quad \mathrm{ksi}:=\frac{1000 \cdot 1 \mathrm{~b}}{\mathrm{in}^{2}} \quad \mathrm{D}_{\mathrm{A}}:=\mathrm{D}_{1}+\mathrm{D}_{2}+\mathrm{D}_{3}+\mathrm{D}_{\mathrm{cc}}
$$

$P:=\frac{D_{A}}{2}+\left(20 \cdot \frac{l b}{\mathrm{ft}^{2}}\right) \cdot 2 \cdot f \cdot 4.5 \cdot f t \quad$ Point load acting on angle

$\mathrm{P}=1.178 \cdot \mathrm{kip}$

$L:=54 \cdot$ in

Angle length

$\mathrm{M}:=\frac{\mathrm{P} \cdot \mathrm{L}}{4}$

$M=15.905 \cdot$ kip $\cdot$ in 
DESIGN ANALYSIS

Client: Westinghouse Hanford Company

Subject: Intake Air Filter Pad and Cooling Coil

Support Frame Analysis

Location: C-Farm. Tank 241-C-106

Date 9/2/94

Checked $3 / 7 / 95$

Revised
WO/Job No. ER4319N-320

Revision 0

Page No. 10 of 10

Use method on Ref. 8, pg. 5-311 section 5.1.1. Assume lateral-torsion restraint along length.

$F_{y}:=36 \cdot k s i$

Try: $\quad F_{b}:=0.66 \cdot F_{y} \quad F_{b}=23.76 \cdot \mathrm{ksi}$

$S:=\frac{M}{F_{b}}$

$\mathrm{s}=0.669 \cdot \mathrm{in}^{3}$

Minimum required section modulus

$s_{y}:=0.833 \cdot$ in $^{3}$

Section modulus of $L 3 \times 3 \times 3 / 8$

$b:=3.0 \cdot$ in $\quad t:=\frac{3}{8} \cdot$ in

$\frac{b}{t}=8$

By JR Booth $\& \Omega \beta$

By faw Foush

By

\section{B}

$\frac{65}{\sqrt{36}}=10.833$

$\frac{b}{t} \leq \frac{65}{\sqrt{F_{y}}}$

Therefore, assumption for allowable bending stress is good.

$f_{b}:=\frac{M}{s_{y}} \quad f_{b}=19.094 \cdot k s i \quad$ OK

b. Check angle under compression. See attachmet B.

$\mathrm{KI}:=3.5 \cdot \mathrm{ft}$

Assume simply supported attachments, conservative.

$P_{a}:=10.3 \cdot k i p$

Allowable Axial Load for L $3 \times 3 \times 3 / 8$ angles.

Since the allowable axial load far exceeds the weight of the Cooling Coil compression will not be a problem.

c. Determine allowable tensile load for $L 3 \times 3 \times 3 / 8$ angles.
$\mathrm{A}_{\mathrm{e}}:=0.88 \cdot \mathrm{in}^{2}$
Area of one leg of angle connected to support per Ref. 8, pg.5-310 section 2.c.
$F_{u}:=58 \cdot \mathrm{ksi}$
$\mathrm{P}_{\text {at }}:=0.5 \cdot \mathrm{F}_{\mathrm{u}} \cdot \mathrm{A}_{\mathrm{e}}$
Ref. 8, pg. 5-310 section 2.
$\mathrm{P}_{\text {at }}=25.52 \cdot \mathrm{kip}$
By inspection it can be seen that tensile force in the diagonal member will be far less than the allowable.

12. Design weld adequate to support any load of this design:

$$
\begin{aligned}
& \mathrm{R}_{\mathrm{w}}:=\frac{1}{4} \cdot \mathrm{in} \cdot(0.707 \cdot 0.3 \cdot 70 \cdot \mathrm{ksi}) \\
& \mathrm{R}_{\mathrm{W}}=3.712 \cdot \frac{\mathrm{kip}}{\mathrm{in}}
\end{aligned}
$$

Welding along one leg of angle (MiN) will be suffient to support any load in this design. 
Calc. No. W320-24-020

Revision: 0

Page No. A2 of A5

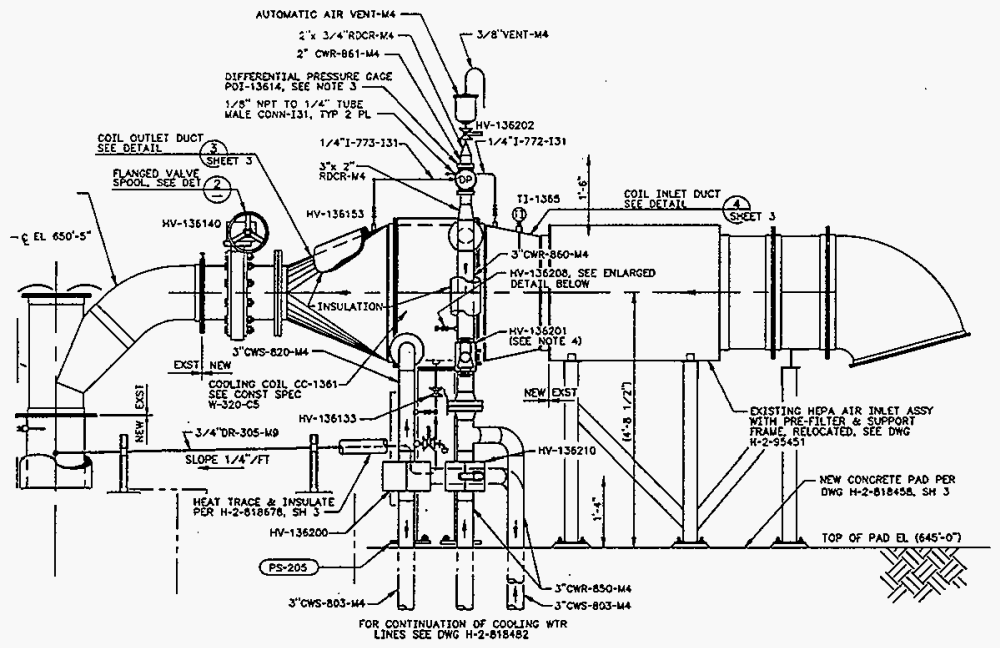

SECTION 8 OF $\mathrm{H}-2-818470, \mathrm{SH}-2$ 


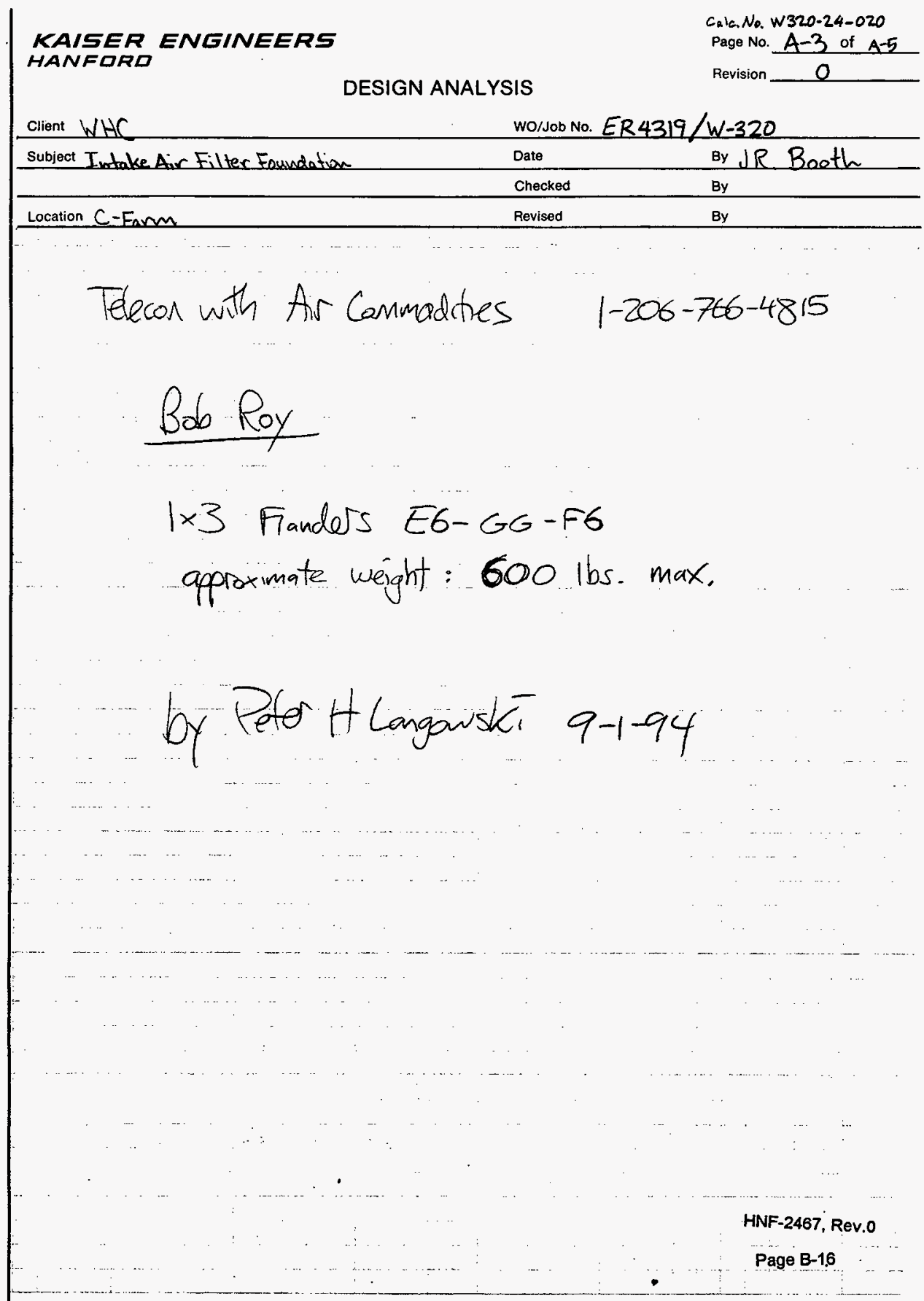




\section{ICF KAISER HANFORD COMPANY}

\begin{tabular}{|c|c|c|c|c|c|c|}
\hline $\begin{array}{l}\text { Ref. Dwg. } \\
\mathrm{H}-2-818473\end{array}$ & $\begin{array}{r}\text { sh. } \\
1\end{array}$ & $\begin{aligned} \text { Rev. } \\
0\end{aligned}$ & $\begin{array}{l}\text { Presoures } \nexists y \\
D \text { NGUYYEN }\end{array}$ & peofle 37296 & $\begin{array}{l}\text { ECN No. } \\
W-320-225\end{array}$ & Page \\
\hline
\end{tabular}
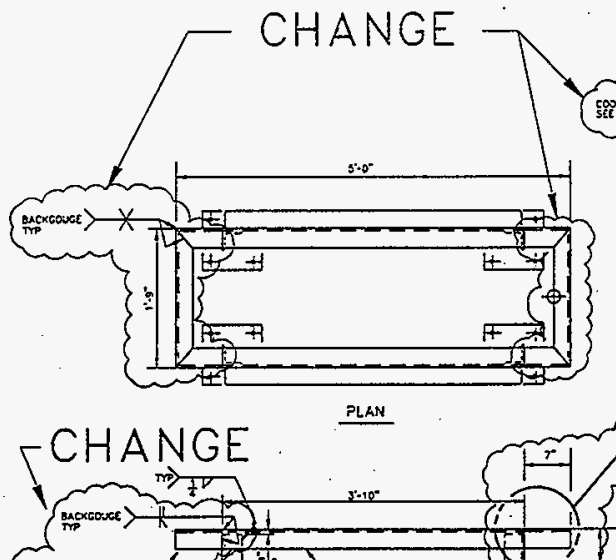

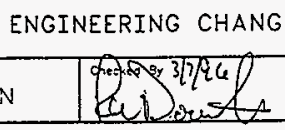

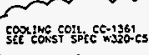
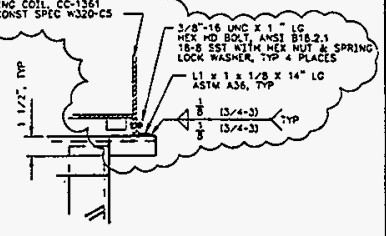

Senef: J"rig:

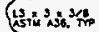
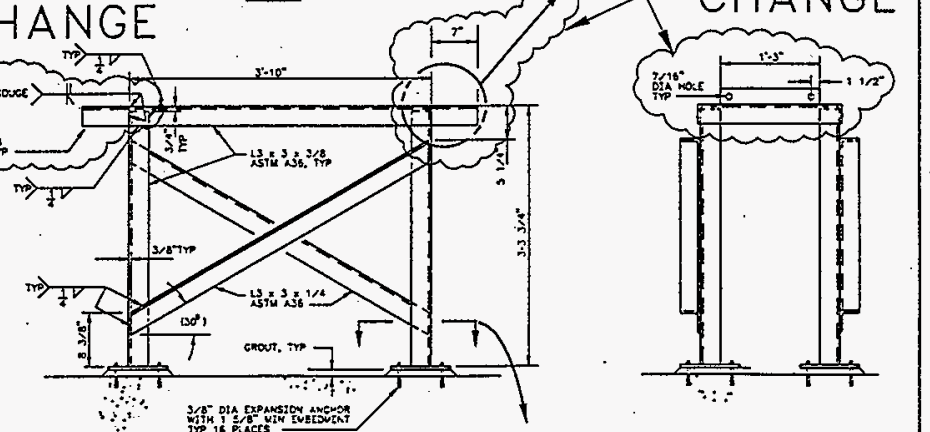
Cä́c. No. W/320-24-020

Revision:

Page No. A5 of A5

HNF-2467, Rev.0

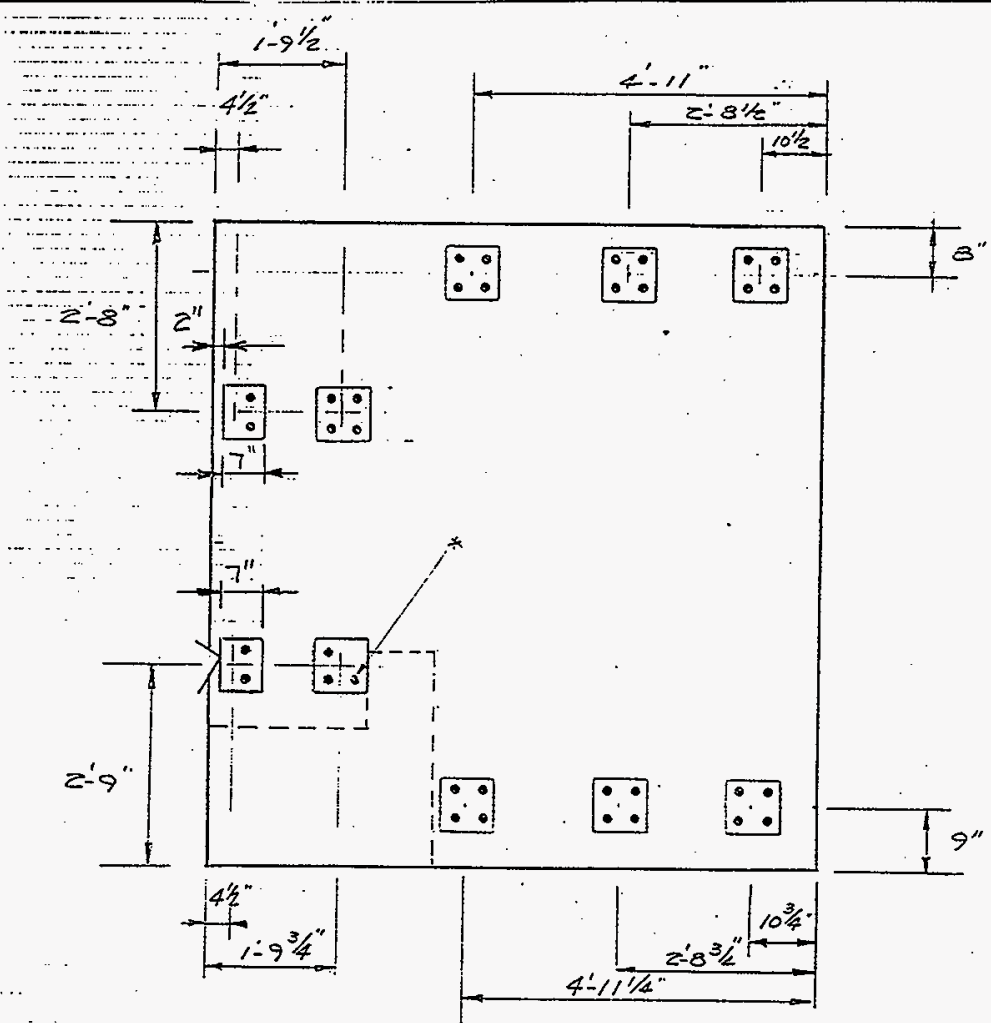

*.THIS ANCLOR WIIC EL SET

AfTER BLOCEOT PUTR JLCK

….............

……............

.....-

........

NITAKE PAD

… ...

:

(..........

\& PLAN

AS- BU/LT

NTS

ラऽ W/O 

Determine the Equivalent $L / r$ for flexural-torsinnal buckling.

$$
\begin{aligned}
& F_{V}=\frac{G J}{A r_{0}^{2}}=\frac{11,200(.0438)}{1.94(2.23)^{2}}=50.8 \mathrm{ksi} \\
& F_{\text {sw }}=\frac{\pi^{2} E}{\left(\frac{L}{r_{w}}\right)^{2}}=\frac{\pi^{2}(29,000)}{\left(\frac{S(12)}{1.58}\right)^{2}}=198.5 \mathrm{ksl}
\end{aligned}
$$

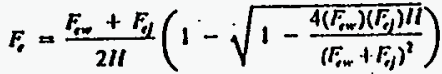

$$
\begin{aligned}
& F_{i}=\frac{198.5+50.8}{2(.627)}\left(1-\sqrt{1-\frac{4(198.5)(50.8)(.627)}{(198.5+50.8)^{2}}}\right)=45.7 \mathrm{ksi} \\
& -\left(\frac{L}{r}\right)_{\text {revir }}=\pi \sqrt{\frac{E}{r_{e}}}=\pi \sqrt{\frac{29,000}{45.7}}=79.1 \\
& \frac{L}{r_{z}}=\frac{5(12)}{.795}=75.5
\end{aligned}
$$

since $79.1>75.5$. Alexural-torsional buckling conitrols.

Determine $F_{\text {. }}$.

$$
C_{r}^{\prime}=\sqrt{\frac{2 \pi^{2} E}{Q F_{\xi}}}=\sqrt{\frac{2 \pi^{7}(29,(000)}{.911(36)}}=132.1 ;
$$

$\frac{U_{r}}{C_{r}^{\prime}}=\frac{79.1}{132.1}=.60$ using Rer. I Thlile $3 \quad c_{*}=.440$

$$
F_{*}=C_{*} Q F_{\mathrm{g}}=.440(.911) .16=14.4 \mathrm{ksi}
$$

Delermine Fr.

$$
F_{\text {st }}=\frac{28,250}{\frac{L}{1}}=\frac{28,250}{\frac{5(12)}{.25}}=117.7 \mathrm{ksi}
$$

since $117.7 \mathrm{ksi}>36 \mathrm{ksi}$, use $r_{-}=\left(0.95-.50 \sqrt{\frac{F_{y}}{F_{i s}}}\right) F_{y} \leq .66 F_{y}$

$$
\begin{gathered}
W 320-24-020 \\
\kappa_{0}=\left(0.95-50 \sqrt{\frac{P_{g}}{116} B-2}\right) 36=24.2 \mathrm{ksi} \\
.66(36)=23.8 \mathrm{ksi}
\end{gathered}
$$

Cleck $b / /$ provisions.

since $b / l>\frac{76}{\sqrt{F}}$, use $\frac{\pi}{7}=.60 Q F_{y}$

$$
r_{\text {s. }}=.60(.911) 36=19.7 \mathrm{ksi}-\text { governs }
$$

Delernine $\pi_{b_{2}}$.

since $b / h>\frac{76}{\sqrt{F}}$ use $\pi_{z}=.60 Q F_{y}=19.7 \mathrm{ksi}$

Solve for the maximum allowable axial load using:

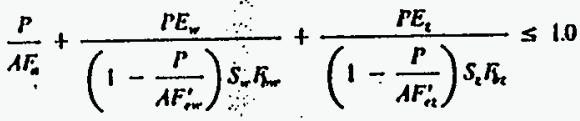

$F_{c m}^{\prime}=103.4 \mathrm{ksi} F_{a t}^{\prime}=26.2 \mathrm{ksi}$ using Ref. 1 Thble 8

$\frac{P}{1.94(14.4)}+\frac{r(1.55)}{\left(1-\frac{P}{1.94(103.4)}\right) 1.71(19.7)}$

$$
+\frac{P(.260)}{\left(1-\frac{P}{1.94(26.2)}\right) .799(19.7)} \leq 1.0
$$

$r=9.6 \mathrm{kijs}$

\section{REFEAFNCES}

1. American Institule of Steel Constructlon, Afamial of Sied Construcfirm, 91t, cd.; (Clicago: AISC, 1989).
HNF-2467, Rev.0

Page B-20 


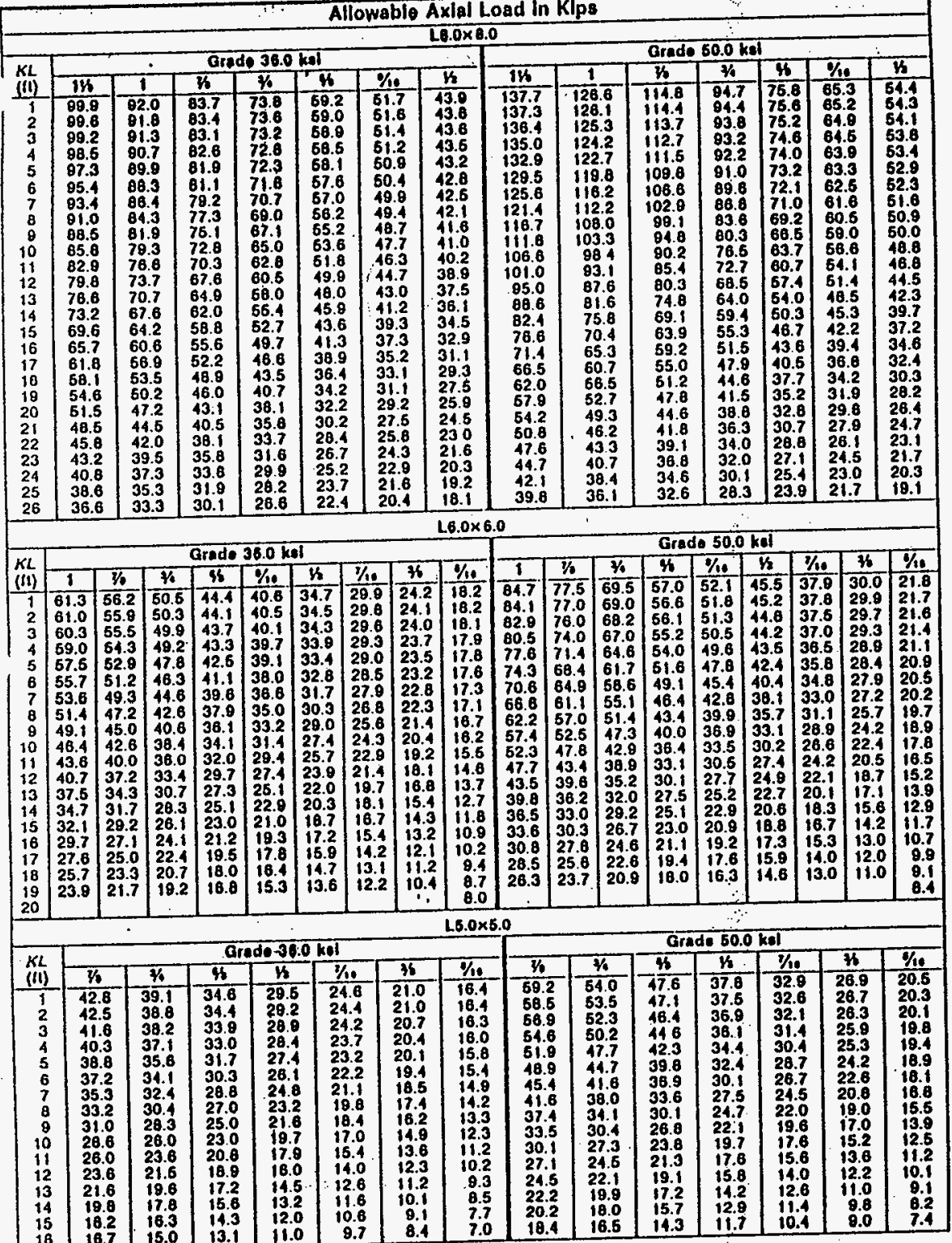




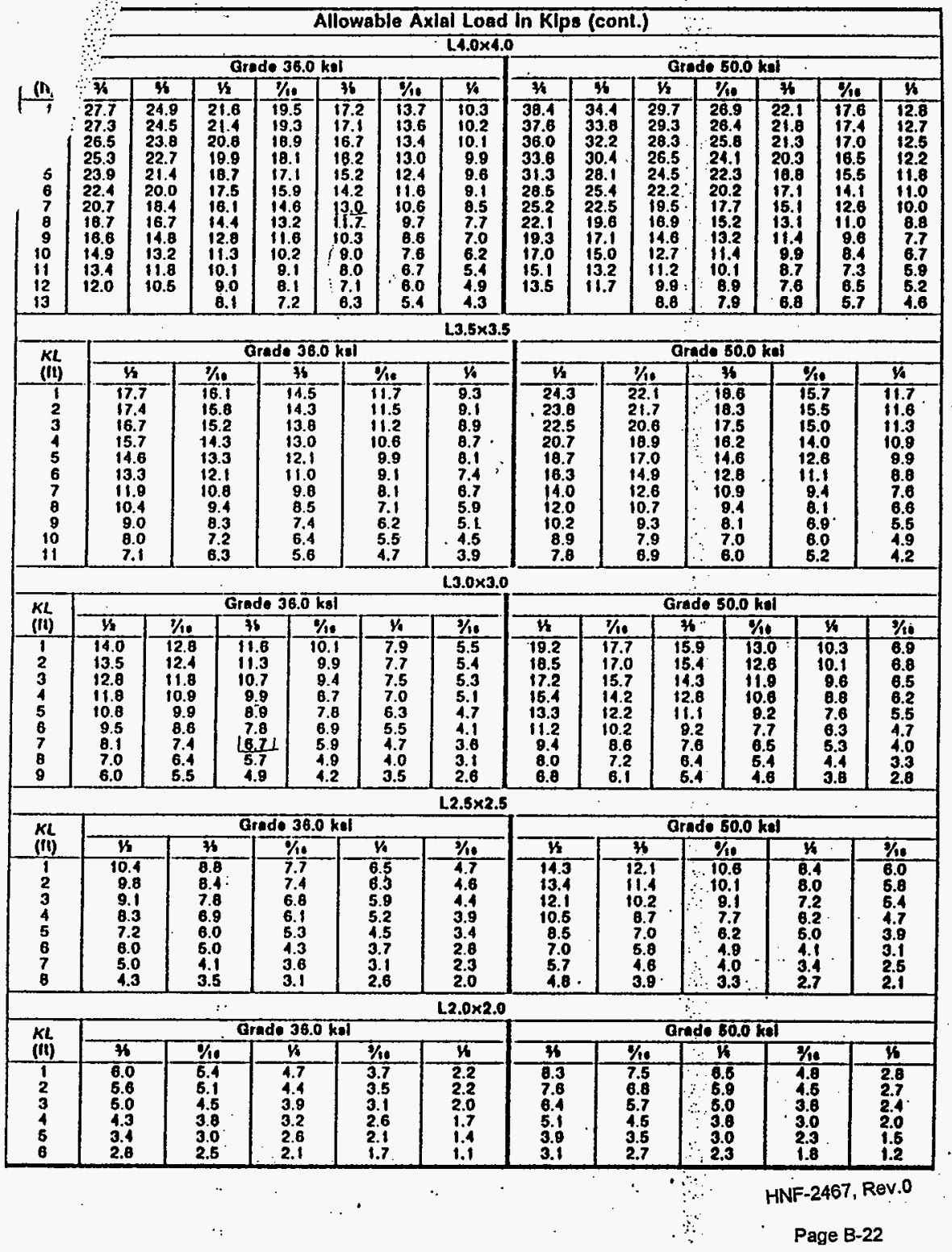


W320-24-021

Misc. Mechanical (HVAC) Equipment Foundation Exhaust Air Cleanup Train Skid 


\section{CALCULATION IDENTIFICATION AND}

INDEX
Page $\mathbf{i}$ of $\mathbf{i} \mathbf{i}$

Date

$07 / 21 / 94$

This sheet shows the status and description of the attached Design Analysis sheets.
Discipline Civil
wo/Job No. W-320/ER-4319
Calculation No. W320-24-021

Project No. \& Name PR0JECT W-320, TANK 241-C-106 WASTE RETRIEVAL

Calculation Item Miscellaneous Mechanical (HVAC) Equipment Foundation Exhaust Air Cleanup Train

These calculations apply to:

Dwg. No. H-2-818458, SHtT, 2

Dwg. No.

other (Study, CDR)

Rev. No.

The status of these calculations is:

[] Prel iminary Calculations

[X] Final Calculations

[ ] Check Calculations (On Calculation Dated)

[ ] Void Calculation (Reason voided)

$\begin{array}{lllll}\text { Incorporated in Final Drawings? } & {[X]} & \text { Yes } & {[] \text { No }} \\ \text { This calculation verified by independent "check" calculations? } & {[]} & \text { Yes } & {[X]} & \text { No }\end{array}$

Original and Revised Calculation Approvals:

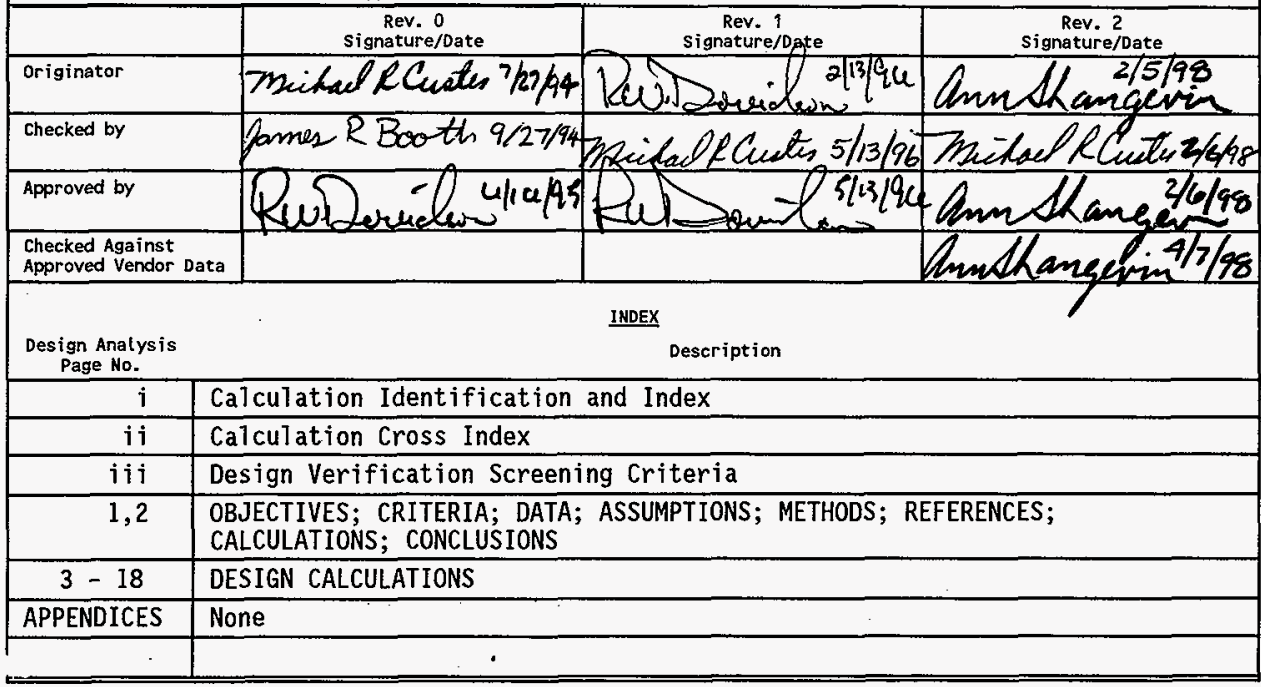

REV 1: ALTERNATE ANCHOR BOLT DESIGN ADDED ON PAGE 15. SEE ECN $W-320-182$, ITEM $2 A$.

SEE ECN W-320-182, ITEM 2A.

Rev $2=$ Add pages 19-22 


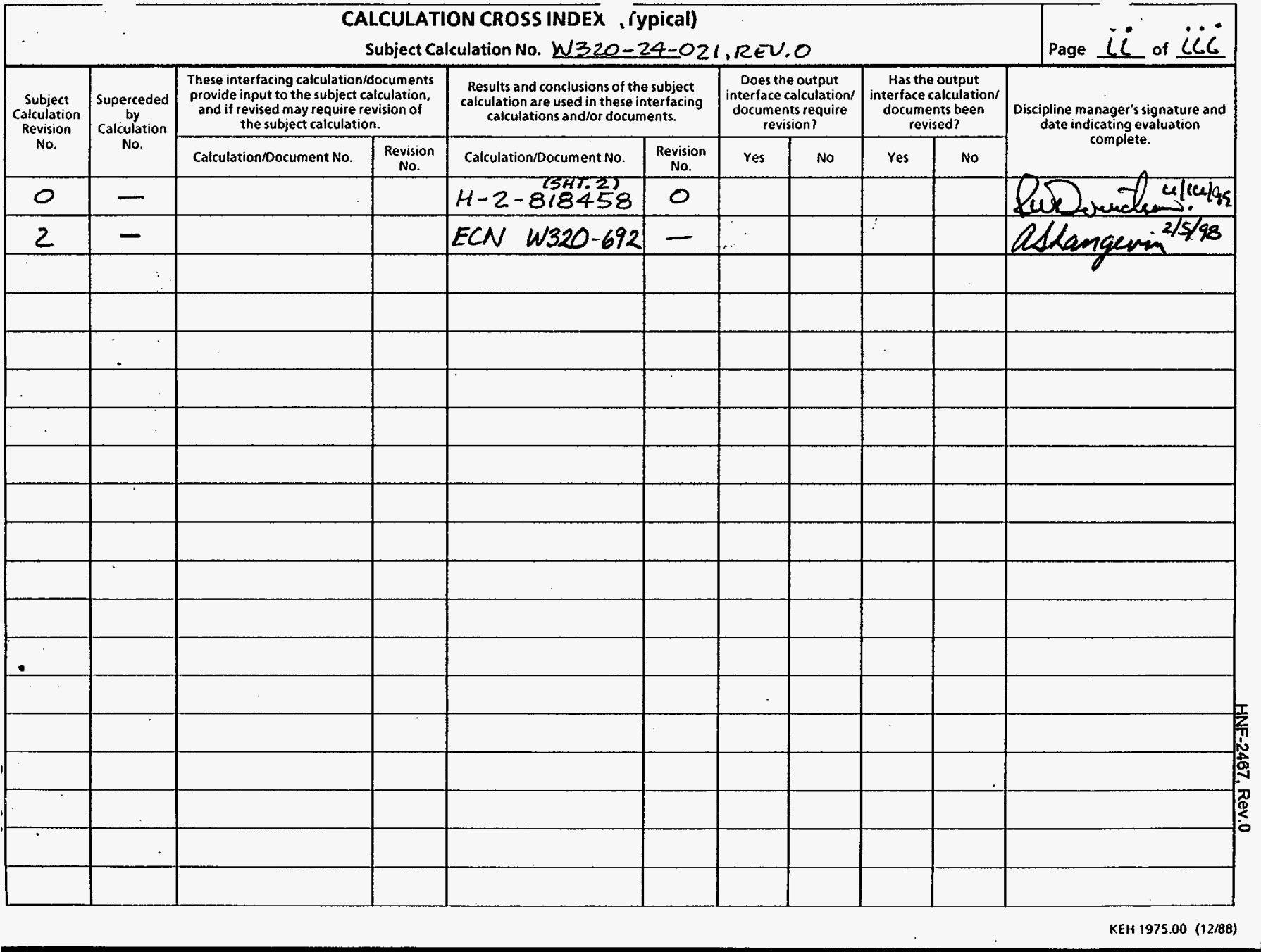




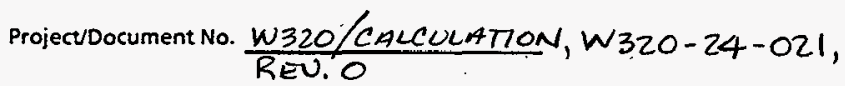

When the design or design change affects hardware, formal design verification must be performed if one or more of the following questions must be answered affirmatively (YES).

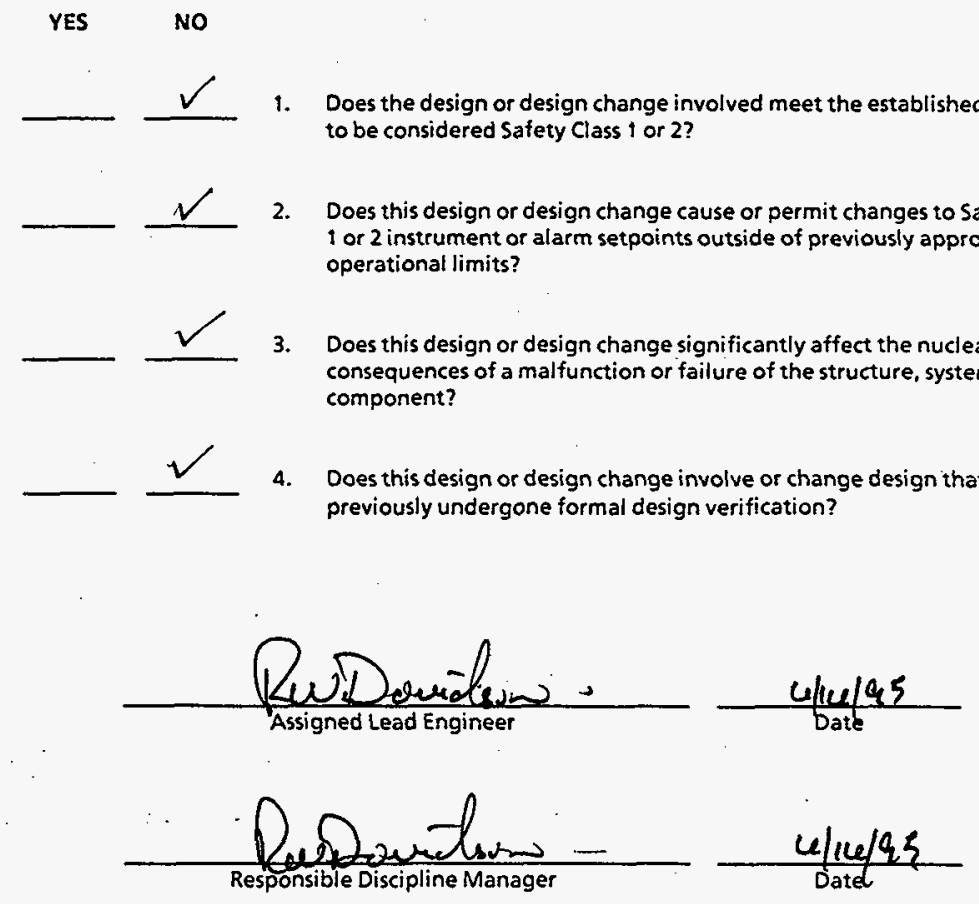

Original Design Package Distribution:

Project Engineer

Chief Design Engineer

Engineering Document Control
Design Change Distribution:

Attach to Engineering Change Notice 


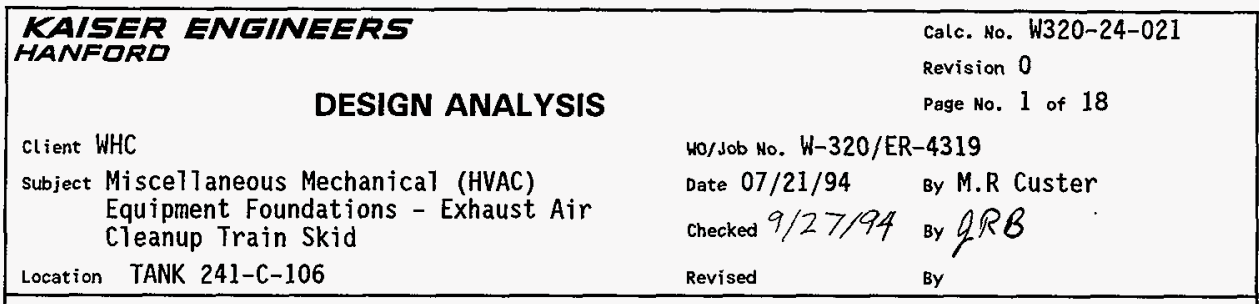

OBJECTIVE:

The objective of this calculation is to provide the detailed design for the Exhaust Air Cleanup Train foundation. The design of the foundation shall be performed in accordance with ACI-318 (Strength Design) and the Uniform Building Code (1991), as required by the project design criteria, SDC-4.4, Rev.11. Since definitive information regarding the weights and geometry of the equipment will not be available, due to long lead time for purchase, conservative estimates of the weights and equipment geometry will be used to perform the analysis and design. The foundation for the Exhaust Air Cleanup Train Skid is located inside the tank farm.

\section{CRITERIA:}

1. DOE ORDER 6430.1A (04/06/89)

2. HANFORD PLANT STANDARDS, STANDARD ARCH./CIVIL DESIGN CRITERIA, SDC-4.1, REV.11

3. FUNCTIONAL DESIGN CRITERIA, WHC-SD-W320-FDC-001, REV.2

4. LETTERS OF INSTRUCTION:

5. ASCE 7-88

DATA:

1. The equipment and foundations for the equipment in this calculation are Safety Class 3 per Reference 4.

ASSUMPTIONS: Weights and equipment geometry will be conservatively assumed and will be verified on receipt and review of the vendor drawings.

METHODS: Hand calculations

REFERENCES:

1. ACI 318-91, Building Code Requirements for Reinforced Concrete.

2. Uniform Building Code (UBC), (1991 Edition).

3. HANFORD PLANT STANDARDS, STANDARD ARCH./ CIVIL DESIGN CRITERIA, SDC-4.1, REV.11.

4. WHC-SD-WM-SEL-033, REV.0-C, Interm Safety Equipment List for 241-C-106 WASTE RETRIEVAL.

5. Foundation Analysis and Design, Joesph E. Bowles (1st Edition).

6. ASCE 7-88 (American Society of Civil Engineers Standard), Minimum Design Loads for Buildings and 0ther Structures. 
KAISER ENGINEERS

HANFARD

\section{DESIGN ANALYSIS}

client WHC

subject Misce11aneous Mechanical (HVAC)

Equipment Foundations - Exhaust Air

Cleanup Train Skid

Location TANK 241-C-106 calc. No. W320-24-021

Revision 0

Page Ho. 2 of 18

Wo/Job No. W-320/ER-4319

Date $07 / 21 / 94$ By M.R Custer

checked $9 / 27 / 94$ вy $20 R$

Revised

By

CALCULATIONS:

Refer to Design Calculation section

CONCLUSIONS :

The foundation for the Exhaust Skid is designed as a slab on grade. The loads developed in the calculation, while conservative are minimal, based on the physical area required by the piping and equipment configuration. The design satisfies the design requirements for the foundation. 


\section{KAISER ENGINEERS}

HANFERE

DESIGN ANALYSIS

client WHC

subject Miscellaneous Mechanical (HVAC)

Equipment Foundations - Exhaust Air

Cleanup Train Skid

Location TANK 241-C-106
Calc. No. W320-24-021

Revision 0

Page No. 3 of 18

Wo/Job No. W-320/ER-4319

Date $07 / 21 / 94$ By M.R Custer MRC

checked $9 / 27 / 94$ вy $\triangle R B$

Revised

By

\section{DESIGN CALCULATIONS:}

The design and analysis of the Exhaust skid foundation consists of developing conservative estimates for the weights of the major equipment, determine relative locations for each equipment on the foundation and design of the concrete foundation to support the load configuration. All equipment supported on the foundation is Safety Class 3 per Reference 4, therefore the design of the foundation is Safety CTass 3 .

\section{DESIGN REQUIREMENTS:}

- Allowable soil pressure for foundation design, $2000 \mathrm{lb} / \mathrm{sq} . \mathrm{ft}$. (Reference 2)

- Coefficient of sliding $=0.25$

(Reference 2)

- Minimum footing dimensions, (Reference 2)

- Thickness of foundation wall, 6"

- Width of footing, 12 "

- Thickness of footing, 6" (Above the reinforcement)

- Depth of footing below undisturbed ground, 12"

- Minimum slab thickness (plain concrete), 3 1/2"

- Snow/Roof Live Load, 20 lbs/sq.ft.

(Reference 3)

- Wind Load, (Reference 3)

- Basic wind speed, $70 \mathrm{mph}$

- Importance Factor, 1.07

- Exposure Category, C 
HNF-2467, Rev.0

\section{KAISER ENGINEERS}

HANFORD

DESIGN ANALYSIS

client WHC

subject Miscellaneous Mechanical (HVAC)

Equipment Foundations - Exhaust Air

Cleanup Train Skid
Calc. No. W320-24-021

Revision 0

Page No. 4 of 18

wo/Job No. W-320/ER-4319

Date $07 / 21 / 94$ By M.R Custer MKC

checked $9 / 27 / 94$ вy $\& R B$

Revised

By

DESIGN LOADS:

DEAD LOADS

- Mechanical equipment (i.e the exhaust fan, stack, HVAC duct/ filters, duct supports, electrical panels and structural skid. Estimated weights for each equipment are:

\section{EQUIPMENT}

a) Exhaust Fan

b) Exhaust Stack

c) (3) Elect.Panels

d) HVAC Filter Assembly

e) Equipment Container and Structural framing

f) Structural Skid

Tota1 Equipment Weight =
WEIGHT

$800 \mathrm{lbs}$

800 1bs

1200 lbs

3200 lbs

2800 lbs

$100001 \mathrm{bs}$

$188001 \mathrm{bs}$

- Dead Load - Foundation (assume 12 "thick slab):

Wt. $s l a b=7.5 \mathrm{ft} \times 27.0 \mathrm{ft} \times 1.0 \mathrm{ft} \times 150 \mathrm{Tbs} / \mathrm{ft}^{3}=$

Total Concrete Weight $=30375$ 1bs $=30.4 \mathrm{Kips}$

\section{EQUIPMENT LIVE LOAD:}

- Equipment Live Load, LLr = Equip. Live Load $\times 1.5$

(Reference 2)

The equipment live load for this design is considered negligible due to the extremely, small magnitude of the loads transmitted by the rotating equipment

(fan unit). An accepted, simplified approach to determine adequacy of a foundation is a comparison of the masses (mass ratio). The mass of the foundation should be 3 to 5 times the wejght of the rotating equipment. For this case the ratio is: (Reference: 5 , page 601)

Mass Ratio $=30.4 / 4.0$ (equipment contributing) $=7.6$ (conservative) 
HNF-2467, Rev.0

\section{KA/SER ENGINEERS} HANFERE

\section{DESIGN ANALYSIS}

Client WHC

subject Miscellaneous Mechanical (HVAC)

Equipment Foundations - Exhaust Air

Cleanup Train Skid
Calc. No. W320-24-02I

Revision 0

Page No. 5 of 18

wo/Job No. W-320/ER-4319

Date 07/21/94 By M.R Custer MRC

checked $9 / 27 / 94$ вy gRB

Location TANK 241-C-106

Revised

By

\section{WIND LOAD:}

- Determine wind load (general):

$$
\begin{aligned}
& q z=0.00256 \mathrm{Kz}(\mathrm{IV}) \text {, where } \\
& \mathrm{Kz}=0.80 \text { (Exposure } \mathrm{C} \text { ) } \\
& I=1.07 \\
& V=70 \mathrm{mph} \\
& q z=0.00256(0.80)[(1.07) 70]^{2}=11.51 \mathrm{~b} / \mathrm{ft}^{2} \\
& F=q z \text { Gh Cf Af, where: } \\
& \mathrm{Gh}=1.32 \\
& C f=2.0 \\
& \text { (Reference 6) } \\
& \text { (Reference 3) } \\
& F=11.5(1.32) \cdot \underset{2}{(2.0)} \mathrm{Af} \text {, } \\
& F_{W}=30.47 \mathrm{bs} / \mathrm{ft} \times \mathrm{Af} \\
& F W=30.4 \mathrm{lbs} / \mathrm{ft}^{2}(4.0 \mathrm{ft} \times 26.0 \mathrm{ft})=3162 \mathrm{lbs}=3.2 \mathrm{k}
\end{aligned}
$$

SNOW LOAD: $20 \mathrm{lbs} / \mathrm{sf}$. ft.

(Reference 3)

- Equipment:

$$
\begin{aligned}
& S=6.5 \mathrm{ft} \times 26.0 \mathrm{ft} \times 20 \mathrm{lbs} / \mathrm{ft}^{2}= \\
& S=3380 \mathrm{lbS}=3.4 \mathrm{k}
\end{aligned}
$$

- Foundation:

$S=7.5 \mathrm{ft} \times 27.0 \mathrm{ft} \times 201 \mathrm{bs} / \mathrm{ft}^{2}=40501 \mathrm{bs}$

$S=4050 \mathrm{lbs}=4.1 \cdot \mathrm{k}$

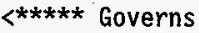


HNF-2467, Rev.0

KA/SER ENGINEERS

HANFDRD

DESIGN ANALYSIS

Client WHC

subject Miscellaneous Mechanical (HVAC)

Equipment Foundations - Exhaust Air

Cleanup Train Skid

Calc. Ho. W320-24-021

Revision 0

Page No. 6 of 18

Wo/Job No. W-320/ER-4319

Date $07 / 21 / 94$ вy M.R Custer MRC

checked $9 / 27 / 94$ вy $9 R B$

Location TANK 241-C-106

Revised

By

EARTHOUAKE LOADS:

- Lateral Seismic Force, Fp = ZICp Wp, where:

(Reference 2)

$Z=0.20$

(Reference 2)

$I=1.25$

(Reference 3)

$C p=.75 \times 2=1.5$

(Reference 3)

$F p=(0.20)(1.25)(1.5) W p=0.375 W p$

$E n-s=V_{n-s}(100 \% \max$.

(Reference 2)

$E$ e-w $=V e-w(30 \% \max$.

(Reference 2)

$E n-s=V n-s=0.375 W p=0.375(18800)=70507 b s=7.1 \mathrm{Kips}$

$E$ e-w $=V_{n-s}=0.375 \mathrm{Wp}(0.3)=0.375(18800)(0.3)=2115 \mathrm{lbs}=2.1 \mathrm{kips}$

$E(S R S S)=\left[7.1^{2}+2.1^{2}\right] \stackrel{1 / 2}{=}$

(Reference $2 \& 3$ )

$E$ (SRSS) $=7.4$ Kips (Apply force in direction providing maximum load conditions)

- Distribution of seismic forces by component:

\section{LOAD IDENTIFIER}

a) Exhaust Fan

b) Exhaust Stack

c) (3) Elect. Panels

d) HVAC Filter Assembly

e) Equip. Container

f) Structural Skid

TOTAL WEIGHT =
VERTICAL COMP * RATIO

$800(7400 / 18800)$

800

1200

3200

2800

10000
HORIZ. COMPONENT

315 lbs

315 lbs

472 lbs

$1260 \mathrm{lbs}$

1102 Ibs

3936 lbs 
DESIGN ANALYSIS

Client WHC

subject Miscellaneous Mechanical (HVAC)

Equipment Foundations - Exhaust Air

Cleanup Train Skid
Calc. Ho. W320-24-021

Revision 0

Page No. 7 of 18

Wo/Job No. W-320/ER-4319

Date $07 / 21 / 94$ By M.R Custer

checked $9 / 27 / 94$ вy $\& R B$

Revised

By

LOCATE THE CENTER OF GRAVITY/CENTER OF MASS FOR THE EQUIPMENT/COMPONENTS:

Component Description

Weight, Ibs

[X-distance,

Z-Distance, H-Distance, (ft.)

a) Exhaust Fan

$800 \mathrm{lbs}$

$23.5 \mathrm{ft}$

$3.5 \mathrm{ft}$

$3.0 \mathrm{ft}$

b) Exhaust Stack

800 lbs

$22.5 \mathrm{ft}$

$4.0 \mathrm{ft}$

$11.0 \mathrm{ft}$

c) (3) Elect. Panels

$1200 \mathrm{lbs}$

$16.5 \mathrm{ft}$

$4.5 \mathrm{ft}$

$4.0 \mathrm{ft}$

d) HVAC Filter Assembly

3200 lbs

$13.5 \mathrm{ft}$

$3.5 \mathrm{ft}$

$4.5 \mathrm{ft}$

e) Equip. Container

2800 1bs

$13.5 \mathrm{ft}$

$3.8 \mathrm{ft}$

$5.0 \mathrm{ft}$

f) Structural Skid

$3.8 \mathrm{ft}$

$0.5 \mathrm{ft}$

Reference points for the $x, z$, and $h$ distances are as follows:

1) $x$-distance, dimension from the west edge of the slab to the approximate center of mass of the component in the east-west direction.

2) z-distance, dimension from the south edge of the slab to the approximate center of mass of the component in the north-south direction.

3) h-distance, dimension from the top of the slab to the approximate center of mass of the component in the vertical direction.

CENTER OF GRAVITY: (Ref. sketch, Figure 1, page 16)

- Center Gravity, East-West direction

$$
\begin{aligned}
& \bar{x}=\Sigma P(x) / \Sigma P=272600 \mathrm{ft}-1 b / 188001 \mathrm{bs} \\
& \bar{x}=14.5 \mathrm{ft}
\end{aligned}
$$

- Center of Gravity, North-South direction

$$
\begin{aligned}
& \bar{z}=\Sigma P(z) / \Sigma P=71240 \mathrm{ft}-1 \mathrm{bs} / 18800 \mathrm{Tbs} \\
& \bar{z}=3.8 \mathrm{ft}
\end{aligned}
$$




\section{DESIGN ANALYSIS}

client WHC

subject Miscellaneous Mechanical (HVAC)

Equipment Foundations - Exhaust Air

Cleanup Train Skid

Calc. No. W320-24-021

Revision 0

Page No. 8 of 18

wo/Job No. W-320/ER-4319

Date $07 / 21 / 94 \quad$ By M.R Custer MRC

checked $9 / 27 / 94$ sy $q R B$

Revised

By

Location TANK 241-C-106

- Eccentricity $(\bar{\hbar})$ :

$$
\begin{aligned}
& \bar{h}=\Sigma P(h) / \Sigma P=19446 \mathrm{~Tb}-\mathrm{ft} / 7400 \mathrm{Tbs} \\
& \bar{h}=2.6 \mathrm{ft}, \text { (Center of mass above the top of the foundation) }
\end{aligned}
$$

DETERMINE GOVERNING LOAD COMBINATION: (Equipment support design)

- Load Combinations:
1) $U=D+L+(L L r$ or $S)$
2) $U=D+L+W$ or $E$
3) $\mathrm{U}=\mathrm{D}+\mathrm{L}+\mathrm{W}+\mathrm{S} / 2$
4) $U=D+L+S+W / 2$
5) $U=D+L+S+E$

Abbreviations:

(Reference 2)

- The evaluation of each load combination is required to determine the maximum reactions for each equipment/ component for design of the supports:

- $\mathrm{L}$ is constant; LLr $=0.0$

- BOLD - designates load combination governing support design

ITEM: $\quad D \quad W \quad S \quad E$ Load Combination:
a)
$0.8 \quad 0.0$
0.0
0.3
1)
2).
3)
0.80
1.10
0.80
4)
5)
b)
$0.8 \quad 0.0$
0.0
0.3
0.08
1.10
0.80
0.80
1.10
c)
$\begin{array}{lll}1.2 & 0.0 & 0.0\end{array}$
0.5
1.20
$1.70 \quad 1.20$
1.20
1.70
d)
$\begin{array}{llll}3.2 & 0.0 & 0.0 & 1.3\end{array}$
3.20
4.50
3.20
3.20
4.50
e)
$\begin{array}{llll}2.8 & 3.2 & 3.4 & 1.1\end{array}$
6.20
6.00
7.70
7.80
7.30
f)
$10.0 \quad 0.0$
$0.0 \quad 3.9$
10.0
$13.9 \quad 10.0$
10.0
13.9 


\section{KA/SER ENGINEERS}

HANFDRD

\section{DESIGN ANALYSIS}

client WHC

subject Miscellaneous Mechanical (HVAC)

Equipment Foundations - Exhaust Air

Cleanup Train Skid
Calc. No. W320-24-021

Revision 0

Page No. 9 of 18 wo/sob No. W-320/ER-4319

Date $07 / 21 / 94$ By M.R Custer MiC checked $9 / 27 / 94$ вy $A R B$

\section{DETERMINE THE GOVERNING LOAD COMBINATION: (Foundation design)}

- Load Combinations:

$D=$ DEAD LOAD, $L=$ LIVE LOAD, $W=$ WIND LOAD, $E=$ EARTHQUAKE LOAD

1) $U=1.4 \mathrm{D}+1.7 \mathrm{~L}$ (Minimum design requirements)

2) $U=0.75(1.4 D+1.7 L+1.7 W)$

3) $U=0.90+1.3 \mathrm{~W}$

In addition, if earthquake loads are included in the design, load combinations of section 2609 (c) (2), (Reference 3) apply, except (1.1E) is substituted for (W), as follows:

4) $U=0.75(1.4 \mathrm{D}+1.7 \mathrm{~L}+1.87 \mathrm{E})$

5) $U=0.9 D+1.43 E$

also for earthquake loading, use the following:

6) $U=1.4(D+L+E)$

7) $U=0.9 D+1-1.4 E$

- Evaluate Load Combinations:

1) $\quad U=1.4(18.8)+1.7(4.1)$

$=33.3 \mathrm{kips}$

2) $U=0.75[1.4(18.8)+1.7(4.1)+1.7(3.2) 2.5 / 6.5]=26.5$ kips

3) $\quad U=0.9(18.8)+1.3[(3.2)(2.5) / 6.5]$

$=18.5 \mathrm{kips}$

4) $\quad U=0.75[1.4(18.8)+1.7(4.1)+1.87(7.4)(2.5) / 6.5]=28.9$ kips

5) $U=0.9(18.8)+[1.43(7.4)(2.5) / 6.5]$

$=21.0 \mathrm{kips}$

6) $U=1.4(18.8+4.1+[(7.4)(2.5) / 6.5)]$

$=36.1$ kips

7) $\quad U=0.9(18.8)+/-1.4[(7.4)(2.5) / 6.5]$

$=20.9$ kips

Page C-12 


\section{KAISER ENGINEERS}

HANFORD

\section{DESIGN ANALYSIS}

Client WHC

subject Miscellaneous Mechanical (HVAC)

Equipment Foundations - Exhaust Air

Cleanup Train Skid
Calc. No. W320-24-021

Revision 0

Page No. 10 of 18

wo/sob No. W-320/ER-4319

Date 07/21/94 sy M.R Custer MRC

checked $9 / 27 / 94$ ву $Q R B$

Location TANK 241-C-106

Revised

By

\section{FOUNDATION DESIGN:}

The foundation required is a mat type on grade. The size of foundation has been predetermined by the physical layout required for the equipment and components.

The foundation dimensions are: $7.5 \mathrm{ft} \times 27.0 \mathrm{ft}$, estimated 12 " thick

(Reference Figures $1,2 \& 3$ for loads and locations)

- Check foundation size: (Reference sketch, Figures 2,3 page 17)

$$
\begin{aligned}
& \text { ge (effective) }=2 \mathrm{k} / \mathrm{ft}-12 / 12(150 \mathrm{lb} / \mathrm{ft})(1 \mathrm{k} / 1000 \mathrm{lb})=1.85 \mathrm{k} / \mathrm{ft} \\
& \text { A req }=(D . L+L . L) / q e=(22.8) / 1.85 \mathrm{k} / \mathrm{ft}^{2}
\end{aligned}
$$

$$
\text { A req }=12 \mathrm{ft}^{2}<169 \mathrm{ft}^{2} \ldots \ldots \text {. . K }
$$

- Check foundation stability:

Safety Factor (Overturning):

$S . F(o t)=[3.75 \mathrm{ft}(30.4 \mathrm{k})+3.75 \mathrm{ft}(18.8 \mathrm{k})] / 2.6 \mathrm{ft}(7.4 \mathrm{k})$

$S . F(o t)=9.6>1.5 \ldots .0 \mathrm{~K}$

Safety Factor (S1iding):

S.F $($ sliding $)=[.25(30.4+18.8)] / 7.4=1.7>1.5 \ldots .0 \mathrm{~K}$ (Loads were doubTed)

- Determine the center of gravity of the loads relative to the kern:

e $(x)$, (length) $=27.0 \mathrm{ft} / 6=4.5 \mathrm{ft}$

e $(z),($ width $)=7.5 \mathrm{ft} / 6=1.3 \mathrm{ft}$

$\bar{x}=14.5 \mathrm{ft}$ and $\bar{z}=3.8 \mathrm{ft}$ (per page 7 ):

$e(x)=14.5-13.5=1.0 \mathrm{ft} .<4.5 / 2=2.25 \mathrm{ft}$.

Load in midd? - $1 / 3$ of $\mathrm{slab}$ )..... OK

$e(z)=3.8 \mathrm{ft} .-3.75 \mathrm{ft} .=0.05 \mathrm{ft}<0.65 \mathrm{ft} .$,

Load in the middle $1 / 3$ of stab..... OK

Use $q=P / A(+/-) M c / I$ approach (two directions) to determine maximum soil bearing $p$ pressures

Page $\mathrm{C}-13$. 


\section{KAISER ENGINEERS \\ HANFRRD}

DESIGN ANALYSIS

client WHC

subject Miscellaneous Mechanical (HVAC)

Equipment Foundations - Exhaust Air

Cleanup Train Skid
Calc. No. W320-24-021

Revision 0

Page No. 11 of 18
wo/Job No. W-320/ER-4319

Date $07 / 21 / 94$ By M.R Custer MaC

checked $9 / 27 / 94$ By $/ 2 B$

Location TANK 241-C-106

Revised

By

- Determine maximum soil bearing pressure:

$$
\begin{aligned}
& M x(e)(n-s)=3.6 \mathrm{ft}(7.1 \mathrm{k})=25.6 \mathrm{k}-\mathrm{ft} \\
& P 1=18.8 \mathrm{k} \text { (Load eccentric with foundation centerline x-direction) } \\
& P 2=4.1 \mathrm{k} \\
& M z(e x)=18.8 \mathrm{k}(1.0 \mathrm{ft})=18.8 \mathrm{k}-\mathrm{ft} \\
& M z(e)(n-s)=3.6 \mathrm{ft}(2.1 \mathrm{k})=7.56 \mathrm{k}-\mathrm{ft}
\end{aligned}
$$

Maximum soil pressures:

$q=P / A(+/-) \sum M x e(z) / I x(+/-) \quad \Sigma M z e(x) / I z$, where:

$$
\begin{aligned}
\mathrm{Ix}= & \mathrm{bd} / 12=\left[7.5(27.0)^{3}\right] / 12=12302 \mathrm{ft}^{4} \\
\mathrm{Iz}= & \mathrm{bd}^{3} / 12=\left[27.0(7.5)^{3}\right] / 12=949 \mathrm{ft}^{4} \\
\mathrm{q}= & (18.8 \mathrm{k}+4.1 \mathrm{k}) / 7.5 \mathrm{ft}(27.0 \mathrm{ft})+/-[(25.6 \mathrm{k}-\mathrm{ft})(0.05 \mathrm{ft})] / 12302 \mathrm{ft} \\
& +/-[(18.8 \mathrm{k}-\mathrm{ft}+7.56 \mathrm{k}-\mathrm{ft})(1.0 \mathrm{ft})] / 949 \mathrm{ft}
\end{aligned}
$$

$q=0.11 \mathrm{k} / \mathrm{ft}^{2}(+/-) \quad 0.0001 \mathrm{k} / \mathrm{ft}^{2}(+/-) .028 \mathrm{k} / \mathrm{ft}, \mathrm{q}(\mathrm{Mx})=0$

$q=(+) 0.11(+) 0.028=+0.138 \mathrm{k} / \mathrm{ft}^{2} \quad$ (maximum)

$q=(+) 0.11(-) 0.028=+0.082 \mathrm{k} / \mathrm{ft}^{2} \quad$ (minimum)

$q$ (maximum) $=0.138 \mathrm{k} / \mathrm{ft}^{2}+0.15$ (conc.) $=0.29 \mathrm{k} / \mathrm{ft}^{2}$

$q(\operatorname{maximum})=0.138 \mathrm{k} / \mathrm{ft}_{2}+0.15$ (conc.) $=0.29 \mathrm{k} / \mathrm{ft} 2$

$q$ (minimum) $=0.082 \mathrm{k} / \mathrm{ft}+0.15$ (conc.) $=0.23 \mathrm{k} / \mathrm{ft}$

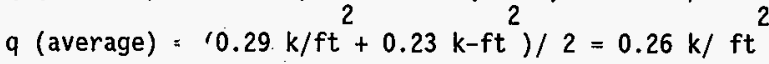

$q\left(\right.$ average) $=0.26 \mathrm{k} / \mathrm{ft}^{2}<\mathrm{q}(\mathrm{a} \text { llow. })^{2}=2.0 \mathrm{k} / \mathrm{ft} \ldots \ldots$ ok 
KAISER ENGINEERS

HANFORD

DESIGN ANALYSIS

client WHC

subject Miscellaneous Mechanical (HVAC)

Equipment Foundations - Exhaust Air

Cleanup Train Skid
Calc. No. W320-24-021

Revision 0

Page No. 12 of 18

Wo/Job No. W-320/ER-4319

Date $07 / 21 / 94$ By M.R Custer MRC

checked $9 / 27 / 94$ вy $/ 2 / B$

Revised

By

- Check slab two way shear:

The critical area for punching shear on the foundation will be at the equipment skid, embedded baseplates, located at each corner of the foundation. The equipment loads are conservatively assumed concentrated at these locations.

Assumed depth of slab, $d=12$ ", then d effective $=(12 "-3 "-0.5)=8.5 "$

bo, (punching shear) $=\left(12^{\prime \prime}+12^{\prime \prime}+8.5^{\prime \prime} / 2\right)=28.25 "=2.35 \mathrm{ft}$

$q u=1.7 \times 0.26 \mathrm{k} / \mathrm{ft}^{2}=0.44 \mathrm{k} / \mathrm{ft}^{2}$

$V u=[(7.5 \mathrm{ft})(27.0 \mathrm{ft})-(2.35 \mathrm{ft})(4)](0.44 \mathrm{k} / \mathrm{ft})=85.0 \mathrm{k}$

$d($ req'd $)=85000 / 0.85(4)(4000)^{1 / 2} 2(28.25)(8.5)=0.82 "$

$d=0.82 "+3 "+0.5 "=4.32^{\prime \prime}<12 " \ldots$ ok

- Check slab one way shear:

$$
\begin{aligned}
& V u=(7.5 \mathrm{ft})(12.8 \mathrm{ft})\left(0.44 \mathrm{k} / \mathrm{ft}^{2}\right)=42.2 \mathrm{k} \\
& d=42200 \mathrm{lbs} /(.85)(2)(4000)^{1 / 2}(90 ")=4.36 " \\
& d=4.36^{\prime \prime}+3 "+0.5 "=7.86^{\prime \prime}<12 \ldots .0 \mathrm{k}
\end{aligned}
$$

- Determine slab flexural reinforcement requirements: (Long Direction)

$M u=(7.5 \mathrm{ft})(6.75 \mathrm{ft})\left(0.44 \mathrm{k} / \mathrm{ft}^{2}\right)(6.75 \mathrm{ft} / 2)=75.2 \mathrm{k}-\mathrm{ft}$

$M u=75.2 \mathrm{k}-\mathrm{ft}=75200 \mathrm{lb}-\mathrm{ft}$

Mu/ $0.90 b d^{2}=12(75200) / 0.90(90)(8.5)^{2}=154, p<p \min =0.0033$ (flexural)

$p(\max )=0.0285, p(\min )=0.0018$ (slab of uniform thickness on grade),

Use $\mathrm{p} \min =\ldots 0018$

As $=0.0018\left(90^{\prime \prime}\right)\left(8.5^{\prime \prime}\right)=1.38 \mathrm{in}^{2}$, As $=0.21 \mathrm{in} / \mathrm{ft}$ (Use width of $6.5 \mathrm{ft}$.)

Use \#5 $012^{\mathrm{n}}$ o.c, As $=0.31 \mathrm{in}^{2} / \mathrm{ft} \ldots$ ok

Page C-15 
HNF-2467, Rev.0

\section{KAISER ENGINEERS}

HANFERO

\section{DESIGN ANALYSIS}

Client WHC

subject Miscellaneous Mechanical (HVAC)

Equipment Foundations - Exhaust Air

CTeanup Train Skid
Calc. No. W320-24-021

Revision 0

Page No. 13 of 18

Ho/Job No. W-320/ER-4319

Date $07 / 21 / 94$ By M.R Custer MKC

checked $9 / 27 / 94$ sy $\mathrm{g} R B$

Revised

By

Location TANK 241-C-106

- Determine slab flexural reinforcement requirements: (Short Direction)

$$
\begin{aligned}
& M u=(3.75 \mathrm{ft})(27 \mathrm{ft})\left(0.44 \mathrm{k} / \mathrm{ft}^{2}\right)(3.75 \mathrm{ft} / \mathrm{2})=83.5 \mathrm{k}-\mathrm{ft} \\
& M u=83500 \mathrm{~Tb}-\mathrm{ft}
\end{aligned}
$$$$
\mathrm{Mu} / 0.90 \mathrm{~b} \mathrm{~d}^{2}=12(83500 \mathrm{lb}-\mathrm{ft}) /(0.90)\left(324^{\prime \prime}\right)(8.5)^{2}=47.6 \text {, }
$$

$p$ design $<p \min =0.0033$ (flexural), Use $p$ min $=0.0018$ (slab of uniform thickness on grade)

$$
\begin{aligned}
& \text { As }=0.0018\left(324^{\prime \prime}\right)\left(8.5^{\prime \prime}\right)=5.0 \mathrm{in}^{2}, A s=0.19 \mathrm{in} / \mathrm{ft} \text { (Use length of } 26.0 \mathrm{ft} \text { ) } \\
& \text { Use \#5012" o.c, As }=0.31 \mathrm{in} / \mathrm{ft} \ldots \text { ok }
\end{aligned}
$$

- Check reinforcement bar development length requirements:

$$
\begin{aligned}
& 1(d b)=[0.04 \mathrm{Ab} f(y)] /\left(f^{\prime} \mathrm{c}\right)^{1 / 2} \quad \text { (Reference 2, section 2612(c), page 469) } \\
& 1(\mathrm{db})=0.04(0.44)(60000) /(4000)^{1 / 2}=16.7^{\prime \prime}(1.0)(0.8)=13.4^{\prime \prime}, \\
& \text { but not }<0.03(\mathrm{db})(\mathrm{fy}) / \mathrm{fc}^{\prime}=0.03(0.75)(60000) / 4000^{1 / 2}=21.3^{\prime \prime} \\
& 1 d \text { (provided) }=12^{\prime \prime}+12^{\prime \prime}-2^{\prime \prime}=22^{\prime \prime}>21.3 \ldots \text { ok }
\end{aligned}
$$

The critical section for both the long and short spans is 24" from the section to the free edge of the slab.

1d (min), per UBC $=12 "<22^{\prime \prime} . \ldots$ ok 
DESIGN ANALYSIS

client WHC

subject Miscel1aneous Mechanical (HVAC)

Equipment Foundations - Exhaust Air

Cleanup Train Skid
Calc. No. W320-24-021

Revision 0

Page No. 14 of 18
wo/Job No. W-320/ER-4319

Date $07 / 21 / 94$ By M.R Custer MLC

checked $9 / 27194$ вy $q R B$

Location TANK 241-C-106

Revised

By

- Determine equipment anchorage requirements: (Loads multiplied by a factor of 2.25 to address the equipment live load for rotating equipment and a conservatism adder.

Equipment

Horizontal Force/ Type

$0.3 k / E$

a) Exhaust Fan

b) Exhaust Stack

$0.30 \mathrm{k} / \mathrm{E}$

$0.20 \mathrm{k} / \mathrm{E}$

c) Elect. Panel/ea.

d) HVAC Filter Assembly

$1.30 \mathrm{k} / \mathrm{E}$

$1.3(2.25) / 4=$

$0.73 k$

$0.20(2.2$

$0.30(2$
$0.17 \mathrm{k}$

$0.30(2$
$0.17 k$

$0.30(2.25)(24)$

$/ 24=0.68 \mathrm{k}$

$0.30(2.25)(96)$

$/(24)=2.7 \mathrm{k}$

$0.20(2.25)(36)$

$/(18)=0.9 \mathrm{k}$

$1.3(2.25)(42)$

$/(24)=5.1 \mathrm{k}$
e) Equipment Container
$3.20 \mathrm{k} / \mathrm{W}$
$3.2(2.25) / 4=$
$1.8 \mathrm{k}$
$3.2(2.25)(30)$
$/(72)=3.0 \mathrm{k}$
f) Structural Skid
$7.4 \mathrm{k} / \mathrm{E}$
$7.4(2.25) / 10=$
$1.7 \mathrm{k}$

\section{LEGEND:}

(E), Indicates earthquake load governs horizontal loading

(W), Indicates wind load governs horizontal loading

\section{NOTES:}

1) Shear loads are assuming a minimum of (4) bolts per each equipment or as indicated.

2) Tension Loads indicated are total reactions per side of each equipment. Anchorage shall be uniformly distributed along each side of each equipment. 
Calc. No. W320-24-021

Revision 6 I

Page No. 15 of 18

Ho/Job No. W-320/ER-4319

client WHC

DESIGN ANALYSIS

subject Miscellaneous Mechanical (HVAC)

Equipment Foundations - Exhaust Air

Cleanup Train Skid

Date $07 / 21 / 94$ By M.R Custer MLC

checked $927 / 94$

By

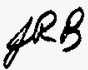

Revised $2\{13 / q 6 e$

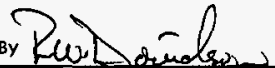

\section{Anchor Bolt requirements:}

The use of anchor bolts with sleeves requires a thickened slab at each anchor bolt location, with a minimum of $12^{\prime \prime}$ beyond the bolt centerlines in all directions.

- Determine size and number of anchor bolts required for the structural skid attachment to the foundation:

\section{BOLT LOADS:}

Tension load $=6.4$ kips $/ 5$ bolts $=1.3 \mathrm{k} /$ anchor bolt Shear load $=1.7 \mathrm{kips} / \mathrm{l}$ bolt $=1.7 \mathrm{k} /$ anchor bolt

Try 3/4" dia. anchor w/ 5" embedment:

Allowable tension load $=4500$ lbs $=4.50 \mathrm{k}$

(Reference 2, Table No. 26-E)

Allowable shear load $=3560 \mathrm{lbs}=3.56 \mathrm{k}$

(Reference 2, Table No. 26-E)

Check unity equation: $\quad 1.3 / 4.50+1.7 / 3.56(<)$ or $(=)$ to 1.0

$0.29+0.48=0.77<1.0 \ldots$. . ok

Provide a minimum of $5-3 / 4^{\prime \prime}$ dia. A 307 anchor bolts $w / 5^{\prime \prime}$ embedment, evenly spaced

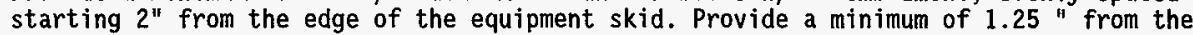
edge of the steel base, typical each side of the skid.

Provide (2), $1 / 4$ " stiffener plates between the flanges of the skid beams at each anchor bolt location.

\section{Alternate Anchor Bolt Design:}

Provide a minimum of (10) $1 / 2^{\prime \prime}$ dia. expansion anchors $w / 3-1 / 2$ " minimum embedment.

Per GC-ANCR-01, Rev 0, Table 21:

Allow. shear $=1,470$ lbs $>7,400$ lbs $/ 10$ bolts $=740$ lbs $/$ bolt

Allow. tension $=2,250$ lbs No Uplift (See Below)

Net uplift $(+)=-18,800 * 0.85 / 10$ bolts $+7,400 * 30 / 78 * 5$ bolts

$$
=-1,589+573=-1016 \text { 1bs down }
$$


KAISER ENGINEERS HANFORD

Client WHC
Calc. No. W320-24-021

Revision

Page No. 16 of 18

SUbject MISC.MECHANICAL (HYAC)EAUIP Date $2 / 27 / 94$ By M.R CUSTRX MLC

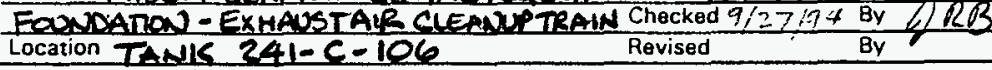
SUMMAFY - FOUNDATION LOADS \& LOCATIONS:
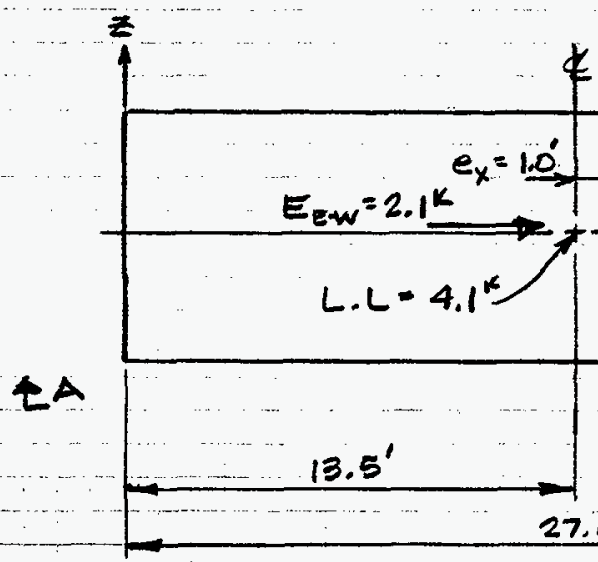

$27,0^{\circ}$

FOUNDATION

FIGUFE

$E_{\text {SRSS }}=7.4^{k} \& \gamma^{\circ} \cdot G$

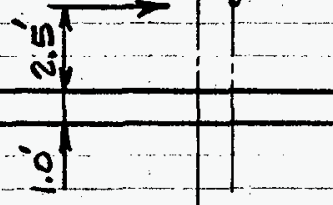

SECTION A-A

Page C-19 
KAISER ENGINEERS HANFORD

DESIGN ANALYSIS

Calc. No. W320-24.021

Revision 0

Page No. $\frac{17 \text { of } 18}{98}$

Client WHC

Wo/Job No. W320/ER-43/9

Subject Misc. MECHANKAC (HVAC)EQule. Date 7/27/94 By M.R. CuSTEX MRE

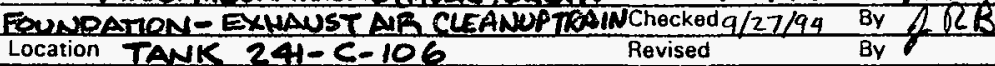

LOADS ON FOUNDATION:

DEAD, LIVE AND SEISMIC LOADS

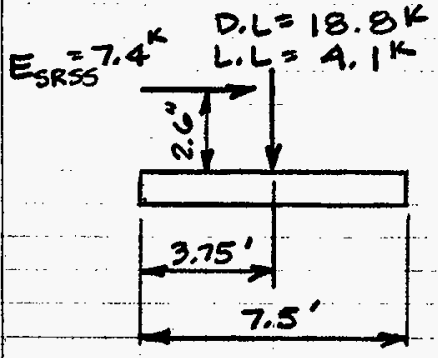

FIGURE 2

\section{DEAD, LIVE AND WIND LOADS}

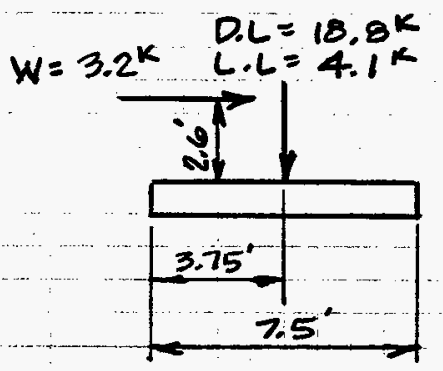

FIGURE 3 


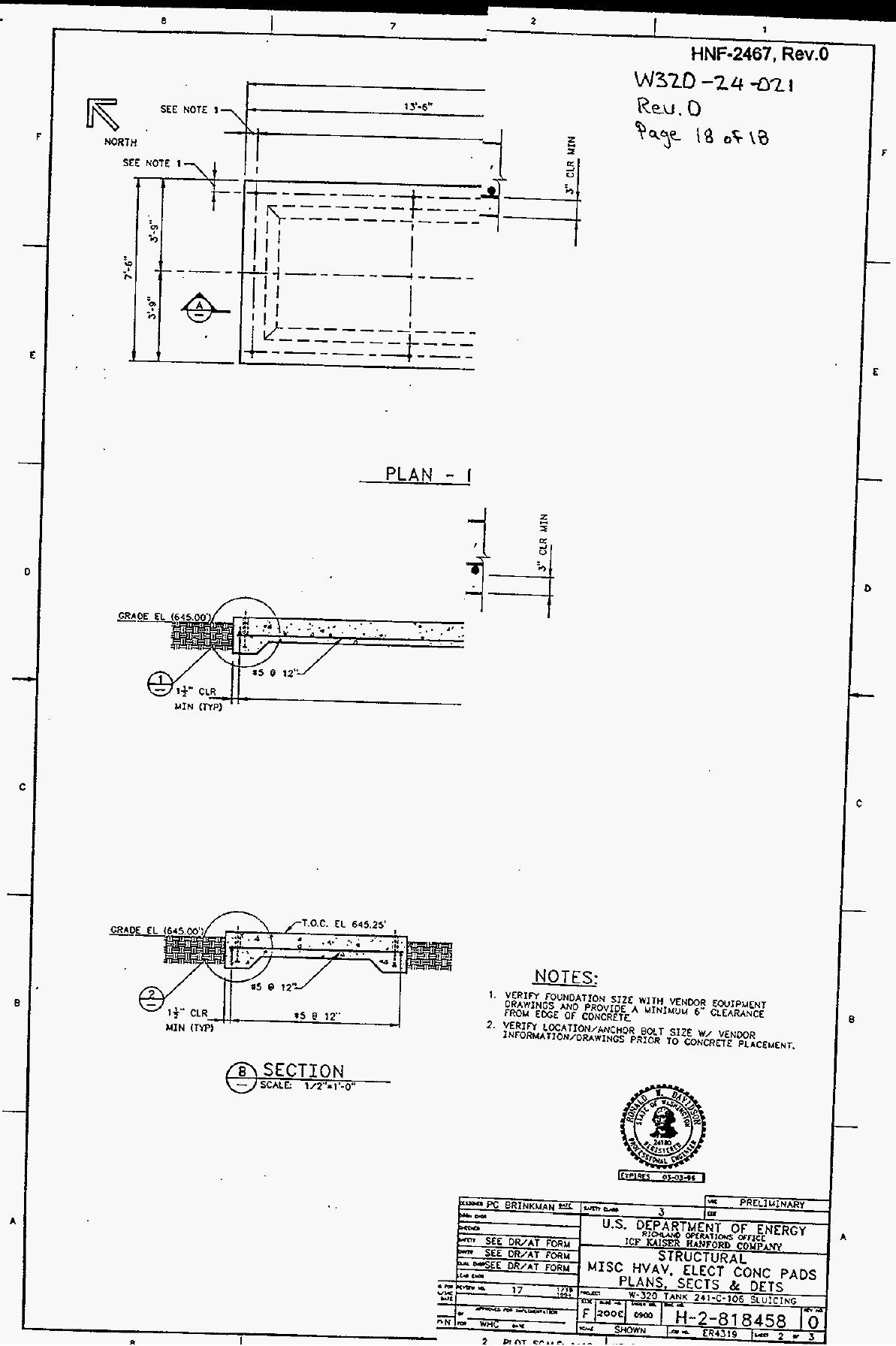


HNF-2467, Rev.0

FLUOR DANIEL NORTHWEST, INC.

Cali. No. W320-24-021

Revision $\frac{2}{19}$ of

Client Numatec

DESIGN ANALYSIS

WO/Job No. $W-320$

Subject Misc Mechanical (HVAC) Date 2/5/98 By CmuAlamgerri Equip Fan - Vacuum Pump Cabinet Checked $2 / 6 / 98$ By

Revised

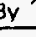

The Vacuum Pump Cabinet Pad was added at the end of the exhaust air cleanup pad by ECN $W-320-692$, due to insufficient space Analyze the foundation and anchorage.

Equipment

Dead load $=600 \mathrm{lb}$. Air Monitor Corp.

$$
\begin{aligned}
& \text { Slab OL }=\left(3.5^{\prime}\right)\left(4.5^{\prime}\right)\left(0.67^{\prime}\right)\left(150 \mu / \mathrm{h} / \mathrm{A}^{3}\right)=1583 \mathrm{lb} \\
& \text { Seismic }=0.375\left(\mathrm{~W}_{\mathrm{p}}\right)=0.375(600)=225 \mu\left(\frac{\mathrm{sec}}{\mathrm{p} .6}\right)
\end{aligned}
$$

Factor for sos $225\left(\frac{7.4}{7.1}\right)=235$ lb (See po)

$$
\begin{aligned}
& \text { Wind }=30.4 \mathrm{lo} / \mathrm{fl}^{2}\left(44^{\prime \prime}\right)\left(285^{\prime \prime}\right) / 144=264 \mathrm{lbg} \\
& \text { Controls } \\
& \text { Snow }=20 \mathrm{los} / \mathrm{fl}^{2}\left(36^{\prime \prime}\right)\left(30^{\prime \prime}\right) / 144=150 \mathrm{lb}
\end{aligned}
$$
Worst case load combination for anchorage

$$
\Delta L+W L
$$

Page C-22 
HNF-2467, Rev.0

FLUOR DANIEL NORTHWEST, INC.

Client Numatec

Subject Misc Mechanical AVAC

Equip

Vacuum Pump Cabinet

Anchorage. -

Neglect $D L$ - conservative

$$
\begin{aligned}
& T=\frac{264 \mathrm{l6}\left(17^{\prime \prime}\right)}{\left(26^{\prime \prime}\right)(2 \text { bolts }} \\
& T=186 \mathrm{l} / \mathrm{bolt} \\
& V=264 \mathrm{lb} / 6 \text { bolt }=44 \mathrm{lb} / \mathrm{bolt} .
\end{aligned}
$$

Bolts are 12 " $\phi$ w 2/4" min embedment

$$
\begin{aligned}
& T_{a}=1358 \mu \\
& V_{a}=1468 \mu \\
& \rightarrow \text { GC-ANCR-OI } \\
& \text { Table } 21 \\
& \frac{T}{T_{a}}+\frac{V}{V a}=\frac{86}{1358}+\frac{44}{1468}=0.09<1.0
\end{aligned}
$$

Loads on anchor bolts are very small $\because$ Anchorage OK

(6) $12 " \phi$ exp anchors with $2 \frac{1}{4}$ "min emp

Page C-23 
HNF-2467, Rev.0

FLUOR DANIEL NORTHWEST, INC.

Laic. No. $\frac{W}{32} 20-24-021$

DESIGN ANALYSIS

Client Numatec

Wo/Job No. W-320

Revision $\frac{2}{21}$

Subject MisC Mechanical (HVAC) Date $215 / 98$ By

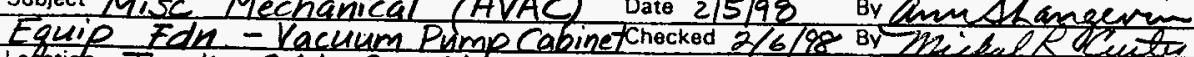
Lobation Tank $241-C-106$

Revised

By

Vacuum Pump Cabinet Slab

Check for overturning

$$
\begin{aligned}
& M=264 \mu\left(\frac{17^{\prime \prime}}{12}\right)=374 \mu-A \\
& P=(600 \mu+1583 \mu) \\
& P=2183 \mu .
\end{aligned}
$$

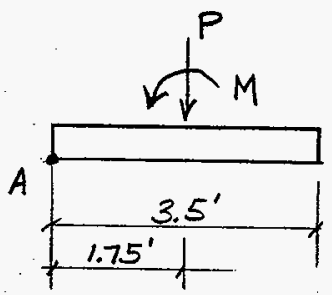

Overturning about pt. $A$ :

$$
\begin{aligned}
& S F=\frac{(0.85) P\left(1.75^{\prime}\right)}{M}=\frac{1855(1.75)}{374}=\frac{8.7>1.5 \text { OK }}{P}=\frac{3.74}{2183}=0.17^{\prime}=2^{\prime \prime} \therefore \frac{\text { Result is within the }}{\text { middle third OK }}
\end{aligned}
$$

Max Soil Pressure:

$$
\begin{aligned}
& p_{\text {max }}=\frac{M}{S}+\frac{P}{A}=\frac{374 \mu-4 f}{(4.5)(3.5)^{2} / 6}+\frac{2183 \mu+150 l 6}{(3.5)(4.5) \text { s include }} \\
&=41+148 \\
& p_{\text {max }}=190 \text { psf soil pressure } \\
& \text { very smalt- oK }
\end{aligned}
$$

Slab Bending Moment:

$$
M_{1}=(190 p s f)(0.5)^{2} / 2
$$

$=-24$ Alb very small

in other direction - (use same pax)

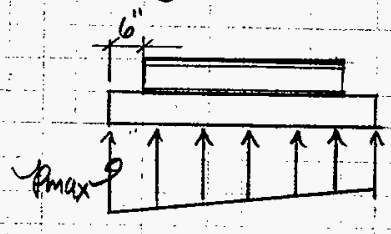

$M_{2}=(190)\left(3^{\prime}\right)^{2} / 12=143 A^{2}-l s$ - also very small

$\rightarrow$ span between supports

Use min steel \#4@12 $\rho=\frac{(0.20)}{12(8)}=0.002>0.0018$ 
W320-24-022

Service Building Foundation

$D-i$ 


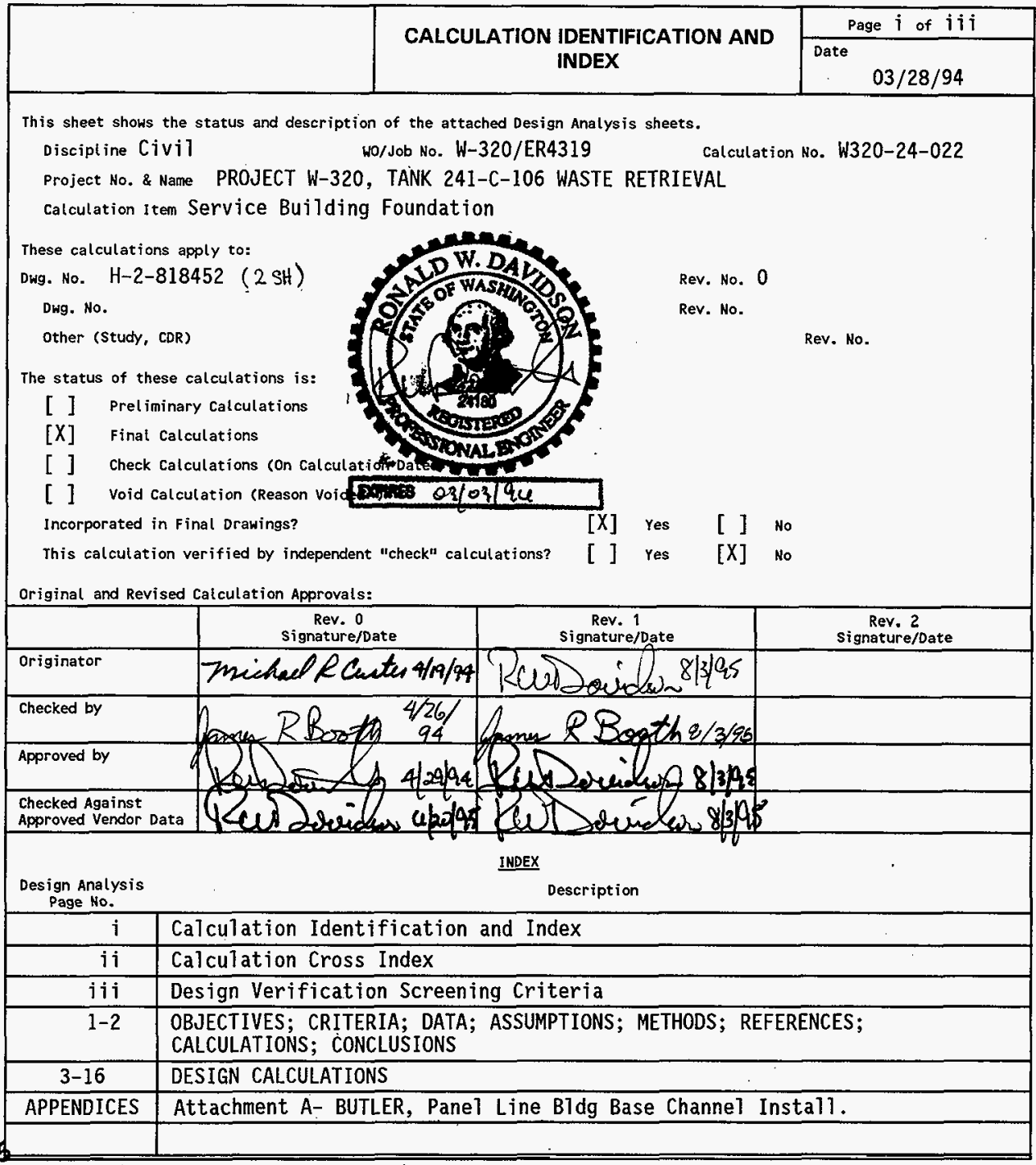

REV 1: CONCLUSION REVISED FOR FDN MODS BASED ON VENDOR APPROVAL DATA FOR BUILDING FRAME INSTALLATION. ATTACHMENT "A" ADDED. REPLACED SH 16 SEE ECN \# W-320-103. 


\section{CALCULATION CROSS INDEX, (Typical)}

Subject Calculation No. $W 320-24-022$

\begin{tabular}{|c|c|c|c|c|c|c|c|c|c|c|}
\hline \multirow{2}{*}{$\begin{array}{l}\text { Subject } \\
\text { Calculation } \\
\text { Revision } \\
\text { No. }\end{array}$} & \multirow{2}{*}{$\begin{array}{l}\text { Superceded } \\
\text { by } \\
\text { Catculation } \\
\text { No. }\end{array}$} & \multicolumn{2}{|c|}{$\begin{array}{l}\text { These interfacing calculation/documents } \\
\text { provide input to the subject calculation, } \\
\text { and if revised may require revision of } \\
\text { the subject calculation. }\end{array}$} & \multicolumn{2}{|c|}{$\begin{array}{l}\text { Results and conclusions of the subject } \\
\text { calculation are used in these interfacing } \\
\text { calculations and/or documents. }\end{array}$} & \multicolumn{2}{|c|}{$\begin{array}{c}\text { Does the output } \\
\text { interface calculation/ } \\
\text { documents require } \\
\text { revision? }\end{array}$} & \multicolumn{2}{|c|}{$\begin{array}{c}\text { Has the output } \\
\text { interface calculation } \\
\text { documents been } \\
\text { revised? }\end{array}$} & \multirow{2}{*}{$\begin{array}{l}\text { Discipline manager's signature and } \\
\text { date indicating evaluation } \\
\text { complete. }\end{array}$} \\
\hline & & Calculation/Document No. & $\begin{array}{c}\text { Revision } \\
\text { No. }\end{array}$ & Calculation/Document No. & $\begin{array}{c}\text { Revision } \\
\text { No. } \\
\end{array}$ & Yes & No & Yes & No & \\
\hline 0 & 一 & · & & $H-2-818452$ & 0 & & $\checkmark$ & & $\checkmark$ & send $4 / 22 / 94$ \\
\hline & & & & & & & & & & \\
\hline & & & & & & & & & & \\
\hline & & & & & & & & & & \\
\hline & & & & & & & & & & \\
\hline & & & & & & & & & & \\
\hline & & & & & & & & & & \\
\hline & & & & & & & & & & \\
\hline & & & & & & & & & & \\
\hline & & & & & & & & & & \\
\hline & & & & & & & & & & \\
\hline & & & & & & & & & & \\
\hline - & & & & & & & & & & \\
\hline & & . & & & & & & & & \\
\hline & & & & & & & & & & \\
\hline & & & & & & & & & & \\
\hline & & & & & & & & & & \\
\hline & & & & & & & & & & \\
\hline & & & & & & & & & & \\
\hline
\end{tabular}




\section{DESIGN VERIFICATION SCREENING CRITERIA}

\section{Project/Document No. W-320, CAL CULATION W320-24-022}

When the design or design change affects hardware, formal design verification must be performed if one or more of the following questions must be answered affirmatively (YES).

YES NO

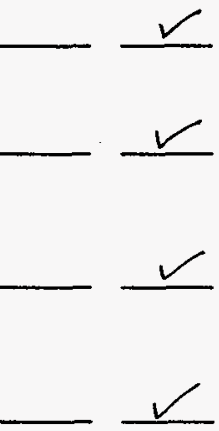

1. Does the design or design change involved meet the established criteria to be considered Safety Class 1 or 2 ?

2. Does this design or design change cause or permit changes to Safety Class 1 or 2 instrument or alarm setpoints outside of previously approved operational limits?

3. Does this design or design change significantly affect the nuclear safety consequences of a malfunction or failure of the structure, system, or component?

4. Does this design or design change involve or change design that has previously undergone formal design verification?
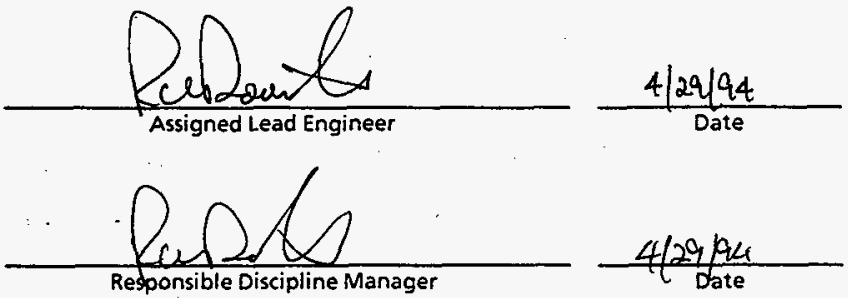

Original Design Package Distribution:

Project Engineer

Chief Design Engineer

Engineering Document Control
Design Change Distribution:

Attach to Engineering Change Notice 
HNF-2467, Rev.0

\begin{tabular}{|c|c|}
\hline $\begin{array}{r}\text { KA/SER ENEINEERS } \\
\text { HANFDRD } \\
\text { DESIGN ANALYSIS }\end{array}$ & $\begin{array}{l}\text { Catc. No. W320-24-022 } \\
\text { Revision } 0 \\
\text { Page No. } 1 \text { of } 16\end{array}$ \\
\hline client WHC & Wo/Job No. W-320/ER-4319 \\
\hline subject Service Building Foundation & $\begin{array}{ll}\text { Date } 03 / 28 / 94 & \text { By M.R Custer MreC } \\
\text { checked } 4 / 26 / 94 & \text { 8y JR Booth fRB }\end{array}$ \\
\hline Location TANK 241-C-106 & By \\
\hline
\end{tabular}

OBJECTIVE:

The objective of this calculation is to provide the detailed design of the building foundation for the Service Building. The design of the foundation shall be performed in accordance with ACI-318 (strength design) and the Uniform Building Code (1991), as required by the project design criteria, SDC-4.1, Rev. 11. In order for the foundation design to proceed, a conservative estimate of the building loads and reactions will be developed, since the building enclosure will be provided later, under a separate purchase specification.

\section{CRITERIA:}

1. DOE ORDER $6430.1 \mathrm{~A}(04 / 06 / 89)$

2. HANFORD PLANT STANDARDS, STANDARD ARCH./CIVIL DESIGN CRITERIA, SDC-4.1, REV.11

3. FUNCTIONAL DESIGN CRITERIA, WHC-SD-W320-FDC-001, REV.2

4. LETTERS OF INSTRUCTION:

5. ASCE $7-88$

DATA:

1. The equipment, structure and foundation for the Service Building is Safety Class 3 per Reference 7.

ASSUMPTIONS: NONE

METHODS:

Hand calculations

REFERENCES:

1. ACI 318-91, Building Code Requirements for Reinforced Concrete

2. Uniform Building Code (UBC), (1991 Edition)

3. ASTM A36-91

4. HANFORD PLANT STANDARDS, STANDARD ARCH./, CIVIL DESIGN CRITERIA, SDC-4.1, REV.11.

5. WHC-SD-WM-SEL-033, REV.0-C, Interm Safety Equipment List for 241-C-106 WASTE RETRIEVAL

6. AWS D1.1-92

7. Foundation Analysis and Design, Joesph E. Bowles (1st Edition)

8. ASCE 7-88 (American Society of Civil Engineers Standard), Minimum Design Loads for Buildings and other Structures 


\section{KAISER ENEINEERS}

HANFORD

\section{DESIGN ANALYSIS}

client WHC

subject Service Building Foundation

Location TANK 241-C-106
Calc. No. W320-24-022

Revision $\not 1$

Page No. 2 of 16

WO/Job No. W-320/ER

Date $03 / 28 / 94$

checked $4 / 26 / 94$

Revised

$8 / 3 / 93$
By M.R Custer MRC

By IR Booth

s Runeridsar

\section{CALCULATIONS:}

Refer to the Design CaTculations section

CONCLUSIONS:

The foundation for the Service Building is designed as a mat type foundation, however, the UBC code as well as the Hanford PI ant Standards (SDC-4.1) requires that a foundation wall be provided, which extends below the frost line to a minimum depth of 30 inches. This requirement has been incorporated in the final design. The foundation loads developed in the calculation are minimal, based on the large, physical area required by the piping and equipment, housed inside the building enclosure. The design satisfies the design requirements for the structure.

Based on a review of vendor approval data of building erection instructions, the current fdn design does not match vendor proposed pre-engineered bldg type. Fdn calculations were based on use of a Butler Panel Frame building system. Vendor has discontinued this line and proposes use of a Panel Line-1 self-framing building system.

Fdn dimensions will match ouside dimensions of building footprint. Width of fdn now $9^{\prime}-4^{\prime \prime}$ was $8^{\prime}-6^{\prime \prime}$ and length now $10^{\prime}-8^{\prime \prime}$ was $10^{\prime}-0^{\prime \prime}$. Other modifications to fdn design require deletion of base plate pedestal and anchor bolts. Panel frame system is to be attached with vendor supplied hardware to fdn curb with $3 / 8$ " dia $\times 21 / 2$ " long expansion anchors at $1^{\prime}-6$ " intervals along top face of $8^{\prime \prime}$ curb using a base channnel/flashing system.

Calculations provided adequately envelope above changes to fdn design. No further analysis is required. 
HNF-2467, Rev.0

KAISER ENGINEERS

HANFORD

DESIGN ANALYSIS

Calc. No. W320-24-022

Page No. 3 of 16

Client WHC Wo/Job No. W320/ eR. 4319

SUbject SERVICE BUILDING EOUNOAFION Date $4 / 11 / 94$ By M/CHARZ R CuSTER

Location TANK 241-C-106 Checked $4 / 26 / 94$ By

FOUNDATION DESIGN REQUIREMENTS

- Minimum depth to the bottom of foundation of PERMANENT STRUCTURES BELOW FINSSHED GRAOE IS 2-6". (RETERENCE 4)

- Allowable soil pressure per chapter zoa vel, (REFEICENCE 2), $1500.16 / \mathrm{AT}^{2}$.

- Coefficient of sliding = 0.25 (Reference 2)

- minimum footing dimensions per reference 2 AS FOLLOWS:

- THICKNESS OF CONCRETE FOUNOATRN WALL, 6 "

- WIDTH OF FOOTING, $12^{\circ}$

- THICKNESS OF FOOTING 6 ".

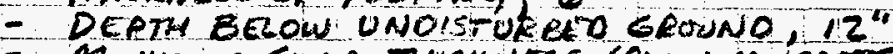

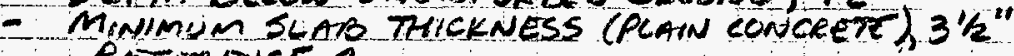
REFERENCE 2 .

- Load combinations: (Reference 2 \$4) $U=1.4 D+1.7 L$ (MINIMUM DE SION REQUIREMENTS) $u=0.75(1.4 D+1.7 L+1.7 W)$ (WIND) $u=0.90+1.3 \mathrm{w}$ $u=0.75(1.40+1.76+1.87 E)$ (SEAL)

IN ADO MONA: IFEARTIQUAKE LOADS INCLUDED IN DESIGN, COMO COMBINATiONS OF SECTION 2609 (C)/2). REFERENCE 2 , APPLY EXCEPT (I .IE) IS SUBSTI TOTED FOR (W).

$u=0.75(1.40+1.7 L+1.87 E)$

$u=0.90+1.43 E$

Page 0.6 
HNF-2467, Rev.0

$\underset{\text { HANFORD }}{\text { HANGER ENGINEERS }}$

Calc. No. $\frac{1320-24-022}{0}$

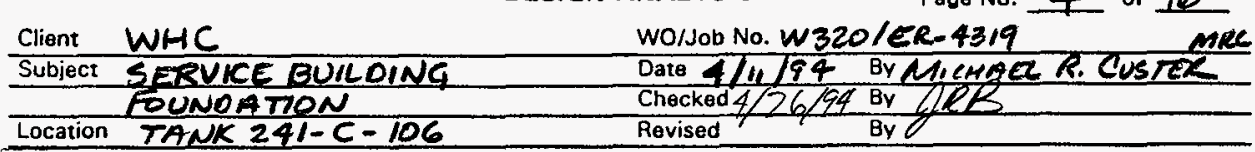

ALSO, FOR EARTHQUAKE LOADING, USE THE FO WOWING:

$$
\begin{aligned}
& U=1.4(D+C+E) \\
& u=0.90 \pm 1.4 E
\end{aligned}
$$

BUILDING DESIGN REQUIREMENTS/LOADS

- RoOF deAd loAd, $2016 / \mathrm{Fr}^{2}$ (REFERENCe 4)

- ROOF COVERING, $50 \mathrm{lb} / \mathrm{FT}^{2}$ OR $200 \mathrm{ib}$ CONCENTRATED

LOAD.

- primary framing and purling, $20 \mathrm{k} / \mathrm{ft}^{2}$

- SNOW / LIVE lOAD, 20 10/ar .... (REFerence 4)

- Wind load,

BASIC WIND SPEED, $70 \mathrm{mi} / \mathrm{h}$

IMPORTANCE FACTOR, 1.07

EXPOSURE CATEGORY, $C$

$$
\begin{aligned}
& \begin{aligned}
q z & =0.00256 K_{z}(I V)^{2} \\
& =0.00256(0.80)[1.07(70)]^{2}
\end{aligned} \\
& q_{z}=11.5 \text {, MORMALY, FoRCES DETERMNADU USING }
\end{aligned}
$$

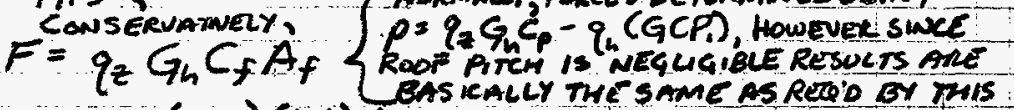

$$
\begin{aligned}
& =11.5(1.32)(1.4) A_{f} \\
& F=21.3 A_{f}, F_{V}=21.3\left(8^{\prime} \times 10^{\prime}\right)=1704^{*}, F_{H}=21.3\left(10 \times 10^{\prime}\right)=2130
\end{aligned}
$$

ESTIMATE DEAD LOAD OF BUILDING ENCLOSURE:

$$
\begin{aligned}
& W_{(B L G)}=[30(2)(516 / F T)]+\left[(8)\left(10^{\circ}\right)\left(2\left(0^{\prime} / F\right)+(8)\left(8^{\prime}\right)(2 \mathrm{~g} / \mathrm{F})\right]+\right. \\
& \text { W } \quad[5)(10)\left(2^{16 / F)}\right]+\left[2\left(10^{\prime}\right)\left(10^{\prime}\right)+2\left(10^{\prime}\right)\left(8^{\prime}\right)+(8)\left(10^{\prime}\right)\right] 1.5 \% / 7^{2}= \\
& W_{D(B L D)}=1348, \text { SAY } 1.5 k
\end{aligned}
$$

ROOF LIVE LOAD:

$$
L_{R}=20^{16} / R^{2}(10 \times 8)=1600^{16} \text { ar } A 6 K
$$

Pageid-7 
HNF-2467, Rev.0

KAISER ENGINEERS

HANFORD

DESIGN ANALYSIS

Cali. No. W320-24-022

Revision 0

Page No. $\frac{0}{5}$ of 16

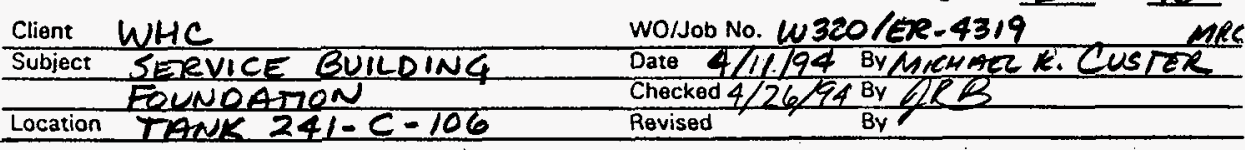

DEAD LOAD, EQUIPMENT 4 PIPING:

- air compressor, $780^{16}$

- air ORYER . . TO

$$
\begin{aligned}
& W_{D L}=W_{A C}+w_{A D}+w_{P I P E}= \\
& W_{O L}=780+70+(4 \mathrm{~b} / \mathrm{FT} \times 14)=906^{16} \mathrm{sAY} / \mathrm{K}
\end{aligned}
$$

DEAD LOAD, FOUNDATION (BASED ON 8'xIO' BLDG DIM.)

$$
\begin{aligned}
& t_{c}=3^{\prime \prime} \text { CONtR }+5^{*}=8^{\prime \prime} \quad \text { (MIN. ThICKNESS } 3^{1 / 2}, \text { PER } \\
& w_{c}=8 / 12 \times 8 \times 10 \times 1501 / 1 / 7^{3}= \\
& w_{C}^{\text {SCAR }}=8000^{\mathrm{b}}=8.0^{\mathrm{K}} \\
& \text { SLAB } \\
& w_{C \text { ALL }}=2\left[\left(2.3^{\prime} \times 1.0^{\circ} \times 10^{\prime}\right)+\left(2.3 \times 1.0^{\prime} \times 6.0^{\prime}\right)\right] 150^{\prime \prime} \mathrm{s}^{3}= \\
& w_{\text {WAW }}=11,040^{\#} \text {, SAY }\|\|^{K} \\
& \dot{w}_{T C}=8 k+11 k=19 k
\end{aligned}
$$

TOTAL DEAD LOADS

$$
\begin{aligned}
& w_{T}=w_{B L O Q}+w_{\text {FWD }}+w_{\text {EQUIP }}= \\
& w_{T}=1.5^{k}+19.0^{k}+1^{k} \\
& 21.5^{k} \text { SAY } 22^{k}
\end{aligned}
$$

Page D:8 
HNF-2467, Rev.0.

KAISER ENGINEERS

HANFORD
Client WHC

Subject BERVICE BUILDINC. Location TONNATMON

DESIGN ANALYSIS

Wo/Job No. W320/ER 4319

Date 4/11/94 ByMICHAER R. CUSTER Checked $4 / 26 / 94$ By GL E

Revised
Call. No.W320.24-022

Revision $\frac{0}{6}$ of 16

EARTHQUAKE LOADS (BLDG)

BUILDING, SAFETY CLASS 3

BASE SHEAR, $V=\frac{Z I C}{R_{w}} w$ wHERE,

$$
\begin{aligned}
& Z=0.20 \\
& \begin{array}{l}
I=1.25 \\
C=2.12
\end{array} \\
& \hat{R}_{w}=6 \\
& \text { (REFERENCE 2) } \\
& \text { (REFERENCE 4) } \\
& \text { (RETERENCE 4) } \\
& \text { (REFERENCE } 2 \text { ) } \\
& E_{N-S}=V_{N-S}(100 \% \text { maX. }) \\
& E_{E-W}=V_{\text {Er }}(30 \% \text { max. }) \\
& \} \text { (REFERENCE 2) } \\
& \text { eRS }=\sqrt{E_{N-S}^{2}+E_{E-W}{ }^{2}} \\
& \text { WELT OF BLDG }=1.5^{\mathrm{K}} \\
& E_{N-S}=V_{N-S}=[0.20(1.25)(2.12) / 6] w_{\text {LOG }}=0.13 \mathrm{~K} \\
& E_{E-\omega}=V_{E-\omega}=[0.20(1.25)(2.12) / 6] \omega_{B L 04}(.3)=0.03^{k} \\
& E_{\text {SoS }}=\sqrt{0.13^{2}+0.03^{2}}=0.13^{K}
\end{aligned}
$$

EARTHLQUAKE LOADS (BLDG 4 FOND.)

$$
\begin{aligned}
& E_{N-S}=V_{N-S}=[0.20(1,25)(2.12) / 6] w_{\text {BLoG }}=2.0 \mathrm{~K} \\
& E_{E-N}=V_{N-S}=[0.20(1.25)(2.12) / 6] w_{\text {BLoG }}(.3)=0.60^{\mathrm{K}} \\
& E_{\text {SaSS }}=\sqrt{2.0^{2}+0.60^{2}}=2.1 \mathrm{~K}+
\end{aligned}
$$

Page D-9 
HNF-2467, Rev.0

$\underset{\text { KANFOER ENGINEERS }}{\text { HANGER }}$

Calc. No. W320-24-022

Revision $\frac{0}{7 \text { of } 16}$

Client $W / H C$

DESIGN ANALYSIS

WO/Job No. W320/ER-4319

SEleCt SEICE BQILDING Date 4/1/94 By Mysyger R. CUSTER FounDations

Location THNK $241-C-106$ Checked $4 / 26 / 94$ By

Determine Governing LOAD COMBINATION For BLCG:

1) $D+L+$ (ROOF LIVE LOAD OR SNOW LOAD)

2) $D+L+W$ ORE

3) $D+L+W+$ SNOW $/ 2$

4) $D+L+$ SNOW $+w / 2$

5) $D+L+$ SNOW $+E$

1) $\begin{aligned} & u=1.7+1.60+2.1(10 / 8) \text { oR } 13(10 / 10)=3.3 \\ & u=1.7+1.7+2.0 \times 3.5\end{aligned}$

3) $u=1.7+1.7+2.1(10 / 8)+1.6 / 2$

4) $u<1.7+1.6+[1.7+2.1(10 / 8) / 2]$

5) $u=1.7+1.6+0.16(10 / 10)$$$
=6.3
$$

MAX. BuLOINGREACTICNS. LOAD COMBINATION (3)

FounOATIN DESIGN:

THE BUILDING LOADS MAY BE SUPPORTED ATT THE FOUR CORNERS ON INDEPENDENT FOUNDATIONS OR ON A MAT FOUNDATION DUE TO THE MAGNTUDE AND SYMMETRY OF THE LOADS AND REQUIRED CONFIGURATION BY UPC.

THE MINIMUM SIZE OF THE FOUNDATION SLAB HAS BEEN DEFINED, BASED ON THE REQUIRED PHYSICAL AREA, $8 \times 10$ '? (EAVE HEIGMT OP BLOL. 10 PT)

THE FOUNDATION DESIGN WILL BE A MAT DESIGN WITH A PERIMETER WALL TO A DEPTH OF $2-C^{\mu}(F I G .1)$

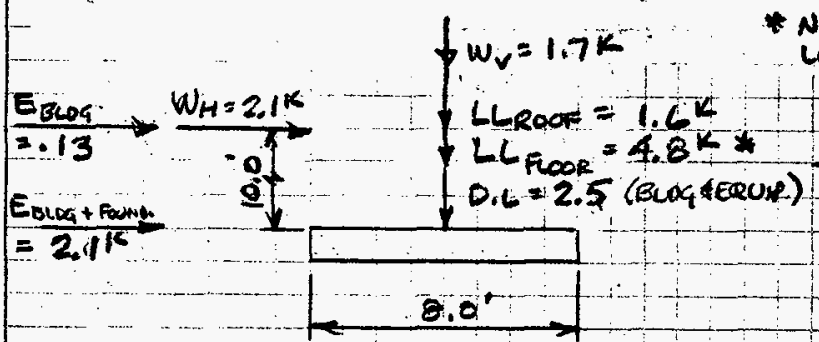

LOADS nominal floor live LOAD $\left(6^{\prime} \times B^{\prime} \times 100^{11} K^{2}=4.8 K\right)$

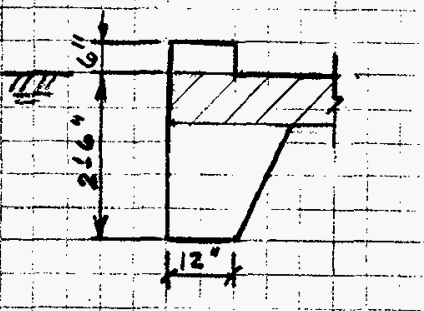

Pic. 1 
HNF-2467, Rev.0

KAISER ENGINEERS

HANFORQ
DESIGN ANALYSIS

Client WHC

Subject SERVICE BULDING WO/Job No. W320/ER-4319 Date 4IIIIA ByMicmier R. CusRer Fenvadoation

Location TANK 241=C-106 Revised

By

FoundeTion DESIGN (CONT.)

FOUNDATON STRBBLITH:

A) SLIOING: COEFFILIENT $=0.25 \quad$ (REFRLNCE 2 , Pg S40) $\underset{\substack{S L 10 w i \\(W W D}}{S F} \frac{R_{L S}}{F_{H}}=\frac{.25(22)}{2.1}=2.6>1.5 \ldots 0 . \mathrm{K}$ $O R$

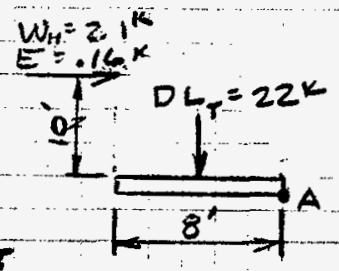

E4. 2

B) OVERTURNIAUS $(0 T)$ :

S.F $=\frac{22^{k}\left(4^{\prime}\right)}{2.1^{k}\left(10^{\prime}\right)}=4.2>1.5 \ldots . . .0 . k$

OR

$$
\text { S.F } F_{\text {OT }}=\frac{22^{k}\left(4^{\circ}\right)}{.16 k\left(10^{\circ}\right)}=55.0>1.5 \ldots \text { NEGLIGBQLE }
$$

LOAD COMBINATIONS (FACTORED LOADS): FOUNDATION DESKGN

1) $u=1.40+1.7 \mathrm{C}$

2) $u=0.75(1.40+1.26+1.7 w)$

3) $U=0.90+1.3 w$

4) MODIFIED FORMULA $9-2$, PER REFERUNCE 2 . SECTION $2609 \cdot \mathrm{C.} 3$

$$
u=0.75(1.40+1.7<+1.1 E)
$$

5) MODified Foemula a-2 PER REFERENCE ?, SECTION 26:25.4, Pg. 522 .

$$
U=14(D+L+E)
$$


HNF-2467, Rev.0

KAISER ENGINEERS

HANFORD

DESIGN ANALYSIS

Calk. No. $\frac{\omega 320-24-022}{0}$

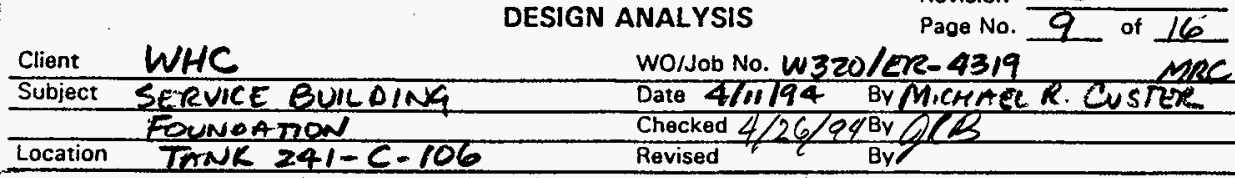

FOUNDATION LOADS: (CONTR.)

6) MODIEIED $9-3$, PER REFERENCE 2 , SECTION $2625,4, \mathrm{pg} \cdot 522$

$$
\begin{aligned}
& w=\frac{2.1^{k}(10)}{8}+1.7^{k} \\
& w=4.3^{k} \\
& E=\frac{.13^{k}\left(10^{\prime}\right)}{8}=0.16^{k}
\end{aligned}
$$$$
U=0.90 \pm 1.4 E
$$

Determine maximum design value FOR "U":

$$
D . L=\left(8^{\prime \prime} / 12\right)\left(8^{\prime}\right)\left(10^{\prime}\right)\left(150 \mathrm{H} / \mathrm{Fr}^{3}\right)+1.5+1.0=10.5_{1}^{*} \text { SAY } 11^{k}
$$

EVALUATE:

$$
\begin{aligned}
& \text { 1) } u=1.4(1 i)+1.7(1.6+4.8)=26.3^{k} \leftarrow \text { soveravs } \\
& \text { 2) } u=0.75 \pi 1.4(11)+1.7(6.4)+1.7(4.3)=25.2 k \\
& \text { 3) } u=0.9(11)+1.3(4.3) \quad=15.5 \mathrm{~K} \\
& 4) u=0.75(1.4(11)+1.7(6.4)+1.1(16)]=19.8 \mathrm{~K} \\
& \text { 5) } \begin{aligned}
u=1.4(11+6.4+.16) & =24.6 \mathrm{k} \\
u=0.9(11) \pm 1.4(616) & =10.1 \mathrm{k}
\end{aligned}
\end{aligned}
$$

DETERMINE FOUNDATION SIZE (CHECK):

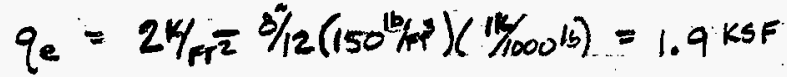

$$
\begin{aligned}
& A_{R E O_{0}}=\frac{0 . L+C . L}{q}=\frac{(1.0+1.5)+(1.6+4.8)}{1.9}=4.7 \mathrm{~F}^{2}<80^{2} \mathrm{~F}^{2}, \mathrm{CK} \\
& M=2,1 \times 10=21 k-1 \text { (WNO Moment) } \\
& P=8.9^{K} \quad(D+L) \text { fROM PG.7 }
\end{aligned}
$$

Determine beAring pressure CONSIDERING LOCATION OF KERN :

$$
e=\frac{M}{V}=\frac{21^{k-1}}{8.9^{-K}}=2.36 \mathrm{FT}, \frac{\omega}{6}=\frac{8}{6}=1.3^{\circ} \text { (LoCATE }
$$

SINCE $e=2.36^{\circ}>1.3^{\circ}$, RESULTANT IS OUTSIDE KEP 
HNF-2467, Rev.0

KAISER ENGINEERS

KAISER
HANFORD

Client WHC

Subject SERVICE BUILOING

FONDATTCN

Location TANK 241-C-106

DESIGN ANALYSIS

Call. No. $10320 \cdot 2907$

Revision

Page No. 10 of 16

Wo/sob No. W320/ek-4319

DETERMINE $q_{\text {max }}$ FROM THE

FOLLOWING (SEE FIG. 3 )

$$
\begin{aligned}
& R(l k+e)=8.9^{k}\left(4^{k+}\right)+21 k-1 \\
& R\left(4+2.36^{\prime}\right)=56.6^{k-1}
\end{aligned}
$$

$R=8.9^{K}$ \& 6.36 FT FROM POUT A

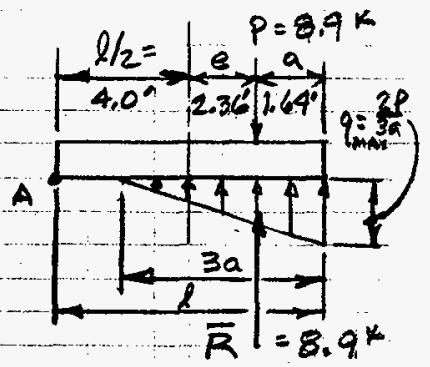

$$
q_{\text {MAX }}=\frac{-2 P}{3 a b}=\frac{2(8.9 \mathrm{~K})}{3(1.64)(10)}=0.36^{\mathrm{KSF}} 1.9 \mathrm{kSF} \quad \mathrm{F} / \mathrm{G.3}
$$

CHECK BEAM AND PUNCHING SHEARS AND DESIGN REINFORCING REQUIREMENTS: (REF, EIG, 4)

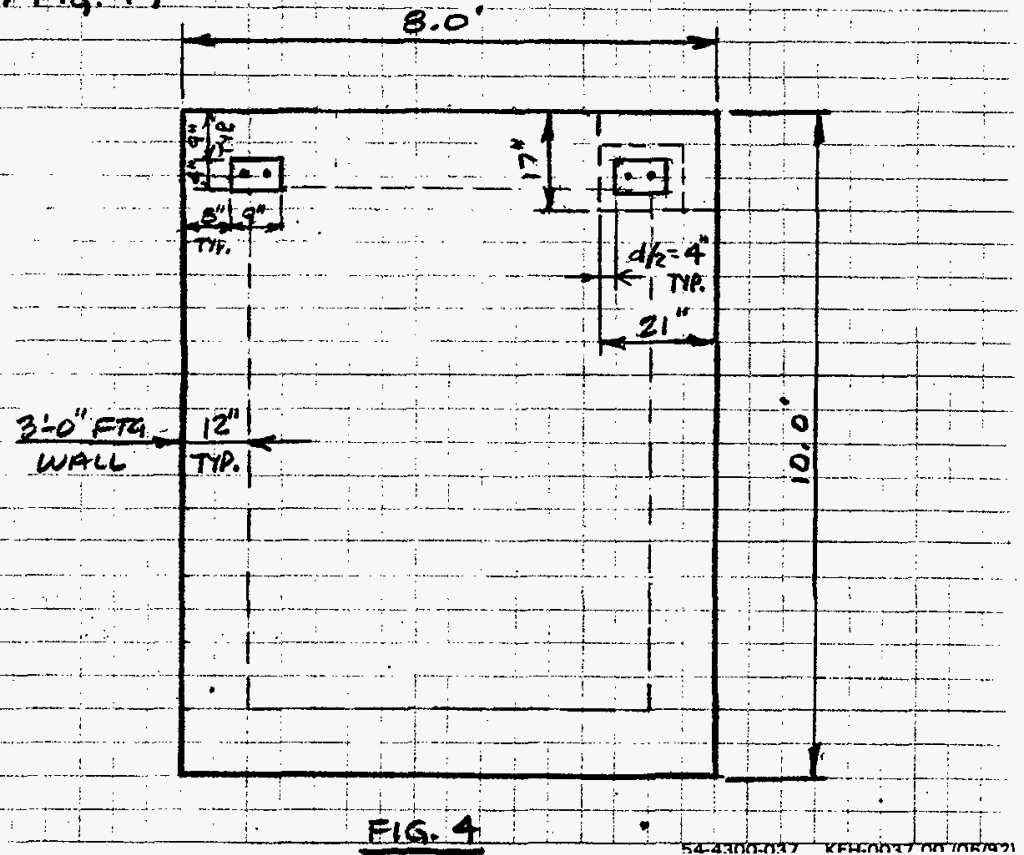


HNF-2467, Rev.0.

KAISER ENGINEERS

Calc. No. W320-24-022

Revision 0

DESIGN ANALYSIS

Page No. 11 of 16

Client WHC

Subject SERVICE BULLDNG CanNATCN
wo/Job No. W320/ER-4319

Date 411194 By MiCHARE QWSTL2

Location TANIL 241-C-106

Revised

BY

CHEZK PUNGHING SHEAR: (THP. SEE FIG,5)

DEPTH OF SLAB $=8^{\prime \prime}-\left(3^{\prime \prime}\right.$ CONER $)=5^{4}$

PERIMETER AT EMCM CORNER $=2 b_{1}+2 b_{2}$

$b_{1}=2\left[2(5 / 2)+9^{\prime \prime}\right]=28^{\prime \prime}=2.3 \mathrm{FT}$

$b_{2}=2[2(5 \%)+4]=18^{\prime \prime}=1,5 \mathrm{FT}$

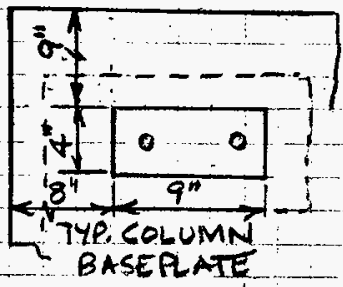

$A_{\text {puncting }}=2.3 \times 1.5=3.45 \mathrm{Fr}^{2}$

FK. 5

$V_{u}=\left(80.0-3.45 \mathrm{~F}^{2}\right)(0.33 \mathrm{ksF})=25.3 \mathrm{~K}$

$d_{\text {Re⿰冫欠 }}=\frac{V_{u}}{\not 4 \sqrt{f_{c} b_{0}}}=\frac{25,300^{16}}{.85(4) \sqrt{4000\left(46^{\prime \prime}\right)}}=$

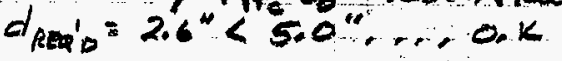

CHEKK BEAM SHEMR: (SEE FI 4.6 )

WIDTH OF FOUNDATIN $=8$ :

LENGTM of FOUNBATMEN = 10

$V_{u}=\left(8.0^{\prime}\right)\left(3.2^{\prime}\right)(0.33 \mathrm{kSF})=8.5^{k}$

$d=\frac{\sqrt{u}}{\rho 2 \sqrt{f e} b_{0}}=\frac{8500^{16}}{85(2) \sqrt{4000}\left(96^{\prime \prime}\right)}$

$d=0.82^{\prime \prime}<5.0^{\prime \prime}, 0 \mathrm{~K}$

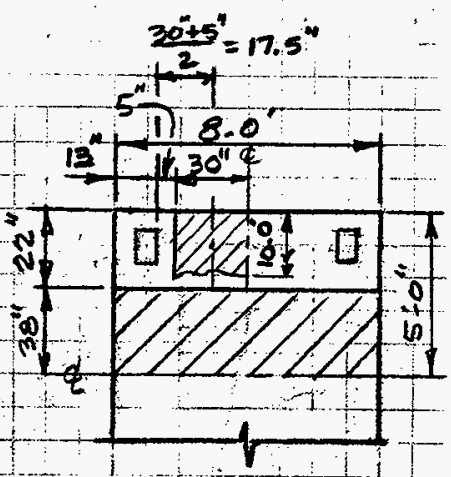

Fia.6

DETERMINE SLAB REINFORCEMENT REQUIREMENTS:

MU ; SHORT DIMENSION

$M_{u}=38^{\prime \prime} / 12\left(8.0^{\prime}\right)$ (.33 kSF) $\left[38^{\prime \prime} / 12(2)\right]=13.2 \mathrm{k-1}$

$6.6^{k}$

q;6k

OR $M_{u}=\frac{\omega_{u} l^{2}}{8}=\frac{165 \mathrm{k} / \mathrm{F}\left(8.0^{\prime}\right)^{2}}{8}=13.2^{\mathrm{K}-1}$

BASED SHEAR /MOMENT DIAGRAMS: (FIG,7)

$$
\begin{aligned}
& M_{u}=1 / 2\left(3.17^{\circ}\right)\left(5.23^{k}\right)-1 / 2(1.36 \mathrm{k})\left(83^{\circ}\right)= \\
& M_{u}=7.73 \mathrm{k}-1
\end{aligned}
$$


HNF-2467, Rev.0

KAISER ENGINEERS

Calc. No. $\frac{\omega 320-24-07 ?}{0}$

ANFORD

DESIGN ANALYSIS

Revision

Page No. 12 of 16

WO/Job No. W320/ER- 4319

Subject SELUVE EUTLOINL

EONNDArTeN

Location THNK $241-C-106$ Date 4/1194 By Mich etch. Pes tex

Revised $\frac{\text { By }}{\text { By }}$

$M_{4}$, LONG DIMENSION :

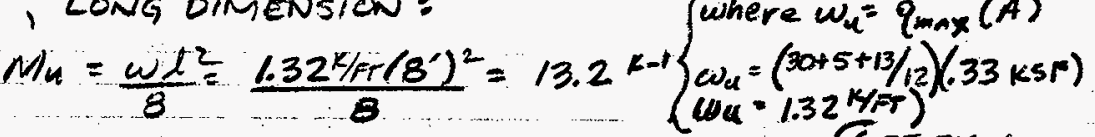

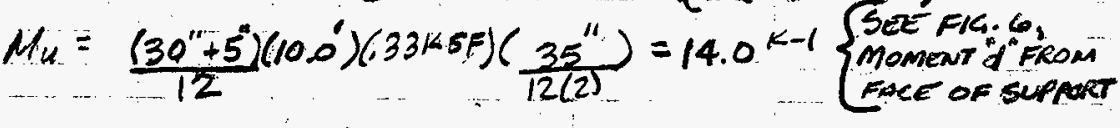

$$
\begin{aligned}
& \frac{M u}{\rho b d^{2}}-\frac{14.000(1 z)}{0.9(120)(2.5)^{2}}=248\left(\frac{M u}{\phi b d^{2}}=248, p=0,0043\right)
\end{aligned}
$$

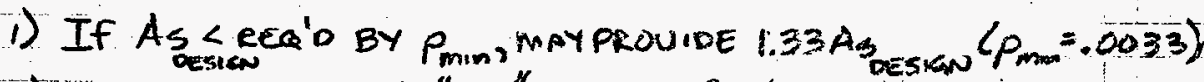

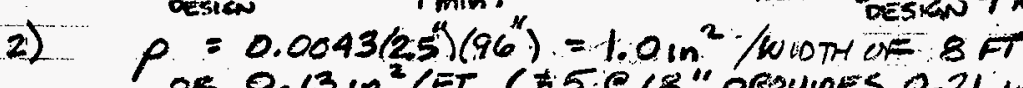

OR $0.13 \mathrm{in}^{2} / F T$, ( 5.5 .18 " PROVIDES $\left.0.21 \mathrm{in}^{2} / F T\right)$

3) TEMP/SHRMKAGE, $\rho=0.0018$, As $=0.43 \mathrm{in}^{2} /$ WIDTH $8.0 \mathrm{FI}$

USE $\$ 5 C 18^{2}, 0.21 \mathrm{~m}^{2} / \mathrm{FT}$

WALL REINFORCEMENT: (FIG.8) (WALL REIN NOT RETD

$$
\begin{aligned}
& M=2.1 k\left(4^{\prime \prime}\right)=8.4^{k-11} \\
& R(l / 2+e)=8.4^{\prime \prime}
\end{aligned}
$$

FOR HORIZONTAL LOADS, HOWEVER MINIMAL As WILl BE

$$
\left.R=0.42 k 15^{\prime \prime}+5\right)=8.4^{k \cdot 11}
$$
provider)

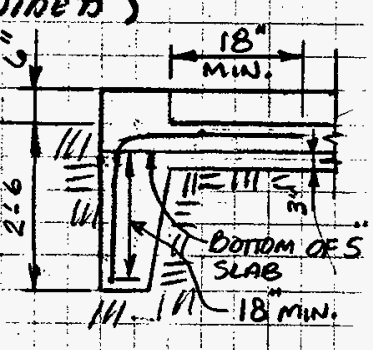

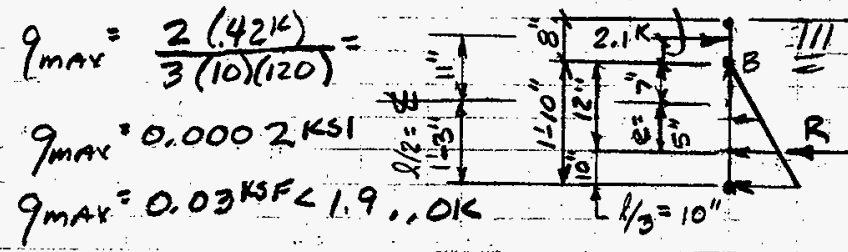

$$
\begin{aligned}
& M_{N}=0.03 \mathrm{ksF}(1.83 \mathrm{Ar})(10.0 \mathrm{~F})(1.0 \mathrm{Fr})=0.55 \mathrm{k-1} / 10 \mathrm{FT} \mathrm{LIGNGM} \\
& M_{u}=0.55 \mathrm{k-1}(1.4)=0.77 \mathrm{k-1}, \frac{\mathrm{Mu}_{u}}{\mathrm{~d}^{6 d^{2}}}=\frac{770(12)}{0.9(96)(6)^{2}}=2.97, \rho^{2} p_{\text {min }}
\end{aligned}
$$

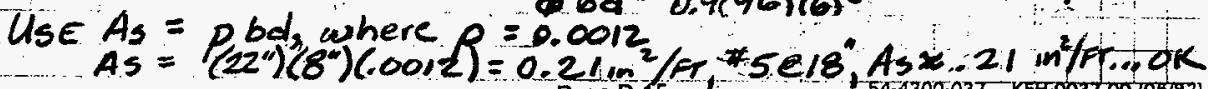


HNF-2467, Rev.0

KAISER ENGINEERS

HANFORD
DESIGN ANALYSIS

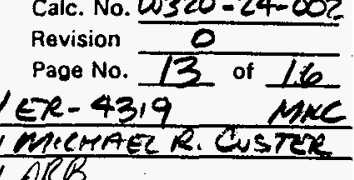
Wo/Job No. W320/Ex-4319 Mate Checked 4/26/94 By ARB

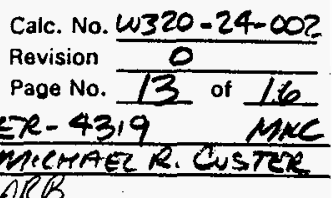
Revised

By

Determine devELOPMENT LENGTH OF SLAB REINF. FOR BOTH POSITIVE AND NEGATIVE MOMENTS:

$$
\begin{aligned}
& \ell_{d b}=\frac{0.04 A_{b} f_{y}}{\sqrt{f_{c}^{\prime}}} \\
& \text { (Reference } 2 \text {, Section 26I2(c) } \\
& p q .469) \\
& l_{d b}=\frac{0.04(307)(60,000)}{\sqrt{4000}} \\
& M_{4}=16.5 \mathrm{~K}-F T=198 \mathrm{k}-\mathrm{rt} \\
& M_{n}=\frac{M_{4}}{0_{2} 9}=220 \mathrm{ks} \\
& f_{d 6 \text { min }}=11.6^{\prime \prime} \text { " providing } 22^{\prime \prime} \\
& l_{d} \leq \frac{M_{n}}{1 / x}+h_{a}, l_{a}=12 d_{b} \\
& l_{d_{6}}=11.6 \text {, provided } 22 \geq 33 " 1 \text {... ok } \\
& l_{d}=\frac{220^{\mathrm{k}-11}+12(.625)}{8.5 \mathrm{k}} \\
& l=33 \pi\left\{\begin{array}{l}
\text { ReFeRENCE } 2 \\
\text { Fetmut } 1: 2
\end{array}\right. \\
& l_{b}=0.0004 d_{6} f_{y} * \\
& =0.0004(625)(60,000)=15^{10} \\
& \ell_{d_{\text {amin }}}=15^{\prime \prime}<22^{\prime \prime}<l_{d_{\text {max }}}=33^{\prime \prime}, \ldots, \text { oK, provioc } l_{d_{\text {min }}}=22^{\prime \prime}
\end{aligned}
$$

DESIGN GRADE BEAM:

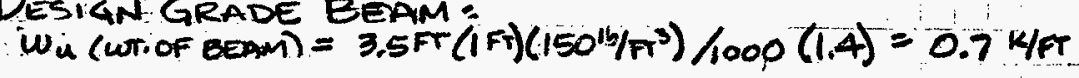

$$
\begin{aligned}
& M_{u}=14.0^{k-1}+\frac{\omega l^{2}}{8}=14.0^{k-1}+\frac{0.7\left(10^{\prime}\right)^{2}}{8}=22.8 \mathrm{~K}-\mathrm{Fr} \\
& P_{\text {men }}=200 / f_{1}=0,0033, P_{\text {mix }}=0.0214 \\
& \frac{M u}{\phi b d^{2}}=\frac{22.800^{k-1}(12)}{090\left(6^{\circ}\right)(19)^{2}}=140<192.2, p_{\min }=0.0033 \\
& A_{s}=0.0033(8)(19)=0.50 \mathrm{in}^{2}, 2-\# 5, A_{s}=0.62 \mathrm{in}^{2}
\end{aligned}
$$

REMAINING AREA, DISTRIRUTE. As $=.1 A_{3}=. \Pi(0.88)=0.09 \mathrm{~m}^{2}$ CPROVIDE 2-" 4SE TOP \& MID-OERTH IA : 


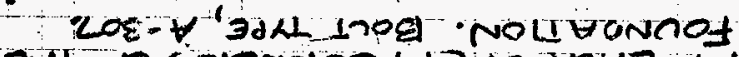

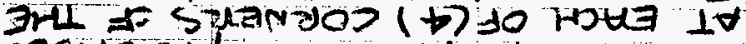

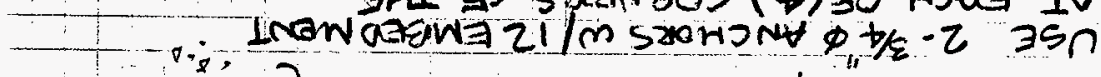

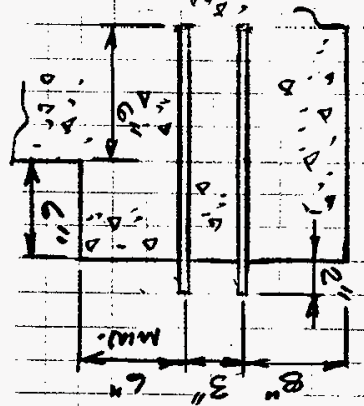

Inowase we
_. 21 onired

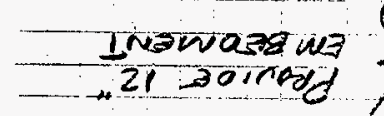

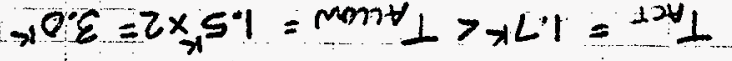

$$
\begin{aligned}
& \left.x^{\prime} t=2 \times x_{x}^{2} Z=\operatorname{mon}_{\Lambda}\right\rangle_{x} 5^{\circ} 0=12 \forall \Lambda
\end{aligned}
$$

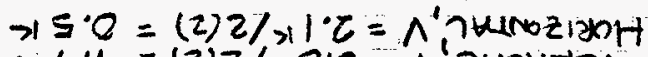

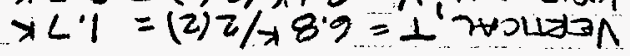

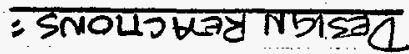

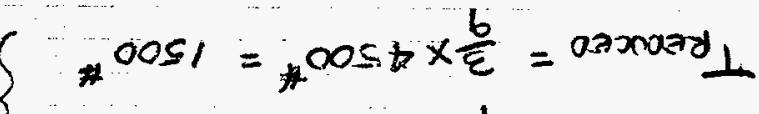

$$
\begin{aligned}
& \angle \varepsilon L \varepsilon z={ }^{2} 021 L \times \frac{b}{\varepsilon}=\operatorname{cosmay} \Lambda \\
& : \text { "E to bNivag jos }
\end{aligned}
$$

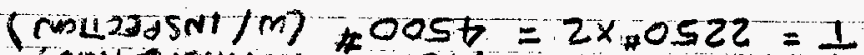

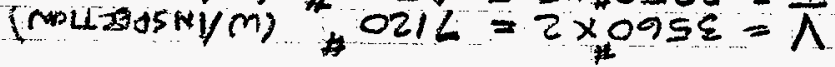

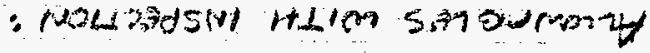

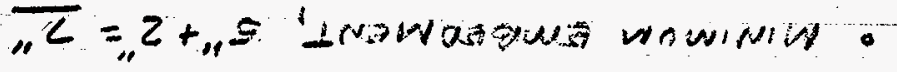

(rolonazy o/M) $\overline{16}=(s L) 21=010 \times 21$ brupbds.

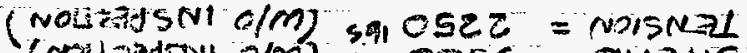

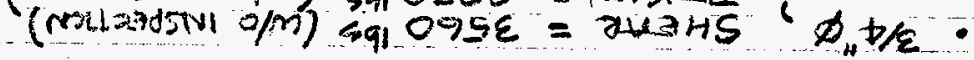

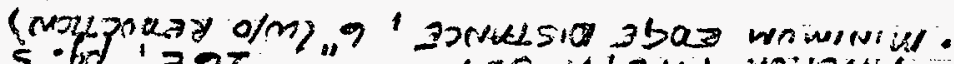

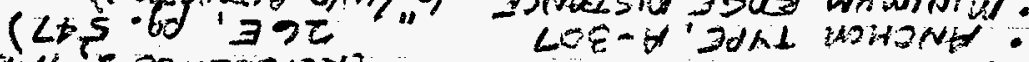

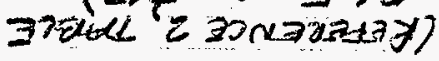
: sLnaw

\section{ב94xnowt briannel}

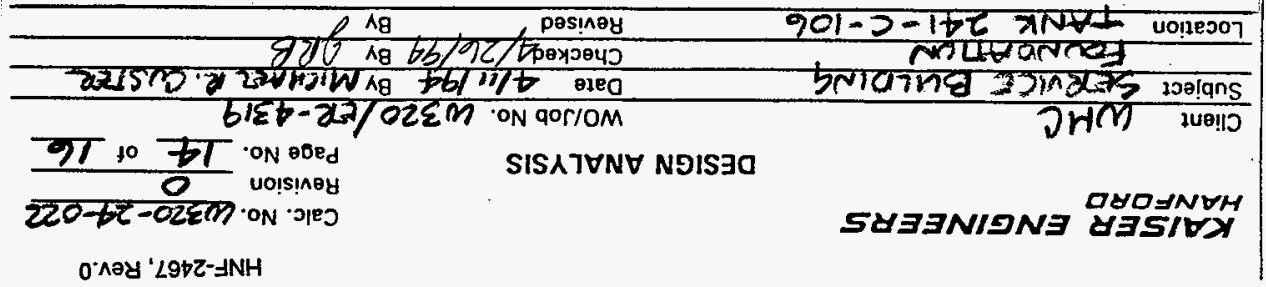


KAISER ENEINEERS MANFORD

Client $\quad$ Subject SARV EOUNDATON

Location TANK 241-C-106
DESIGN ANALYSIS

Wo/Job No. $10320 / E R-4319$ Date $4 / 1194$ By Mecmate Checked $4 / 26 / 44$ Bv $/ 2 R 3$

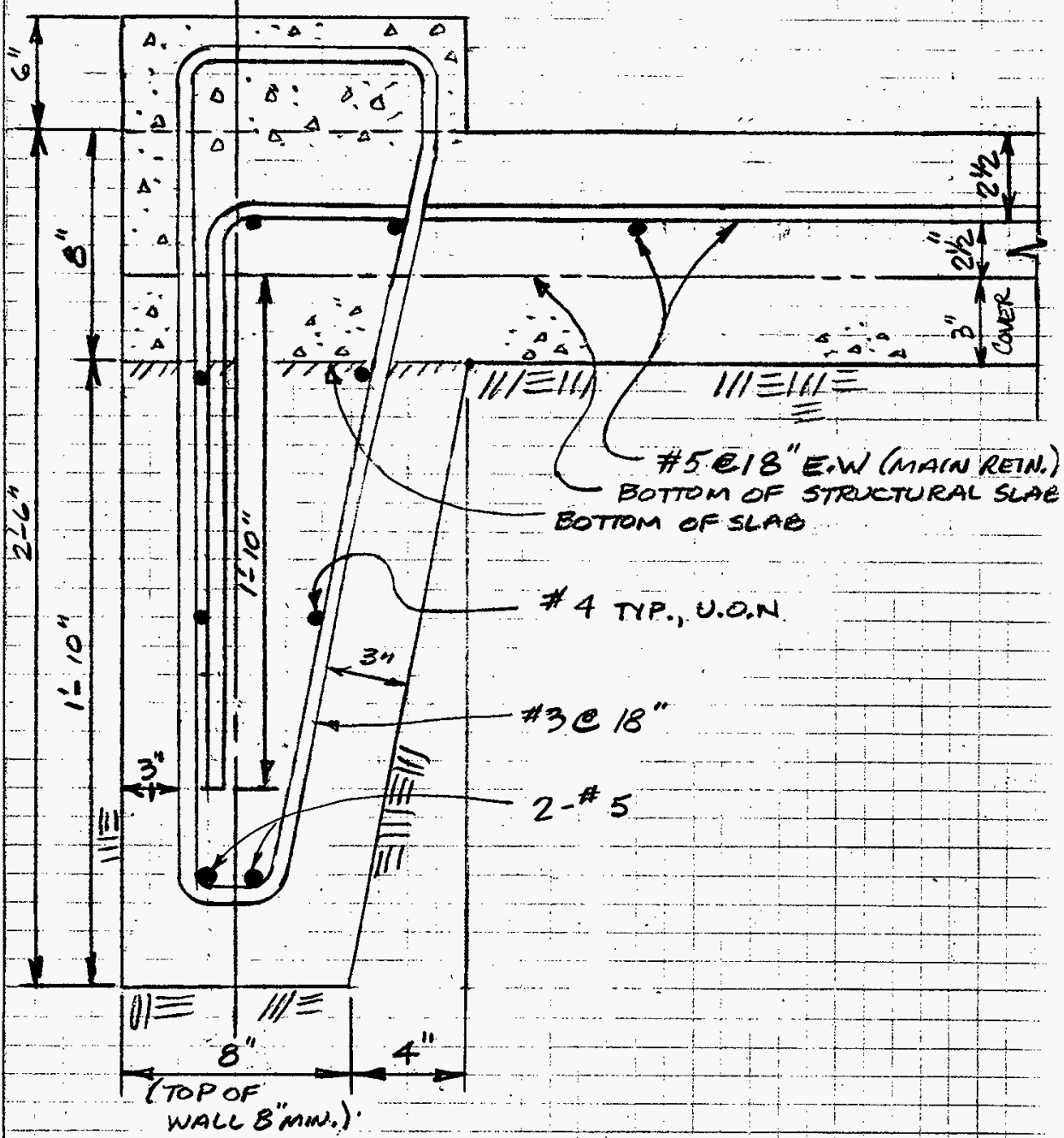

WALC B"Mm.). 


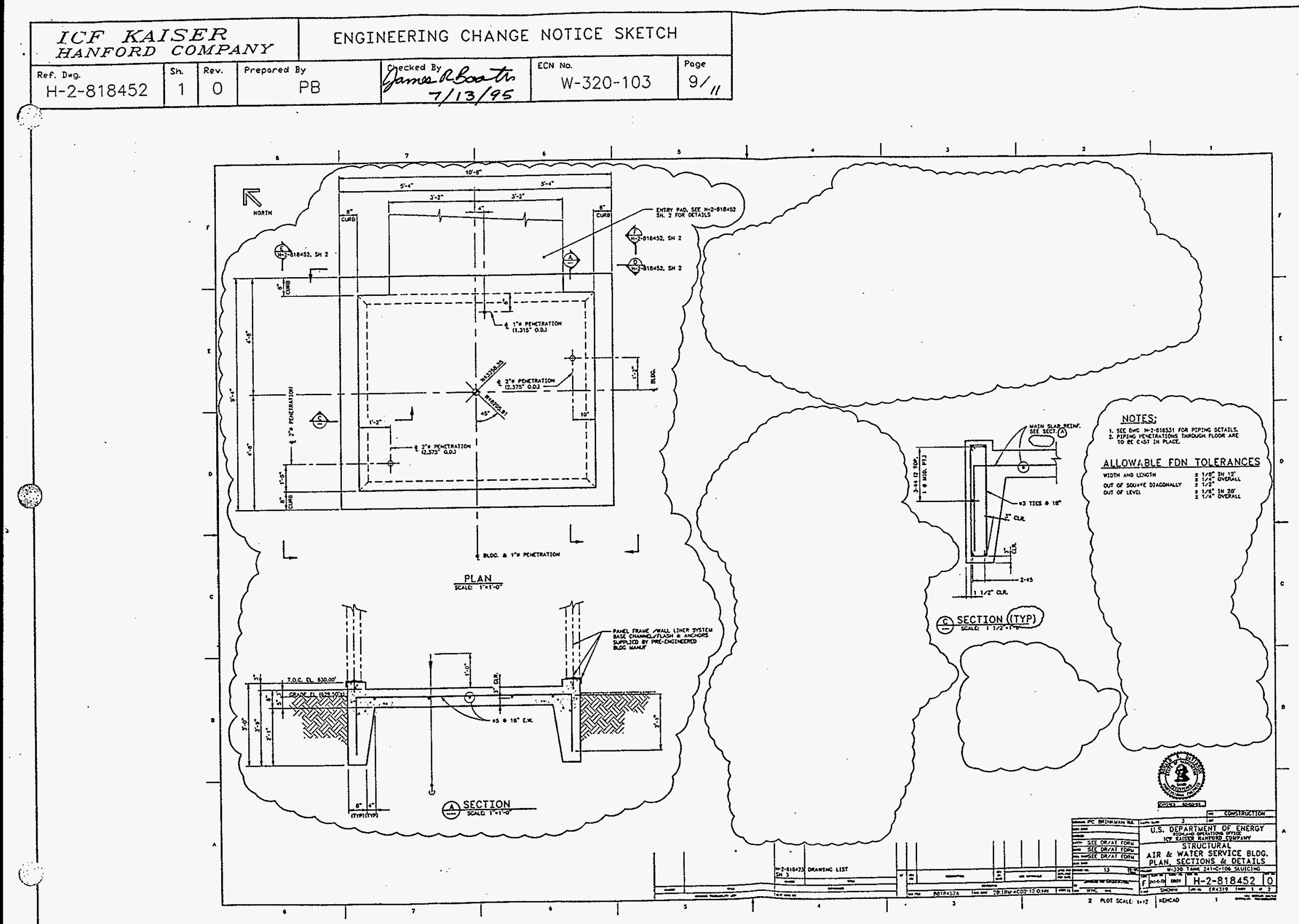




\section{ATTACHMENT A}




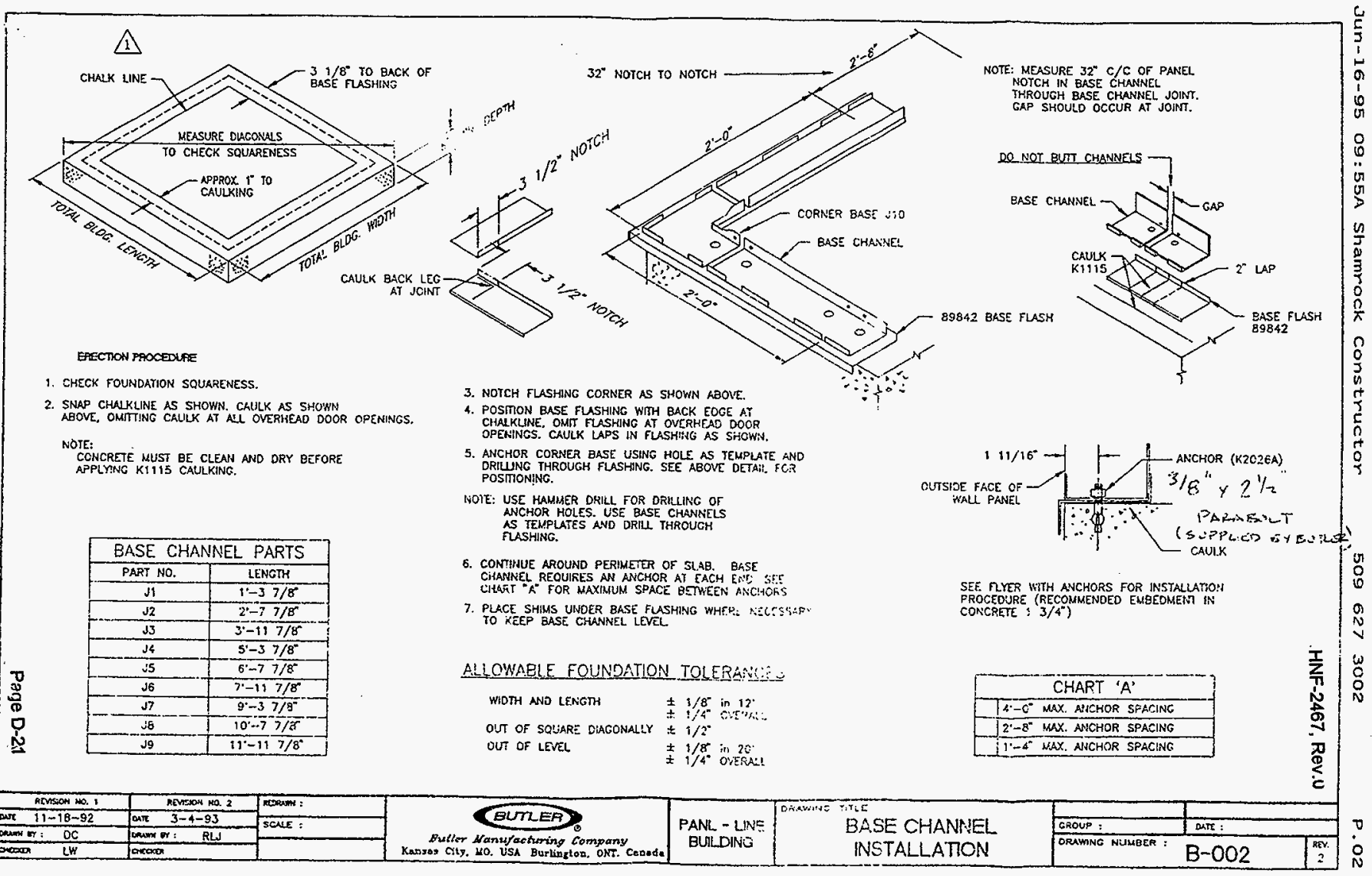


HNF-2467, Rev.0

W320-24-023

Misc. Mechanical (HVAC) Equipment Foundation Chiller and Pumps 


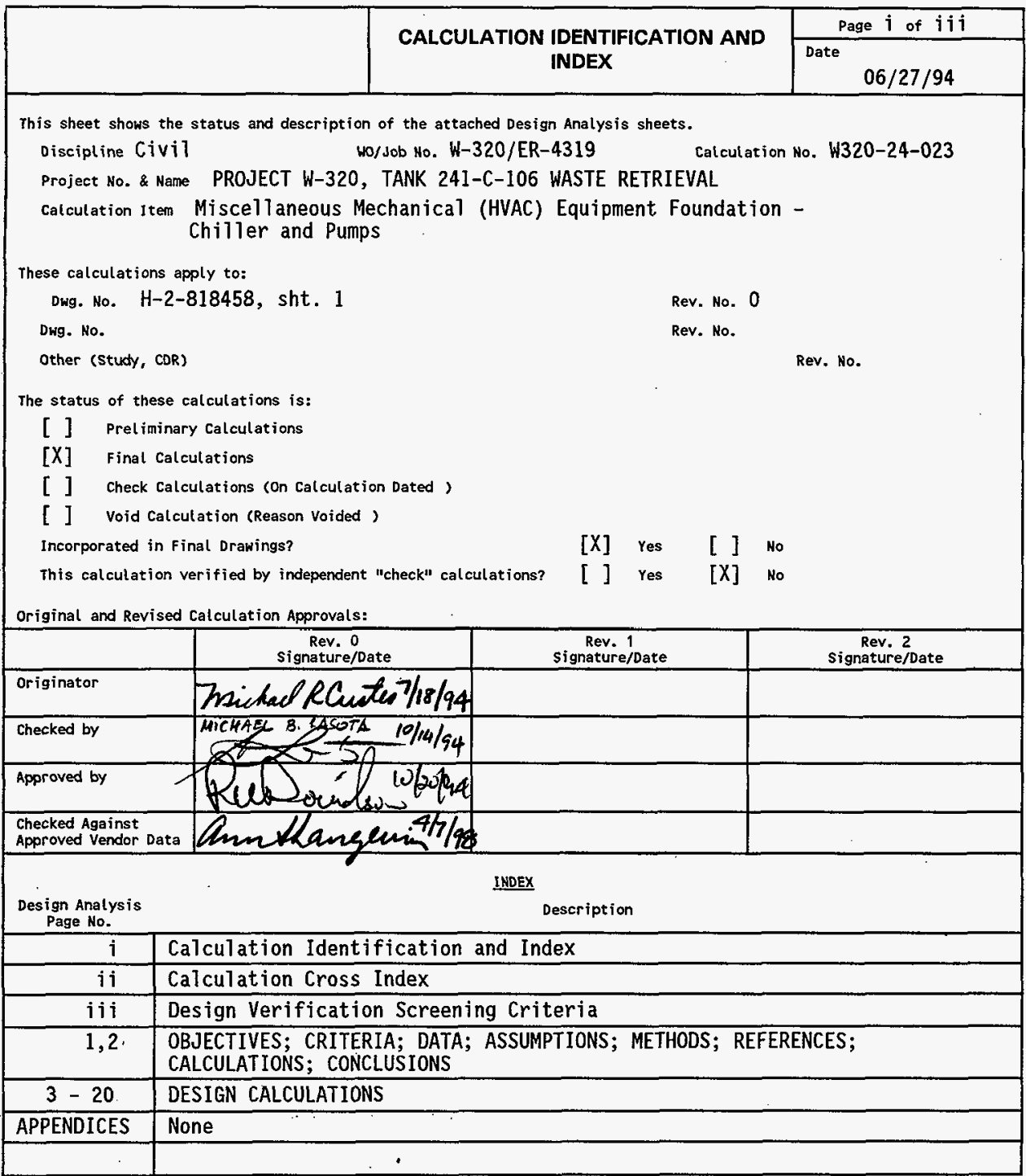




\section{CALCULATION CROSS INDEX , iypical)}

Subject Calculation No. W320-24-023, REV.O

\begin{tabular}{|c|c|c|c|c|c|c|c|c|c|c|}
\hline \multirow{2}{*}{$\begin{array}{l}\text { Subject } \\
\text { Calculation } \\
\text { Revision } \\
\text { No. }\end{array}$} & \multirow{2}{*}{$\begin{array}{l}\text { Superceded } \\
\text { by } \\
\text { Calculation } \\
\text { No. }\end{array}$} & \multicolumn{2}{|c|}{$\begin{array}{l}\text { These interfacing calculation/documents } \\
\text { provide input to the subject calculation, } \\
\text { and if revised may require revision of } \\
\text { the subject calculation. }\end{array}$} & \multicolumn{2}{|c|}{$\begin{array}{l}\text { Results and conclusions of the subject } \\
\text { calculation are used in these interfacing } \\
\text { calculations and/or documents. }\end{array}$} & \multicolumn{2}{|c|}{$\begin{array}{c}\text { Does the output } \\
\text { interface calculation } \\
\text { documents require } \\
\text { revision? }\end{array}$} & \multicolumn{2}{|c|}{$\begin{array}{l}\text { Has the output } \\
\text { interface calculation/ } \\
\text { documents been } \\
\text { revised? }\end{array}$} & \multirow{2}{*}{$\begin{array}{c}\text { Discipline manager's signature and } \\
\text { date indicating evaluation } \\
\text { complete. }\end{array}$} \\
\hline & & Calculation/Document No. & $\begin{array}{c}\text { Revision } \\
\text { No. }\end{array}$ & Calculation/Document No. & $\begin{array}{c}\text { Revision } \\
\text { No. }\end{array}$ & Yes & No & Yes & No & \\
\hline 0 & - & & & $H-2-81845^{\text {sमm. }}$ & 0 & & $V$ & & & 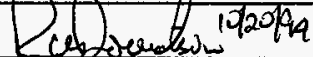 \\
\hline & & & & & & & & & & \\
\hline & & & & & & & & & & \\
\hline & . & & & & & & & & & \\
\hline & & & & & & & & & & \\
\hline & & & & & & & & & & \\
\hline & & & & & & & & & & \\
\hline & & & & & & & & & & \\
\hline & & & & & & & & & & \\
\hline & & & & & & & & & & \\
\hline$\bullet$ & & & & & & & & & & \\
\hline & & & & & & & & & & \\
\hline & & & & & & & & & & \\
\hline & & & & & & & & & & \\
\hline & & & & & & & & & & \\
\hline & . & ' & & & & & & & & \\
\hline
\end{tabular}




\section{DESIGN VERIFICATION SCREENING CRITERIA}

Project/Document No. $\frac{W-320 / C A L C U L A T Z O N}{\text { REV. O }}$ W $20-24-023$,

When the design or design change affects hardware, formal design verification must be performed if one or more of the following questions must be answered affirmatively (YES).

YES NO

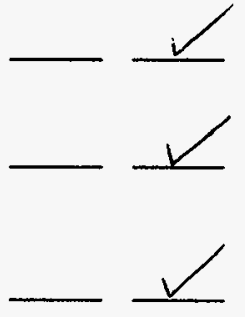

1. Does the design or design change involved meet the established criteria to be considered Safety Class 1 or 2?

2. Does this design or design change cause or permit changes to Safety Class 1 or 2 instrument or alarm setpoints outside of previousiy approved operational limits?

3. Does this design or design change significantly affect the nuclear safety consequences of a malfunction or failure of the structure, system, or component?

4. Does this design or design change involve or change design that has previously undergone formal design verification?
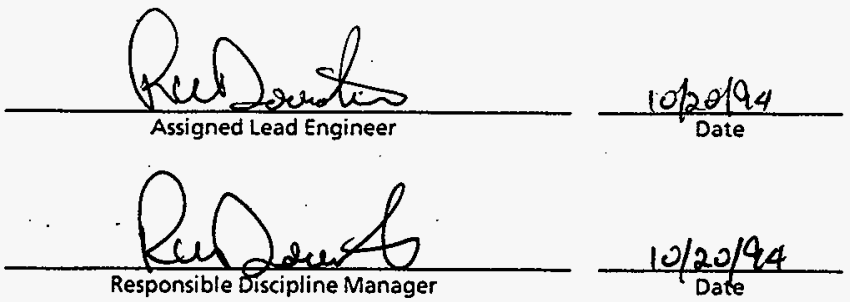

Original Design Package Distribution:

Project Engineer

Chief Design Engineer

Engineering Document Control
Design Change Distribution:

Attach to Engineering Change Notice 


\section{KAISER ENEINEERS} HANFORD

\section{DESIGN ANALYSIS}

Client WHC

subject Miscellaneous Mechancial (HVAC)

Equipment Foundations

Location TANK 241-C-106
Calc. No. W320-24-023

Revision 0

Page Ho. 1 of 20

Wo/Job No. W-320/ER-4319

Date $06 / 27 / 94$

By M.R Custer MRC

Checked $10 / 14 / 94$

Revised

\section{OBJECTIVE:}

The objective of this calculation is to provide the detailed design for the Chiller and Pump, equipment foundation. The design of the foundation shall be performed in accordance with ACI-318 (Strength Design) and the Uniform Building Code (1991), as required by the project design criteria, SDC-4.4, Rev.11. Since definitive information regarding the weights and geometry of the equipment will not be available, due to long lead time for purchase, conservative estimates of the weights and equipment geometry will be used to perform the analysis and design. This calculation provides the Chiller and Pump foundation part of the active ventilation system (Outside tank farm).

\section{CRITERIA:}

1. DOE ORDER 6430.1A (04/06/89)

2. HANFORD PLANT STANDARDS, STANDARD ARCH./CIVIL DESIGN CRITERIA, SDC-4.1, REV.11

3. FUNCTIONAL DESIGN CRITERIA, WHC-SD-W320-FDC-001, REV.2

4. WHC-SD-WM-SEL-033, REV.0-C, Interim Safety Equipment List for 241-C-106 WASTE RETRIEVAL

5. ASCE $7-88$

DATA:

1. The equipment and foundation for the equipment in this caiculation are Safety

Class 3 per Criteria 4. (See Active Ventilation System classification)

ASSUMPTIONS: Weights and equipment geometry will be conservatively assumed and will be verified on receipt and review of the vendor drawings.

METHODS: Hand calculations

REFERENCES:

1. ACI 318-91, Building Code Requirements for Reinforced Concrete.

2. Uniform Building Code (UBC), (1991 Edition).

3. HANFORD PLANT STANDARDS, STANDARD ARCH./ CIVIL DESIGN CRITERIA, SDC-4.1, REV.11.

4. Foundation Analysis and Design, Joesph E. Bowles (1st Edition).

5. ASCE 7-88 (American Society of Civit Engineers Standard), Minimum Design Loads for Buildings and 0ther Structures. 
HNF-2467, Rev.0

\section{KA/SER ENGINEERS}

HANFERE

DESIGN ANALYSIS

client WHC

subject Miscellaneous Mechanical (HVAC)

Equipment Foundations

Location TANK 241-C-106
Calc. No. W320-24-023

Revision 0

Page No. 2 of 20
Wo/Job No. W-320/ER-4319

Date $06 / 27 / 94$

By M.R Custer MRE

Checked $10 / 14 / 94$

BY M.B. LASOTA Mre

Revised

By

\section{CALCULATIONS:}

Refer to Design Calculation Section

CONCLUSIONS:

The foundation for the chiller and pumps is designed as a slab on grade. The loads developed in the calculation, while conservative are minimal, based on the extensive, physical area required by the piping and equipment configuration. The design satisfies the design requirements for the foundation. 
HNF-2467, Rev.0

KAISER ENGINEERS

HANFDRD

DESIGN ANALYSIS

client WHC

subject Miscellaneous Mechanical (HVAC)

Equipment Foundations
Calc. No. W320-24-023

Revision 0

Page No. 3 of 20

wo/Job No. W-320/ER-4319

Date 06/27/94 By M.R Custer MRC

checked 10/14/94 By M. B. LASOTA YRe

Revised

By

\section{DESIGN CALCULATIONS:}

The design and analysis of the chiller and pump foundation consists of developing conservative estimates for the weights of the major equipment, determine relative locations for each equipment on the foundation and design of the concrete foundation to support the load configuration. All equipment supported on the foundation is Safety Class 3 per Reference 4, therefore the design of the foundation is Safety Class 3 .

\section{DESIGN REQUIREMENTS:}

- Soi1 Category reference drawings: $\mathrm{H}-2-70495$ and $\mathrm{H}-2-70498$

Soil slightly silty, very coarse to coarse sand

- Allowable soil pressure (foundation design), $1500 \mathrm{lb} / \mathrm{ft}^{2}$. (Reference 2, Table 29-B)

- Coefficient of Sliding $=0.25$

(Reference 2, Table 29-B)

- Minimum footing dimensions,

(Reference 2, Table 29-B)

- Snow/Roof Live Load, $20 \mathrm{lbs} / \mathrm{ft}^{2}$

(Reference 3)

- Wind Load,

(Reference 3)

- Basic wind speed, $70 \mathrm{mph}$

- Importance Factor, 1.07

- Exposure Category, C 


\section{KA/SER ENGINEERS} HANFDRD

\section{DESIGN ANALYSIS}

client WHC

subject Miscellaneous Mechanical (HVAC)

Equipment Foundations
Calc. No. W320-24-023

Revision 0

Page No. 4 of 20
wo/Job No. W-320/ER-4319

Date 06/27/94 By M.R Custer MeC

Checked 10/14/94 By M.B. LA50TA Yfue

Location TANK 24I-C-106

Reyised

By

\section{DESIGN LOADS:}

\section{DEAD LOADS}

- Mechanical equipment (i.e the chitler, expansion tanks, air separator and pumps), piping/supports and an electrical distribution panel. Estimated weights for each equipment are:

\section{EQUIPMENT}

a) Chiller $(\mathrm{CH}-\mathrm{I})$

b) Expansion Tank (ET-1)

c) Expansion Tank (ET-2)

d) Pump $(P-1)$

e) Pump $(P-2)$

f) Air Separator Tank

g) Elect. Dist. Panel

h) Piping/Supports

Total Equipment Weight $=$
WEIGHT

$10,0007 \mathrm{bs}$

1000 lbs

500 1bs

500 lbs

$500 \mathrm{lbs}$

200 lbs

300 1bs

500 lbs

$13500 \mathrm{ibs}$

\section{AREA (sf.ft)}

54

100

Totaled in (b) Totaled in (b)

NOTE: Slab critical area for loading is located under the Chiller

- Dead Load - Foundation (assume 12 "thick slab):

Wt. $s 1 \mathrm{ab}=10.0 \mathrm{ft} \times 25.5 \mathrm{ft} \times 1.0 \mathrm{ft} \times 150 \mathrm{lbs} / \mathrm{ft}^{3}=$

Total Concrete Weight $=38250$ 1bs $/ 38.3 \mathrm{Kips}$ 
HNF-2467, Rev.0

\section{KAISER ENGINEERS}

HANFDRE

DESIGN ANALYSIS

client WHC

subject Miscellaneous Mechanical (HVAC)

Equipment Foundations
Calc. No. W320-24-023

Revision 0

Page No. 5 of 20
wo/Job No. W-320/ER-4319

Date 06/27/94 By M.R Custer MRC

checked 10/14/94 By H.B. LASOTA Mre

Location TANK 241-C-106

Revised

By

LIVE LOAD: $\quad$ L.L $=60 \mathrm{psf}$

$L . L=0.06(10 \mathrm{ft}).(25.5 \mathrm{ft})=.15.3 \mathrm{k}$

(Reference 5)

EQUIPMENT LIVE LOAD:

- Equipment Live Load, $\mathrm{LL}_{e}=$ Equip. Live Load $\times 1.5$

(Reference 2)

The equipment live load for this design is considered negligible due to the extremely, small magnitude of the loads transmitted by the rotating equipment (pumps) and chiller unit in addition to consideration of the orientation of the fan units of the chiller. An accepted, simplified approach to determine adequacy of a foundation is a comparison of the masses (mass ratio). The weight of the foundation should be 3 to 5 times the weight of the rotating equipment. For this case the ratio is: (Reference 5, page 601)

Mass Ratio $=40.6 / 11.2$ (equipment contributing) $=3.6$ (conservative)

Actual equipment loads transmitted to the foundation are considered in addition to the other loads on the equipment to design the equipment supports.

WIND LOAD:

- Determine wind load (genera1):

$\mathrm{q}_{\mathrm{z}}=0.00256 \mathrm{Kz}(\text { IV })^{2}$, where

(Reference 6)

$K_{2}=0.80$ (Exposure $C$ )

(Reference 3)

$I=1.07$

$V=70 \mathrm{mph}$

$q_{z}=0.00256(0.80)[(1.07) 70]^{2}=11.5 \mathrm{lb} / \mathrm{ft}^{2}$

$F=q_{z} G_{h} C_{f} A_{f}$, where:

(Reference 5)

$G_{h}=1.32$

(Ref. 5, Table 8)

$C_{f}=1.4$

(Ref. 5, Table 12)

$F=11.5(1.32)(1.4) A_{f}$,

$F=21.25 \mathrm{lbs} / \mathrm{ft}^{2} \times A_{f}$

Page E-8 
KAISER ENGINEERS

HANFDRE

DESIGN ANALYSIS

client WHC

subject Miscellaneous Mechanical (HVAC)

Equipment Foundations

Calc. No. W320-24-023

Revision 0

Page No. 6 of 20

Wo/Job No. W-320/ER-4319

Date $06 / 27 / 94$ By M.R Custer MRC

checked 10/14/94 ву M.B. LASOTA Yue

Revised

By

- Wind forces on major equipment:

$\begin{array}{llc}\text { Equipment Item } & \text { Projected Area } & \text { Wind Force } \\ \text { Chiller } & 6.5 \mathrm{ft} \times 7.5 \mathrm{ft}=48.8 \mathrm{sq} . \mathrm{ft} & 1036 \mathrm{lbs} \\ \text { Tank, TK-1 } & 4.2 \mathrm{ft} \times 2.0 \mathrm{ft}=8.3 \mathrm{sq} . \mathrm{ft} & 177 \mathrm{lbs} \\ \text { Tank, TK-2 } & 1.0 \mathrm{ft} \times 2.0 \mathrm{ft}=2.0 \mathrm{sq} . \mathrm{ft} & 43 \mathrm{lbs} \\ \text { Pumps (simi1iar to TK-2) } \times(2) & 86 \mathrm{lbs} \\ \text { Elect.Distr.Pn1 } & 4.0 \mathrm{ft} \times 6.0 \mathrm{ft}=24 \mathrm{sf} . \mathrm{ft} & 510 \mathrm{lbs} \\ & & \text { Total }=1852 \mathrm{lbs} \text { or } 1.9 \mathrm{k}\end{array}$

The location of the lateral wind force is reasonably close to the center of gravity of the equipment, therefore the wind forces will coincide with the seismic forces.

NOTE: C-CW-TK-3 not considered since it is sheltered by TK-1 in the critical wind direction.

SNOW LOAD: $20 \mathrm{lbs} / \mathrm{ft}^{2}$

(Reference 3)

$S=10.0 \mathrm{ft} \times 25.5 \mathrm{ft} \times 20 \mathrm{lbs} / \mathrm{ft}^{2}$

$S=5100 \mathrm{lbs}=5.1 \mathrm{k}$

EARTHQUAKE LOADS:

- Lateral Seismic Force, $F_{p}=Z I C_{p} W_{p}$, where:

(Reference 2, Eq.36-1)

$Z=0.20$

$I=1.25$

$C_{p}=.75 \times 2=1.5$, (for non rigid equipment)

$F_{p}^{p}=(0.20)(1.25)(1.5) W_{p}=0.375 W_{p}$

$E_{n-s}^{p}=V_{n-s}(100 \% \max$.

(Reference 2, Table 23-I)

(Reference 3)

(Reference 3, Table 23-P)

$E_{e-w}=V_{e-w}(30 \% \max$.

(Reference 2)

(Reference 2)

$E_{n-s}=V_{n-s}=0.375 W_{p}=0.375(13500)=5063$ lbs $=\underline{5.1 \mathrm{Kips}}$

$E_{e-w}=V_{n-s}=0.375 W_{p}(0.3)=0.375(13500)(0.3)=1518$ lbs $=1.5$ Kips

$E_{\text {(SRss) }}^{.}=\left[5.1^{2}+1.5^{2}\right]^{1 / 2}=$

(Reference $2 \& 3$ )

$E_{\text {(sRss) }}=5.3$ Kips (Apply force in direction providing maximum load conditions) 
HNF-2467, Rev.0

KAISER ENGINEEFS

HANFERE

DESIGN ANALYSIS

Cliene WHC

subject Miscellaneous Mechanical (HVAC)

Equipment Foundations

Calc. No. W320-24-023

Revision 0

Page No. 7 of 20

Wo/sob No. W-320/ER-4319

Date $06 / 27 / 94$ By M.R Custer MRC

checked 10/14/94 By H.B. LASOTA Mul

Revised

By

- Distribution of seismic forces by component:

LOAD IDENTIFIER

a) Chiller (R-1)

b) Expansion Tank (TK-1)

c) Expansion Tank (TK-2)

d) Pump (P-1)

e) Pump (P-2)

f) Air Separator Tank (TK-3) 200

g) Elect. Dist. Pane]

h) Piping/Supports

TOTAL WEIGHT $=$
VERTICAL COMP./ RATIO

$10,000(5300 / 13500)$

1000

500

500

500

300

500

$13,5001 \mathrm{bs}$
HORIZ. COMPONENT

3925 lbs/ 3.90 Kips

$393 \quad 0.39$

196 " 0.20

$196 " 0.20$

$196 " 0.20$

$79 \quad 0.08$

$118 \quad 0.12$

$196 " 0.20$

5300 lbs/ 5.3 Kips

LOCATE THE CENTER OF GRAVITY/CENTER OF MASS FOR THE EQUIPMENT/COMPONENTS:

Component Description

a) Chiller, R-I

b) Tank, TK-1

c) Tank, TK-2

d) Pump, P-1

e) Pump, P-2

f) Air Sep.Tank TK-3

g) Elect.Pane]

h) Pipe/Supports
Weight, 1 bs $\quad[X$-distance,

10000 lbs $5.67 \mathrm{ft}$

1000 lbs $14.42 \mathrm{ft}$

$500 \quad 1 \mathrm{bs} \quad 17.58 \mathrm{ft}$

500 lbs $18.83 \mathrm{ft}$

500 lbs $22.33 \mathrm{ft}$

200 lbs $14.42 \mathrm{ft}$

300 lbs $15.58 \mathrm{ft}$

$500 \cdot$ lbs $16.25 \mathrm{ft}$
Z-Distance, H-Distance, (ft.)

$5.83 \mathrm{ft}$

$4.0 \mathrm{ft}$

$5.25 \mathrm{ft}$

$2.0 \mathrm{ft}$

$3.25 \mathrm{ft}$

$1.5 \mathrm{ft}$

$6.50 \mathrm{ft} \quad 1.5 \mathrm{ft}$

$6.50 \mathrm{ft} \quad 1.5 \mathrm{ft}$

$1.25 \mathrm{ft} \quad 2.0 \mathrm{ft}$

$7.42 \mathrm{ft} \quad 3.5 \mathrm{ft}$

$3.00 \mathrm{ft} \quad 3.0 \mathrm{ft}$ 
HNF-2467, Rev.0

KA/SER ENGINEERS

HANFDRD

DESIGN ANALYSIS

client WHC

subject Miscellaneous Mechanical (HVAC)

Equipment Foundations
Calc. No. W320-24-023

Revision 0

Page No. 8 of 20
wo/Job No. W-320/ER-4319

Date 06/27/94 By M.R Custer MLC

checked 10/14194 By M.B. LA507A Mue

Revised By

Reference points for the $x, z$, and $h$ distances are as follows:

1) $x$-distance, dimension from the west edge of the slab to the approximate center of mass of the component in the east-west direction.

2) z-distance, dimension from the south edge of the slab to the approximate center of mass of the component in the north-south direction.

3) h-distance, dimension from the top of the slab to the approximate center of mass of the component in the vertical direction.

CENTER OF GRAVITY: (Ref. sketch, Figure 1, page 16)

- Center Gravity, East-West direction

$$
\begin{aligned}
& \bar{x}=\Sigma P_{x} / \Sigma P=116273 \mathrm{ft}-1 \mathrm{~b} / 13500 \mathrm{lbs} \\
& \overline{\mathbf{x}}=8.61 \mathrm{ft}, \text { eccentricity, } \mathrm{e}(\mathrm{x})=25.5 / 2-8.61=4.14
\end{aligned}
$$

- Center of Gravity, North-South direction

$$
\begin{aligned}
& \bar{z}=\Sigma P_{z} / \Sigma P=75651 \mathrm{ft}-1 \mathrm{bs} / 13500 \mathrm{lbs} \\
& \bar{z}=5.60 \mathrm{ft}, \text { eccentricity, } e(z)=10.0 / 2-5.60=-0.60
\end{aligned}
$$

CENTER OF GRAVITY: (Above the top of the foundation) (Ref. sketch, Figure 1, page 16)

- Eccentricity $(\bar{h})$ :

$\hbar=\Sigma P_{h} / \Sigma P=47200 \mathrm{ft}-1 \mathrm{bs} / 135001 \mathrm{bs}$

$\bar{h}=3.50 \mathrm{ft}$, Center of Gravity above the top of the foundation 


\section{KAISER ENGINEERS}

HANFםRD

DESIGN ANALYSIS

client WHC

subject Miscellaneous Mechanical (HVAC)

Equipment Foundations
Calc. No. W320-24-023

Revision 0

Page No. 9 of 20

wo/Job No. W-320/ER-4319

Date $06 / 27 / 94$ By M.R Custer MRC

Checked 10/14/94 By H,B, LASOTAMJUC

Revised

By

DETERMINE GOVERNING LOAD COMBINATION: (Equipment support design)

- Load Combinations:
1) $U=D+L+(L L r$ or $S$ )
2) $\mathrm{U}=\mathrm{D}+\mathrm{L}+\mathrm{W}$ or $\mathrm{E}$
3) $U=D+L+W+S / 2$
4) $U=D+L+S+W / 2$
5) $U=D+L+S+E$

Abbreviations:

(Reference 2)

- The evaluation of each load combination is required to determine the maximum reactions for each equipment/ component for design of the supports:

- $\mathrm{L}$ is constant; $\mathrm{LL}_{r}=0.0$

- UNDERLINE - designates load combination governing support design

ITEM: SQ FT D $\quad W \quad$ S $\quad E \quad L$ Load Combination:
1) 2) 3)
4)

a) $\quad \begin{array}{llllll}115 & 10.0 & 1.04 & 2.30 & 3.90 & 6.90\end{array}$

a) 115

b) $\quad 21 \quad 1.0$

$0.18 \quad 0.42$

$0.39 \quad 1.26$

19.20

$16.90 / 18.05 /$

c)

c) $12 \quad 0.5$

$\begin{array}{llll}0.04 & 0.24 & 0.20 & 0.72\end{array}$

0.00

3.90

1.04

$19.20 / 19.20 /$

$0.52 \quad \underline{3.90}$

\begin{tabular}{|c|c|c|c|c|c|c|c|c|c|c|c|}
\hline d) & 21 & 0.5 & 0.04 & 0.42 & 0.20 & 1.26 & $\begin{array}{l}2.18 / \\
0.00\end{array}$ & $\begin{array}{l}1.76 / \\
0.20\end{array}$ & $\begin{array}{l}1.97 / \\
0.04\end{array}$ & $\begin{array}{l}2.18 / \\
0.02\end{array}$ & $\frac{2.18 /}{\underline{0.20}}$ \\
\hline e) & 21 & 0.5 & 0.04 & 0.42 & 0.20 & 1.26 & $\begin{array}{l}2.18 / \\
0.00\end{array}$ & $\begin{array}{l}1.76 / \\
0.20\end{array}$ & $\begin{array}{l}1.97 / \\
0.04\end{array}$ & $\begin{array}{l}2.18 / \\
0.02\end{array}$ & $\frac{2.18 /}{\underline{0.20}}$ \\
\hline f) & 14 & 0.2 & 0.00 & 0.28 & 0.08 & 0.84 & $\begin{array}{l}1.32 / \\
0.00\end{array}$ & $\begin{array}{l}1.04 / \\
0.08\end{array}$ & $\begin{array}{l}1.18 / \\
0.00\end{array}$ & $\begin{array}{l}1.32 / \\
0.00\end{array}$ & $\frac{1.321}{0.08}$ \\
\hline g) & 21 & 0.3 & 0.51 & 0.42 & 0.12 & 1.26 & $\begin{array}{l}1.98 / \\
0.00\end{array}$ & $\begin{array}{l}1.56 / \\
\underline{0.51}\end{array}$ & $\begin{array}{l}1.77 / \\
0.51\end{array}$ & $\begin{array}{l}1.98 / \\
0.26\end{array}$ & $\frac{1.98 /}{0.12}$ \\
\hline h) & 30 & 0.5 & 0.20 & 0.60 & 0.20 & 1.80 & $\begin{array}{l}2.90 / \\
0.00\end{array}$ & $\begin{array}{l}2.30 / \\
0.20\end{array}$ & $\begin{array}{l}2.60 / \\
0.20 \\
\text { TOTAL }\end{array}$ & $\begin{aligned} & 2.90 / \\
& 0.10 \\
&= 33.9 /\end{aligned}$ & $\frac{\frac{2.90 /}{0.20}}{5.29}$ \\
\hline
\end{tabular}

Page E-12 
DESIGN ANALYSIS

Client WHC

subject Miscellaneous Mechanical (HVAC)

Equipment Foundations
Calc. Ho. W320-24-023

Revision 0

Page No. 10 of 20

WO/Job No. W-320/ER-4319

Date $06 / 27 / 94$ By M.R Custer MRC

checked 10/14194 By M.B.LASOTA Bul

Location TANK 241-C-106

Revised

By

\section{DETERMINE THE GOVERNING LOAD COMBINATION: (Foundation design)}

- Load Combinations:

(Reference 1)

$(D=$ DEAD LOAD, $L=$ LIVE LOAD, $W=$ WIND LOAD, $E=$ EARTHQUAKE LOAD)

1) $U=1.40+1.7 \mathrm{~L}$ (Minimum design requirements)

2) $U=0.75(1.4 D+1.7 \mathrm{~L}+1.7 \mathrm{~W})$

3) $U=0.9 D+1.3 W$

In addition, if earthquake loads are included in the design, load combinations of section 2609(c)(2), (Reference 3) apply, except (I.IE) is substituted for (W), as follows:

4) $U=0.75(1.4 \mathrm{D}+1.7 \mathrm{~L}+1.87 \mathrm{E})$

5) $U=0.9 D+1.43 E$

also for earthquake loading, use the following:

6) $U=1.4(D+L+E)$

7) $U=0.9 D+/-1.4 E$

- Evaluate Load Combinations:
1) $\quad U=1.4(13.5)+1.7(15.3)$
$=44.9$ kips
2) $U_{\text {(VERT.) }}=0.75[1.4(13.5)+1.7(15.3)]$
$=33.7 \mathrm{kips}$
$U_{\text {(HORIZ.) }}=0.75[1.7(1.9)]$
$=2.4$ kips
3) $\quad U_{\text {(VERT.) }}^{U}=0.9(13.5)$
$=12.2 \mathrm{kips}$
4) $\quad U_{U}^{U}($ VERT.) $=0.75[1.4(13.5)+1.7(15.3)$
$=2.5 \mathrm{kips}$
5) $\quad \begin{aligned} U & U_{(\text {VERT.) }}=0.9(13.5) \\ U_{(\text {HORIZ.) }} & =1.43(5.3)\end{aligned}$
$=33.7 \mathrm{kips}$
$=7.4 \mathrm{kips}$
6) $\quad U_{\text {(VERT.) }}^{U}=1.4(13.5+15.3)$
$=12.2 \mathrm{kips}$
$=7.6 \mathrm{kips}$
7) $\quad U_{\text {(HORIZ. })}=0.9(13.5) \quad$ (SEE EQUATION 5)
$U_{\text {(HORIZ.) }}^{\text {(HORIZ.) }}=(+/-) 1.4(5.3)$
$=40.3 \mathrm{kips}$
$=7.4$ kips
$=12.2$ kips
$=(+/-) 7.4$ kips

Equation 6: Maximum design load value to be applied to develop foundation design. 
HNF-2467, Rev.0

KAISER ENGINEERS

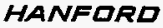

\section{DESIGN ANALYSIS}

client WHC

subject Miscellaneous Mechanical (HVAC) Equipment Foundations
Calc. No. W320-24-023

Revision 0

Page No. 11 of 20

Wo/Job No. W-320/ER-4319

Date $06 / 27 / 94 \quad$ By M.R Custer MRC

checked 10/14/94 ву M.B. Lasota Mul

Location TANK 241-C-106

Revised

By

\section{FOUNDATION DESIGN:}

The foundation required is a mat type on grade. The size of foundation has been predetermined by the physical layout required for the equipment and components.

The foundation dimensions are: $10.0 \mathrm{ft} \times 25.5 \mathrm{ft}$, estimated $12^{11}$ thick

(Reference Figures $1,2 \& 3$ for loads and locations)

- Check foundation size: (Reference sketch, Figures 2,3 page 17)

$$
\begin{aligned}
& q_{e} \text { (effective) }=1.5 \mathrm{k} / \mathrm{ft}^{2}-12^{\prime \prime} / 12^{\prime \prime}\left(150 \mathrm{lb} / \mathrm{ft}^{3}(1 \mathrm{k} / 1000 \mathrm{lb})=1.35 \mathrm{k} / \mathrm{ft}^{2}\right. \\
& \text { A req }=(D . L+L . L) / \mathrm{q}_{\mathrm{e}}=(13.5+15.3) / 1.35 \mathrm{k} / \mathrm{ft}^{2} \\
& \text { A req }=21 \mathrm{ft}^{2}<255 \mathrm{ft}^{2} \quad \ldots \ldots .0 \mathrm{~K}
\end{aligned}
$$

- Check foundation stability:

Safety Factor (Overturning):

$S . F(o t)=[5.0 \mathrm{ft}(38.3 \mathrm{k})(0.85)+4.5 \mathrm{ft}(13.5 \mathrm{k})(0.85)] /$ (Ref.2, Section 2337)

$$
4.5 \mathrm{ft}(5.3 \mathrm{k})
$$

$\underline{S . F}($ ot $)=8.99>1.5 \ldots$. ok

Safety Factor (Sliding):

$\underline{S . F}$ (sliding) $=[.25(0.85)(38.3+13.5)] / 5.3=2.08>1.5 \ldots$ ok

- Determine the center of gravity of the loads relative to the kern:

$k_{x},($ length) $=25.5 \mathrm{ft} / 6=4.25 \mathrm{ft}$

$k_{z},($ width $)=10.0 \mathrm{ft} / 6=1.67 \mathrm{ft}$

$e_{x}=4.14 \mathrm{ft}$ and $e(z)=0.60 \mathrm{ft}$ (per page 8),

$\underline{k}_{x}=4.25 \mathrm{ft}>-\bar{x}=4.14 \mathrm{ft}$. Load in middle $1 / 3$ of slab...OK

$k_{z}=1.67 \mathrm{ft}>-z=0.60 \mathrm{ft}$. . Load in the middle $1 / 3$ of $\mathrm{slab} . \ldots . \mathrm{K}$

Use $q=P / A(+/-) M_{c} / I$ approach (two directions) to determine maximum soil bearing $p$ pressures. 
HNF-2467, Rev.0

\section{KAISER ENGINEERS}

HANFDRD

\section{DESIGN ANALYSIS}

client WHC

subject Miscellaneous Mechanical (HVAC)

Equipment Foundations
Calc. No. W320-24-023

Revision 0

Page No. 12 of 20
Wo/sob No. W-320/ER-4319

Date $06 / 27 / 94 \quad$ By M.R Custer MRC

Checked 10/14194 By M.B. LASOTA bgue

Location TANK 241-C-106

Revised

By

- Determine maximum soil bearing pressure:

$\mathrm{P}_{1}=13.5 \mathrm{k}$ (Equipment load, eccentric with foundation centerlines, $\mathrm{n}-\mathrm{s} / \mathrm{e}-\mathrm{w}$ )

$\mathrm{P}_{2}=15.3 \mathrm{k}$ (Live load at centerline of foundation)

$\mathrm{P}_{3}^{2}=38.3 \mathrm{k}$ (Dead weight of concrete at centerline of foundation)

$M_{x}^{3}=13.5 \mathrm{k}(0.60 \mathrm{ft})=8.1 \mathrm{k}-\mathrm{ft}$ (Moment due to eccentric equipment load)

$M_{z}^{x}=13.5 \mathrm{k}(4.14 \mathrm{ft})=55.9 \mathrm{k}-\mathrm{ft}$ (Moment due to eccentric equipment load)

$M_{e(x)}^{2}=4.5 \mathrm{ft}(5.1 \mathrm{k})=23.0 \mathrm{k}-\mathrm{ft}$ (Moment due to $n-s$ seismic load)

$M_{e(z)}^{e(x)}=4.5 \mathrm{ft}(1.5 \mathrm{k})=6.8 \mathrm{k}-\mathrm{ft}$ (Moment due to e-w seismic 1oad)

Maximum soil pressures:

$q=\Sigma P / A(+/-) \Sigma M_{x}(c) / I_{x}(+/-) \Sigma M_{z}$ (c)/ $I_{z}$, where:

$I_{x}=b d^{3} / 12=\left[25.5(10)^{3}\right] / 12=2125 \mathrm{ft}^{4}$

$I_{z}=b d^{3} / 12=[10(25.5)]^{3} / 12=13818 \mathrm{ft}^{4}$

$q=(13.5 k+15.3 k+38.3 k) / 10 \mathrm{ft}(25.5 \mathrm{ft})$

$+/-[(55.9 k-f t+6.8 k-f t)](12.75 f t) / 13818 \mathrm{ft}^{4}$

$t /-[(8.1 k-f t+23.0 k-f t)](5.0 \mathrm{ft}) / 2125 \mathrm{ft}^{4}$

$q=0.263 \mathrm{k} / \mathrm{ft}^{2}(+/-) \quad 0.058 \mathrm{k} / \mathrm{ft}^{2} \quad(+/-) \quad 0.073 \mathrm{k} / \mathrm{ft}^{2}$

$q_{a}=(t) 0.263(t) 0.058(t) 0.073=+0.394 \mathrm{k} / \mathrm{ft}^{2}$ (maximum)

$q_{b}=(+) 0.263(-) 0.058(+) 0.073=+0.278 \mathrm{k} / \mathrm{ft}^{2}$

$q_{c}=(+) 0.263(+) 0.058(-) 0.073=+0.248 \mathrm{k} / \mathrm{ft}^{2}$

$q_{d}=(+) 0.263(-) 0.058(-) 0.073=+0.132 \mathrm{k} / \mathrm{ft}^{2}$ (minimum)

$\mathrm{q}($ maximum $)=0.394 \mathrm{k} / \mathrm{ft}^{2}$

$q($ minimum $)=0.132 \mathrm{k} / \mathrm{ft}^{2}$.

$q($ maximum $)=0.394 \mathrm{k} / \mathrm{ft}^{2}<q($ all ow. $)=1.5 \mathrm{k} / \mathrm{ft}^{2} \ldots \ldots$ ok 
HNF-2467, Rev.0

\section{KAISER ENGINEERS}

HANFDRD

\section{DESIGN ANALYSIS}

client WHC

subject MisceTlaneous Mechanicat (HVAC)

Equipment Foundations
Calc. No. W320-24-023

Revision 0

Page No. 13 of 20

Wo/Job No. W-320/ER-4319

Date 06/27/94 By M.R Custer MRC

Checked 10114194 вy MiB. Lasota Myl

Revised

By

- Check slab two way shear:

The critical area for punching shear is located at the chiller unit, since this equipment represents approximately $80 \%$ of the load on the foundation.

$q($ ultimate $)=1.7(0.394 \mathrm{k} / \mathrm{ft})^{2}=0.67 \mathrm{k} / \mathrm{ft}^{2}$

Assumed depth of slab, $d=12^{\prime \prime}$, then $d$ effective $=12 " 2^{\prime \prime}=9^{\prime \prime}$

$b_{o}$, (punching shear) $=4(7.33+d / 2)=4\left(7.33 \mathrm{ft}+9^{11} / 2\left(12^{\prime \prime}\right) \mathrm{ft}\right)=30.8 \mathrm{ft}$

$V_{u}=\left[(10.0 \mathrm{ft})(25.5 \mathrm{ft})-7.7 \mathrm{ft}^{2}\right]\left(0.67 \mathrm{k} / \mathrm{ft}^{2}\right)=131 \mathrm{k}$

$\mathrm{d}=131000 \mathrm{1bs} /\left[0.85(4)(4000)^{1 / 2}\left(30.8 \mathrm{ft} \times 12^{\prime \prime}\right)\right]=1.65 "$

$d=1.65 "+3^{\prime \prime}+1^{\prime \prime}=5.65^{\prime \prime}<12^{\prime \prime} \ldots$ ok

- Check slab one way shear:

$$
\begin{aligned}
& V u=(10.0 \mathrm{ft})(2.0 \mathrm{ft})\left(0.67 \mathrm{k} / \mathrm{ft}^{2}\right)=13.4 \mathrm{k} \\
& d=134001 \mathrm{bs} /(.85)(2)(4000)^{1 / 2}\left(120^{\prime \prime}\right)=1.04^{\prime \prime} \\
& d=1.04^{\prime \prime}+3^{\mathrm{n}}+1^{\prime \prime}=5.04^{\prime \prime}<12 \ldots .0 \mathrm{k}
\end{aligned}
$$

- Determine slab flexural reinforcement requirements: (Long Dimension)

$$
\begin{aligned}
& M u=(2.00 \mathrm{ft})(10.0 \mathrm{ft})\left(0.67 \mathrm{k} / \mathrm{ft}^{2}\right)(2.00 \mathrm{ft} / 2)=13.4 \mathrm{k}-\mathrm{ft} \\
& \mathrm{Mu}=13.4 \mathrm{k}-\mathrm{ft}=13400 \mathrm{lb}-\mathrm{ft} \\
& \mathrm{Mu} / 0.90 \mathrm{~b} \mathrm{~d} 2=12(13400) / 0.90(120)(8.5)^{2}=20.6 \text {, Use } \mathrm{p}(\mathrm{min})=0.0018 \\
& \text { As }=0.0018\left(120^{\prime \prime}\right)\left(8.5^{\prime \prime}\right)=\cdot 1.84 \mathrm{in}^{2}, 1.84 \mathrm{in}^{2} / 10 \mathrm{ft}=0.18 \mathrm{in}^{2} / 1 \mathrm{ft} \text { width }
\end{aligned}
$$

Use \#4 $12^{\prime \prime}$ o.c, $\left(0.20 \mathrm{in}^{2} / 1 \mathrm{ft}\right.$. width), ....ok 
HNF-2467, Rev.0

KAISER ENGINEERS

HANFORD

DESIGN ANALYSIS

client WHC

subject Miscellaneous Mechanical (HVAC)

Equipment Foundations
Calc. No. W320-24-023

Revision 0

Page No. 14 of 20
wo/Job No. W-320/ER-4319

Date $06 / 27 / 94 \quad$ By M.R Custer M CC

checked 10114,94 вy M.B.LASOTA Pul

Location TANK 241-C-106

Revised

By

- Determine slab flexural reinforcement requirements: (Short dimension)

$$
\begin{aligned}
& M u=(2.17 \mathrm{ft})(25.5 \mathrm{ft})\left(0.67 \mathrm{k} / \mathrm{ft}^{2}\right)(2.17 \mathrm{ft} / 2)=40.2 \mathrm{k}-\mathrm{ft} \\
& M u=40200 \mathrm{lb}-\mathrm{ft}
\end{aligned}
$$

$\mathrm{Mu} / 0.90 \mathrm{~b} \mathrm{~d} \mathrm{~d}^{2}=12(40200 \mathrm{lb}-\mathrm{ft}) /(0.90)\left(306^{\prime \prime}\right)(8.5)^{2}=24.2$, Use $\mathrm{p}(\min )=0.0018$

As $=0.0018\left(306^{\prime \prime}\right)\left(8.5^{\prime \prime}\right)=4.7 \mathrm{in}^{2}, 4.7 \mathrm{in}^{2} / 25.5 \mathrm{ft}=0.18 \mathrm{in}^{2} / 1 \mathrm{ft}$ width

Use \#4 12" o.c, $\left(0.20 \mathrm{in}^{2} / 1 \mathrm{ft}\right.$. width $), \ldots$. . ok

- Check reinforcement bar development length requirements:

$$
\begin{aligned}
& 1(\mathrm{db})=[0.04 \mathrm{Ab} f(y)] /\left(f^{\prime} \mathrm{c}\right)^{1 / 2} \quad \text { (Reference 2, section 2612(c), page 469) } \\
& 1(\mathrm{db})=0.04(.20)(60000) /(4000)^{1 / 2}=7.6^{11}
\end{aligned}
$$

Id $($ provided $)=\left(2.17 \mathrm{ft} \times 12^{\prime \prime}\right)-3^{\prime \prime}=23^{\prime \prime}>7.6^{\prime \prime} \ldots$. ok

1d (min), per UBC $=12^{\prime \prime}<23^{\prime \prime} \ldots$ ok 
DESIGN ANALYSIS

client WHC

subject Miscellaneous Mechanical (HVAC)

Equipment Foundations
Revision 0

Page No. 15 of 20
WO/Job No. W-320/ER-4319

Date $06 / 27 / 94$

By M.R Custer

MRC

Checked $10 / 14 / 94$ By H.B. LASSTA

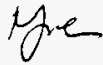

Location TANK 241-C-106

Revised

By

- Determine individual equipment anchorage requirements (equipment to skid): (Loads are multiplied by a factor of 2.5 to address equipment Live Load and an increase factor of 1.5 for rotating equipment).

Equipment

a) Chiller (R-1)

b) Exp. Tank (TK-1)

$0.39 k / E$

c) Exp. Tank (TK-2)

$0.20 k / E$

d) Pump (P-1)

$0.20 k / E$

e) Pump (P-2)

$0.20 \mathrm{k} / \mathrm{E}$

f) Air Separator Tank

$0.08 k / E$
Shear Force

$3.9(2.5) / 4=$

$2.4 \mathrm{k}$

$0.39(2.5) / 4=$

$0.24 \mathrm{k}$

$0.20(2.5) / 4=$

$0.125 k$

It

II
Tension Force

$3.9(2.5)(3.5) /$

$(7.3)(2)=2.3 \mathrm{k}$

$0.39(2.5)(2.0) /$

(2) $(2)=0.49 \mathrm{k}$

$0.20(2.5)(1.0) /$

$(0.75)(2)=0.33 \mathrm{k}$
g) Elect. Dist. Pane1
$0.51 \mathrm{k} / \mathrm{W}$
g) Elect. Dist. Panel $0.51 \mathrm{k} / \mathrm{W}$

$0.08(2.5) / 4=$

$0.08(2.5)(2) /$

$0.05 \mathrm{k}$

(2) $(0.75)=0.27 k$

(E), Earthquake Toad governs; (W), Wind load governs

$0.51(2.5) / 4=$

$0.51(2.5)(3.5) /$

$(2)(1.25)=1.79 \mathrm{k}$

- Anchorage requirements: Cast-in-piace anchor bolts or an equivalent capacity embedded $\mathrm{p} 7 \mathrm{ate} \mathrm{w} /$ welded studs, as required by the equipment vendor.

Anchor Bolt requirements (Skid to Foundation): The anchor bolts will be located $9.0 \mathrm{ft}$ center to center ( $6^{\prime \prime}$ from the edge of the foundation). The shear and tension loads on the anchors are: (Special inspection of the anchor bolts required)

- Shear, $V=5.3 \mathrm{kips}$ (total), providing 10 anchors; $5.3 \mathrm{k} / 10=0.53 \mathrm{k} /$ anchor

- Tension, $T=(5.3 \mathrm{k})(3.5 \mathrm{ft}.) / 9.0 \mathrm{ft}=2.1 \mathrm{k}$, or $2.1 \mathrm{k} / 5=0.42 \mathrm{k} /$ anchor

Try 1/2 dia. cast-in-place anchors, ASTM A $307 \mathrm{w} / 4$ "min. embedment:

(Reference 2, Table No. 26-E), Allowable loads: Shear, $V_{a}=2 \mathrm{k}$, Tension, $T_{a}=1.9 \mathrm{k}$

$$
\begin{aligned}
& V / V_{a}+T / T_{a}<1.0, \\
& 0.53 / 2.0+0.42 / 1.9= \\
& 0.26 k+0.22 k=0.48<1.0 \ldots . .0 k
\end{aligned}
$$

Provide $10-1 / 2^{\prime \prime}$ dia. A 307 anchor bolts, 5 bolts each side along the $25.5 \mathrm{ft}$ dimension $W / 6^{\prime \prime}$ min. edge distance and $4^{\prime \prime}$ min. embedment depth, beyond the bottom of the anchor sleeve.

Page E-18: 
HNF-2467, Rev.0

KAISER ENGINEERS

HANFORD
DESIGN ANALYSIS
Calc. No.W320-24-023

Revision $\frac{0}{16 \text { of } 20}$

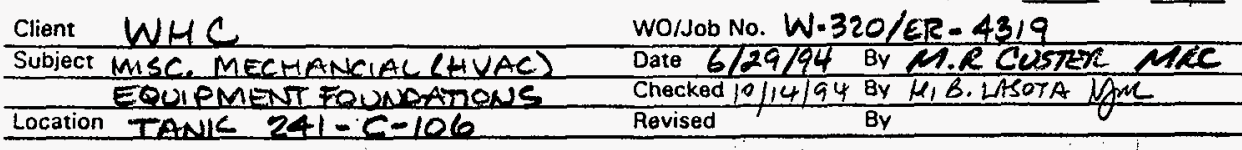

SUMMARY - FOUNDATION LOADS/LOCATIONS

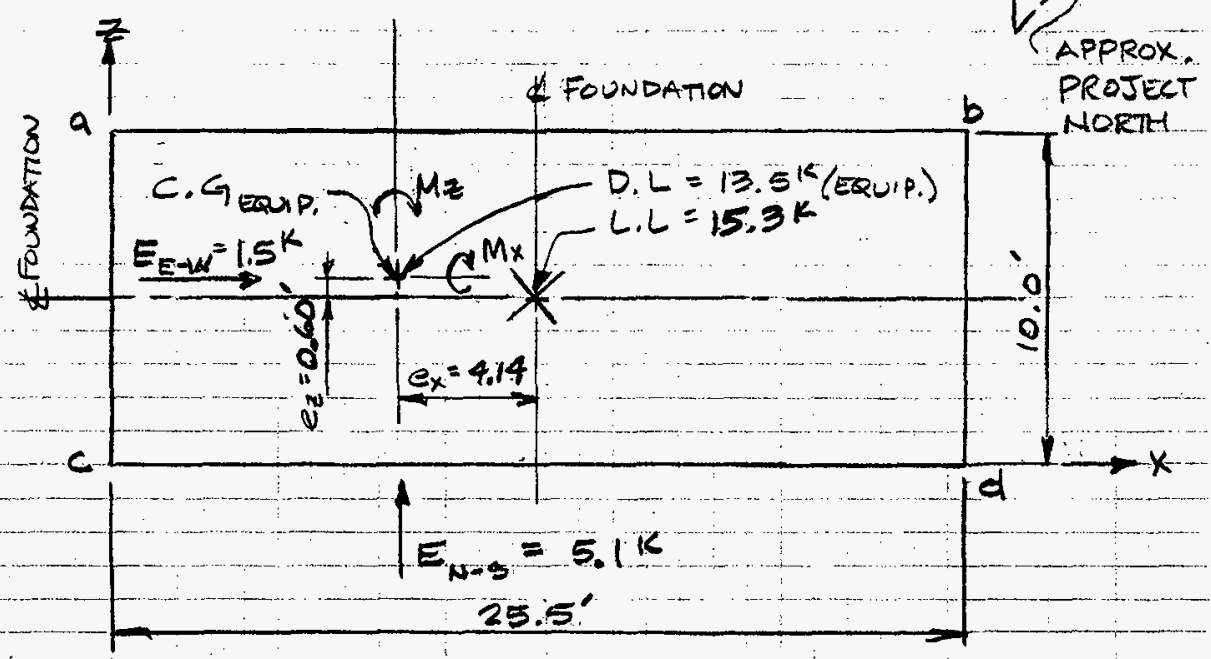

EIGURE 1 $\quad E_{\text {SRSS }}=5.3^{k}$ (DIRECTION of $E_{5 \mathrm{eSS}}=5.3 \mathrm{~K} \& \mathrm{CA}$ MARimum Lontas)

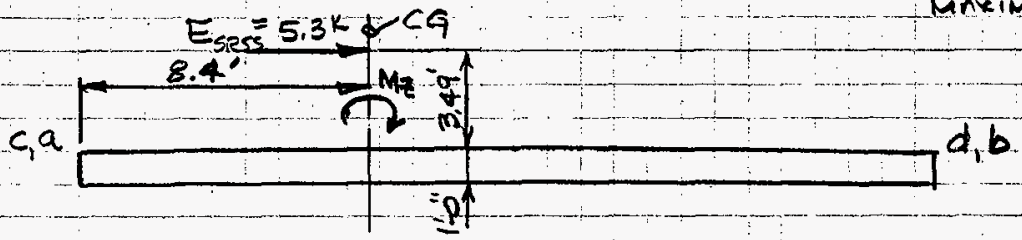


HNF-2467, Rev.0

KAISER ENGINEERS

Calc. No. $w_{320} 24.023$

Revision $\frac{0}{17 \text { of } 20}$

Client W(-

DESIGN ANALYSIS

WO/Job No.

Subject MISCELANEOUS MECHANICAL Date $6 / 29 / 94$ By M.R CUSTEX MAP

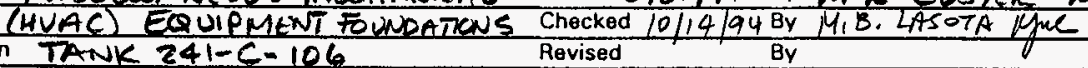
Location TANK $241-C=106$

LOADS ON FOUNDATION:

DEAD, LIVE SEISMIC LOADS

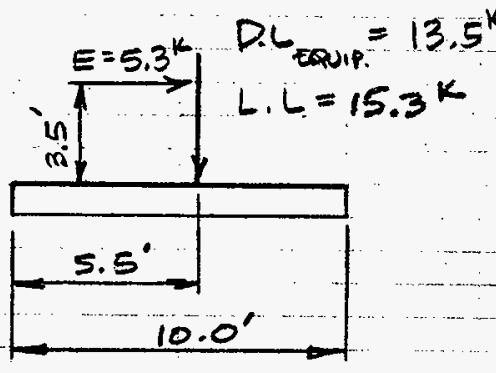

FIGURE 2
DEAD, LIVE F WIND LOADS

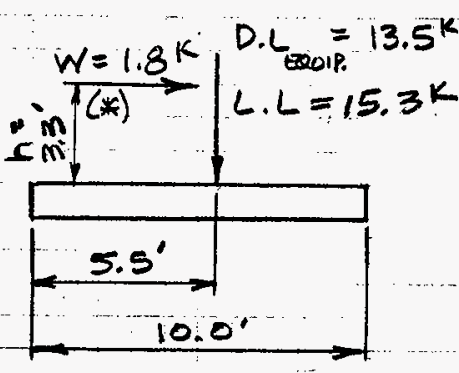

Figure 3

(*) Location of tHe RESULTANT WIND forces IS DETERMINED AS FOLLOWS:

$\sum F_{w 1} h=\left[4.0^{\prime}\left(.912^{k}\right)+1.0^{\prime}\left(.12^{k}\right)+1.0^{\prime}\left(.06^{k}\right)+3.0^{\prime}(.731)\right]$

$\Sigma F_{w} h=6.02, I F E F_{w}=1.8^{\mathrm{k}}$

$h: \frac{6.02}{1.8}=3.3 \mathrm{FT}$. 


\section{DESIGN ANALYSIS}

WO/Job No. ER-4319

Client WHC

Subject Miscellaneous Mechanical (HVAC)

Equipment Foundations

Location: Tank Farm 241-C
Date $9 / 28 / 94$

Revised
Checked $10 / 14 / 94$
Calc. No. W320-24-023

Revision 0

Page No. 18 of 20

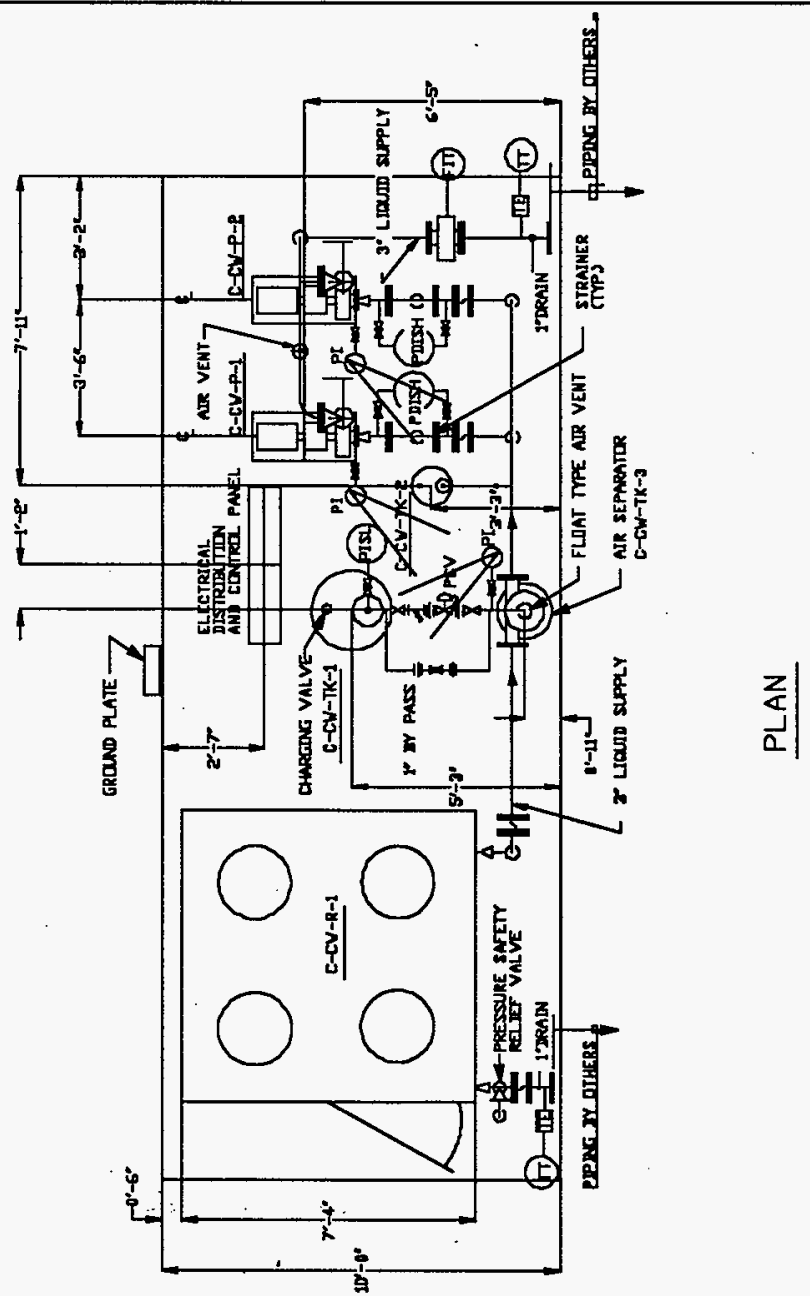


Calc. No. W320-24-023

\section{DESIGN ANALYSIS}

WO/Job No. ER-4319

Client WHC

Subject Miscellaneous Mechanical (HVAC)

Equipment Foundations

Location: Tank Farm 241-C
Date $9 / 28 / 94$

Checked 10/14/94
Revision 0

Page No. 19 of 20
M.R. CUSTer Mare By M.B. LASOTA YuKC By

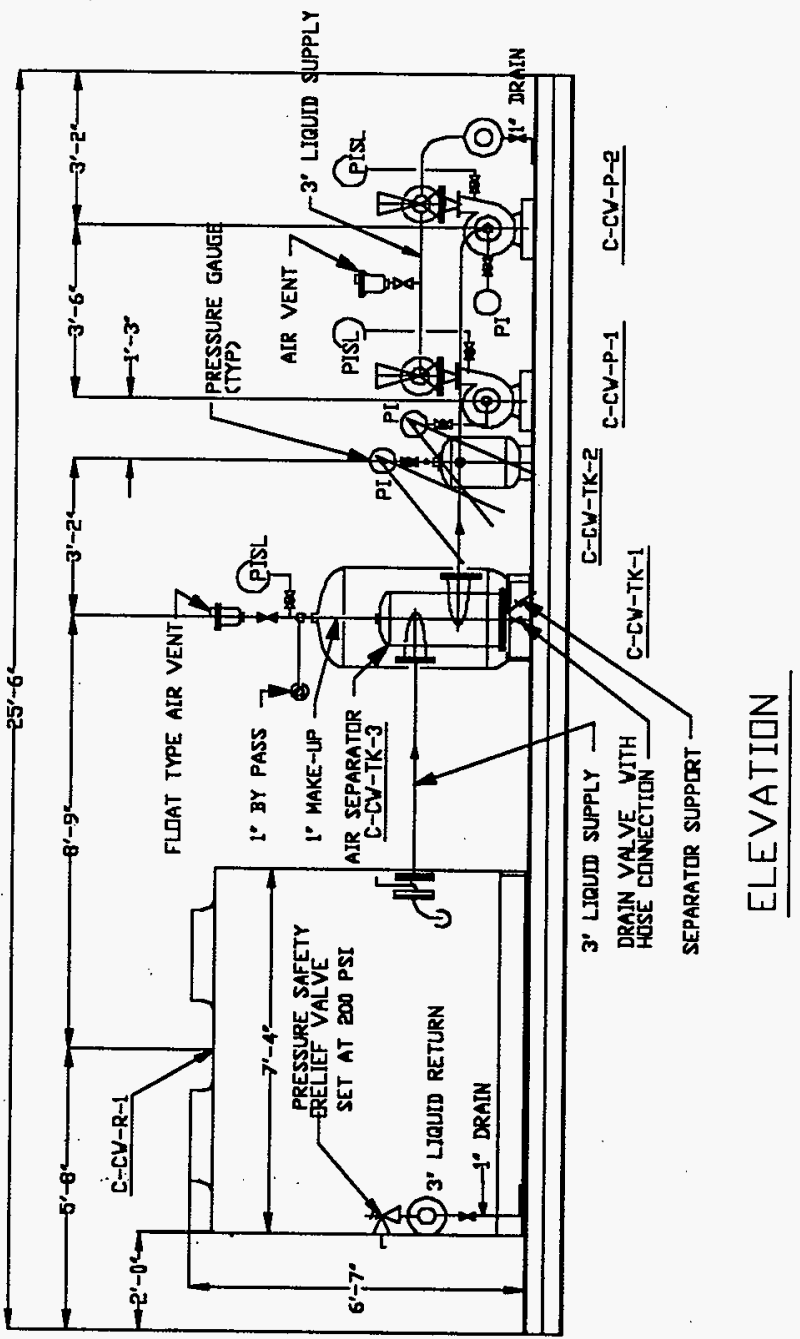




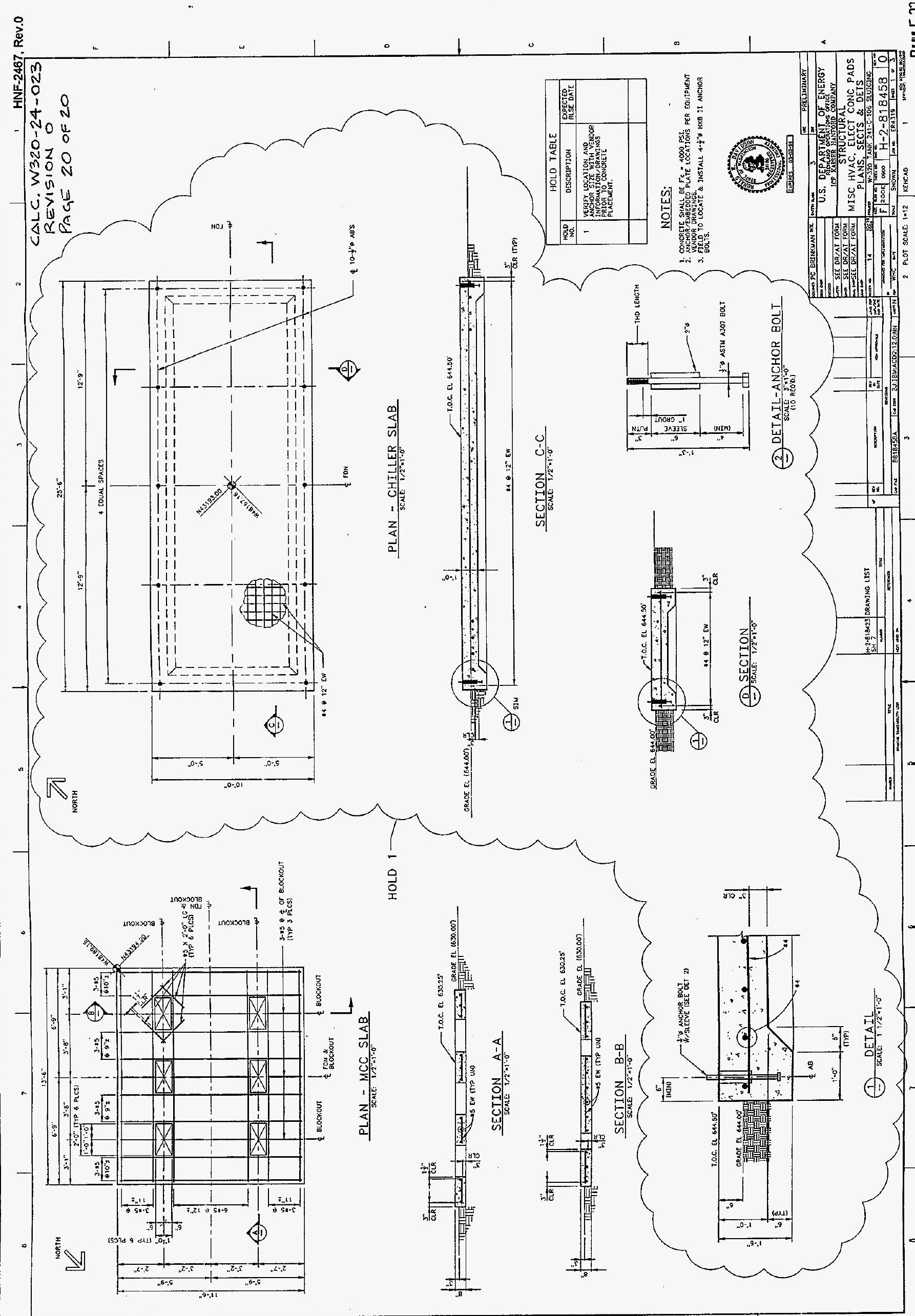


JOHN TUNISON

- STRUCTURAL ENGINEER
PHONE ISOO) CO6-4345

SOUTH 422 CONIFER COUAT

BPOKANE, WASH, DOOOS

PROJECT_-_SHILCER_SLLIP

BY_._ USI__ DATE $2 / 2$

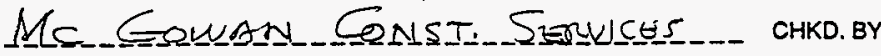
JOB NO. SHT. $I Z 1--$

H) BRSE_SUMANIARY-

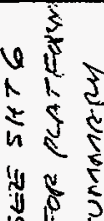

है
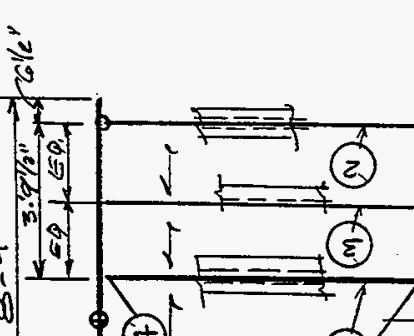

$$
=
$$
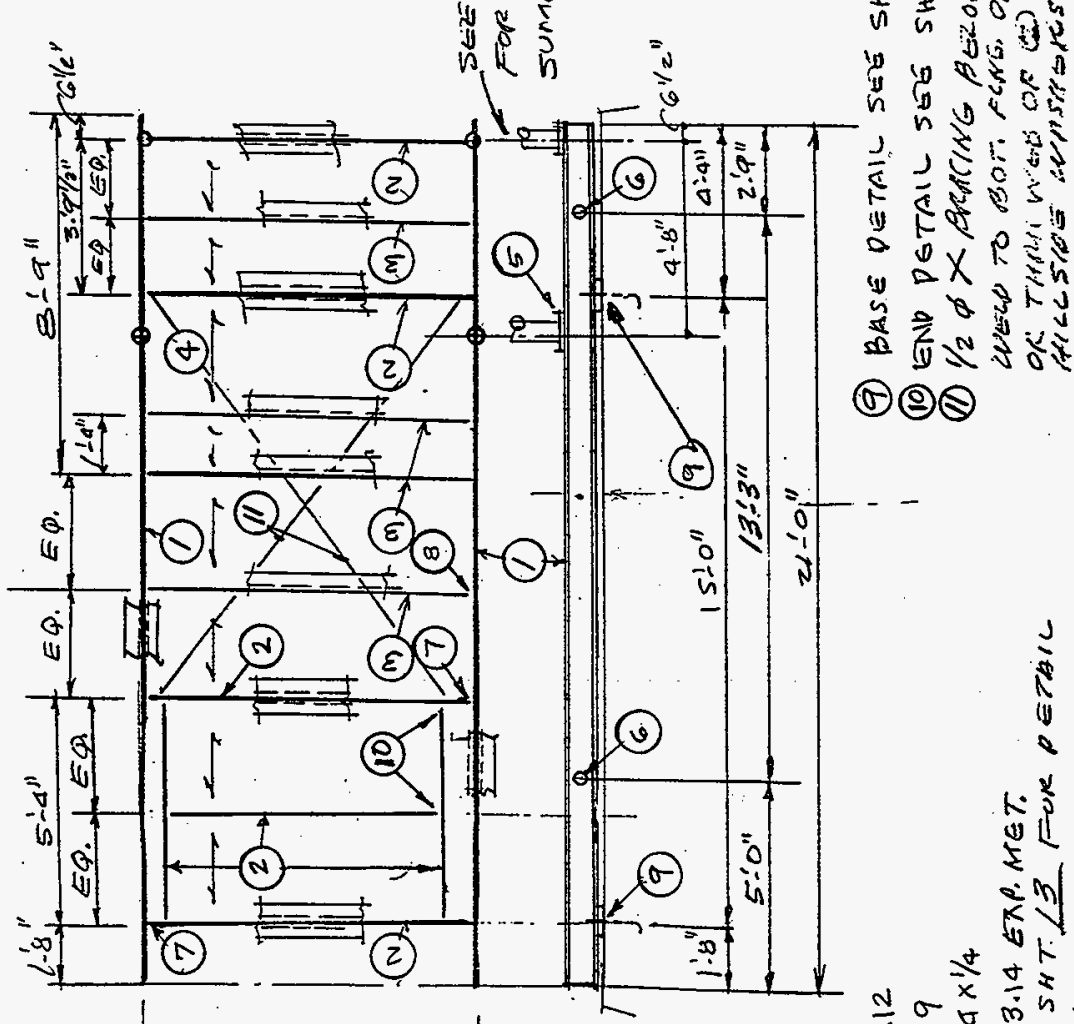

(b)

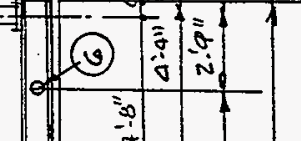

J.

E ह ते है

i) $E$ ए

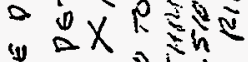

400
30

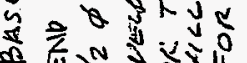

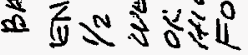

()ㅇ

N 
HNF-2467, Rev.0

JOHN TUNISON

PHONE (5OO) OUTH 4122 CONIFEA COURT SPOKANE. WASH. EOES

$\therefore \quad$ STRUCTURAL ENGINEER BY_LSIT_DATE $7 / 30 / 95$

PROJECT _ EHISEEZL_LEIL. CHKD. BY DATE

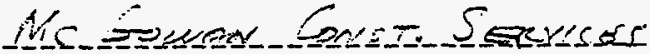

$9 \leq 3 \leq 2$

SHT. $L S T_{-}$

a

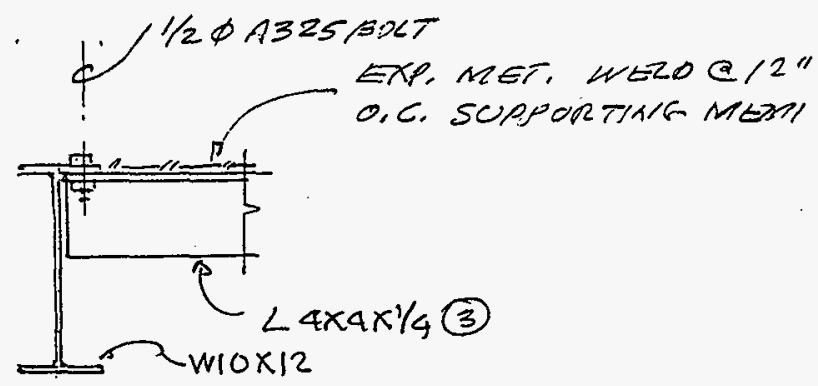

ENO cond:

$$
\begin{aligned}
& V=\sqrt{1.0 C^{2}+(33 \times 1.46)^{2}}=1,54 K \\
& \text { USE } 5 / 3 \text { D HAN. BOLT. }
\end{aligned}
$$

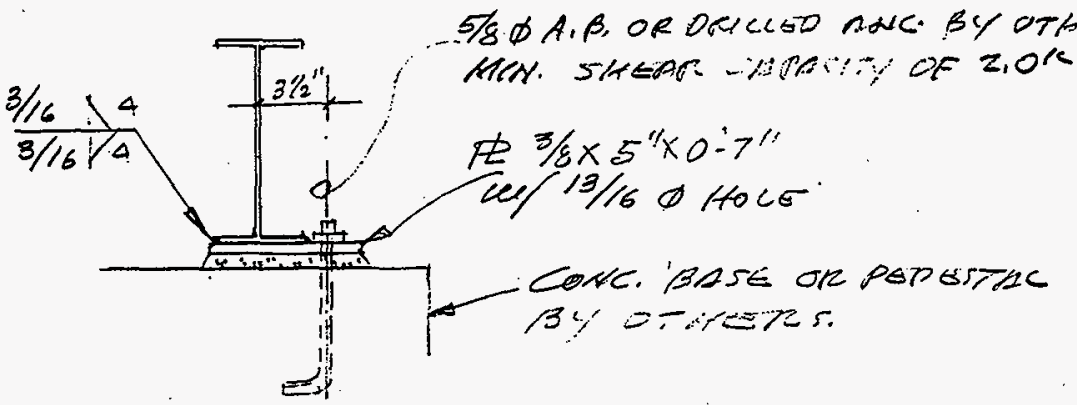

BASE CONAE:TION

Page E-25 
(20)

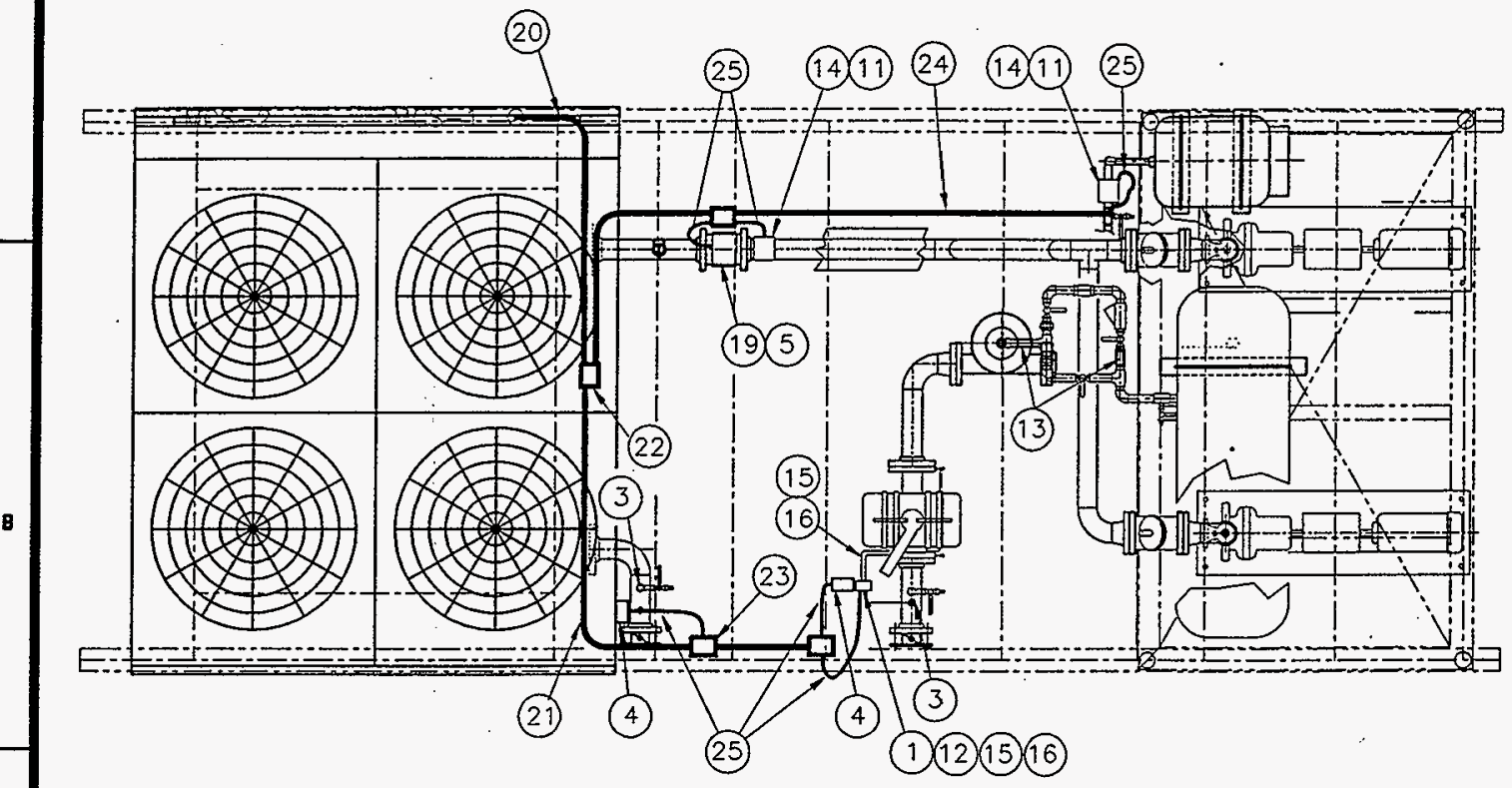

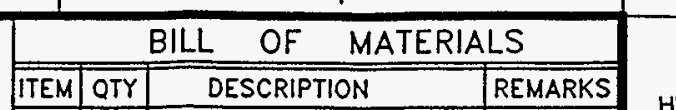

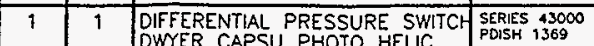

\begin{tabular}{|c|c|l|l|}
\hline & & DWTER CAPSU PHOTO HELIC & \\
\hline 2 & & & \\
\hline 3 & 2 & $\begin{array}{l}\text { CHROMOLOX CPWC-1 } \\
\text { PROTECTIVE WELL }\end{array}$ & TS4 13518.13619 \\
\hline
\end{tabular}

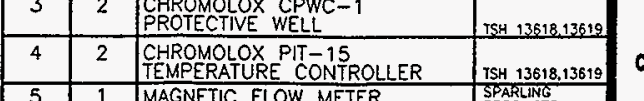

FM625-03-4-8-1-0-0

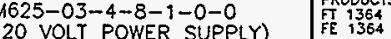

\begin{tabular}{l|l|l|l}
\hline 6 & 40 & $1 / 2^{n}$ & $R G D$ \\
\hline
\end{tabular}

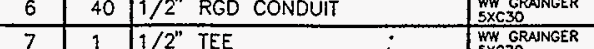

\begin{tabular}{|c|c|c|c|c|}
\hline 7 & 1 & 1 & $5 \times C 70$ \\
\hline 8 & 21 & $1 / 2^{\prime \prime}$ & SEALTIGHT CONNECTION & WW GRANGER \\
\hline
\end{tabular}

\begin{tabular}{|l|l|l|l}
\hline 9 & QTY & 14 THHN/THWN MTW & WW GEANGER \\
5 SC21
\end{tabular}

\begin{tabular}{ll|l} 
& & STRANDED COPPER CONDUCTOR \\
\hline 10 & OTY & $1 / ?^{\prime \prime}$
\end{tabular} \begin{tabular}{l|l|l|l|}
\hline 10 & OTY & $1 / 2^{\prime \prime}$ JIFFY STRAP \\
\hline 11 & 4 & 2 \\
\hline
\end{tabular}

\begin{tabular}{l|l|l|l}
\hline 11 & 4 & $\begin{array}{l}2 \text { VALVE MANIFOLD } \\
\text { M507-CMT ASSEMBLY }\end{array}$ \\
\hline 1 & 12 & 5 VALVE MANFOLD \\
\hline
\end{tabular}

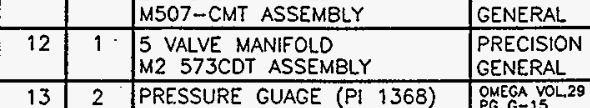

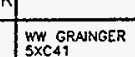

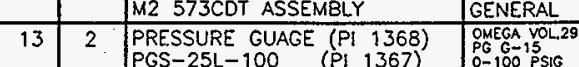

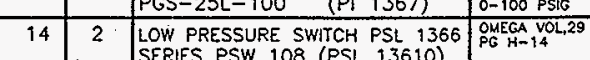

\begin{tabular}{l|l|l|l|l}
15 & 2 & $61 / 2^{\circ}$ NPT $T 06.375^{\circ}$ COMP. FIT \\
\hline
\end{tabular}

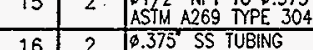

\begin{tabular}{|c|c|l|l|}
\hline 16 & 2 & $\begin{array}{l}0.375^{\circ} \text { SS TUBNG } \\
\text { ASTM A269 TPPE } 304\end{array}$ & \\
\hline 17 & & &
\end{tabular}

\begin{tabular}{|l|l|l|}
\hline 17 & & \\
\hline 18 & & \\
\hline 19 & OTY & \\
\hline
\end{tabular}

\begin{tabular}{l|l|l}
\hline 19 & OTY & 8000 SERIES TWISTED \\
\hline
\end{tabular}

\begin{tabular}{l|l|l} 
& & SHELLDED PAIR \\
\hline 20 & OTY $1 "$ RIGID CONDUI \\
\hline
\end{tabular}

\begin{tabular}{|l|l} 
& MDUSTRES \\
\hline
\end{tabular}

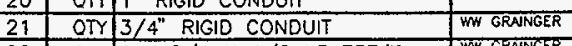

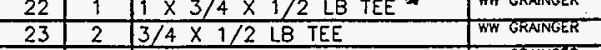

$24,1 / 2 \times 1 / 2$ LB TEE

25 OTY $1 / 2$ " SEALTIGHT FLEX

\begin{tabular}{|l|l|}
\hline 26 & OTY \\
\hline 27 & OTY \\
\hline
\end{tabular}

NOTE: SEE DWG DIAGRAM-WIRING ACDR45B 200,230,460

SEE DWG. DIAGRAM WWRING ACDRA5B 200,230,460 V. DWG. NO 593440101 SHT 1 THROUGH 4 FOR WIRING DIAGRAM.

$\cdots$

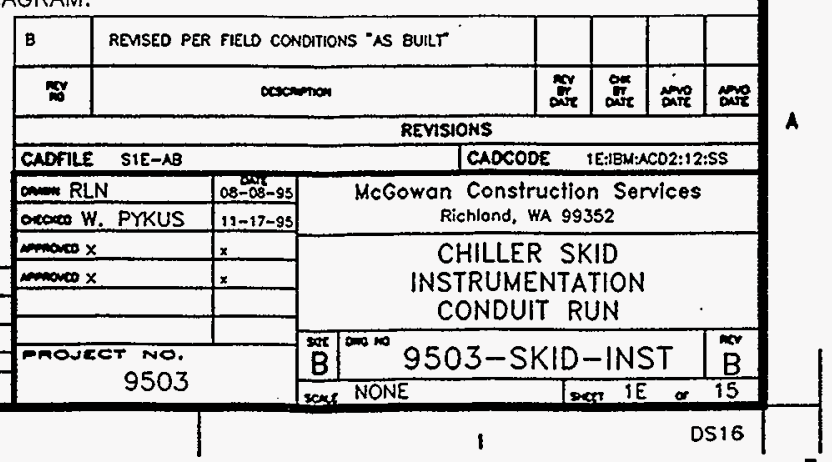




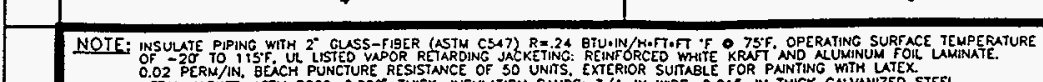

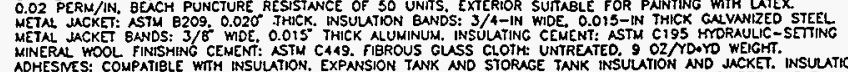

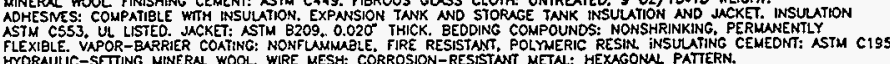

$E$
(4)

(6) (1) (5) 跑运 (5) 1 .

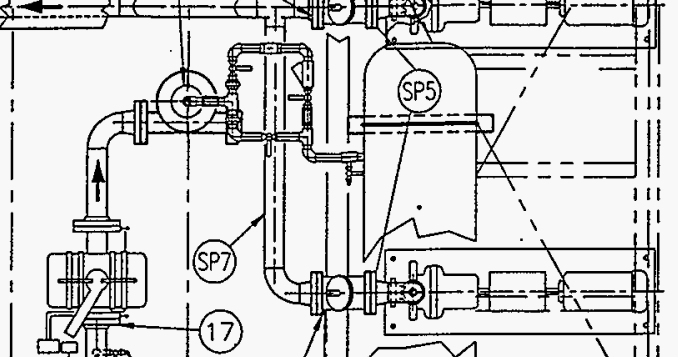

$=-1$

(1)

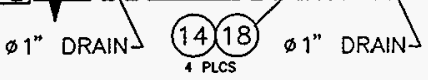

(5)

(3)

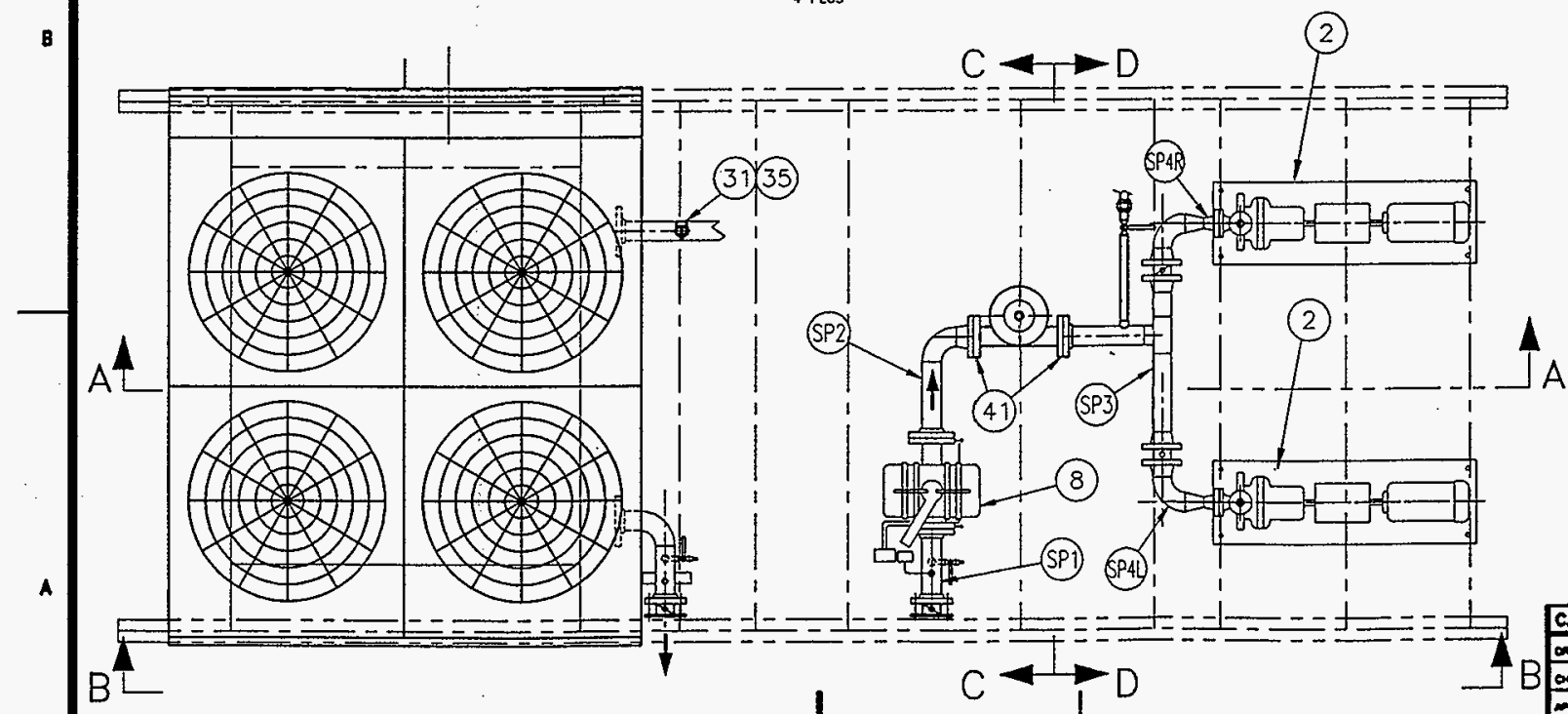

$\checkmark \mathrm{A}$

TI BIL OF MATERIALS

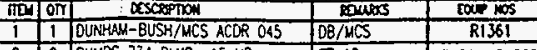

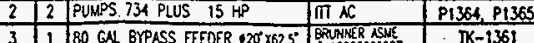

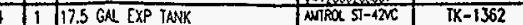

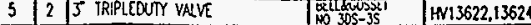

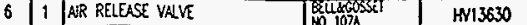

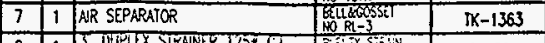

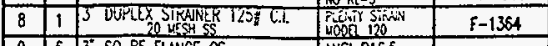
\begin{tabular}{l|l|l|l|l}
9 & 6 & $3^{\circ}$ SO RF FUWGE CS & ANSI 16.5 & \\
\hline
\end{tabular}

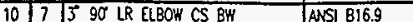
\begin{tabular}{|l|l|l|l|l|l}
\hline 11 & 2 & 3 & 45 LR ELOOH CS BH & ANSI B16.9 & \\
\hline 12 & & 5 & &
\end{tabular}

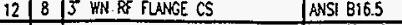

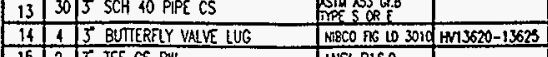

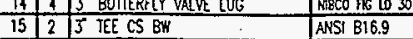
ASSI B16.5 \begin{tabular}{|l|l}
\hline ASIM A307 \\
\hline
\end{tabular}

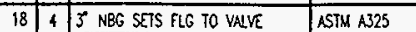
40

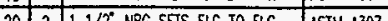

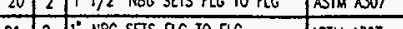

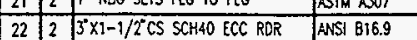
\begin{tabular}{l|l|l|}
\hline 23 & $211 / 2 \%$ KN RF FI6 CS & ANSI B16.5 \\
\hline
\end{tabular} \begin{tabular}{|l|l|l|}
\hline 25 & 2 & $3^{\circ} \times 1^{\circ}$ CS SCH 40 CONC ROR \\
\hline 26 & 4 & 3000 TM \\
\hline
\end{tabular} \begin{tabular}{l|l|l}
26 & 4 & $0^{\circ} 3000$ I THREDLLT
\end{tabular}

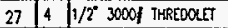

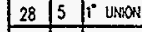

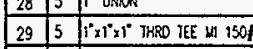
30 - 9 i" 90 ELL MI 150 : BII \begin{tabular}{|l|l|l|l}
\hline 31 & 1 & $1^{\circ} \mathrm{CS}$ SCH 40 MPPPIES \\
\hline 32 & 2 & I/ \\
\hline
\end{tabular}

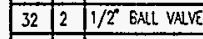
\begin{tabular}{|l|l|l|l|l}
\hline 33 & 8 & 1. \\
\hline 3 & BALL YAVE \\
\hline
\end{tabular}

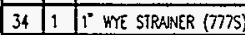

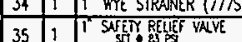

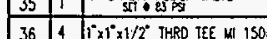

913 Bezu 01 PST 1362

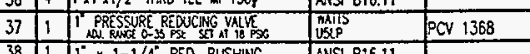

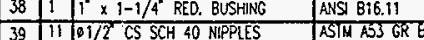

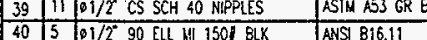

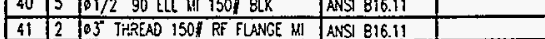

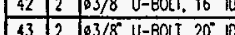

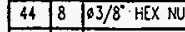

$45266^{6} \times 5$ ST $\times .120$ HALL $\times 22$ -

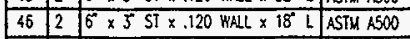

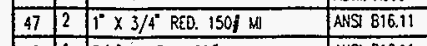

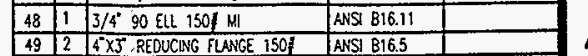




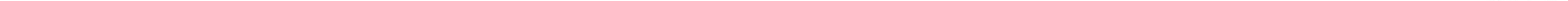




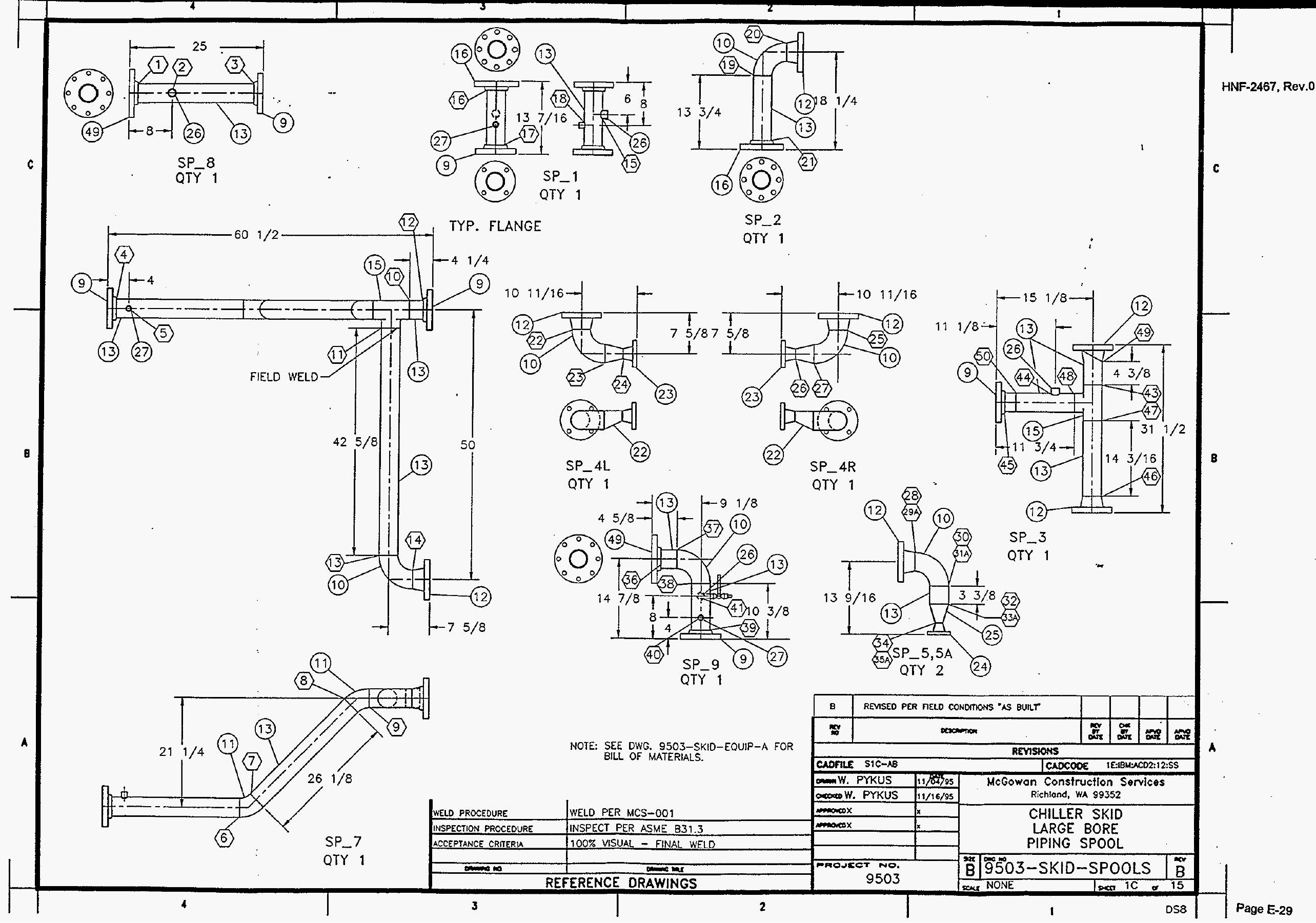


$\left.\right|_{\text {BOTIOU ENTRY POWER INET } z \bullet} ^{-4}$

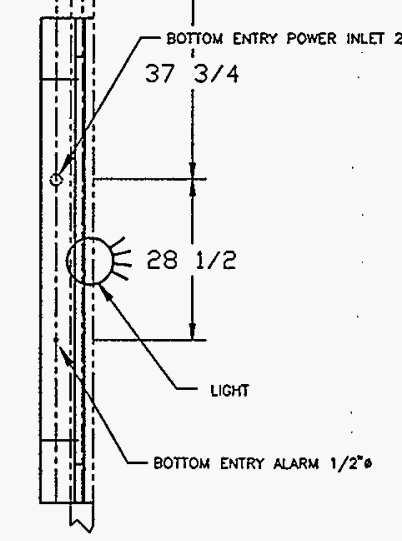

CDNDUIT DPENINGS FOR PDWER AND ALARM

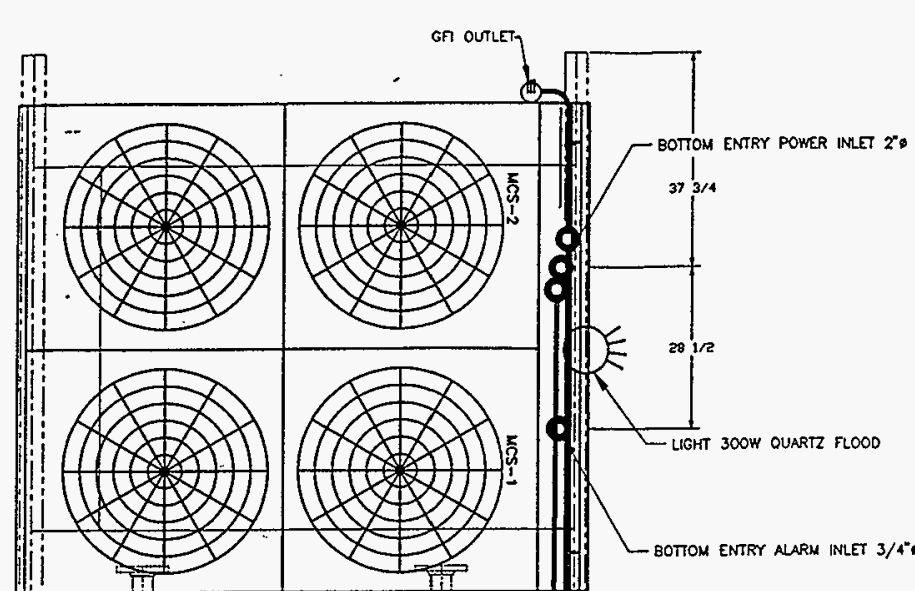

BILL OF MATERIALS

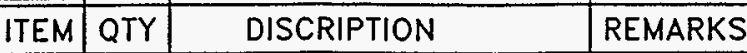

\begin{tabular}{|c|c|l|l|}
\hline 1 & 30 & $1 / 2 " R G D, C O N D U I T$ & WW GRANGER \\
\hline
\end{tabular}

\begin{tabular}{|c|c|}
\hline & \begin{tabular}{l|l|}
30 & $3 / 4 " R G D, C O N D U I T$ \\
\end{tabular} \\
\hline
\end{tabular}

\begin{tabular}{|l|l|l|}
\hline 3 & 3 & $1 / 2^{n}$ TEE \\
\hline
\end{tabular}

\begin{tabular}{|l|l|l|l|}
\hline 4 & 1 & $3 / 4^{\prime \prime}$ TEE & $5 \times C$ TO \\
\hline WW GRAINGER \\
\hline
\end{tabular}

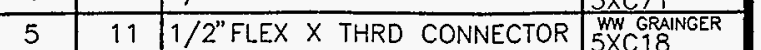

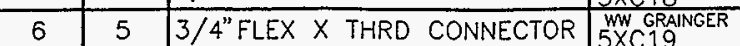

\begin{tabular}{|l|l|l|l|l|}
\hline 7 & $5 / 2^{\prime \prime}$ & COUPLING & WW GRAINGER \\
\hline 7 & 3 & $1 / 2^{\prime \prime}$ & WW \\
\hline
\end{tabular}

\begin{tabular}{l|l|l|l}
7 & 3 & $1 / 2$ & COUPLING \\
\hline 8 & 3 & $3 / 4 "$ COUPLING
\end{tabular}

\begin{tabular}{|l|l|ll|l|}
\hline 9 & 2 & RECT SAL GANG BOX & $5 X$ XC22 \\
\hline 15758$)$ & WW GRAINGER \\
\hline
\end{tabular}

\begin{tabular}{c|c|l|l}
\hline 10 & 2 & VERT RAINPROOF GFCI COVER $(5738)$ & WW GRAINGER \\
\hline
\end{tabular}

\begin{tabular}{l|l|l|l|}
\hline 11 & QTY & $1 / 2 "$ JIFFY STRAP & WW 510 \\
\hline WW GRAIGER
\end{tabular}

\begin{tabular}{c|c|l|l|l}
\hline 12 & QTY & $3 / 4$ " JIFFY STRAP & WW GRAINGER \\
\hline 13 & 2 & GFCI OUTLETS (DUPLEX) (52752) & WW GRA GAINGER \\
\hline
\end{tabular}

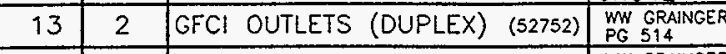

\begin{tabular}{l|l|l}
14 & $350 L$ \#10 THHN/TWN STRANDED
\end{tabular} COPPER CONDUCTOR

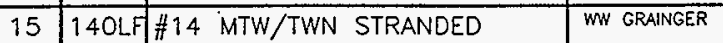

$1 / 2$ condur
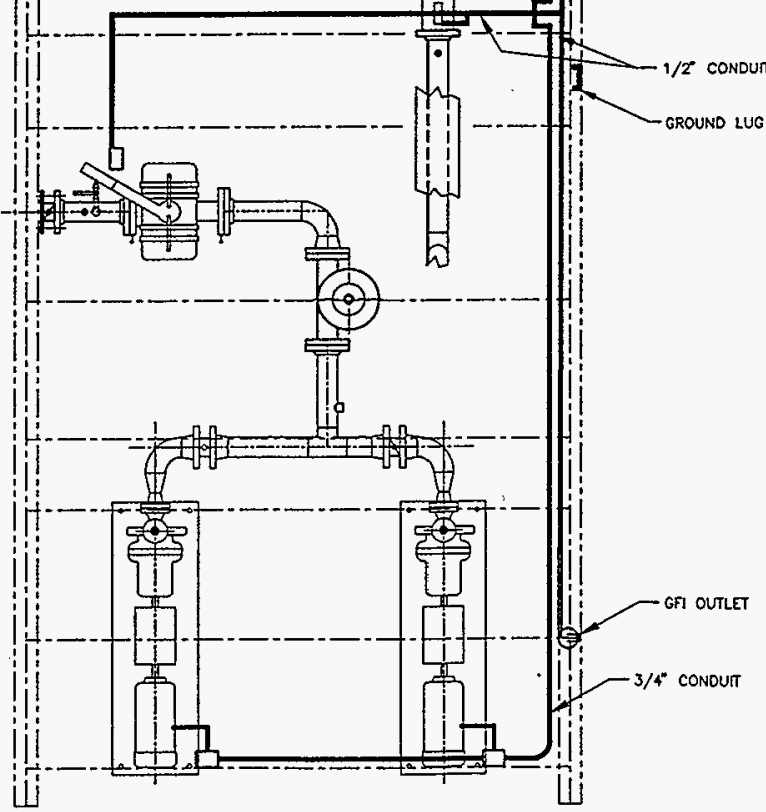

COPPER CONDUCTOR

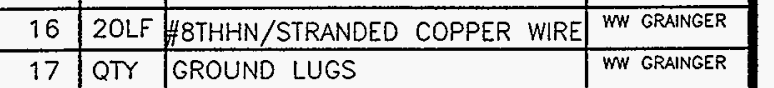

\begin{tabular}{c|c|l}
18 & 1 & $\begin{array}{l}300 \text { WATT QUARTZ } \\
\text { FLOOD LIGHT W/OPTIONAL } \\
\text { SURFACE ADAPTER }\end{array}$ \\
\hline
\end{tabular}

\begin{tabular}{|l|l|l|l|}
\hline 19 & QTY & $1 / 2 "$ SEALTIGHT FLEX & w GRAINGER \\
\hline 20 & QTY & $3 / 4^{\prime \prime}$ SEALTIGHT FLEX & w GRAIMGER \\
\hline 21 & 1 & $2 "$ CONDUIT SPOOL - (TBE) & - \\
\hline
\end{tabular}

NOTE: SEE DWG. DIAGRAM-WIRING ACDR45B 200,230,460 V. DWG. No 593440101 SHT 1 THROUGH 4 FOR WIRING DIAGRAM.

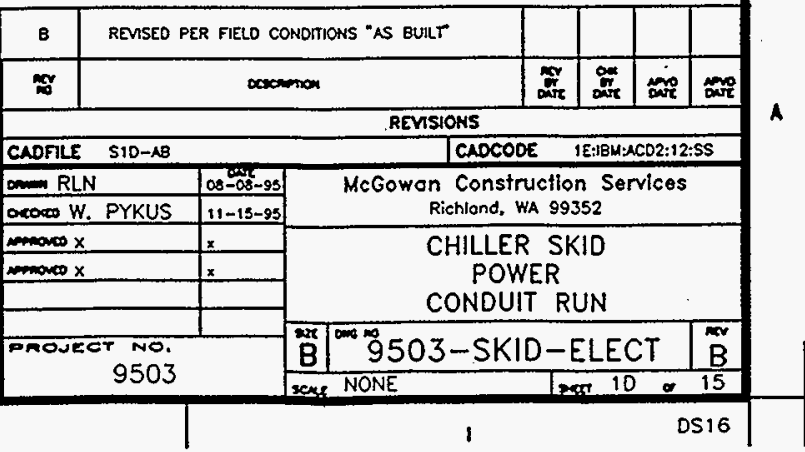




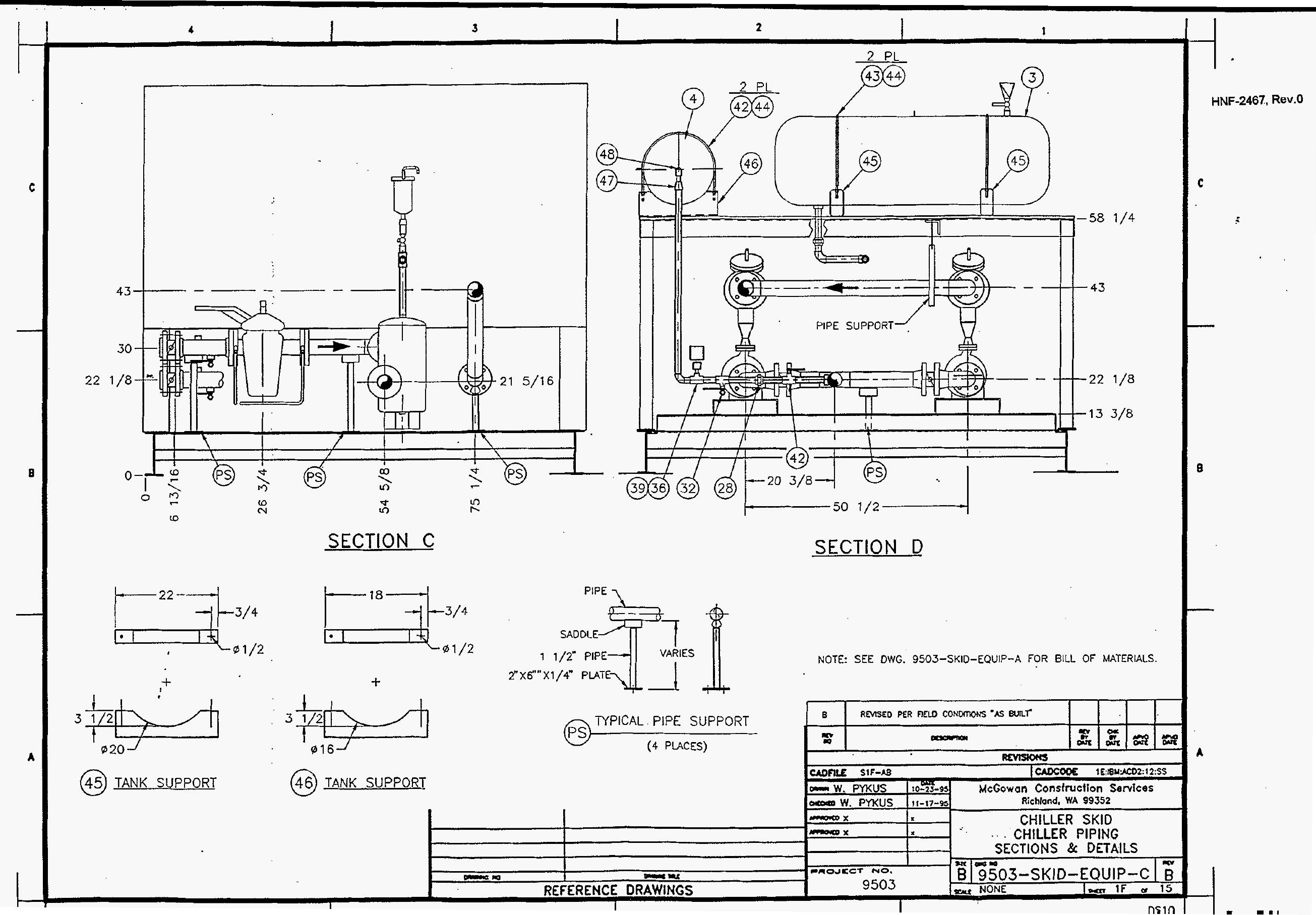




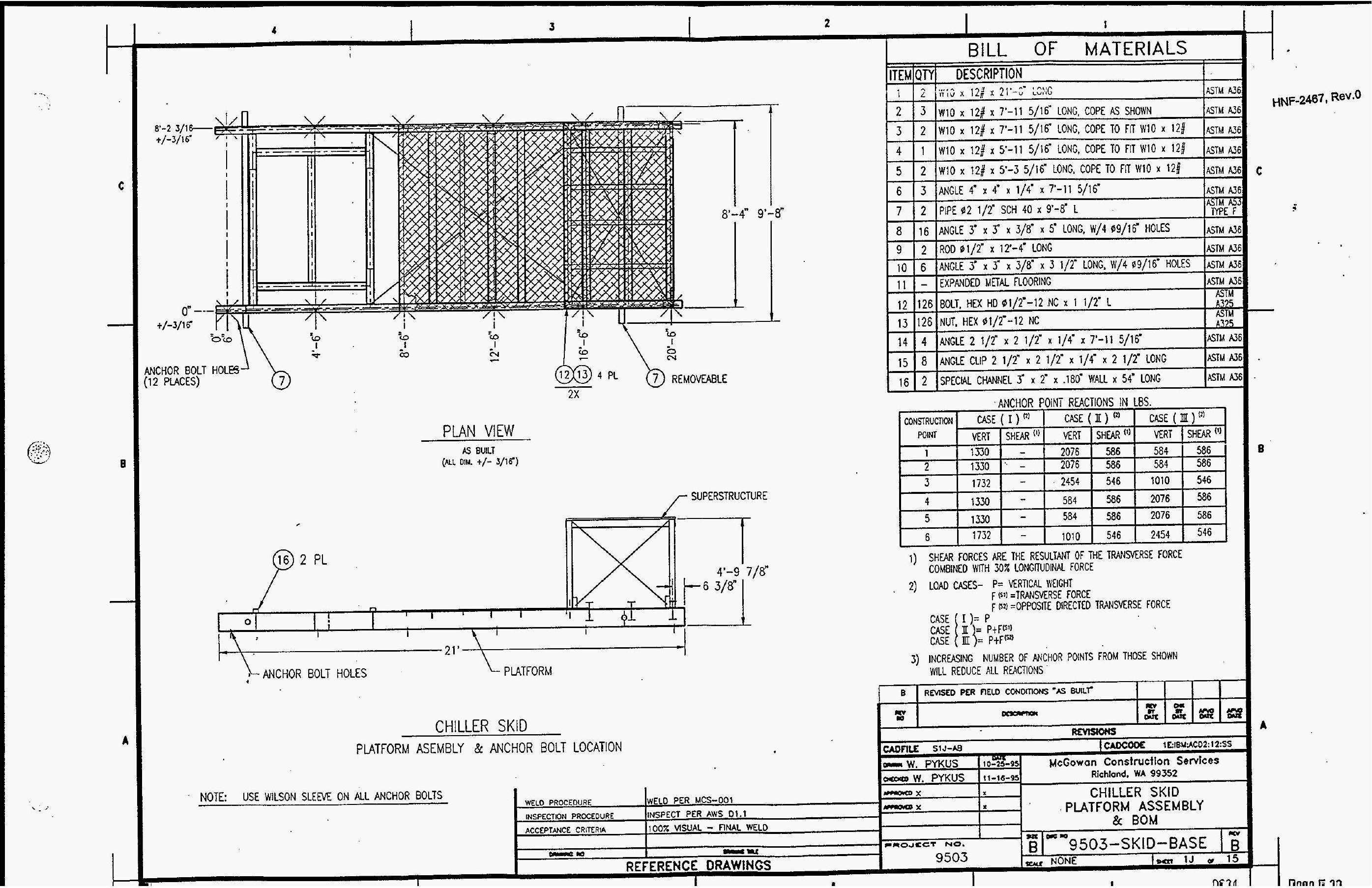




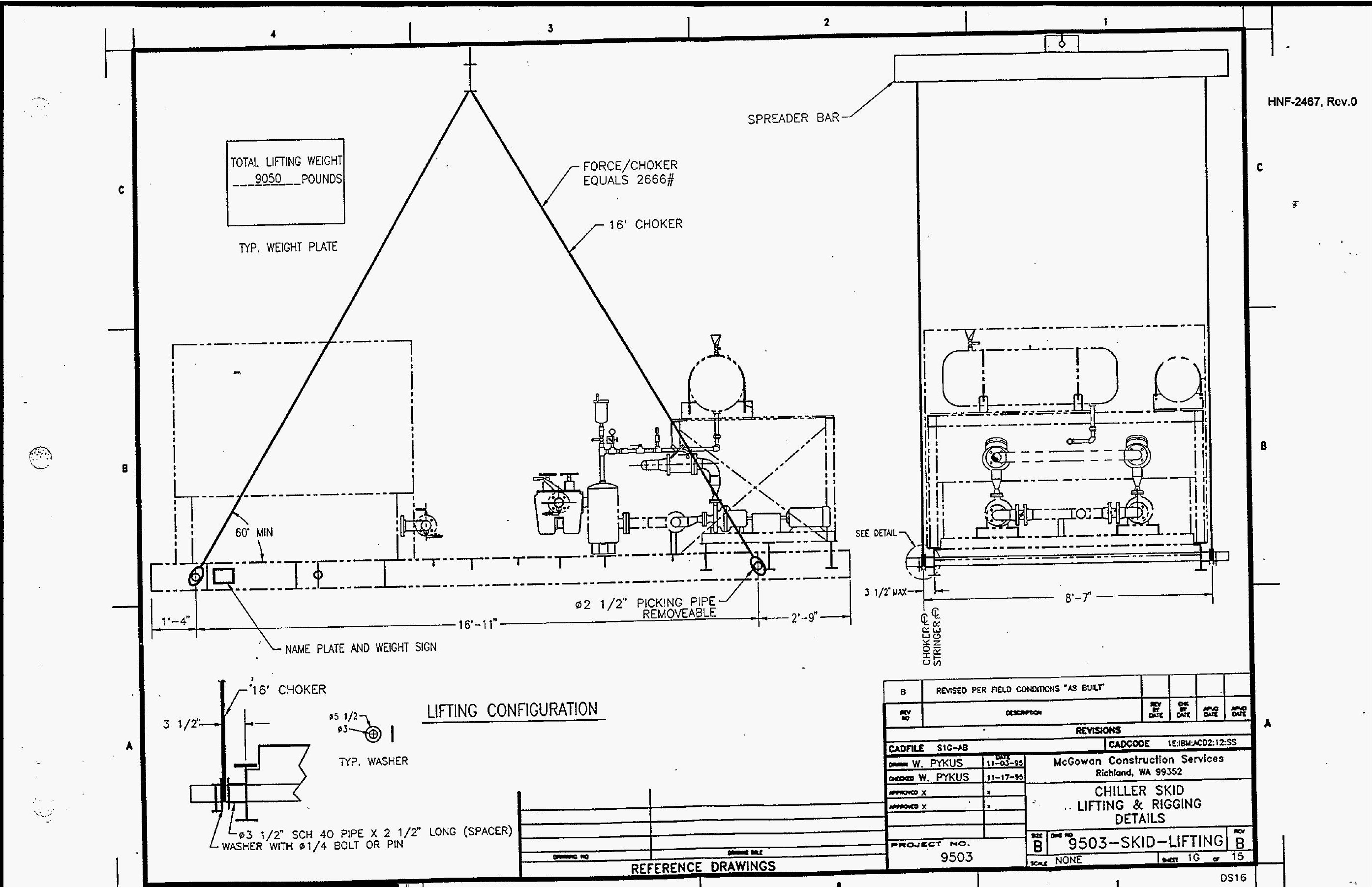


HNF-2467, Rev.0

W320-24-024

Electrical Equipment Pad (241-C-51)

F-i 


\section{KAISER ENGINEERS HANFORD}

\section{CALCULATION IDENTIFICATION AND INDEX}

Page $i$ of $i \mathrm{i}$

$7-29-94$

This sheet shows the status and description of the attached Design Analysis sheets.
Discipline Structural
Ho/Job No. ER4319/W-320
Calculation No. W320-24-024

Project No. \& Nane Project W-320, Tank 241-C-106 S1uicing

Calculation Item Electrical Equipment pad (241-C-51)

These calculations apply to:

Dwg. No. $\mathrm{H}-2-818458 \mathrm{sh} . \mathrm{I}$ \& $\mathrm{H}-2-818461$

Rev. No. 0

Dwg. No. N/A

Rev. No.

other (Study, CDR) N/A

Rev. No.

The status of these calculations is:

[] Preliminary Calculations

[X] Finat Calculations

[] Check Calculations (On Calculation Dated)

[] Void Calculation (Reason Voided )

$\begin{array}{lllll}\text { Incorporated in Final Drawings? } & {[\mathrm{X}]} & \text { Yes } & {[]} & \text { No } \\ \text { This calculation verified by independent "check" calculations? } & {[]} & \text { Yes } & {[X]} & \text { No }\end{array}$

Original and Revised Calculation Approvals:

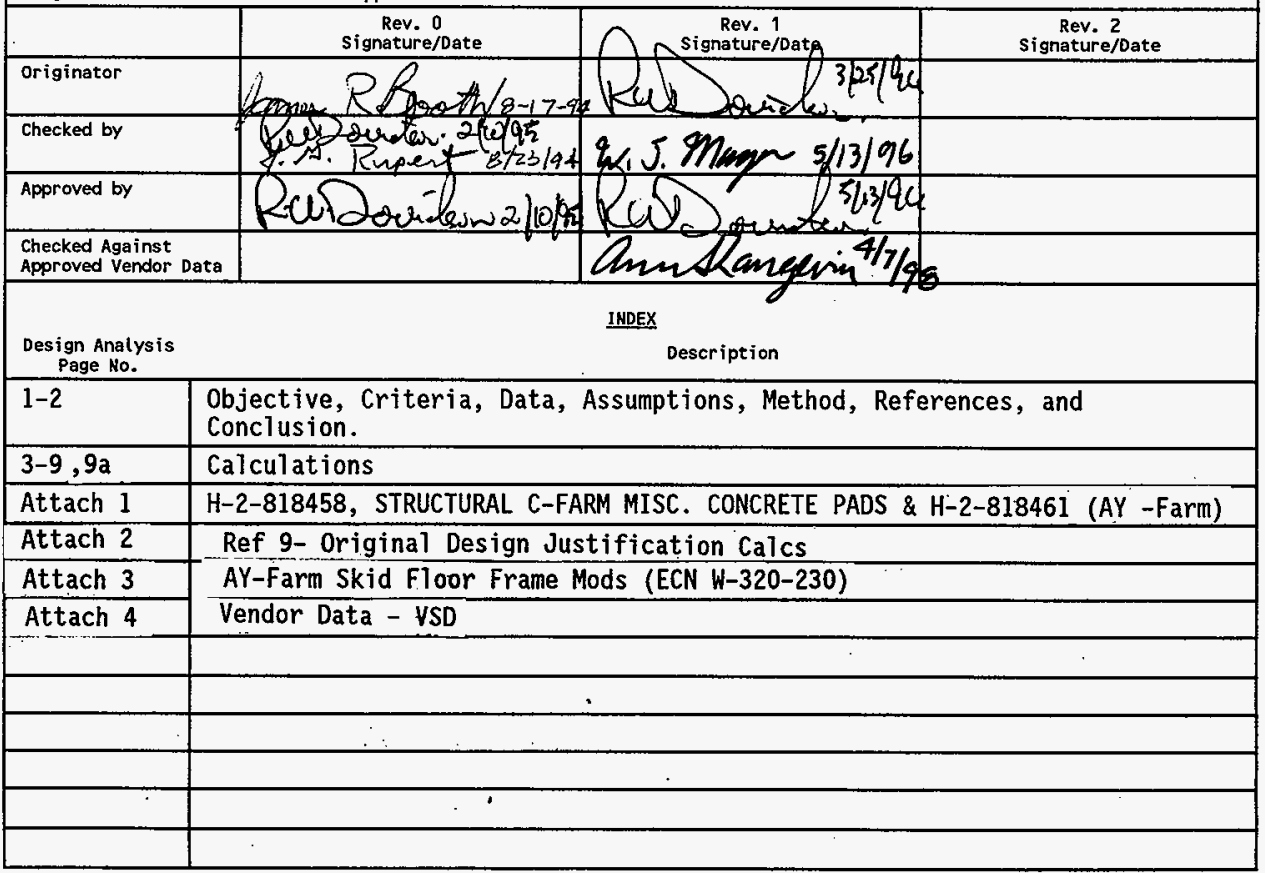




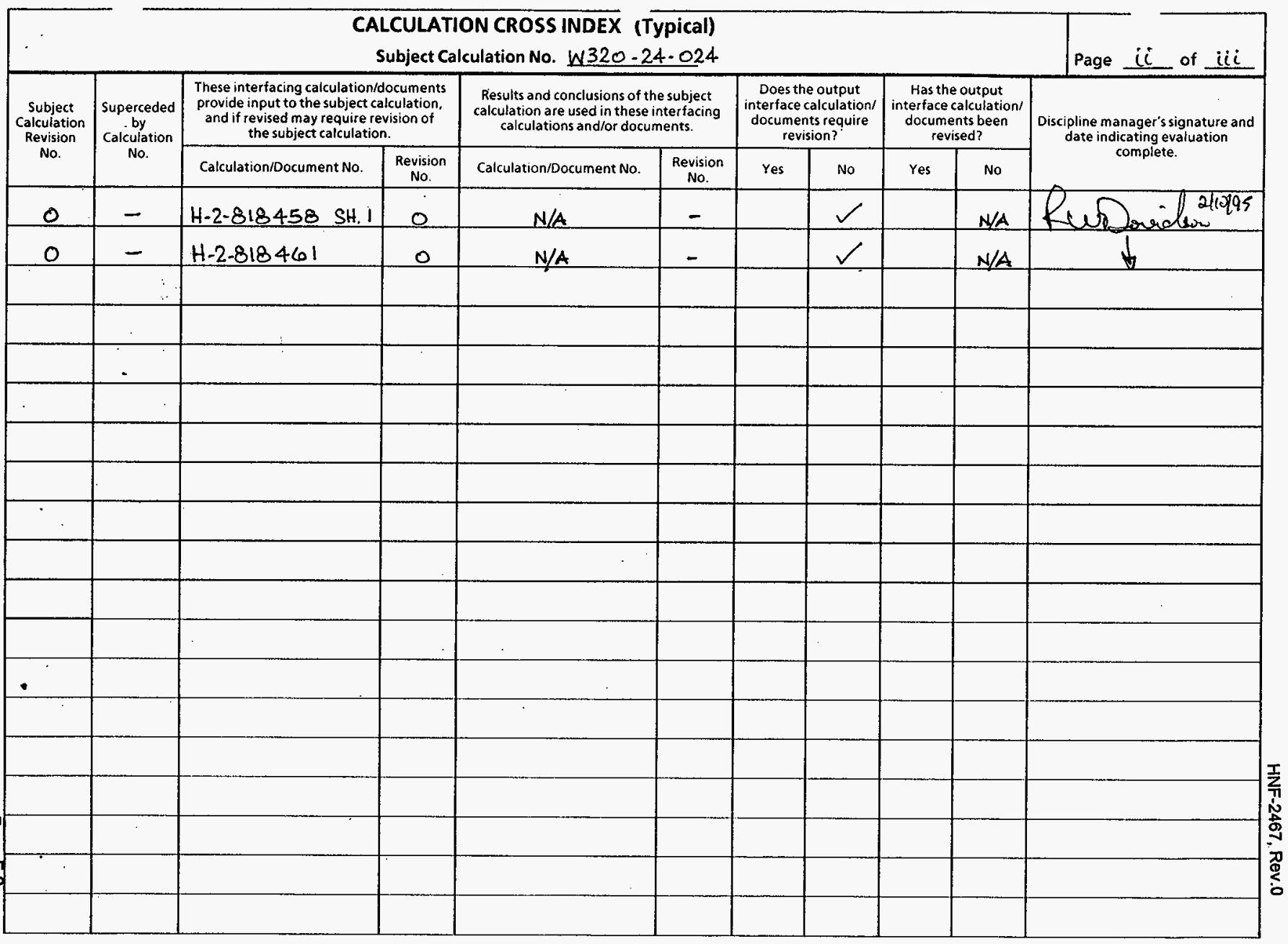


Project/Document No. W-320/W320-24-024

When the design or design change affects hardware, formal design verification must be performed if one or more of the following questions must be answered affirmatively (YES).

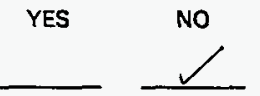

1. Does the design or design change involved meet the established criteria to be considered Safety Class 1 or 2 ?

2. Does this design or design change cause or permit changes to Safety Class 1 or 2 instrument or alarm setpoints outside of previously approved operational limits?

3. Does this design or design change significantly affect the nuclear safety consequences of a malfunction or failure of the structure, system, or component?

4. Does this design or design change involve or change design that has previously undergone formal design verification?

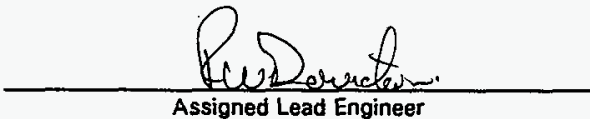

Assigned Lead Engineer

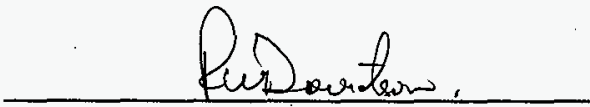

Responsible Discipline Manager
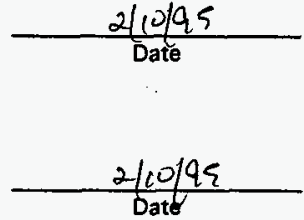

Original Design Package Distribution:

Project Manager

Design Verification Officer

Engineering Document Control
Design Change Distribution:

Attach to Engineering Change Notice 
HNF-2467, Rev.0

KAISER ENGINEERS

HANFDRD

DESIGN ANALYSIS

Client WHC

subject Electrical Equipment Pad (241-C-51) Date 7-29-94

Location 200E

Checked

Revised $3-2<-94$
Calc. No. W320-24-024

Revision 1

Page No. 1 of 9

Ho/Job No. ER4319/W-320

OBJECTIVE: $\quad$ The objective of this calculation is to design SC3 structural concrete pads and determine expansion anchor requirements to support and secure SC3 Electrical Enclosures.

CRITERIA:

1. Functional Design Criteria for Tank 241-C-106 Waste Retrieval, Project $W-320$; Document No. WHC-W320-FDC-001, Rev.2.

2. SDC 4.1, Rev. 11 .

3. DOE order $6430.1 \mathrm{a}$

DATA: $\quad$ 1. $f^{\prime} \mathrm{c}=4000 \mathrm{psi}$

2. $f y=60 \mathrm{ksi}$ for rebar

3. $1 / 2^{\prime \prime}$ dia. expansion anchors with $21 / 4^{\prime \prime}$ minimum embedment.

Values for tension and shear assumed from SDC 4.2 are conservative.

ASSUMPTIONS: $\quad$ See body of calc.

METHOD: $\quad$ MATHCAD 4.0

REFERENCES: $\quad$ 1. American Concrete Institute (ACI). ACI 318-89 (Revised 1992).

2. American Concrete Institute (ACI). ACI SP-17(91).

3. American Society of Civil Engineers (ASCE). ASCE 7-88.

4. Standard ARCH-Civi Tesign Criteria, SDC-4.1. Design Loads for Facilities. Rev. 11 .

5. Standard Arch-Civi1 Design Criteria, SDC-4.2. Design and Installation of Expansion Anchors. Rev. 0.

6. Uniform Building Code (UBC-91).

7. Design and Evaluation Guidelines for Department of Energy Facilities. Subjected to Natural Phenomena Hazards. UCRL 15910, June 1990.

8. Final Report of Geotechnical Engineering Studies, W-320 Waste Retrieval and Sluicing System, 200 East Area, Hanford Site. Prepared by Shannon and Wilson, Inc. for Kaiser Engineers Hanford Company. April 1994.

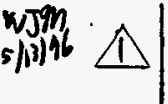

9. Vendor Information File: OTympic Tool \& Engineering (AY-Farm Electrical Equipment Skid/Enclosure- Dwgs 51001, Sht 1-3 \& Calculations. 


\section{KAISER ENEINEERS \\ HANFORD}

client WHC

subject Electrical

Location $200 \mathrm{E}$

CONCLUSION:

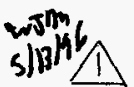

DESIGN ANALYSIS

Calc. No. W320-24-024

Revision Q 1

Page No. 2 of 9

wo/Job No. ER4319/W-320

Equipment Pad (241-C-51) Date 7-29-94

checked

Revised $3-25.94$ вy her. Booth $Q R B$

By

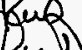

The concrete slab $\$$ will be $8^{\prime \prime}$ thick and be reinforced with \#5 rebar distributed as shown on $\mathrm{H}-2-818458$, sh. 1 (Equivalent to 0.31

sq.in./ft). Foun 1/21 dia. expansion anchons with-2 1/411 min, ombed, one at oach cornor, will bo sufficiont to anchor the ckid to tho Tho 1/2" dia. exp anchors with $21 / 4^{\prime \prime}$ min embed. on all

(4) sides will be used to anchor skid to the stab.

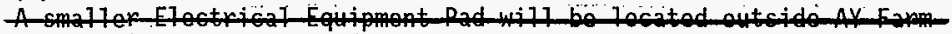

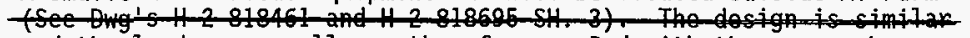

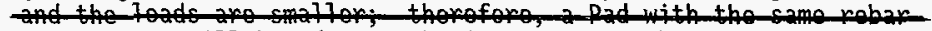
requipementwill bo dequate by inspoction. Tho now Eloctrical Equipent for AK Fam is shown +2 2 818461.

A similar Electrical Equipment Pad will be required outside AY-Farm (See Dwg's H-2-818461 and H-2-818695 SH 3) Loading conditions are similar. Calculations for C-Farm Electrical Equipment Pad adequately envelope loads for AY-Farm Pad. Provide same pad thickness and rebar size/pattern as C-farm.

See page $9 a$ for an evaluation of required field modifications to the AY-Farm Electrical Equipment Skid floor framing. The Ay-Farm Electrical Equipment Skid/Enclosure design/fabrication was provided by 0lympic Tool \& Engineering. 


\section{DESIGN ANALYSIS}

Client: Westinghouse Hanford_Company WO/Job No. ER4319M-320

Calc. No. W320-24-024

Revision 0

Page No. 3 of 9

Subject Electrical Equipment Pad _Date 6/24/94

Location: 200E $(241-C-51)$

\section{DESIGN MCC CONCRETE PAD}

Determine Design Loads:

1. Snow (REF. ASCE 7-88, pg. 23-27)

$\mathrm{C}_{\mathrm{e}}:=0.9$

$\mathrm{C}_{\mathrm{t}}:=1.1$

$\mathrm{I}:=1.2$

$\mathrm{p}_{\mathrm{g}}:=15 \cdot \frac{\mathrm{lb}}{\mathrm{ft}^{2}}$

$\mathrm{p}_{\mathrm{f}}:=0.7 \cdot \mathrm{C}_{\mathrm{e}} \cdot \mathrm{C}_{\mathrm{t}} \cdot \mathrm{I} \cdot \mathrm{p}_{\mathrm{g}}$

$\mathrm{p}_{\mathrm{f}}=12.474 \cdot \frac{\mathrm{lb}}{\mathrm{ft}^{2}} \quad$ Use $\mathrm{p}_{\mathrm{f}}:=20 \cdot \frac{\mathrm{lb}}{\mathrm{ft}^{2}}$

$S:=p_{f}(10 \cdot f t \cdot 12 \cdot f t)$

2. Wind: (Ref. SDC 4.1 and ASCE 7-88, section 6)

Gross dimensions: $12^{\prime}$ wide $\times 10^{\prime}$ wide $\times 10^{\prime}$ Eve height.

Exposure Class=C (SDC 4.1, pg.13)

Wind Speed=70 $\mathrm{mph}$ (SDC 4.1, pg.13)

Importance Factor=1.07 (SDC 4.1, pg.13)

Sign convention: + pressure acting on surface, - pressure acting away from surface.

$q z=q h$

$I:=1.07$

$\mathrm{V}:=70$

$\mathrm{K}_{\mathrm{Z}}:=0.80$

$\mathrm{q}_{\mathrm{z}}:=0.00256 \cdot \mathrm{K}_{\mathrm{z}} \cdot(\mathrm{I} \cdot \mathrm{v})^{2} \cdot \frac{\mathrm{lb}}{\mathrm{ft}^{2}}$

$\mathrm{q}_{\mathrm{z}}=11.489 \cdot \frac{\mathrm{lb}}{\mathrm{ft}^{2}}$

$\mathrm{G}_{\mathrm{h}}:=1.32$

$\mathrm{C}_{\text {pw }}:=0.8 \quad$ Windward

$\mathrm{C}_{\mathrm{pl}}:=-0.5$. Leeward

$C_{p s}:=-0.7 \quad$ Sidewall

$\mathrm{GC}_{\text {piw }}:=0.75$

$\mathrm{GC}_{\text {pil }}:=-0.25$

Wall Pressure

$\mathrm{W}_{\mathrm{wwl}}:=\mathrm{q}_{\mathrm{z}} \cdot \mathrm{G}_{\mathrm{h}} \cdot \mathrm{C}_{\mathrm{pw}}-\mathrm{q}_{\mathrm{z}} \cdot \mathrm{GC}$ piw

$\mathrm{w}_{\mathrm{ww1}}=3.516 \cdot \frac{\mathrm{lb}}{\mathrm{ft}^{2}}$

$\mathrm{w}_{\mathrm{ww} 2}:=\mathrm{q}_{\mathrm{z}} \cdot \mathrm{G}_{\mathrm{h}} \cdot \mathrm{C}_{\mathrm{pw}}-\mathrm{q}_{\mathrm{z}} \cdot \mathrm{GC}$ pil .

$\mathrm{w}_{\mathrm{ww} 2}=15.005 \cdot \frac{\mathrm{lb}}{\mathrm{ft}^{2}}$

(Windward Wall Pressure, Table 4)
Ref. SDC 4.1
(Minimum Snow Load per SDC 4.1)
(Gust Response Factor, Table 8)
(External Pressure Coeff., Fig. 2)
(Table 6 of ASCE 7-88)
Nelocity pressure, Eq.3 of ASCE 7-88)
ressure acting away from surface.

Ref. SDC 4.1
(Minimum Snow Load per SDC 4.1)
(Gust Response Factor, Table 8)
(External Pressure Coeff., Fig. 2)
(Table 6 of ASCE 7-88)
Nelocity pressure, Eq.3 of ASCE 7-88)
ressure acting away from surface.

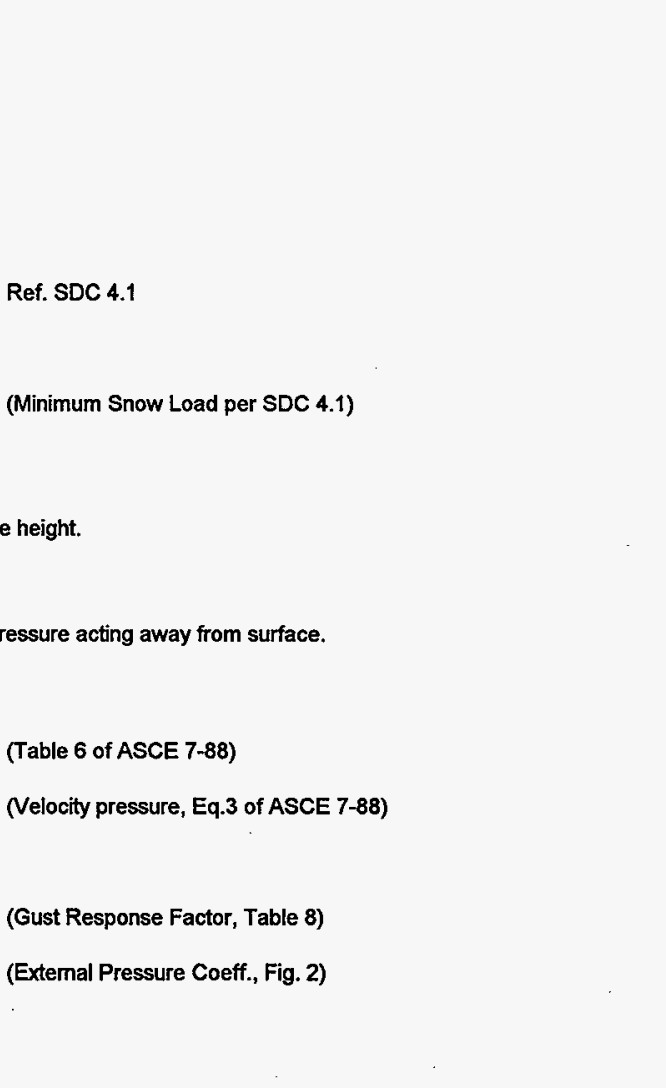

Ref. SDC 4.1
(Minimum Snow Load per SDC 4.1)
(Gust Response Factor, Table 8)
(External Pressure Coeff., Fig. 2)
(Table 6 of ASCE 7-88)
Nelocity pressure, Eq.3 of ASCE 7-88)
ressure acting away from surface.

Ref. SDC 4.1
(Minimum Snow Load per SDC 4.1)
(Gust Response Factor, Table 8)
(External Pressure Coeff., Fig. 2)
(Table 6 of ASCE 7-88)
Nelocity pressure, Eq.3 of ASCE 7-88)
ressure acting away from surface.

(Internal Pressure Coeff., Table 9 leeward) 
Client: Westinghouse Hanford Company

Subject Electrica! Equipment $(241-C-51)$ Location: $200 \mathrm{E}$

WO/Job No. ER4319M-320

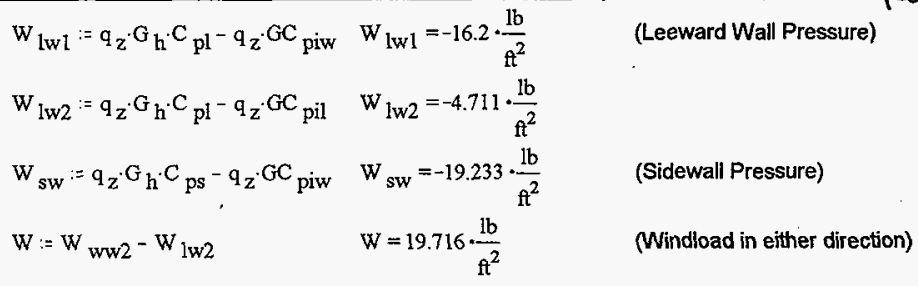$$
\text { Checked }
$$

3-2रीQ By

\section{Roof Pressure}

$\mathbf{h}:=10 \cdot \mathrm{ft}$

$\mathrm{L}:=12 \cdot \mathrm{ft}$

$\frac{\mathrm{h}}{\mathrm{L}}=0.833$

$C_{p r}:=-0.7$

$$
\begin{aligned}
& \mathrm{W}_{\mathrm{r}}:=\mathrm{q}_{\mathrm{z}} \cdot \mathrm{G}_{\mathrm{h}} \cdot \mathrm{C}_{\mathrm{pr}}-\mathrm{q}_{\mathrm{z}} \cdot \mathrm{GC}_{\text {piw }} \\
& \mathrm{W}_{\mathrm{r}}=-19.233 \cdot \frac{\mathrm{lb}}{\mathrm{ft}^{2}}
\end{aligned}
$$

3. Live Floor Load

$$
\begin{aligned}
& \mathrm{L}:=\left(100 \cdot \frac{\mathrm{lb}}{\mathrm{ft}^{2}}\right) \cdot(10 \cdot \mathrm{ft} \cdot 12 \cdot \mathrm{ft}) \quad \text { (Nominal floor load) } \\
& \mathrm{L}=1.2 \cdot 10^{4} \cdot \mathrm{lb}
\end{aligned}
$$

\section{Eave HT.}

Length

Roof pressure coeff. same for both directions since roof slope $=0 \mathrm{deg}$.

Pressure acting away from surface

4. Dead Loads.

a. C-Farm: Slab dimensions $13^{\prime}-6^{\prime \prime} \times 11^{\prime}-6^{\prime \prime} \times 0^{\prime}-8^{\prime \prime}(\mathrm{H}-2-818458$, sh )

$$
\begin{array}{ll}
\mathrm{Wc}:=150 \cdot \frac{\mathrm{lb}}{\mathrm{ft}^{3}} \cdot\left(13.5 \cdot \mathrm{ft} \cdot 11.5 \cdot \mathrm{ft} \cdot \frac{8}{12} \cdot \mathrm{t}\right) & \mathrm{Wc}=1.552 \cdot 10^{4} \cdot \mathrm{lb} \quad \text { (Weight of concrete sla } \\
\mathrm{We}:=3073 \cdot \mathrm{lb} & \begin{array}{l}
\text { Weight of electrical equipment in enclosure per electrical } \\
\text { estimated weights) }
\end{array} \\
\mathrm{Ws}:=3425 \cdot \mathrm{lb} & \text { (Weight of skid and attached enclosure, estimated) } \\
\mathrm{D}:=\mathrm{We}+\mathrm{Ws} & \begin{array}{l}
\text { (Total dead load acting on slab at C-Farm controls dead } \\
\text { loading and shall be used for load cases) } \\
\mathrm{g}^{i}-0^{\prime \prime}
\end{array}
\end{array}
$$

b. AY-Farm: Slab dimensions 15'-0" x'

$$
\mathrm{W}_{\text {ay }}:=150 \cdot \frac{\mathrm{lb}}{\mathrm{ft}^{3}} \cdot\left(15 \cdot \mathrm{f} \cdot \mathrm{x}^{\mathrm{g}} \mathrm{\textrm {ft }} \cdot \frac{8}{12} \cdot \mathrm{ft}\right)
$$

$$
w_{\text {ay }} \text { 13,500 Ibs (Weight of concrete slab) }
$$

$\mathrm{W}_{\text {eay }} 2800$ lbs.

$$
\begin{aligned}
& W_{\text {say }}:=3200 \cdot 1 \mathrm{~b} \\
& D_{\text {ay }}:=W_{\text {eay }}+W_{\text {say }} \\
& D_{\text {ay }}=4.2+0^{3} \text { elb } 6000 \text { lbs }
\end{aligned}
$$

Weight of electrical equipment in enclosure per electrical estimated weights)

(Weight of skid and attached enclosure, estimated. Reduced based on smaller overall dimensions) 
Client: Westinghouse Hanford Company WO/Job No. ER4319N-320

Subject Electrical Equipment Pad
(241-C-5I)
Location: $200 E$

Date 7/29/94

Page No. 5 of 9

5. Seismic: Ref. SDC 4.1, UCRL 15910, and UBC-91.

Ground motion shall be taken $100 \%$ in one perpendicular direction plus $30 \%$ in the other direction combined in a SRSS basis.

Critical Damping $=5 \%$ for Safety class 3 per SDC 4.1 .
$\mathrm{Z}:=0.12$
$\mathrm{I}:=1.25$
Peak Ground Acceleration (SDC 4.1)
$\mathrm{C}:=2.12$
Importance Factor (SDC 4.1)
Ws $:=\mathrm{D}$
Spectral amplification factor (SCD 4.1)
$R_{\mathrm{W}}:=6$
Weight of enclosure acting on slab.
$\mathrm{V}:=\frac{\mathrm{Z} \cdot \mathrm{I} \cdot \mathrm{C} \cdot \mathrm{Ws}}{\mathrm{R}_{\mathrm{W}}}$
Reduction Factor (UBC 91, Table No. 23-O)
$\mathrm{V}_{\text {ew }}:=\mathrm{V}$
Base Shear
$\mathrm{V}_{\text {ew }}=\mathbf{3 4 4 . 3 9 4 \cdot \mathrm { lb }}$
$\mathrm{V}_{\mathrm{ns}}:=0.3 \cdot \mathrm{V}$
$\mathrm{V}_{\mathrm{ns}}=103.318 \cdot \mathrm{lb}$
$\mathrm{E}:=\sqrt{\mathrm{V}_{\mathrm{ew}}{ }^{2}+\mathrm{V}_{\mathrm{ns}}{ }^{2}}$
$E=359.558 \cdot 1 b$
Earthquake Load

6. Load Combinations

a. UBC 91 load combinations, pg. 148. The zero loads below are to make the MathCAD equations work.

$$
\begin{aligned}
& D_{1}=6.498 \cdot 10^{3} \cdot 1 \mathrm{~b} \\
& D_{1}:=0 \cdot 1 \mathrm{~b} \\
& L_{r}:=0 \cdot 1 \mathrm{~b} \\
& L_{r}=0 \cdot 1 \mathrm{~b} \\
& L_{1}:=0 \cdot 1 \mathrm{~b} \\
& E_{1}:=0 \cdot 1 \mathrm{~b} \\
& \mathrm{~s}_{1}:=0 \cdot 1 \mathrm{~b} .
\end{aligned}
$$

Dead Load

Dead Load (horizontal)

Snow Load and Ashfall-Roof Live Load

1. $\mathrm{U}:=\mathrm{D}+\mathrm{L}+\mathrm{L}_{\mathrm{T}}$

$$
\mathrm{U}=1.85 \cdot 10^{4} \cdot \mathrm{lb}
$$

2. $\mathrm{U}:=\mathrm{D}+\mathrm{L}+\mathrm{W}_{\mathrm{r}} \cdot(10 \cdot \mathrm{ft} \cdot 12 \cdot \mathrm{ft})$

$$
\mathrm{U}=1.619 \cdot 10^{4} \cdot \mathrm{lb}
$$

$\mathrm{U}_{11}:=\mathrm{D}_{1}+\mathrm{L}_{1}+\mathrm{W} \cdot(10 \cdot \mathrm{ft} \cdot 10 \cdot \mathrm{ft}) \quad$ (Wind controls horizontal loading)

$$
\mathrm{U}_{11}=1.972 \cdot 10^{3 \cdot} \cdot 1 \mathrm{~b}
$$

Horizontal live load

Vertical seismic

0 snow load horizontal

$\mathrm{U}_{12}:=\mathrm{D}_{1}+\mathrm{L}_{1}+\mathrm{W} \cdot(10 \cdot \mathrm{ft}: 12 \cdot \mathrm{ft})$

$\mathrm{U}_{12}=2.366 \cdot 10^{3} \cdot \mathrm{lb}$

$\mathrm{U}:=\mathrm{D}_{1}+\mathrm{L}_{1}+\mathrm{E}$

$\mathrm{U}=359.558 \cdot \mathrm{lb}$ 
Client: Westinghouse Hanford Company WO/Job No. ER $4319 M-320$

Subject Electrical Equipment Pad_Date 7/29/94

Location: 200E

$$
(241-C-5 I)
$$

3. $\mathrm{U}:=\mathrm{D}+\mathrm{L}+\mathrm{W}_{\mathrm{r}} \cdot(12 \cdot \mathrm{ft} \cdot 10 \cdot \mathrm{ft})+\frac{\mathrm{S}}{2}$

$\mathrm{U}=1.739 \cdot 10^{4} \cdot \mathrm{lb}$

4. $\mathrm{U}_{13}:=\mathrm{D}+\mathrm{L}+\mathrm{S}+\frac{\mathrm{W}_{\mathrm{T}} \cdot(12 \cdot \mathrm{f} \cdot 10 \cdot \mathrm{ft})}{2}$ Checked 8/23/94 Revised

$$
\mathrm{U}_{13}=1.974 \cdot 10^{4} \cdot 1 \mathrm{~b}
$$

5. $\mathrm{U}:=\mathrm{D}_{1}+\mathrm{L}_{1}+\mathrm{S}+\mathrm{E}_{1}$

$$
\mathrm{U}=2.4 \cdot 10^{3} \cdot \mathrm{lb}
$$

b. Strength Design load cases. ASCE 7-88.

1. $U:=1.4-\mathrm{D}$

$$
\mathrm{U}=9.097 \cdot 10^{3} \cdot \mathrm{lb}
$$

2. $U_{21}:=1.2 \cdot \mathrm{D}+1.6 \cdot \mathrm{L}+0.5 \cdot \mathrm{S}$

$$
U_{21}=2.82 \cdot 10^{4} \cdot 1 b
$$

3. $\mathrm{U}:=1.2 \cdot \mathrm{D}+1.6 \cdot \mathrm{S}+0.5 \cdot \mathrm{L}$

$$
\mathrm{U}=1.764 \cdot 10^{4} \cdot 1 \mathrm{~b}
$$

4. $\mathrm{U}_{22}:=1.2 \cdot \mathrm{D}_{1}+1.3 \cdot \mathrm{W} \cdot(12 \cdot \mathrm{ft} \cdot 10 \cdot \mathrm{ft})+0.5 \cdot \mathrm{L}_{1}+0.5 \cdot \mathrm{S}_{1} \quad$ (Controlling side loads)

$\mathrm{U}_{22}=3.076 \cdot 10^{3} \cdot \mathrm{lb}$

$$
\begin{aligned}
& \mathrm{U}_{23}:=1.2 \cdot \mathrm{D}_{1}+1.3 \cdot \mathrm{W} \cdot(10 \cdot \mathrm{ft} \cdot 10 \cdot \mathrm{ft})+0.5 \cdot \mathrm{L}_{1}+0.5 \cdot \mathrm{S}_{1} \\
& U_{23}=2.563 \cdot 10^{3} \cdot \mathrm{lb}
\end{aligned}
$$

5. $U:=1.2 \cdot \mathrm{D}_{1}+1.5 \cdot \mathrm{E}+0.5 \cdot \mathrm{L}$

$$
U=6.539 \cdot 10^{3} \cdot 1 \mathrm{~b}
$$

6. $\mathrm{U}:=0.9 \cdot \mathrm{D}_{1}-1.3 \cdot \mathrm{W} \cdot(12 \cdot \mathrm{ft} \cdot 10 \cdot \mathrm{ft})$

$$
\mathrm{U}=-3.076 \cdot 10^{3} \cdot 1 \mathrm{~b}
$$

c. Determine controlling vertical ACl load case.

1. $\mathrm{U}_{31}:=1.4 \cdot \mathrm{D}+1 \cdot 7 \cdot \mathrm{L}$

$$
\mathrm{U}_{31}=2.95 \cdot 10^{4} \cdot 1 \mathrm{~b}
$$

\section{Check Soil Bearing Properties}

a. Check Qmax against Qapplied.

$$
\begin{aligned}
& Q_{\max }:=4000 \cdot \frac{\mathrm{lb}}{\mathrm{f}^{2}} \\
& \text { (Shannon an Wilson Report pg. 10) } \\
& \mathrm{Q}_{\text {applied }}:=\frac{\mathrm{U}_{13}}{(13.5} \frac{\mathrm{ft}}{\mathrm{f}} \cdot \frac{\mathrm{Wc}}{11.5 \cdot \mathrm{ft})} \\
& \mathrm{Q}_{\text {applied }}=227.176 \cdot \frac{\mathrm{lb}}{\mathrm{ft}^{2}} \quad \text {. Foundation Bearing pressure OK. }
\end{aligned}
$$

Note: Due to the low bearing pressure and minimal overturning moment, eccentric fondation loading is not considered. 
KAISER ENGINEERS

HANFORD
Client: Westinghouse Hanford Company

\section{Subject Electrical Equipment Pad} (241-C-5i)

Location: 200E
WO/Job No. ER4319M-320

\section{Date 7/29/94}

Checked $8 / 23 / 94$ Revised
(Lateral Sliding coefficient given in UBC, pg. 590)

Resistance to sliding by friction is sufficient in both directions.

8. Check overturning moment (See figure below)

$$
\begin{aligned}
& M_{\text {ot }}:=(W \cdot 12 \cdot f \cdot 10 \cdot \mathrm{ft}) \cdot 5 \cdot \mathrm{ft} \\
& M_{\text {ot }}=1.183 \cdot 10^{4} \cdot \mathrm{lb} \cdot \mathrm{ft} \\
& M_{I}:=\mathrm{D} \cdot 5.75 \cdot \mathrm{ft} \\
& M_{I}=3.736 \cdot 10^{4} \cdot \mathrm{lb} \cdot \mathrm{ft} \\
& \frac{M_{\text {ot }}}{M_{\mathrm{I}}}=0.317
\end{aligned}
$$$$
\text { Resisting moment to overturning }
$$

Overtuming moment applied at the estimated mid-height of the enclosure.

OK, since less than $2 / 3$.

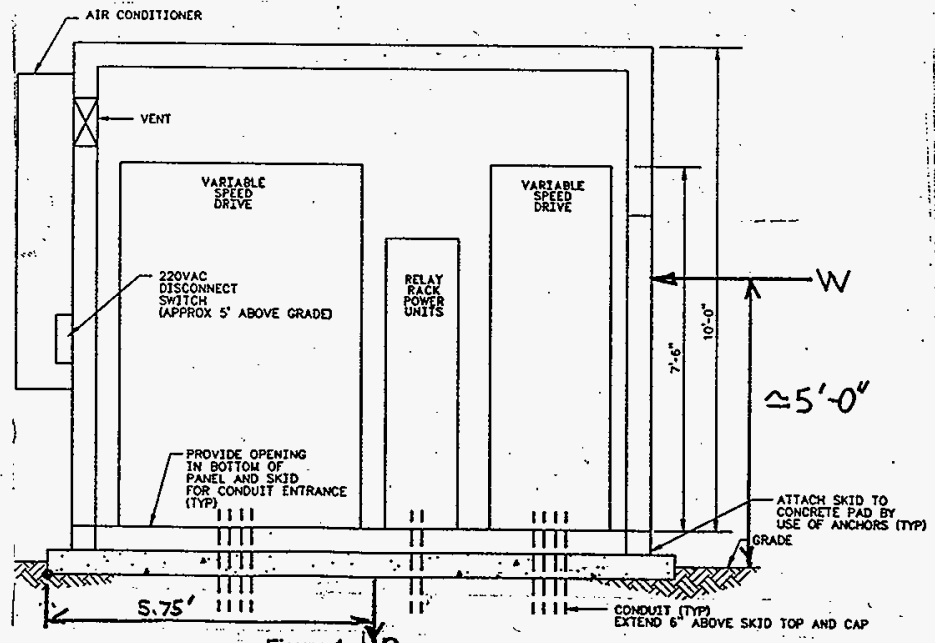

Figure $1: D$

9. Concrete Slab Design. Using the design proceedures of ACl SP-17(91).

$$
\begin{aligned}
& \text { kip }:=1000 \cdot \mathrm{lb} \quad \mathrm{f}_{\mathrm{c}}:=4000 \mathrm{psi} \\
& \mathrm{I}:=11.5 \cdot \mathrm{ft} \\
& \mathrm{w}_{\mathrm{u}}:=1.4 \cdot\left(150 \cdot \frac{\mathrm{lb}}{\mathrm{ft}^{3}} \cdot \frac{8}{12} \cdot \mathrm{ft} \cdot 1 \cdot \mathrm{ft}\right) \\
& \mathrm{w}_{\mathrm{u}}=140 \cdot \frac{\mathrm{lb}}{\mathrm{ft}}
\end{aligned}
$$$$
f_{y}:=60000 \text { psi }
$$$$
\text { Length of slab in } \mathrm{ft} \text {. }
$$ 
KAISER ENGINEERS

\section{HANFORD}

Client: Westinghouse Hanford Company

Subject Electrical Equipment Pad

$$
(241-C-51) L
$$

Location: 200E

\section{DESIGN ANALYSIS}

Revision 0

Page No. 8 of 9

WO/Job No. ER4319NV-320

Date 7/29/94.

By JR Booth

Checked $8 / 23 / 94$

Revised

By f.A. Rmpent

$\mathrm{M}_{\mathrm{u}}:=\frac{\left(\frac{\mathrm{U}_{31}}{8} \cdot \frac{9}{12} \cdot \mathrm{ft}\right)}{1000 \cdot \mathrm{lb} \cdot \mathrm{l} \cdot \mathrm{f}}$

$\mathrm{M}_{\mathrm{UL}}=5.08$

$h:=8$

(Estimated Slab thicknes in inches)

The skid will rest on a total of 8 plates welded along the bottom of the skigs to distribute the weight throughout the.

slab.

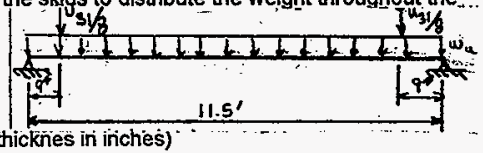

$d:=h-3-0.625 \quad d=4.375 \quad$ (Effective depth of slab with $3^{\prime \prime}$ min. cover for rebar)

$b:=12$

(Unit width of slab in inches)

$\rho_{\max }:=0.0214$

SP17, Flexure 2.2

$F:=\frac{d^{2}}{1000}$

$\mathrm{F}=0.019$

$\mathrm{K}_{\mathrm{n}}:=\frac{\mathrm{M}_{\mathrm{u}}}{\mathrm{F}} \quad \mathrm{K}_{\mathrm{n}}=265.39$

$a_{n}:=4.29$

$A_{s}:=\frac{M}{d \cdot a}$

Area of steel required per $12^{n}$ strip of concrete, sq. in

$\mathrm{A}_{\mathrm{s}}=0.271 \mathrm{in}^{2}$

$\rho_{\min }:=0.0018$

For grade 60 steel

$A_{\text {smin }}:=\rho_{\min }-b \cdot h$

Required steel area is greater than minimum required.

$A_{\operatorname{smin}}=0.173$ in $^{2}$

Check shear

$$
\begin{aligned}
& \mathrm{V}_{\mathrm{u}}:=\frac{\mathrm{U}_{13}}{8}+\mathrm{w}_{\mathrm{u}} \cdot \frac{1}{2} \\
& \mathrm{~V}_{\mathrm{u}}=3.273 \cdot 10^{3} \cdot \mathrm{lb}
\end{aligned}
$$

$\mathrm{b}_{\mathrm{w}}:=12$ in $\phi:=0.85$

$\mathrm{V}_{\mathrm{c}}:=\phi \cdot\left(2 \cdot \sqrt{\mathrm{f}_{\mathrm{c}}}\right) \cdot \mathrm{b}_{\mathrm{w}} \cdot \mathrm{d}$

$v_{c}=5.645 \cdot 10^{3} \mathrm{lb}$

Ok

Use $\# 5$ bars spaced $12^{\prime \prime}$ O.C. See H-2-818458 for sketch of reinforcing. 


\section{KAISER ENGINEERS} HANFORD

Client: Westinghouse Hanford Company Subiect Electrical Equipment $(241-C-51)$

Location: 200E
DESIGN ANALYSIS

Calc. No. W320-24-024

Revision $g$

Page No. 9 of 9

WO/Job No. ER4319NN-320

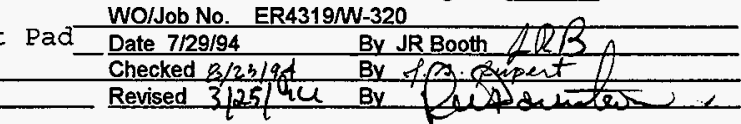

10. Expansion Anchor Design. Per SDC 4.2, Rev. 0$$
\text { Tension couple divided by } 2 \text { caused from overturning. }
$$

$$
\begin{aligned}
& \mathrm{V}_{\text {applied }}:=\frac{\sqrt{\mathrm{U}_{11}{ }^{2}+\mathrm{U}_{12}{ }^{2}}}{4} \\
& \mathrm{~V}_{\text {applied }}=0.77 \cdot \mathrm{kip} \\
& \mathrm{T}_{\text {applied }}:=\frac{\mathrm{U}_{12} \cdot 5 \cdot \mathrm{ft}}{11.5 \cdot \mathrm{ft}} \cdot 0.5 \\
& \mathrm{~T}_{\text {applied }}=0.514 \cdot \mathrm{kip}
\end{aligned}
$$

Factored shear force per corner of enclosure.(conservative)

Try 1/2" HILTI KWIK-BOLT II with min. embed of $21 / 4 "$.

$\mathrm{T}:=1.36 \cdot \mathrm{kip} \quad$ (Allowable)

$\mathrm{V}:=1.47 \cdot \mathrm{kip}$ (Allowable)

$\frac{\mathrm{V}_{\text {applied }}}{\mathrm{V}}+\frac{\mathrm{T}_{\text {applied }}}{\mathrm{T}} \leq 1.0$

$\frac{\mathrm{V}_{\text {applied }}}{\mathrm{V}}+\frac{\mathrm{T}_{\text {applied }}}{\mathrm{T}}=0.902$
Final: Provide a minimum of 2-1/2" exp anchors on a11 (4) sides of each skid. ok 
HNF-2467, Rev. 0

\section{RAISER ENEINEERS \\ HANFERE}

DESIGN ANALYSIS

client WHC

subject AY-Farm ETectrical Equipment Pad/Skid Modifications

Location 200E/ 241-AY
Calc. Ho. W320-24-024

Revision 1

Page No. $9 a$ of 9

wo/Job Ho. ER6159/W-320

Date 03-11-96 By RW Davidson

Checked By

Revised By

\section{AY - Farm Electrical Equipment Pad Mods:}

a. Reinforcement details modified to add diagonal rebars at blockouts and clarify rebar configuration. (See ECN No. W-320-123)

b. Expansion anchoring requirements for skid increased and overall dimensions of pad increased by 6 -inches in each direction to satisfy expansion anchor edge distances. (See ECN No. W-320-125)

The structural integrity of AY-farm Electrical Equipment Pad not affected by these changes. No further calculations required.

\section{AY-Farm Electrical Equipment Skid Modifications:}

Modifications to floor framing are needed to clear conduit stub-up's through equipment pad blockouts. (See ECN W-320-230)

Additionally the weight of the VSD has gone from $13001 \mathrm{bs}$ to 3000 1bs.

Assumo $80 \%$ of the woight is contained in cabinets A \& B. Cabinet C is sepatate for AY Farm.

Ref 9: ( See Attach 2 for Original Design Justification Calc, Page 5)

Chk floor loading: Assume max equip load over 3 beams due to framing mods

$$
\begin{aligned}
& M \max =3000 * .8 * 2 * 6 / 8 * 12=43,200 \text { in-lbs } \\
& \mathrm{fb}=43,200 / 3 * 1.02=14,118 \text { psi }>12,667 \quad\langle N G\rangle \text { using } S F=3, F y=38 k s i
\end{aligned}
$$

Try load distributed over 3.5 beams:

$$
\mathrm{fb}=43,200 / 3.5 * 1.02=12,590<12,667\langle 0 \mathrm{~K}\rangle
$$

Chk shear e coped formed channe]: Use max shear, conserv.

$$
\begin{aligned}
& \text { Cross-section, } 14 \mathrm{ga}=.0747 * 6=.45 \mathrm{sq} \text { in } \\
& v=3000 * .8 / 3 * 6 / 8=600 \mathrm{lbs} \\
& \mathrm{fv}=600 / .45=1333 \mathrm{psi} \text { oK }
\end{aligned}
$$

Notes:

1. Provide $C 6 \times 8.2$ cross braces a coped formed channels, both sides.

2. Provide $C 6 \times 8.2$ beams/braces cut formed channels, both sides.

3. Provide $1 / 2^{11}$ dia plug weld between perimeter top plate and formed channels ( 5 plcs) s'

4. Use min 1/8" fillet weld for beam/brace connections

5. Perform a11 work per Constr Spec W-320-C7. Section 05500 .

6. See Attach 3 for required mods.

7. As a precaution, add a nameplate on bldg. to remove VSD cabinets before lifting skid/housing enclosure. 


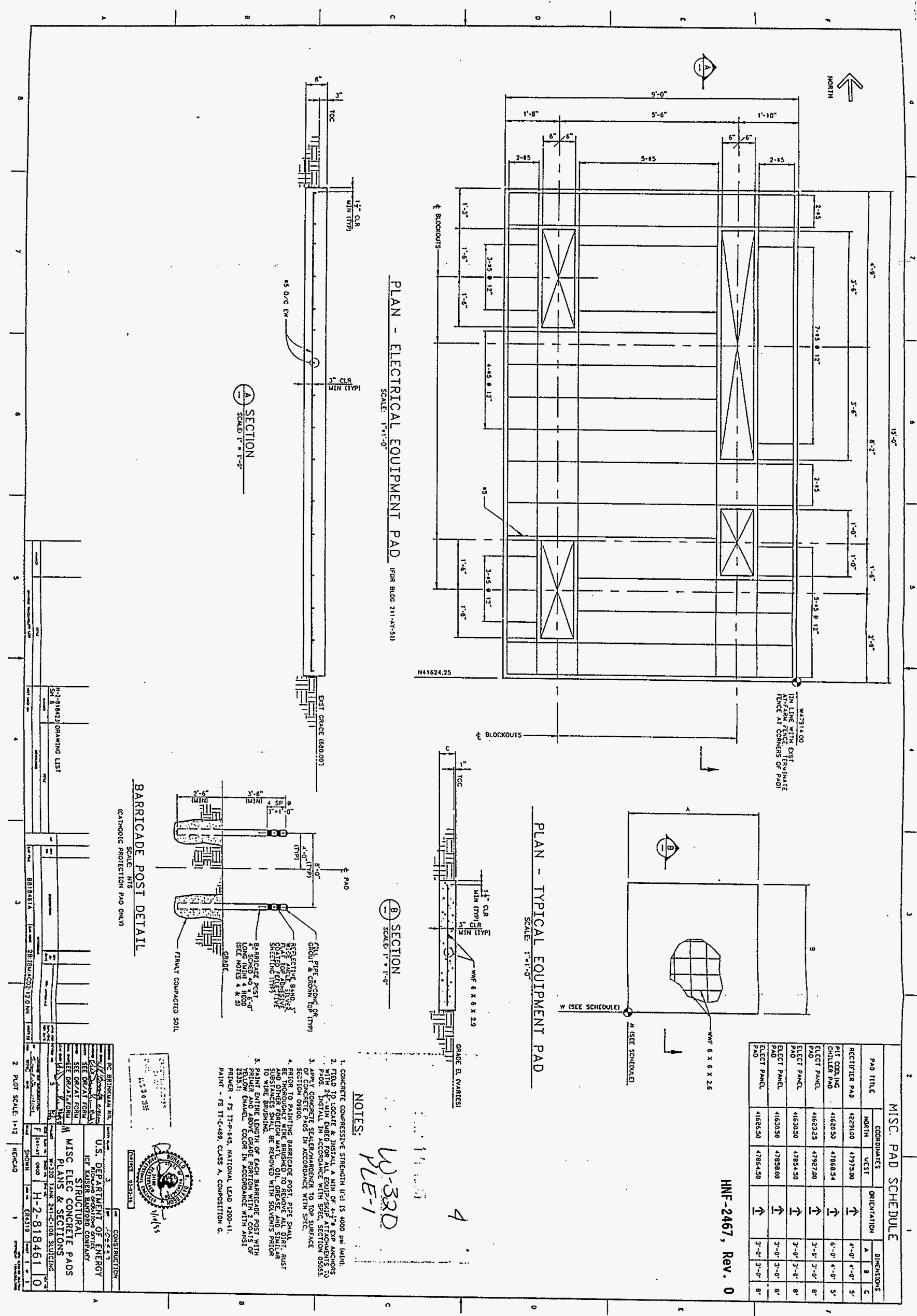


DESIGN JUSTIFICATION CALCULATIONS

REF : KAISER HANEORD SPEC 320 P33 REV 1

INDEX

PAGE CONTENTS

2 WIND LOADING, WIND OVERTURNING

3 WIND SHEAR STRESSES

4 ROOF STRUCTURAL STRESSES \& DEFLECTION

5 FLOOR LOADING, STRESSES

6 SEISMIC LOADS

7 HEAT LOAD CHECK

8 HEIGHT CALCULATION

ATTACH 2. TOTAL OF 8 PAGES

CONCLUSIONS :

1. . STRUCTURE WILL EXCEED THE SPEC REQUIREMENTS

2. HEAT PUMP SYSTEM WILL BE ADEQUATE

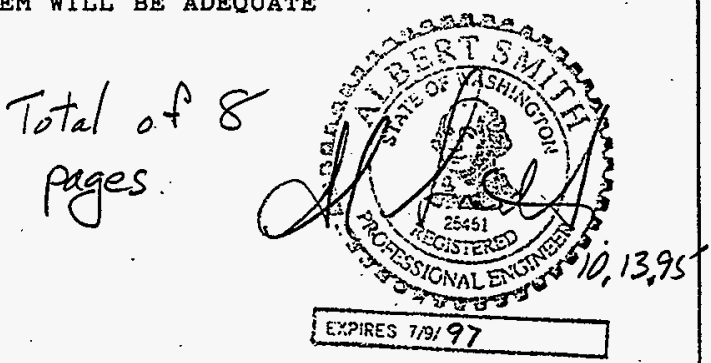


AL SMITH PE

6341 50th Ave Si

2

Seattle, HA 9813

(206) 9378557

HNF-2467, Rev. 0

WIND LOADING

(UBC method)

By examination worst case is full face exposure to 70 mph wind.

$$
\begin{aligned}
& P(\text { pressure psf })=C_{e} C_{q} q_{s} I \\
& C_{e}=1.06(0.15 \text { fl -high }) \\
& C_{q}=.8 \text { worst case } \\
& \text { Stag. pressure } q_{I}=12.6 \text { pst a } 70 \text { mph } \\
& I=1.07 \text { constant }
\end{aligned}
$$

Then $P=11.4$ psf.

$$
\begin{aligned}
& \text { Exposed area }=10.5 \times 14 . \\
&=147 \\
& \mathrm{ft}^{2} . \\
& \text { Total wind load }=147 \times 11.4 . \\
&=1675 \text { tbs. }
\end{aligned}
$$

Wind OVerturning.

$$
\begin{aligned}
& R_{\text {front } 2 \text { corner }}=\frac{1675 \times \frac{6}{2} \times \frac{8}{8}}{628} \\
& \frac{\square}{\uparrow .8^{\prime} b_{R}} \\
& \frac{1675^{\circ} \mathrm{ks} .}{4} \\
& \text { G'assume } \\
& \begin{array}{c}
R=628 \\
\text { ('lissome } \\
\text { eff, } \left.C_{0} f p\right) \therefore R<\frac{\omega t}{4} \quad(\omega t=4000 \mathrm{lbs})
\end{array}
\end{aligned}
$$

Al

Page F-17 
AL. SMITH P E

3

Seattle, HA 98136

(206) 9378557

HNF-2467, Rev. 0

WIND SHEAR.

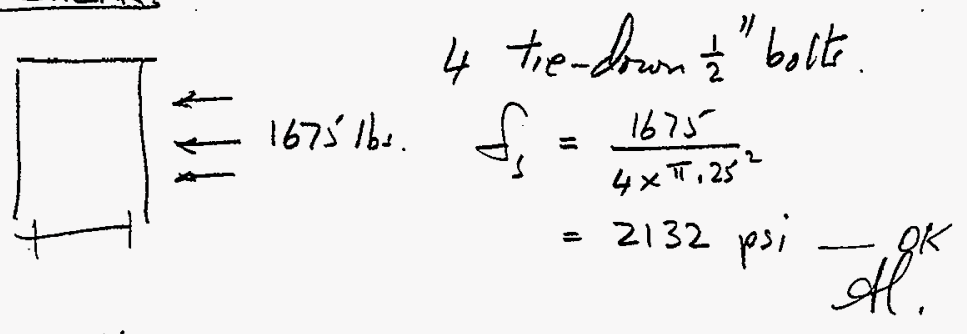

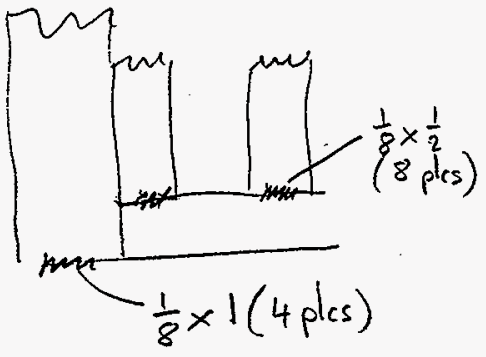

Total weld in shear $\simeq 2 \mathrm{inch}^{2}$

$$
\begin{array}{r}
f_{s}=837 \text { psi }- \text { ok } \\
\text { gl. }
\end{array}
$$

Page F-18 
AL. SMITH P E

6341 50th Ave SY

Seattle, HA 98135

(206)9378557

HNF-2467, Rev. 0

Roof Structure

lecce

LoADing. span $\rightarrow$ $8 \times 14$ Area,

$5016 / A t^{2}+2016 / A t^{2}+1016 / A t^{2}$ live snow aux. $=80 \mathrm{~m} / \mathrm{ft}^{2}$.

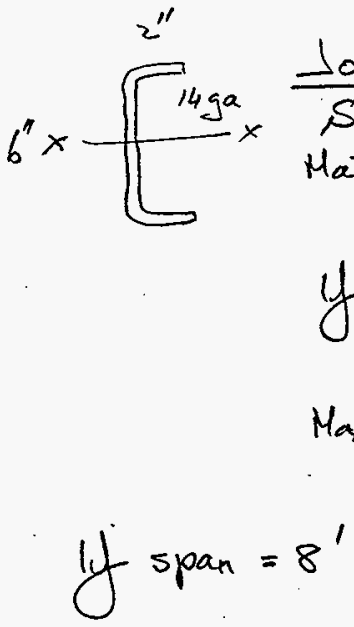

if $S_{.} F_{0} \geqslant 3$.

$f_{\text {allowable }}=12000 \mathrm{psi}$.

Max. B.H. $=f_{a l} \times S$

$=12840 \mathrm{lb} \mathrm{m}$.

$$
\text { If span }=8^{\prime} \quad \begin{aligned}
\text { B.H. } & =8016 / 44^{2} \times \frac{4}{3} \times \frac{8^{2}}{8} \times 12 \quad\left(16^{\prime \prime} 0 . C\right) \\
& \left.=10,24016 \text {.ing. } \quad \frac{0 K}{(10,240}<12,840 .\right)
\end{aligned}
$$

Deflection.

$$
\begin{aligned}
& y_{\text {mat }}=\frac{5 w e^{3}}{384 E I} \\
& \text { E. } 2910^{6} \\
& I \simeq 1.07 \times 3 \\
& W=\omega \ell \text {. } \\
& =\frac{5 \times 80 \times 4}{384 \times \frac{4}{3} \times \frac{8^{4} \times 12^{3}}{29 \times 10^{6} \times 1.07 \times 3}} \\
& =.1056 \mathrm{im} \text {. } \\
& =\frac{1}{900} \times \text { span. - OK } \\
& \left(y_{n_{4}}<\frac{1}{180}\right)
\end{aligned}
$$

Page F-19 
AL. SMIth PE

634150 th Ave 54

Seattle, HA 9813

(205) 9378557

5

HNF-2467, Rev.' 0

Floor LoADING.

Worst case - sling into position.

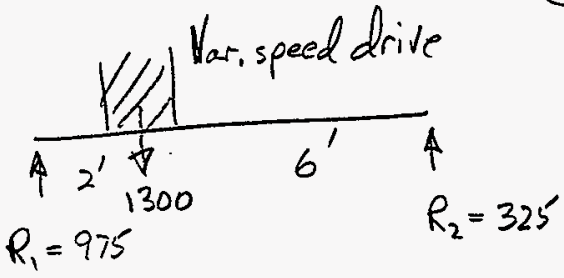

$$
\begin{aligned}
\text { Mat BM } & =975^{\circ} \times 2 \times 12 \\
& =2340016 \text { ins. }
\end{aligned}
$$

Carried on 4 joists.

Section in bending.

$$
6 \times\left[\begin{array}{l}
2 \\
x 14 \mathrm{ga} . \\
2
\end{array}\right.
$$

$$
\begin{aligned}
& S_{x}=1.02 \mathrm{~m}^{3} \\
& \begin{aligned}
f_{b} & =\frac{23400}{4 \times 1.02} \\
& =5735 \mathrm{psi}-\text { ok }
\end{aligned} \\
&
\end{aligned}
$$

lengthwise channel.$$
\begin{array}{ccc}
4 & 14^{\prime} \quad 7^{4} \\
208 & 766
\end{array}
$$

$$
\begin{aligned}
\phi^{975} 3^{\prime} & \left.B H_{\text {max }}\right)=27576 \mathrm{lb} \text {. ins. } \\
\therefore 766 & S_{x}=4.38 \mathrm{in}^{3}(\mathrm{C} 6 \times 8,2) \\
& f_{b}=6295^{\prime} \text { psi OK OK }
\end{aligned}
$$

Page F-20 


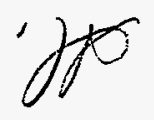

Haר

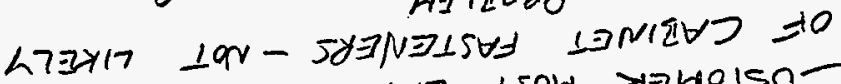

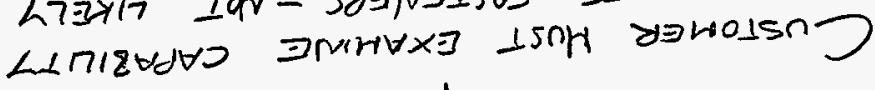

magged rangrus on $\because$

rooys pmin $>$ says onesios

$W_{219 ! B_{1} l^{2 u}}$

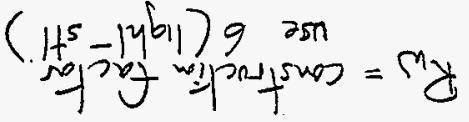

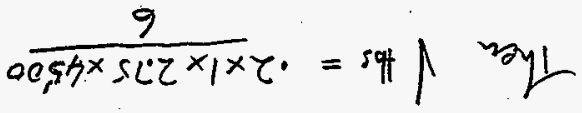

91 oost $\approx$ pool papp $=M$

arpes xam $\mathrm{SL} \cdot \mathrm{C}=\mathrm{J}$

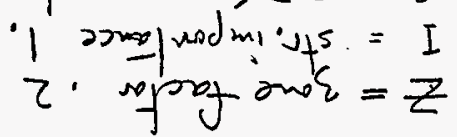

$m \frac{m y}{31 z}=1200+5>\forall 031 \nabla 7$

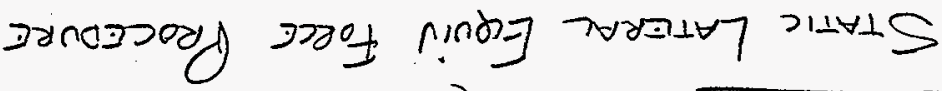

( $8861($ binny.12) $\forall \exists S$ )

jintor ग11513S

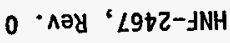

LSSB LE6(90Z)

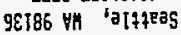


AL SMITH PE

7

Seattle, WA 9813

HNF-2467, Rev. 0

Heat Load.

Check of Customer Spec.

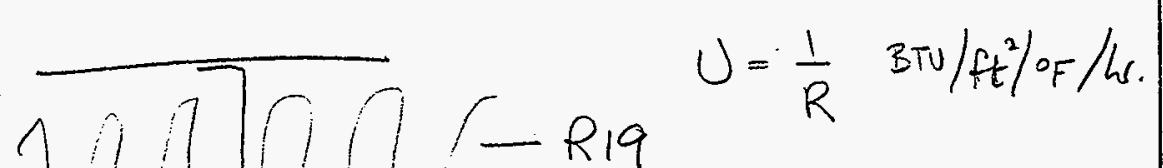

Total Ares $\simeq(8+14+16) \times 10+8 \times 14$ $\cong 500 \mathrm{ft}^{2}$ insulated ural

Therefore. $U=\frac{500}{19}+$ loss thru door + loss thru studs + joist e.

$$
\begin{aligned}
U_{S+1} & =\underbrace{\frac{.12}{6} \times 26}_{\text {cot }} \times \underbrace{\frac{.075}{12}(25 \times 10+24 \times 8)}_{\text {Area of steel webs. }} \text { (no wind) } \\
& =1.44 \text { BTu/ho/ } /{ }^{\circ} \\
\Sigma U & =27.8+\text { door loss } 8 T 0 / \mathrm{hs} /{ }^{\circ} \mathrm{F}
\end{aligned}
$$

Heating

$$
\begin{aligned}
\text { load }= & (50-2) \times\left(27.8+v_{d 1}\right) \\
= & 1332 \text { BTu } / h_{1}+\text { door losses }+ \text { wind } . \\
& (8,000 \text { araikble })
\end{aligned}
$$

Cooling

$$
\begin{aligned}
\text { load } & =(101-85)(27.8+0.01) \\
& =444+8 . T 0 / h \text { (16,000 available) }
\end{aligned}
$$

$\rightarrow$ Performance margin looks oK of l. 
AL SMITH PE

634150 th Ave 5

Seattle, HA 98136

(206) 9378557

8

HNF-2467, Rev. 0

Weight Calculation.

use bill of material - see dug 51001 . sh 1 .

F/A channel $230 \mathrm{lbs}$.

$2-4$ changes. 1370

5 girts. 60

6 floor 974

7 tie-douns 10

11 heat pump 300

$15^{\prime}$ reefing 120

16 watt kit 300

22 liner 375

17 door 150 est.

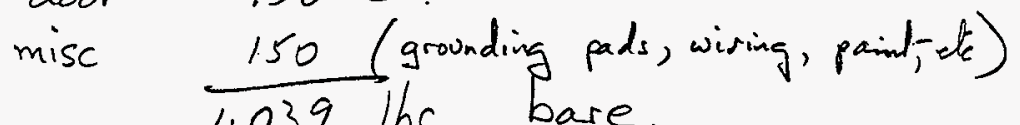
4039 lbs. bare.

Page F-23 


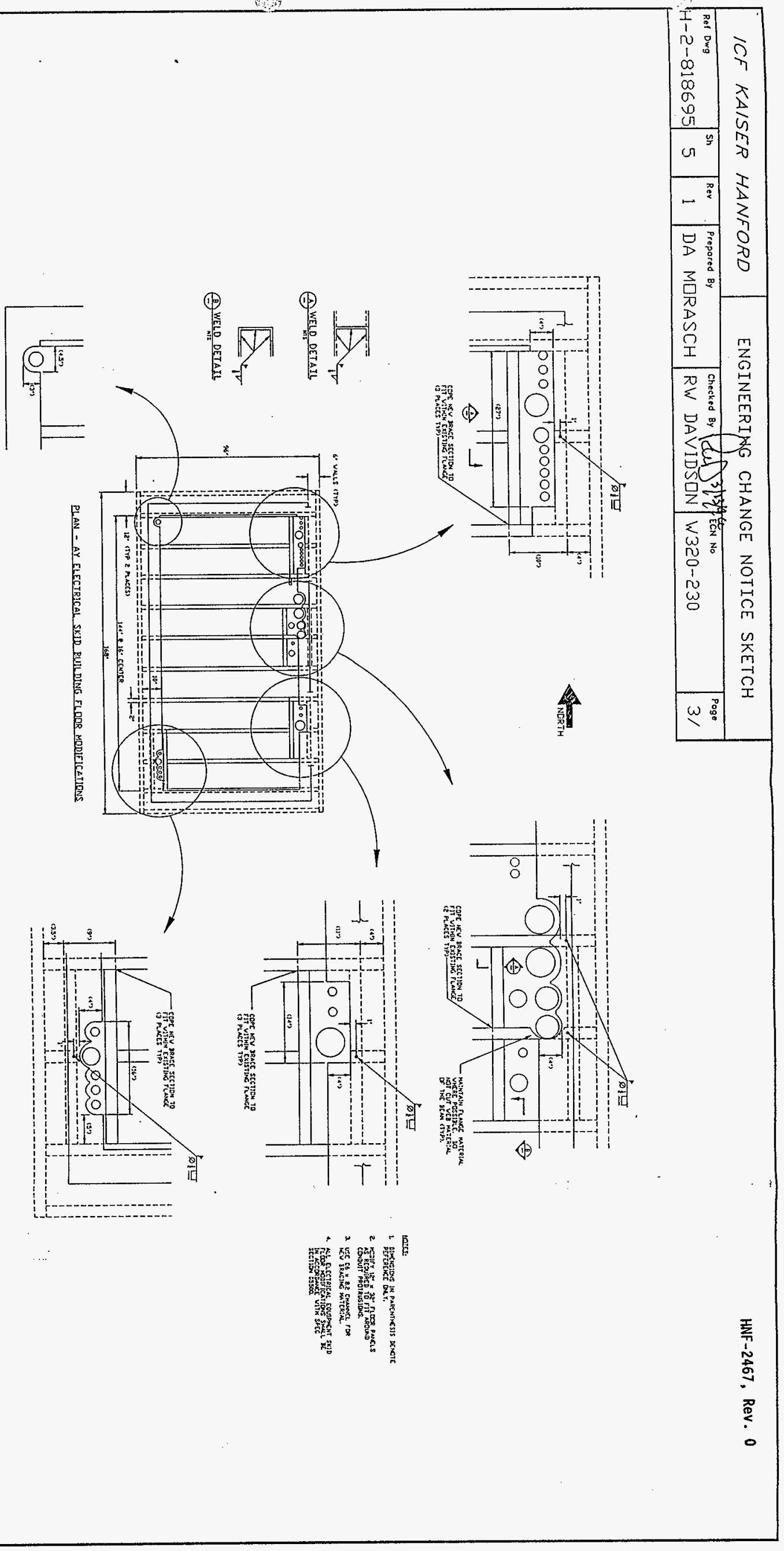

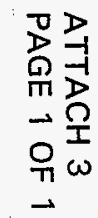




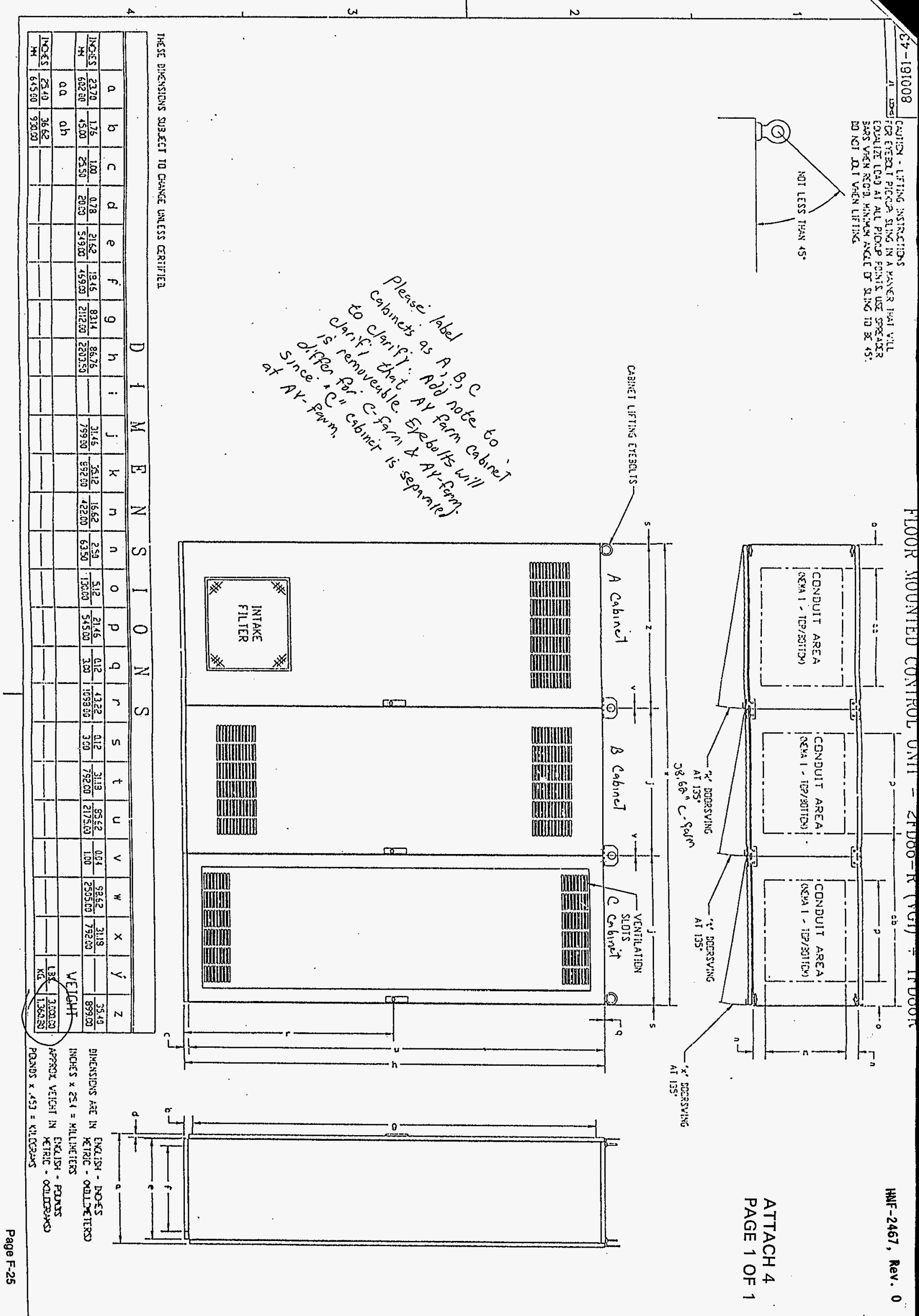




\section{DISTRIBUTION SHEET}

\begin{tabular}{|c|c|c|c|c|c|}
\hline \multirow{2}{*}{$\begin{array}{l}\text { To } \\
\text { Distribution }\end{array}$} & \multirow{2}{*}{\multicolumn{3}{|c|}{$\begin{array}{l}\text { From } \\
\text { JW Bailey }\end{array}$}} & \multicolumn{2}{|c|}{ Page 1 of 1} \\
\hline & & & & \multicolumn{2}{|c|}{ Date 19 July 1998} \\
\hline \multicolumn{4}{|l|}{ Project Title/Work Order } & \multicolumn{2}{|c|}{ EDT No. 622225} \\
\hline \multicolumn{4}{|c|}{$\begin{array}{l}\text { Project W-320 Tank 106-C, HNF-2467, Civil/Structural } \\
\text { Calculations, Vol. } 7\end{array}$} & \multicolumn{2}{|c|}{ ECN No. $n / a$} \\
\hline Name & MSIN & $\begin{array}{c}\text { Text } \\
\text { With All } \\
\text { Attach. }\end{array}$ & Text Only & $\begin{array}{l}\text { Attach./ } \\
\text { Appendix } \\
\text { Only }\end{array}$ & $\begin{array}{l}\text { EDT/ECN } \\
\text { Only. }\end{array}$ \\
\hline $\begin{array}{l}\text { JW Bailey } \\
\text { W-320 Project Files }\end{array}$ & $\begin{array}{l}S 2-48 \\
\text { R1 }-29\end{array}$ & & & & \\
\hline
\end{tabular}

